THIS PAGE INTENTIONALLY LEFT BLANK 
Nevada

Environmental

Restoration

Project

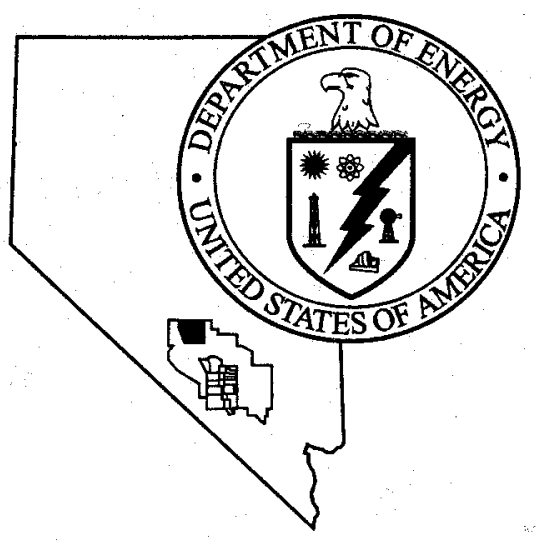

Streamlined Approach for

Environmental Restoration

Closure Report for Corrective

Action Unit 461: Joint Test

Assembly Sites

Tonopah Test Range, Nevada

Controlled Copy No.

Revision: 0

September 1999

Approved for public release; further distribution is authorized

Environmental Restoration

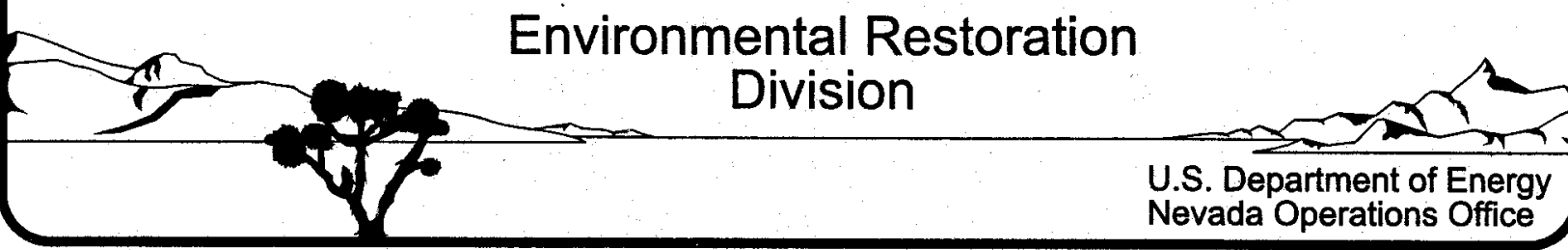


Available to the public from:

U.S. Department of Commerce

National Technical Information Service

5285 Port Royal Road

Springfield, VA, 22161-0002

(703) $487-4650$

Available electronically at http://www.doe.gov/bridge. Available to U.S. Department of Energy and its contractors in paper from:

U.S. Department of Energy

Office of Scientific and Technical Information

P.O. Box 62

Oak Ridge, TN, 37831-0062

(423) 576-8401

Reference herein to any specific commercial product, process, or service by trade name, trademark, manufacturer, or otherwise, does not necessarily constitute or imply its endorsement, recommendation, or favoring by the U.S. Government or any agency thereof or its contractors or subcontractors. 


\title{
STREAMLINED APPROACH FOR ENVIRONMENTAL RESTORATION CLOSURE REPORT FOR CORRECTIVE ACTION UNIT 461: JOINT TEST ASSEMBLY SITES TONOPAH TEST RANGE, NEVADA
}

\author{
Controlled Copy No.
}

Revision: 0

September 1999

Prepared for the U. S. Department of Energy

Nevada Operations Office

Work Performed Under Contract No. DE-AC08-96NV11718 
THIS PAGE INTENTIONALLY LEFT BLANK 


\section{STREAMLINED APPROACH FOR ENVIRONMENTAL RESTORATION CLOSURE REPORT FOR CORRECTIVE ACTION UNIT 461: JOINT TEST ASSEMBLY SITES TONOPAH TEST RANGE, NEVADA}

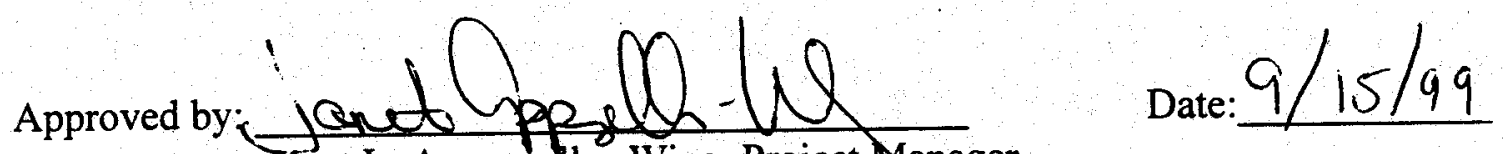
Janet L. Appenzeller-Wing, Project Manager Industrial Sites Project
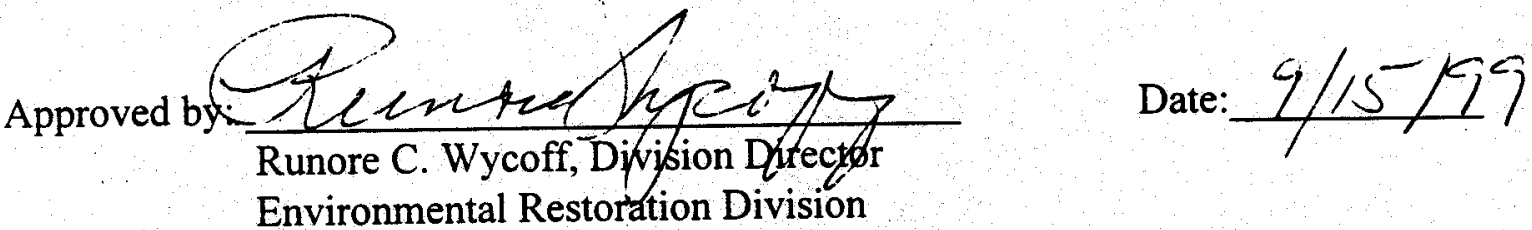
THIS PAGE INTENTIONALLY LEFT BLANK 
ACRONYMS AND ABBREVIATIONS $\ldots \ldots \ldots \ldots \ldots \ldots \ldots \ldots \ldots \ldots$ vii

EXECUTIVE SUMMARY $\ldots \ldots \ldots \ldots \ldots \ldots \ldots \ldots \ldots \ldots \ldots \ldots \ldots \ldots$ ix

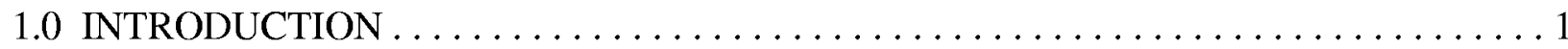

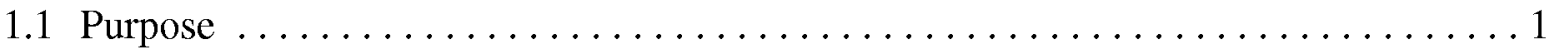

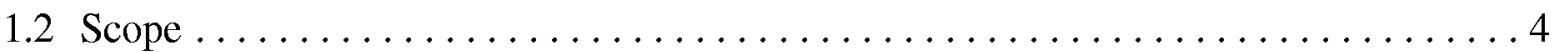

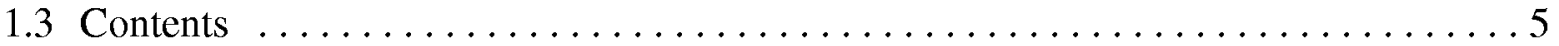

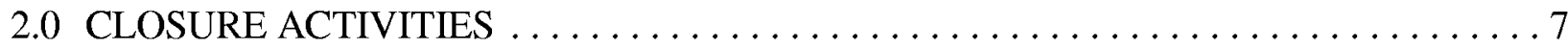

2.1 Description of Corrective Action Activities $\ldots \ldots \ldots \ldots \ldots \ldots \ldots \ldots \ldots \ldots \ldots \ldots \ldots \ldots \ldots \ldots$

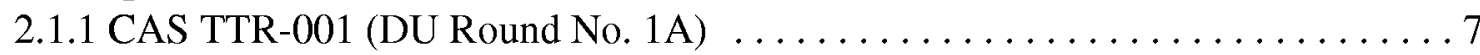

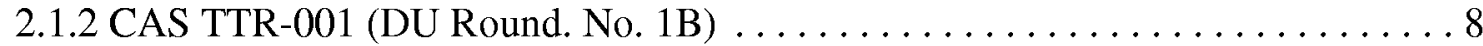

2.1.3 CAS TA-52-003-0960 (DU Artillery Round No. 2) . . . . . . . . . . . . 8

2.1.4 CAS TA-52-002-TAML (DU Impact Site) . . . . . . . . . . . . . . . 9

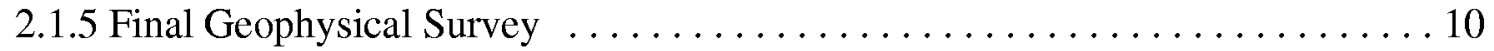

2.2 Deviations from SAFER Closure Plan as Approved $\ldots \ldots \ldots \ldots \ldots \ldots \ldots$

2.3 Corrective Action Schedule as Completed $\ldots \ldots \ldots \ldots \ldots \ldots \ldots \ldots \ldots \ldots \ldots \ldots \ldots \ldots \ldots$

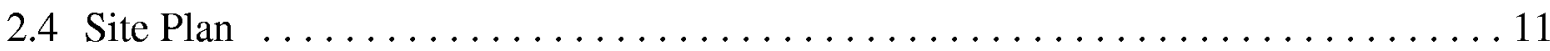

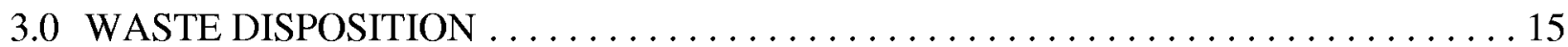

4.0 CLOSURE VERIFICATION RESULTS $\ldots \ldots \ldots \ldots \ldots \ldots \ldots \ldots \ldots \ldots \ldots \ldots$

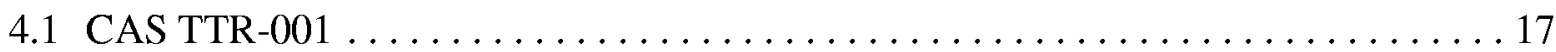

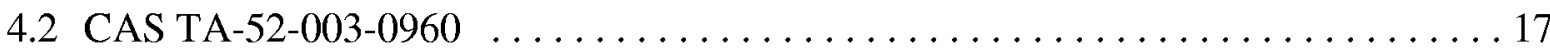

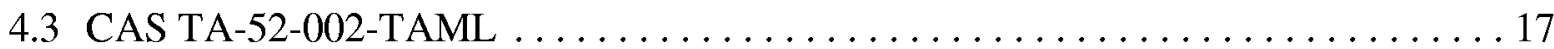

4.4 Land Use Restrictions . . . . . . . . . . . . . . . . . . . . . . . . . . 19

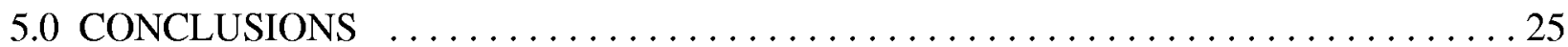

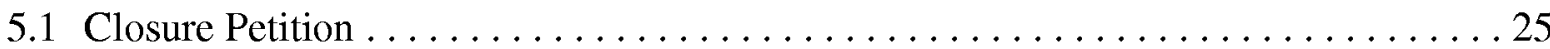

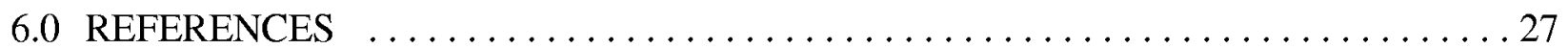

APPENDIX A - RESULTS OF FINAL GEOPHYSICAL SURVEY

APPENDIX B - SURVEY RESULTS

APPENDIX C - BACKFILL COMPACTION TEST RESULTS

APPENDIX D - VERIFICATION SAMPLE RESULTS 


\section{TABLE OF CONTENTS (continued)}

APPENDIX E - RADIOLOGICAL SURVEY RESULTS

APPENDIX F - TECHNICAL CHANGES

APPENDIX G - SHIPPING MANIFESTS

APPENDIX H - NEDEP COMMENT RESPONSE

DISTRIBUTION LIST

\section{FIGURES}

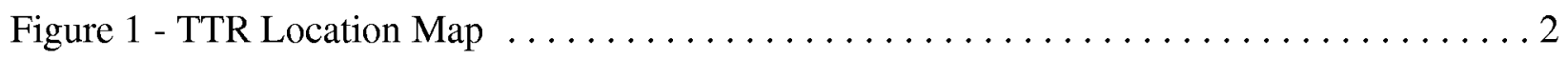

Figure 2 - Location of Corrective Action Unit $461 \ldots \ldots \ldots \ldots \ldots \ldots \ldots \ldots$

Figure 3 - Surface Sample Grid - DU Impact Site $\ldots \ldots \ldots \ldots \ldots \ldots \ldots \ldots \ldots \ldots$

\section{TABLE}

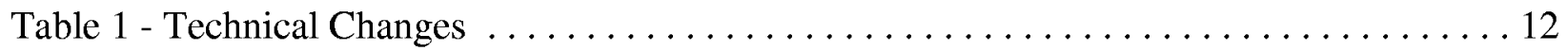

Table 2 - CAU 461 Closure Schedule as Completed $\ldots \ldots \ldots \ldots \ldots \ldots \ldots \ldots \ldots$

Table 3 - DU Round 2 Verification Sample Summary $\ldots \ldots \ldots \ldots \ldots \ldots \ldots$

Table 4 - DU Impact Site Verification Sample Summary $\ldots \ldots \ldots \ldots \ldots \ldots \ldots \ldots \ldots \ldots$ 


\section{ACRONYMS AND ABBREVIATIONS}

\begin{tabular}{|c|c|}
\hline CAS & Corrective Action Site \\
\hline CAU & Corrective Action Unit \\
\hline $\mathrm{cm}$ & centimeter \\
\hline $\mathrm{CR}$ & Closure Report \\
\hline DOE & U.S. Department of Energy \\
\hline DU & Depleted Uranium \\
\hline $\mathrm{EOD}$ & Explosive Ordnance Disposal \\
\hline FFACO & Federal Facility Agreement and Consent Order \\
\hline $\mathrm{ft}$ & feet \\
\hline $\mathrm{HE}$ & High Explosive \\
\hline in & inches \\
\hline JTA & Joint Test Assembly \\
\hline $\mathrm{m}$ & meter \\
\hline $\mathrm{m}^{3}$ & cubic meters \\
\hline NDEP & Nevada Division of Environmental Protection \\
\hline NTS & Nevada Test Site \\
\hline No. & Number \\
\hline RWMS & Radioactive Waste Management Site \\
\hline
\end{tabular}




\section{ACRONYMS AND ABBREVIATIONS (continued)}

SAFER

SNL

TCLP

TTR

$\mathrm{UXO}$

$\mathrm{yd}^{3}$
Streamlined Approach for Environmental Restoration

Sandia National Laboratories

Toxicity Characteristic Leaching Procedure

Tonopah Test Range

Unexploded Ordnance

cubic yards 


\section{EXECUTIVE SUMMARY}

This report addresses the closure of Corrective Action Unit (CAU) 461, Joint Test Assembly (JTA) Sites identified in Appendix III of the Federal Facility Agreement and Consent Order (FFACO). Closure was completed following the approved Streamlined Approach for Environmental Restoration Work Plan. The site is located at the Tonopah Test Range in south central Nevada. CAU 461 consists of three Corrective Action Sites (CASs): CAS Number (No.) TTR-001 (1987 W79 JTA), CAS No. TA-52-003-0960 (Depleted Uranium [DU] Artillery Round No. 2), and CAS No. TA-52002-TAML (DU Impact Site). The 1987 W79 JTA was believed to be the impact site of a failed artillery round test package containing DU and high explosives (HE). Based on review of historical documentation two potential sites were identified. The two sites were designated as DU Round $1 \mathrm{~A}$ and 1B. DU Artillery Round No. 2 was a detonated, buried W79 20-centimeter (cm) (8-inch [in]) diameter DU artillery round located under two witness bars. The DU Impact Site is an area 55 meters (m) by 50 meters $(\mathrm{m})$ (180 feet [ft] by 160 feet [ft] of disturbed ground containing metal fragments and DU impacted soil from a failed weapons test. Historical documentation and fragment examination suggest that during the 1960s, a 150-cm (60-in) diameter weapons test unit incurred parachute failure, impacted, and released DU.

Closure activities began on November 16,1998, with the installation of three-strand barbed wire fence around each site. Waste characterization samples were collected at the DU Impact Site on November 18, 1998. Excavation activities began on November 22, 1998, at CAS TTR-001 (1987 W79 JTA). A backhoe was used to excavate the two areas that were possible locations for the artillery round. Excavation revealed no radiologically impacted soil, ordnance related debris, or HE residue at DU Round 1A. Excavation at DU Round 1B revealed various pieces of metallic debris. This debris was not related to air drop packages or projectiles. No unexploded ordnance, HE residue, or radiological contamination was present on the debris or the surrounding soil.

Closure activities at CAS TA-52-003-0960 (DU Artillery Round No. 2) began on November 30, 1998. Radiologically impacted soil and debris were excavated to a depth of $5.3 \mathrm{~m}(17.5 \mathrm{ft})$ and placed into nine B-25 boxes. Verification samples were collected on December 8, 1998. The analytical results did not detect any constituents of concern in any of the verification samples. A final radiological survey was conducted and the results indicate the site is free of radiological contamination.

Closure activities at CAS TA-52-002-TAML (DU Impact Site) began on November 18, 1998 with the collection of waste characterization samples. Closure activities began on January 13, 1999 and included scraping the top 5-7.6 $\mathrm{cm}(2-3 \mathrm{in})$ of soil and excavating any remaining hot spots. A central hot spot was excavated to $2.7 \mathrm{~m}(9 \mathrm{ft})$. Soil and debris were placed into 35 burrito-lined 15.3 cubic meter $\left(\mathrm{m}^{3}\right)$ (20 cubic yard $\left.\left[\mathrm{yd}^{3}\right]\right)$ side dump trucks. After all impacted soil was removed, verification samples were collected. The analytical results indicate no DU 
remaining. A final radiological survey was conducted and the results indicate the site is free of depleted uranium.

After determining that no impacted soil was present at any of the CASs, the fencing was removed and the excavations were backfilled, compacted to 80 percent, and graded. Each excavation was surveyed to provide locations for future reference.

A final geophysical survey was done. The surveys were completed on March 3 (DU Round 1A) and July 7, 1999, (DU Rounds 1B, DU Round 2, and the DU Impact Site). The geophysical survey results indicated no unaccounted anomalies were present at any of the sites.

Closure for this CAU was completed following the approved Streamlined Approach for Environmental Restoration Work Plan as a clean closure. Therefore, the U.S. Department of Energy, Nevada Operations Office requests that a Notice of Completion be issued by the Nevada Department of Environmental Protection, and the Joint Test Assembly Sites, CAU 461, be moved from Appendix III to Appendix IV of the FFACO "Closed Corrective Action Units." 


\subsection{INTRODUCTION}

This closure report (CR) provides documentation for the closure of Corrective Action Unit (CAU) 461, Joint Test Assembly (JTA) Sites. The CAU is currently listed in Appendix III of the Federal Facility Agreement and Consent Order (FFACO) (Nevada Division of Environmental Protection [NDEP] et. al, 1996). The CAU is located at the Tonopah Test Range (TTR) in south central Nevada (Figure 1). CAU 461 consists of three Corrective Action Sites (CASs), CAS Number (No.) TTR001 (1987 W79 JTA), CAS No. TA-52-003-0960 (Depleted Uranium [DU] Artillery Round No. 2), and CAS No. TA-52-002-TAML (DU Impact Site) (Figure 2).

CAS No. TTR-001 consisted of a potentially unexploded W79 JTA 20-centimeter (cm) (8-inch [in]) diameter test artillery projectile containing high explosives (HE) and DU. Two areas (designated as DU Round No. 1A and 1B) were identified as possible locations for this CAS. DU Round 1A consisted of two low soil mounds and DU Round 1B consisted of a small depression.

CAS No. TA-52-003-0960 consisted of a detonated, buried W79 20-cm (8-in) diameter DU artillery round which is located under two witness bars.

CAS No. TA-52-002-TAML is an area 55 meters $(\mathrm{m})$ by 50 meters $(\mathrm{m})$ (180 feet [ft] by 160 feet [ft] of disturbed ground containing metal fragments and DU with soil from a failed weapons test. Historical documentation and examination of metal fragments suggest that during the $1960 \mathrm{~s}$, a $150-\mathrm{cm}(60-\mathrm{in})$ diameter weapons test unit incurred parachute failure, impacted, and released DU.

\subsection{PURPOSE}

The purpose of this CR is to:

C Document the closure activities as proposed in the Streamlined Approach for Environmental Restoration (SAFER) Closure Plan (U.S. Department of Energy [DOE], 1998a).

C Obtain a Notice of Completion from the NDEP.

C Recommend the movement of CAU 461 from Appendix III to Appendix IV of the FFACO. 


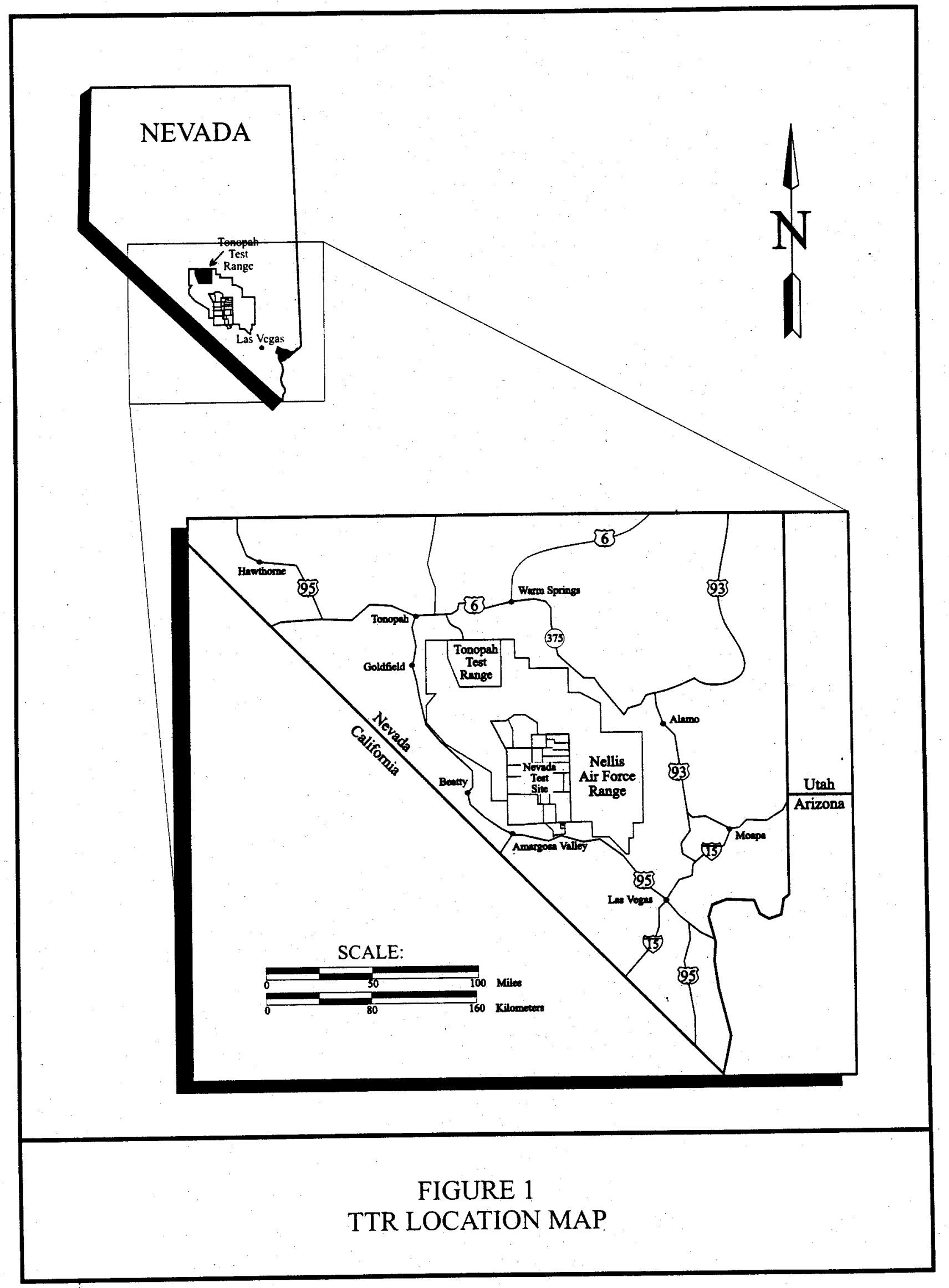




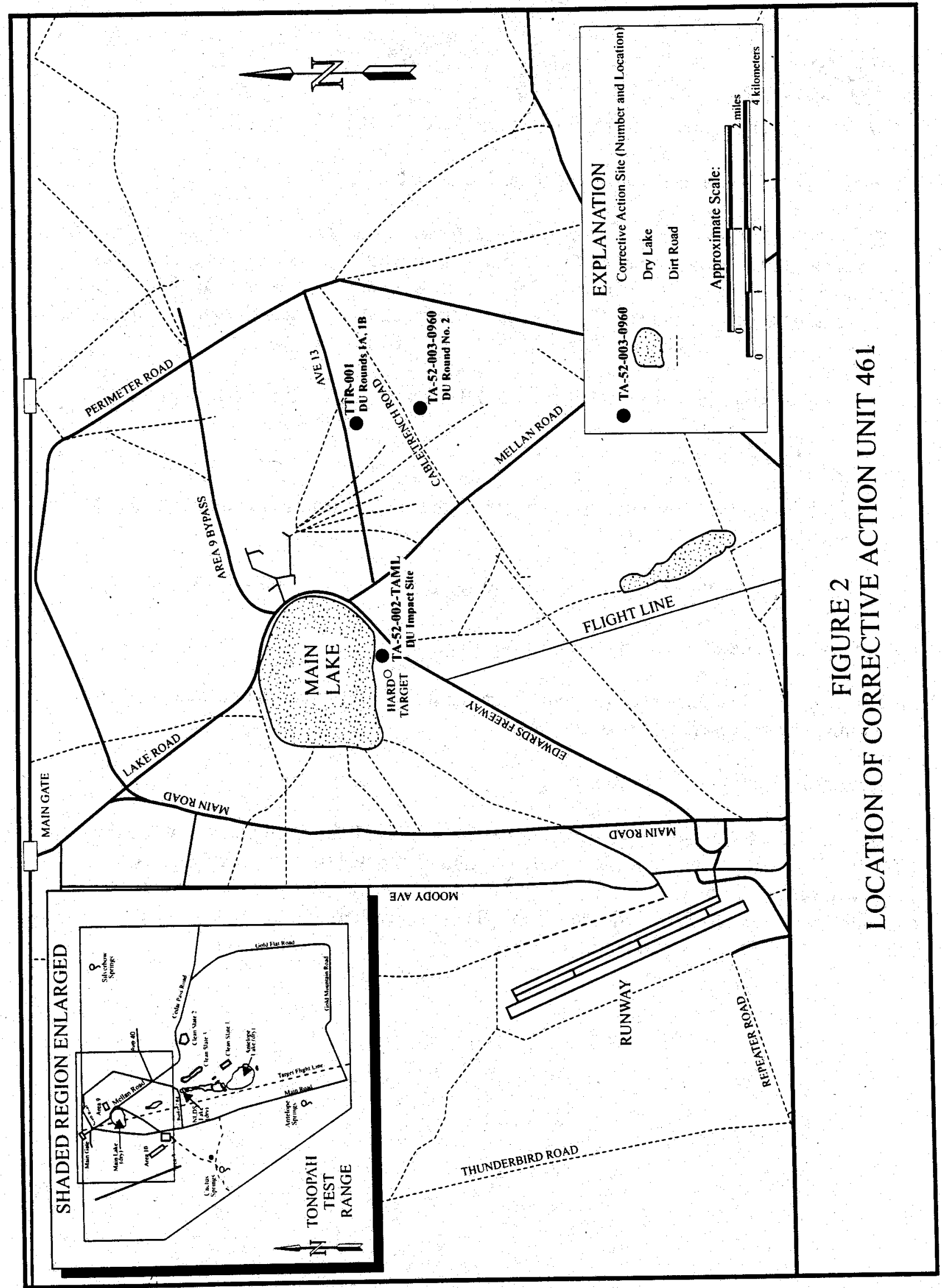




\subsection{SCOPE}

The following is the scope of the closure actions for CAU 461 :

C Install barbed wire fence around the areas to be excavated.

C Excavate identified magnetic anomalies. Each area was excavated in $0.3-\mathrm{m}(1.0-\mathrm{ft})$ lifts and the excavated soil was surveyed for radiological constituents and magnetic anomalies. Excavation activities were stopped if DU rounds were not detected at a depth of $3 \mathrm{~m}(10 \mathrm{ft})$. Excavation at DU Round No. 2 was stopped after all the DU impacted soil was removed. This occurred at a depth of $5.3 \mathrm{~m}(17.5 \mathrm{ft})$ below ground surface.

C Verification soil samples were collected at DU Round No. 2 and the DU Impact site.

C Remove barbed wire fence.

C Backfill the excavations with either the excavated soil or clean soil and compacted the soil to 80 percent.

C Perform a final radiological survey of DU Round No. 2 and DU Impact sites.

C Survey corners and center point of excavations.

C Perform a final geophysical survey on excavations to verify that no unaccounted magnetic anomalies are present at any of the sites.

C Return the site to unrestricted use.

Closure of CAU 461 was completed using the SAFER Work Plan, Revision 0, dated August 1998 (DOE, 1998a). This SAFER closure is based on the results of a geophysical survey (SAIC, 1998) and process knowledge of the Constituents of Concern. Verification samples were collected since the SAFER Work Plan (DOE, 1998a) specified that they are required if artillery rounds or impacted soil was encountered. 


\subsection{CONTENTS}

This CR is divided into the following sections:

C Section 1.0 - Introduction

C Section 2.0-Closure Activities

C Section 3.0 - Waste Disposition

C Section 4.0 - Closure Verification Results

C Section 5.0-Conclusions

C Section 6.0-References 
THIS PAGE INTENTIONALLY LEFT BLANK 


\subsection{CLOSURE ACTIVITIES}

This section of the CR details the specific activities involved in the closure of CAU 461 Joint Test Assembly Sites (CAS No. TTR-001, CAS No. TA-52-003-0960, and CAS No. TA-52-002TAML).

\subsection{DESCRIPTION OF CORRECTIVE ACTION ACTIVITIES}

Before field activities began, the following pre-field activities were completed:

C Preparation of National Environmental Policy Act documentation.

C Preparation of the Site-Specific Health \& Safety Plan (Bechtel Nevada, 1998), Radiological Work Permit and Bioassay Plan.

C Preparation of the Field Management Plan (DOE, 1998b).

C An As Low As Reasonably Achievable Committee review of the proposed activities.

\subsubsection{CAS TTR-001 (DU Round No. 1A)}

Closure activities at CAU 461 began on November 16, 1998, with the installation of a three-strand barbed wire fence around each site. Excavation activities began on November 22, 1998 at CAS No. TTR-001 (DU Round No. 1A). A backhoe was used to excavate the possible artillery round areas that were identified in the geophysics report (SAIC, 1998). A Sandia National Laboratories (SNL) Explosive Ordnance Disposal (EOD) specialist using a hand-held magnetometer was on-site during excavation activities. The EOD specialist was on-site to provide technical assistance in identifying any ordnance-related debris. The excavations were conducted in $0.3-\mathrm{m}$ (1-ft ) lifts. Radiological Control Technicians ran radiological instrument surveys on the excavated soil and inside the excavation prior to excavation of each subsequent lift. All personnel not directly involved with excavation activities were located at a safe distance of $914 \mathrm{~m}(3,000 \mathrm{ft})$ away from excavation activities.

Excavation proceeded until a depth of $3 \mathrm{~m}(10 \mathrm{ft})$ was reached. The approved SAFER Work Plan (DOE, 1998a) specifies a depth of $3 \mathrm{~m}(10 \mathrm{ft})$ as a hold/decision point. If no elevated radiologic or magnetometer readings are detected then excavation activities are stopped. During the DU Round No. 1A site excavation activities radiologically impacted soil or ordnance-related debris was not found at either of the two soil mounds. The hand-held magnetometer did not detect any magnetic anomalies in the excavation below the 3-m (10-ft) depth. However, the final geophysical survey (Appendix A) indicated an anomaly at DU Round 1A South. The site was revisited on April 20-21, 1999 and the area of the anomaly was reexcavated. The area was 
excavated to a depth of $1.7 \mathrm{~m}(5.5 \mathrm{ft})$. No artillery round, debris, or radiologically impacted soil was observed. A lens of iron-bearing gravel was located by the geophysical instruments which corresponded with the anomaly identified at the surface. Instrument sweeps indicated no anomalies below this level. Based on this information, it was determined that an artillery round did not exist at this site.

After it was determined that an artillery round was not present at this site, the barbed wire fence was removed and the excavation was backfilled, compacted to 80 percent, and graded. The site was then staked and surveyed (Appendix B). Backfill compaction test results are provided in Appendix C.

\subsubsection{CAS TTR-001 (DU Round No. 1B)}

Excavation activities began at CAS No. TTR-001 (DU Round No. 1B) on November 23, 1998, using the same procedures used at DU Round No. 1A. Various metallic objects were uncovered at a depth

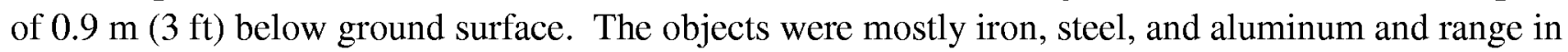
size from $5 \mathrm{~cm}(2 \mathrm{in})$ to $0.5 \mathrm{~m}(1.5 \mathrm{ft})$. The SNL EOD specialist identified the debris as rocket parts, not air-drop packages or projectiles (DU artillery rounds). Most of the debris was subjected to a highorder detonation. There was no unexploded ordnance (UXO) associated with any of this debris and an instrument and swipe survey indicated that radiological contamination was not present. The surrounding soil was also free from radiological contamination and HE residue based on instrument surveys and visual observations, respectively.

Excavation activities were stopped at a depth of $1.5 \mathrm{~m}(5 \mathrm{ft})$ when no more debris was uncovered. The hand-held magnetometer confirmed that no additional magnetic anomalies were located below this depth. This was later confirmed during the final geophysical survey (Appendix A). Based on the large variety of debris and lack of HE residue and radiological contamination, it is unlikely that a DU round was detonated here. This was probably an area where debris from other demilitarization activities and scrap items were disposed. Based on this information, it was determined that an artillery round did not exist at this site.

After it was determined that artillery rounds were not present at this site, the barbed wire fence was removed and the excavation was backfilled, compacted to 80 percent, and graded. The site was then staked and surveyed (Appendix B). Backfill compaction test results are provided in Appendix C.

\subsubsection{CAS TA-52-003-0960 (DU Round No. 2)}

Excavation activities began at DU Round 2 on November 30, 1998 using the same procedures as DU Round 1. At this site, radiologically impacted soil, including pieces of oxidized DU, were uncovered from approximately $0.3 \mathrm{~m}(1 \mathrm{ft})$ below ground surface to the final excavation depth of 
$5.3 \mathrm{~m}(17.5 \mathrm{ft})$. Several small pieces of shell casing were also recovered. The EOD specialist indicated they had been subjected to a high order detonation consistent with what would typically be done during demilitarization activities. In addition, the witness bars also showed evidence of detonation. The bottom $1.5 \mathrm{~m}(5 \mathrm{ft})$ of the witness bars were bent, twisted, and embedded with DU. There was no evidence of UXO or HE residue in the excavation.

All of the radiologically impacted soil, debris, and compactable waste was placed into nine B-25 Boxes for disposal in the Nevada Test Site (NTS) Area 5 Radioactive Waste Management Site (RWMS).

Because radiologically impacted soil and debris was present at this site, verification soil samples were collected as specified in Section 3.4.1 of the approved SAFER Work Plan. See Section 4.0 Closure Verification Results for details on sampling activities.

Once verification samples were collected, the excavation was backfilled, compacted to 80 percent, and graded. Backfilling was done prior to receiving sample results because the site was sufficiently over excavated so that there was no visually or instrument detected DU remaining. The site was then staked and surveyed (Appendix B). Backfill compaction test results are found in Appendix C. A final radiological survey was conducted on March 17, 1999. The results showed that the site was free of radiological contamination, and the site signs and fencing were removed. Results of the radiological survey are found in Appendix E.

\subsubsection{CAS TA-052-002-TAML}

Closure activities began at the DU Impact site (TAML Site) on November 18, 1998 with the collection of waste characterization samples. Eight soil samples (plus one duplicate) were collected from radiological hot spots identified during pre-characterization field activities. The purpose of these samples was to characterize and create a waste profile for disposal purposes. Sample results are found in Appendix D.

Soil removal activities began on January 13, 1999. Activities included the scraping of approximately 5$7.6 \mathrm{~cm}$ (2-3 in) of surface soil. All impacted soil was loaded into burrito-lined 15.3 cubic meter $\left(\mathrm{m}^{3}\right)$ (20 cubic yard $\left.\left[\mathrm{yd}^{3}\right]\right)$ side dump trucks. After the area was scraped, a radiological survey was done to identify any remaining hot spots. Sixteen hot spots were identified and 15 were overexcavated.

A hot spot in the center of the site was more extensive than anticipated. Additional investigation revealed that the extent and volume of impacted soil was much greater than anticipated. At that point work was placed on hold and the closure methodology revised.

Work resumed on June 14, 1999. The center hot spot was excavated to a total depth of $2.7 \mathrm{~m}(9 \mathrm{ft})$ and all the radiologically impacted soil removed. After all impacted soil was excavated, a final surface scraping was done. About $10 \mathrm{~cm}$ (4 in) of soil was removed during this scraping. 
Three small hot spots were identified and excavated. Surveys with a metal detector and radiological instrumentation revealed no significant debris fields, depleted uranium, or ordnance-size debris. The EOD specialist indicated that there is no UXO on the site.

All soil was loaded into burrito-lined side-dump trucks and transported to the NTS Area 3 RWMS.

Because radiologically impacted soil and debris was present at this site, verification soil samples were collected as specified in Section 3.4.1 of the approved SAFER Work Plan. See Section 4.0 Closure Verification Results for details on sampling activities.

Once verification samples were collected, the final radiological survey was done. Results showed the site to be free of radiological contamination and the site signs and fencing was removed. Then the excavation was backfilled with clean backfill material, compacted to 80 percent, and graded.

The site was also surveyed (Appendix B). Backfill compaction test results are found in Appendix C. Results of the radiological survey are found in Appendix E.

\subsubsection{Final Geophysical Survey}

Once the excavations were backfilled and surveyed, the final geophysical surveys using a Geometrics G858 cesium vapor magnetometer configured as a gradiometer were conducted by a geophysicist. The final geophysical survey for DU Round 1A was conducted on March 3, 1999 and the final geophysical surveys for DU Round 1B, DU Round 2, and the DU Impact site were conducted on July 7,1999 . This survey provided more accurate and precise data than could be obtained with a handheld magnetometer. The results indicate that ordnance-related magnetic anomalies do not occur below the excavated areas. Results of the geophysical survey are found in Appendix A. 


\subsection{DEVIATIONS FROM SAFER CLOSURE PLAN AS APPROVED}

The approved SAFER Work Plan (DOE, 1998a) was modified in the field to adjust to unexpected conditions, simplify activities, or increase worker safety. Eight technical changes to the work plan were approved (Table 1). The technical change notices are found in Appendix F.

\subsection{CORRECTIVE ACTION SCHEDULE AS COMPLETED}

The corrective action activities for CAS TTR-001 and CAS TA-52-003-0960 were completed in a timely manner. Excavation activities for CAS TTR-001 began on November 22, 1998, and were completed on November 24, 1998. Excavation activities for CAS TA-52-003-0960 began on December 1, 1998 and were completed on December 4, 1998. Backfilling the excavations and fence removal was completed by April 22, 1999. The final geophysical surveys were completed on July 7 , 1999.

Corrective action activities for CAS TA-52-002-TAML began on November 18, 1998 and were completed on July 1, 1998. The major impact to the original corrective action schedule was the increased volume of impacted soil that required excavation and disposal. The volume of soil requiring excavation and disposal increased from $145 \mathrm{~m}^{3}\left(190 \mathrm{yd}^{3}\right)$ to $573 \mathrm{~m}^{3}\left(750 \mathrm{yd}^{3}\right)$.

A detailed schedule as completed for the corrective action is provided in Table 2.

\subsection{SITE PLAN}

CAS TTR-001 and CAS TA-52-003-0960 were surveyed on December 14, 1998. CAS TA-52002-TAML was surveyed on July 7, 1999. The corners of each excavation were surveyed for location and elevation. Survey data are found in Appendix B. 
TABLE 1 - TECHNICAL CHANGES

\begin{tabular}{|c|c|c|}
\hline CHANGE & REASON & IMPACT ON CLOSURE \\
\hline Excavation Sampling at DU Impact Site & $\begin{array}{l}\text { Work Plan did not address large excavation } \\
\text { sampling at DU Impact site }\end{array}$ & Confirm all DU impacted soil was removed \\
\hline $\begin{array}{l}\text { Subsurface excavation activities confined to upper } 3 \mathrm{~m} \\
(10 \mathrm{ft}) \text { rather than upper } 0.61 \mathrm{~m}(2 \mathrm{ft})\end{array}$ & Account for large subsurface excavation & Confirm all DU impacted soil was removed \\
\hline Soil was loaded into trailers using a conveyor system & Easier and safer to use a front end loader & Expedited closure activities \\
\hline Additional $420 \mathrm{~m}^{3}\left(550 \mathrm{yd}^{3}\right)$ of waste generated & $\begin{array}{l}\text { Additional soil discovered during excavation } \\
\text { activities }\end{array}$ & $\begin{array}{l}\text { Delay in closure schedule due to increased } \\
\text { volume of soil }\end{array}$ \\
\hline $\begin{array}{l}\text { Hot spot excavation can be conducted using shovel as } \\
\text { well as backhoe. Hot spot soil placed into burrito-lined } \\
\text { trailers instead of B- } 25 \text { boxes }\end{array}$ & $\begin{array}{l}\text { Some hot spots were too small to use a backhoe. } \\
\text { B- } 25 \text { boxes not necessary since trailers are already } \\
\text { in place. }\end{array}$ & None \\
\hline $\begin{array}{l}\text { Non-impacted stockpiled soil not placed on plastic or } \\
\text { covered with plastic and sprayed with water to prevent } \\
\text { wind erosion }\end{array}$ & $\begin{array}{l}\text { Not necessary to cover or place non-impacted soil } \\
\text { on plastic }\end{array}$ & None \\
\hline $\begin{array}{l}\text { Excavation activities at the DU Impact Site will follow } \\
\text { Section } 3.1 \text { of the Work Plan. Impacted soil will be } \\
\text { placed on plastic and sprayed with water to prevent } \\
\text { wind erosion }\end{array}$ & $\begin{array}{l}\text { Account for large subsurface excavation at the DU } \\
\text { Impact Site. DU was determined not to be an } \\
\text { airborne hazard based on the continuous air } \\
\text { monitoring filter data collected during the } \\
\text { excavation activities. In addition, no elevated DU } \\
\text { levels were detected in any on-site personnel's } \\
\text { breathing zone air sampler filter or nasal swipe. } \\
\text { Plastic cover would create additional waste and } \\
\text { possible personnel exposure during cover } \\
\text { maintenance. }\end{array}$ & $\begin{array}{l}\text { Reduction in waste volume and personnel } \\
\text { exposure. }\end{array}$ \\
\hline $\begin{array}{l}\text { Use front end loader instead of a backhoe to excavate } \\
\text { DU Impact Site }\end{array}$ & $\begin{array}{l}\text { Front end loader is faster and safer than a } \\
\text { backhoe. }\end{array}$ & Expedited closure schedule \\
\hline
\end{tabular}




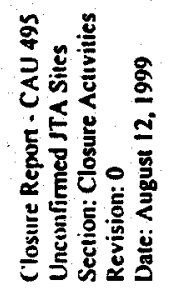

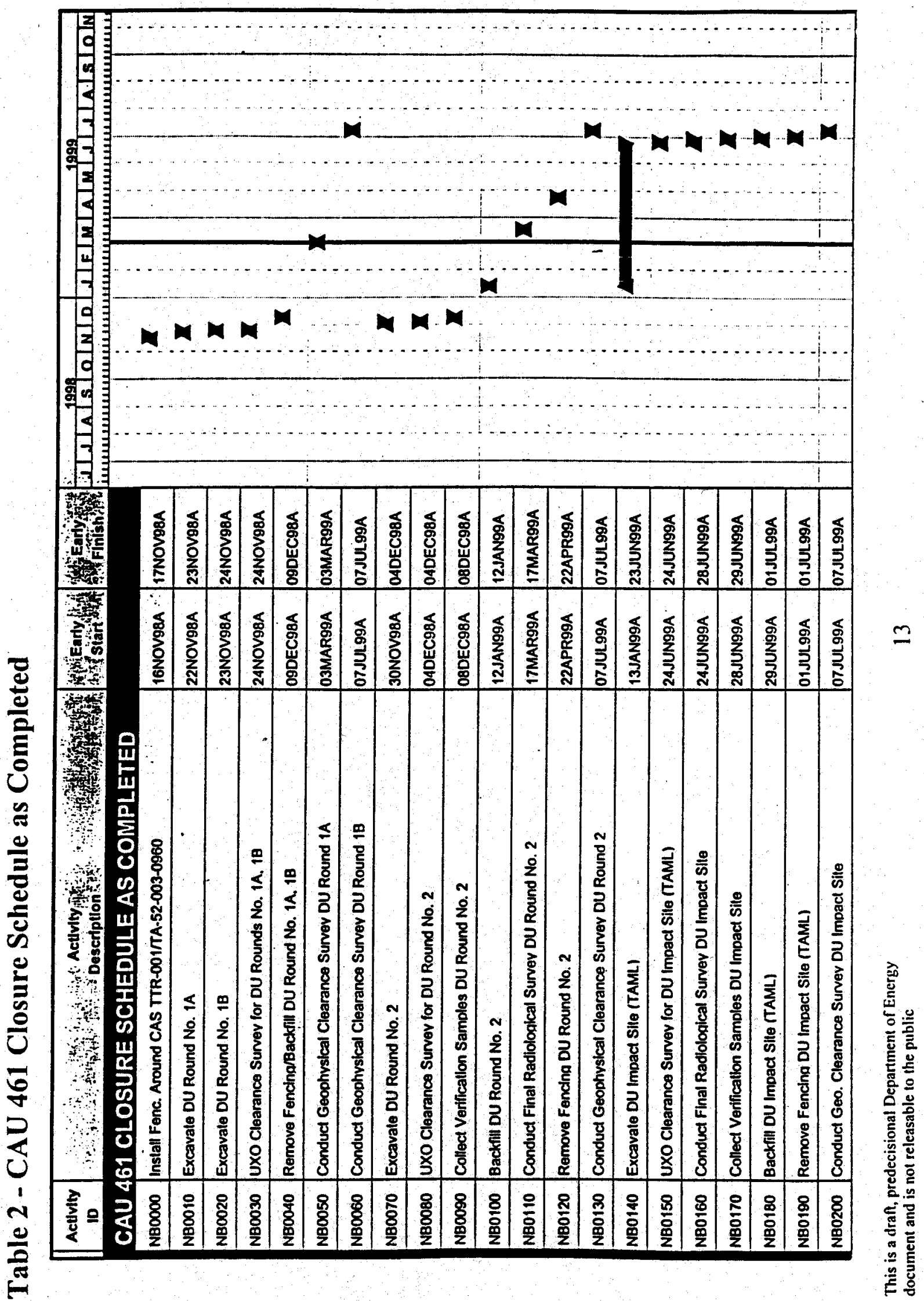




\section{THIS PAGE INTENTIONALLY LEFT BLANK}




\subsection{WASTE DISPOSITION}

No artillery rounds were discovered in the excavations at DU Rounds 1A and 1B. Therefore, radioactive or hazardous waste was not generated from closure activities at this CAS. Depleted uranium and radiologically impacted soil and debris were identified and excavated at both DU Round 2 and the DU Impact Site. Radioactive waste and compactable trash from DU Round 2 was placed into nine B-25 boxes and disposed at the NTS Area 5 RWMS. The radioactive waste and compactable trash from the DU Impact site was placed into side-dump trucks lined with $15.3 \mathrm{~m}^{3}\left(20 \mathrm{yd}^{3}\right)$ burrito bags. The intact bags were disposed at the NTS Area 3 RWMS. A total of 35 truck loads (approximately $561 \mathrm{~m}^{3}\left[734 \mathrm{yd}^{3}\right]$ ) were disposed. Copies of the shipping manifests are provided in Appendix G. Reusable personal protective equipment was sent to the NTS Area 6 Decontamination facility for laundering. Sanitary waste was properly disposed in the TTR Sanitary Landfill. 


\section{THIS PAGE INTENTIONALLY LEFT BLANK}




\subsection{CLOSURE VERIFICATION RESULTS}

This section of the CR details the specific activities involved in the verification of closure of CAU 461 Joint Test Assembly Sites (CAS No. TTR-001, CAS No. TA-52-003-0960, and CAS No. TA-52002-TAML).

\subsection{CAS TTR-001}

CAS TTR-001 (DU Rounds 1A and 1B) was closed by verifying that artillery rounds were not present in any excavation. As specified in Section 3.4.1 of the approved SAFER Work Plan, verification sampling was not required if artillery rounds or impacted soil was not present. Instrument surveys indicated that no radiological contamination was present in the excavation or in the excavated soil. Swipe and instrument survey results indicate no radiological contamination on the metal debris from DU Round No. 1B. There was no evidence of explosive detonations or EOD activities. Geophysical surveys using hand-held instrumentation indicated no anomalies below the bottom of the excavations. A more extensive geophysical survey, using a Geometrics G858 cesium vapor magnetometer, also indicated no anomalies below the excavated areas. Appendix A provides the results of the geophysical survey.

\subsection{CAS TA-52-003-0960}

CAS TA-52-003-0960 (DU Round 2) was closed by excavating radiologically impacted soil and debris. Since impacted soil was present, soil verification samples were collected. One sample was collected from each of the four walls and bottom of the excavation. A total of seven samples were collected including one duplicate and one equipment blank. The samples were analyzed for TCLP metals, TCLP semi-volatile, nitroaromatics and nitroamines, and 20 minute gamma scan.

The results of the sample analysis indicate that there are no detectable levels of analyzed constituents present in the soil (Table 3). Laboratory data reports are found in Appendix D.

\subsection{CAS TA-52-002-TAML}

CAS TA-52-002-TAML (DU Impact Site) was also closed by excavating radiologically impacted soil and debris. Verification samples were collected after all the impacted soil had been removed. Since DU was present on the surface prior to clean up activities, samples were collected from the surface as well as from the excavation. 
TABLE 3 - DU ROUND 2 VERIFICATION SAMPLE SUMMARY

\begin{tabular}{|c|c|c|c|c|}
\hline SAMPLE NUMBER & $\begin{array}{c}\text { TCLP SVOC } \\
(\mathrm{mg} / \mathrm{L})\end{array}$ & $\begin{array}{c}\text { TCLP METALS } \\
(\mathrm{mg} / \mathrm{L})\end{array}$ & $\begin{array}{c}\text { NITROAROMATICS/ } \\
\text { NITROAMINES } \\
(\mathrm{mg} / \mathrm{Kg})\end{array}$ & $\begin{array}{c}\text { GAMMA }^{\mathrm{D}} \\
(\mathrm{U} 238) \\
(\mathrm{pCi} / \mathrm{g}) \\
\end{array}$ \\
\hline DU RD \#2-1 & $\mathrm{ND}^{\mathrm{E}}$ & ND & ND & $<26$ \\
\hline DU RD \#2-2 & ND & ND & ND & $<22$ \\
\hline DU RD \#2-3 & ND & ND & ND & $<22$ \\
\hline DU RD \#2-4 & ND & ND & $\mathrm{ND}$ & $<23$ \\
\hline DU RD \#2-5 & ND & ND & ND & $<25$ \\
\hline DU RD \#2-6 & ND & $\mathrm{ND}$ & $\mathrm{ND}$ & $<27$ \\
\hline
\end{tabular}

\section{NOTES}

A - TCLP SVOCs: TCLP Semi-Volatile Organic Compounds analyzed using EPA Method 8270C.

Extraction by Method 1311. (EPA, 1996)

B - TCLP Metals: Metals analyzed using EPA Methods 6010 and 7470A. Extraction by Method 1311. (EPA, 1996)

C - Nitroaromatics/Nitroamines analyzed using EPA Method 8330. (EPA, 1996)

D - 20-minute gamma spectroscopy for U238. Values are Minimum Detectable Activities (MDA). Results are less than MDAs.

E - ND: Not detected above laboratory detection limits. See Appendix D for Laboratory Reports.

( $\mathrm{mg} / \mathrm{L}$ or $\mathrm{mg} / \mathrm{Kg}$ or $\mathrm{pCi} / \mathrm{g}$ ): Milligrams per liter (for TCLP extraction), Milligrams per kilogram (for soil), Picocuries per gram for gamma spectroscopy. 
A total of eleven soil samples (including one duplicate) were collected from the excavation. Each of the four sides and the bottom of the excavation was divided into nine sections. Two of the sections were then randomly selected using a random number generator for sampling.

The surface verification samples were selected by dividing the entire site into 233, $3 \mathrm{~m} \times 3 \mathrm{~m}$ (10 ft x 10 $\mathrm{ft}$ ) grids. Sixteen of the grids were randomly selected and sampled (Figure 3). A total of 18 samples were collected including one duplicate and one equipment blank. The samples were analyzed for TCLP metals, TCLP semi-volatile, nitroaromatics and nitroamines, and 300 minute gamma scan.

The sample results indicate that there are no hazardous or radiological constituents present in the excavation or on the surface (Table 4). Laboratory data reports are found in Appendix D.

\subsection{LAND-USE RESTRICTIONS}

A clean closure was performed at all three CASs. Land use is unrestricted. A Post-Closure Plan is not necessary for this site. 


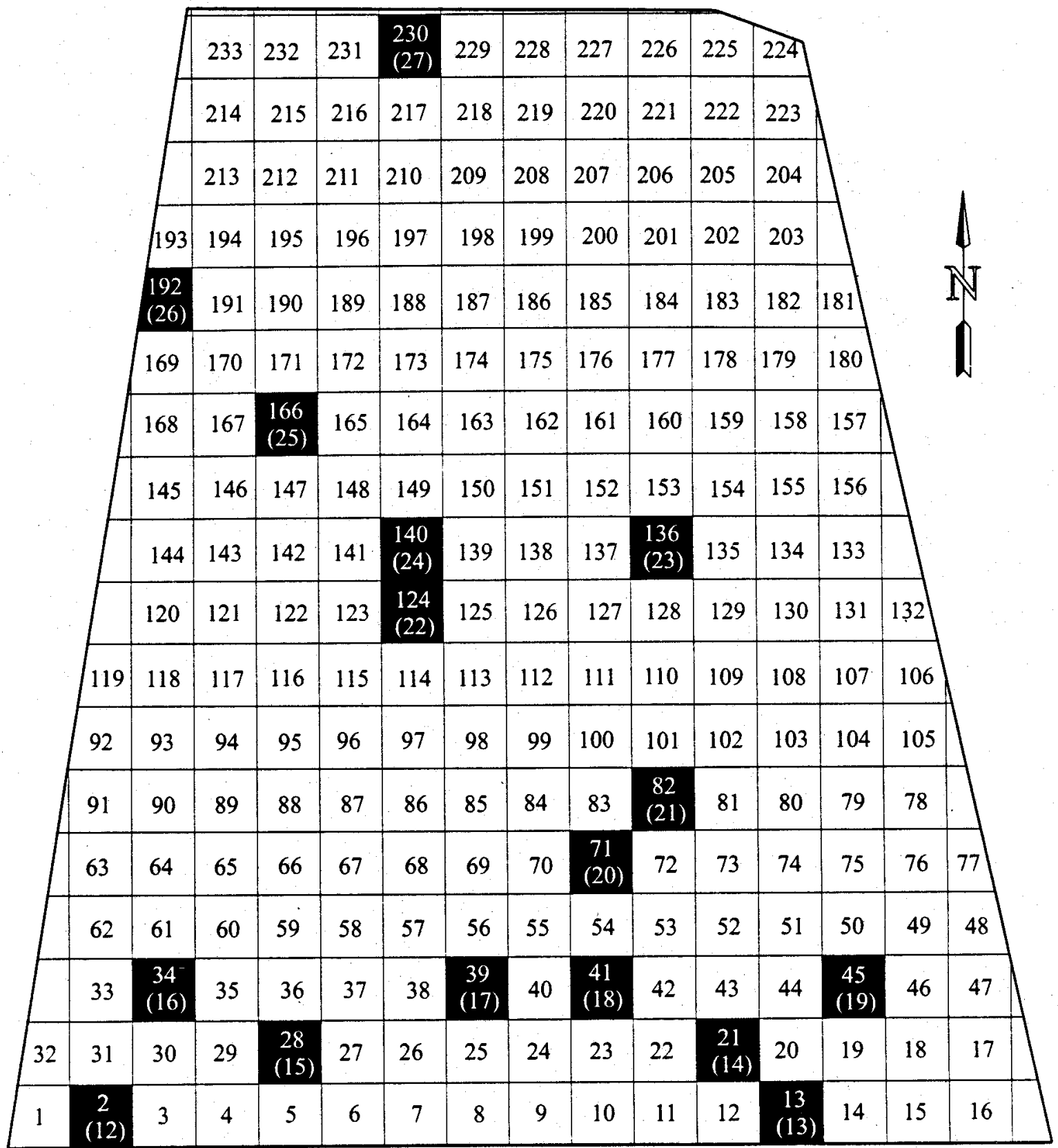

SCALE:

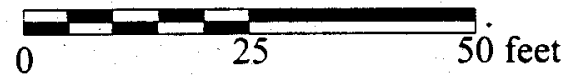

Numbers in parentheses are sample numbers

FIGURE 3

Surface Sample Grid - DU Impact Site 
TABLE 4 - DU IMPACT SITE VERIFICATION SAMPLE SUMMARY

\begin{tabular}{|c|c|c|c|c|}
\hline SAMPLE NUMBER & $\begin{array}{c}\text { TCLP SVOC } \\
(\mathrm{mg} / \mathrm{L})\end{array}$ & $\begin{array}{l}\text { TCLP METALSB } \\
(\mathrm{mg} / \mathrm{L})\end{array}$ & $\begin{array}{c}\text { NITROAROMATICS/ } \\
\text { NITROAMINES } \\
(\mathbf{m g} / \mathrm{Kg})\end{array}$ & $\begin{array}{c}\text { GAMMA }^{\mathrm{D}} \\
(\mathrm{U} 238) \\
(\mathrm{pCi} / \mathrm{g}) \\
\end{array}$ \\
\hline TAML-VER-01 & ND & Chromium 0.071 & ND & ND \\
\hline TAML-VER-02 & ND & ND & ND & ND \\
\hline TAML-VER-03 & ND & ND & ND & ND \\
\hline TAML-VER-04 & ND & ND & ND & ND \\
\hline TAML-VER-05 & ND & ND & ND & ND \\
\hline TAML-VER-06 & ND & ND & $\mathrm{ND}$ & ND \\
\hline TAML-VER-07 & ND & ND & ND & ND \\
\hline TAML-VER-08 & $\mathrm{ND}$ & ND & $\mathrm{ND}$ & 19.1 \\
\hline TAML-VER-09 & ND & ND & ND & 13.4 \\
\hline TAML-VER-10 & $\mathrm{ND}$ & ND & $\mathrm{ND}$ & ND \\
\hline TAML-VER-11 & $\mathrm{ND}$ & $\mathrm{ND}$ & $\mathrm{ND}$ & 7.81 \\
\hline TAML-VER-12 & ND & ND & ND & ND \\
\hline
\end{tabular}


TABLE 4 - DU IMPACT SITE VERIFICATION SAMPLE SUMMARY

\begin{tabular}{|c|c|c|c|c|}
\hline SAMPLE NUMBER & $\begin{array}{c}\text { TCLP SVOCA } \\
(\mathrm{mg} / \mathrm{L})\end{array}$ & $\begin{array}{c}\text { TCLP METALS } \\
(\mathrm{mg} / \mathrm{L})\end{array}$ & $\begin{array}{c}\text { NITROAROMATICS/ } \\
\text { NITROAMINES }{ }^{\text {NTRg }} \\
(\mathrm{mg} / \mathrm{Kg})\end{array}$ & $\begin{array}{c}\text { GAMMA }^{\mathbf{D}} \\
(\mathrm{U238}) \\
(\mathrm{pCi} / \mathrm{g}) \\
\end{array}$ \\
\hline TAML-VER-13 & ND & ND & ND & ND \\
\hline TAML-VER-14 & ND & ND & ND & 11.2 \\
\hline TAML-VER-15 & ND & ND & ND & 6.54 \\
\hline TAML-VER-16 & ND & ND & ND & 7.27 \\
\hline TAML-VER-17 & ND & ND & ND & 11.7 \\
\hline TAML-VER-18 & ND & ND & ND & ND \\
\hline TAML-VER-19 & ND & ND & ND & 3.95 \\
\hline TAML-VER-20 & ND & ND & ND & ND \\
\hline TAML-VER-21 & ND & ND & ND & 4.73 \\
\hline TAML-VER-22 & ND & ND & ND & 5.55 \\
\hline TAML-VER-23 & ND & ND & ND & 4.54 \\
\hline TAML-VER-24 & ND & ND & ND & 7.97 \\
\hline
\end{tabular}


TABLE 4 - DU IMPACT SITE VERIFICATION SAMPLE SUMMARY

\begin{tabular}{||l|c|c|c|c||}
\hline \hline SAMPLE NUMBER & $\begin{array}{c}\text { TCLP SVOC } \\
(\mathbf{m g} / \mathbf{L})\end{array}$ & $\begin{array}{c}\text { TCLP METALS }^{\mathbf{B}} \\
(\mathbf{m g} / \mathbf{L})\end{array}$ & $\begin{array}{c}\text { NITROAROMATICS/ }_{\text {NITROAMINES }^{\mathbf{c}}} \\
(\mathbf{m g} / \mathbf{K g})\end{array}$ & $\begin{array}{c}\text { GAMMA }^{\mathbf{D}} \\
(\mathbf{U} \text { 238) } \\
(\mathbf{p C i} / \mathbf{g})\end{array}$ \\
\hline \hline TAML-VER-25 & $\mathrm{ND}^{\mathrm{E}}$ & $\mathrm{ND}$ & $\mathrm{ND}$ & 8.64 \\
\hline TAML-VER-26 & $\mathrm{ND}$ & $\mathrm{ND}$ & $\mathrm{ND}$ & 7.82 \\
\hline TAML-VER-27 & $\mathrm{ND}$ & $\mathrm{ND}$ & $\mathrm{ND}$ & 23.8 \\
\hline TAML-VER-28 & $\mathrm{ND}$ & Mercury 0.0021 & $\mathrm{ND}$ & $\mathrm{ND}$ \\
\hline
\end{tabular}

NOTES:

A - TCLP SVOCs: TCLP Semi-Volatile Organic Compounds analyzed using EPA Method 8270C.

Extraction by Method 1311. (EPA, 1996)

B - TCLP Metals: Metals analyzed using EPA Methods 6010 and 7470A. Extraction by Method 1311. (EPA, 1996)

C - Nitroaromatics/Nitroamines analyzed using EPA Method 8330. (EPA, 1996)

D - 300-minute gamma spectroscopy for U238.

E - ND: Not detected above laboratory detection limits. See Appendix D for Laboratory Reports.

$(\mathrm{mg} / \mathrm{L}$ or $\mathrm{mg} / \mathrm{Kg}$ or $\mathrm{pCi} / \mathrm{g}$ ): Milligrams per liter (for TCLP extraction), Milligrams per kilogram (for soil), Picocuries per gram for gamma spectroscopy. 


\section{THIS PAGE INTENTIONALLY LEFT BLANK}




\subsection{CONCLUSIONS}

CAU 461, Joint Test Assembly Sites, which includes CAS TTR-001 (DU Round No. 1A and 1B), CAS TA-52-003-0960 (DU Round No. 2), and CAS TA-52-002-TAML (DU Impact Site) were clean closed. CAS TTR-001 was excavated and found not to contain DU artillery rounds.

Radiological surveys of the excavations and excavated soil indicate no radiological contamination. The excavations were backfilled with the excavated soil to 80 percent compaction. The sites were graded and the barbed wire fences were removed. A final geophysical survey indicates no anomalies below the excavated areas.

CAS TA-52-003-0960 and CAS TA-52-002-TAML were excavated and found to contain radiologically impacted soil and debris. All of the impacted material was excavated and disposed in either the NTS Area 5 or Area 3 RWMS, respectively. Verification samples were collected and the analytical results indicate that all impacted material was removed. The excavations were backfilled with clean soil to 80 percent compaction and graded. Final radiological surveys were done and show the sites to be free of radiological contamination. The sites were de-posted and the barbed wire fences were removed. A final geophysical survey indicates no anomalies below the excavated areas.

Closure was completed following the approved SAFER Closure Plan (DOE, 1998a). Verification sampling was not required at CAS TTR-001 since artillery rounds or impacted soil were not discovered. Verification sampling at CAS TA-52-003-0960 and TA-52-002-TAML indicate that all impacted soil and debris was removed. Therefore, both sites are clean closed.

\subsection{CLOSURE PETITION}

This closure was completed following the approved SAFER Closure Plan (DOE, 1998a). Based upon the completion of site closure activities, the U.S. Department of Energy, Nevada Operations Office requests that a Notice of Completion be issued by the NDEP for the Joint Test Assembly Sites, CAU 461, and that CAU 461 be moved from Appendix III to Appendix IV of the FFACO, "Closed Corrective Action Units." 


\section{THIS PAGE INTENTIONALLY LEFT BLANK}




\subsection{REFERENCES}

ASTM, see American Society for Testing and Materials

DOE, see U.S. Department of Energy

EPA, see U.S. Environmental protection Agency

NDEP, see Nevada Division of Environmental Protection

SAIC, see Science Applications International Corporation

American Society for Testing and Materials, 1997b, D 2922-96 Standard Test Methods for Density of Soil and Soil-Aggregate in Place by Nuclear Methods (Shallow Depth), West Conshohocken, Pennsylvania.

Bechtel Nevada, 1998, Site-Specific Health and Safety Plan for Corrective Action Unit 461: Joint Test Assembly Sites and Corrective Action Unit 495: Unconfirmed Joint Test Assembly Sites Tonopah Test Range, Nevada, Las Vegas, Nevada.

Nevada Division of Environmental Protection, U.S. Department of Energy, and U.S. Department of Defense, April 1996, Federal Facilities Agreement and Consent Order (FFACO) of 1996.

Science Applications International Corporation, 1998, First Quarter 1998 Surficial Geophysical Survey Report. Depleted Uranium Impact Site (CAU 461), 1987 W79 Joint Test Assembly (CAU 461) Sites A and B, and Buried Artillery Rounds (CAU 495) \#3 and \#4, Tonopah Test Range, Tonopah, Nevada, Middletown, Pennsylvania.

U.S. Department of Energy, 1998a, Streamlined Approach for Environmental Restoration Work Plan for Corrective Action Unit 461: Joint Test Assembly Sites and Corrective Action Unit 495: Unconfirmed Joint Test Assembly Sites Tonopah Test Range, Nevada, Revision 0, DOE/NV/11718--247, Las Vegas, Nevada.

U.S. Department of Energy, 1998b, Field Management Plan for Corrective Action Unit 461: Joint Test Assembly Sites and Corrective Action Unit 495: Unconfirmed Joint Test Assembly Sites Tonopah Test Range. Nevada, Las Vegas, Nevada.

U.S. Environmental Protection Agency,1996, Test Methods for Evaluating Solid Waste. Physical/Chemical Methods, SW-846, CD ROM, Washington, DC. 


\section{THIS PAGE INTENTIONALLY LEFT BLANK}


APPENDIX A

\section{RESULTS OF FINAL GEOPHYSICAL SURVEY}


THIS PAGE INTENTIONALLY LEFT BLANK 


\section{CONFIRMATION GEOPHYSICAL SURVEY CORRECTIVE ACTION UNIT 461}

Magnetometer surveys were conducted over the excavations as well as the areas immediately surrounding the excavations. The magnetometer surveys were conducted for the purpose of confirming and documenting that the site had been cleared of any subsurface projectiles or associated debris. Surveys were conducted on CAS TTR-001 (Depleted Uranium (DU) Round 1A North, DU Round 1A South, DU Round 1B), CAS TA-52-003-0960 (DU Round 2), and on CAS TA-52-002-TAML (DU Impact site).

CAS TTR-001 and CAS TA-52-003-0960 locations were geophysical targets thought to be associated with artillery round impact sites of either warmer rounds or W79 Joint Test Assemblies. All sites were excavated to determine the nature of the target and remediate those sites as required.

Target identification relied on calibrating to a know target at varying depths. Calibration magnetic signatures were determined by burying a dummy $155 \mathrm{~mm}$ artillery shell at $0 \mathrm{~m}(0 \mathrm{ft}), 0.6$ $\mathrm{m}(2 \mathrm{ft}), 1.2 \mathrm{~m}(4 \mathrm{ft})$, and $2.4 \mathrm{~m}(8 \mathrm{ft})$ depths. For each depth the round was laid flat with an orientation of $\mathrm{N} 45^{\circ} \mathrm{W}$, covered with soil, and a survey conducted over the round. While the actual W79 and warmer rounds were $200 \mathrm{~mm}$ ( 8 in) shells, the smaller $155 \mathrm{~mm}$ (6 in) calibration round provided a conservative estimate of the magnetic signature that could be expected from the target artillery round. The results of these calibrations are presented in Figures 1 through 5.

Magnetometer surveys where conducted using a Geometrics G858 Cesium Vapor magnetometer configured as a Gradiometer. Results were recorded using continuous profiling with a 0.1 seconds/sample sample rate. The magnetometer recorded both total field and the vertical gradient over a $56 \mathrm{~cm}$ sensor separation. An integrated Global Position System (GPS) was used with the magnetometer system to provide line survey position data. Earlier surveys used a presurveyed grid for line and station control. The survey boundaries were laid out to include all surface disturbed ground within the surveyed area.

\section{CAS TTR-001 (DU Round 1A)}

This site consisted of two magnetic anomalies identified in the geophysical report (SAIC, 1998). No EM anolmalies were noted at this site. The southern magnetic anomaly, as noted in the geophyical report, was the stronger of the two with a peak positive gradient of approximately 90 $\mathrm{nT} / \mathrm{m}$. The northern anomaly peak was approximately $15 \mathrm{nT} / \mathrm{m}$. Excavations to $3.3 \mathrm{~m}(10 \mathrm{ft})$ in depth and the use of a handheld magnaetomter revealed no ordnance related debris or radiologically impacted soils. The confirmation survey (Figure 6 ) indicated a dipolar anomaly $(+30,-60 \mathrm{nT} / \mathrm{m})$ located approximately 5 feet from the Round $1 \mathrm{~A}$ south excavation site. The magnitude of this anomaly was large enough to require further investigation. Depth calculations indicated a target at approximately $1.2 \mathrm{~m}(4 \mathrm{ft})$ and about $0.9 \mathrm{~m}(3 \mathrm{ft})$ long. The site was revisited 
THIS PAGE INTENTIONALLY LEFT BLANK 


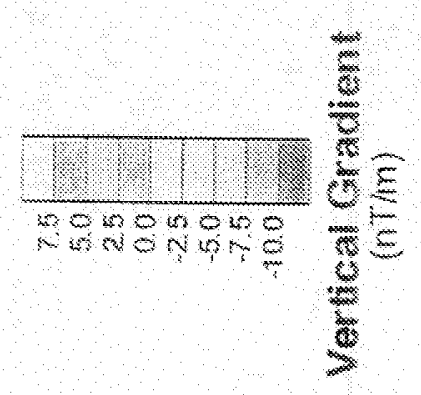

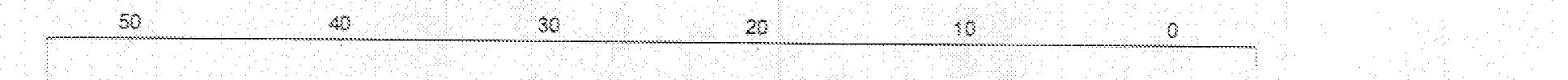

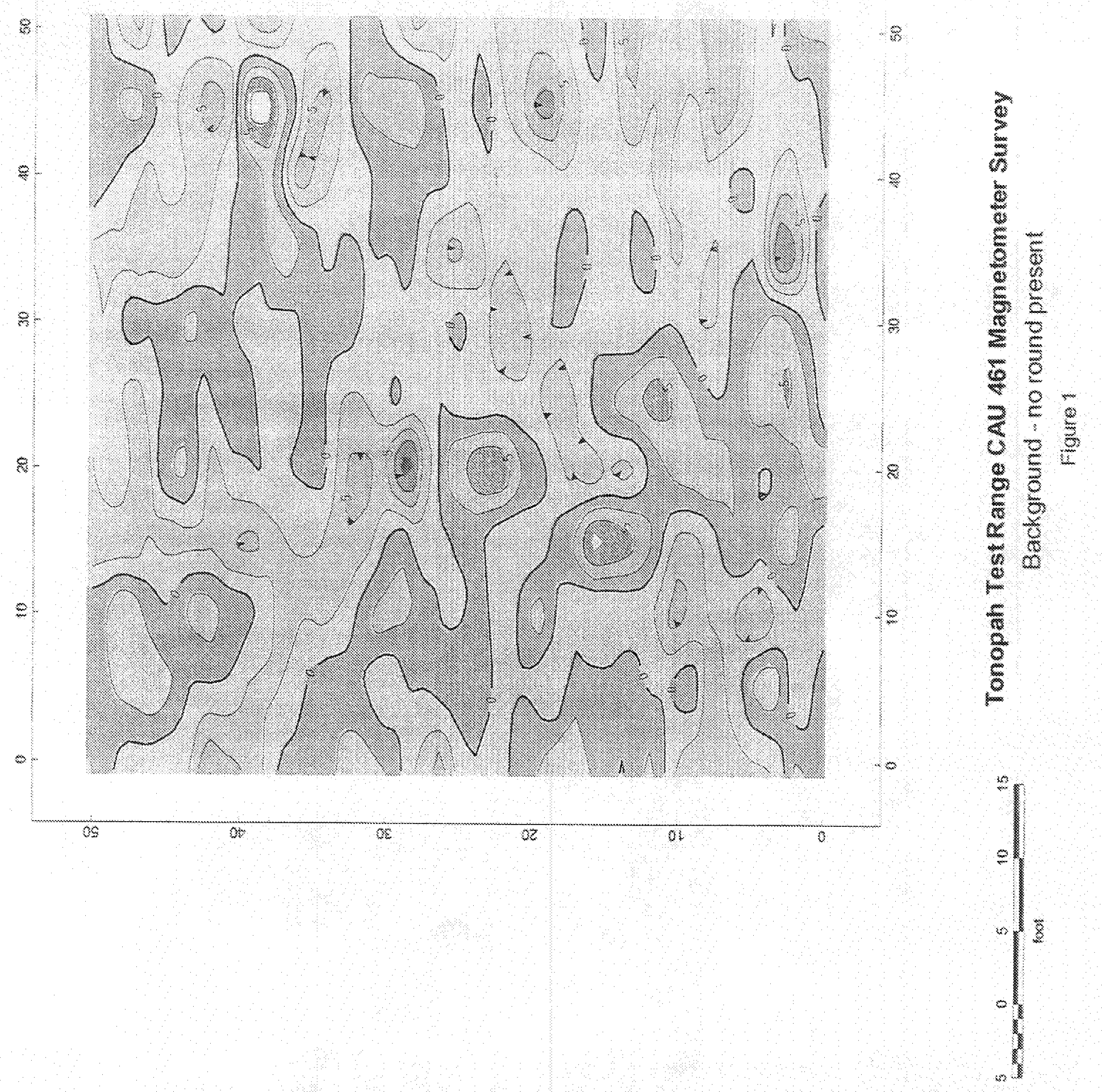


THIS PAGE INTENTIONALLY LEFT BLANK 


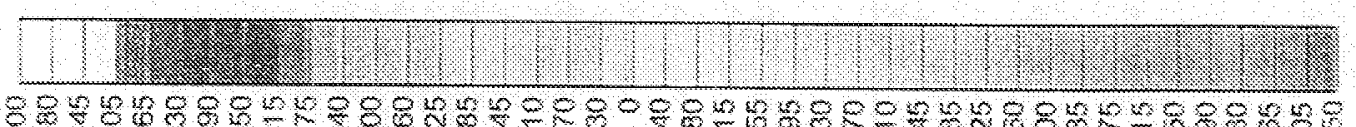

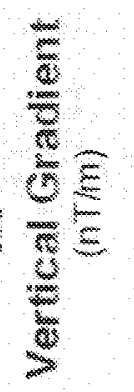

st $30 \quad 30 \quad 2000$

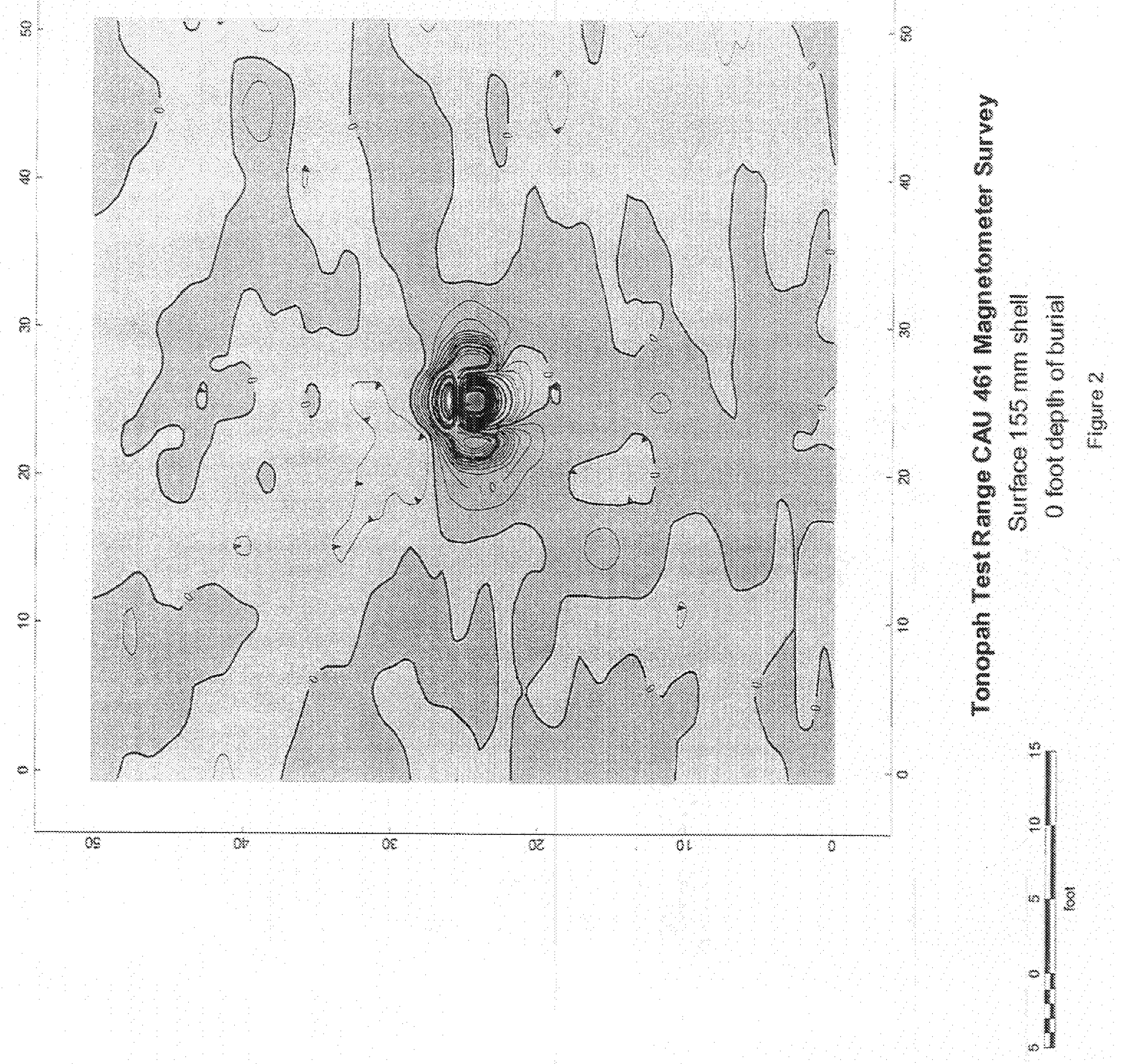


THIS PAGE INTENTIONALLY LEFT BLANK 

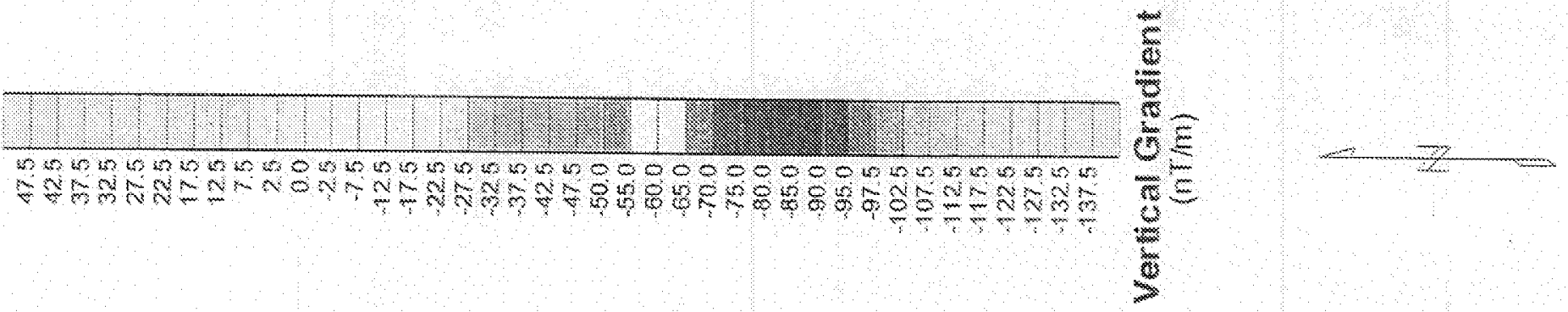

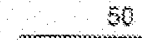

$\Delta$ 30 20
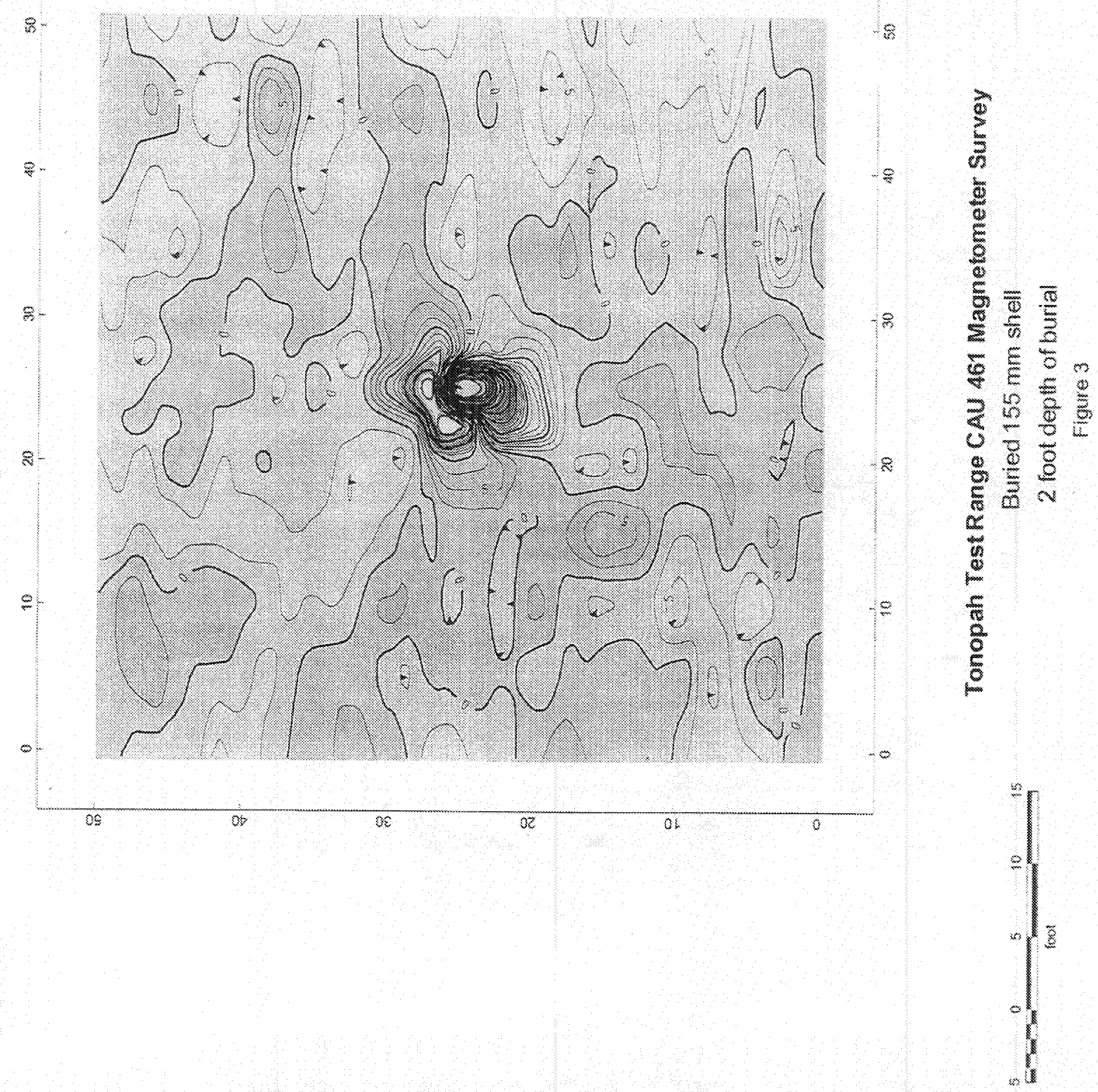
THIS PAGE INTENTIONALLY LEFT BLANK 


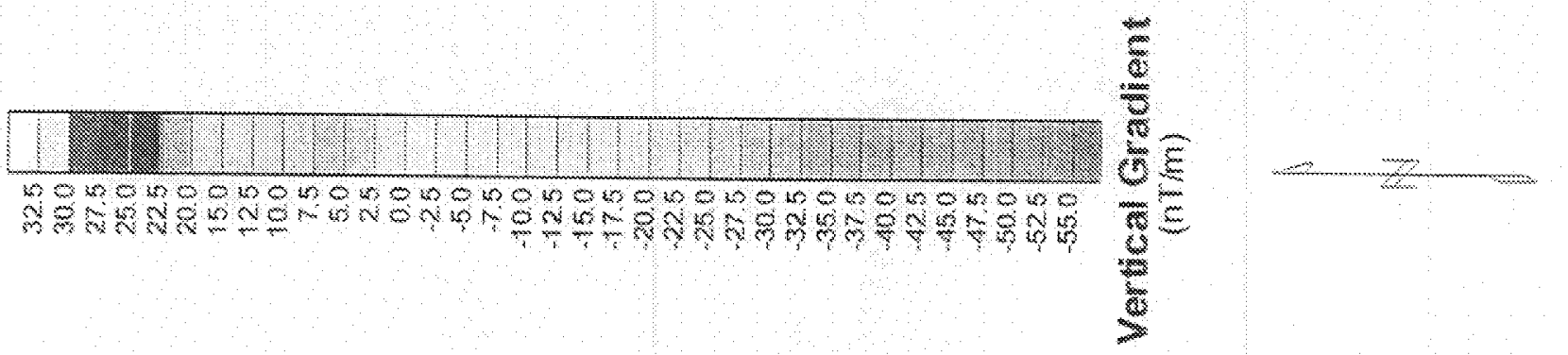

50

45

30

20

0

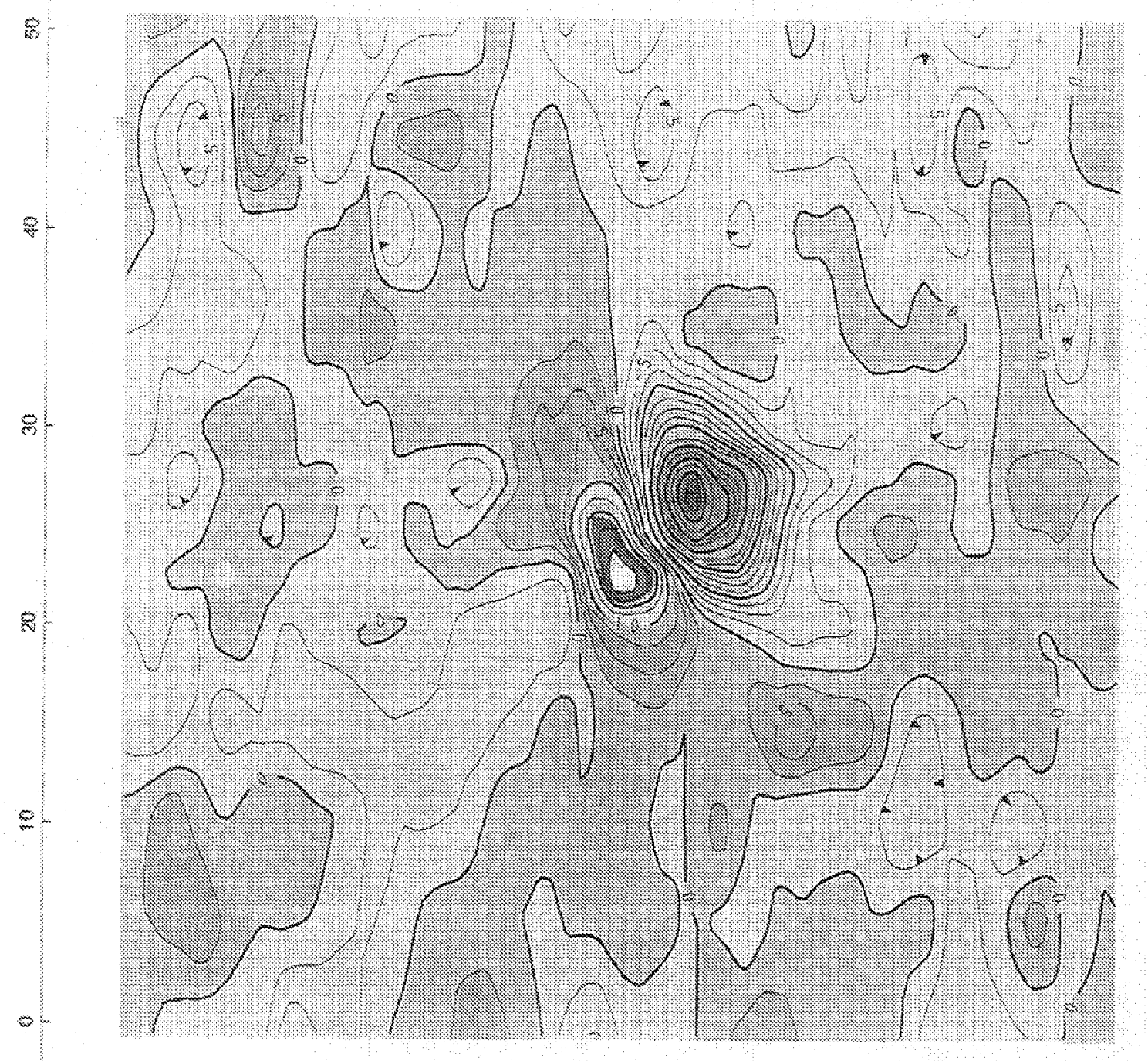

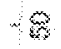

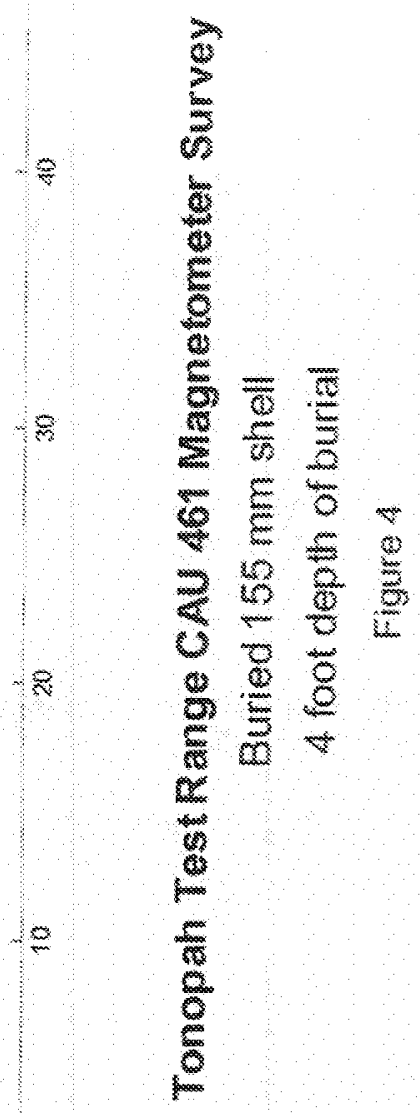

65

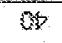

ge

II

$0)$

)

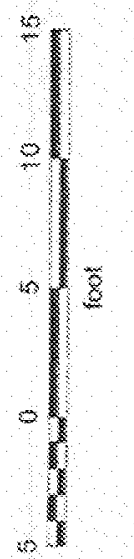



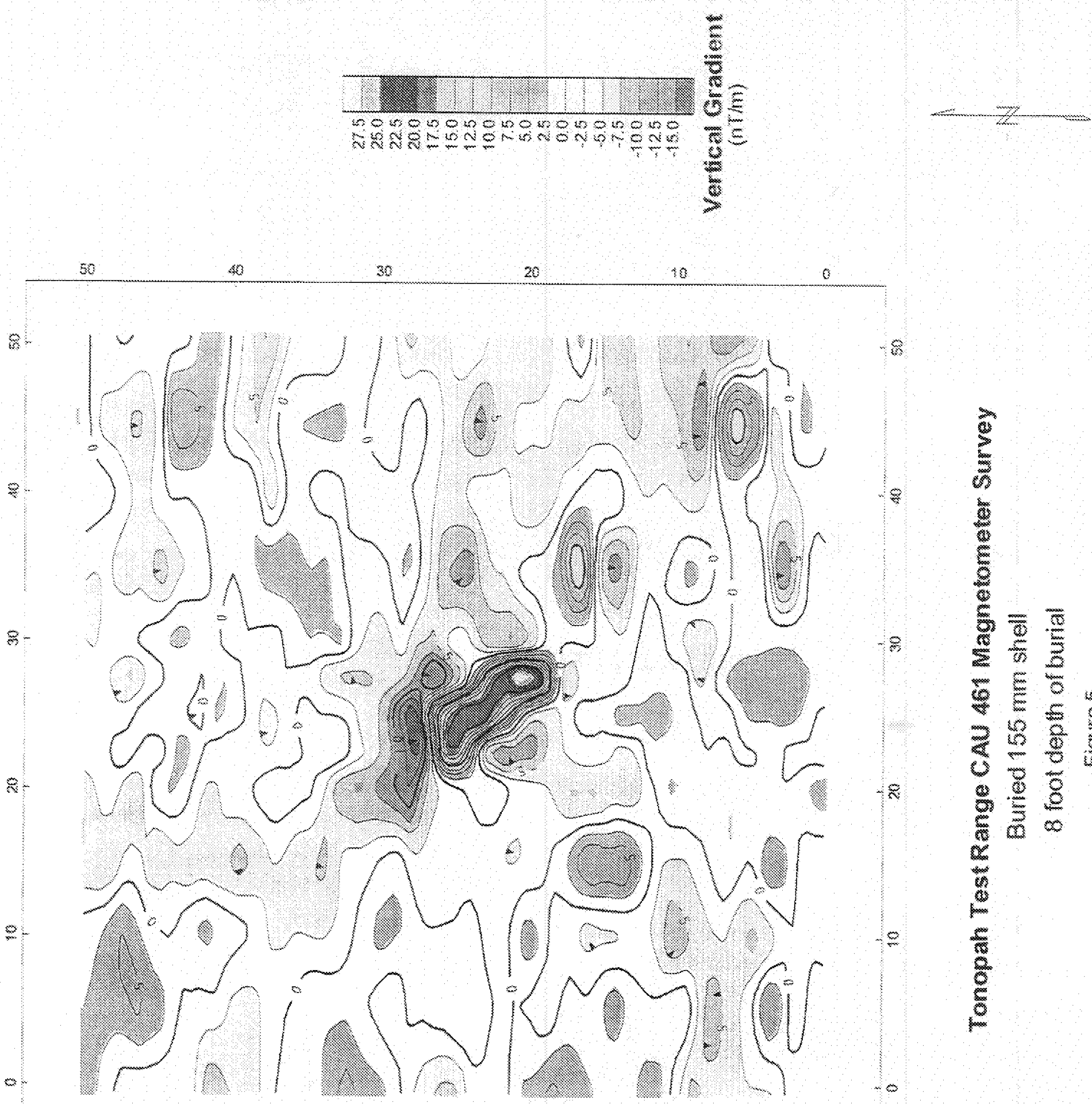

8

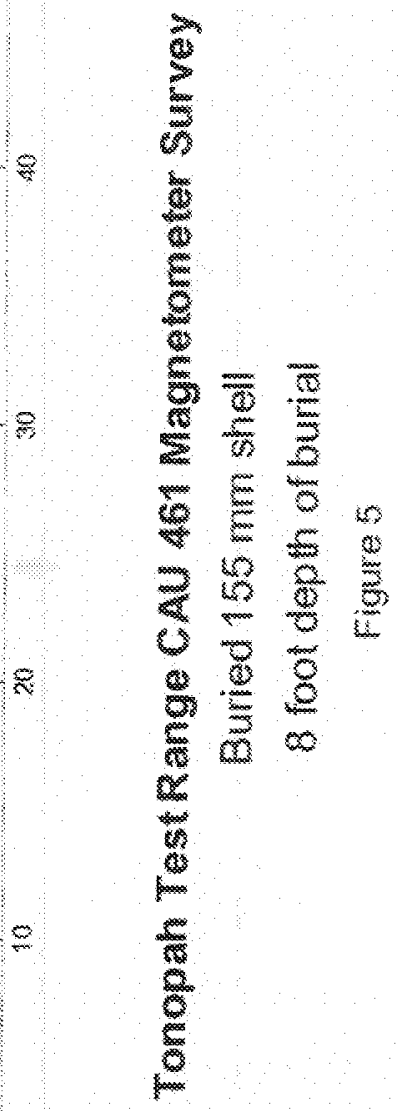

as

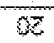

03

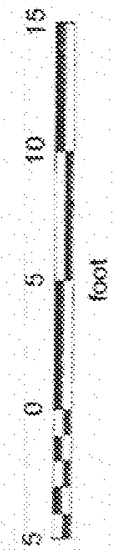


THIS PAGE INTENTIONALLY LEFT BLANK 


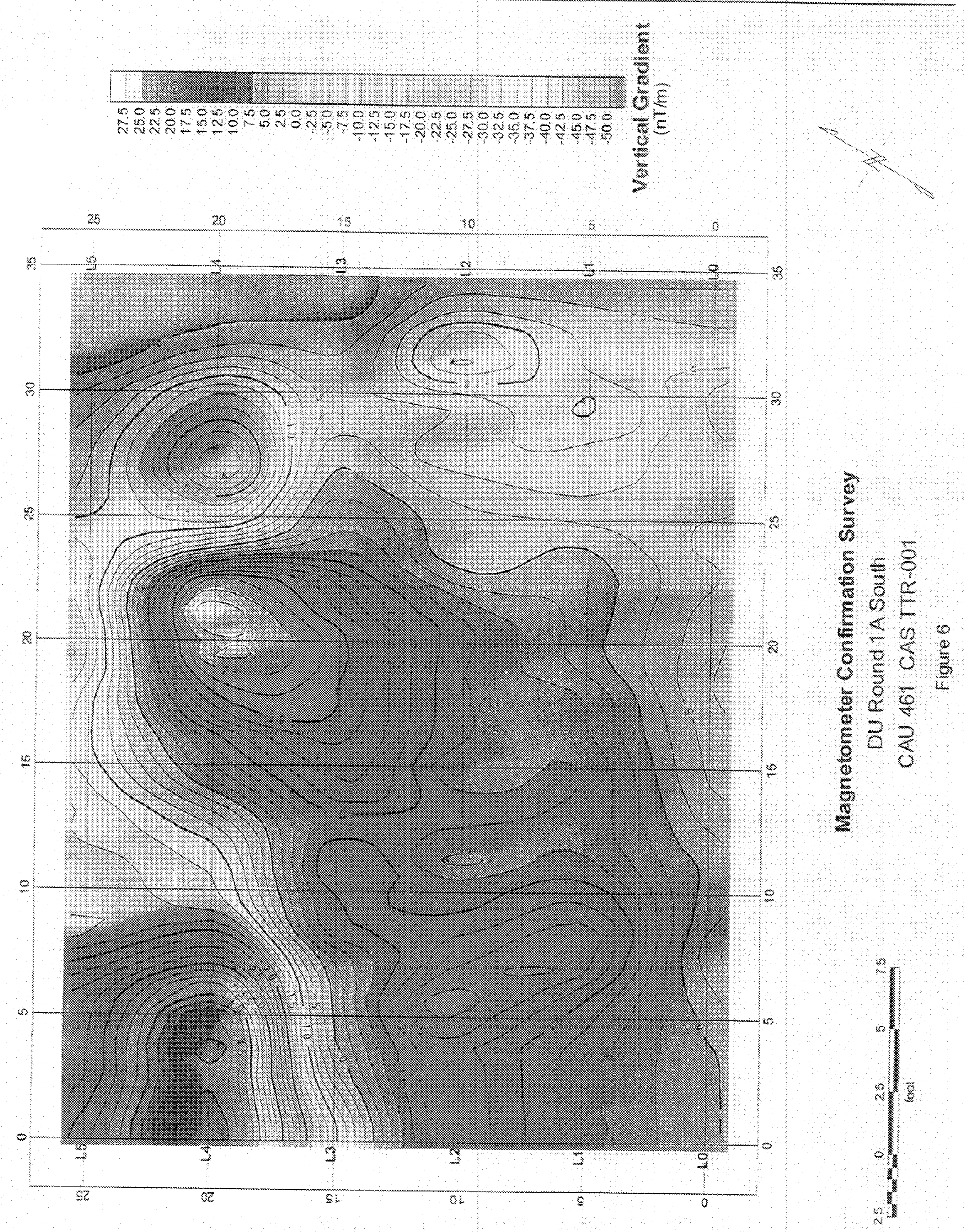


THIS PAGE INTENTIONALLY LEFT BLANK 
and excavated. A lense of iron rich gravel was found at a depth of approximately 3.5 feet and confirmed with use of a handheld magnetometer. No debris or signs of disturbed materials were found.

The confirmation survey at DU Round 1A North (Figure 7) indicates a weak magnetic feature that can also be associated with native materials. No magnetic signatures indicative of an ordnance size object are observed.

\section{CAS TTR-001 (DU Round 1B)}

The initial geophysical surveys of this area indicated strong EM and Magnetic anomalies confined to a localized area. Excavation of this area produced numerous metallic objects ranging in size from $5 \mathrm{~cm}(2 \mathrm{in}$.) to $0.5 \mathrm{~m}(1.5 \mathrm{ft})$ down to a depth of $1.5 \mathrm{~m}(5 \mathrm{ft})$ which is consistant with the geophysical signatures observed. The confirmation survey (Figure 8 ) indicates some small near surface pieces of iron located between $528255 \mathrm{E}$ to $528257.5 \mathrm{E}$ along $4187674.5 \mathrm{~N}$. The cause of the strongest signature $(+40 /-38$ nanoTesla/meter $[\mathrm{nT} / \mathrm{m}])$ located at $528257.34 \mathrm{E}, 4187674.4 \mathrm{~N}$ is a small piece of iron shrapnel visible at the surface.

No magnetic targets were observed on this confirmatory survey that would be representative of a buried target at or below the excavation location.

\section{CAS TA-52-003-0960 (DU Round 2)}

Excavation at this site produced soils that were radiologically impacted, pieces of oxidized DU, and some pieces of shell casings. Evidence of a high order detonation was observed at a depth of $1.5 \mathrm{~m}(5 \mathrm{ft})$. Excavation indicated clean material at a depth of $5.3 \mathrm{~m}(17.5 \mathrm{ft})$.

Several ferrous objects are evident along the periphery of the site (Figure 9) and are associated with near surface survey markers (IT72, IT69) and iron debris. The larger anomaly seen at $528719 \mathrm{E}, 41876323 \mathrm{~N}$ was due to an iron T-Post plate just beneath the surface.

No magnetic signatures indicative of a buried ordnance size object are observed.

\section{CAS TA-52-002-TAML}

The TAML site consisted of a Depleted Uranium impact site measuring approximately $55 \mathrm{~m}$ by 50 $\mathrm{m}(180 \mathrm{ft}$ by $160 \mathrm{ft})$. This site hosted numerous small pieces of metallic debris along with DU contaminated soils originally though to be very near surface. While the majority of the site was found to meet these conditions a central pit area was characterized and cleaned up to a depth of $3.3 \mathrm{~m}(10 \mathrm{ft})$. The pit was comprised of an area of disturbed ground hosting metal fragments and DU contaminated soils from a failed weapons test. Hand held metal detectors (Whites Spectrum $\mathrm{XLT}$ and a Schonstedt GA 52C Flux gate magnetometer) were used to clear the surface and excavation area of metallic and ferrous debris. 
THIS PAGE INTENTIONALLY LEFT BLANK 

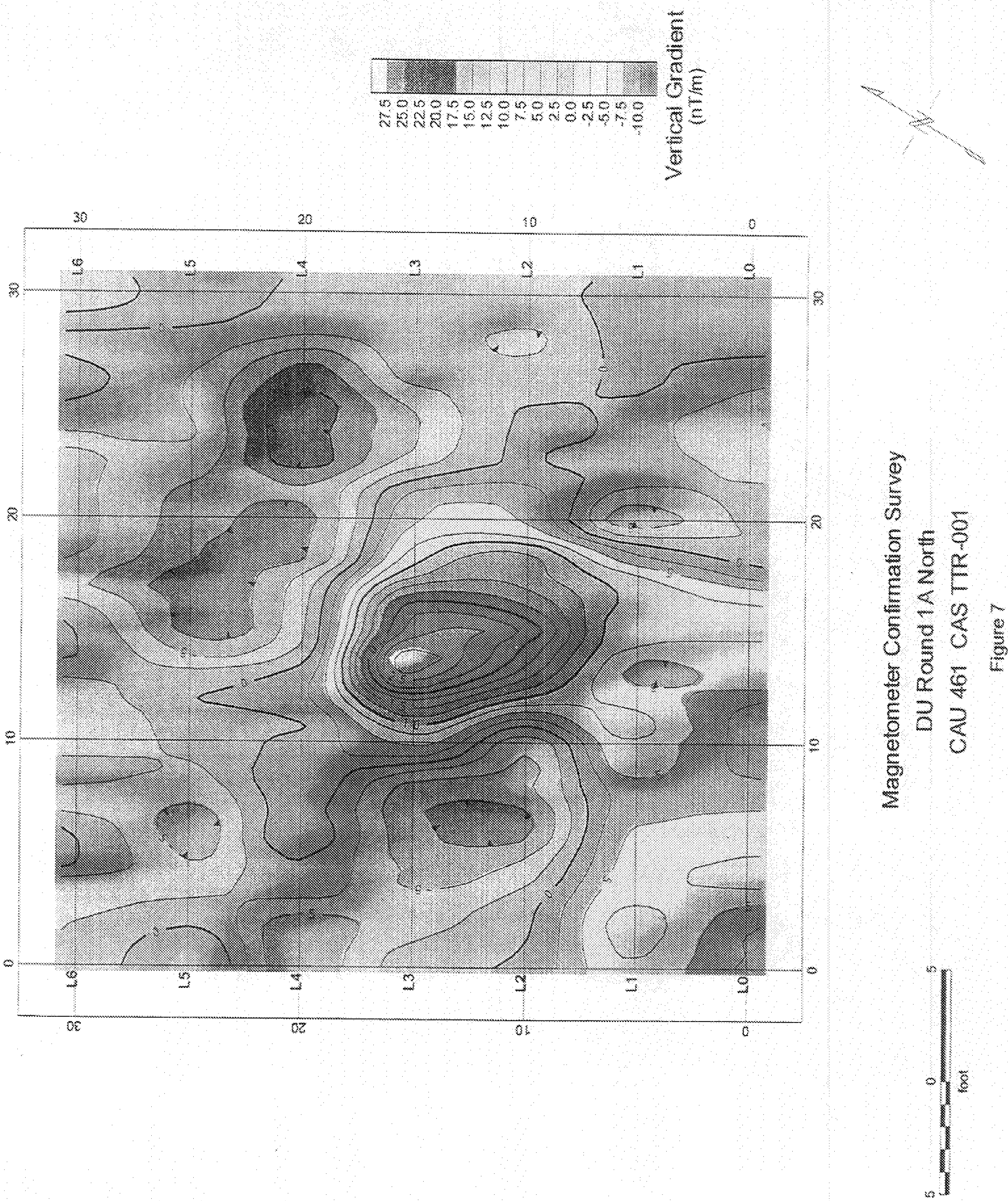
THIS PAGE INTENTIONALLY LEFT BLANK 


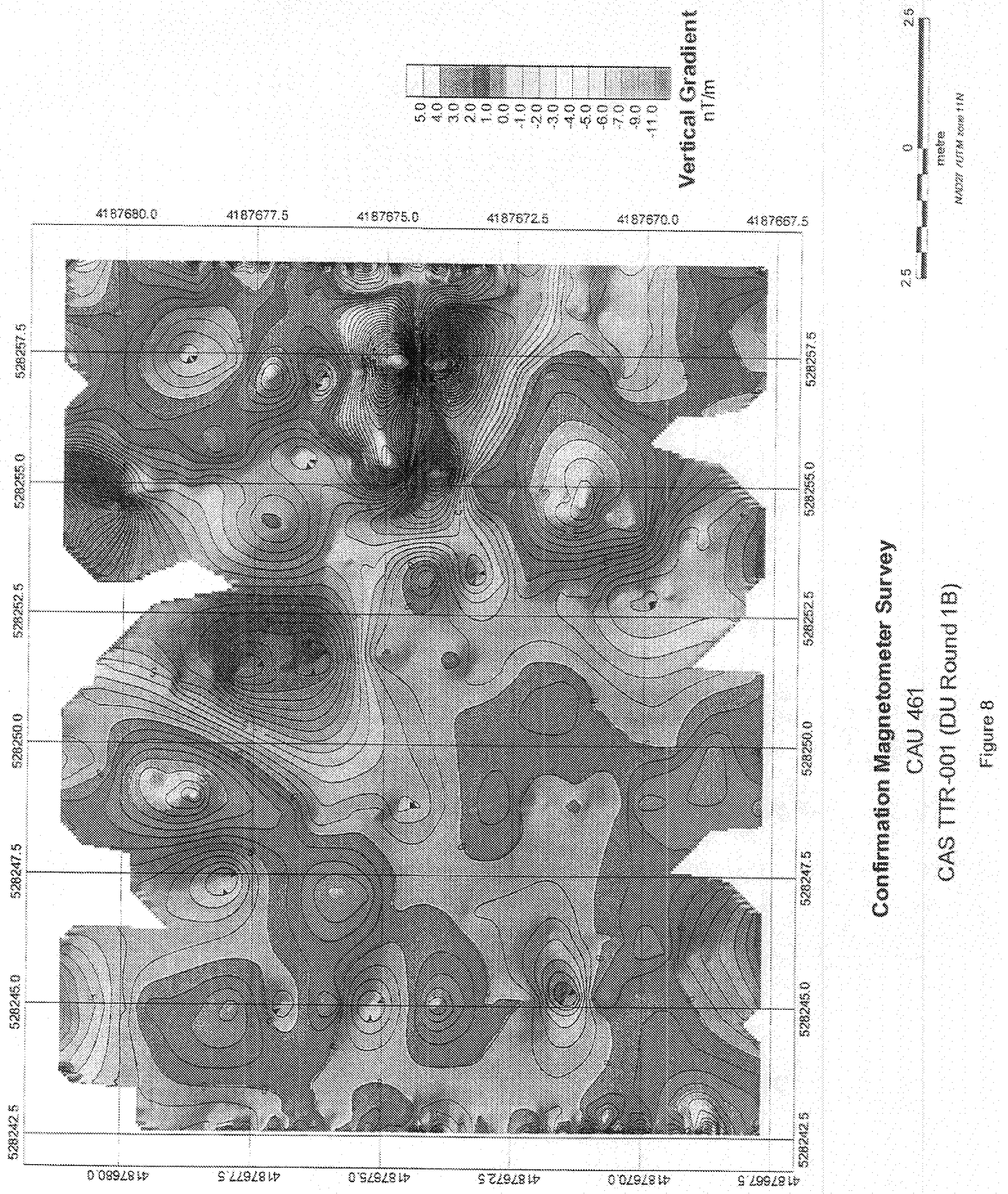


THIS PAGE INTENTIONALLY LEFT BLANK 


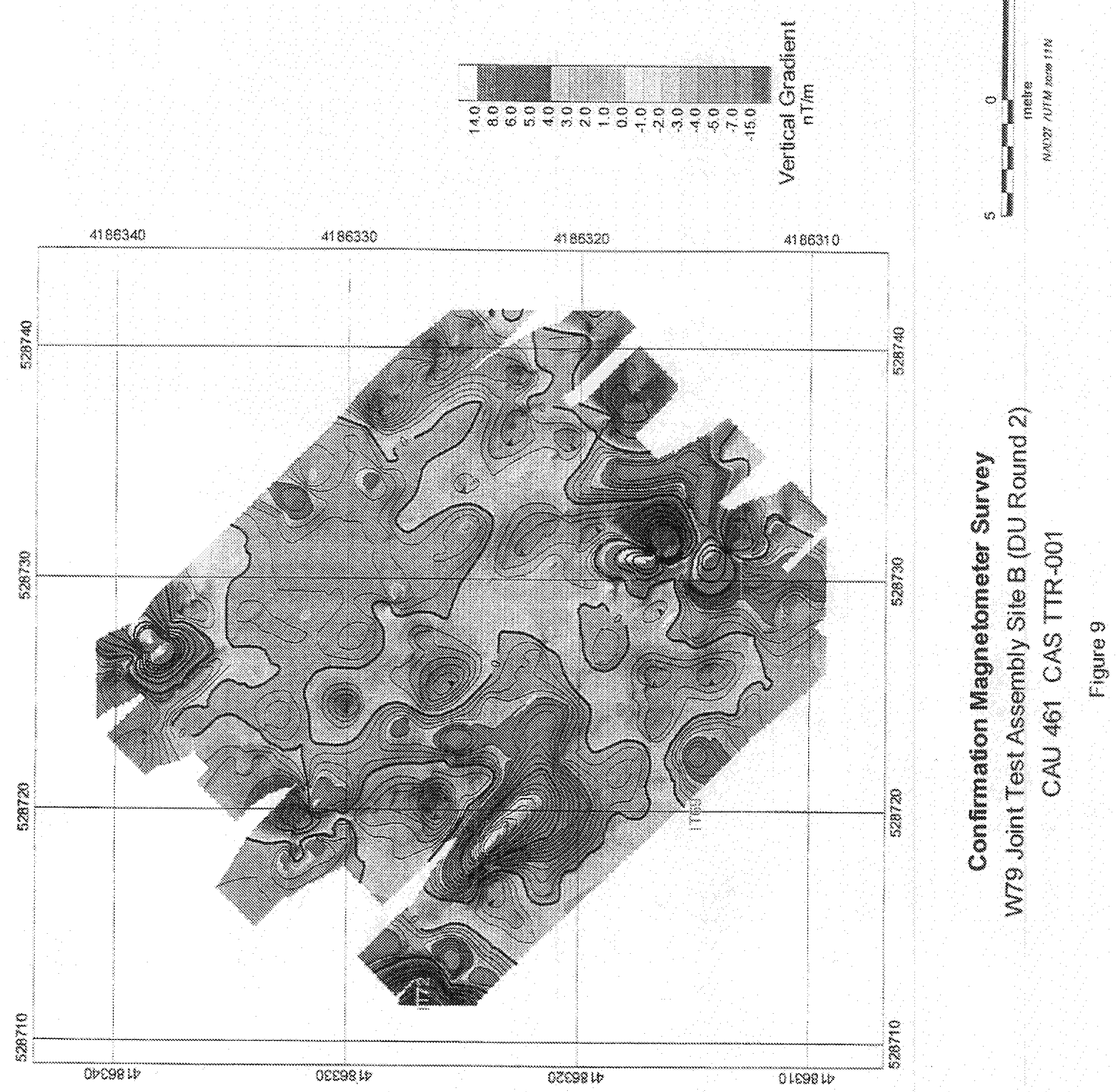


THIS PAGE INTENTIONALLY LEFT BLANK 
A confirmation survey was conducted over the area to be certain no larger deeply buried objects remained. The magnetometer results (Figure 10) indicate the central pit area and the area surrounding this excavation are free of any deeply buried objects.

Some smaller pieces of iron can be seen in the near surface along the eastern corner of the survey area. The large features noted around the periphry of the site are iron fence posts and survey markers.

No magnetic signatures indicative of an ordnance size object are observed. 
THIS PAGE INTENTIONALLY LEFT BLANK 


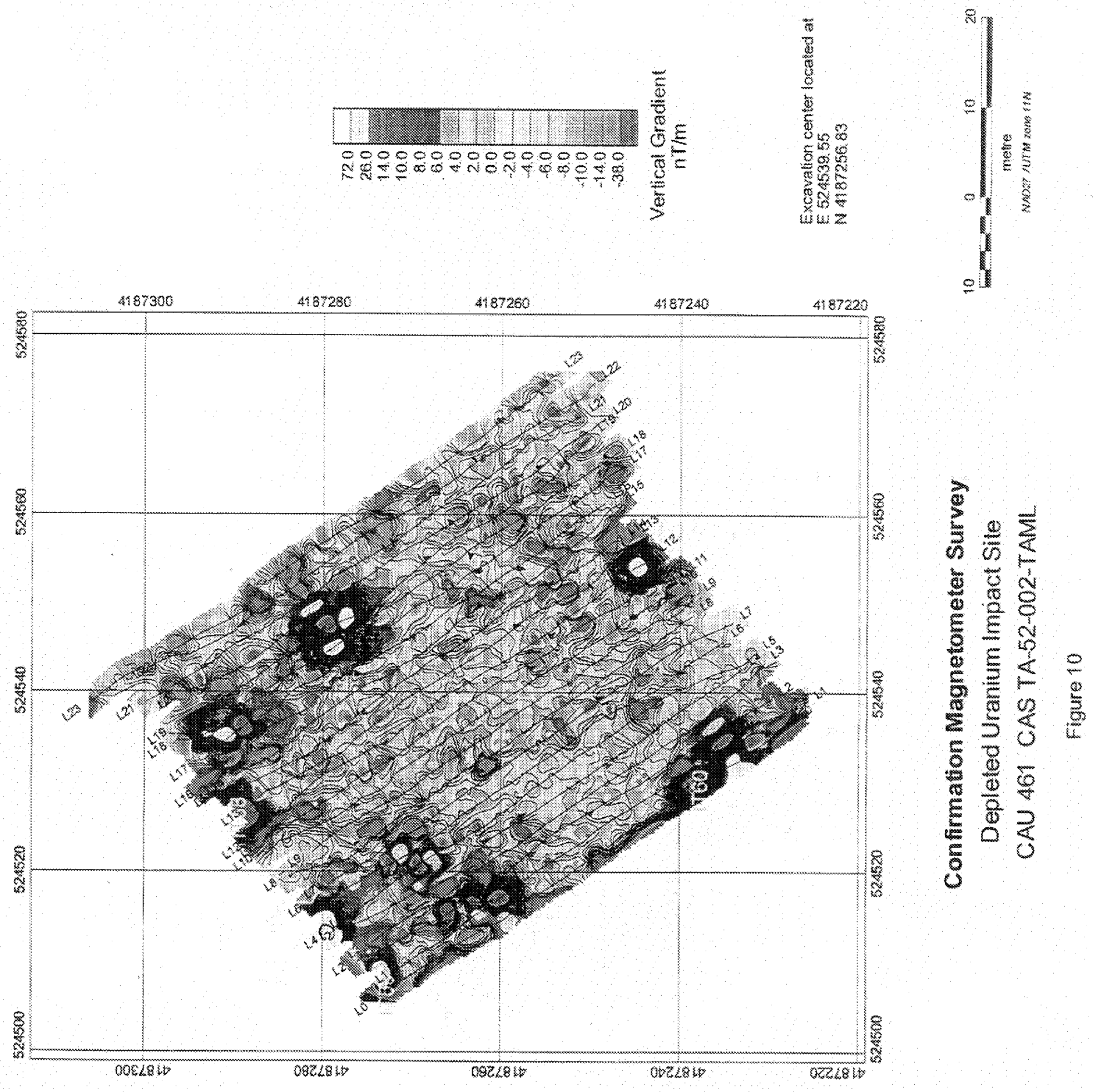


THIS PAGE INTENTIONALLY LEFT BLANK 


\section{APPENDIX B}

\section{SURVEY RESULTS}


THIS PAGE INTENTIONALLY LEFT BLANK 
BECHTEL NEVADA CORPORATION

SURVEY DEPARTMENT

(702) 295-3487

Date: 01-13-99

Time: 15:45:21

Page: 1

Coordinate File: TTRDURAB.CRD List of Coordinate Points

* Denotes Contouring Masspoint

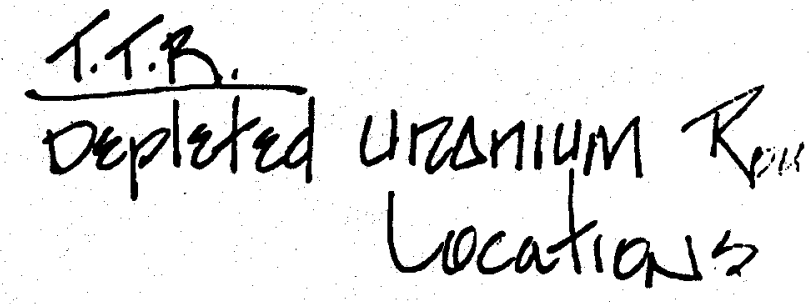

ELEV

1647.8270

1647.7800

1647.7880

1647.7940

1643.8160

1643.7210

1643.6550

1643.7570

1643.6500

1643.6790

1643.7370

1643.7170

1644.5160

1644.6020

1644.6600

1644.6480

1639.6100

1638.7030

1638.8160

1638.7720

1638.6990

1638.7050

1638.7100

1638.7080

1639.4110

1639.3790

1639.3200

1639.3970

1639.2070

1639.1680

1639.2250

1639.2420

1639.2450

1639.2790

1639.3540

1639.3160
Descriptor

COR. D.U.SITE 2

COR. D.U.SITE \#2

COR. D.U.SITE 2

COR. D.U.SITE 2

COR. D.U.SITE $1-A, N$ N COR.D.U.SITE I-A, NOK: COR.D.U.SITE I-A, NOR! COR.D.U.SITE $1-A$, NOIR?! COR.D.U.SITE 1-A, SOI!" COR.D.U.SITE 1-A, SOTI COR.D.U.SITE I-A, SOT! COR.D.U.SITE $\# 1-\lambda, \quad S O::$ COR.D.U.SITE \#1-B, COR. D.U. SITE \#1-B COR. D.U. SITE 1-B COR. D.U.SITE \#1-B COR.D.U.SITE 3, EAST COR.D.U. SITE 3, EASIJ COR. D.U.SITE \#3, EAS\% COR. D.U.SITE 3, EAS: COR. D.U.SITE \#3, WFSI! COR. D.U.SITE \#, WE:E! COR. D.U. SITE \#3, WES! COR. D.U. SITE 3, WL: COR. D.U. SITE 4 , E:A COR. D.U. SITE \#4, EA. COR. D.U. SITE \#4, FAST COR. D.U. SITE 4, EXE! COR. D.U.SITE 4 , SOUII: COR. D.U. SITE *4, SOl!" COR. D.U. SITE 4, SOII! COR. D.U. SITE \#4, SCIY, COR. D.U. SITE 4 , hl: COR. D.U. SITE \#4 WES!: COR. D.U. SITE \#4, KE COR. D.U. SITE \#4, WF 
THIS PAGE INTENTIONALLY LEFT BLANK 


\section{APPENDIX C}

\section{BACKFILL COMPACTION TEST RESULTS}


THIS PAGE INTENTIONALLY LEFT BLANK 


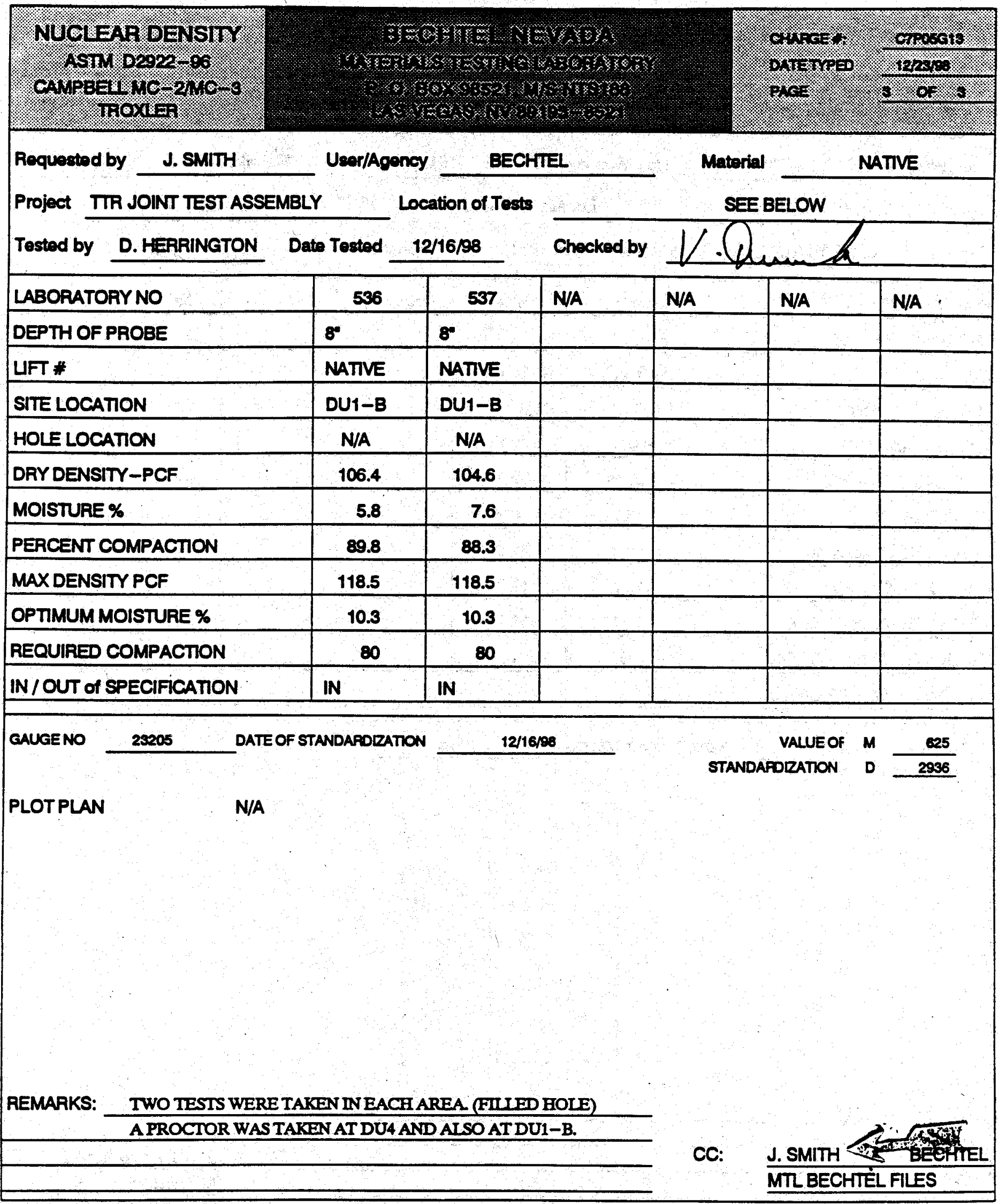




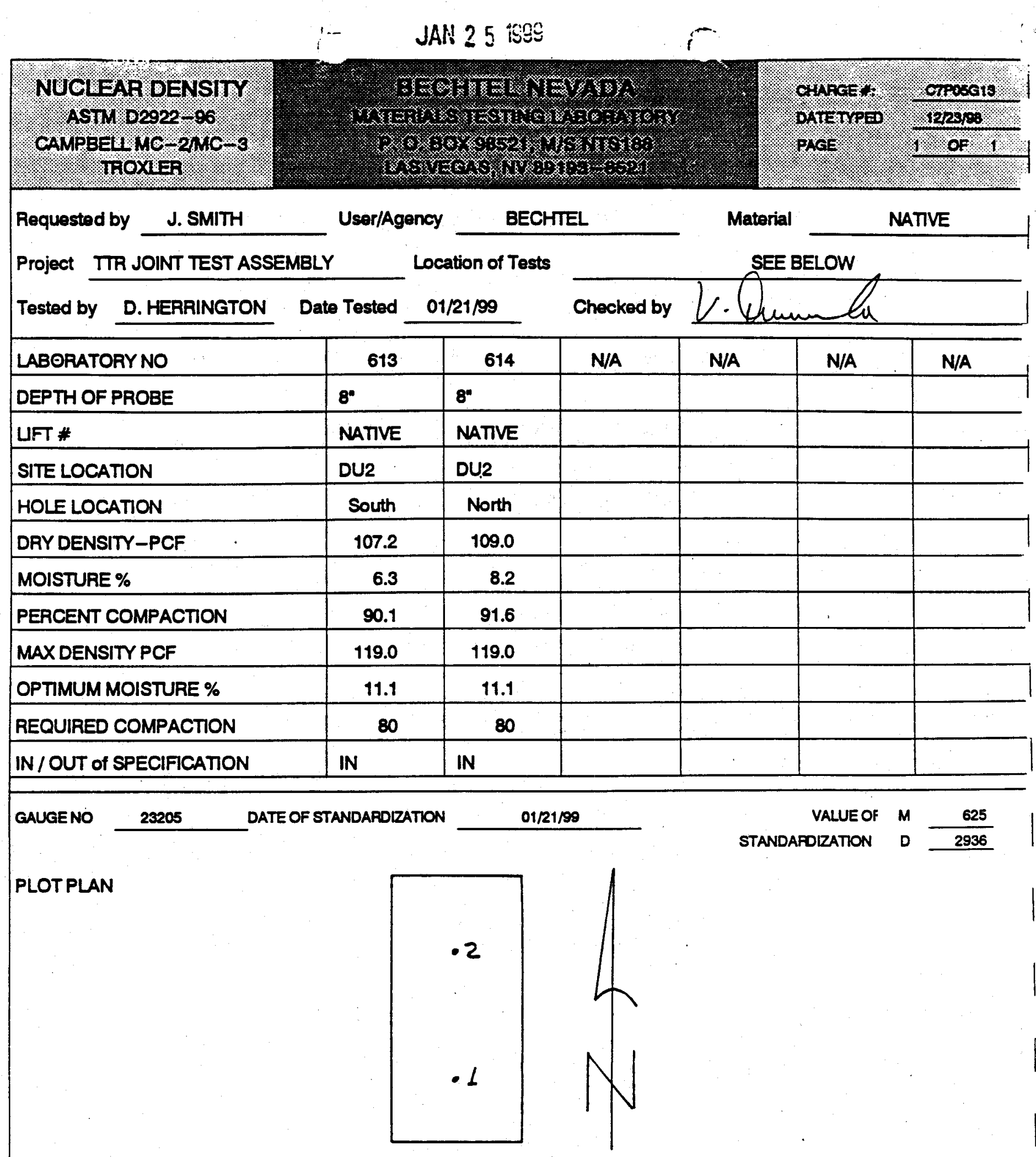

REMARKS: N/A

CC: J. SMITH MTL BECHTEL FILES 


\section{NUCLEAR DENSITY ASTM D2922-96 \\ CAMPBELLLMC-2MC.3 TROXLER \\ in 100

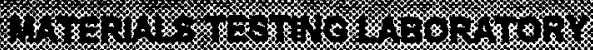

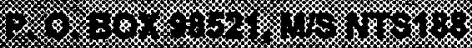 \\ 3.}

CHARGE: DATE TYPED

PAGE

Material SAINDIA BORROW

Requested by

D. TOBIASSON

User/Agency

BECHTEL Location of Tests

BACKFILL OF EXCAVATION

Project TA-52-002-TAML

Date Tested $\quad 08 / 24 / 99$

Checked by $V \cdot Q$, b

Tested by D. HERRINGTON Date Tested 08/24/99

LABORATORY NO

\begin{tabular}{|r|r}
2135 & 2136 \\
\hline 1 & 2
\end{tabular}

TEST \#

LIFT \#

Final Grade $\quad$ Final Grad

DEPTH OF PROBE

$8^{n}$

DRY DENSITY-PCF

MOISTURE $\%$

PERCENT COMPACTION

MAX DENSITY PCF (Rock Correction)

OPTIMUM MOISTURE \%

REQUIRED COMPACTION \%

IN / OUT OF SPECIFICATION

\begin{tabular}{l|l}
$8^{n}$ & 8 \\
\hline
\end{tabular}

114.3

$8^{n}$

114.4

4.8

3.8

2137

3

2138

\begin{tabular}{|l|l}
\hline N/A & N/A \\
\hline & \\
\hline &
\end{tabular}

Final Grade Final Grade

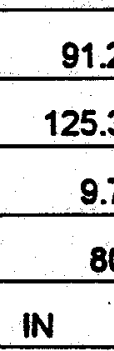

91.3

$8^{n}$

GAUGE NO 23205 DATE OF STANDARDIZATION

$08 / 2499$

\begin{tabular}{rr} 
VALUE O & $M$ \\
STANDARDIZATION & D \\
\hline 2929 \\
\hline
\end{tabular}

PLOT PLAN
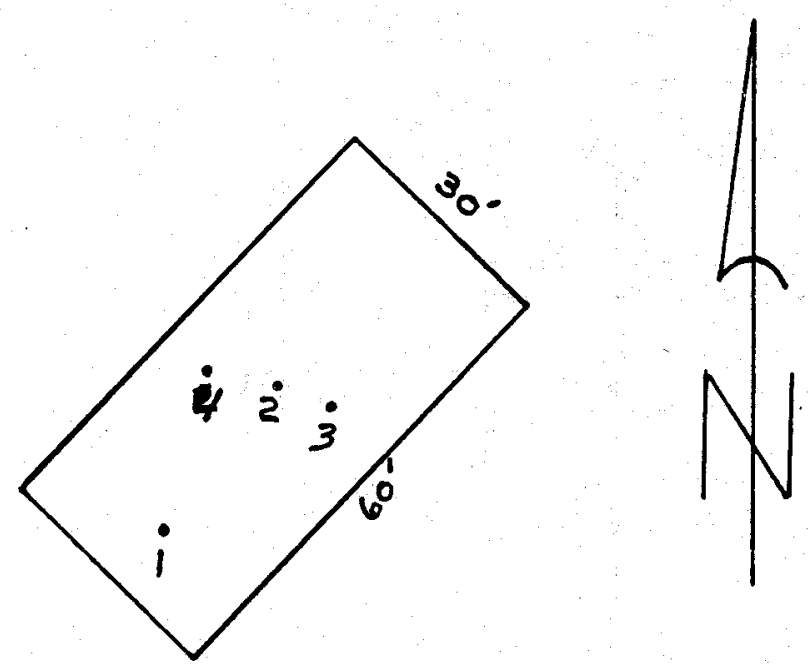

REMARKS: TEST LOCATIONS PROVIDED BYD. TOBIASSON.

CC: $\frac{\text { J. SMITH }}{\text { D. TOBIASSON JECHTEL }}$




\begin{tabular}{|c|c|c|c|c|c|}
\hline NUCLEAR DENSITY & & CHARGE : & \multicolumn{3}{|c|}{ C7POSH13 } \\
\hline ASTM D2922-96 / / & 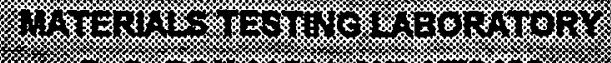 & DATE TYPED & \multicolumn{3}{|c|}{$08 / 25 / 99$} \\
\hline $\begin{array}{l}\text { CAMPBELL MC-2MC-3 } \\
\text { TROXLER }\end{array}$ & 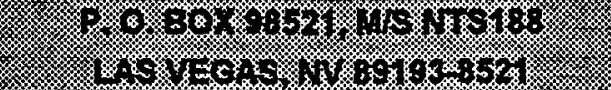 & PAGE 1 : & 1 & OF & 1 \\
\hline
\end{tabular}

Requested by

D. TOBIASSON

User/Agency .

BECHTEL

Material NATIVE

Project TTR - 001 DU RD 1A Location of Tests

BACKFILL OF EXCAVATION

Tested by D. HERRINGTON Date Tested 08/24/99 Checked by

V.

\begin{tabular}{|l|c|c|c|c|c|c|}
\hline LABORATORY NO & 2139 & 2140 & 2141 & 2142 & N/A & N/A \\
\hline TEST \# & 1 & 2 & 3 & 4 & & \\
\hline LIFT \# & Final Grade & Final Grade & Final Grade & Final Grade & & \\
\hline DEPTH OF PROBE & $8^{n}$ & $8^{n}$ & $8^{n}$ & $8^{n}$ & & \\
\hline DRY DENSITY-PCF & 104.5 & 102.8 & 104.2 & 105.0 & & \\
\hline MOISTURE \% & 3.0 & 3.1 & 3.4 & 3.7 & & \\
\hline PERCENT COMPACTION & 88.2 & 86.8 & 87.9 & 88.6 & & \\
\hline MAX DENSITY PCF (Rock Correction) & 118.5 & 118.5 & 118.5 & 118.5 & & \\
\hline OPTIMUM MOISTURE \% & 10.3 & 10.3 & 10.3 & 10.3 & & \\
\hline REQUIRED COMPACTION \% & 80 & 80.0 & 80.0 & 80.0 & & \\
\hline IN / OUT Of SPECIFICATION & $\mathbb{N}$ & $\mathbb{N}$ & $\mathbb{N}$ & IN & & \\
\hline
\end{tabular}

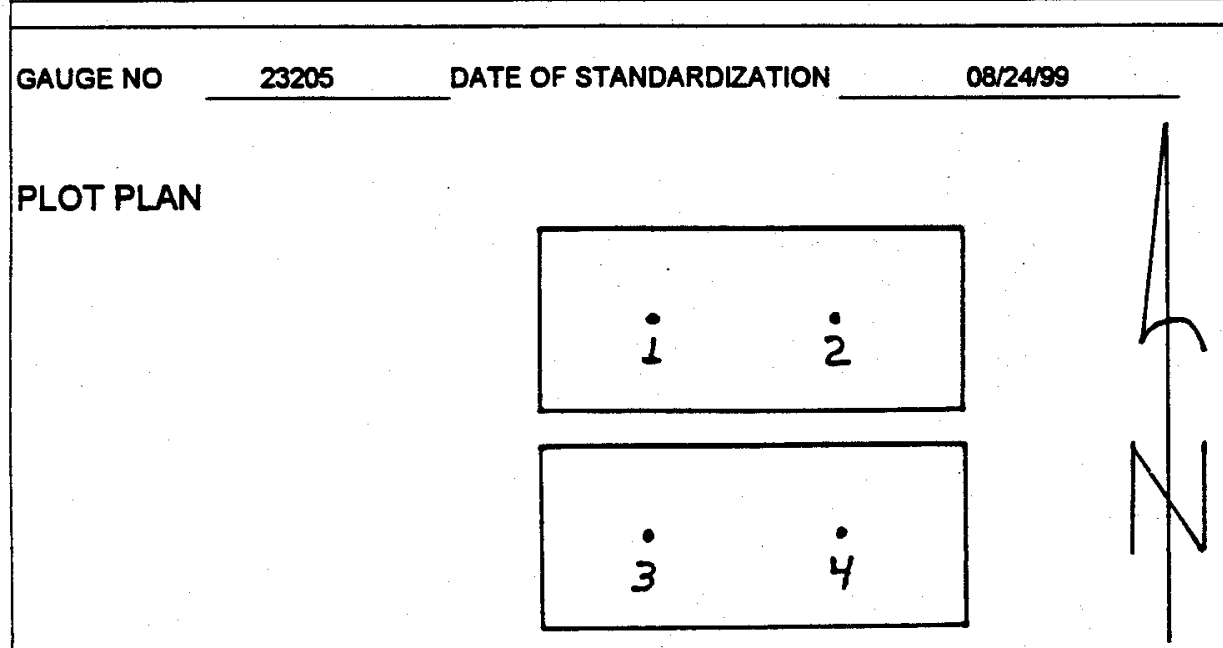

REMARKS: TEST LOCATIONS AND PROCTOR RESULTS PROVIDED BY D. TOBIASSON.

$$
\begin{array}{rr}
\text { VALUE O } & M \\
\text { STANDARDIZATION } & \text { D } \\
\hline \\
\hline
\end{array}
$$

cc:

J. SMITH BECHTEL
D. TOBIASSOA
MTL BECHTEL FILES




\section{APPENDIX D}

\section{VERIFICATION SAMPLE RESULTS}


THIS PAGE INTENTIONALLY LEFT BLANK 


\section{SAMPLE}

\section{DELIVERY}

GROUP

V557 
THIS PAGE INTENTIONALLY LEFT BLANK 
CLIENT: Bechtel Nevada

P.O. Box 98521, M/S NTS273

Las Vegas, NV 89193-8521

ATTN: Ted Redding

PROJECT NAME: V557

PROJECT NUMBER: 17777

NEL ORDER ID: L9812133

Attached are the analytical results for samples in support of the above referenced project.

Samples were received by NEL in good condition, under chain of custody on 12/11/98.

Samples were analyzed as received.

Should you have any questions or comments, please feel free to contact our Client Services department at (702) 657-1010.

Some results have been flagged as follows:

$\mathrm{J}$ - This concentration should be considered an estimate due laboratory control sample failure.

Some $Q A$ results have been flagged as follows:

$\mathrm{J}$ - This concentration should be considered an estimate due laboratory control sample failure.
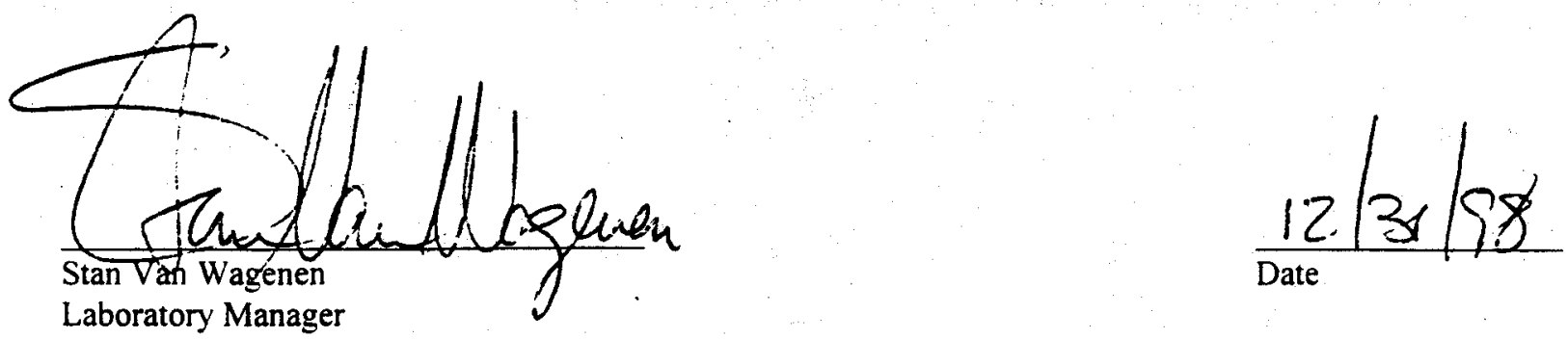

CERTIFICATIONS:

\begin{tabular}{|c|c|c|c|c|c|c|c|}
\hline & Reno & Las Vegas & S. California & & Reno & Las Vegas & S. California \\
\hline Arizona & $\overline{\mathrm{AZ} 0520}$ & AZ0518 & AZ0583 & Idaho & $\overline{\text { Certified }}$ & Certified & \\
\hline California & 1707 & 2002 & 2264 & Montana & Certified & Certified & \\
\hline $\begin{array}{l}\text { US Army Corps } \\
\text { of Engineers }\end{array}$ & Certified & Certified & Certified & Nevada & NV033 & NV052 & CA084 \\
\hline
\end{tabular}




\begin{tabular}{llll}
\hline CLIENT: & Bechtel Nevada & CLIENT ID: & DURD \# 2-1 \\
PROJECT ID: & V557/17777 & DATE SAMPLED: $12 / 8 / 98$ \\
PROJECT \#: & 17777 & NEL SAMPLE ID: & L9812133-01
\end{tabular}

TEST:

TCLP by EPA 1311, July 1992 \& Semivolatile Organics by EPA Method 8270C, Dec. 1996

METHOD: $\quad$ EPA 8270

MATRIX: $\quad$ Solid

TCLP EXTRACT DATE: $12 / 17 / 98$

DILUTION: 1

EXTRACTED: $\quad 12 / 18 / 98$

ANALYZED: $\quad 12 / 21 / 98$

\section{PARAMETER}

1.4-Dichlorobenzene (p-DCB)

2.4-Dinitrotoluene (DNT)

Hexachlorobenzene

\begin{tabular}{c}
$\begin{array}{c}\text { Result } \\
\mathbf{m g} / \mathbf{L}\end{array}$ \\
\hline $\mathrm{ND}$ \\
$\mathrm{ND}$ \\
$\mathrm{ND}$ \\
$\mathrm{ND}$ \\
$\mathrm{ND}$ \\
$\mathrm{ND}$ \\
$\mathrm{ND}$ \\
$\mathrm{ND}$ \\
$\mathrm{ND}$ \\
$\mathrm{ND}$ \\
$\mathrm{ND}$ \\
$\mathrm{ND}$
\end{tabular}

Reporting

Limit

$0.1 \mathrm{mg} / \mathrm{L}$

$0.1 \mathrm{mg} / \mathrm{L}$

$0.1 \mathrm{mg} / \mathrm{L}$

$0.1 \mathrm{mg} / \mathrm{L}$

$0.1 \mathrm{mg} / \mathrm{L}$

$0.1 \mathrm{mg} / \mathrm{L}$

$0.1 \mathrm{mg} / \mathrm{L}$

$0.1 \mathrm{mg} / \mathrm{L}$

$0.1 \mathrm{mg} / \mathrm{L}$

$0.1 \mathrm{mg} / \mathrm{L}$

$0.1 \mathrm{mg} / \mathrm{L}$

$0.1 \mathrm{mg} / \mathrm{L}$

QUALITY CONTROL DATA:

\section{Surrogate}

2.4,6-Tribromophenol

2-Fluorobiphenyl

2-Fluorophenol

Nitrobenzene-d5

p-Terphenyl-d14

Phenol-d5

\section{\% Recovery}

72

63

53

60

80

33
Acceptable Range

$10-123$

$43-116$

2] -100

$35-114$

$33-141$

10. 94 
CLIENT: Bechtel Nevada

PROJECT NAME: V557

PROJECT NUMBER: 17777
CLIENT ID: DURD \# 2-1

DATE SAMPLED: $12 / 8 / 98$

NEL SAMPLE ID: L9812133-01

TEST: Nitroaromatics and Nitroamines by EPA 8330

MATRIX: Solid

EXTRACTED: $12 / 18 / 98$

DILUTION: 1

ANALYST: YW

\section{PARAMETER}

1.3-DNB

$\mathrm{HMX}$

Nitrobenzene

2-Nitrotoluene

3-Nitrotoluene

4-Nitrotoluene

RDX

Tetryl

1.3,5-TNB

2.4.6-TNT

\section{Result}

ND

ND

ND

ND

ND

ND

ND

ND

ND

ND
Reporting

Limit

$0.25 \mathrm{mg} / \mathrm{kg}$

2. $\mathrm{mg} / \mathrm{Kg}$

$0.25 \mathrm{mg} / \mathrm{Kg}$

$0.25 \mathrm{mg} / \mathrm{Kg}$

$0.25 \mathrm{mg} / \mathrm{Kg}$

$0.25 \mathrm{mg} / \mathrm{Kg}$

2. $\mathrm{mg} / \mathrm{Kg}$

$0.65 \mathrm{mg} / \mathrm{Kg}$

$0.25 \mathrm{mg} / \mathrm{Kg}$

$0.25 \mathrm{mg} / \mathrm{Kg}$

ND - Not Detected

This report shall not be reproduced except in full, without the written approval of the laboratory. 
CLIENT: Bechtel Nevada

PROJECT NAME: V557

PROJECT NUMBER: 17777
CLIENT ID: $\quad$ DURD \# 2-1

DATE SAMPLED: $12 / 8 / 98$

NEL SAMPLE ID: L9812133-01

\section{TEST: $\quad$ TCLP-8 Metals \\ MATRIX: Solid}

\begin{tabular}{|c|c|c|c|c|c|c|c|}
\hline PARAMETER & $\begin{array}{c}\text { RESULT } \\
\mathrm{mg} / \mathrm{L} \\
\end{array}$ & $\begin{array}{c}\text { REPORTING } \\
\text { LIMIT }\end{array}$ & D. F. & METHOD & $\begin{array}{c}\text { TCLP/STLC } \\
\text { EXTRACTION } \\
\text { DATE } \\
\end{array}$ & DIGESTED & ANALYZED \\
\hline Arsenic & ND & $0.5 \mathrm{mg} / \mathrm{k}$ & 5 & EPA 6010 & $12 / 17 / 98$ & $12 / 18 / 98$ & $12 / 18 / 98$ \\
\hline Barium & ND & $5 . \mathrm{mg} / \mathrm{L}$ & 5 & EPA $6010^{\circ}$ & $12 / 17 / 98$ & $12 / 18 / 98$ & $12 / 18 / 98$ \\
\hline Cadmium & ND & $0.05 \mathrm{mg} / \mathrm{L}$ & 5 & EPA 6010 & $12 / 17 / 98$ & $12 / 18 / 98$ & $12 / 18 / 98$ \\
\hline Chromium & ND & $0.05 \mathrm{mg} / \mathrm{L}$ & 5 & EPA 6010 & $12 / 17 / 98$ & $12 / 18 / 98$ & $12 / 18 / 98$ \\
\hline Lead & ND & $0.25 \mathrm{mg} / \mathrm{L}$ & 5 & EPA 6010 & $12 / 17 / 98$ & $12 / 18 / 98$ & $12 / 18 / 98$ \\
\hline Mercury & ND & $0.002 \mathrm{mg} / \mathrm{L}$ & 10 & EPA $7470 A$ & $12 / 17 / 98$ & $12 / 18 / 98$ & $12 / 18 / 98$ \\
\hline Selenium & ND & $0.5 \mathrm{mg} / \mathrm{L}$ & 5 & EPA 6010 & $12 / 17 / 98$ & $12 / 18 / 98$ & $12 / 18 / 98$ \\
\hline Silver & ND & $0.1 \mathrm{mg} / \mathrm{L}$ & 5 & EPA 6010 & $12 / 17 / 98$ & $12 / 18 / 98$ & $12 / 18 / 98$ \\
\hline
\end{tabular}

D.F. - Dilution Factor

ND - Not Detected

This report shall not be reproduced except in full, without the written approval of the laboratory. 


$\begin{array}{llll}\text { CLIENT: } & \text { Bechtel Nevada } & \text { CLIENT ID: } & \text { DURD \# 2-2 } \\ \text { PROJECT ID: } & \text { V557/17777 } & \text { DATE SAMPLED: } 12 / 8 / 98 \\ \text { PROJECT \#: } & 17777 & \text { NEL SAMPLE ID: } & \text { L9812133-02 }\end{array}$

TEST:

METHOD: EPA 8270

MATRIX: Solid

DILUTION: 1

TCLP by EPA 1311, July 1992 \& Semivolatile Organics by EPA Method 8270C, Dec. 1996

TCLP EXTRACT DATE: $12 / 17 / 98$

EXTRACTED:

$12 / 18 / 98$

ANALYZED:

$12 / 21 / 98$

\section{PARAMETER}

1.4-Dichlorobenzene ( $\mathrm{p}-\mathrm{DCB})$

2.4-Dinitrotoluene (DNT)

Hexachlorobenzene

Hexachlorobutadiene

Hexachloroethane

2-Methylphenol

3.4-Methylphenol (isomeric pair)

Vitrobenzene

Pentachlorophenol

Pridine

$\therefore$ 4.5-Trichlorophenol

2.4.6-Trichlorophenol

$\begin{array}{cc}\begin{array}{c}\text { Result } \\ \mathbf{m g} / \mathbf{L}\end{array} & \begin{array}{c}\text { Reporting } \\ \text { Limit }\end{array} \\ \text { ND } & 0.1 \mathrm{mg} / \mathrm{L} \\ \text { ND } & 0.1 \mathrm{mg} / \mathrm{L} \\ \text { ND } & 0.1 \mathrm{mg} / \mathrm{L} \\ \text { ND } & 0.1 \mathrm{mg} / \mathrm{L} \\ \text { ND } & 0.1 \mathrm{mg} / \mathrm{L} \\ \text { ND } & 0.1 \mathrm{mg} / \mathrm{L} \\ \text { ND } & 0.1 \mathrm{mg} / \mathrm{L} \\ \text { ND } & 0.1 \mathrm{mg} / \mathrm{L} \\ \text { ND } & 0.1 \mathrm{mg} / \mathrm{L} \\ \text { ND } & 0.1 \mathrm{mg} / \mathrm{L} \\ \text { ND } & 0.1 \mathrm{mg} / \mathrm{L} \\ \text { ND } & 0.1 \mathrm{mg} / \mathrm{L}\end{array}$

JUALITY CONTROL DATA:

iurrogate

4,6-Tribromophenol

\% Recovery

-Fluorobiphenyl

87

-Fluorophenol

itrobenzene-d5

-Terphenyl-d 14

'henol-d5
93

58

84

87

35
Acceptable Range

$10-123$

$43-116$

$21-100$

$35-114$

$33-141$

$10-94$ 
CLIENT: Bechtel Nevada

PROJECT NAME: . V557

PROJECT NUMBER: 17777
CLIENT ID: DURD \# 2-2

DATE SAMPLED: $12 / 8 / 98$

NEL SAMPLE ID: L9812133-02

TEST: Nitroaromatics and Nitroamines by EPA 8330

MATRIX: Solid

DILUTION: 1

\section{PARAMETER}

1.3-DNB

HMX

Nitrobenzene

2-Nitrotoluene

3-Nitrotoluene

4-Nitrotoluene

RDX

Tetryl

1.3,5-TNB

2.4,6-TNT
EXTRACTED: $12 / 18 / 98$

ANALYZED: $12 / 22 / 98$
ANALYST: YW

Reporting

Limit

$0.25 \mathrm{mg} / \mathrm{Kg}$

2. $\mathrm{mg} / \mathrm{Kg}$

$0.25 \mathrm{mg} / \mathrm{Kg}$

$0.25 \mathrm{mg} / \mathrm{Kg}$

$0.25 \mathrm{mg} / \mathrm{Kg}$

$0.25 \mathrm{mg} / \mathrm{Kg}$

2. $\mathrm{mg} / \mathrm{Kg}$

$0.65 \mathrm{mg} / \mathrm{Kg}$

$0.25 \mathrm{mg} / \mathrm{Kg}$

$0.25 \mathrm{mg} / \mathrm{Kg}$

ND - Not Detected

This report shall not be reproduced except in full, without the written approval of the laboratory. 
NEL LABORATORIES

CLIENT: Bechtel Nevada

PROJECT NAME: V557

PROJECT NUMBER: 17777
CLIENT ID: $\quad$ DURD \# 2-2

DATE SAMPLED: $12 / 8 / 98$

NEL SAMPLE ID: L9812133-02

TEST: TCLP-8 Metals

MATRIX: $\quad$ Solid

\begin{tabular}{|c|c|c|c|c|c|c|c|}
\hline PARAMETER & $\begin{array}{c}\text { RESULT } \\
\mathrm{mg} / \mathrm{L} \\
\end{array}$ & $\begin{array}{c}\text { REPORTING } \\
\text { LIMIT } \\
\end{array}$ & D. F. & METHOD & $\begin{array}{c}\text { TCLP/STLC } \\
\text { EXTRACTION } \\
\text { DATE } \\
\end{array}$ & DIGESTED & ANALYZED \\
\hline Arsenic & ND & $0.5 \mathrm{mg} / \mathrm{k}$ & 5 & EPA 6010 & $12 / 17 / 98$ & $12 / 18 / 98$ & $12 / 18 / 98$ \\
\hline Barium & ND & $5 . \mathrm{mg} / \mathrm{L}$ & 5 & EPA 6010 & $12 / 17 / 98$ & $12 / 18 / 98$ & $12 / 18 / 98$ \\
\hline Cadmium & ND & $0.05 \mathrm{mg} / \mathrm{L}$ & 5 & EPA 6010 & $12 / 17 / 98$ & $12 / 18 / 98$ & $12 / 18 / 98$ \\
\hline Chromium & ND & $0.05 \mathrm{mg} / \mathrm{L}$ & 5 & EPA 6010 & $12 / 17 / 98$ & $12 / 18 / 98$ & $12 / 18 / 98$ \\
\hline Lead & ND & $0.25 \mathrm{mg} / \mathrm{L}$ & 5 & EPA 6010 & $12 / 17 / 98$ & $12 / 18 / 98$ & $12 / 18 / 98$ \\
\hline Mercury & ND & $0.002 \mathrm{mg} / \mathrm{L}$ & 10 & EPA $7470 \mathrm{~A}$ & $12 / 17 / 98$ & $12 / 18 / 98$ & $12 / 18 / 98$ \\
\hline Selenium & ND & $0.5 \mathrm{mg} / \mathrm{L}$ & 5 & EPA 6010 & $12 / 17 / 98$ & $12 / 18 / 98$ & $12 / 18 / 98$ \\
\hline Silver & ND & $0.1 \mathrm{mg} / \mathrm{L}$ & 5 & EPA 6010 & $12 / 17 / 98$ & $12 / 18 / 98$ & $12 / 18 / 98$ \\
\hline
\end{tabular}

D.F. - Dilution Factor

ND - Not Detected

This report shall not be reproduced except in full, without the written approval of the laboratory. 
NEL LABORATORIES

$\begin{array}{llll}\text { CLIENT: } & \text { Bechtel Nevada } & \text { CLIENT ID: } & \text { DURD \# 2-3 } \\ \text { PROJECT ID: } & \text { V557/17777 } & \text { DATE SAMPLED: } & 12 / 8 / 98 \\ \text { PROJECT \#: } & 17777 & \text { NEL SAMPLE ID: } & \text { L9812133-03 }\end{array}$

TEST: $\quad$ TCLP by EPA 1311, July 1992 \& Semivolatile Organics by EPA Method 8270C, Dec. 1996

METHOD: EPA $8270 \quad$ TCLP EXTRACT DATE: $12 / 17 / 98$

MATRIX: Solid

EXTRACTED: $\quad 12 / 18 / 98$

DILUTION: 11 ANALYZED: $12 / 21 / 98$

\section{PARAMETER}

1.4-Dichlorobenzene (p-DCB)

2.4-Dinitrotoluene (DNT)

Hexachlorobenzene

Hexachlorobutadiene

Hexachloroethane

2-Methylphenol

Result

3.4-Methylphenol (isomeric pair)

\begin{tabular}{cc}
$\mathbf{m g} / \mathbf{L}$ \\
\hline ND \\
ND \\
ND \\
ND \\
ND \\
ND \\
ND \\
ND \\
ND \\
ND \\
ND \\
ND
\end{tabular}

\begin{tabular}{c}
$\begin{array}{c}\text { Reporting } \\
\text { Limit }\end{array}$ \\
\hline $0.1 \mathrm{mg} / \mathrm{L}$ \\
$0.1 \mathrm{mg} / \mathrm{L}$ \\
$0.1 \mathrm{mg} / \mathrm{L}$ \\
$0.1 \mathrm{mg} / \mathrm{L}$ \\
$0.1 \mathrm{mg} / \mathrm{L}$ \\
$0.1 \mathrm{mg} / \mathrm{L}$ \\
$0.1 \mathrm{mg} / \mathrm{L}$ \\
$0.1 \mathrm{mg} / \mathrm{L}$ \\
$0.1 \mathrm{mg} / \mathrm{L}$ \\
$0.1 \mathrm{mg} / \mathrm{L}$ \\
$0.1 \mathrm{mg} / \mathrm{L}$ \\
$0.1 \mathrm{mg} / \mathrm{L}$
\end{tabular}

Nitrohenzene

Pentachlorophenol

Pyridine

$0.1 \mathrm{mg} / \mathrm{L}$

\section{QUALITY CONTROL DATA:}

Surrogate

2.4.6-Tribromophenol

2-Fluorobiphenyl

2-Fluorophenol

Nitrobenzene-d5

p-Terphenyl-d 14

Phenol-d5
$\%$ Recovery

93

93

55

87

106

34
Acceptable Range

$10-123$

$43-116$

$21-100$

$35-114$

$33-141$

$10-94$ 
CLIENT: Bechtel Nevada

PROJECT NAME: V557

PROJECT NUMBER: 17777
CLIENT ID: DURD \# 2-3

DATE SAMPLED: $\quad 12 / 8 / 98$

NEL SAMPLE ID: L9812133-03

TEST: Nitroaromatics and Nitroamines by EPA 8330

MATRIX: Solid

DILUTION: 1

\section{PARAMETER}

1.3-DNB

HMX

Nitrobenzene

2-Nitrotoluene

3-Nitrotoluene

4-Nitrotoluene

RDX

Tetryl

1.3.5-TNB

2.4,6-TNT
EXTRACTED: $12 / 18 / 98$

ANALYZED: $12 / 22 / 98$

\begin{tabular}{l} 
Result \\
\hline ND \\
ND \\
ND \\
ND \\
ND \\
ND \\
ND \\
ND \\
ND \\
ND
\end{tabular}

ANALYST: YW

Reporting

Limit

$0.25 \mathrm{mg} / \mathrm{Kg}$

2. $\mathrm{mg} / \mathrm{Kg}$

$0.25 \mathrm{mg} / \mathrm{Kg}$

$0.25 \mathrm{mg} / \mathrm{Kg}$

$0.25 \mathrm{mg} / \mathrm{Kg}$

$0.25 \mathrm{mg} / \mathrm{Kg}$

2. $\mathrm{mg} / \mathrm{Kg}$

$0.65 \mathrm{mg} / \mathrm{Kg}$

$0.25 \mathrm{mg} / \mathrm{Kg}$

$0.25 \mathrm{mg} / \mathrm{Kg}$

ND - Not Detected

This report shall not be reproduced except in full, without the written approval of the laboratory. 
CLIENT: Bechtel Nevada

PROJECT NAME: V557

PROJECT NUMBER: 17777
CLIENT ID: DURD \# 2-3

DATE SAMPLED: $12 / 8 / 98$

NEL SAMPLE ID: L9812133-03

TEST: $\quad$ TCLP-8 Metals

MATRIX: Solid

\begin{tabular}{|c|c|c|c|c|c|c|c|}
\hline PARAMETER & $\begin{array}{c}\text { RESULT } \\
\mathrm{mg} / \mathrm{L} \\
\end{array}$ & $\begin{array}{c}\text { REPORTING } \\
\text { LIMIT } \\
\end{array}$ & D. F. & METHOD & $\begin{array}{c}\text { TCLP/STLC } \\
\text { EXTRACTION } \\
\text { DATE } \\
\end{array}$ & DIGESTED & ANALYZED \\
\hline Arsenic & ND & $0.5 \mathrm{mg} / \mathrm{t}$ & 5 & EPA 6010 & $12 / 17 / 98$ & $12 / 18 / 98$ & $12 / 18 / 98$ \\
\hline Barium & ND & 5. $\mathrm{mg} / \mathrm{L}$ & 5 & EPA 6010 & $12 / 17 / 98$ & $12 / 18 / 98$ & $12 / 18 / 98$ \\
\hline Cadmium & ND & $0.05 \mathrm{mg} / \mathrm{L}$ & 5 & EPA 6010 & $12 / 17 / 98$ & $12 / 18 / 98$ & $12 / 18 / 98$ \\
\hline Chromium & ND & $0.05 \mathrm{mg} / \mathrm{L}$ & 5 & EPA 6010 & $12 / 17 / 98$ & $12 / 18 / 98$ & $12 / 18 / 98$ \\
\hline Lead & ND & $0.25 \mathrm{mg} / \mathrm{L}$ & 5 & EPA 6010 & $12 / 17 / 98$ & $12 / 18 / 98$ & $12 / 18 / 98$ \\
\hline Mercury & ND & $0.002 \mathrm{mg} / \mathrm{L}$ & 10 & EPA 7470A & $12 / 17 / 98$ & $12 / 18 / 98$ & $12 / 18 / 98$ \\
\hline Selenium & ND & $0.5 \mathrm{mg} / \mathrm{L}$ & 5 & EPA 6010 & $12 / 17 / 98$ & $12 / 18 / 98$ & $12 / 18 / 98$ \\
\hline Silver & ND & $0.1 \mathrm{mg} / \mathrm{L}$ & 5 & EPA 6010 & $12 / 17 / 98$ & $12 / 18 / 98$ & $12 / 18 / 98$ \\
\hline
\end{tabular}

D.F. - Dilution Factor

ND - Not Detected

This report shall not be reproduced except in full, without the written approval of the laboratory. 
NEL LABORATORIES

$\begin{array}{llll}\text { CLIENT: } & \text { Bechtel Nevada } & \text { CLIENT ID: } & \text { DURD \# 2-4 } \\ \text { PROJECT ID: } & \text { V557/17777 } & \text { DATE SAMPLED: } & 12 / 8 / 98 \\ \text { PROJECT \#: } & 17777 & \text { NEL SAMPLE ID: } & \text { L9812133-04 }\end{array}$

TEST: $\quad$ TCLP by EPA 1311, July 1992 \& Semivolatile Organics by EPA Method 8270C, Dec. 1996

METHOD: $\quad$ EPA 8270

MATRIX: Solid

DILUTION: $\quad 1$

TCLP EXTRACT DATE: $12 / 17 / 98$

EXTRACTED: $\quad 12 / 18 / 98$

ANALYZED: $\quad 12 / 21 / 98$

\section{PARAMETER}

1.4-Dichlorobenzene (p-DCB)

2.4-Dinitrotoluene (DNT)

Hexachlorobenzene

Hexachlorobutadiene

Hexachloroethane

2-Methylphenol

3.4-Methylphenol (isomeric pair)

Nitrobenzene

Pentachlorophenol

Pyridine

2.4.5-Trichlorophenol

2.4.6-Trichlorophenol

\begin{tabular}{cc}
$\begin{array}{c}\text { Result } \\
\text { mg/L }\end{array}$ \\
\cline { 1 - 2 } ND \\
ND & J \\
ND & \\
ND & \\
ND & \\
ND & J \\
ND & J \\
ND & \\
ND & J \\
ND & \\
ND & J \\
ND & J
\end{tabular}

\begin{tabular}{c}
$\begin{array}{c}\text { Reporting } \\
\text { Limit }\end{array}$ \\
\hline $0.1 \mathrm{mg} / \mathrm{L}$ \\
$0.1 \mathrm{mg} / \mathrm{L}$ \\
$0.1 \mathrm{mg} / \mathrm{L}$ \\
$0.1 \mathrm{mg} / \mathrm{L}$ \\
$0.1 \mathrm{mg} / \mathrm{L}$ \\
$0.1 \mathrm{mg} / \mathrm{L}$ \\
$0.1 \mathrm{mg} / \mathrm{L}$ \\
$0.1 \mathrm{mg} / \mathrm{L}$ \\
$0.1 \mathrm{mg} / \mathrm{L}$ \\
$0.1 \mathrm{mg} / \mathrm{L}$ \\
$0.1 \mathrm{mg} / \mathrm{L}$ \\
$0.1 \mathrm{mg} / \mathrm{L}$
\end{tabular}

\section{JUALITY CONTROL DATA:}

Surrogate

2.4,6-Tribromophenol

-Fluorobiphenyl

2-Fluorophenol

vitrobenzene-d5

. -Terphenyl-d 14

Phenol-d5
$\%$ Recovery

0

83

0

78

72

0

\section{Acceptable Range}

$10-123$

$43-116$

$21-100$

$35-114$

$33-141$

$10-94$ 
CLIENT: Bechtel Nevada

PROJECT NAME: V557

PROJECT NUMBER: 17777
CLIENT ID: DURD \# 2-4

DATE SAMPLED: $12 / 8 / 98$

NEL SAMPLE ID: $\quad$ L9812133-04

TEST: Nitroaromatics and Nitroamines by EPA 8330

MATRIX: Solid

DILUTION: 1
EXTRACTED: $12 / 18 / 98$

ANALYZED: $12 / 22 / 98$

ANALYST: YW

\section{PARAMETER}

1.3-DNB

HMX

Nitrobenzene -

2-Nitrotoluene

3-Nitrotoluene

4-Nitrotoluene

RDX

Tetryl

1,3.5-TNB

2.4,6-TNT

\section{Result}

ND

ND

ND

ND

ND

ND

ND

ND

ND

ND
Reporting

Limit

$0.25 \mathrm{mg} / \mathrm{Kg}$

2. $\mathrm{mg} / \mathrm{Kg}$

$0.25 \mathrm{mg} / \mathrm{Kg}$

$0.25 \mathrm{mg} / \mathrm{Kg}$

$0.25 \mathrm{mg} / \mathrm{kg}$

$0.25 \mathrm{mg} / \mathrm{Kg}$

2. $\mathrm{mg} / \mathrm{Kg}$

$0.65 \mathrm{mg} / \mathrm{Kg}$

$0.25 \mathrm{mg} / \mathrm{Kg}$

$0.25 \mathrm{mg} / \mathrm{Kg}$

ND - Not Detected

This report shall not be reproduced except in full, without the written approval of the laboratory. 
CLIENT: Bechtel Nevada

PROJECT NAME: V557

PROJECT NUMBER: 17777
CLIENT ID:

DATE SAMPLED: 12/8/98

NEL SAMPLE ID: L9812133-04

\section{TEST: \\ TCLP-8 Metals \\ MATRIX: \\ Solid}

\section{PARAMETER}

Arsenic

Barium

Cadmium

Chromium

Lead

Mercury

Selenium

Silver

\section{RESULT REPORTING} mg/L

ND

ND

ND

ND

ND

ND

ND

ND
LIMIT

$0.5 \mathrm{mg} / \mathrm{L}$

$$
\text { 5. } \mathrm{mg} / \mathrm{L}
$$

$0.05 \mathrm{mg} / \mathrm{L}$

$0.05 \mathrm{mg} / \mathrm{L}$

$0.25 \mathrm{mg} / \mathrm{L}$

$0.002 \mathrm{mg} / \mathrm{L}$

$0.5 \mathrm{mg} / \mathrm{L}$

$0.1 \mathrm{mg} / \mathrm{L}$

\section{TCLP/STLC}

EXTRACTION

D. F. METHOD

EPA 6010

DATE

DIGESTED ANALYZED

12/17/98

$12 / 18 / 98$

$12 / 18 / 98$

$12 / 17 / 98$.

$12 / 18 / 98$

$12 / 18 / 98$

EPA 6010

$12 / 17 / 98$

$12 / 18 / 98$

$12 / 18 / 98$

EPA 6010

$12 / 17 / 98$

$12 / 18 / 98$

$12 / 18 / 98$

EPA 6010

$12 / 17 / 98$

$12 / 18 / 98$

$12 / 18 / 98$

EPA $7470 A$

$12 / 17 / 98$

$12 / 18 / 98$

$12 / 18 / 98$

EPA 6010

$12 / 17 / 98$

$12 / 18 / 98$

$12 / 18 / 98$

$12 / 18 / 98$

EPA 6010

$12 / 17 / 98$

$12 / 18 / 98$

D.F. - Dilution Factor

ND - Not Detected

This report shall not be reproduced except in full, without the written approval of the laboratory. 


$\begin{array}{llll}\text { CLIENT: } & \text { Bechtel Nevada } & \text { CLIENT ID: } & \text { DURD \# 2-5 } \\ \text { PROJECT ID: } & \text { V557/17777 } & \text { DATE SAMPLED: } 12 / 8 / 98 \\ \text { PROJECT \#: } & 17777 & \text { NEL SAMPLE ID: } \text { L9812133-05 }\end{array}$

TEST: $\quad$ TCLP by EPA 1311, July 1992 \& Semivolatile Organics by EPA Method 8270C, Dec. 1996

$\begin{array}{llll}\text { METHOD: } & \text { EPA } 8270 & \text { TCLP EXTRACT DATE: } 12 / 17 / 98 \\ \text { MATRIX: } & \text { Solid } & \text { EXTRACTED: } & 12 / 18 / 98 \\ \text { DILUTION: } & 1 & \text { ANALYZED: } & 12 / 21 / 98\end{array}$

\section{PARAMETER}

1.4-Dichlorobenzene (p-DCB)

2.4-Dinitrotoluene (DNT)

Hexachlorobenzene

Hexachlorobutadiene

Hexachloroethane

2-Methylphenol

3.4-Methylphenol (isomeric pair)

Nitrobenzene

Pentachlorophenol

Pyridine

2.4.5-Trichlorophenol

2.4.6-Trichlorophenol

$\begin{array}{cc}\begin{array}{c}\text { Result } \\ \text { mg/L }\end{array} & \begin{array}{c}\text { Reporting } \\ \text { Limit }\end{array} \\ \text { ND } & 0.1 \mathrm{mg} / \mathrm{L} \\ \text { ND } & 0.1 \mathrm{mg} / \mathrm{L} \\ \text { ND } & 0.1 \mathrm{mg} / \mathrm{L} \\ \text { ND } & 0.1 \mathrm{mg} / \mathrm{L} \\ \text { ND } & 0.1 \mathrm{mg} / \mathrm{L} \\ \text { ND } & 0.1 \mathrm{mg} / \mathrm{L} \\ \text { ND } & 0.1 \mathrm{mg} / \mathrm{L} \\ \text { ND } & 0.1 \mathrm{mg} / \mathrm{L} \\ \text { ND } & 0.1 \mathrm{mg} / \mathrm{L} \\ \text { ND } & 0.1 \mathrm{mg} / \mathrm{L} \\ \text { ND } & 0.1 \mathrm{mg} / \mathrm{L} \\ \text { ND } & 0.1 \mathrm{mg} / \mathrm{L}\end{array}$

QUALITY CONTROL DATA:

\section{Surrogate}

2,4,6-Tribromophenol

2-Fluorobiphenyl

2-Fluorophenol

Nitrobenzene-d5

p-Terphenyl-d14

Phenol-d5

\section{$\%$ Recovery}

86

92

58

84

103

36
Acceptable Range

$10-123$

$43-116$

$21-100$

$35-114$

$33-141$

$10-94$ 
CLIENT: Bechtel Nevada

PROJECT NAME: $\quad$ V557

PROJECT NUMBER: 17777
CLIENT ID: DURD \# 2-5

DATE SAMPLED: $12 / 8 / 98$

NEL SAMPLE ID: L9812133-05

TEST: Nitroaromatics and Nitroamines by EPA 8330

MATRIX: Solid

DILUTION: 1

\section{PARAMETER}

1.3-DNB

HMX

Nitrobenzene

2-Nitrotoluene

3-Nitrotoluene

4-Nitrotoluene

RDX

Tetryl

1.3.5-TNB

2.4,6-TNT
EXTRACTED: $12 / 18 / 98$

ANALYZED: $12 / 22 / 98$

\begin{tabular}{l} 
Result \\
\hline ND \\
ND \\
ND \\
ND \\
ND \\
ND \\
ND \\
ND \\
ND \\
ND
\end{tabular}

ANALYST: YW

Reporting

Limit

$0.25 \mathrm{mg} / \mathrm{Kg}$

2. $\mathrm{mg} / \mathrm{Kg}$

$0.25 \mathrm{mg} / \mathrm{Kg}$

$0.25 \mathrm{mg} / \mathrm{Kg}$

$0.25 \mathrm{mg} / \mathrm{Kg}$

$0.25 \mathrm{mg} / \mathrm{Kg}$

2. $\mathrm{mg} / \mathrm{Kg}$

$0.65 \mathrm{mg} / \mathrm{Kg}$

$0.25 \mathrm{mg} / \mathrm{Kg}$

$0.25 \mathrm{mg} / \mathrm{Kg}$

ND - Not Detected

This report shall not be reproduced except in full, without the written approval of the laboratory. 
CLIENT: Bechtel Nevada

PROJECT NAME: V557

PROJECT NUMBER: 17777
CLIENT ID:

DATE SAMPLED: $12 / 8 / 98$

NEL SAMPLE ID: L9812133-05

\section{TEST: $\quad$ TCLP-8 Metals \\ MATRIX: Solid}

\begin{tabular}{|c|c|c|c|c|c|c|c|}
\hline PARAMETER & $\begin{array}{c}\text { RESULT } \\
\mathrm{mg} / \mathrm{L} \\
\end{array}$ & $\begin{array}{c}\text { REPORTING } \\
\text { LIMIT } \\
\end{array}$ & D. $\mathbf{F}$. & METHOD & $\begin{array}{c}\text { TCLP/STLC } \\
\text { EXTRACTION } \\
\text { DATE } \\
\end{array}$ & DIGESTED & ANALYZED \\
\hline Arsenic & ND & $0.5 \mathrm{mg} / \mathrm{t}$ & 5 & EPA 6010 & $12 / 17 / 98$ & $12 / 18 / 98$ & $12 / 18 / 98$ \\
\hline Barium & ND & $5 . \mathrm{mg} / \mathrm{L}$ & 5 & EPA 6010 & $12 / 17 / 98$ & $12 / 18 / 98$ & $12 / 18 / 98$ \\
\hline Cadmium & ND & $0.05 \mathrm{mg} / \mathrm{L}$ & 5 & EPA 6010 & $12 / 17 / 98$ & $12 / .18 / 98$ & $12 / 18 / 98$ \\
\hline Chromium & ND & $0.05 \mathrm{mg} / \mathrm{L}$ & 5 & EPA 6010 & $12 / 17 / 98$ & $12 / 18 / 98$ & $12 / 18 / 98$ \\
\hline Lead & ND & $0.25 \mathrm{mg} / \mathrm{L}$ & 5 & EPA 6010 & $12 / 17 / 98$ & $12 / 18 / 98$ & $12 / 18 / 98$ \\
\hline Mercury & ND & $0.002 \mathrm{mg} / \mathrm{L}$ & 10 & EPA $7470 A$ & $12 / 17 / 98$ & $12 / 18 / 98$ & $12 / 18 / 98$ \\
\hline Selenium & ND & $0.5 \mathrm{mg} / \mathrm{L}$ & 5 & EPA 6010 & $12 / 17 / 98$ & $12 / 18 / 98$ & $12 / 18 / 98$ \\
\hline Silver & ND & $0.1 \mathrm{mg} / \mathrm{L}$ & 5 & EPA 6010 & $12 / 17 / 98$ & $12 / 18 / 98$ & $12 / 18 / 98$ \\
\hline
\end{tabular}

D.F. - Dilution Factor

ND - Not Detected

This report shall not be reproduced except in full, without the written approval of the laboratory. 
NEL LABORATORIES

$\begin{array}{llll}\text { CLIENT: } & \text { Bechtel Nevada } & \text { CLIENT ID: } & \text { DURD \# 2-6 } \\ \text { PROJECT ID: } & \text { V557/17777 } & \text { DATE SAMPLED: } 12 / 8 / 98 \\ \text { PROJECT \#: } & 17777 & \text { NEL SAMPLE ID: } \text { L9812133-06 } \\ & & & \\ \text { TEST: } & \text { TCLP by EPA 1311, July } 1992 ~ \& \text { Semivolatile Organics by EPA Method 8270C, Dec. } 1996 \\ \text { METHOD: } & \text { EPA } 8270 & \text { TCLP EXTRACT DATE: } 12 / 17 / 98 \\ \text { MATRIX: } & \text { Solid } & \text { EXTRACTED: } & 12 / 18 / 98 \\ \text { DILUTION: } & 1 & \text { ANALYZED: } & 12 / 21 / 98\end{array}$

\section{PARAMETER}

1.4-Dichlorobenzene ( $p$-DCB)

2.4-Dinitrotoluene (DNT)

Hexachlorobenzene

Hexachlorobutadiene

Hexachloroethane

2-Methylphenol

\begin{tabular}{c} 
Result \\
mg/L \\
\cline { 2 - 2 } \\
ND \\
ND J \\
ND \\
ND \\
ND \\
ND \\
ND \\
ND \\
ND J \\
ND \\
ND \\
ND
\end{tabular}

3.4-Methylphenol (isomeric pair)

Nitrobenzene

Pentachlorophenol

Pyridine

2.4.5-Trichlorophenol

2.4.6-Trichlorophenol

pair)

\section{ZUALITY CONTROL DATA:}

\section{Surrogate}

:.4.6-Tribromophenol

-Fluorobiphenyl

2-Fluorophenol

vitrobenzene-d5

i-Terphenyl-d14

Phenol-d5

\section{\% Recovery}

89

93

65

89

103

39
Acceptable Range

$10-123$

$43-116$

$21-100$

$35-114$

$33-141$

$10-94$

'D - Not Detected

his report shall not be reproduced except in full, without the written approval of the laboratory. 

CLIENT: Bechtel Nevada
PROJECT NAME: V557
CLIENT ID: DURD \# 2-6
PROJECT NUMBER: 17777
DATE SAMPLED: $12 / 8 / 98$
NEL SAMPLE ID: L9812133-06

TEST: Nitroaromatics and Nitroamines by EPA 8330

MATRIX: Solid

DILUTION: 1
EXTRACTED: $12 / 18 / 98$

ANALYZED: $12 / 22 / 98$
ANALYST: YW

Reporting

Limit

$0.25 \mathrm{mg} / \mathrm{Kg}$

2. $\mathrm{mg} / \mathrm{Kg}$

$0.25 \mathrm{mg} / \mathrm{Kg}$

$0.25 \mathrm{mg} / \mathrm{Kg}$

$0.25 \mathrm{mg} / \mathrm{Kg}$

$0.25 \mathrm{mg} / \mathrm{Kg}$

2. $\mathrm{mg} / \mathrm{Kg}$

$0.65 \mathrm{mg} / \mathrm{Kg}$

$0.25 \mathrm{mg} / \mathrm{Kg}$

$0.25 \mathrm{mg} / \mathrm{Kg}$

ND - Not Detected

This report shall not be reproduced except in full, without the written approval of the laboratory. 
NEL LABORATORIES

CLIENT: Bechtel Nevada

PROJECT NAME: V557

PROJECT NUMBER: 17777
CLIENT ID: $\quad$ DURD \# 2-6

DATE SAMPLED: 12/8/98

NEL SAMPLE ID: L9812133-06

TEST: $\quad$ TCLP-8 Metals

MATRIX: . Solid

\section{PARAMETER}

Arsenic

Barium

Cadmium

Chromium

Lead

Mercury

Selenium

Silver

\section{RESULT REPORTING} mg/L

ND

ND

ND

ND

ND

ND

ND

ND

\section{LIMIT}

$0.5 \mathrm{mg} / \mathrm{k}$

5. $\mathrm{mg} / \mathrm{L}$

$0.05 \mathrm{mg} / \mathrm{L}$

$0.05 \mathrm{mg} / \mathrm{L}$

$0.25 \mathrm{mg} / \mathrm{L}$

$0.002 \mathrm{mg} / \mathrm{L}$

$0.5 \mathrm{mg} / \mathrm{L}$

$0.1 \mathrm{mg} / \mathrm{L}$
TCLPISTLC EXTRACTION

D.F. METHOD

5. EPA 6010 DATE

$12 / 17 / 98$

DIGESTED ANALYZED

$12 / 17 / 98$

$12 / 18 / 98$

$12 / 18 / 98$

EPA 6010

$12 / 17 / 98$

$12 / 18 / 98$

$12 / 18 / 98$

$12 / 18 / 98$

$12 / 18 / 98$

$12 / 17 / 98$

$12 / 18 / 98$

$12 / 18 / 98$

$12 / 18 / 98$

$12 / 17 / 98$

$12 / 18 / 98$

$12 / 18 / 98$

$12 / 17 / 98$

$12 / 17 / 98$

$12 / 18 / 98$

$12 / 18 / 98$

$12 / 18 / 98$

$12 / 18 / 98$

$12 / 18 / 98$

D.F. - Dilution Factor

ND - Not Detected

This report shall not be reproduced except in full, without the written approval of the laboratory. 
CLIENT: Bechtel Nevada

PROJECT NAME: V557

PROJECT NUMBER: 17777
CLIENT ID: DURD \# 2-ER

DATE SAMPLED: $12 / 8 / 98$

NEL SAMPLE ID: L9812133-09

TEST: Nitroaromatics and Nitroamines by EPA 8330

MATRIX: Aqueous

DILUTION: 1

PARAMETER

2-Am-DNT \& 4-Am-DNT

1.3-DNB

2.4-DNT \& 2,6-DNT

HMX

Nitrobenzene

2-Nitrotoluene

3-Nitrotoluene

4-Nitrotoluene

RDX

Tetryl

1.3.5-TNB

2.4.6-TNT
EXTRACTED: $12 / 15 / 98$

ANALYZED: $12 / 22 / 98$

Result

ND

ND

ND

ND

ND

ND

ND

ND

ND

ND J

ND

ND
ANALYST: YW

Reporting

Limit

$1.5 \mu \mathrm{g} / \mathrm{L}$

$0.25 \mu \mathrm{g} / \mathrm{L}$

$1.5 \mu \mathrm{g} / \mathrm{L}$

2. $\mu \mathrm{g} / \mathrm{L}$

$0.25 \mu \mathrm{g} / \mathrm{L}$

$0.25 \mu \mathrm{g} / \mathrm{L}$

$0.25 \mu \mathrm{g} / \mathrm{L}$

$0.25 \mu \mathrm{g} / \mathrm{L}$

2. $\mu \mathrm{g} / \mathrm{L}$

$0.65 \mu \mathrm{g} / \mathrm{L}$

$0.25 \mu \mathrm{g} / \mathrm{L}$

$0.25 \mu \mathrm{g} / \mathrm{L}$

ND - Not Detected

This report shall not be reproduced except in full, without the written approval of the laboratory. 
CLIENT: Bechtel Nevada

PROJECT NAME: V557

PROJECT NUMBER: 17777
CLIENT ID: $\quad$ DURD \# 2-ER

DATE SAMPLED: $12 / 8 / 98$

NEL SAMPLE ID: L9812133-09

TEST: $\quad$ TCLP-8 Metals

MATRIX: Aqueous

\begin{tabular}{|c|c|c|c|c|c|c|c|}
\hline PARAMETER & $\begin{array}{c}\text { RESULT } \\
\text { mg/L } \\
\end{array}$ & $\begin{array}{l}\text { REPORTING } \\
\text { LIMIT }\end{array}$ & D. F. & METHOD & $\begin{array}{c}\text { TCLP/STLC } \\
\text { EXTRACTION } \\
\text { DATE } \\
\end{array}$ & DIGESTED & ANALYZED \\
\hline Arsenic & ND & $0.1 \mathrm{mg} / \mathrm{k}$ & 1 & EPA 6010 & NA & $12 / 18 / 98$ & $12 / 18 / 98$ \\
\hline Barium & ND & $1 . \mathrm{mg} / \mathrm{L}$ & 1 & EPA 6010 & NA & $12 / 18 / 98$ & $12 / 18 / 98$ \\
\hline Cadmium & ND & $0.01 \mathrm{mg} / \mathrm{L}$ & 1 & EPA 6010 & $\mathrm{NA}$ & $12 / 18 / 98$ & $12 / 18 / 98$ \\
\hline Chromium & ND & $0.01 \mathrm{mg} / \mathrm{L}$ & 1 & EPA 6010 & NA & $12 / 18 / 98$ & $12 / 18 / 98$ \\
\hline Lead & ND & $0.05 \mathrm{mg} / \mathrm{L}$ & 1 & EPA 6010 & NA & $12 / 18 / 98$ & $12 / 18 / 98$ \\
\hline Mercury & ND & $0.002 \mathrm{mg} / \mathrm{L}$ & 10 & EPA 7470A & NA & $12 / 18 / 98$ & $12 / 18 / 98$ \\
\hline Selenium & ND & $0.1 \mathrm{mg} / \mathrm{L}$ & 1 & EPA 6010 & NA & $12 / 18 / 98$ & $12 / 18 / 98$ \\
\hline Silver & ND & $0.02 \mathrm{mg} / \mathrm{L}$ & 1 & EPA 6010 & NA & $12 / 18 / 98$ & $12 / 18 / 98$ \\
\hline
\end{tabular}

D.F. - Dilution Factor

ND - Not Detected

This report shall not be reproduced except in full, without the written approval of the laboratory. 


\begin{tabular}{llll}
\hline CLIENT: & Bechtel Nevada & CLIENT ID: & Method Blank \\
PROJECT ID: & Soil Testing Apex Industrial Use Zone/3745 & DATE SAMPLED: & NA \\
PROJECT \#: & $37456-002-16$ & NEL SAMPLE ID: & 121598-E1-BLK
\end{tabular}

TEST:

METHOD:

TCLP by EPA 1311, July 1992 \& Semivolatile Organics by EPA Method 8270C, Dec. 1996

MATRIX:

EPA 8270

TCLP Extract
TCLP EXTRACT DATE: NA

EXTRACTED: $\quad 12 / 15 / 98$

ANALYZED: $\quad 12 / 29 / 98$

\begin{tabular}{lcc}
\hline PARAMETER & $\begin{array}{c}\text { Result } \\
\mathbf{m g} / \mathrm{L}\end{array}$ & $\begin{array}{c}\text { Reporting } \\
\text { Limit }\end{array}$ \\
\cline { 2 - 3 } 1.4-Dichlorobenzene (p-DCB) & $\mathrm{ND}$ & $0.1 \mathrm{mg} / \mathrm{L}$ \\
2.4-Dinitrotoluene(DNT) & $\mathrm{ND}$ & $0.1 \mathrm{mg} / \mathrm{h}$ \\
Hexachlorobenzene & $\mathrm{ND}$ & $0.1 \mathrm{mg} / \mathrm{L}$ \\
Hexachlorobutadiene & $\mathrm{ND}$ & $0.1 \mathrm{mg} / \mathrm{L}$ \\
Hexachloroethane & $\mathrm{ND}$ & $0.1 \mathrm{mg} / \mathrm{h}$ \\
2-Methylphenol & $\mathrm{ND}$ & $0.1 \mathrm{mg} / \mathrm{L}$ \\
Nitrobenzene & $\mathrm{ND}$ & $0.1 \mathrm{mg} / \mathrm{h}$ \\
Pentachlorophenol & $\mathrm{ND}$ & $0.1 \mathrm{mg} / \mathrm{L}$ \\
P.ridine & $\mathrm{ND}$ & $0.1 \mathrm{mg} / \mathrm{L}$ \\
2.4.5-Trichlorophenol & $\mathrm{ND}$ & $0.1 \mathrm{mg} / \mathrm{h}$ \\
2.4.6-Trichlorophenol & $\mathrm{ND}$ & $0.1 \mathrm{mg} / \mathrm{h}$
\end{tabular}

QUALITY CONTROL DATA:

\section{Surrogate}

2.4.6-Tribromophenol

2-Fluorobiphenyl

2-Fluorophenol

Nitrobenzene-d5

p-Terphenyl-d 14

Phenol-d5

ND - Not Detected
\% Recovery

54

84

48

77

101

28
Acceptable Range

$10-123$

$43-116$

$21-100$

$35-114$

$33-141$

$10-94$

This report shall not be reproduced except in full, without the written approval of the laboratory. 


\begin{tabular}{llll}
\hline CLIENT: & Bechtel Nevada & CLIENT ID: & Method Blank \\
PROJECT ID: & Soil Testing Apex Industrial Use Zone/3745 & DATE SAMPLED: & NA \\
PROJECT \#: & $37456-002-16$ & NEL SAMPLE ID: & 121898-E3-TCLP-BLK
\end{tabular}

TEST:

METHOD:

TCLP by EPA 1311, July 1992 \& Semivolatile Organies by EPA Method 8270C, Dec. 1996

MATRIX:

EPA 8270

TCLP Extract

TCLP EXTRACT DATE: $12 / 17 / 98$

EXTRACTED:

$12 / 18 / 98$

ANALYZED:

$12 / 21 / 98$

\section{PARAMETER}

1.4-Dichlorobenzene ( $\mathrm{p}-\mathrm{DCB})$

2.4-Dinitrotoluene (DNT)

Hexachlorobenzene

Hexachlorobutadiene

Hexachloroethane

2-Methylphenol

Nitrobenzene

Pentachlorophenol

Pyridine

2.4.5-Trichlorophenol

2.4.6-Trichlorophenol

\begin{tabular}{c}
$\begin{array}{c}\text { Result } \\
\text { mg/L }\end{array}$ \\
\hline ND \\
ND \\
ND \\
ND \\
ND \\
ND \\
ND \\
ND \\
ND \\
ND \\
ND
\end{tabular}

\% Recovery

15

44

14

43

45

14
Reporting

Limit

$0.1 \mathrm{mg} / \mathrm{L}$

$0.1 \mathrm{mg} / \mathrm{L}$

$0.1 \mathrm{mg} / \mathrm{L}$

$0.1 \mathrm{mg} / \mathrm{L}$

$0.1 \mathrm{mg} / \mathrm{L}$

$0.1 \mathrm{mg} / \mathrm{L}$

$0.1 \mathrm{mg} / \mathrm{L}$

$0.1 \mathrm{mg} / \mathrm{L}$

$0.1 \mathrm{mg} / \mathrm{L}$

$0.1 \mathrm{mg} / \mathrm{l}$

$0.1 \mathrm{mg} / \mathrm{L}$

\section{Surrogate}

3.4.6-Tribromophenol

?-Fluorobiphenyl

2-Fluorophenol

vitrobenzene-d5

-Terphenyl-d14

Phenol-d5

VD - Not Detected

his report shall not be reproduced except in full, without the written approval of the laboratory. 
CLIENT: $\quad$ Bechtel Nevada

PROJECT ID: V557/17777

PROJECT \#: $\quad 17777$
CLIENT ID:

DATE SAMPLED: NA

NEL SAMPLE ID: $\quad$ 121598-H1-BLK

TEST: $\quad$ Nitroaromatics and Nitroamines by EPA 8330

METHOD: - EPA 8330

MATRIX: Aqueous

ANALYST: $\quad Y W$

EXTRACTED: $\quad 12 / 15 / 98$

ANALYZED: $\quad 12 / 22 / 98$

PARAMETER

2-Am-DNT \& 4-Am-DNT

1.3-DNB

2,4-DNT \& 2,6-DNT

HMX

Nitrobenzene

2-Nitrotoluene

3-Nitrotoluene

4-Nitrotoluene

RDX

Tetryl

1.3.5-TNB

2.4.6-TNT

\section{Result}

ND

ND

ND

ND

ND

ND

ND

ND

ND

ND

ND

ND
Reporting

Limit

$0.25 \mu \mathrm{g} / \mathrm{L}$

$1.5 \mu \mathrm{g} / \mathrm{L}$

2. $\mu \mathrm{g} / \mathrm{L}$

$0.25 \mu \mathrm{g} / \mathrm{L}$

$0.25 \mu \mathrm{g} / \mathrm{L}$

$0.25 \mu \mathrm{g} / \mathrm{L}$

$0.25 \mu \mathrm{g} / \mathrm{L}$

2. $\mu \mathrm{g} / \mathrm{L}$

$0.65 \mu \mathrm{g} / \mathrm{L}$

$0.25 \mu \mathrm{g} / \mathrm{L}$

$0.25 \mu \mathrm{g} / \mathrm{L}$

ND - Not Detected

This report shall not be reproduced except in full, without the written approval of the laboratory. 
NEL LABORATORIES

$\begin{array}{llll}\text { CLIENT: } & \text { Bechtel Nevada } & \text { CLIENT ID: } & \text { Method Blank } \\ \text { PROJECT ID: } & \text { V557/17777 } & \text { DATE SAMPLED: NA } \\ \text { PROJECT \#: } & 17777 & \text { NEL SAMPLE ID: } 121898 \text {-H1-BLK }\end{array}$

TEST: $\quad$ Nitroaromaties and Nitroamines by EPA 8330

METHOD: $\quad$ EPA 8330

MATRIX: $\quad$ Solid

ANALYST: $\quad$ YW

EXTRACTED: ' $12 / 18 / 98$

ANALYZED: $\quad 12 / 21 / 98$

\section{PARAMETER}

1.3-DNB

HMX

Nitrobenzene

2-Nitrotoluene

3-Nitrotoluene

4-Nitrotoluene

RDX

Tetryl

1.3.5-TNB

2.4.6-TNT

\section{Result}

ND

ND

ND

ND

ND

ND

ND

ND

ND

ND
Reporting

$\frac{\text { Limit }}{0.25 \mathrm{mg} / \mathrm{K}^{\prime} \mathrm{g}}$

2. $\mathrm{mg} / \mathrm{Kg}$

$0.25 \mathrm{mg} / \mathrm{Kg}$

$0.25 \mathrm{mg} / \mathrm{Kg}$

$0.25 \mathrm{mg} / \mathrm{Kg}$

$0.25 \mathrm{mg} / \mathrm{Kg}$

2. $\mathrm{mg} / \mathrm{Kg}$

$0.65 \mathrm{mg} / \mathrm{Kg}$

$0.25 \mathrm{mg} / \mathrm{Kg}$

$0.25 \mathrm{mg} / \mathrm{kg}$

ND - Not Detected

This report shall not be reproduced except in full, without the written approval of the laboratory. 
CLIENT:

Bechtel Nevada

PROJECT ID: V557/17777

PROJECT \#: $\quad 17777$
CLIENT ID:

DATE SAMPLED: NA

NEL SAMPLE ID: L12133i-09A-BLK

TEST: $\quad$ TCLP by EPA 1311, July $1992 \& 7$ Metals by EPA 6010A, July 1992

MATRIX: TCLP Extract

\begin{tabular}{|c|c|c|c|c|c|c|c|}
\hline \multirow[b]{2}{*}{ PARAMETER } & \multirow[b]{2}{*}{ RESULT } & \multirow{2}{*}{$\begin{array}{c}\text { REPORTING } \\
\text { LIMIT } \\
\end{array}$} & \multicolumn{4}{|c|}{$\begin{array}{c}\text { TCLP/STLC } \\
\text { EXTRACTION }\end{array}$} & \multirow[b]{2}{*}{ ANALYZED } \\
\hline & & & D. F. & METHOD & DATE & DIGESTED & \\
\hline Arsenic & ND & $0.1 \mathrm{mg} / \mathrm{L}$ & 1 & EPA 6010 & NA & $12 / 18 / 98$ & $12 / 18 / 98$ \\
\hline Barium & ND & $1 \mathrm{mg} / \mathrm{L}$ & 1 & EPA 6010 & NA & $12 / 18 / 98$ & $12 / 18 / 98$ \\
\hline Cadmium - & ND & $0.01 \mathrm{mg} / \mathrm{L}$ & 1 & EPA 6010 & $\mathrm{NA}$ & $12 / 18 / 98$ & $12 / 18 / 98$ \\
\hline Chromium & ND & $0.01 \mathrm{mg} / \mathrm{L}$ & 1 & EPA 6010 & $\mathrm{NA}$ & $12 / 18 / 98$ & $12 / 18 / 98$ \\
\hline Lead & ND & $0.05 \mathrm{mg} / \mathrm{L}$ & 1 & EPA 6010 & $\mathrm{NA}$ & $12 / 18 / 98$ & $12 / 18 / 98$ \\
\hline Selenium & ND & $0.1 \mathrm{mg} / \mathrm{L}$ & 1 & EPA 6010 & NA & $12 / 18 / 98$ & $12 / 18 / 98$ \\
\hline Silver & ND & $0.02 \mathrm{mg} / \mathrm{L}$ & 1 & EPA 6010 & NA & $12 / 18 / 98$ & $12 / 18 / 98$ \\
\hline
\end{tabular}

D.F. - Dilution Factor

ND - Not Detected

This report shall not be reproduced except in full, without the written approval of the laboratory. 
NEL LABORATORIES

$\begin{array}{llll}\text { CLIENT: } & \text { Bechtel Nevada } & \text { CLIENT ID: } & \text { Method Blank } \\ \text { PROJECT ID: } & \text { V557/17777 } & \text { DATE SAMPLED: } & \text { NA } \\ \text { PROJECT \#: } & 17777 & \text { NEL SAMPLE ID: } & \text { L12133i-BLK }\end{array}$

TEST: $\quad$ TCLP by EPA 1311, July 1992 \& 7 Metals by EPA 6010A, July 1992

MATRIX: $\quad$ TCLP Extract

\begin{tabular}{|c|c|c|c|c|c|c|c|}
\hline \multirow[b]{2}{*}{ PARAMETER } & \multirow[b]{2}{*}{ RESULT } & \multirow{2}{*}{$\begin{array}{c}\text { REPORTING } \\
\text { LIMIT } \\
\end{array}$} & \multicolumn{5}{|c|}{$\begin{array}{c}\text { TCLP/STLC } \\
\text { EXTRACTION }\end{array}$} \\
\hline & & & D. F. & METHOD & DATE & DIGESTED & ANALYZED \\
\hline Arsenic & ND & $0.5 \mathrm{mg} / \mathrm{L}$. & 5 & EPA 6010 & $12 / 17 / 98$ & $12 / 18 / 98$ & $12 / 18 / 98$ \\
\hline Barium & ND & $5 \mathrm{mg} / \mathrm{L}$ & 5 & EPA 6010 & $12 / 17 / 98$ & $12 / 18 / 98$ & $12 / 18 / 98$ \\
\hline Cadmium & ND & $0.05 \mathrm{mg} / \mathrm{L}$ & 5 & EPA 6010 & $12 / 17 / 98$ & $12 / 18 / 98$ & $12 / 18 / 98$ \\
\hline Chromium & ND & $0.05 \mathrm{mg} / \mathrm{L}$ & 5 & EPA 6010 & $12 / 17 / 98$ & $12 / 18 / 98$ & $12 / 18 / 98$ \\
\hline Lead & ND & $0.25 \mathrm{mg} / \mathrm{L}$ & 5 & EPA 6010 & $12 / 17 / 98^{\circ}$ & $12 / 18 / 98$ & $12 / 18 / 98$ \\
\hline Selenium & ND & $0.5 \mathrm{mg} / \mathrm{L}$ & 5 & EPA 6010 & $12 / 17 / 98$ & $12 / 18 / 98$ & $12 / 18 / 98$ \\
\hline Silver & ND & $0.1 \mathrm{mg} / \mathrm{L}$ & 5 & EPA 6010 & $12 / 17 / 98$ & $12 / 18 / 98$ & $12 / 18 / 98$ \\
\hline
\end{tabular}

D.F. - Dilution Factor

ND - Not Detected

This report shall not be reproduced except in full, without the written approval of the laboratory. 
NEL LABORATORIES

$\begin{array}{llll}\text { CLIENT: } & \text { Bechtel Nevada } & \text { CLIENT ID: } & \text { Method Blank } \\ \text { PROJECT ID: } & \text { V557/17777 } & \text { DATE SAMPLED: NA } \\ \text { PROJECT \#: } & 17777 & \text { NEL SAMPLE ID: } & \text { L12133-Hg-BLK }\end{array}$

TEST: $\quad$ TCLP by EPA 1311, July 1992 \& Mercury by EPA 7470A, July 1992

MATRIX: $\quad$ TCLP EXtract

\begin{tabular}{llll}
\hline & \multicolumn{2}{c}{ TCLP/STLC } \\
$\frac{\text { PARAMETER }}{\text { Mercury }}$ & $\frac{\text { RESULT }}{\mathrm{ND}} \frac{\text { LIMIT }}{0.002 \mathrm{mg} / \mathrm{L} .}$ & $\frac{\text { D. F. }}{10}$ & $\frac{\text { METHOD }}{\text { EPA } 7470 \mathrm{~A}} \frac{\text { DATE }}{12 / 17 / 98} \frac{\text { DIGESTED }}{12 / 18 / 98} \frac{\text { ANALYZED }}{12 / 18 / 98}$
\end{tabular}

D.F. - Dilution Factor

ND - Not Detected

This report shall not be reproduced except in full, without the written approval of the laboratory. 


\section{CLIENT: Bechtel Nevada \\ PROJECT ID: V557/17777 \\ PROJECT \#: $\quad 17777$}

TEST: Nitroaromatics and Nitroamines by EPA 8330

MATRIX: $\quad$ Solid

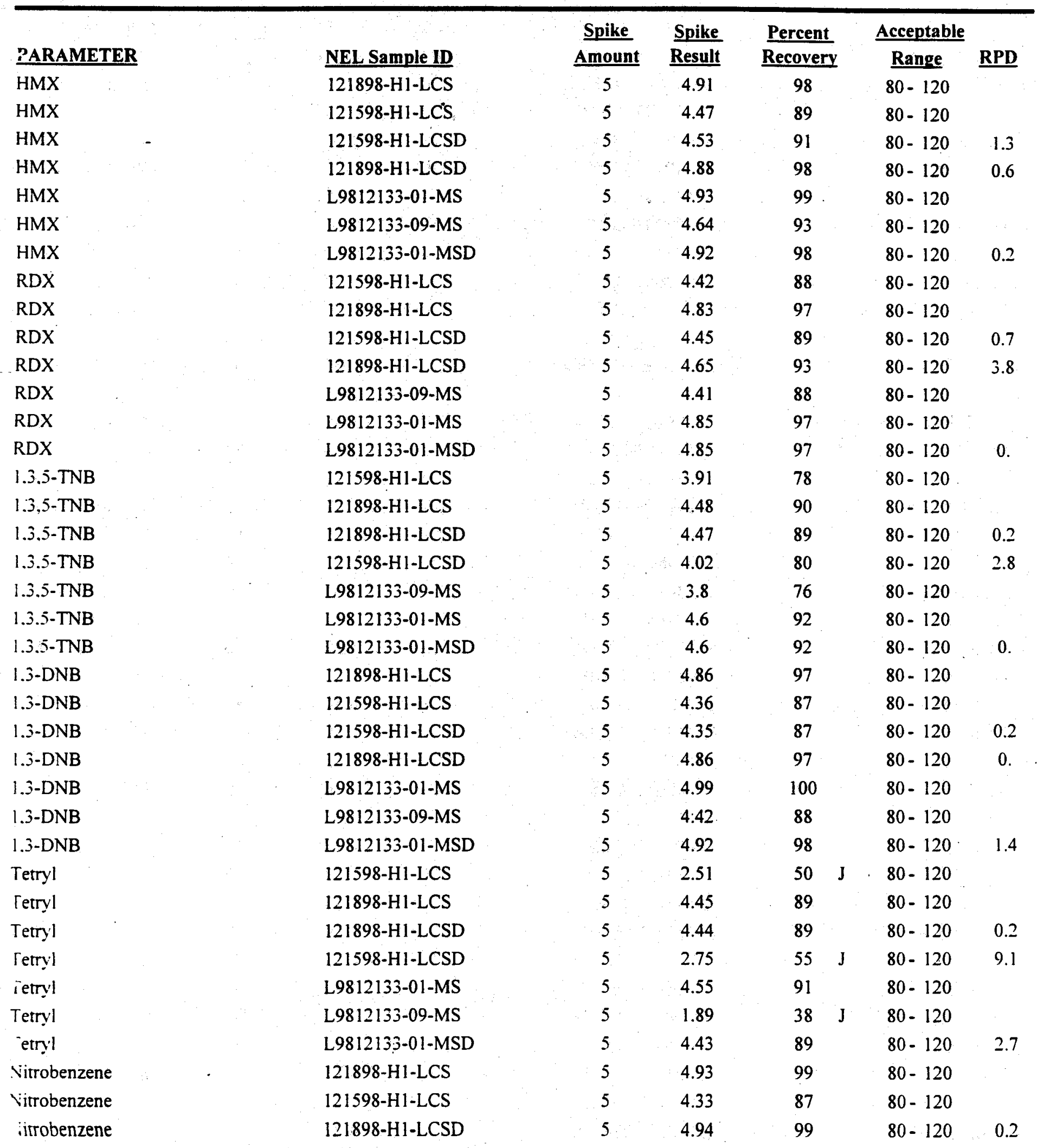

:D - Not Detected

- his report shall not be reproduced except in full, without the written approval of the laboratory. 


$\begin{array}{ll}\text { CLIENT: } & \text { Bechtel Nevada } \\ \text { PROJECT ID: } & \text { V557/17777 } \\ \text { PROJECT \#: } & 17777 \\ \text { TEST: } & \text { Nitroaromatics and Nitroamines by EPA } 8330 \\ \text { MATRIX: } & \text { Aqueous }\end{array}$

\section{PARAMETER}

Nitrobenzene

Nitrobenzene

Nitrobenzene

Nitrobenzene

2,4,6-TNT

2.4,6-TNT

2.4,6-TNT

2.4,6-TNT

2.4,6-TNT

2.4,6-TNT

2.4,6-TNT

2-Am-DNT \& 4-Am-DNT

2-Am-DNT \& 4-Am-DNT

2-Am-DNT \& 4-Am-DNT

2.4-DNT \& 2,6-DNT

2.4-DNT \& 2,6-DNT

2.4-DNT \& 2,6-DNT

2-Nitrotoluene

2-Nitrotoluene

2-Nitrotoluene

2-Nitrotoluene

2-Nitrotoluene

2-Nitrotoluene

2-Nitrotoluene

4-Nitrotoluene

4-Nitrotoluene

4-Nitrotoluene

4-Nitrotoluene

t-Nitrotoluene

t-Nitrotoluene

+-Nitrotoluene

3-Nitrotoluene

3-Nitrotoluene

3 -Nitrotoluene

3-Nitrotoluene

3-Nitrotoluene

3-Nitrotoluene

3-Nitrotoluene

\section{NEL Sample ID}

121598-H1-LCSD

L9812133-09-MS

L9812133-01-MS

L9812133-01-MSD

121898-H1-LCS

121598-H1-LCS

121898-H1-LCSD

121598-H1-LCSD

L9812133-01-MS

L9812133-09-MS

L9812133-01-MSD

121598-H1-LCS

121598-H1-LCSD

L9812133-09-MS

121598-H1-LCS

121598-H1-LCSD

L9812133-09-MS

121598-H1-LCS

121898-H1-LCS

121898-H1-LCSD

121598-H1-LCSD

L9812133-01-MS

L9812133-09-MS

L9812133-01-MSD

121598-H1-LCS

121898-H1-LCS

121898-H1-LCSD

121598-H1-LCSD

L9812133-01-MS

L9812133-09-MS

L9812133-01-MSD

121598-H1-LCS

121898-H1-LCS

121898-H1-LCSD

121598-H1-LCSD

L9812133-01-MS

L9812133-09-MS

L9812133-01-MSD

S

\section{Spike} Amount

Spike

Result

5

5

4.4

4.29

5.01

5.05

5.29

5.41

5.26

5.28

5.37

5.59

5.37

9.16

9.12

9.34

8.75

8.7

8.96

4.49

4.97

5.1

4.51

4.96

4.44

4.99

4.33

4.96

5.06

4.42

4.83

4.58

4.89

4.29

4.93

5

4.36

4.85

4.76

4.91 \begin{tabular}{c} 
Percen \\
\hline Recove \\
\hline 88 \\
86
\end{tabular}

100

101

106

108

105

106

107

112

107

92

91

93

88

87

90

90

99

102

90

99

89

100

87

99

101

88

97

92

98

86

99

100

87

97

95

98
Acceptable

\section{Range RPD}

$80-120 \quad 1.6$

$80-120$

$80-120$

$80-120$

0.8

$80-120$

$80-120$

$80-120$

$80-120$

0.6

2.4

$80-120$

$80-120$

$80-120$

0.

$80-120$

$80-120$

0.4

$80-120$

$80-120$

$80-.120$

0.6

$80-120$

$80-120$

$80-120^{\circ}$

$80-120$

2.6

$80-120$

0.4

$80-120$

$80-120$

$80-120$

0.6

$80-120$

$80-120$

$80-120$

2.

$80-120$

2.1

$80-120$

$80-120$

$80-120$

1.2

$80-120$

$80-120$

$80-120$

1.4

$80-120$

1.6

$80-120$

$80-120$

$80-120$

ND - Not Detected

This report shall not be reproduced except in full, without the written approval of the laboratory. 


\section{CLIENT: $\quad$ Bechtel Nevada \\ PROJECT ID: V557/17777 \\ PROJECT \#: $\quad 17777$ \\ TEST: \\ TCLP/STLC Metals \\ MATRIX: Solid}

\begin{tabular}{|c|c|c|c|c|c|c|}
\hline PARAMETER & NEL Sample ID & $\frac{\text { Spike }}{\text { Amount }}$ & $\frac{\text { Spike }}{\text { Result }}$ & $\begin{array}{l}\text { Percent } \\
\text { Recoverv }\end{array}$ & $\frac{\text { Acceptable }}{\text { Range }}$ & $\underline{\text { RPD }}$ \\
\hline Arsenic & L12133i-LCS & 0.5 & 0.52 & 104 & $85-115$ & \\
\hline Arsenic & L12133i-09A-LECS & 0.5 & 0.528 & 106 & $85-115$ & \\
\hline Arsenic & L9812133-01-MS & 0.5 & 0.477 & 95 & $75-125$ & \\
\hline Arsenic & L9812133-01-MSD & 0.5 & 0.533 & 107 & $75-125$ & 11.1 \\
\hline Mercury & L12133-Hg-LCS & 0.005 & 0.00483 & 97 & $85-115$ & \\
\hline Mercury & L9812133-01-MS & 0.05 & 0.0565 & 113 & $75-125$ & \\
\hline Mercury & L9812133-01-MSD & 0.05 & 0.0497 & 99 & $75-125$ & 12.8 \\
\hline Silver & L12133i-LCS & 0.5 & 0.467 & 93 & $85-115$ & \\
\hline Silver & L9812133-01-MS & 0.5 & 0.459 & 92 & $75-125$ & \\
\hline Silver & L9812133-01-MSD & 0.5 & 0.47 & 94 & $75-125$ & 2.4 \\
\hline Barium & L12133i-LCS & 1 & 1.13 & 113 & $85-115$ & \\
\hline Barium & L12133i-09A-LCS & 1 & 1.01 & 101 & $85 .-115$ & \\
\hline Barium & L9812133-01-MS & 1 & 2.55 & 95 & $75-125$ & \\
\hline Barium & L9812133-01-MSD & 1 & 2.58 & 98 & $75-125$ & 3.1 \\
\hline Cadmium & L12133i-LCS & 0.2 & 0.18 & 90 & $85-115$ & \\
\hline Cadmium & L12133i-09A-LCS & 0.2 & 0.197 & 99 & $85-115$ & \\
\hline Cadmium & L9812133-01-MS & 0.2 & 0.187 & 94 & $75-125$ & \\
\hline Cadmium & L9812133-01-MSD & 0.2 & 0.192 & 96 & $75-125$ & 2.6 \\
\hline Chromium & L12133i-09A-LCS & 0.5 & 0.503 & 101 & $85-115$ & \\
\hline Chromium & L12133i-LCS & 0.5 & 0.477 & 95 & $85-115$ & \\
\hline Chromium & L9812133-01-MS & 0.5 & 0.476 & 95 & $75-125$ & \\
\hline Zhromium & L9812133-01-MSD & 0.5 & 0.49 & 98 & $75-125$ & 2.9 \\
\hline Lead & L12133i-09A-LCS & 1 & 1.03 & 103 & $85-115$ & \\
\hline Lead & L12133i-LCS & 1 & 0.953 & 95 & $85-115$ & \\
\hline Lead & L9812133-01-MS & 1 & 0.939 & 94 & $75-125$ & \\
\hline Lead & L9812133-01-MSD & 1 & 1.01 & 101 & $75-125$ & 7.3 \\
\hline jelenium & L12133i-LCS & 0.5 & 0.56 & 112 & $85-115$ & \\
\hline Selenium & L12133i-09A-LCS & 0.5 & 0.51 & 102 & $85-115$ & \\
\hline Selenium & L9812133-01-MS & 0.5 & 0.471 & 94 & $75-125$ & \\
\hline ielenium & L9812133-01-MSD & 0.5 & 0.451 & 90 & $75-125$ & 4.3 \\
\hline Silver & L12133i-09A-LCS & 0.5 & 0.498 & 100 & $85-115$ & \\
\hline
\end{tabular}




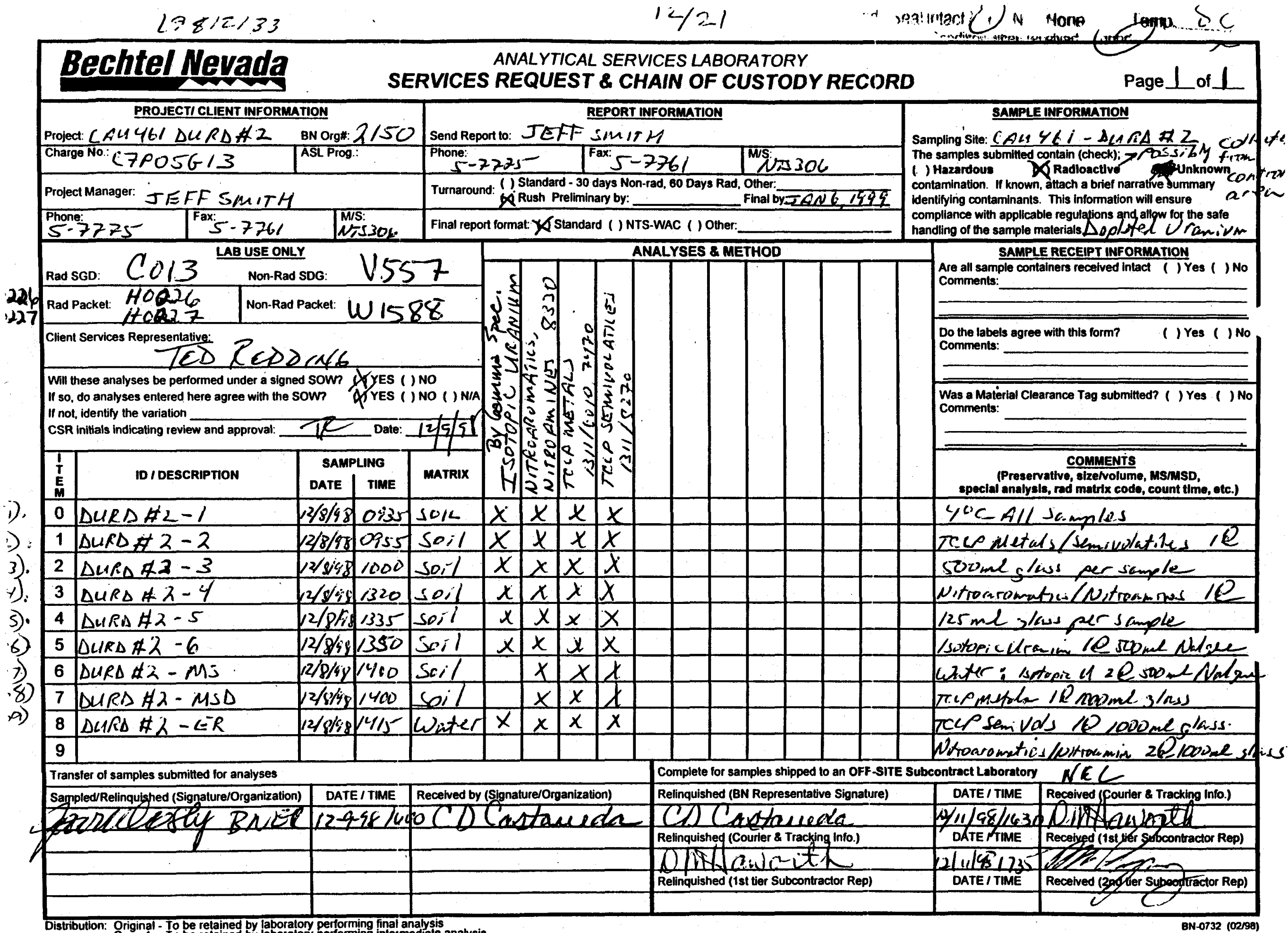

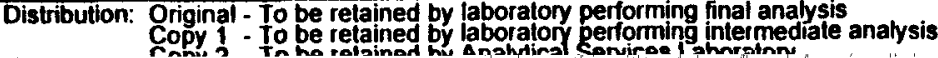




\section{GENERAL INFORMATION}

\begin{tabular}{|c|c|c|c|c|c|c|}
\hline Sumple Delivery Group: & V557 & Client: & Becht & 1 Nevada & & \\
\hline Laboratory: & NEL & Reviewed by: & Mars & c. Webb & Date: August 9, 19 & \\
\hline Requested Analysis: & sVo & Methods: & action & 1311 & Analysis & 8270 \\
\hline
\end{tabular}

Matrix: $\quad$ Soil +1 water

\section{COMPLETENESS}
A. Were all deliverables submitted?
B. Are forms properly completed and legible?
C. Were all requested analyses performed by the laboratory?
D. Were the requested analytical methods used for analysis?

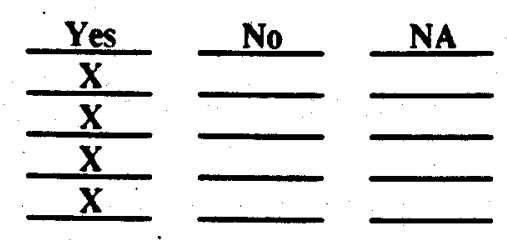

\section{DATA ASSESSMENT SUMMARY}

( ) A. Field and QA/QC sample data have been generated in accordance with method requirements and within quality control limits.

(X) B. QA/QC problems were encountered during analysis of the samples. However, the data are considered acceptable.

( ) C. Significant $Q A / Q C$ problems were encountered during analysis of the samples. Data for the following samples may be rejected.

\section{HOLDING TIME COMPLIANCE}
A. Sampled
B. ASL Received:
C. Contract Lab Received:
D. Latest TCLP Extraction:
E. Latest Preparative Extraction:
F. Latest Date Analyzed:
Were holding time criteria met?

\begin{tabular}{|c|c|}
\hline Date & \# Days \\
\hline $12 / 8 / 98$ & NA \\
\hline $12 / 9 / 98$ & 1 \\
\hline $12 / 11 / 98$ & 3 \\
\hline $12 / 17 / 98$ & 9 \\
\hline $12 / 18 / 98$ & 1 \\
\hline $12 / 21 / 98$ & 3 \\
\hline
\end{tabular}

() No

\begin{tabular}{c}
$\frac{\text { Acceptable }}{0-2}$ \\
\hline 1 \\
\hline 7 \\
\hline 14 \\
\hline 7 \\
\hline 40
\end{tabular}

\section{INSTRUMENT \& SAMPLE QA/QC CRITERIA}

\section{A. INSTRUMENT QUALITY CONTROL}

1. Were all samples analyzed within a valid 12-hour tune period?

2. Tune - Decafluorotriphenylphosphine

3. Calibrations Initial:

Verification:
Ion Abundances

SPCC RRF values

CCC \% RSD, CofV 20.99

SPCC RRF values

CCC \% D, Conc. \% Drift

IS Area and Retention Times

\section{Criteria met}

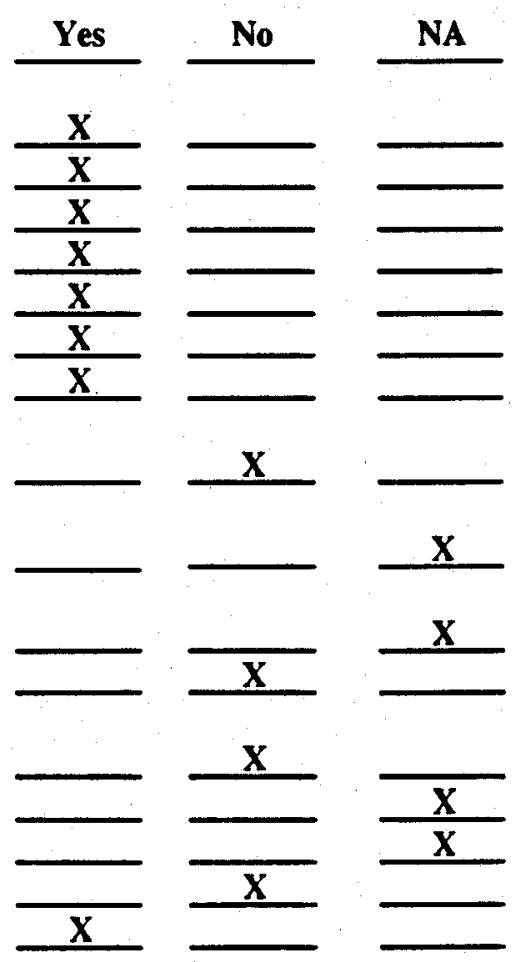

B. SAMPLE ASSOCIATED QUALITY CONTROL

1. Blanks: Were target compounds detected in field or laboratory blanks?

If so, were they detected:

a. at $>5$ times the CRDL / PQL / Reporting Limit?

b. in the samples at $>5$ times the blank value (non-common lab contaminants) or $>$ 10 times the blank value (common lab contaminants)?

2. Lab Control Sample: Were recoveries within acceptable limits?

3. Samples

a. Were target compounds detected in the samples?

b. If so, were the raw EICPs provided which confirm the qualification?

c. Were concentrations calculated accurately?

4. Were all Surrogate recoveries acceptable?

5. Were all Internal Standard Areas and Retention Times within control limits? 


\section{INSTRUMENT \& SAMPLE QA/QC CRITERIA}

6. As appropriate, were the following within quality control limits:

a. MS/MSD recoveries and RPDs

b. Lab and/or field duplicate RPDs

= indeterminant

COMMENTS: $\quad$ LCS/LCSD substituted for MS/MSD; samples qualified based on LCS and surrogate recoveries.

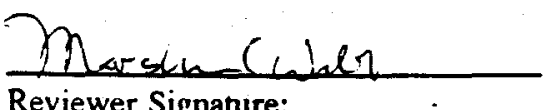

Reviewer Signanire:

$\bar{\delta} / \sigma_{l} / \sigma_{l} c_{i}$ 


\section{GENERAL INFORMATION}

Sample Delivery Group:

Laboratory:

Requested Analysis:

Matrix:

\begin{tabular}{ll} 
V557 & $\begin{array}{l}\text { Client: } \\
\text { NEL }\end{array}$ \\
\hline Neviewed by: \\
Soitroaromatics & Methods:
\end{tabular}

Bechtel Nevada
Date: $8 / 9 / 99$

Extraction NA Analysis 8330

\section{COMPLETENESS}

A. Were all deliverables submitted?

B. Are forms properly completed and legible?

C. Were all requested analyses performed by the laboratory?

D. Were the requested analytical methods used for analysis?

Criteria Met

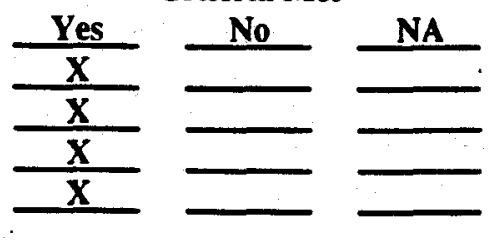

\section{DATA ASSESSMENT SUMMARY}

(X) A. Field and QA/QC sample data have been generated in accordance with method requirements and within quality control limits.

0 B. QA/QC problems were encountered during analysis of the samples. However, the data are considered acceptable.

0 C. Significant QA/QC problenss were encountered during analysis of the samples. Data for the following samples may be rejected.

IV. HOLDING TIME COMPLIANCE

A. Sampled

B. ASL Received:

C. Contract Lab Received:

D. Latest Extraction:

E. Latest Date Analyzed:

Were holding time criteria met?
(X) Yes

$\frac{\frac{\text { Date }}{12 / 8 / 98}}{\frac{12 / 9 / 98}{12 / 11 / 98}} \frac{12 / 18 / 98}{\frac{12 / 21 / 98}{0 \text { No }}}$

Acceptable

\begin{tabular}{c} 
Acceptable \\
\hline $0-2$ \\
\hline 1 \\
\hline 7 \\
\hline 14 \\
\hline 40
\end{tabular}

\section{INSTRUMIENT \& SAMPLE QA/QC COMPLIANCE}

\section{A. INSTRUMENT QUALITY CONTROL}

1. Calibrations
Within $3 \mathrm{X}$ of the IDL (< reporting limit)

\section{Initial:}

3 Standards minimum

Verification:

B. SAMPLE ASSOCIATED QUALITY CONTROL

\section{$\mathrm{R}^{2}>0.995$ or \% RSD met}

ICV within $\pm 20 \%$

CCV every 10 samples within $\pm 20 \%$

\section{Criteria met}
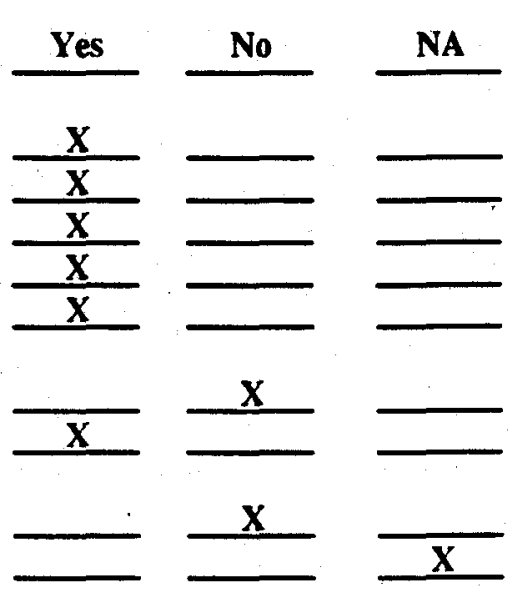
4. As appropriate, were the following within quality control limits:
a. MS/MSD recoveries (75.125 \%) MS/MSD RPD (20\%)
b. Lab and/or field duplicate RPDs ( $<20 \%)$
c. Surrogate recoveries within quality control limits
a. Were target compounds detected in the samples?
b. Were concentrations calculated correctly?

1. Prep blank: Were target compounds detected in field or laboratory blanks?

2. Lab Control Sample: Were recoveries within acceptable limits? $( \pm 20 \%)$

3. Samples

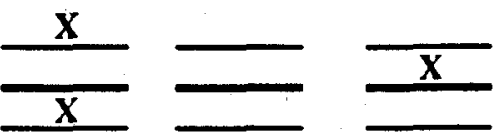


COMMENTS: The run log for 12/22/98 is included in the package, but the data is NOT. DURD\#2-MS and DURD\#2-MSD were not analyzed nor reported...

Marshn Chindr

Reviewer Signature:
August 9.1999 (2.09pm)

Date: 


\section{GENERAL INFORMATION}

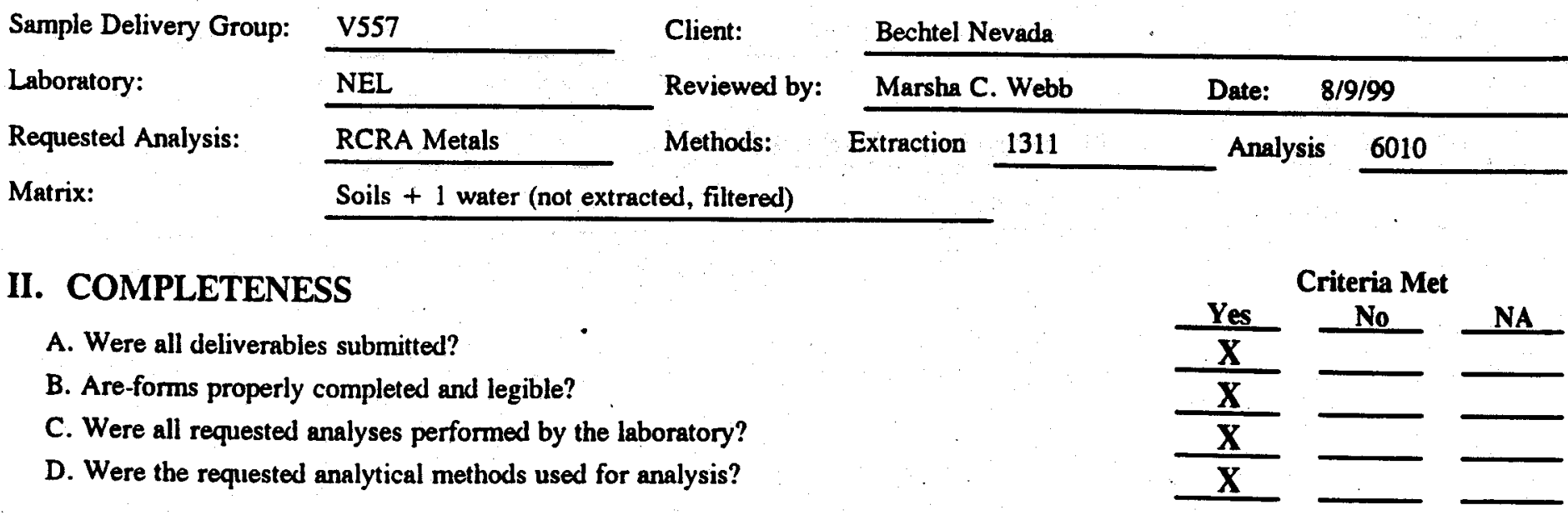

\section{DATA ASSESSMENT SUMMARY}

( ) A. Field and QA/QC sample data have been generated in accordance with method requirements and within quality control limits.

(X) B. QA/QC problems were encountered during analysis of the samples. However, the data are considered acceptable.

( ) C. Significant QA/QC problems were encountered during analysis of the samples. Data for the following samples may be rejected.

\section{HOLDING TIME COMPLIANCE}
A. Sampled
B. ASL Received:
C. Contract Lab Received:
D. Latest Extraction:
E. Latest Date Analyzed:

Were holding time criteria met?

\begin{tabular}{|c|}
\hline Date \\
\hline $12 / 8 / 98$ \\
\hline $12 / 9 / 98$ \\
\hline $12 / 11 / 98$ \\
\hline $12 / 17 / 98$ \\
\hline $12 / 18 / 98$ \\
\hline
\end{tabular}

$\frac{\frac{\text { \# Days }}{\text { NA }}}{\frac{1}{3}}$

( ) No

\begin{tabular}{c} 
Acceptable \\
\hline $0-2$ \\
\hline$\frac{1}{7}$ \\
\hline 14 \\
\hline 180
\end{tabular}

\section{INSTRUMENT \& SAMPLE QA/QC COMPLIANCE}

\section{A. INSTRUMENT QUALITY CONTROL \\ 1. Calibrations \\ Blank: \\ Initial: \\ Verification:}

2. Interference Check Sample
Within 3X of the IDL

Minimum of Cal Blank and 1 Standard

ICV, CCVs, check standard within $\pm 10 \%$

RSD $<5 \%$ for 22 replicate integrations

within $\pm 20 \%$ true value
Criteria met

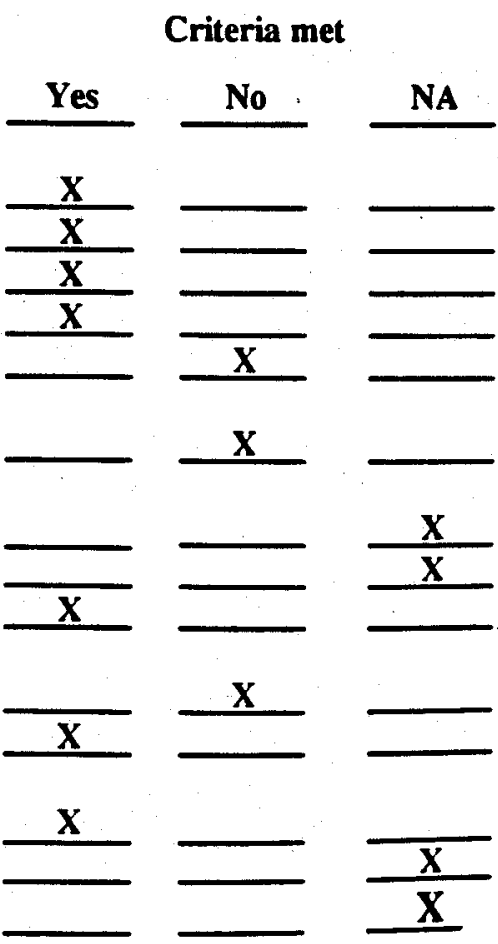

B. SAMPLE ASSOCIATED QUALITY CONTROL

1. Blanks: Were target compounds detected in field or laboratory blanks?

If so, were they detected:

a. at levels $2 \mathrm{CRDL}$ ?

b. in the samples at $<5$ times the blank value?

2. Lab Control Sample: Were recoveries within acceptable limits?

3. Sumples

a. Were target compounds detected in the samples?

b. Were concentrations calculated correctly?

4. As appropriate, were the following within quality control limits:
a. MS/MSD recoveries and RPDs
b. Lab and/or field duplicate RPDs
c. Serial dilution within $\pm 10 \%$ for undiluted concentrations $\geq 10 \mathrm{X}$ the IDL 


\section{COMMENTS:}

The TCLP extraction was done on $25 \mathrm{~g}$ rather than $100 \mathrm{~g}$. The extraction sheet did NOT have sample pH, nuid \# used, post pH, pH of extraction fluid used, start time; end time; room temp; rpm check; etc.

ICSA/ICSAB As did not pass. The standards were not rerun.

Mardinciser

Reviewer Signature:
August 9.1999 (1:44pm)

Date: 


\section{GENERAL INFORMATION}

Sample Delivery Group:

Laboratory:

Requested Analysis:

Matrix:

$\frac{\mathrm{V} 557}{\mathrm{NEL}}$

Client:

Reviewed by:

Methods:
Bechtel Nevada

Marsha C. Webb

Date: $8 / 9 / 99$

Soil +1 water

\section{COMPLETENESS}
A. Were all deliverables submitted?
B. Are forms properly completed and legible?
C. Were all requested analyses performed by the laboratory?
D. Were the requested analytical methods used for analysis?

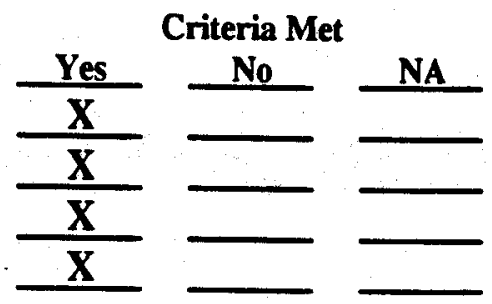

\section{DATA ASSESSMENT SUMMARY}

( ) A. Field and QA/QC sample data have been generated in accordance with method requirements and within quality control limits.

(X) B. QA/QC problems were encountered during analysis of the samples. However, the data are considered acceptable.

( ) C. Significant QA/QC problems were encountered during analysis of the samples. Data for the following samples may be rejected.

IV. HOLDING TIME COMPLIANCE

A. Sampled

B. ASL Received:

C. Contract Lab Received:

D. Latest Extraction:

E. Latest Date Analyzed:

Were holding time criteria met?

(X) Yes

\begin{tabular}{c} 
Date \\
\hline$\frac{12 / 8 / 98}{12 / 9 / 98}$ \\
\hline $12 / 11198$ \\
\hline $12 / 17 / 98$ \\
\hline $12 / 18 / 98$ \\
\hline ( ) No
\end{tabular}

$\frac{\frac{\text { \# Davs }}{\text { NA }}}{\frac{1}{3}}$

\section{INSTRUMENT \& SAMPLE QA/QC COMPLIANCE}

\section{A. INSTRUMENT QUALITY CONTROL}

1. Calibrations

Blank:
Initial:
Verification:

B. SAMPLE ASSOCIATED QUALITY CONTROL

Within $3 X$ of the IDL

Cal Blank and 5 Standards

$\mathrm{R}^{2}>0.995$

ICV within $\pm 10 \%$

CCV every 10 samples within $\pm 20 \%$

1. Prep blank: Were target compounds detected in field or laboratory blanks?

2. Lab Control Sample: Were recoveries within acceptable limits? (80-120\%)

3. Samples

a. Were target compounds detected in the samples?

b. Were concentrations calculated correctly?

4. As appropriate, were the following within quality control limits:

a. MS/MSD recoveries (75-125\%)

b. Lab and/or field duplicate RPDs $(<20 \%)$

c. Serial dilution within $\pm 10 \%$ for undiluted concentrations $\geq 10 \mathrm{X}$ the IDL
Acceptable

\begin{tabular}{|c|}
\hline $0-2$ \\
\hline 1 \\
\hline 7 \\
\hline 14 \\
\hline 28 \\
\hline
\end{tabular}

\section{Criteria met}

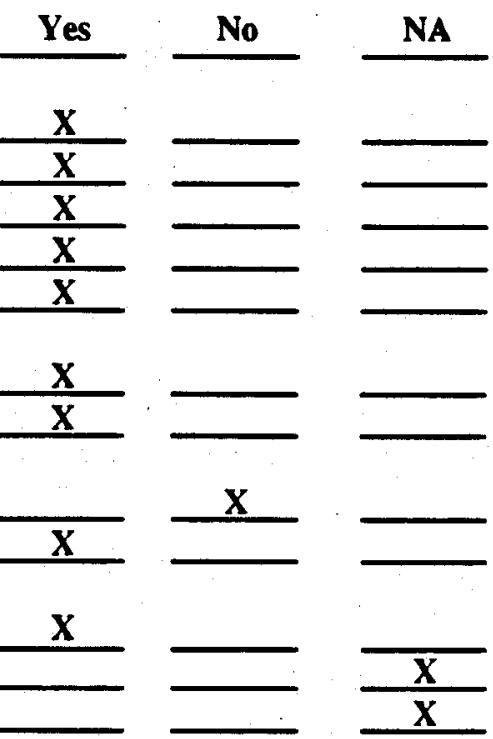


COMMENTS:

The TCLP extraction was done on $25 \mathrm{~g}$ rather than $100 \mathrm{~g}$. The extraction sheet did NOT have sample $\mathrm{pH}$, fluid \# used, post pH, pH of extraction fluid used, start time; end time; room temp; rpm check; etc.

Marshic viot

Reviewer Signature:
August 9. 1999 (1:38pm)

Date: 


\section{Bechtel Nevada Corporation}

\section{ANALYTICAL SERVICES LABORATORY}

P.O.Box 3936, N. Las Vegas, NV 89036

Reported to: Environmental Restoration

CAU 461 \& 495 DU Site

J. L. Smith, M/S NTS306

Report Date: 14-DEC-98 Sample Delivery Group: $\mathrm{C} 013$

Batch: 0578

Program: 720

Report No. :

\begin{tabular}{|c|c|c|c|c|c|c|c|c|c|c|c|c|c|c|c|c|}
\hline $\begin{array}{c}\text { Sample } \\
\ldots . . . \\
\text { Identification ..... }\end{array}$ & Isolope & Analysis & Result & $\begin{array}{c}\text { Error } \\
\%\end{array}$ & \begin{tabular}{|l|} 
Oual \\
Flag
\end{tabular} & MDA & $\begin{array}{l}\text { Result } \\
\text { Units }\end{array}$ & $\begin{array}{l}\text { Analysis } \\
\text { Date }\end{array}$ & $\begin{array}{c}\text { Sample } \\
\text { Coll Date }\end{array}$ & Size & $\begin{array}{r}\text { Size } \\
\text { Units }\end{array}$ & $\begin{array}{l}\text { Type } \\
\text { Matrix }\end{array}$ & $\left|\begin{array}{l}\text { Tracer } \\
\text { Yield \% }\end{array}\right|$ & $\begin{array}{l}\text { Spike } \\
\text { Recr \% }\end{array}$ & \begin{tabular}{|l|} 
System \\
Detector
\end{tabular} & $\begin{array}{l}\text { Packet-Item } \\
\text { Sample }\end{array}$ \\
\hline DURD $/ 2$-ER & No Nucl Det & GAM20 & $0.00 E+00$ & $0.0 E+00$ & U & $0.0 E+00$ & NA & 12-09-98 & $12-08-98$ & $5.00 E+02$ & $\mathrm{ml}$ & Water & & & 08.01 & HO227-0.75293 \\
\hline DURD \#2-ER & U 238 & GAM20 & $0.00 E+00$ & $0.0 E+\infty$ & U & $1.2 E+01$ & $\mathrm{pCl} / \mathrm{s}$ & 12.09.98 & $12-08-98$ & $5.00 E+02$ & $\mathrm{ml}$ & Water & & & 08.01 & HO227-0-75293 \\
\hline OA BKG WATER UOB & No Nucl Det & GAM 20 & $0.00 E+00$ & $0.0 \mathrm{E}+00$ & U & $0.0 E+00$ & NA & 12.09.98 & $07-01-98$ & $5.00 E+02$ & $\mathrm{ml}$ & QUAL & & & 08.01 & $00624-0.00343$ \\
\hline QA BKG WATER UOB & AM241 & GAM20 & $0.00 E+00$ & $0.0 \mathrm{E}+00$ & $\mathbf{u}$ & $7.9 E+02$ & $\mathrm{pCi}$ & 12.09-98 & $07-01-98$ & $1.00 E+00$ & $\mathrm{sm}$ & QUAL & & & $08-01$ & $00624 \cdot 0.00343$ \\
\hline OA BKG WATER UOB & $\mathrm{CO60}$ & GAM20 & $0.00 E+00$ & $0.0 E+\infty 0$ & U & $3.6 E+01$ & $\rho C i$ & 12.09-98 & $07.01-98$ & $1.00 E+00$ & $\mathrm{sm}$ & QUAL & & & 08.01 & $00624-0.00343$ \\
\hline OA BKG WATER UOB & CS137 & GAM20 & $0.00 E+00$ & $0.0 E+00$ & U & $3.8 E+01$ & $\mathrm{pCi}$ & 12.09.98 & 07-01-98 & $1.00 E+00$ & sm & OUAL & & & 08-01 & 00624-0.00343 \\
\hline QA SP:QAW-8 & AM241 & GAM 20 & $7.14 E+04$ & $9.9 E+00$ & & $1.3 E+03$ & $\mathrm{pCi}$ & $12.09-98$ & $08-01-90$ & $1.00 E+00$ & $\mathrm{sm}$ & QUAL & & 102.1 & $08-01$ & 00619-3-16258 \\
\hline QA SP:QAW-8 & $\mathrm{CO60}$ & GAM20 & $7.04 E+04$ & $8.5 E+00$ & & $2.2 E+02$ & $\mathrm{pCi}$ & $12-09-98$ & $08.01-90$ & $1.00 E+00$ & $\mathrm{sm}$ & QUAL & & 97.4 & 08.01 & $00619-3-16258$ \\
\hline QA SP:OAW-8 & CS137 & GAM20 & $5.97 E+04$ & $7.9 E+00$ & & $1.1 E+02$ & $\mathrm{pCi}$ & 12.09 .98 & $08-01-90$ & $1.00 E+00$ & sm & QUAL & & 96.1 & 08-01 & $00619-3-16258$ \\
\hline
\end{tabular}

\section{Comment:}

Data generated from analyses performed on samples submitted 12/9/98. Gamma spectroscopy was performed in order to analyze for U-238 within the turnaround requested by the project.

Prepared by:

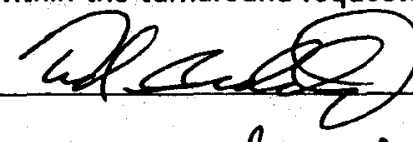

Date: $12 / 14 / 68$

Approved by: Stany folsos Date: $12 / 15 / 98$
Qualification Flags:

$E=$ Estimated Quantity

$H=$ High Recovery for Sample

$\mathrm{J}=$ Result is less than the RDL

$\mathrm{L}=$ Low Recovery for Sample

$P=$ Preliminary Results

$\mathrm{Q}=$ Bad Instrument Quality Control, Result is OK

$R=$ Results are Unusable, Resampling is Necessary

$U=$ Result is less than Minimum Detectable Activity
Note: \% Error is the 2.0 Sigma Error 


\section{Bechtel Nevada Corporation}

ANALYTICAL SERVICES LABORATORY

P.O.Box 3936, N. Las Vegas, NV 89036

Reported to: Environmental Restoration

CAU 461 \& 495 DU Site

J. L. Smith, M/S NTS306

Report Date: 14-DEC-98

Sample Delivery Group: $\mathrm{C013}$

Batch: 0862

Program: 720

Report No. :

\begin{tabular}{|c|c|c|c|c|c|c|c|c|c|c|c|c|c|c|c|c|}
\hline $\begin{array}{c}\text { Sample } \\
\ldots \ldots \\
\text {... Identification } \cdots . . .\end{array}$ & Isotope & Analysis & Result & $\begin{array}{l}\text { Error } \\
\%\end{array}$ & $\begin{array}{l}\text { Qual } \\
\text { Flag }\end{array}$ & MDC & $\begin{array}{l}\text { Result } \\
\text { Units }\end{array}$ & $\begin{array}{c}\text { Analysis } \\
\text { Date }\end{array}$ & $\begin{array}{l}\text { Sample } \\
\text { Coll Date }\end{array}$ & Size & $\begin{array}{l}\text { Size } \\
\text { Units }\end{array}$ & $\begin{array}{c}\text { Type } \\
\text { Matrix }\end{array}$ & \begin{tabular}{|l|} 
Tracer \\
Yield \%
\end{tabular} & $\begin{array}{l}\text { Spike } \\
\text { Hecv \% }\end{array}$ & \begin{tabular}{|l} 
System \\
Detector
\end{tabular} & $\begin{array}{c}\text { Packet-ftem } \\
\text { Sample }\end{array}$ \\
\hline DURD $\# 2-1$ & K 40 & GAM 20 & $2.85 E+01$ & $1.8 E+01$ & & $1.2 E+00$ & pCi/g & 12-09-98 & 12.08 .98 & $7.56 E+02 \mid$ & gm & soll G & & & 05.01 & HO226-0-75287 \\
\hline DURD $12-1$ & RA226 & GAM20 & $1.50 E+00$ & $2.6 E+01$ & & $1.5 E-01$ & pCi/g & 12-09.98 & $12-08-98$ & $7.56 E+02$ & gm & soll G & & & 05.01 & H0226-0.75287 \\
\hline DURD $\# 2-1$ & TH228 & GAM20 & $2.66 E+00$ & $1.8 \mathrm{E}+01$ & & $1.7 \mathrm{E}-01$ & pCi/g & $12-09-98$ & 12.08 .98 & $7.56 \mathrm{E}+02$ & $g m$ & SOIL G & & & 05.01 & HO226-0.75287 \\
\hline DURD $12-1$ & TH232 & GAM20 & $2.57 E+00$ & $2.9 E+01$ & & 3.8E-01 & pCi/g & 12-09-98 & 12.08 .98 & $7.56 E+02$ & gm & SOIL G & & & $05-01$ & HO226-0.75287 \\
\hline DURD $* 2-1$ & U 238 & GAM 20 & $0.00 E+\infty 0$ & $0.0 E+00$ & u & $2.6 E+01$ & pCi/g & 12.09-98 & $12-08.98$ & $7.56 E+02$ & gm & soll $\mathrm{G}$ & & & 05.01 & HO226-0-75287 \\
\hline DURD $12-2$ & K 40 & GAM20 & $2.55 E+01$ & $1.9 E+01$ & & $1.3 E+00$ & $\mathrm{pCi} / \mathrm{g}$ & $12-09.98$ & 12.08 .98 & $6.50 E+02$ & gm & soll G & & & 05.01 & HO226-1.75288 \\
\hline DURD \#2-2 & RA226 & GAM20 & $1.52 E+00$ & $2.4 E+01$ & & $1.5 \mathrm{E}-01$ & $\mathrm{pCi} / \mathrm{g}$ & $12-09.98$ & $12.08-98$ & $6.50 E+02$ & gm & SOIL G & & & $05-01$ & HO226-1-75288 \\
\hline DURD \#2-2 & TH228 & GAM20 & $2.00 E+00$ & $2.0 E+01$ & & $1.7 \mathrm{E}-01$ & pCi/g & 12.09 .98 & 12.08-98 & $6.50 E+02$ & gm & SOIL G & & & 05.01 & HO226-1-75288 \\
\hline DURD $\# 2-2$ & TH232 & GAM20 & $1.90 E+00$ & $3.3 E+01$ & & $3.9 E-01$ & pCi/g & 12-09-98 & $12.08-98$ & $6.50 E+02 \mid$ & $\mathrm{gm}$ & SOll G & & & 05.01 & H0226-1-75288 \\
\hline DURD $\# 2-2$ & U 238 & GAM20 & $0.00 E+00$ & $0.0 E+00$ & $U$ & $2.2 E+01$ & pCilg & $12-09-98$ & $12-08-98$ & $6.50 E+02 \mid$ & gm & SOHL G & & & 05.01 & H0226-1-75288 \\
\hline
\end{tabular}

Comment:

Data generated from analyses performed on samples submitted

12/9/98. Gamma spectroscopy was performed in order to analyze

for U-238 within the turnaround requested by the project.

Prepared by:

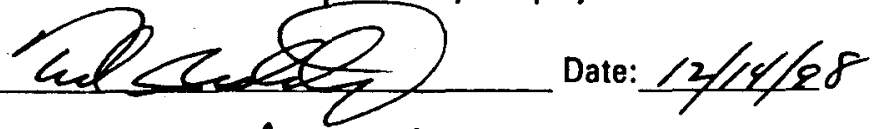

Approved by: Jany ff atoke Date: 12/15/98
Qualification Flags:

Note: \% Error is the 2.0 Sigma Error

$E$ = Estimated Quantity

$H=$ High Recovery for Sample

$\mathrm{J}=$ Result is less than the RDL

$\mathrm{L}=$ Low Recovery for Sample

$P=$ Preliminary Results

$\mathrm{Q}=$ Bad Instrument Quality Control, Result is OK

$\mathrm{R}=$ Results are Unusable, Resampling is Necessary

$\mathrm{U}=$ Result is less than Minimum Detectable Activity 


\section{Bechtel Nevada Corporation}

\section{ANALYTICAL SERVICES LABORATORY}

P.O.Box 3936, N. Las Vegas, NV 89036

Reported to: Environmental Restoration CAU 461 \& 495 DU Site

Report Date: 14-DEC-98

J. L. Smith, M/S NTS306

Sample Delivery Group: $\mathrm{C} 013$

Batch: 0862

Program: 720

Report No. :

\begin{tabular}{|c|c|c|c|c|c|c|c|c|c|c|c|c|c|c|c|c|}
\hline Sample & Isotope & Analysis & Result & $\begin{array}{c}\text { Error } \\
\%\end{array}$ & $\begin{array}{l}\text { Qual } \\
\text { Flag }\end{array}$ & MDC & $\begin{array}{l}\text { Result } \\
\text { Units }\end{array}$ & $\begin{array}{c}\text { Analysis } \\
\text { Date }\end{array}$ & $\begin{array}{l}\text { Sample } \\
\text { Coll Date }\end{array}$ & Size & $\begin{array}{l}\text { Size } \\
\text { Units }\end{array}$ & \begin{tabular}{c|} 
Type \\
Matrix
\end{tabular} & $\begin{array}{l}\text { Tracer } \\
\text { Yield \% }\end{array}$ & $\begin{array}{l}\text { Spike } \\
\text { Recv \% }\end{array}$ & $\begin{array}{l}\text { System } \\
\text { Detector }\end{array}$ & $\begin{array}{l}\text { Packet-ltem } \\
\text { Sample }\end{array}$ \\
\hline DURD $12-3$ & K 40 & GAM20 & $2.33 E+01$ & $1.9 E+01$ & & $1.2 E+00$ & pCi/g & $12-09-98$ & $12.08-98$ & $7.06 E+02$ & gm & soll G & & & 05.01 & HO226-2.75289 \\
\hline DUAD $\approx 2-3$ & RA226 & GAM20. & $1.69 E+00$ & $2.3 E+01$ & & $1.8 \mathrm{E}-01$ & pCi/g & $12-09-98$ & $12-08-98$ & $7.06 E+02$ & gm & SOIL G & & & 05.01 & HO226-2-75289 \\
\hline DUAD $12-3$ & TH228 & GAM20 & $2.09 E+00$ & $2.0 E+01$ & & $1.7 \mathrm{E}-01$ & pCi/g & $12.09-98$ & 12-08-98 & $7.06 E+02$ & gm & SOIL G & & & 05.01 & HO226-2-75289 \\
\hline DURD 12.3 & TH232 & GAM20 & $1.87 E+00$ & $3.4 E+01$ & & $3.8 \mathrm{E}-01$ & $\mathrm{pCi} / \mathrm{g}$ & 12.09-98 & $12-08-98$ & $7.06 E+02$ & gm & SOIL G & & & 05-01 & HO226-2-75289 \\
\hline DURD 12.3 & U. 238 & GAM20 & $0.00 E+00$ & $0.0 E+00$ & U & $2.2 E+01$ & pCi/g & $12-09.98$ & $12-08-98$ & $7.06 E+02$ & gm & SOIL G & & & 05-01 & H0226-2-75289 \\
\hline DURD 12.4 & K 40 & GAM2O & $3.22 E+01$ & $1.8 E+01$ & & $1.2 E+00$ & pCi/g & 12-09-98 & $12.08-98$ & $7.86 E+02$ & gm & SOIL G & & & 05.01 & H0226-3-75290 \\
\hline DURD $12-4$ & RA226 & GAM20 & $1.02 E+00$ & $3.3 E+01$ & & $1.8 \mathrm{E}-01$ & pCi/g & 12-09-98 & $12-08-98$ & $7.86 E+02$ & gm & soll G & & & 05.01 & HO226-3-75290 \\
\hline DURD $\approx 2-4$ & TH228 & GAM20 & $2.58 E+00$ & $1.8 E+01$ & & $1.7 \mathrm{E}-01$ & pCi/g & 12.09 .98 & $12-08-98$ & $7.86 E+02$ & $g m$ & SOLL G & & & 05.01 & H0226-3-7529O \\
\hline DURD \#2-4 & TH232 & GAM20 & $2.02 E+00$ & $3.2 E+01$ & & $3.8 \mathrm{E}-01$ & pCi/g & $12-09-98$ & $12-08-98$ & $7.86 E+02$ & gm & soll G & & & 05.01 & HO226-3-7529O \\
\hline DURD $\approx 2-4$ & U 238 & GAM20 & $0.00 E+00$ & $0.0 E+\infty$ & U & $2.3 E+01$ & pCi/g & 12-09-98 & $12-08-98$ & $7.86 E+02$ & $g m$ & SOIL G & & & 05-01 & HO226-3.7529O \\
\hline
\end{tabular}

Comment:

Data generated from analyses performed on samples submitted 12/9/98. Gamma spectroscopy was performed in order to analyze for $\mathrm{U}-238$ within the turnaround requested by the project.

Prepared by

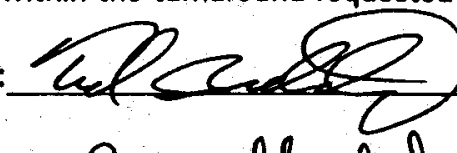

Approved by: Sary ffatso

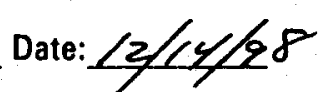
Date: $12 / 15 / 98$

\section{Qualification Flags:}

$E=$ Estimated Quantity

$\mathrm{H}=$ High Recovery for Sample

$J=$ Result is less than the RDL

$L=$ Low Recovery for Sample

$P=$ Preliminary Results

Q = Bad Instrument Quality Control, Result is OK

$R=$ Results are Unusable, Resampling is Necessary

$U=$ Result is less than Minimum Detectable Activity

Note: \% Error is the 2.0 Sigma Error 


\section{Bechtel Nevada Corporation}

\section{ANALYTICAL SERVICES LABORATORY}

P.O.Box 3936, N. Las Vegas, NV 89036

Reported to: Environmental Restoration

CAU $461 \& 495$ DU Site

Report Date: 14-DEC-98

J. L. Smith, M/S NTS306

Sample Delivery Group: $\mathrm{C} 013$

Batch: 0862

Program: 720

Report No. :

\begin{tabular}{|c|c|c|c|c|c|c|c|c|c|c|c|c|c|c|c|c|}
\hline $\begin{array}{c}\text { Sample } \\
\text {..... Identification ...... }\end{array}$ & Isotope & Analysis & Result & $\begin{array}{c}\text { Error } \\
\%\end{array}$ & $\begin{array}{l}\text { Qual } \\
\text { Flag }\end{array}$ & MDC & $\begin{array}{l}\text { Result } \\
\text { Units }\end{array}$ & $\begin{array}{c}\text { Analysis } \\
\text { Date }\end{array}$ & $\begin{array}{l}\text { Sample } \\
\text { Coll Date }\end{array}$ & Size & $\begin{array}{l}\text { Size } \\
\text { Units }\end{array}$ & \begin{tabular}{|c|} 
Type \\
Matrix \\
\end{tabular} & \begin{tabular}{|l|} 
Tracer \\
Yield \%
\end{tabular} & $\begin{array}{l}\text { Spike } \\
\text { Pecv \% }\end{array}$ & $\begin{array}{l}\text { System } \\
\text { Detector }\end{array}$ & $\begin{array}{l}\text { Packet-Item } \\
\text { Sample }\end{array}$ \\
\hline DURD 12.5 & K 40 & GAM2O & $2.96 E+01$ & $1.8 E+01$ & & $1.2 E+00$ & pCi/g & 12-09-98 & $12.08-98$ & $|7.79 E+02|$ & gm & SOIL G & & & 05.01 & Ho226-4-75291 \\
\hline DURD $12-5$ & RA226 & GAM 20 & $1.33 E+\infty$ & $2.8 E+01$ & & $1.5 \mathrm{E}-01$ & pCi/g & 12-09-98 & $12.08-98$ & $7.79 E+02$ & $\mathrm{gm}$ & SOIL G & & & 05.01 & H0226-4-75291 \\
\hline DURD $¥ 2.5$ & TH228 & GAM20 & $2.56 E+00$ & $1.8 E+01$ & & $1.7 \mathrm{E}-01$ & pCi/g & 12.09-98 & $12-08-98$ & $7.79 E+02$ & $\mathrm{gm}$ & SOIL G & & & 05.01 & HO226-4-75291 \\
\hline DURD $\$ 2.5$ & TH232 & GAM20 & $2.05 E+00$ & $3.2 E+01$ & & 3.8E-01 & pCi/g & 12-09-98 & $12-08-98$ & $|7.79 E+02|$ & gm & SOIL G & & & 05-01 & H0226-4-75291 \\
\hline DURD $12-5$ & U 238 & GAM20 & $0.00 E+00$ & $0.0 E+00$ & u & $2.5 E+01$ & pCi/g & 12-09-98 & 12.08 .98 & $7.79 E+02$ & $\mathrm{gm}$ & SOIL G & & & 05.01 & HO226-4-75291 \\
\hline DURD $\$ 2.6$ & K 40 & GAM20 & $2.68 \mathrm{E}+01$ & $1.9 E+01$ & & $1.2 E+\infty 0$ & pCi/g & 12-09-98 & 12.08 .98 & $6.94 E+02$ & gm & SOIL G & & & 05-01 & HO226-5-75292 \\
\hline DURD $\$ 2-6$ & RA226 & GAM20. & $1.24 E+00$ & $2.7 E+01$ & & $1.8 \mathrm{E}-01$ & pCi/g & 12-09-98 & $12.08-98$ & $6.94 E+02 \mid$ & gm & SOIL G & & & 05.01 & HO226-5-75292 \\
\hline DURD $\# 2.6$ & TH228 & GAM20 & $2.57 E+00$ & $1.8 E+01$ & & $1.7 E-01$ & pCi/g & 12-09-98 & $12-08-98$ & $6.94 E+02 \mid$ & gm & SOIL G & & & 05.01 & HO226-5.75292 \\
\hline DURD $\# 2-6$ & TH232 & GAM 20 & $2.47 E+00$ & $3.0 E+01$ & & $3.8 E-01$ & $\mathrm{pC} / \mathrm{g}$ & $12-09-98$ & 12.08 .98 & $|6.94 E+02|$ & $g m$ & SOIL G & & & 05.01 & HO226-5-75292 \\
\hline DURD $12-6$ & U 238 & GAM2O & $0.00 E+00$ & $0.0 E+00$ & $u$ & $2.7 E+01$ & $\mathrm{pCi} / \mathrm{g}$ & 12-09-98 & $12-08-98$ & $6.94 E+02$ & $\mathrm{gm}$ & SOIL G & & & 05-01 & HO226-5.75292 \\
\hline
\end{tabular}

\section{Comment:}

Data generated from analyses performed on samples submitted 12/9/98. Gamma spectroscopy was performed in order to analyze for $\mathrm{U}-238$ within the turnaround requested by the project.

Prepared by:
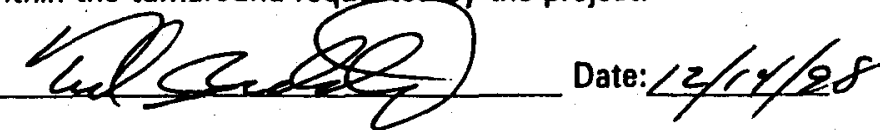

Approved by: Sarngffetax Date: $12 / 15 / 98$

\section{Qualification Flags:}

$E$ = Estimated Quantity

$H=$ High Recovery for Sample

$\mathrm{J}=$ Result is less than the RDL

$L=$ Low Recovery for Sample

$P=$ Preliminary Results

$\mathrm{Q}=$ Bad Instrument Quality Control, Result is OK

$R=$ Results are Unusable, Resampling is Necessary

$\mathrm{U}=$ Result is less than Minimum Detectable Activity
Note: \% Error is the 2.0 Sigma Error 


\section{Bechtel Nevada Corporation}

\section{ANALYTICAL SERVICES LABORATORY}

P.O.Box 3936, N. Las Vegas, NV 89036

Reported to: Environmental Restoration

CAU $461 \& 495$ DU Site

Report Date: 14-DEC-98

J. L. Smith, M/S NTS306

Sample Delivery Group: $\mathrm{C} 013$

Batch: 0862

Program: 500

Report No. :

\begin{tabular}{|c|c|c|c|c|c|c|c|c|c|c|c|c|c|c|c|c|}
\hline $\begin{array}{c}\text { Sample } \\
\ldots . . .\end{array}$ & Isotope & Analysis & Result & $\begin{array}{c}\text { Error } \\
\%\end{array}$ & $\begin{array}{l}\text { Qual } \\
\text { Flag }\end{array}$ & MDA & $\begin{array}{l}\text { Result } \\
\text { Units }\end{array}$ & $\begin{array}{c}\text { Analysis } \\
\text { Date }\end{array}$ & $\begin{array}{c}\text { Sample } \\
\text { Coll Date }\end{array}$ & Size & $\begin{array}{c}\text { Size } \\
\text { Units }\end{array}$ & $\begin{array}{c}\text { Type } \\
\text { Matrix }\end{array}$ & $\mid \begin{array}{l}\text { Tracer } \\
\text { Yield \% }\end{array}$ & $\begin{array}{l}\text { Spike } \\
\text { Recv \% }\end{array}$ & $\begin{array}{l}\text { System } \\
\text { Detector }\end{array}$ & $\begin{array}{l}\text { Packet-Item } \\
\text { Sample }\end{array}$ \\
\hline QA BKG EMPTY BTL UO5 & No Nucl Det & GAM20 & $0.00 E+\infty 0$ & $0.0 E+00$ & u & $0.0 E+00$ & NA & $12-09.98$ & $07-01-98$ & $1.00 E+\infty \mid$ & gm & QUAL & & & $05-01$ & $00631 \cdot 0.00344$ \\
\hline QA BKG EMPTY BTL U05 & AM241 & GAM20 & $0.00 E+00$ & $0.0 E+00$ & $u$ & $2.3 E+01$ & $\mathrm{pCi}$ & $12-09.98$ & $07-01-98$ & $1.00 E+\infty \mid$ & sm & QUAL & & & $05-01$ & 00631.0 .00344 \\
\hline QA BKG EMPTY BTL U05 & $\mathrm{CO60}$ & GAM20 & $0.00 E+00$ & $0.0 E+00$ & u & $9.9 E+\infty 0$ & $\mathrm{pCi}$ & $12-09-98$ & $07.01-98$ & $|1.00 E+00|$ & sm & QUAL & & & $05-01$ & $00631 \cdot 0.00344$ \\
\hline OA BKG EMPTY BTL U05 & CS137 & GAM20 & $0.00 E+00$ & $0.0 E+00$ & $u$ & $7.2 E+\infty 0$ & $\mathrm{pCi}$ & 12-09-98 & $07.01-98$ & $1.00 E+00$ & $\mathbf{s m}$ & QUAL & & & 05.01 & $00631 \cdot 0-00344$ \\
\hline QA SP:NAS-A0271 & AM241 & GAM20 & $1.80 E+05$ & $8.8 E+00$ & & $2.9 E+02$ & $\mathrm{pCi}$ & $12.09-98$ & 04-01-92 & $1.00 E+00$ & $\mathbf{s m}$ & QUAL & & 111.0 & $05-01$ & $00630-3-16259$ \\
\hline QA SP:NAS-A0271 & $\mathrm{CO60}$ & GAM2O & $3.01 E+05$ & $8.6 E+\infty$ & & $1.9 E+02$ & $\mathrm{pCi}$ & $12-09-98$ & $04-01-92$ & $1.00 E+00 \mid$ & $\mathrm{sm}$ & QUAL & & 101.0 & 05.01 & $00630-3-16259$ \\
\hline QA SP:NAS-A0271. & CS137 & GAM20 & $2.06 E+05$ & $8.1 E+00$ & & $6.8 E+01$ & $\mathrm{pCi}$ & 12.09-98 & $04-01-92$ & $1.00 E+00 \mid$ & $\mathrm{sm}$ & QUAL & & 101.3 & 05.01 & $00630-3-16259$ \\
\hline
\end{tabular}

\section{Comment:}

Data generated from analyses performed on samples submitted $12 / 9 / 98$. Gamma spectroscopy was performed in order to analyze for U-238 within the turnaround requested by the project.

Prepared by:

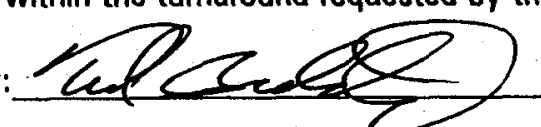
Date: $/ 2 / 14 / 28$ approved by: Sasuyffeteta Date: $12 / 15 / 98$
Qualification Flags:

$E$ = Estimated Quantity

$H=$ High Recovery for Sample

$\mathrm{J}=$ Result is less than the RDL

$L=$ Low Recovery for Sample

$P=$ Preliminary Results

$\mathrm{Q}=$ Bad Instrument Quality Control, Result is OK

$R=$ Results are Unusable, Resampling is Necessary

$\mathrm{U}=$ Result is less than Minimum Detectable Activity
Note: \% Error is the 2.0 Sigma Error 
THIS PAGE INTENTIONALLY LEFT BLANK 


\section{SAMPLE}

\section{DELIVERY}

GROUP

V653 
THIS PAGE INTENTIONALLY LEFT BLANK 
CLIENT: Bechtel Nevada

P.O. Box 98521, M/S NTS273

Las Vegas, NV 89193-8521

ATTN: Ted Redding

PROJECT NAME: V653

NEL ORDER ID: .L9907011

PROJECT NUMBER: 17777

Attached are the analytical results for samples in support of the above referenced project.

Samples were received by NEL in good condition, under chain of custody on 7/1/99.

Samples were analyzed as received.

Should you have any questions or comments, please feel free to contact our Client Services department at (702) 657-1010.

Some results have been flagged as follows:

J - This concentration should be considered an estimate due laboratory control sample failure.

Some $Q A$ results have been nagged as follows:

J - This concentration should be considered an estimate due laboratory control sample failure.

Stan $\operatorname{Tan}$ Wagenen

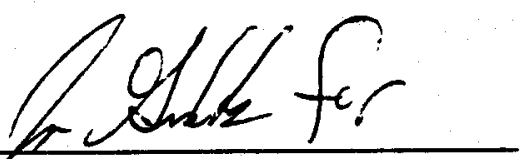

Gooratory Manager

CERTIFICATIONS:

\begin{tabular}{|c|c|c|c|c|c|c|c|}
\hline & Reno & Las Vegas & S. California & & Reno & Las Vegas & S. California \\
\hline Arizona & AZ0520 & AZ0518 & AZ0605 & Idaho & Certified & Certified & \\
\hline California & 1707 & 2002 & 2264 & Montana & Certified & Certified & \\
\hline $\begin{array}{l}\text { US Army Corps } \\
\text { of Engineers }\end{array}$ & Certified & Certified. & Certified & $\begin{array}{l}\text { Nevada } \\
\text { L.A.C.S.D. }\end{array}$ & NV033 & NV052 & $\begin{array}{l}\text { CA084 } \\
10228\end{array}$ \\
\hline
\end{tabular}




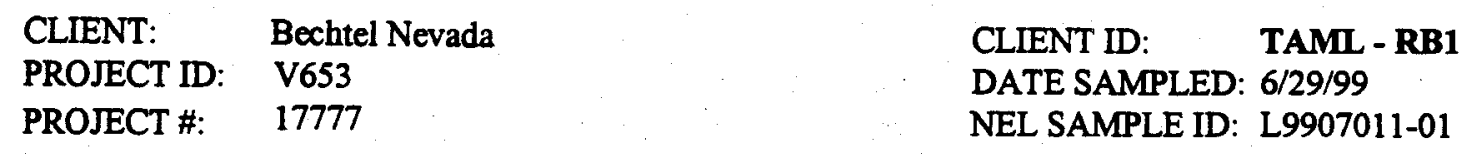

TEST: Nitroaromatics and Nitroamines by EPA 8330

$\begin{array}{llll}\text { MATRIX: } & \text { Aqueous } & \text { ANALYST: } & \text { YW - Reno Division } \\ \text { DILUTION: } & 1 & \text { EXTRACTED: } & 7 / 2 / 99 \\ & & \text { ANALYZED: } & 7 / 7 / 99\end{array}$

\begin{tabular}{lcc}
\hline PARAMETER & Result & $\begin{array}{c}\text { Reporting } \\
\text { Limit }\end{array}$ \\
\cline { 2 - 3 } 2-Am-DNT \& 4-Am-DNT & $\mathrm{ND}$ & $1.5 \mu \mathrm{g} / \mathrm{L}$ \\
2,4-DNT \& 2,6-DNT & $\mathrm{ND}$ & $1.5 \mu \mathrm{g} / \mathrm{L}$ \\
1,3-DNB & $\mathrm{ND}$ & $1.5 \mu \mathrm{g} / \mathrm{L}$ \\
HMX & $\mathrm{ND}$ & $1.5 \mu \mathrm{g} / \mathrm{L}$ \\
Nitrobenzene & $\mathrm{ND}$ & $1.5 \mu \mathrm{g} / \mathrm{L}$ \\
2-Nitrotoluene & $\mathrm{ND}$ & $2 . \mu \mathrm{g} / \mathrm{L}$ \\
3-Nitrotoluene & $\mathrm{ND}$ & $2.5 \mu \mathrm{g} / \mathrm{L}$ \\
4-Nitrotoluene & $\mathrm{ND}$ & $2 . \mu \mathrm{g} / \mathrm{L}$ \\
RDX & $\mathrm{ND}$ & $4 . \mu \mathrm{g} / \mathrm{L}$ \\
Tetryl & $\mathrm{ND}$ & $3 . \mu \mathrm{g} / \mathrm{L}$ \\
1,3,5-TNB & $\mathrm{ND}$ & $1 . \mu \mathrm{g} / \mathrm{L}$ \\
2,4,6-TNT & $\mathrm{ND}$ & $2 . \mu \mathrm{g} / \mathrm{L}$ \\
\hline QUALITY CONTROL DATA: & & Acceptable Range \\
Surrogate & Recovery & $70-130$
\end{tabular}

ND - Not Detected

This report shall not be reproduced except in full, without the written approval of the laboratory. 


\begin{tabular}{|c|c|c|c|}
\hline $\begin{array}{l}\text { ILIENT: } \\
\text { ROJECT ID: } \\
\text { ROJECT \#: }\end{array}$ & $\begin{array}{l}\text { Bechtel Nevada } \\
\text { V653 } \\
17777\end{array}$ & $\begin{array}{l}\text { CLIENT ID: } \\
\text { DATE SAMPLED: } \\
\text { NEL SAMPLE ID: }\end{array}$ & $\begin{array}{l}\text { Method Blank } \\
\text { NA } \\
\text { 070299-H1-BLK }\end{array}$ \\
\hline
\end{tabular}

TEST: Nitroaromatics and Nitroamines by EPA 8330

$\begin{array}{llll}\text { MATRIX: } & \text { Aqueous } & \text { ANALYST: } & \text { YW - Reno Division } \\ & & \text { EXTRACTED: } & 7 / 2 / 99 \\ & \text { ANALYZED: } & 7 / 7 / 99\end{array}$

\section{PARAMETER}

2-Am-DNT \& 4-Am-DNT

1,3-DNB

2,4-DNT \& 2,6-DNT

$\mathrm{HMX}$

Nitrobenzene

2-Nitrotoluene

3-Nitrotoluene

4-Nitrotoluene

$\mathrm{RDX}$

Tetryl

1,3,5-TNB

2,4,6-TNT

QUALITY CONTROL DATA:

Surrogate

1,2-Dinitrobenzene

$\%$ Recovery

93
Reporting

Result

ND

ND

ND

ND

$\mathrm{ND}$

ND

ND

ND

ND

ND

ND

$\mathrm{ND}$
Limit

$1.5 \mu \mathrm{g} / \mathrm{L}$

$1.5 \mu \mathrm{g} / \mathrm{L}$

$1.5 \mu \mathrm{g} / \mathrm{L}$

$1.5 \mu \mathrm{g} / \mathrm{L}$

$1.5 \mu \mathrm{g} / \mathrm{L}$

2. $\mu \mathrm{g} / \mathrm{L}$

$2.5 \mu \mathrm{g} / \mathrm{L}$

2. $\mu \mathrm{g} / \mathrm{L}$

4. $\mu \mathrm{g} / \mathrm{L}$

3. $\mu g / L$

1. $\mu \mathrm{g} / \mathrm{L}$

2. $\mu \mathrm{g} / \mathrm{L}$

Acceptable Range

$70-130$

ND - Not Detected

This report shall not be reproduced except in full, without the written approval of the laboratory. 


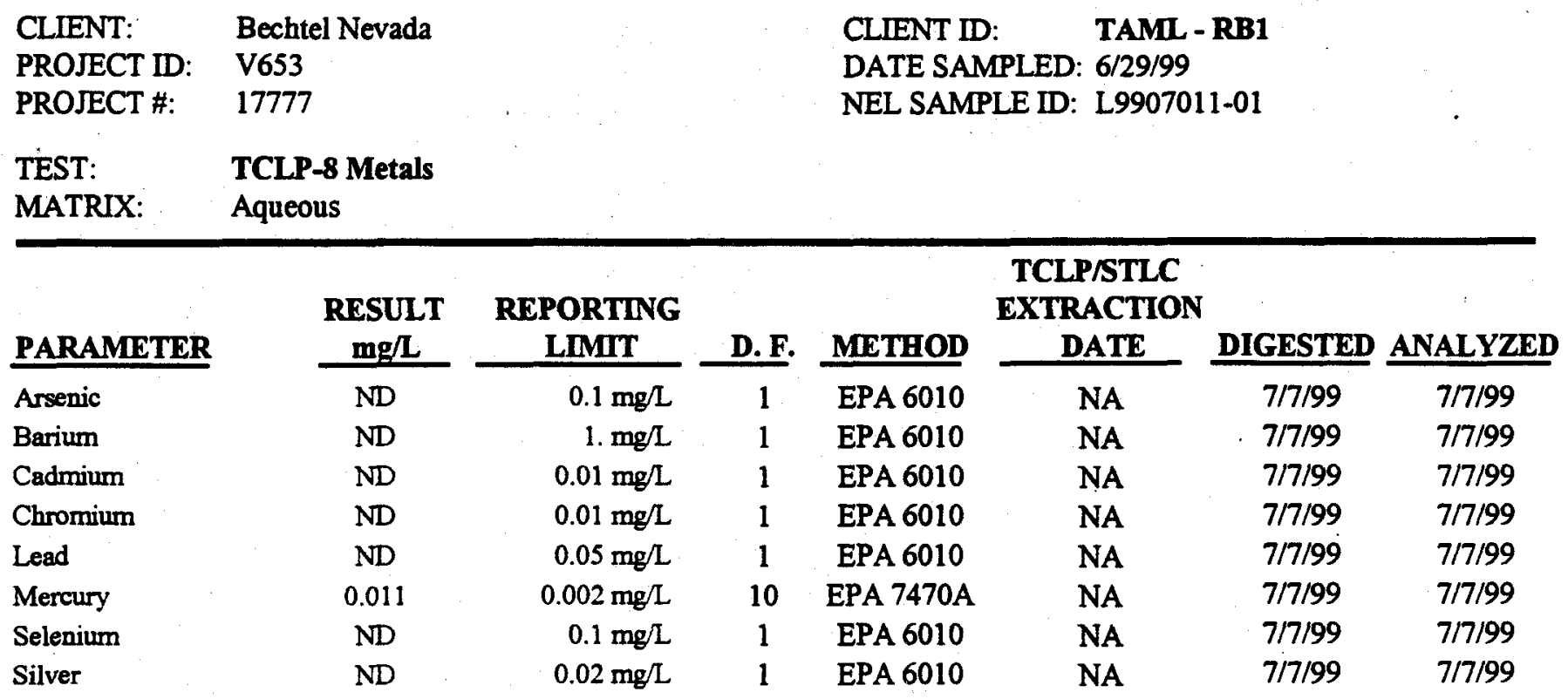

D.F. - Dilution Factor

ND - Not Detected

This report shall not be reproduced except in full, without the written approval of the laboratory. 


\begin{tabular}{|c|c|c|c|}
\hline $\begin{array}{l}\text { ZLIENT: } \\
\text { 'ROJECT ID: } \\
\text { 'ROJECT \#: }\end{array}$ & $\begin{array}{l}\text { Bechtel Nevada } \\
\text { V653 } \\
17777\end{array}$ & $\begin{array}{l}\text { CLIENT ID: } \\
\text { DATE SAMPLED: } \\
\text { NEL SAMPLE ID: }\end{array}$ & $\begin{array}{l}\text { Method Blank } \\
\text { NA } \\
\text { L07011i-BLK }\end{array}$ \\
\hline
\end{tabular}

[EST: $\quad$ TCLP by EPA 1311, July 1992 \& 7 Metals by EPA 6010A, July 1992

MATRIX: TCLP Extract

\begin{tabular}{|c|c|c|c|c|c|c|c|}
\hline \multirow[b]{2}{*}{ ?ARAMETER } & \multirow[b]{2}{*}{ RESULT } & \multirow{2}{*}{$\begin{array}{l}\text { REPORTING } \\
\text { LIMIT }\end{array}$} & \multicolumn{5}{|c|}{$\begin{array}{c}\text { TCLP/STLC } \\
\text { EXTRACTION }\end{array}$} \\
\hline & & & D. $\mathbf{F}$. & METHOD & DATE & DIGESTED & ANALYZED \\
\hline trsenic & $\mathrm{ND}$ & $0.1 \mathrm{mg} / \mathrm{L}$ & 1 & EPA 6010 & NA & 7/7/99 & 7/7/99 \\
\hline 3arium & ND & $1 \mathrm{mg} / \mathrm{L}$ & 1 & EPA 6010 & NA & 7/7/99 & $7 / 7 / 99$ \\
\hline Jadmium & $\mathrm{ND}$ & $0.01 \mathrm{mg} / \mathrm{L}$ & 1 & EPA 6010 & NA & 7/7/99 & 7/7/99 \\
\hline Yhromium & ND & $0.01 \mathrm{mg} / \mathrm{L}$ & 1 & EPA 6010 & NA & 7/7/99 & $7 / 7 / 99$ \\
\hline ead & ND & $0.05 \mathrm{mg} / \mathrm{L}$ & 1 & EPA 6010 & NA & 7/7/99 & 7/7/99 \\
\hline ielenium & ND & $0.1 \mathrm{mg} / \mathrm{L}$ & 1 & EPA 6010 & NA & 7/7/99 & $7 / 7 / 99$ \\
\hline jilver & ND & $0.02 \mathrm{mg} / \mathrm{L}$ & 1 & EPA 6010 & NA & $7 / 7 / 99$ & 7/7/99 \\
\hline
\end{tabular}

J.F. - Dilution Factor

VD - Not Detected

This report shall not be reproduced except in full, without the written approval of the laboratory. 


$\begin{array}{llll}\text { CLIENT: } & \text { Bechtel Nevada } & \text { CLIENT ID: } & \text { Method Blank } \\ \text { PROJECT ID: } & \text { V653 } & \text { DATE SAMPLED: } & \text { NA } \\ \text { PROJECT \#: } & 17777 & \text { NEL SAMPLE ID: } \text { P07003HG-BLK }\end{array}$

TEST: $\quad$ TCLP by EPA 1311, July 1992 \& Mercury by EPA 7470A, July 1992

MATRIX: TCLP Extract

\begin{tabular}{|c|c|c|c|c|c|c|c|}
\hline \multirow[b]{2}{*}{ PARAMETER } & \multicolumn{3}{|c|}{ REPORTING } & \multirow{2}{*}{\multicolumn{3}{|c|}{$\begin{array}{c}\text { TCLPSSTLC } \\
\text { EXTRACTION }\end{array}$}} & \\
\hline & RESULT & LIMIT & D. F. & & & DIGESTED & ANALYZED \\
\hline Mercury & ND & $0.002 \mathrm{mg} / \mathrm{L}$ & 10 & EPA 7470A & NA & $7 / 7 / 99$ & $7 / 7 / 99$ \\
\hline
\end{tabular}

D.F. - Dilution Factor

ND - Not Detected

This report shall not be reproduced except in full, without the written approval of the laboratory. 


$\begin{array}{llll}\text { CLIENT: } & \text { Bechtel Nevada } & \text { CLIENT ID: } & \text { TAML - RB1 } \\ \text { PROJECT ID: } & \text { V653 } & \text { DATE SAMPLED: } & 6 / 29 / 99 \\ \text { PROJECT \#: } & 17777 & \text { NEL SAMPLE ID: } & \text { L9907011-01 }\end{array}$

TEST: $\quad$ TCLP by EPA 1311, July 1992 \& Semivolatile Organics by EPA Method 8270C, Dec. 1996

MATRIX: Aqueous TCLP EXTRACT DATE: NA

DILUTION: 1

EXTRACTED $\quad 7 / 2 / 99$

ANALYZED: $\quad 7 / 7 / 99$

\section{PARAMETER}

1,4-Dichlorobenzene (p-DCB)

2,4-Dinitrotoluene (DNT)

Hexachlorobenzene

Hexachlorobutadiene

Hexachloroethane

2-Methylphenol

Nitrobenzene

Pentachlorophenol

Pyridine

2,4,5-Trichlorophenol

2,4,6-Trichlorophenol

\begin{tabular}{c}
$\begin{array}{c}\text { Result } \\
\text { mg/L }\end{array}$ \\
\hline ND \\
ND \\
ND \\
ND \\
ND \\
ND \\
ND \\
ND \\
ND \\
ND \\
ND
\end{tabular}

Reporting

Limit

$0.1 \mathrm{mg} / \mathrm{L}$

$0.1 \mathrm{mg} / \mathrm{L}$

$0.1 \mathrm{mg} / \mathrm{L}$

$0.1 \mathrm{mg} / \mathrm{L}$

$0.1 \mathrm{mg} / \mathrm{L}$

$0.1 \mathrm{mg} / \mathrm{L}$

$0.1 \mathrm{mg} / \mathrm{L}$

$0.1 \mathrm{mg} / \mathrm{L}$

$0.1 \mathrm{mg} / \mathrm{L}$

$0.1 \mathrm{mg} / \mathrm{L}$

$0.1 \mathrm{mg} / \mathrm{L}$

QUALITY CONTROL DATA:

\section{Surrogate}

2,4,6-Tribromophenol

2-Fluorobiphenyl

2-Fluorophenol

Nitrobenzene-d5

p-Terphenyl-d14

Phenol-d5
$\%$ Recovery

66

54

32

53

105

21
Acceptable Range

$10-123$

$43-116$

$21-100$

$35-114$

$33-141$

10 - 94 


$\begin{array}{llll}\text { CLIENT: } & \text { Bechtel Nevada } & \text { CLIENT ID: } & \text { Method Blank } \\ \text { PROJECT ID: } & \text { V653 } & \text { DATE SAMPLED: NA } \\ \text { PROJECT \#: } & 17777 & \text { NEL SAMPLE ID: } & \text { 070299-E2-BLK }\end{array}$

TEST: $\quad$ TCLP by EPA 1311, July 1992 \& Semivolatile Organics by EPA Method 8270C, Dec. 1996

METHOD: EPA $8270 \quad$ TCLP EXTRACT DATE: NA

$\begin{array}{lll}\text { MATRIX: TCLP Extract } & \text { EXTRACTED }\end{array}$

ANALYZED: $\quad 7 / 7 / 99$

\section{PARAMETER}

1,4-Dichlorobenzene (p-DCB)

2,4-Dinitrotoluene (DNT)

Hexachlorobenzene

Hexachlorobutadiene

Hexachloroethane

2-Methylphenol

Nitrobenzene

Pentachlorophenol

Pyridine

2,4,5-Trichlorophenol

2,4,6-Trichlorophenol

\begin{tabular}{c}
$\begin{array}{c}\text { Result } \\
\text { mg/L }\end{array}$ \\
\hline $\mathrm{ND}$ \\
$\mathrm{ND}$ \\
$\mathrm{ND}$ \\
$\mathrm{ND}$ \\
$\mathrm{ND}$ \\
$\mathrm{ND}$ \\
$\mathrm{ND}$ \\
$\mathrm{ND}$ \\
$\mathrm{ND}$ \\
$\mathrm{ND}$ \\
$\mathrm{ND}$
\end{tabular}

Reporting

Limit

$0.1 \mathrm{mg} / \mathrm{L}$

$0.1 \mathrm{mg} / \mathrm{L}$

$0.1 \mathrm{mg} / \mathrm{L}$

$0.1 \mathrm{mg} / \mathrm{L}$

$0.1 \mathrm{mg} / \mathrm{L}$

$0.1 \mathrm{mg} / \mathrm{L}$

$0.1 \mathrm{mg} / \mathrm{L}$

$0.1 \mathrm{mg} / \mathrm{L}$

$0.1 \mathrm{mg} / \mathrm{L}$

$0.1 \mathrm{mg} / \mathrm{L}$

$0.1 \mathrm{mg} / \mathrm{L}$

QUALITY CONTROL DATA:

\section{Surrogate}

2,4,6-Tribromophenol

2-Fluorobiphenyl

2-Fluorophenol

Nitrobenzene-d5

p-Terphenyl-d14

Phenol-d5
\% Recovery

63

56

32

54

108

21
Acceptable Range

$10-123$

$43-116$

$21-100$

$35-114$

$33-141$

$10-94$

ND - Not Detected

This report shall not be reproduced except in full. without the written approval of the laboratory. 


\section{GENERAL INFORMATION}

Sample Delivery Group: V653

Client: Bechtel Nevada

Laboratory:

NEL

Reviewed by:

Marsha C. Webb

Date: August 25, 1999

Requested Analysis:

SVO (TCLP)

Methods: Extraction

$44 \pi 1311$
Analysis 8270

Matrix: Water

II. COMPLETENESS

A. Were all deliverables submitted?

B. Are forms properly completed and legible?

C. Were all requested analyses performed by the laboratory?

D. Were the requested analytical methods used for analysis?

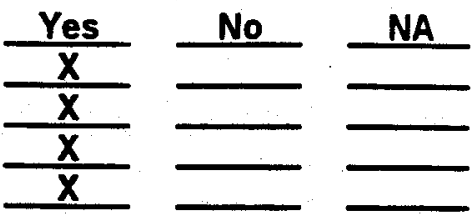

III. DATA ASSESSMENT SUMMARY

( ) A. Field and QAQC sample data have been generated in accordance with method requirements and within quality control limits.

(XF B. QAQC problems were encountered during analysis of the samples. However, the data are considered acceptable.

( ) C. Significant QAQC problems were encountered during analysis of the samples. Data for the following samples may be rejected.

IV. HOLDING TIME COMPLIANCE
A. Sampled
B. ASL Received:
C. Contract Lab Received:
D. Latest TCLP Extraction:
E. Latest Preparative Extraction:
F. Latest Date Analyzed:
Were holding time criteria met?

\section{INSTRUMENT \& SAMPLE QAVQC CRITERIA}

\section{A. INSTRUMENT QUALITY CONTROL}

1. Were all samples analyzed within a valid 12 -hour tune period?

2. Tune - Decafluorotriphenylphosphine

Ion Abundances

3. Calibrations

Initial:

Verification:

B. SAMPLE ASSOCIATED QUALITY CONTROL
CCC \% RSD, COFV $>0.99$

SPCC RRF values

CCC \% D, Conc. \% Drift

IS Area and Retention Times
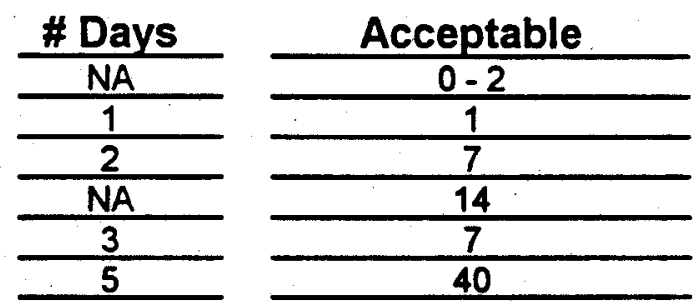

\section{Criteria met}

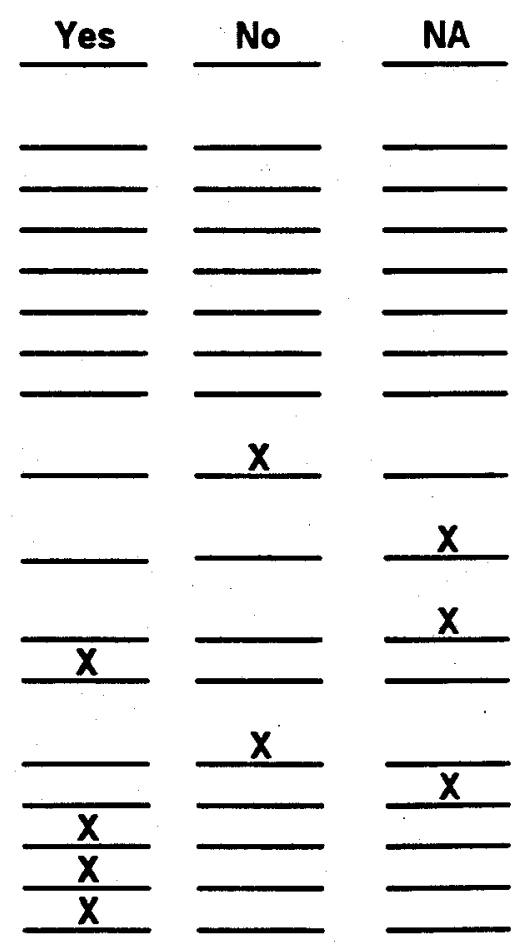




\section{INSTRUMENT \& SAMPLE QAQC CRITERIA}

6. As appropriate, were the following within quality control limits:

a. MSMSD recoveries and RPDs

b. Lab and/or field duplicate RPDs

\section{Criteria met}

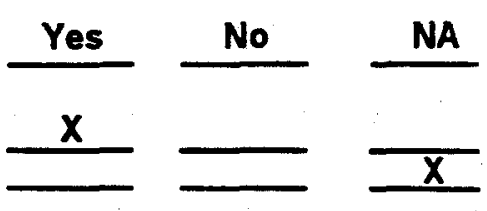

$1^{*}=$ indeterminant

COMMENTS: Tune data was not found in the data package. The $50 \mathrm{ng}$ std was run, but the continuing calibration report was not included. LCS and MSMSD spiking compounds and spiking levels would be useful information!

Marshechelo

Reviewer Signature:
Auqust 26.1999 (3:54pm)

Date: 


\section{GENERAL INFORMATION}

Sample Delivery Group: V653

Client: Bechtel Nevada

Laboratory:

NEL

Reviewed by: Marsha C. Webb

Date: $8 / 26 / 99$

Requested Analysis:

Nitroaromatics

Methods: Extraction NA

Analysis 8330

Matrix:

Water

\section{COMPLETENESS}

A. Were all deliverables submitted?

B. Are forms properly completed and legible?

C. Were all requested analyses performed by the laboratory?

D. Were the requested analytical methods used for analysis?

Criteria Met

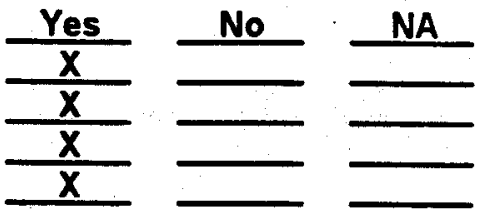

III. DATA ASSESSMENT SUMMARY

0 A. Field and QAQC sample data have been generated in accordance with method requirements and within quality control limits.

(X) B. QAVC problems were encountered during analysis of the samples. However, the data are considered acceptable.

0 C. Significant QAQC problems were encountered during analysis of the samples. Data for the following samples may be rejected.

IV. HOLDING TIME COMPLIANCE
A. Sampled
B. ASL Received:
C. Contract Lab Received:
D. Latest Extraction:
E. Latest Date Analyzed:

Were holding time criteria met?

(X) Yes

\begin{tabular}{c} 
Date \\
\hline $6 / 29 / 99$ \\
\hline $6 / 30 / 99$ \\
\hline $7 / 1 / 99$ \\
\hline $7 / 2 / 99$ \\
\hline $7 / 7 / 99$ \\
\hline $0 / N 0$
\end{tabular}

$\frac{\frac{\text { \# Days }}{\text { NA }}}{\frac{1}{2}}$

V. INSTRUMENT \& SAMPLE QAQC COMPLIANCE

A. INSTRUMENT QUALITY CONTROL
1. Calibrations

\begin{abstract}
Blank:
Initial:
\end{abstract}
Verification:
Within $3 X$ of the IDL (< reporting timit)
3 Standards minimum
$R^{2}>0.995$ or $\% R S D$ met
ICV within $\pm 20 \%$
CCV beginning, ending within $\pm 20 \%$

B. SAMPLE ASSOCIATED QUALITY CONTROL

1. Prep blank: Were target compounds detected in field or laboratory blanks?

2. Lab Control Sample: Were recoveries within acceptable limits? $( \pm 20 \%)$

3. Samples

a. Were target compounds detected in the samples?

b. Were concentrations calculated correctly?

4. As appropriate, were the following within quality control limits:

a. MSMSD recoveries and RPDs

b. Lab and/or field duplicate RPDs

c. Surrogate recoveries within quality control limits $(70-130 \%)$
Acceptable

\begin{tabular}{c}
\hline $0-2$ \\
\hline 1 \\
\hline 7 \\
\hline 14 \\
\hline 40
\end{tabular}

\section{Criteria met}

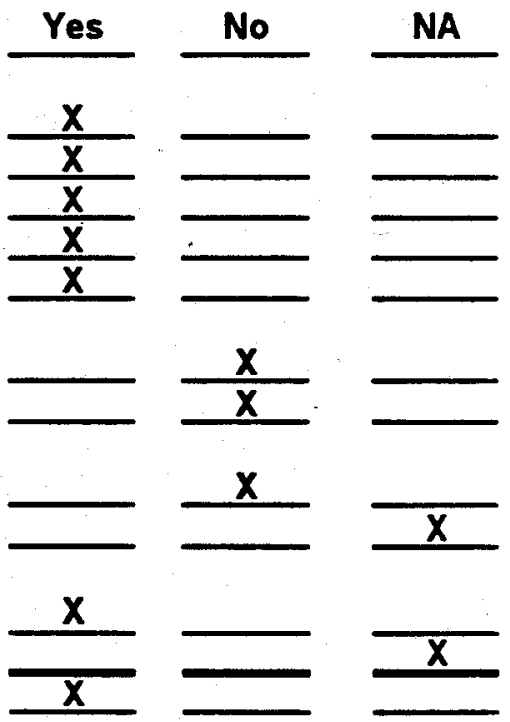


COMMENTS: Low recovery for Tetryl; sample result qualified J, estimated, by the laboratory.

Mras a d led

Reviewer Signature:
Auqust 26. $1999(1: 21 \mathrm{pm})$

Date: 


\section{GENERAL INFORMATION}

Sample Delivery Group: V653

Client:

Bechtel Nevada

Laboratory:

Requested Analysis:

NEL

Reviewed by:

\begin{tabular}{ll}
\hline Marsha C. Webb & Date: $8 / 26 / 99$ \\
\hline Extraction $\quad$ Analysis 6010
\end{tabular}

Matrix:

RCRA Metals Dotaf)TC<pMethods:

Water

\section{COMPLETENESS}

A. Were all deliverables submitted?

B. Are forms properly completed and legible?

C. Were all requested analyses performed by the laboratory?

D. Were the requested analytical methods used for analysis?

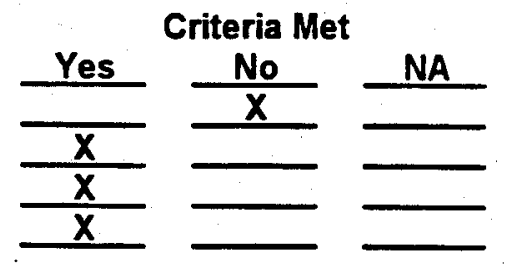

\section{DATA ASSESSMENT SUMMARY}

( ) A. Field and QAQC sample data have been generated in accordance with method requirements and within quality control limits.

X B. QAQC problems were encountered during analysis of the samples. However, the data are considered acceptable.

( ) C. Significant QAVC problems were encountered during analysis of the samples. Data for the.following samples may be rejected.

\section{HOLDING TIME COMPLIANCE}
A. Sampled
B. ASL Received:
C. Contract Lab Received:
D. Latest Extraction:
E. Latest Date Analyzed:

Were holding time criteria met?

\section{INSTRUMENT \& SAMPLE QAQC COMPLIANCE}

\section{A. INSTRUMENT QUALITY CONTROL}

\section{Calibrations

Blank:
Initial:
Verification:

2. Interference Check Sample

Within $3 X$ of the IDL

Minimum of Cal Blank and 1 Standard

ICV, CCVs, check standard within $\pm 10 \%$

RSD $<5 \%$ for 22 replicate integrations

within $\pm 20 \%$ true value
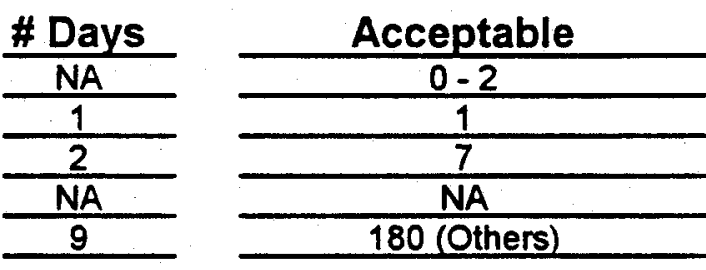

B. SAMPLE ASSOCIATED QUALITY CONTROL

1. Blanks: Were target compounds detected in field or laboratory blanks?

If so, were they detected:

a. at levels 2 CRDL?

b. in the samples at $<5$ times the blank value?

2. Lab Control Sample: Were recoveries within acceptable limits?

3. Samples

a. Were target compounds detected in the samples?

b. Were concentrations calculated correctly?

4. As appropriate, were the following within quality control limits:

a. MSMSD recoveries and RPDs

b. Lab and/or field duplicate RPDs

c. Serial dilution within $\pm 10 \%$ for undiluted concentrations $\geq 10 \times$ the IDL

Criteria met

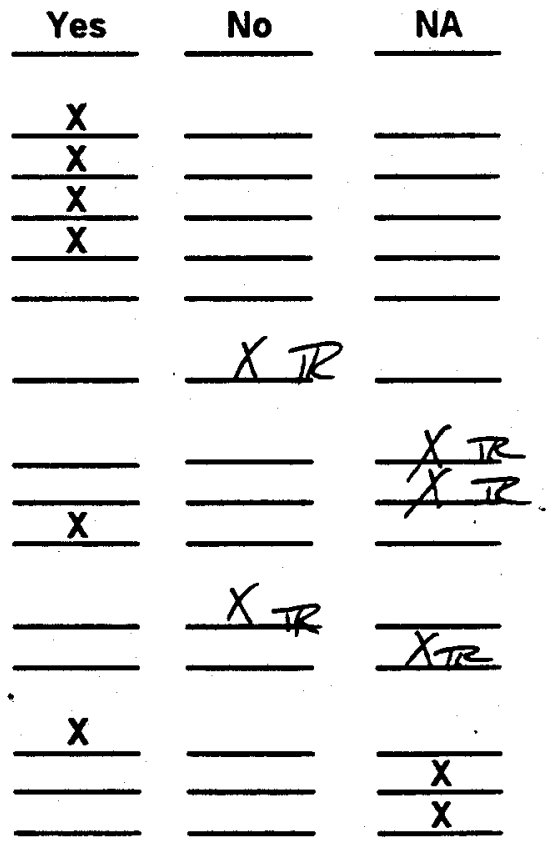


COMMENTS: The laboratory included the 1311 blank as the method blank for this sdg. This blank does sotapply to this water sample. The submitted CCB blank @ 14:48 is an incomplete copy. The raw data submitted for the sample was run $7 / 7 / 99$ at 16:27. Barium and Cadmium are detected; As is marked for a re-run. The sample is reported as ND for all analytes. Submit the correct raw data for the sample. Missing ending bracketing QC and ending ICSA, ICSAB runs.

Cased on reacellysis on $7 / 7 / 99016: 3 / \cdot R$

March civile

Reviewer Signature:
August 26. $1999(1: 34 \mathrm{pm})$ Date: 


\section{GENERAL INFORMATION}

\begin{tabular}{|c|c|c|c|c|c|c|}
\hline Sample Delivery Group: & $\forall 503 \mathbb{R} V 653$ & Client: & Bechtel & Jevada & & \\
\hline Laboratory: & NEL & Reviewed by: & Marsha & Webb & Date: $8 / 2$ & 199 \\
\hline Requested Analysis: & $\mathrm{Hg}$ & Methods: & Extraction & NA & Analysis & 7470 \\
\hline Matrix: & Water & & & & & \\
\hline
\end{tabular}

II. COMPLETENESS
A. Were all deliverables submitted?
B. Are forms properly completed and legible?
C. Were all requested analyses performed by the laboratory?
D. Were the requested analytical methods used for analysis?

Criteria Met

\begin{tabular}{|c|c|}
\hline \multirow{2}{*}{$\frac{\text { Yes }}{\mathrm{X}}$} & No \\
\hline & \\
\hline$\frac{x}{x}$ & \\
\hline$x$ & \\
\hline
\end{tabular}

III. DATA ASSESSMENT SUMMARY
(X) A. Field and QAVC sample data have been generated in accordance with method requirements and within quality control limits.
( ) B. QAVQC problems were encountered during analysis of the samples. However, the data are considered acceptable.
( ) C. Significant QAQC problems were encountered during analysis of the samples. Data for the following samples may be rejected.

IV. HOLDING TIME COMPLIANCE
A. Sampled
B. ASL Received:
C. Contract Lab Received:
D. Latest Extraction:
E. Latest Date Analyzed:

Were holding time criteria met?

(X) Yes

$\frac{\frac{\text { Date }}{6 / 29 / 99}}{\frac{6 / 30 / 99}{7 / 1 / 99}}$

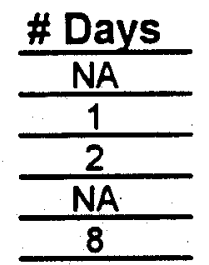

( ) No

\section{INSTRUMENT \& SAMPLE QAQC COMPLIANCE}

A. INSTRUMENT QUALITY CONTROL
1. Calibrations
Within $3 X$ of the IDL

Cal Blank and 5 Standards

$R^{2}>0.995$

ICV within $\pm 10 \%$

CCV every 10 samples within $\pm 20 \%$

B. SAMPLE ASSOCIATED QUALITY CONTROL

\begin{tabular}{ll} 
Initial: & Cal Blank and 5 Standards \\
& $R^{2}>0.995$ \\
Verification: & ICV within $\pm 10 \%$ \\
\hline & CCV every 10 samples within $\pm 20 \%$
\end{tabular}

1. Prep blank: Were target compounds detected in field or laboratory blanks?

2. Lab Control Sample: Were recoveries within acceptable limits? (80-120\%)

3. Samples
a. Were target compounds detected in the samples?
b. Were concentrations calculated correctly?

4. As appropriate, were the following within quality control limits:
a. MSMSD recoveries (75-125\%)
b. Lab and/or field duplicate RPDs ( $<20 \%)$
c. Serial dilution within $\pm 10 \%$ for undiluted concentrations $\geq 10 X$ the IDL

\section{Criteria met}
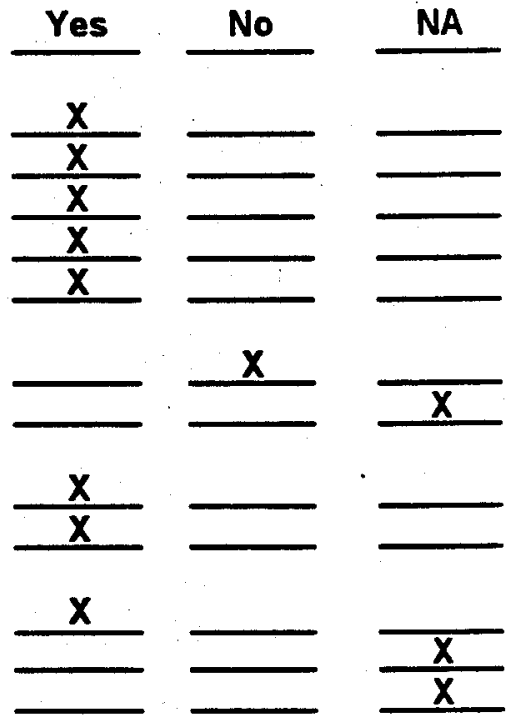
COMMENTS: Batch QC was not performed on this sample; batch QC was acceptable.

Mercincrion

Reviewer Signature:
August 26. 1999 (8:59am)

Date: 
CLIENT: Bechtel Nevada

P.O. Box 98521 , M/S NTS273

Las Vegas, NV 89193-8521

ATTN: Ted Redding

PROJECT NAME: V653

PROJECT NUMBER: 17777

NEL ORDER ID: L9907046

Attached are the analytical results for samples in support of the above referenced project.

Samples were received by NEL in good condition, under chain of custody on 7/7/99.

Samples were analyzed as received.

Should you have any questions or comments, please feel free to contact our Client Services department at (702) 657-1010.

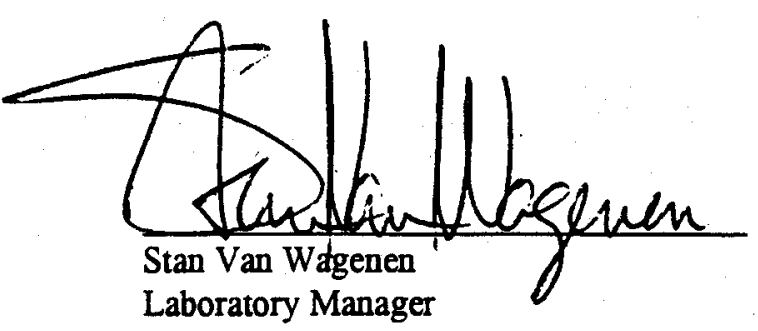

CERTIFICATIONS:

Arizona

California

$$
\text { Reno }
$$
AZ0520 1707 Las Vegas S. California US Army Corps of Engineers

Certified AZ0518 2002 AZ0605 2264

Certified Certified

Reno

Idaho Certified Montana

Nevada

Certified

NV033

L.A.C.S.D.

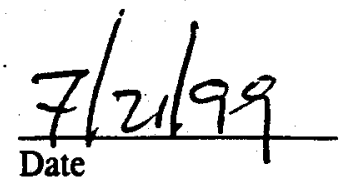

- 


\begin{tabular}{lll} 
CLIENT: & Bechtel Nevada & CLIENT ID: \\
PROJECT ID: & V653 & TAML - VER - 01 \\
PROJECT \#: & 17777 & DATE SAMPLED: $6 / 28 / 99$ \\
\hline
\end{tabular}

TEST: $\quad$ Nitroaromatics and Nitroamines by EPA 8330

$\begin{array}{llll}\text { MATRIX: } & \text { Solid } & \text { ANALYST: } & \text { YW - Reno Division } \\ \text { DILUTION: } & 1 & \text { EXTRACTED: } & 7 / 8 / 99 \\ & & \text { ANALYZED: } & 7 / 9 / 99\end{array}$

\section{PARAMETER}

1,3-DNB

$\mathrm{HMX}$

Nitrobenzene

2-Nitrotoluene

3-Nitrotoluene

4-Nitrotoluene

$\mathrm{RDX}$

Tetryl

1,3,5-TNB

2,4,6-TNT

\begin{tabular}{l} 
Result \\
\hline $\mathrm{ND}$ \\
$\mathrm{ND}$ \\
$\mathrm{ND}$ \\
$\mathrm{ND}$ \\
$\mathrm{ND}$ \\
$\mathrm{ND}$ \\
$\mathrm{ND}$ \\
$\mathrm{ND}$ \\
$\mathrm{ND}$ \\
$\mathrm{ND}$
\end{tabular}

ND - Not Detected

This report shall not be reproduced except in full, without the written approval of the laboratory. 


$\begin{array}{lll}\text { CLIENT: } & \text { Bechtel Nevada } & \text { CLIENT ID: } \quad \text { TAML - VER - 02 } \\ \text { PROJECT ID: } & \text { V653 } & \text { DATE SAMPLED: 6/28/99 } \\ \text { PROJECT \#: } & 17777 & \text { NEL SAMPLE ID: } \text { L9907046-02 }\end{array}$

TEST: $\quad$ Nitroaromatics and Nitroamines by EPA 8330

MATRIX: Solid

ANALYST: $\quad$ YW - Reno Division

DILUTION: 1

EXTRACTED: $\quad 7 / 8 / 99$

ANALYZED: $\quad 7 / 9 / 99$

\section{PARAMETER}

1,3-DNB

IMX

vitrobenzene

-Nitrotoluene

3-Nitrotoluene

h-Nitrotoluene

DDX

-etryl

,3,5-TNB

:,4,6-TNT

\section{Result}

ND

ND

ND

ND

ND

ND

ND

ND

ND

ND

\section{Reporting}

Limit

$0.25 \mathrm{mg} / \mathrm{Kg}$

2. $\mathrm{mg} / \mathrm{Kg}$

$0.25 \mathrm{mg} / \mathrm{Kg}$

$0.25 \mathrm{mg} / \mathrm{Kg}$

$0.25 \mathrm{mg} / \mathrm{Kg}$

$0.25 \mathrm{mg} / \mathrm{Kg}$

2. $\mathrm{mg} / \mathrm{Kg}$

$0.65 \mathrm{mg} / \mathrm{Kg}$

$0.25 \mathrm{mg} / \mathrm{Kg}$

$0.25 \mathrm{mg} / \mathrm{Kg}$

JD - Not Detected

his report shall not be reproduced except in full, without the written approval of the laboratory. 


\begin{tabular}{|c|c|c|}
\hline CLIENT: & Bechtel Nevada & CLIENT ID: $\quad$ TAML - VER - 03 \\
\hline PROJECT ID: & V653 & DATE SAMPLED: 6/28/99 \\
\hline PROJECT \#: & 17777 & NEL SAMPLE ID: L9907046-03 \\
\hline
\end{tabular}

TEST: $\quad$ Nitroaromatics and Nitroamines by EPA 8330

MATRIX: Solid

DILUTION: 1

ANALYST: $\quad$ YW - Reno Division

EXTRACTED: $\quad$ 7/8/99

ANALYZED: $\quad 7 / 9 / 99$

\section{PARAMETER}

1,3-DNB

\begin{tabular}{l} 
Result \\
\hline ND \\
ND \\
ND \\
ND \\
ND \\
ND \\
ND \\
ND \\
ND \\
ND
\end{tabular}

Reporting

HMX

Nitrobenzene

2-Nitrotoluene

3-Nitrotoluene

4-Nitrotoluene

\begin{tabular}{r}
\multicolumn{1}{c}{ Limit } \\
\hline $0.25 \mathrm{mg} / \mathrm{Kg}$ \\
$2 . \mathrm{mg} / \mathrm{Kg}$ \\
$0.25 \mathrm{mg} / \mathrm{Kg}$ \\
$0.25 \mathrm{mg} / \mathrm{Kg}$ \\
$0.25 \mathrm{mg} / \mathrm{Kg}$ \\
$0.25 \mathrm{mg} / \mathrm{Kg}$ \\
$2 . \mathrm{mg} / \mathrm{Kg}$ \\
$0.65 \mathrm{mg} / \mathrm{Kg}$ \\
$0.25 \mathrm{mg} / \mathrm{Kg}$ \\
$0.25 \mathrm{mg} / \mathrm{Kg}$
\end{tabular}

ND - Not Detected

This report shall not be reproduced except in full, without the written approval of the laboratory. 


\begin{tabular}{|c|c|c|}
\hline CLIENT: & Bechtel Nevada & CLIENT ID: $\quad$ TAML - VER - 04 \\
\hline PROJECT \#: & 17777 & NEL SAMPLE ID: L9907046-04 \\
\hline
\end{tabular}

TEST: $\quad$ Nitroaromatics and Nitroamines by EPA 8330

MATRIX: Solid

DILUTION: 1

ANALYST: $\quad$ YW - Reno Division

EXTRACTED: $\quad 7 / 8 / 99$

ANALYZED: $\quad 7 / 9 / 99$

\section{PARAMETER}

1,3-DNB

$\mathrm{TMX}$

Vitrobenzene

--Nitrotoluene

3-Nitrotoluene

H-Nitrotoluene

2DX

[etryl

,3,5-TNB

$:, 4,6-\mathrm{TNT}$

\begin{tabular}{l} 
Result \\
\hline $\mathrm{ND}$ \\
$\mathrm{ND}$ \\
$\mathrm{ND}$ \\
$\mathrm{ND}$ \\
$\mathrm{ND}$ \\
$\mathrm{ND}$ \\
$\mathrm{ND}$ \\
$\mathrm{ND}$ \\
$\mathrm{ND}$ \\
$\mathrm{ND}$
\end{tabular}

ID - Not Detected

his report shall not be reproduced except in full, without the written approval of the laboratory. 


\begin{tabular}{lll} 
CLIENT: & Bechtel Nevada & CLIENT ID: \\
PROJECT ID: & V653 & TAML - VER - 05 \\
PROJECT \#: & 17777 & DATE SAMPLED: 6/28/99 \\
\hline
\end{tabular}

TEST: $\quad$ Nitroaromatics and Nitroamines by EPA 8330

\begin{tabular}{llll} 
MATRIX: & Solid & ANALYST: & YW - Reno Division \\
DILUTION: & 1 & EXTRACTED: & $7 / 8 / 99$ \\
& & ANALYZED: & $7 / 9 / 99$ \\
\hline
\end{tabular}

\section{PARAMETER}

1,3-DNB

HMX

Nitrobenzene

2-Nitrotoluene

3-Nitrotoluene

4-Nitrotoluene

RDX

Tetryl

$1,3,5-\mathrm{TNB}$

$2,4,6-\mathrm{TNT}$

\begin{tabular}{l} 
Result \\
\hline ND \\
ND \\
ND \\
ND \\
ND \\
ND \\
ND \\
ND \\
ND \\
ND
\end{tabular}

\begin{tabular}{l}
$\begin{array}{l}\text { Reporting } \\
\text { Limit }\end{array}$ \\
\hline $0.25 \mathrm{mg} / \mathrm{Kg}$ \\
$2 . \mathrm{mg} / \mathrm{Kg}$ \\
$0.25 \mathrm{mg} / \mathrm{Kg}$ \\
$0.25 \mathrm{mg} / \mathrm{Kg}$ \\
$0.25 \mathrm{mg} / \mathrm{Kg}$ \\
$0.25 \mathrm{mg} / \mathrm{Kg}$ \\
$2 . \mathrm{mg} / \mathrm{Kg}$ \\
$0.65 \mathrm{mg} / \mathrm{Kg}$ \\
$0.25 \mathrm{mg} / \mathrm{Kg}$ \\
$0.25 \mathrm{mg} / \mathrm{Kg}$
\end{tabular}

ND - Not Detected

This report shall not be reproduced except in full, without the written approval of the laboratory. 
NEL LABORATORIES

$\begin{array}{llll}\text { CLIENT: } & \text { Bechtel Nevada } & \text { CLIENT ID: } & \text { TAML - VER - 06 } \\ \text { PROJECT ID: } & \text { V653 } & \text { DATE SAMPLED: 6/28/99 } \\ \text { PROJECT \#: } & 17777 & \text { NEL SAMPLE ID: L9907046-06 }\end{array}$

TEST: Nitroaromatics and Nitroamines by EPA 8330

MATRIX: Solid

DILUTION: $\quad 1$

ANALYST: $\quad$ YW - Reno Division

EXTRACTED: $\quad$ 7/8/99

ANALYZED: $\quad 7 / 9 / 99$

\section{PARAMETER}

,3-DNB

IMX

vitrobenzene

--Nitrotoluene

;-Nitrotoluene

-Nitrotoluene

iDX

ietryl

,3,5-TNB

$:, 4,6-\mathrm{TNT}$

\section{Result}

ND

ND

ND

ND

ND

ND

ND

ND

ND

ND

\section{Reporting}

Limit

$0.25 \mathrm{mg} / \mathrm{Kg}$

2. $\mathrm{mg} / \mathrm{Kg}$

$0.25 \mathrm{mg} / \mathrm{Kg}$

$0.25 \mathrm{mg} / \mathrm{Kg}$

$0.25 \mathrm{mg} / \mathrm{Kg}$

$0.25 \mathrm{mg} / \mathrm{Kg}$

2. $\mathrm{mg} / \mathrm{Kg}$

$0.65 \mathrm{mg} / \mathrm{Kg}$

$0.25 \mathrm{mg} / \mathrm{Kg}$

$0.25 \mathrm{mg} / \mathrm{Kg}$

ID - Not Detected

his report shall not be reproduced except in full, without the written approval of the laboratory. 


\begin{tabular}{|c|c|c|}
\hline CLIENT: & Bechtel Nevada & CLIENT ID: $\quad$ TAML - VER - 07 \\
\hline PROJECT ID: & V653 & DATE SAMPLED: $6 / 28 / 99$ \\
\hline PROJECT \#: & 17777 & NEL SAMPLE ID: L9907046-07 \\
\hline
\end{tabular}

TEST: $\quad$ Nitroaromatics and Nitroamines by EPA 8330

MATRIX: Solid

DILUTION: $\quad 1$

ANALYST: $\quad$ YW - Reno Division

EXTRACTED: $\quad 7 / 8 / 99$

ANALYZED: $\quad 7 / 9 / 99$

\section{PARAMETER}

1,3-DNB

HMX

Nitrobenzene

2-Nitrotoluene

3-Nitrotoluene

4-Nitrotoluene

RDX

Tetryl

1,3,5-TNB

2,4,6-TNT

\begin{tabular}{l} 
Result \\
\hline ND \\
ND \\
ND \\
ND \\
ND \\
ND \\
ND \\
ND \\
ND \\
ND
\end{tabular}

Reporting

Limit

$0.25 \mathrm{mg} / \mathrm{Kg}$
$2 . \mathrm{mg} / \mathrm{Kg}$
$0.25 \mathrm{mg} / \mathrm{Kg}$
$0.25 \mathrm{mg} / \mathrm{Kg}$
$0.25 \mathrm{mg} / \mathrm{Kg}$
$0.25 \mathrm{mg} / \mathrm{Kg}$
$2 . \mathrm{mg} / \mathrm{Kg}$
$0.65 \mathrm{mg} / \mathrm{Kg}$
$0.25 \mathrm{mg} / \mathrm{Kg}$
$0.25 \mathrm{mg} / \mathrm{Kg}$

2. $\mathrm{mg} / \mathrm{Kg}$

$0.25 \mathrm{mg} / \mathrm{Kg}$

$0.25 \mathrm{mg} / \mathrm{Kg}$

$0.25 \mathrm{mg} / \mathrm{Kg}$

$.25 \mathrm{mg} / \mathrm{Kg}$

2. $\mathrm{mg} / \mathrm{Kg}$

$0.65 \mathrm{mg} / \mathrm{Kg}$

$0.25 \mathrm{mg} / \mathrm{Kg}$

ND - Not Detected

This report shall not be reproduced except in full, without the written approval of the laboratory. 


$\begin{array}{lll}\text { CLIENT: } & \text { Bechtel Nevada } & \text { CLIENT ID: } \quad \text { TAML - VER - 08 } \\ \text { PROJECT ID: } & \text { V653 } & \text { DATE SAMPLED: 6/28/99 } \\ \text { PROJECT \#: } & 17777 & \text { NEL SAMPLE ID: } \text { L9907046-08 }\end{array}$

TEST: $\quad$ Nitroaromatics and Nitroamines by EPA 8330

MATRIX: Solid

DILUTION: 1

ANALYST: $\quad$ YW - Reno Division

EXTRACTED: $\quad$ 7/8/99

ANALYZED: $\quad 7 / 9 / 99$

\section{PARAMETER}

1,3-DNB

$\mathrm{HMX}$

Nitrobenzene

2-Nitrotoluene

3-Nitrotoluene

4-Nitrotoluene

$\mathrm{RDX}$

Tetryl

1,3,5-TNB

2,4,6-TNT

\begin{tabular}{l} 
Result \\
\hline ND \\
ND \\
ND \\
ND \\
ND \\
ND \\
ND \\
ND \\
ND \\
ND
\end{tabular}

ND - Not Detected

This report shall not be reproduced except in full, without the written approval of the laboratory. 


$\begin{array}{lll}\text { CLIENT: } & \text { Bechtel Nevada } & \text { CLIENT ID: } \quad \text { TAMR - VER - 09 } \\ \text { PROJECT ID: } & \text { V653 } & \text { DATE SAMPLED: } 6 / 28 / 99 \\ \text { PROJECT \#: } & 17777 & \text { NEL SAMPLE ID: } \text { L9907046-09 }\end{array}$

TEST: Nitroaromatics and Nitroamines by EPA 8330

\begin{tabular}{llll} 
MATRIX: & Solid & ANALYST: & YW - Reno Division \\
DILUTION: & 1 & EXTRACTED: & $7 / 8 / 99$ \\
& & ANALYZED: & $7 / 9 / 99$ \\
\hline
\end{tabular}

\section{PARAMETER}

1,3-DNB

\begin{tabular}{l} 
Result \\
\hline $\mathrm{ND}$ \\
$\mathrm{ND}$ \\
$\mathrm{ND}$ \\
$\mathrm{ND}$ \\
$\mathrm{ND}$ \\
$\mathrm{ND}$ \\
$\mathrm{ND}$ \\
$\mathrm{ND}$ \\
$\mathrm{ND}$ \\
$\mathrm{ND}$ \\
$\mathrm{ND}$ \\
$\mathrm{ND}$
\end{tabular}

\begin{tabular}{l}
$\begin{array}{l}\text { Reporting } \\
\text { Limit }\end{array}$ \\
\hline $0.25 \mathrm{mg} / \mathrm{Kg}$ \\
$0.25 \mathrm{mg} / \mathrm{kg}$ \\
$0.25 \mathrm{mg} / \mathrm{kg}$ \\
$2 . \mathrm{mg} / \mathrm{Kg}$ \\
$0.25 \mathrm{mg} / \mathrm{Kg}$ \\
$0.25 \mathrm{mg} / \mathrm{Kg}$ \\
$0.25 \mathrm{mg} / \mathrm{Kg}$ \\
$0.25 \mathrm{mg} / \mathrm{Kg}$ \\
$2 . \mathrm{mg} / \mathrm{Kg}$ \\
$0.65 \mathrm{mg} / \mathrm{Kg}$ \\
$0.25 \mathrm{mg} / \mathrm{Kg}$ \\
$0.25 \mathrm{mg} / \mathrm{Kg}$
\end{tabular}

ID - Not Detected

his report shall not be reproduced except in full, without the written approval of the laboratory. 


\begin{tabular}{|c|c|c|}
\hline CLIENT: & Bechtel Nevada & TAML - VER - 10 \\
\hline PROJECT ID: & V653 & DATE SAMPLED: $6 / 28 / 99$ \\
\hline PROJECT \#: & 1.7777 & NEL SAMPLE ID: L9907046-10 \\
\hline
\end{tabular}

TEST: $\quad$ Nitroaromatics and Nitroamines by EPA 8330

MATRIX: Solid

DILUTION: $\quad 1$

ANALYST: $\quad$ YW - Reno Division

EXTRACTED: $\quad 7 / 8 / 99$

ANALYZED: $\quad 7 / 9 / 99$

\section{PARAMETER}

1,3-DNB

HMX

Nitrobenzene

2-Nitrotoluene

3-Nitrotoluene

4-Nitrotoluene

RDX

Tetryl

$1,3,5-\mathrm{TNB}$

2,4,6-TNT

\section{Result}

ND

ND

ND

ND

ND

ND

ND

ND

ND

ND

\section{Reporting}

Limit

$0.25 \mathrm{mg} / \mathrm{Kg}$

2. $\mathrm{mg} / \mathrm{Kg}$

$0.25 \mathrm{mg} / \mathrm{Kg}$

$0.25 \mathrm{mg} / \mathrm{Kg}$

$0.25 \mathrm{mg} / \mathrm{Kg}$

$0.25 \mathrm{mg} / \mathrm{Kg}$

2. $\mathrm{mg} / \mathrm{Kg}$

$0.65 \mathrm{mg} / \mathrm{Kg}$

$0.25 \mathrm{mg} / \mathrm{Kg}$

$0.25 \mathrm{mg} / \mathrm{Kg}$

ND - Not Detected

This report shall not be reproduced except in full, without the written approval of the laboratory. 


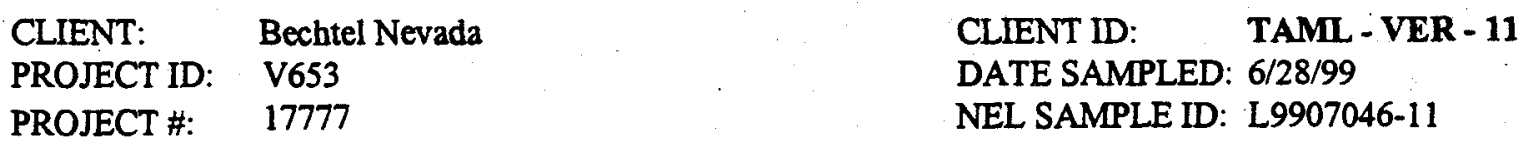

TEST: $\quad$ Nitroaromatics and Nitroamines by EPA 8330

$\begin{array}{llll}\text { MATRIX: } & \text { Solid } & \text { ANALYST: } & \text { YW - Reno Division } \\ \text { DILUTION: } & 1 & \text { EXTRACTED: } & 7 / 8 / 99 \\ & & \text { ANALYZED: } & 7 / 9 / 99\end{array}$

\section{PARAMETER}

1,3-DNB

HMX

Nitrobenzene

2-Nitrotoluene

3-Nitrotoluene

4-Nitrotoluene

$\mathrm{RDX}$

Tetryl

1,3,5-TNB

2,4,6-TNT

\begin{tabular}{l} 
Result \\
\hline ND \\
ND \\
ND \\
ND \\
ND \\
ND \\
ND \\
ND \\
ND \\
ND
\end{tabular}

Reporting

Limit

$0.25 \mathrm{mg} / \mathrm{Kg}$

2. $\mathrm{mg} / \mathrm{Kg}$

$0.25 \mathrm{mg} / \mathrm{Kg}$

$0.25 \mathrm{mg} / \mathrm{Kg}$

$0.25 \mathrm{mg} / \mathrm{Kg}$

$0.25 \mathrm{mg} / \mathrm{Kg}$

2. $\mathrm{mg} / \mathrm{Kg}$

$0.65 \mathrm{mg} / \mathrm{Kg}$

$0.25 \mathrm{mg} / \mathrm{Kg}$

$0.25 \mathrm{mg} / \mathrm{Kg}$

ND - Not Detected

This report shall not be reproduced except in full, without the written approval of the laboratory. 


\begin{tabular}{|c|c|c|}
\hline CLIENT: & Bechtel Nevada & TAML - VER - 12 \\
\hline PROJECT ID: & V653 & DATE SAMPLED: $6 / 29 / 99$ \\
\hline PROJECT \#: & 17777 & NEL SAMPLE ID: L9907046-12 \\
\hline
\end{tabular}

TEST: $\quad$ Nitroaromatics and Nitroamines by EPA 8330

\begin{tabular}{|c|c|c|c|}
\hline $\begin{array}{l}\text { MATRIX: } \\
\text { DILUTION: }\end{array}$ & $\begin{array}{l}\text { Solid } \\
1\end{array}$ & $\begin{array}{l}\text { ANALYST: } \\
\text { EXTRACTED: } \\
\text { ANALYZED: }\end{array}$ & $\begin{array}{l}\text { YW - Reno Division } \\
7 / 8 / 99 \\
7 / 10 / 99\end{array}$ \\
\hline
\end{tabular}

\section{PARAMETER}

1,3-DNB

HMX

Nitrobenzene

2-Nitrotoluene

3-Nitrotoluene

4-Nitrotoluene

RDX

Tetryl

$1,3,5-\mathrm{TNB}$

2,4,6-TNT

\begin{tabular}{l} 
Result \\
\hline ND \\
ND \\
ND \\
ND \\
ND \\
ND \\
ND \\
ND \\
ND \\
ND
\end{tabular}

\section{Reporting \\ Limit}

$0.25 \mathrm{mg} / \mathrm{Kg}$

2. $\mathrm{mg} / \mathrm{Kg}$

$0.25 \mathrm{mg} / \mathrm{Kg}$

$0.25 \mathrm{mg} / \mathrm{Kg}$

$0.25 \mathrm{mg} / \mathrm{Kg}$

$0.25 \mathrm{mg} / \mathrm{Kg}$

2. $\mathrm{mg} / \mathrm{Kg}$

$0.65 \mathrm{mg} / \mathrm{Kg}$

$0.25 \mathrm{mg} / \mathrm{Kg}$

$0.25 \mathrm{mg} / \mathrm{Kg}$

ND - Not Detected

This report shall not be reproduced except in full, without the written approval of the laboratory. 
CLIENT: Bechtel Nevada

PROJECT ID: V653

PROJECT \#: $\quad 17777$
CLIENT ID:

DATE SAMPLED: $6 / 28 / 99$

NEL SAMPLE ID: L9907046-13

TEST: Nitroaromatics and Nitroamines by EPA 8330

MATRIX: Solid

DILUTION: 1
ANALYST: $\quad$ YW - Reno Division

EXTRACTED: $\quad 7 / 8 / 99$

ANALYZED: $\quad 7 / 10 / 99$

\section{PARAMETER}

1,3-DNB

$\mathrm{HMX}$

Nitrobenzene

2-Nitrotoluene

3-Nitrotoluene

4-Nitrotoluene

$\mathrm{RDX}$

Tetryl

1,3,5-TNB

2,4,6-TNT

\begin{tabular}{l} 
Result \\
\hline $\mathrm{ND}$ \\
$\mathrm{ND}$ \\
$\mathrm{ND}$ \\
$\mathrm{ND}$ \\
$\mathrm{ND}$ \\
$\mathrm{ND}$ \\
$\mathrm{ND}$ \\
$\mathrm{ND}$ \\
$\mathrm{ND}$ \\
$\mathrm{ND}$
\end{tabular}

$\mathrm{ND}$
Reporting

Limit $0.25 \mathrm{mg} / \mathrm{Kg}$

2. $\mathrm{mg} / \mathrm{Kg}$

$0.25 \mathrm{mg} / \mathrm{Kg}$

$0.25 \mathrm{mg} / \mathrm{Kg}$

$0.25 \mathrm{mg} / \mathrm{Kg}$

$0.25 \mathrm{mg} / \mathrm{Kg}$

2. $\mathrm{mg} / \mathrm{Kg}$

$0.65 \mathrm{mg} / \mathrm{Kg}$

$0.25 \mathrm{mg} / \mathrm{Kg}$

$0.25 \mathrm{mg} / \mathrm{Kg}$

ND - Not Detected

This report shall not be reproduced except in full, without the written approval of the laboratory. 


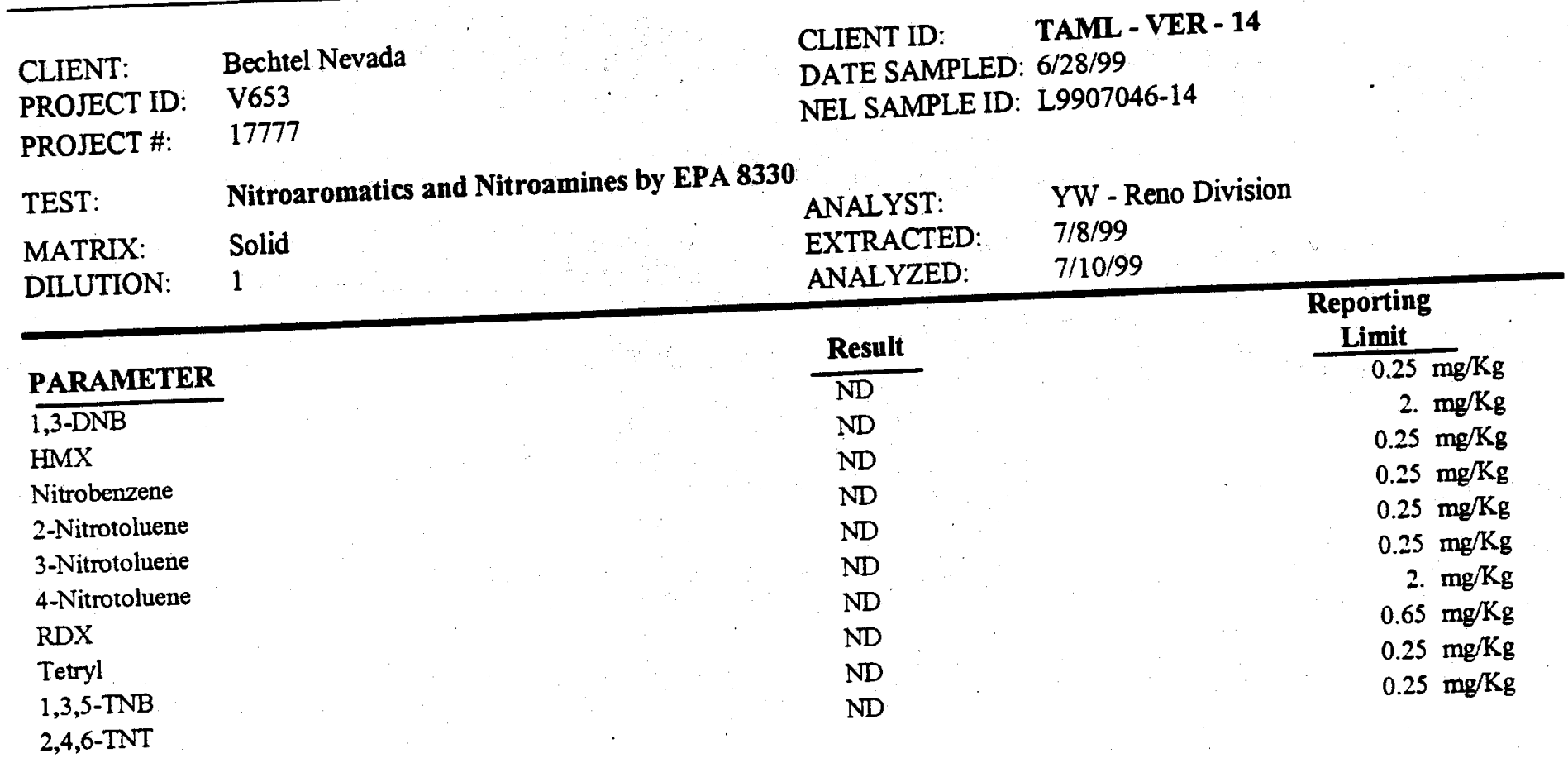

ND - Not Detected

This report shall not be reproduced except in full, without the written approval of the laboratory. 


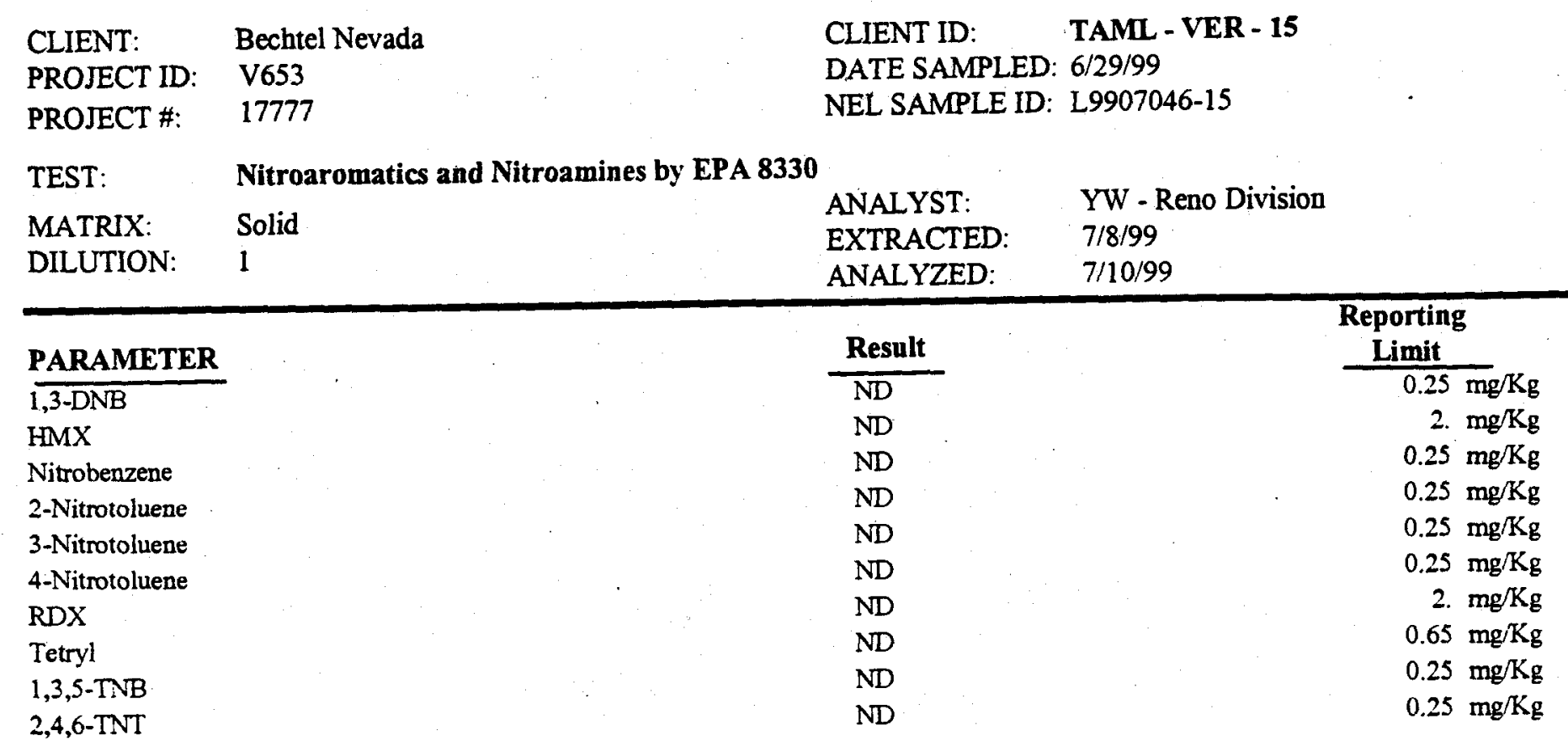

ND - Not Detected

This report shall not be reproduced except in full, without the written approval of the laboratory. 


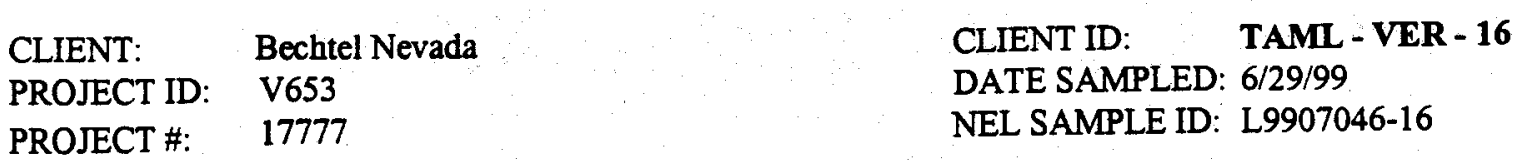

TEST: $\quad$ Nitroaromatics and Nitroamines by EPA 8330

MATRIX: Solid

DILUTION: 1

ANALYST: $\quad$ YW - Reno Division

EXTRACTED: $\quad 7 / 8 / 99$

ANALYZED: $\quad 7 / 10 / 99$

\section{PARAMETER}

1,3-DNB

$\mathrm{HMX}$

Nitrobenzene

2-Nitrotoluene

3-Nitrotoluene

4-Nitrotoluene

$\mathrm{RDX}$

Tetryl

1,3,5-TNB

2,4,6-TNT

\begin{tabular}{l} 
Result \\
\hline $\mathrm{ND}$ \\
$\mathrm{ND}$ \\
$\mathrm{ND}$ \\
$\mathrm{ND}$ \\
$\mathrm{ND}$ \\
$\mathrm{ND}$ \\
$\mathrm{ND}$ \\
$\mathrm{ND}$ \\
$\mathrm{ND}$ \\
$\mathrm{ND}$
\end{tabular}

\section{Reporting}

Limit

$0.25 \mathrm{mg} / \mathrm{Kg}$

2. $\mathrm{mg} / \mathrm{Kg}$

$0.25 \mathrm{mg} / \mathrm{Kg}$

$0.25 \mathrm{mg} / \mathrm{Kg}$

$0.25 \mathrm{mg} / \mathrm{Kg}$

$0.25 \mathrm{mg} / \mathrm{Kg}$

2. $\mathrm{mg} / \mathrm{Kg}$

$0.65 \mathrm{mg} / \mathrm{Kg}$

$0.25 \mathrm{mg} / \mathrm{Kg}$

$0.25 \mathrm{mg} / \mathrm{Kg}$

ND - Not Detected

This report shall not be reproduced except in full, without the written approval of the laboratory. 
CLIENT: Bechtel Nevada

PROJECT ID: V653

PROJECT \#: 17777
CLIENT ID: $\quad$ TAML - VER - 17

DATE SAMPLED: 6/29/99

NEL SAMPLE ID: L9907046-17

TEST: Nitroaromatics and Nitroamines by EPA 8330

MATRIX: Solid

DILUTION: 1

$\begin{array}{ll}\text { ANALYST: } & \text { YW - Reno Division } \\ \text { EXTRACTED: } & 7 / 8 / 99 \\ \text { ANALYZED: } & 7 / 10 / 99\end{array}$

PARAMETER

1,3-DNB

HMX

Nitrobenzene

2-Nitrotoluene

3-Nitrotoluene

4-Nitrotoluene

$\mathrm{RDX}$

Tetryl

1,3,5-TNB

2,4,6-TNT

\begin{tabular}{l} 
Result \\
\hline ND \\
ND \\
ND \\
ND \\
ND \\
ND \\
ND \\
ND \\
ND \\
ND
\end{tabular}

Reporting

Limit

$0.25 \mathrm{mg} / \mathrm{Kg}$

2. $\mathrm{mg} / \mathrm{Kg}$

$0.25 \mathrm{mg} / \mathrm{Kg}$

$0.25 \mathrm{mg} / \mathrm{Kg}$

$0.25 \mathrm{mg} / \mathrm{Kg}$

$0.25 \mathrm{mg} / \mathrm{Kg}$

2. $\mathrm{mg} / \mathrm{Kg}$

$0.65 \mathrm{mg} / \mathrm{Kg}$

$0.25 \mathrm{mg} / \mathrm{Kg}$

$0.25 \mathrm{mg} / \mathrm{Kg}$

ND - Not Detected

This report shall not be reproduced except in full. without the written approval of the laboratory. 


\section{CLIENT: Bechtel Nevada}

PROJECT ID: V653

PROJECT \#: $\quad 17777$
CLIENT ID:

TAMI - VER - 18

DATE SAMPLED: 6/28/99

NEL SAMPLE ID: L9907046-18

TEST: Nitroaromatics and Nitroamines by EPA 8330

MATRIX: Solid

DILUTION: 1
ANALYST: $\quad$ YW - Reno Division

EXTRACTED: $\quad 7 / 8 / 99$

ANALYZED: $\quad 7 / 10 / 99$

\section{PARAMETER}

1,3-DNB

$\mathrm{HMX}$

Nitrobenzene

2-Nitrotoluene

3-Nitrotoluene

4-Nitrotoluene

RDX

Tetryl

1,3,5-TNB

2,4,6-TNT

\begin{tabular}{l} 
Result \\
\hline $\mathrm{ND}$ \\
$\mathrm{ND}$ \\
$\mathrm{ND}$ \\
$\mathrm{ND}$ \\
$\mathrm{ND}$ \\
$\mathrm{ND}$ \\
$\mathrm{ND}$ \\
$\mathrm{ND}$ \\
$\mathrm{ND}$ \\
$\mathrm{ND}$
\end{tabular}

\section{Reporting}

Limit

$0.25 \mathrm{mg} / \mathrm{Kg}$

2. $\mathrm{mg} / \mathrm{Kg}$

$0.25 \mathrm{mg} / \mathrm{Kg}$

$0.25 \mathrm{mg} / \mathrm{Kg}$

$0.25 \mathrm{mg} / \mathrm{Kg}$

$0.25 \mathrm{mg} / \mathrm{Kg}$

2. $\mathrm{mg} / \mathrm{Kg}$

$0.65 \mathrm{mg} / \mathrm{Kg}$

$0.25 \mathrm{mg} / \mathrm{Kg}$

$0.25 \mathrm{mg} / \mathrm{Kg}$

VD - Not Detected

This report shall not be reproduced except in full, without the written approval of the laboratory. 


\begin{tabular}{|c|c|c|}
\hline CLIENT: & Bechtel Nevada & TAML - VER - 19 \\
\hline PROJECT ID: & V653 & DATE SAMPLED: $6 / 28 / 99$ \\
\hline PROJECT \#: & 17777 & NEL SAMPLE ID: L9907046-19 \\
\hline
\end{tabular}

TEST: $\quad$ Nitroaromatics and Nitroamines by EPA 8330

$\begin{array}{llll}\text { MATRIX: } & \text { Solid } & \text { ANALYST: } & \text { YW - Reno Division } \\ \text { DILUTION: } & 1 & \text { EXTRACTED: } & 7 / 8 / 99 \\ & & \text { ANALYZED: } & 7 / 10 / 99\end{array}$

\section{PARAMETER}

1,3-DNB

$\mathrm{HMX}$

Nitrobenzene

2-Nitrotoluene

3-Nitrotoluene

4-Nitrotoluene

RDX

Tetryl

1,3,5-TNB

2,4,6-TNT

\begin{tabular}{l} 
Result \\
\hline ND \\
ND \\
ND \\
ND \\
ND \\
ND \\
ND \\
ND \\
ND \\
ND
\end{tabular}

Reporting

Limit

$0.25 \mathrm{mg} / \mathrm{Kg}$

2. $\mathrm{mg} / \mathrm{Kg}$

$0.25 \mathrm{mg} / \mathrm{Kg}$

$0.25 \mathrm{mg} / \mathrm{Kg}$

$0.25 \mathrm{mg} / \mathrm{Kg}$

$0.25 \mathrm{mg} / \mathrm{Kg}$

2. $\mathrm{mg} / \mathrm{Kg}$

$0.65 \mathrm{mg} / \mathrm{Kg}$

$0.25 \mathrm{mg} / \mathrm{Kg}$

$0.25 \mathrm{mg} / \mathrm{Kg}$

ND - Not Detected

This report shall not be reproduced except in full, without the written approval of the laboratory. 
ILIENT: Bechtel Nevada

'ROJECT ID: V653

'ROJECT \#: 17777
CLIENT ID:

DATE SAMPLED: 6/29/99

NEL SAMPLE ID: L9907046-20

TEST: $\quad$ Nitroaromatics and Nitroamines by EPA 8330

MATRIX: Solid

JILUTION: 1
ANALYST: $\quad$ YW - Reno Division

EXTRACTED: $\quad 7 / 8 / 99$

ANALYZED: $\quad 7 / 10 / 99$

\section{Reporting \\ Limit}

$0.25 \mathrm{mg} / \mathrm{Kg}$

2. $\mathrm{mg} / \mathrm{Kg}$

$0.25 \mathrm{mg} / \mathrm{Kg}$

$0.25 \mathrm{mg} / \mathrm{Kg}$

$0.25 \mathrm{mg} / \mathrm{Kg}$

$0.25 \mathrm{mg} / \mathrm{Kg}$

2. $\mathrm{mg} / \mathrm{Kg}$

$0.65 \mathrm{mg} / \mathrm{Kg}$

$0.25 \mathrm{mg} / \mathrm{Kg}$

$0.25 \mathrm{mg} / \mathrm{Kg}$

ID - Not Detected

his report shall not be reproduced except in full, without the written approval of the laboratory. 


$\begin{array}{llll}\text { CLIENT: } & \text { Bechtel Nevada } & \text { CLIENT ID: } & \text { TAML - VER - 21 } \\ \text { PROJECT ID: } & \text { V653 } & \text { DATE SAMPLED: 6/29/99 } \\ \text { PROJECT \#: } & 17777 & \text { NEL SAMPLE ID: } \text { L9907046-21 }\end{array}$

TEST: $\quad$ Nitroaromatics and Nitroamines by EPA 8330

MATRIX: Solid

DILUTION: 1

ANALYST: $\quad$ YW - Reno Division

EXTRACTED: $\quad 7 / 8 / 99$

ANALYZED: $\quad 7 / 10 / 99$

PARAMETER

1,3-DNB

$\mathrm{HMX}$

Nitrobenzene

2-Nitrotoluene

3-Nitrotoluene

4-Nitrotoluene

$\mathrm{RDX}$

Tetryl

1,3,5-TNB

2,4,6-TNT
Result

ND

$\mathrm{ND}$

ND

ND

ND

ND

ND

ND

ND

ND
Reporting

Limit

$0.25 \mathrm{mg} / \mathrm{Kg}$

2. $\mathrm{mg} / \mathrm{Kg}$

$0.25 \mathrm{mg} / \mathrm{Kg}$

$0.25 \mathrm{mg} / \mathrm{Kg}$

$0.25 \mathrm{mg} / \mathrm{Kg}$

$0.25 \mathrm{mg} / \mathrm{Kg}$

2. $\mathrm{mg} / \mathrm{Kg}$

$0.65 \mathrm{mg} / \mathrm{Kg}$

$0.25 \mathrm{mg} / \mathrm{Kg}$

$0.25 \mathrm{mg} / \mathrm{Kg}$

ND - Not Detected

This report shall not be reproduced except in full, without the written approval of the laboratory. 


$\begin{array}{lll}\text { CLIENT: } & \text { Bechtel Nevada } & \text { CLIENT ID: } \quad \text { TAML - VER - 22 } \\ \text { PROJECT ID: } & \text { V653 } & \text { DATE SAMPLED: } 6 / 28 / 99 \\ \text { PROJECT \#: } & 17777 & \text { NEL SAMPLE ID: } \text { L9907046-22 }\end{array}$

TEST: $\quad$ Nitroaromatics and Nitroamines by EPA 8330

MATRIX: Solid

DILUTION: 1

ANALYST: $\quad$ YW - Reno Division

EXTRACTED: $\quad 7 / 8 / 99$

ANALYZED: $\quad 7 / 10 / 99$

\section{PARAMETER}

1,3-DNB

TMX

Vitrobenzene

2-Nitrotoluene

3-Nitrotoluene

-Nitrotoluene

2DX

retryl

, 3,5-TNB

:,4,6-TNT

\begin{tabular}{l} 
Result \\
\hline ND \\
ND \\
ND \\
ND \\
ND \\
ND \\
ND \\
ND \\
ND \\
ND
\end{tabular}

\section{Reporting}

Limit

$0.25 \mathrm{mg} / \mathrm{Kg}$

2. $\mathrm{mg} / \mathrm{Kg}$

$0.25 \mathrm{mg} / \mathrm{Kg}$

$0.25 \mathrm{mg} / \mathrm{Kg}$

$0.25 \mathrm{mg} / \mathrm{Kg}$

$0.25 \mathrm{mg} / \mathrm{Kg}$

2. $\mathrm{mg} / \mathrm{Kg}$

$0.65 \mathrm{mg} / \mathrm{Kg}$

$0.25 \mathrm{mg} / \mathrm{Kg}$

$0.25 \mathrm{mg} / \mathrm{Kg}$

JD - Not Detected

his report shall not be reproduced except in full, without the written approval of the laboratory. 


\begin{tabular}{|c|c|c|}
\hline CLIENT: & Bechtel Nevada & CLIENT ID: $\quad$ TAML - VER - 23 \\
\hline PROJECT ID: & V653 & DATE SAMPLED: $6 / 28 / 99$ \\
\hline PROJECT \#: & 17777 & NEL SAMPLE ID: L9907046-23 \\
\hline
\end{tabular}

TEST: $\quad$ Nitroaromatics and Nitroamines by EPA 8330

MATRIX: Solid

DILUTION: 1

ANALYST: $\quad$ YW - Reno Division

EXTRACTED: $\quad 7 / 8 / 99$

ANALYZED: $\quad 7 / 10 / 99$

\begin{tabular}{lr} 
Result & \multicolumn{1}{c}{$\begin{array}{c}\text { Reporting } \\
\text { Limit }\end{array}$} \\
\cline { 2 - 2 } ND & $0.25 \mathrm{mg} / \mathrm{Kg}$ \\
$\mathrm{ND}$ & $2 . \mathrm{mg} / \mathrm{Kg}$ \\
$\mathrm{ND}$ & $0.25 \mathrm{mg} / \mathrm{Kg}$ \\
$\mathrm{ND}$ & $0.25 \mathrm{mg} / \mathrm{Kg}$ \\
$\mathrm{ND}$ & $0.25 \mathrm{mg} / \mathrm{Kg}$ \\
$\mathrm{ND}$ & $0.25 \mathrm{mg} / \mathrm{Kg}$ \\
$\mathrm{ND}$ & $2 . \mathrm{mg} / \mathrm{Kg}$ \\
$\mathrm{ND}$ & $0.65 \mathrm{mg} / \mathrm{Kg}$ \\
$\mathrm{ND}$ & $0.25 \mathrm{mg} / \mathrm{Kg}$ \\
$\mathrm{ND}$ & $0.25 \mathrm{mg} / \mathrm{Kg}$
\end{tabular}

ND - Not Detected

This report shall not be reproduced except in full, without the written approval of the laboratory. 
CLIENT: Bechtel Nevada

PROJECT ID: V653

PROJECT \#: 17777
CLIENT ID:

\section{TAMI - VER - 24}

DATE SAMPLED: $6 / 28 / 99$

NEL SAMPLE ID: L9907046-24

TEST: $\quad$ Nitroaromatics and Nitroamines by EPA 8330

MATRIX: Solid

DILUTION: 1

ANALYST: $\quad$ YW - Reno Division

EXTRACTED: $\quad 7 / 8 / 99$

ANALYZED; $\quad 7 / 10 / 99$

\section{PARAMETER}

1,3-DNB

$\mathrm{HMX}$

Nitrobenzene

2-Nitrotoluene

3-Nitrotoluene

4-Nitrotoluene

RDX

Tetryl

$1,3,5-\mathrm{TNB}$

2,4,6-TNT

\begin{tabular}{l} 
Result \\
\hline $\mathrm{ND}$ \\
$\mathrm{ND}$ \\
$\mathrm{ND}$ \\
$\mathrm{ND}$ \\
$\mathrm{ND}$ \\
$\mathrm{ND}$ \\
$\mathrm{ND}$ \\
$\mathrm{ND}$ \\
$\mathrm{ND}$ \\
$\mathrm{ND}$
\end{tabular}

\section{Reporting}

Limit

$0.25 \mathrm{mg} / \mathrm{Kg}$

2. $\mathrm{mg} / \mathrm{Kg}$

$0.25 \mathrm{mg} / \mathrm{Kg}$

$0.25 \mathrm{mg} / \mathrm{Kg}$

$0.25 \mathrm{mg} / \mathrm{Kg}$

$0.25 \mathrm{mg} / \mathrm{Kg}$

2. $\mathrm{mg} / \mathrm{Kg}$

$0.65 \mathrm{mg} / \mathrm{Kg}$

$0.25 \mathrm{mg} / \mathrm{Kg}$

$0.25 \mathrm{mg} / \mathrm{Kg}$

ND - Not Detected

This report shall not be reproduced except in full, without the written approval of the laboratory. 


$\begin{array}{lll}\text { CLIENT: } & \text { Bechtel Nevada } & \text { CLIENT ID: } \quad \text { TAML - VER - 25 } \\ \text { PROJECT ID: } & \text { V653 } & \text { DATE SAMPLED: } 6 / 28 / 99 \\ \text { PROJECT \#: } & 17777 & \text { NEL SAMPLE ID: } \text { L9907046-25 }\end{array}$

TEST: - Nitroaromatics and Nitroamines by EPA 8330

\begin{tabular}{llll} 
MATRIX: & Solid & ANALYST: & YW - Reno Division \\
DILUTION: & 1 & EXTRACTED: & $7 / 8 / 99$ \\
& & ANALYZED: & $7 / 10 / 99$ \\
\hline
\end{tabular}

\section{PARAMETER}

1,3-DNB

HMX

Nitrobenzene

2-Nitrotoluene

3-Nitrotoluene

4-Nitrotoluene

$\mathrm{RDX}$

Tetryl

1,3,5-TNB

2,4,6-TNT

\begin{tabular}{l} 
Result \\
\hline $\mathrm{ND}$ \\
$\mathrm{ND}$ \\
$\mathrm{ND}$ \\
$\mathrm{ND}$ \\
$\mathrm{ND}$ \\
$\mathrm{ND}$ \\
$\mathrm{ND}$ \\
$\mathrm{ND}$ \\
$\mathrm{ND}$ \\
$\mathrm{ND}$
\end{tabular}

Reporting

Limit $0.25 \mathrm{mg} / \mathrm{Kg}$

2. $\mathrm{mg} / \mathrm{Kg}$

$0.25 \mathrm{mg} / \mathrm{Kg}$

$0.25 \mathrm{mg} / \mathrm{Kg}$

$0.25 \mathrm{mg} / \mathrm{Kg}$

$0.25 \mathrm{mg} / \mathrm{Kg}$

2. $\mathrm{mg} / \mathrm{Kg}$

$0.65 \mathrm{mg} / \mathrm{Kg}$

$0.25 \mathrm{mg} / \mathrm{Kg}$

$0.25 \mathrm{mg} / \mathrm{Kg}$

ND - Not Detected

This report shall not be reproduced except in full. without the written approval of the laboratory. 


\begin{tabular}{|c|c|c|}
\hline ILIENT: & Bechtel Nevada & TAML - VER - 26 \\
\hline PROJECT ID: & V653 & DATE SAMPLED: $6 / 28 / 99$ \\
\hline ROJECT \#: & 17777 & NEL SAMPLE ID: L9907046-26 \\
\hline
\end{tabular}

IEST: $\quad$ Nitroaromatics and Nitroamines by EPA 8330

VATRIX: Solid

OILUTION: 1 .

ANALYST: YW - Reno Division

EXTRACTED: $\quad 7 / 8 / 99$

ANALYZED: $\quad 7 / 10 / 99$

\section{PARAMETER}

,3-DNB

$\mathrm{IMX}$

vitrobenzene

:-Nitrotoluene

-Nitrotoluene

-Nitrotoluene

¿DX

Cetryl

$, 3,5-\mathrm{TNB}$

$\therefore, 4,6-T N T$

\begin{tabular}{l} 
Result \\
\hline ND \\
ND \\
ND \\
ND \\
ND \\
ND \\
ND \\
ND \\
ND \\
ND
\end{tabular}

\section{Reporting}

Limit

$0.25 \mathrm{mg} / \mathrm{Kg}$

2. $\mathrm{mg} / \mathrm{Kg}$

$0.25 \mathrm{mg} / \mathrm{Kg}$

$0.25 \mathrm{mg} / \mathrm{Kg}$

$0.25 \mathrm{mg} / \mathrm{Kg}$

$0.25 \mathrm{mg} / \mathrm{Kg}$

2. $\mathrm{mg} / \mathrm{Kg}$

$0.65 \mathrm{mg} / \mathrm{Kg}$

$0.25 \mathrm{mg} / \mathrm{Kg}$

$0.25 \mathrm{mg} / \mathrm{Kg}$

JD - Not Detected

his report shall not be reproduced except in full. without the written approval of the laboratory. 


\begin{tabular}{|c|c|c|}
\hline CLIENT: & Bechtel Nevada & CLIENT ID: $\quad$ TAML - VER - 27 \\
\hline PROJECT ID: & V653 & DATE SAMPLED: $6 / 28 / 99$ \\
\hline PROJECT \#: & 17777 & NEL SAMPLE ID: L9907046-27 \\
\hline
\end{tabular}

TEST: Nitroaromatics and Nitroamines by EPA 8330

\begin{tabular}{llll} 
MATRIX: & Solid & ANALYST: & YW - Reno Division \\
DILUTION: & 1 & EXTRACTED: & $7 / 8 / 99$ \\
& & ANALYZED: & $7 / 10 / 99$ \\
\hline
\end{tabular}

\section{PARAMETER}

1,3-DNB

HMX

Nitrobenzene

2-Nitrotoluene

3-Nitrotoluene

4-Nitrotoluene

RDX

Tetryl

$1,3,5-\mathrm{TNB}$

2,4,6-TNT

\begin{tabular}{l} 
Result \\
\hline $\mathrm{ND}$ \\
$\mathrm{ND}$ \\
$\mathrm{ND}$ \\
$\mathrm{ND}$ \\
$\mathrm{ND}$ \\
$\mathrm{ND}$ \\
$\mathrm{ND}$ \\
$\mathrm{ND}$ \\
$\mathrm{ND}$ \\
$\mathrm{ND}$
\end{tabular}

Reporting

Limit $0.25 \mathrm{mg} / \mathrm{Kg}$

2. $\mathrm{mg} / \mathrm{Kg}$

$0.25 \mathrm{mg} / \mathrm{Kg}$

$0.25 \mathrm{mg} / \mathrm{Kg}$

$0.25 \mathrm{mg} / \mathrm{Kg}$

$0.25 \mathrm{mg} / \mathrm{Kg}$

2. $\mathrm{mg} / \mathrm{Kg}$

$0.65 \mathrm{mg} / \mathrm{Kg}$

$0.25 \mathrm{mg} / \mathrm{Kg}$

$0.25 \mathrm{mg} / \mathrm{Kg}$

ND - Not Detected

This report shall not be reproduced except in full, without the written approval of the laboratory. 


\section{ILIENT: Bechtel Nevada}

'ROJECT ID: V653

'ROJECT \#: 17777
CLIENT ID:

DATE SAMPLED: $6 / 28 / 99$

NEL SAMPLE ID: L9907046-28

IEST: $\quad$ Nitroaromatics and Nitroamines by EPA 8330

MATRIX: Solid

JILUTION: 1
ANALYST: $\quad$ YW - Reno Division

EXTRACTED: $\quad 7 / 8 / 99$

ANALYZED: $\quad 7 / 10 / 99$

\section{PARAMETER}

, 3-DNB

IMX

Jitrobenzene

:-Nitrotoluene

-Nitrotoluene

-Nitrotoluene

JDX

etryl

,3,5-TNB

:,4,6-TNT

\begin{tabular}{l} 
Result \\
\hline ND \\
ND \\
ND \\
ND \\
ND \\
ND \\
ND \\
ND \\
ND \\
ND
\end{tabular}

\section{Reporting}

Limit

$0.25 \mathrm{mg} / \mathrm{Kg}$

2. $\mathrm{mg} / \mathrm{Kg}$

$0.25 \mathrm{mg} / \mathrm{Kg}$

$0.25 \mathrm{mg} / \mathrm{Kg}$

$0.25 \mathrm{mg} / \mathrm{Kg}$

$0.25 \mathrm{mg} / \mathrm{Kg}$

2. $\mathrm{mg} / \mathrm{Kg}$

$0.65 \mathrm{mg} / \mathrm{Kg}$

$0.25 \mathrm{mg} / \mathrm{Kg}$

$0.25 \mathrm{mg} / \mathrm{Kg}$

ID - Not Detected

his report shall not be reproduced except in full, without the uritten approval of the laboratory. 


\begin{tabular}{|c|c|c|c|}
\hline $\begin{array}{l}\text { CLIENT: } \\
\text { PROJECT ID: } \\
\text { PROJECT \#: }\end{array}$ & $\begin{array}{l}\text { Bechtel Nevada } \\
\text { V653 } \\
17777\end{array}$ & $\begin{array}{l}\text { CLIENT ID: } \\
\text { DATE SAMPLED: } \\
\text { NEL SAMPLE ID: }\end{array}$ & $\begin{array}{l}\text { Method Blank } \\
\text { NA } \\
\text { 070899-H1-BLK }\end{array}$ \\
\hline
\end{tabular}

TEST: $\quad$ Nitroaromatics and Nitroamines by EPA 8330

\begin{tabular}{|c|c|c|c|}
\hline MATRIX: & Solid & $\begin{array}{l}\text { ANALYST: } \\
\text { EXTRACTED: } \\
\text { ANALYZED: }\end{array}$ & $\begin{array}{l}\text { YW - Reno Division } \\
7 / 8 / 99 \\
7 / 9 / 99\end{array}$ \\
\hline
\end{tabular}

\section{PARAMETER}

1,3-DNB

HMX

Nitrobenzene

2-Nitrotoluene

3-Nitrotoluene

4-Nitrotoluene

RDX

Tetryl

$1,3,5-\mathrm{TNB}$

2,4,6-TNT

\begin{tabular}{l} 
Result \\
\hline $\mathrm{ND}$ \\
$\mathrm{ND}$ \\
$\mathrm{ND}$ \\
$\mathrm{ND}$ \\
$\mathrm{ND}$ \\
$\mathrm{ND}$ \\
$\mathrm{ND}$ \\
$\mathrm{ND}$ \\
$\mathrm{ND}$ \\
$\mathrm{ND}$
\end{tabular}

Reporting

Limit

$\overline{0.25 \mathrm{mg} / \mathrm{Kg}}$

2. $\mathrm{mg} / \mathrm{Kg}$

$0.25 \mathrm{mg} / \mathrm{Kg}$

$0.25 \mathrm{mg} / \mathrm{Kg}$

$0.25 \mathrm{mg} / \mathrm{Kg}$

$0.25 \mathrm{mg} / \mathrm{Kg}$

2. $\mathrm{mg} / \mathrm{Kg}$

$0.65 \mathrm{mg} / \mathrm{Kg}$

$0.25 \mathrm{mg} / \mathrm{Kg}$

$0.25 \mathrm{mg} / \mathrm{Kg}$

ND - Not Detected

This report shall not be reproduced except in full, without the written approval of the laboratory. 


$\begin{array}{llll}\text { CLIENT: } & \text { Bechtel Nevada } & \text { CLIENT ID: } & \text { Method Blank } \\ \text { PROJECT ID: } & \text { V653 } & \text { DATE SAMPLED: NA } \\ \text { PROJECT \#: } & 17777 & \text { NEL SAMPLE ID: } & \text { 070899-H2-BLK }\end{array}$

TEST: $\quad$ Nitroaromatics and Nitroamines by EPA 8330

MATRIX: Solid

ANALYST: $\quad$ YW - Reno Division

EXTRACTED: $\quad 7 / 8 / 99$

ANALYZED: $\quad 7 / 10 / 99$

\section{PARAMETER}

1,3-DNB

TMX

Vitrobenzene

-Nitrotoluene

i-Nitrotoluene

b-Nitrotoluene

एDX

Cetryl

,3,5-TNB

$:, 4,6-\mathrm{TNT}$

\section{Result}

ND

$\mathrm{ND}$

$\mathrm{ND}$

ND

$\mathrm{ND}$

ND

ND

ND

$\mathrm{ND}$

ND
Reporting

Limit

$0.25 \mathrm{mg} / \mathrm{Kg}$

2. $\mathrm{mg} / \mathrm{Kg}$

$0.25 \mathrm{mg} / \mathrm{Kg}$

$0.25 \mathrm{mg} / \mathrm{Kg}$

$0.25 \mathrm{mg} / \mathrm{Kg}$

$0.25 \mathrm{mg} / \mathrm{Kg}$

2. $\mathrm{mg} / \mathrm{Kg}$

$0.65 \mathrm{mg} / \mathrm{Kg}$

$0.25 \mathrm{mg} / \mathrm{Kg}$

$0.25 \mathrm{mg} / \mathrm{Kg}$

ID - Not Detected

his report shall not be reproduced except in full, without the written approval of the laboratory. 


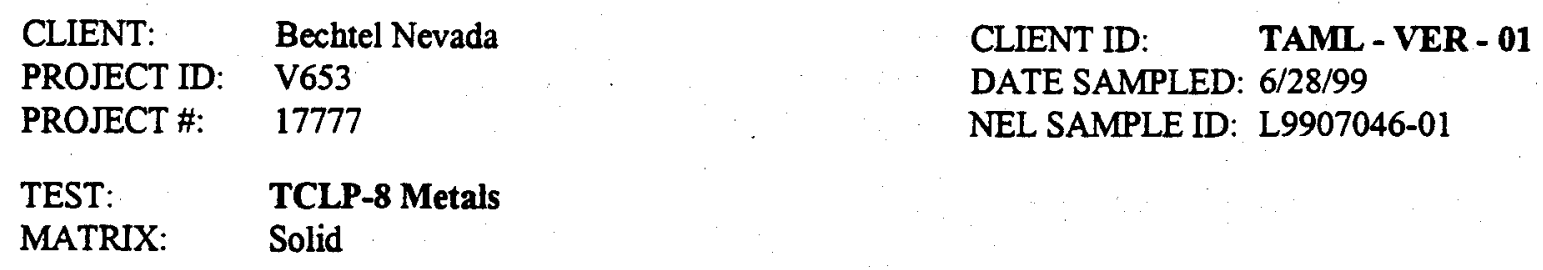

\begin{tabular}{|c|c|c|c|c|c|c|c|}
\hline PARAMETER & $\begin{array}{c}\text { RESULT } \\
\text { mg/L } \\
\end{array}$ & $\begin{array}{c}\text { REPORTING } \\
\text { LIMIT } \\
\end{array}$ & D. F. & METHOD & $\begin{array}{c}\text { TCLP/STLC } \\
\text { EXTRACTION } \\
\text { DATE } \\
\end{array}$ & DIGESTED & ANALYZED \\
\hline Arsenic & ND & $0.1 \mathrm{mg} / \mathrm{L}$ & 1 & EPA 6010 & 7/8/99 & 7/9/99 & $7 / 12 / 99$ \\
\hline Barium & ND & 1. $\mathrm{mg} / \mathrm{L}$ & 1 & EPA 6010 & $7 / 8 / 99$ & 7/9/99 & $7 / 12 / 99$ \\
\hline Cadmium & ND & $0.01 \mathrm{mg} / \mathrm{L}$ & 1 & EPA 6010 & $7 / 8 / 99$ & $7 / 9 / 99$ & $7 / 12 / 99$ \\
\hline Chromium & 0.071 & $0.01 \mathrm{mg} / \mathrm{L}$ & 1 & EPA 6010 & $7 / 8 / 99$ & 7/9/99. & $7 / 12 / 99$ \\
\hline Lead & $\mathrm{ND}$ & $0.05 \mathrm{mg} / \mathrm{L}$ & 1 & EPA 6010 & $7 / 8 / 99$ & $7 / 9 / 99$ & $7 / 12 / 99$ \\
\hline Mercury & $\mathrm{ND}$ & $0.002 \mathrm{mg} / \mathrm{L}$ & 10 & EPA $7470 A$ & $7 / 8 / 99$ & $7 / 11 / 99$ & $7 / 11 / 99$ \\
\hline Selenium & ND & $0.1 \mathrm{mg} / \mathrm{L}$ & 1 & EPA 6010 & $7 / 8 / 99$ & 7/9/99 & $7 / 12 / 99$ \\
\hline Silver & ND & $0.02 \mathrm{mg} / \mathrm{L}$ & 1 & EPA 6010 & $7 / 8 / 99$ & $7 / 9 / 99$ & $7 / 12 / 99$ \\
\hline
\end{tabular}

D.F. - Dilution Factor

ND - Not Detected

This report shall not be reproduced except in full. without the written approval of the laboratory. 


$\begin{array}{lll}\text { CLIENT: } & \text { Bechtel Nevada } & \text { CLIENT ID: } \\ \text { PROJECT ID: } & \text { V653 } & \text { TAML - VER - 02 } \\ \text { PROJECT \#: } & 17777 & \text { NATE SAMPLED: } 6 / 28 / 99 \\ & & \text { NEL SAMPLE ID: } \text { L9907046-02 }\end{array}$

TEST: $\quad$ TCLP-8 Metals

MATRIX: Solid

\begin{tabular}{|c|c|c|c|c|c|c|c|}
\hline PARAMETER & $\begin{array}{c}\text { RESULT } \\
\mathrm{mg} / \mathrm{L} \\
\end{array}$ & $\begin{array}{c}\text { REPORTING } \\
\text { LIMIT } \\
\end{array}$ & D. F. & METHOD & $\begin{array}{c}\text { TCLP/STLC } \\
\text { EXTRACTION } \\
\text { DATE } \\
\end{array}$ & DIGESTED & ANALYZED \\
\hline trsenic & ND & $0.1 \mathrm{mg} / \mathrm{L}$ & 1 & EPA 6010 & $7 / 8 / 99$ & 7/9/99 & $7 / 12 / 99$ \\
\hline 3arium & ND & 1. $\mathrm{mg} / \mathrm{L}$ & 1 & EPA 6010 & $7 / 8 / 99$ & 7/9/99 & $7 / 12 / 99$ \\
\hline Cadmium & ND & $0.01 \mathrm{mg} / \mathrm{L}$ & 1 & EPA 6010 & $7 / 8 / 99$ & $7 / 9 / 99$ & $7 / 12 / 99$ \\
\hline Zhromium & ND & $0.01 \mathrm{mg} / \mathrm{L}$ & 1 & EPA 6010 & $7 / 8 / 99$ & 7/9/99 & $7 / 12 / 99$ \\
\hline ead & ND & $0.05 \mathrm{mg} / \mathrm{L}$ & 1 & EPA 6010 & $7 / 8 / 99$ & $7 / 9 / 99$ & $7 / 12 / 99$ \\
\hline Mercury & ND & $0.002 \mathrm{mg} / \mathrm{L}$ & 10 & EPA 7470A & 7/8/99 & $7 / 11 / 99$ & $7 / 11 / 99$ \\
\hline ielenium & $\mathrm{ND}$ & $0.1 \mathrm{mg} / \mathrm{L}$ & 1 & EPA 6010 & $7 / 8 / 99$ & 7/9/99 & $7 / 12 / 99$ \\
\hline iilver & $\mathrm{ND}$ & $0.02 \mathrm{mg} / \mathrm{L}$ & 1 & EPA 6010 & $7 / 8 / 99$ & $7 / 9 / 99$ & $7 / 12 / 99$ \\
\hline
\end{tabular}

F. - Dilution Factor

ND - Not Detected

is report shall not be reproduced except in full, without the written approval of the laboratory. 


\begin{tabular}{|c|c|c|c|c|c|c|c|}
\hline $\begin{array}{l}\text { CLIENT: } \\
\text { PROJECT ID: } \\
\text { PROJECT \#: }\end{array}$ & $\begin{array}{l}\text { Bechtel Nevada } \\
\text { V653 } \\
17777\end{array}$ & & & $\begin{array}{l}\text { CLIENT ID } \\
\text { DATE SAI } \\
\text { NEL SAMI }\end{array}$ & $\begin{array}{l}\text { TAML - } \\
\text { APLED: } 6 / 28 / 99 \\
\text { LE ID: } \\
\text { L990704 }\end{array}$ & $\begin{array}{l}\text { VER - } 03 \\
6-03\end{array}$ & \\
\hline $\begin{array}{l}\text { TEST: } \\
\text { MATRIX: }\end{array}$ & $\begin{array}{l}\text { TCLP-8 Metals } \\
\text { Solid }\end{array}$ & & & & & & \\
\hline PARAMETER & $\begin{array}{c}\text { RESULT } \\
\mathrm{mg} / \mathrm{L} \\
\end{array}$ & $\begin{array}{c}\text { REPORTING } \\
\text { LIMIT } \\
\end{array}$ & D. F. & METHOD & $\begin{array}{c}\text { TCLP/STLC } \\
\text { EXTRACTION } \\
\begin{array}{c}\text { DATE } \\
\end{array}\end{array}$ & DIGESTED & ANALYZED \\
\hline Arsenic & ND & $0.1 \mathrm{mg} / \mathrm{L}$ & 1 & EPA 6010 & $7 / 8 / 99$ & 7/9/99 & $7 / 12 / 99$ \\
\hline Barium & ND & 1. $\mathrm{mg} / \mathrm{L}$ & 1 & EPA 6010 & $7 / 8 / 99$ & 7/9/99 & $7 / 12 / 99$ \\
\hline Cadmium & ND & $0.01 \mathrm{mg} / \mathrm{L}$ & 1 & EPA 6010 & $7 / 8 / 99$ & 7/9/99 & $7 / 12 / 99$ \\
\hline Chromium & ND & $0.01 \mathrm{mg} / \mathrm{L}$ & 1 & EPA 6010 & $7 / 8 / 99$ & 7/9/99 & $7 / 12 / 99$ \\
\hline Lead & ND & $0.05 \mathrm{mg} / \mathrm{L}$ & 1 & EPA 6010 & 7/8/99. & $7 / 9 / 99$ & $7 / 12 / 99$ \\
\hline Mercury & $\mathrm{ND}$ & $0.002 \mathrm{mg} / \mathrm{L}$ & 10 & EPA $7470 A$ & $7 / 8 / 99$ & $7 / 11 / 99$ & $7 / 11 / 99$ \\
\hline Selenium & ND & $0.1 \mathrm{mg} / \mathrm{L}$ & 1 & EPA 6010 & $7 / 8 / 99$ & $7 / 9 / 99$ & $7 / 12 / 99$ \\
\hline Silver & $\mathrm{ND}$ & $0.02 \mathrm{mg} / \mathrm{L}$ & 1 & EPA 6010 & $7 / 8 / 99$ & $7 / 9 / 99$ & $7 / 12 / 99$ \\
\hline
\end{tabular}

D.F. - Dilution Factor

ND - Not Detected

This report shall not be reproduced except in full, without the written approval of the laboratory. 


\begin{tabular}{|c|c|c|}
\hline CLIENT: & Bechtel Nevada & CLIENT ID: $\quad$ TAML - VER - 04 \\
\hline PROJECT ID: & V653 & DATE SAMPLED: $6 / 28 / 99$ \\
\hline PROJECT \#: & 17777 & NEL SAMPLE ID: L9907046-04 \\
\hline $\begin{array}{l}\text { TEST: } \\
\text { MATRIX: }\end{array}$ & $\begin{array}{l}\text { TCLP-8 Metals } \\
\text { Solid }\end{array}$ & \\
\hline
\end{tabular}

\begin{tabular}{|c|c|c|c|c|c|c|c|}
\hline PARAMETER & $\begin{array}{c}\text { RESULT } \\
\mathrm{mg} / \mathrm{L} \\
\end{array}$ & $\begin{array}{c}\text { REPORTING } \\
\text { LIMIT } \\
\end{array}$ & D. F. & METHOD & $\begin{array}{c}\text { TCLP/STLC } \\
\text { EXTRACTION } \\
\text { DATE } \\
\end{array}$ & DIGESTED & ANALYZED \\
\hline Arsenic & ND & $0.1 \mathrm{mg} / \mathrm{L}$ & 1 & EPA 6010 & $7 / 8 / 99$ & 7/9/99 & $7 / 12 / 99$ \\
\hline 3arium & ND & 1. $\mathrm{mg} / \mathrm{L}$ & 1 & EPA 6010 & $7 / 8 / 99$ & 7/9/99 & $7 / 12 / 99$ \\
\hline Cadmium & ND & $0.01 \mathrm{mg} / \mathrm{L}$ & 1 & EPA 6010 & $7 / 8 / 99$ & $7 / 9 / 99$ & $7 / 12 / 99$ \\
\hline Thromium & ND & $0.01 \mathrm{mg} / \mathrm{L}$ & 1 & EPA 6010 & $7 / 8 / 99$ & 7/9/99 & $7 / 12 / 99$ \\
\hline ead & ND & $0.05 \mathrm{mg} / \mathrm{L}$ & 1 & EPA 6010 & $7 / 8 / 99$ & $7 / 9 / 99$ & $7 / 12 / 99$ \\
\hline Mercury & $\mathrm{ND}$ & $0.002 \mathrm{mg} / \mathrm{L}$ & 10 & EPA 7470A & 7/8/99 & $7 / 11 / 99$ & $7 / 11 / 99$ \\
\hline Yelenium & ND & $0.1 \mathrm{mg} / \mathrm{L}$ & 1 & EPA 6010 & 7/8/99 & $7 / 9 / 99$ & $7 / 12 / 99$ \\
\hline 'ilver & ND & $0.02 \mathrm{mg} / \mathrm{L}$ & 1 & EPA 6010 & $7 / 8 / 99$ & $7 / 9 / 99$ & $7 / 12 / 99$ \\
\hline
\end{tabular}

F. - Dilution Factor

ND - Not Detected

is report shall not be reproduced except in full, without the uritten approval of the laboratory. 


\begin{tabular}{|c|c|c|c|c|c|c|c|}
\hline $\begin{array}{l}\text { CLIENT: } \\
\text { PROJECT ID: } \\
\text { PROJECT \#: }\end{array}$ & $\begin{array}{l}\text { Bechtel Nevada } \\
\text { V653 } \\
17777\end{array}$ & & & $\begin{array}{l}\text { CLIENT II } \\
\text { DATE SAI } \\
\text { NEL SAM }\end{array}$ & $\begin{array}{l}\text { TAML - } \\
\text { APLED: } 6 / 28 / 99 \\
\text { LLE ID: } \\
\text { L990704 }\end{array}$ & VER - 05 & \\
\hline $\begin{array}{l}\text { TEST: } \\
\text { MATRIX: }\end{array}$ & $\begin{array}{l}\text { TCLP-8 Metals } \\
\text { Solid }\end{array}$ & & & & & & \\
\hline PARAMETER & $\begin{array}{c}\text { RESULT } \\
\mathrm{mg} / \mathrm{L} \\
\end{array}$ & $\begin{array}{c}\text { REPORTING } \\
\text { LIMIT } \\
\end{array}$ & D. F. & METHOD & 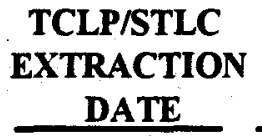 & DIGESTED & ANALYZED \\
\hline Arsenic & ND & $0.1 \mathrm{mg} / \mathrm{L}$ & 1 & EPA 6010 & $7 / 8 / 99$ & 7/9/99 & $7 / 12 / 99$ \\
\hline Barium & ND & 1. $\mathrm{mg} / \mathrm{L}$ & 1 & EPA 6010 & $7 / 8 / 99$ & 7/9/99 & $7 / 12 / 99$ \\
\hline Cadmium & ND & $0.01 \mathrm{mg} / \mathrm{L}$ & 1 & EPA 6010 & $7 / 8 / 99$ & $7 / 9 / 99$ & $7 / 12 / 99$ \\
\hline Chromium & ND & $0.01 \mathrm{mg} / \mathrm{L}$ & 1 & EPA 6010 & 7/8/99 & $7 / 9 / 99$ & $7 / 12 / 99$ \\
\hline Lead & ND & $0.05 \mathrm{mg} / \mathrm{L}$ & 1 & EPA 6010 & 7/8/99 & 7/9/99 & $7 / 12 / 99$ \\
\hline Mercury & ND & $0.002 \mathrm{mg} / \mathrm{L}$ & 10 & EPA 7470A & 7/8/99 & $7 / 11 / 99$ & $7 / 11 / 99$ \\
\hline Selenium & $\mathrm{ND}$ & $0.1 \mathrm{mg} / \mathrm{L}$ & 1 & EPA 6010 & $7 / 8 / 99$ & 7/9/99 & $7 / 12 / 99$ \\
\hline Silver : & ND & $0.02 \mathrm{mg} / \mathrm{L}$ & 1. & EPA 6010 & $7 / 8 / 99$ & $7 / 9 / 99$ & $7 / 12 / 99$ \\
\hline
\end{tabular}

D.F. - Dilution Factor

ND - Not Detected

This report shall not be reproduced except in full, without the written approval of the laboratory. 


\begin{tabular}{|c|c|c|}
\hline CLIENT: & Bechtel Nevada & CLIENT ID: $\quad$ TAMI - VER - 06 \\
\hline PROJECT ID: & V653 & DATE SAMPLED: $6 / 28 / 99$ \\
\hline PROJECT \#: & 17777 & NEL SAMPLE ID: L9907046-06 \\
\hline $\begin{array}{l}\text { TEST: } \\
\text { MATRIX: }\end{array}$ & $\begin{array}{l}\text { TCLP-8 Metals } \\
\text { Solid }\end{array}$ & \\
\hline
\end{tabular}

\begin{tabular}{|c|c|c|c|c|c|c|c|}
\hline PARAMETER & $\begin{array}{c}\text { RESULT } \\
\mathrm{mg} / \mathrm{L} \\
\end{array}$ & $\begin{array}{c}\text { REPORTING } \\
\text { LIMIT } \\
\end{array}$ & D. F. & METHOD & $\begin{array}{c}\text { TCLP/STLC } \\
\text { EXTRACTION } \\
\text { DATE } \\
\end{array}$ & DIGESTED & ANALYZED \\
\hline trsenic & ND & $0.1 \mathrm{mg} / \mathrm{L}$ & 1 & EPA 6010 & $7 / 8 / 99$ & $7 / 9 / 99$ & $7 / 12 / 99$ \\
\hline 3arium & ND & 1. $\mathrm{mg} / \mathrm{L}$ & 1 & EPA 6010 & $7 / 8 / 99$ & $7 / 9 / 99$ & $7 / 12 / 99$ \\
\hline Cadmium & ND & $0.01 \mathrm{mg} / \mathrm{L}$ & 1 & EPA 6010 & $7 / 8 / 99$ & $7 / 9 / 99$ & $7 / 12 / 99$ \\
\hline 'hromium & ND & $0.01 \mathrm{mg} / \mathrm{L}$ & 1 & EPA 6010 & $7 / 8 / 99$ & $7 / 9 / 99$ & $7 / 12 / 99$ \\
\hline ead & $\mathrm{ND}$ & $0.05 \mathrm{mg} / \mathrm{L}$ & 1 & EPA 6010 & $7 / 8 / 99$ & $7 / 9 / 99$ & $7 / 12 / 99$ \\
\hline Mercury & $\mathrm{ND}$ & $0.002 \mathrm{mg} / \mathrm{L}$ & 10 & EPA 7470A & $7 / 8 / 99$ & $7 / 11 / 99$ & $7 / 11 / 99$ \\
\hline Telenium & ND & $0.1 \mathrm{mg} / \mathrm{L}$ & 1 & EPA 6010 & $7 / 8 / 99$ & $7 / 9 / 99$ & $7 / 12 / 99$ \\
\hline ilver & ND & $0.02 \mathrm{mg} / \mathrm{L}$ & 1 & EPA 6010 & $7 / 8 / 99$ & $7 / 9 / 99$ & $7 / 12 / 99$ \\
\hline
\end{tabular}

3. F. - Dilution Factor

ND - Not Detected

is report shall not be reproduced except in full, without the written approval of the laboratory. 


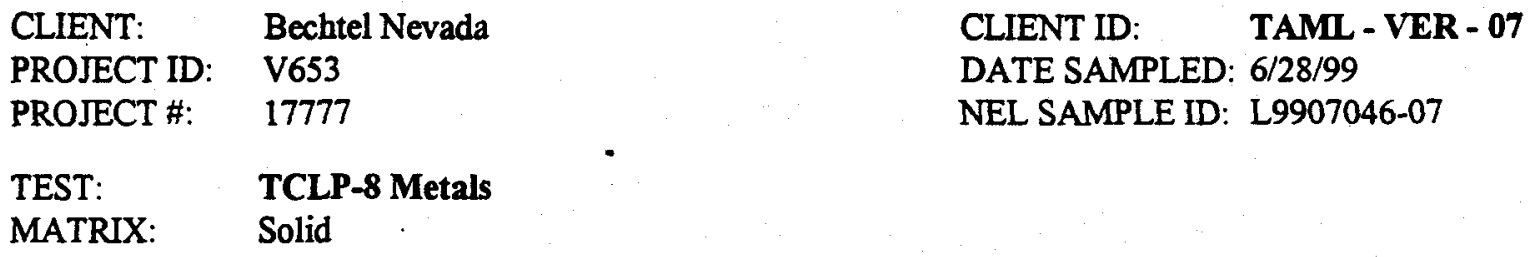

\begin{tabular}{|c|c|c|c|c|c|c|c|}
\hline PARAMETER & $\begin{array}{c}\text { RESULT } \\
\text { mg/L } \\
\end{array}$ & $\begin{array}{c}\text { REPORTING } \\
\text { LIMIT } \\
\end{array}$ & D. F. & METHOD & $\begin{array}{c}\text { TCLP/STLC } \\
\text { EXTRACTION } \\
\text { DATE } \\
\end{array}$ & DIGESTED & ANALYZED \\
\hline Arsenic & ND & $0.1 \mathrm{mg} / \mathrm{L}$ & 1 & EPA 6010 & 7/8/99 & 7/9/99 & $7 / 12 / 99$ \\
\hline Barium & $\mathrm{ND}$ & 1. $\mathrm{mg} / \mathrm{L}$ & 1 & EPA 6010 & 7/8/99 & 7/9/99 & $7 / 12 / 99$ \\
\hline Cadmium & ND & $0.01 \mathrm{mg} / \mathrm{L}$ & 1 & EPA 6010 & $7 / 8 / 99$ & 7/9/99 & $7 / 12 / 99$ \\
\hline Chromium & $\mathrm{ND}$ & $0.01 \mathrm{mg} / \mathrm{L}$ & 1 & EPA 6010 & $7 / 8 / 99$ & $7 / 9 / 99$ & $7 / 12 / 99$ \\
\hline Lead & $\mathrm{ND}$ & $0.05 \mathrm{mg} / \mathrm{L}$ & 1 & EPA 6010 & $7 / 8 / 99$ & 7/9/99 & $7 / 12 / 99$ \\
\hline Mercury & $\mathrm{ND}$ & $0.002 \mathrm{mg} / \mathrm{L}$ & 10 & EPA 7470A & $7 / 8 / 99$ & $7 / 11 / 99$ & $7 / 11 / 99$ \\
\hline Selenium & $\mathrm{ND}$ & $0.1 \mathrm{mg} / \mathrm{L}$ & 1 & EPA 6010 & $7 / 8 / 99$ & $7 / 9 / 99$ & $7 / 12 / 99$ \\
\hline Silver & $\mathrm{ND}$ & $0.02 \mathrm{mg} / \mathrm{L}$ & 1. & EPA 6010 & $7 / 8 / 99$ & 7/9/99 & $7 / 12 / 99$ \\
\hline
\end{tabular}

D.F. - Dilution Factor

ND - Not Detected

This report shall not be reproduced except in full, without the written approval of the laboratony. 


$\begin{array}{lll}\text { CLIENT: } & \text { Bechtel Nevada } & \text { CLIENT ID: } \quad \text { TAML - VER - 08 } \\ \text { PROJECT ID: } & \text { V653 } & \text { DATE SAMPLED: 6/28/99 } \\ \text { PROJECT \#: } & 17777 & \text { NEL SAMPLE ID: L9907046-08 } \\ & \\ \text { IEST: } & \text { TCLP-8 Metals } & \\ \text { MATRIX: } & \text { Solid } & \end{array}$

\begin{tabular}{|c|c|c|c|c|c|c|c|}
\hline PARAMETER & $\begin{array}{c}\text { RESULT } \\
\mathrm{mg} / \mathrm{L} \\
\end{array}$ & $\begin{array}{c}\text { REPORTING } \\
\text { LIMIT } \\
\end{array}$ & D. F. & METHOD & $\begin{array}{c}\text { TCLPISTLC } \\
\text { EXTRACTION } \\
\text { DATE } \\
\end{array}$ & DIGESTED & ANALYZED \\
\hline Irsenic & ND & $0.1 \mathrm{mg} / \mathrm{L}$ & 1 & EPA 6010 & $7 / 8 / 99$ & $7 / 9 / 99$ & $7 / 12 / 99$ \\
\hline 3arium & ND & 1. $\mathrm{mg} / \mathrm{L}$ & 1 & EPA 6010 & $7 / 8 / 99$ & $7 / 9 / 99$ & $7 / 12 / 99$ \\
\hline Cadmium & ND & $0.01 \mathrm{mg} / \mathrm{L}$ & 1 & EPA 6010 & $7 / 8 / 99$ & $7 / 9 / 99$ & $7 / 12 / 99$ \\
\hline 'hromium & ND & $0.01 \mathrm{mg} / \mathrm{L}$ & 1 & EPA 6010 & $7 / 8 / 99$ & 7/9/99 & $7 / 12 / 99$ \\
\hline ead & $\mathrm{ND}$ & $0.05 \mathrm{mg} / \mathrm{L}$ & 1 & EPA 6010 & $7 / 8 / 99$ & $7 / 9 / 99$ & $7 / 12 / 99$ \\
\hline Mercury & $\mathrm{ND}$ & $0.002 \mathrm{mg} / \mathrm{L}$ & 10 & EPA 7470A & 7/8/99 & $7 / 11 / 99$ & $7 / 11 / 99$ \\
\hline 'elenium & ND & $0.1 \mathrm{mg} / \mathrm{L}$ & 1 & EPA 6010 & $7 / 8 / 99$ & $7 / 9 / 99$ & $7 / 12 / 99$ \\
\hline ilver & ND & $0.02 \mathrm{mg} / \mathrm{L}$ & 1 & EPA 6010 & $7 / 8 / 99$ & 7/9/99 & $7 / 12 / 99$ \\
\hline
\end{tabular}

I F. - Dilution Factor

ND - Not Detected

is report shall not be reproduced except in full, without the written approval of the laboratony. 


\begin{tabular}{|c|c|c|c|c|c|c|c|}
\hline \multirow{2}{*}{$\begin{array}{l}\text { CLIENT: } \\
\text { PROJECT ID: } \\
\text { PROJECT \#: } \\
\text { TEST: } \\
\text { MATRIX: }\end{array}$} & \multirow{2}{*}{$\begin{array}{l}\text { Bechtel Nevada } \\
\text { V653 } \\
17777 \\
\text { TCLP-8 Metals } \\
\text { Solid }\end{array}$} & & & \multicolumn{3}{|c|}{$\begin{array}{ll}\text { CLIENT ID: } & \text { TAML - VER - } 09 \\
\text { DATE SAMPLED: } & \text { 6/28/99 } \\
\text { NEL SAMPLE ID: } & \text { L9907046-09 }\end{array}$} & \\
\hline & & & & & & & \\
\hline PARAMETER & $\begin{array}{c}\text { RESULT } \\
\mathrm{mg} / \mathbf{L} \\
\end{array}$ & $\begin{array}{c}\text { REPORTING } \\
\text { LIMIT } \\
\end{array}$ & D. F. & METHOD & $\begin{array}{c}\text { TCLP/STLC } \\
\text { EXTRACTION } \\
\text { DATE } \\
\end{array}$ & DIGESTED & ANALYZED \\
\hline Arsenic & ND & $0.1 \mathrm{mg} / \mathrm{L}$ & 1 & EPA 6010 & $7 / 8 / 99$ & 7/9/99 & $7 / 12 / 99$ \\
\hline Barium & ND & 1. $\mathrm{mg} / \mathrm{L}$ & 1 & EPA 6010 & $7 / 8 / 99$ & $7 / 9 / 99$ & $7 / 12 / 99$ \\
\hline Cadmium & $\mathrm{ND}$ & $0.01 \mathrm{mg} / \mathrm{L}$ & 1 & EPA 6010 & $7 / 8 / 99$ & 7/9/99 & $7 / 12 / 99$ \\
\hline Chromium & ND & $0.01 \mathrm{mg} / \mathrm{L}$ & 1 & EPA 6010 & $7 / 8 / 99$ & $7 / 9 / 99$ & $7 / 12 / 99$ \\
\hline Lead & $\mathrm{ND}$ & $0.05 \mathrm{mg} / \mathrm{L}$ & 1 & EPA 6010 & 7/8/99 & 7/9/99 & $7 / 12 / 99$ \\
\hline Mercury & $\mathrm{ND}$ & $0.002 \mathrm{mg} / \mathrm{L}$ & 10 & EPA 7470A & $7 / 8 / 99$ & $7 / 11 / 99$ & $7 / 11 / 99$ \\
\hline Selenium & $\mathrm{ND}$ & $0.1 \mathrm{mg} / \mathrm{L}$ & 1 & EPA 6010 & $7 / 8 / 99$ & $7 / 9 / 99$ & $7 / 12 / 99$ \\
\hline Silver & ND & $0.02 \mathrm{mg} / \mathrm{L}$ & 1 & EPA 6010 & 7/8/99 & $7 / 9 / 99$ & $7 / 12 / 99$ \\
\hline
\end{tabular}

D.F. - Dilution Factor

ND - Not Detected

This report shall not be reproduced except in full, without the written approval of the laboratory. 


\begin{tabular}{|c|c|c|}
\hline $\begin{array}{l}\text { ILIENT: } \\
\text { PROJECT ID: } \\
\text { PROJECT \#: }\end{array}$ & $\begin{array}{l}\text { Bechtel Nevada } \\
\text { V653 } \\
17777\end{array}$ & $\begin{array}{ll}\text { CLIENT ID: } & \text { TAML - VER - } 10 \\
\text { DATE SAMPLED: } & 6 / 28 / 99 \\
\text { NEL SAMPLE ID: } & \text { L9907046-10 }\end{array}$ \\
\hline $\begin{array}{l}\text {-IEST: } \\
\text { MATRIX: }\end{array}$ & $\begin{array}{l}\text { TCLP-8 Metals } \\
\text { Solid }\end{array}$ & \\
\hline
\end{tabular}

\begin{tabular}{|c|c|c|c|c|c|c|c|}
\hline PARAMETER & $\begin{array}{c}\text { RESULT } \\
\text { mg/L } \\
\end{array}$ & $\begin{array}{l}\text { REPORTING } \\
\text { LIMIT } \\
\end{array}$ & D. F. & METHOD & $\begin{array}{c}\text { TCLP/STLC } \\
\text { EXTRACTION } \\
\text { DATE } \\
\end{array}$ & DIGESTED & ANALYZED \\
\hline Irsenic & ND & $0.1 \mathrm{mg} / \mathrm{L}$ & 1 & EPA 6010 & $7 / 8 / 99$ & $7 / 9 / 99$ & $7 / 12 / 99$ \\
\hline 3arium & ND & 1. $\mathrm{mg} / \mathrm{L}$ & 1 & EPA 6010 & $7 / 8 / 99$ & 7/9/99 & $7 / 12 / 99$ \\
\hline Cadmium & ND & $0.01 \mathrm{mg} / \mathrm{L}$ & 1 & EPA 6010 & 7/8/99 & 7/9/99 & $7 / 12 / 99$ \\
\hline 'thromium & ND & $0.01 \mathrm{mg} / \mathrm{L}$ & 1 & EPA 6010 & 7/8/99 & 7/9/99 & $7 / 12 / 99$ \\
\hline ead & ND & $0.05 \mathrm{mg} / \mathrm{L}$ & 1 & EPA 6010 & $7 / 8 / 99$ & 7/9/99 & $7 / 12 / 99$ \\
\hline Mercury & ND & $0.002 \mathrm{mg} / \mathrm{L}$ & 10 & EPA 7470A & 7/8/99 & $7 / 11 / 99$ & $7 / 11 / 99$ \\
\hline Telenium & ND & $0.1 \mathrm{mg} / \mathrm{L}$ & 1 & EPA 6010 & $7 / 8 / 99$ & 7/9/99 & $7 / 12 / 99$ \\
\hline ilver & ND & $0.02 \mathrm{mg} / \mathrm{L}$ & 1 & EPA 6010 & 7/8/99 & 7/9/99 & $7 / 12 / 99$ \\
\hline
\end{tabular}

I F. - Dilution Factor

ND - Not Detected

: is report shall not be reproduced except in full, without the written approval of the laboratory. 


\begin{tabular}{|c|c|c|c|c|c|c|c|}
\hline $\begin{array}{l}\text { CLIENT: } \\
\text { PROJECT ID: } \\
\text { PROJECT \#: }\end{array}$ & $\begin{array}{l}\text { Bechtel Nevada } \\
\text { V653 } \\
17777\end{array}$ & & & $\begin{array}{l}\text { CLIENT II } \\
\text { DATE SAI } \\
\text { NEL SAM }\end{array}$ & $\begin{array}{l}\quad \text { TAML - } \\
\text { PPLED: } 6 / 28 / 99 \\
\text { LE ID: } \quad \text { L9907046 }\end{array}$ & $\begin{array}{l}\text { VER - } 11 \\
6-11\end{array}$ & \\
\hline $\begin{array}{l}\text { TEST: } \\
\text { MATRIX: }\end{array}$ & $\begin{array}{l}\text { TCLP-8 Metals } \\
\text { Solid }\end{array}$ & & & & & & \\
\hline PARAMETER & $\begin{array}{c}\text { RESULT } \\
\text { mg/L }\end{array}$ & $\begin{array}{c}\text { REPORTING } \\
\text { LMIT } \\
\end{array}$ & D. F. & METHOD & $\begin{array}{c}\text { TCLP/STLC } \\
\text { EXTRACTION } \\
\text { DATE } \\
\end{array}$ & DIGESTED & ANALYZED \\
\hline Arsenic & $\mathrm{ND}$ & $0.1 \mathrm{mg} / \mathrm{L}$ & 1 & EPA 6010 & 7/8/99 & $7 / 9 / 99$ & $7 / 12 / 99$ \\
\hline Barium & $\mathrm{ND}$ & 1. $\mathrm{mg} / \mathrm{L}$ & 1 & EPA 6010 & $7 / 8 / 99$ & 7/9/99 & $7 / 12 / 99$ \\
\hline Cadmium & ND & $0.01 \mathrm{mg} / \mathrm{L}$ & 1 & EPA 6010 & $7 / 8 / 99$ & $7 / 9 / 99$ & $7 / 12 / 99$ \\
\hline Chromium & ND & $0.01 \mathrm{mg} / \mathrm{L}$ & 1 & EPA 6010 & $7 / 8 / 99$ & $7 / 9 / 99$ & $7 / 12 / 99$ \\
\hline Lead & ND & $0.05 \mathrm{mg} / \mathrm{L}$ & 1 & EPA 6010 & $7 / 8 / 99$ & $7 / 9 / 99$ & $7 / 12 / 99$ \\
\hline Mercury & ND & $0.002 \mathrm{mg} / \mathrm{L}$ & 10 & EPA 7470A & $7 / 8 / 99$ & $7 / 11 / 99$ & $7 / 11 / 99$ \\
\hline Selenium & ND & $0.1 \mathrm{mg} / \mathrm{L}$ & 1 & EPA 6010 & 7/8/99 & $7 / 9 / 99$ & $7 / 12 / 99$ \\
\hline Silver & ND & $0.02 \mathrm{mg} / \mathrm{L}$ & 1 & EPA 6010 & 7/8/99 & $7 / 9 / 99$ & $7 / 12 / 99$ \\
\hline
\end{tabular}

D.F. - Dilution Factor

ND - Not Detected

This report shall not be reproduced except in full, without the written approval of the laboratory. 


\begin{tabular}{|c|c|c|}
\hline ILIENT: & Bechtel Nevada & CLIENT ID: $\quad$ TAML - VER - 12 \\
\hline ?ROJECT ID: & V653 & DATE SAMPLED: 6/29/99 \\
\hline PROJECT \#: & 17777 & NEL SAMPLE ID: L9907046-12 \\
\hline $\begin{array}{l}\text { IEST: } \\
\text { MATRIX: }\end{array}$ & $\begin{array}{l}\text { TCLP-8 Metals } \\
\text { Solid }\end{array}$ & \\
\hline
\end{tabular}

\begin{tabular}{|c|c|c|c|c|c|c|c|}
\hline PARAMETER & $\begin{array}{c}\text { RESULT } \\
\text { mg/L } \\
\end{array}$ & $\begin{array}{l}\text { REPORTING } \\
\text { LIMIT } \\
\end{array}$ & D. F. & METHOD & $\begin{array}{c}\text { TCLP/STLC } \\
\text { EXTRACTION } \\
\text { DATE } \\
\end{array}$ & DIGESTED & ANALYZED \\
\hline Irsenic & ND & $0.1 \mathrm{mg} / \mathrm{L}$ & 1 & EPA 6010 & $7 / 8 / 99$ & 7/9/99 & 7/12/99 \\
\hline 3arium & ND & $1 . \mathrm{mg} / \mathrm{L}$ & 1 & EPA 6010 & 7/8/99 & 7/9/99 & 7/12/99 \\
\hline Cadmium & ND & $0.01 \mathrm{mg} / \mathrm{L}$ & 1 & EPA 6010 & $7 / 8 / 99$ & 7/9/99 & $7 / 12 / 99$ \\
\hline 'hromium & ND & $0.01 \mathrm{mg} / \mathrm{L}$ & 1 & EPA 6010 & $7 / 8 / 99$ & 7/9/99 & $7 / 12 / 99$ \\
\hline ead & ND & $0.05 \mathrm{mg} / \mathrm{L}$ & 1 & EPA 6010 & $7 / 8 / 99$ & 7/9/99 & $7 / 12 / 99$ \\
\hline Mercury & ND & $0.002 \mathrm{mg} / \mathrm{L}$ & 10 & EPA $7470 A$ & $7 / 8 / 99$ & $7 / 11 / 99$ & $7 / 11 / 99$ \\
\hline Telenium & ND & $0.1 \mathrm{mg} / \mathrm{L}$ & 1 & EPA 6010 & 7/8/99 & 7/9/99 & $7 / 12 / 99$ \\
\hline ilver & ND & $0.02 \mathrm{mg} / \mathrm{L}$ & 1 & EPA 6010 & $7 / 8 / 99$ & 7/9/99 & $7 / 12 / 99$ \\
\hline
\end{tabular}

F. - Dilution Factor

ND - Not Detected

is report shall not be reproduced except in full, without the written approval of the laboratory. 


\begin{tabular}{|c|c|c|c|c|c|c|c|}
\hline $\begin{array}{l}\text { CLIENT: } \\
\text { PROJECT ID: } \\
\text { PROJECT \#: }\end{array}$ & $\begin{array}{l}\text { Bechtel Nevada } \\
\text { V653 } \\
17777\end{array}$ & & & \multicolumn{3}{|c|}{$\begin{array}{ll}\text { CLIENT ID: } & \text { TAML - VER - } 13 \\
\text { DATE SAMPLED: } & 6 / 28 / 99 \\
\text { NEL SAMPLE ID: } & \text { L9907046-13 }\end{array}$} & \\
\hline $\begin{array}{l}\text { TEST: } \\
\text { MATRIX: }\end{array}$ & $\begin{array}{l}\text { TCLP-8 Metals } \\
\text { Solid }\end{array}$ & & & & & & \\
\hline PARAMETER & $\begin{array}{c}\text { RESULT } \\
\mathrm{mg} / \mathrm{L} \\
\end{array}$ & $\begin{array}{c}\text { REPORTING } \\
\text { LIMIT } \\
\end{array}$ & D. F. & METHOD & $\begin{array}{l}\text { TCLP/STLC } \\
\text { EXTRACTION } \\
\text { DATE } \\
\end{array}$ & DIGESTED & ANALYZED \\
\hline Arsenic & ND & $0.1 \mathrm{mg} / \mathrm{L}$ & 1 & EPA 6010 & $7 / 8 / 99$ & 7/9/99 & $7 / 12 / 99$ \\
\hline Barium & ND & 1. $\mathrm{mg} / \mathrm{L}$ & 1 & EPA 6010 & $7 / 8 / 99$ & $7 / 9 / 99$ & $7 / 12 / 99$ \\
\hline Cadmium & $\mathrm{ND}$ & $0.01 \mathrm{mg} / \mathrm{L}$ & 1 & EPA 6010 & $7 / 8 / 99$ & 7/9/99 & $7 / 12 / 99$ \\
\hline Chromium & ND & $0.01 \mathrm{mg} / \mathrm{L}$ & 1 & EPA 6010 & $7 / 8 / 99$ & 7/9/99 & $7 / 12 / 99$ \\
\hline Lead & $\mathrm{ND}$ & $0.05 \mathrm{mg} / \mathrm{L}$ & 1 & EPA 6010 & $7 / 8 / 99$ & 7/9/99 & $7 / 12 / 99$ \\
\hline Mercury & $\mathrm{ND}$ & $0.002 \mathrm{mg} / \mathrm{L}$ & 10 & EPA 7470A & 7/8/99 & $7 / 11 / 99$ & $7 / 11 / 99$ \\
\hline Selenium & ND & $0.1 \mathrm{mg} / \mathrm{L}$ & 1 & EPA 6010 & $7 / 8 / 99$ & $7 / 9 / 99$ & $7 / 12 / 99$ \\
\hline Silver & $\mathrm{ND}$ & $0.02 \mathrm{mg} / \mathrm{L}$ & 1 & EPA 6010 & $7 / 8 / 99$ & $7 / 9 / 99$ & $7 / 12 / 99$ \\
\hline
\end{tabular}

D.F. - Dilution Factor

ND - Not Detected

This report shall not be reproduced except in full, without the written approval of the laboratory. 


\begin{tabular}{|c|c|c|c|c|c|c|c|}
\hline $\begin{array}{l}\text { CLIENT: } \\
\text { PROJECT ID: } \\
\text { PROJECT \#: }\end{array}$ & $\begin{array}{l}\text { Bechtel Nevada } \\
\text { V653 } \\
17777\end{array}$ & & & $\begin{array}{l}\text { CLIENT ID: } \\
\text { DATE SAM } \\
\text { NEL SAMP }\end{array}$ & $\begin{array}{l}\quad \text { TAMI - } \\
\text { PLED: } 6 / 28 / 99 \\
\text { LE ID: } \text { L990704 }\end{array}$ & $\begin{array}{l}\text { VER - } 14 \\
6-14\end{array}$ & • \\
\hline $\begin{array}{l}\text { TEST: } \\
\text { MATRIX: }\end{array}$ & $\begin{array}{l}\text { TCLP-8 Metals } \\
\text { Solid }\end{array}$ & & & & & & \\
\hline PARAMETER & $\begin{array}{c}\text { RESULT } \\
\text { mg/L } \\
\end{array}$ & $\begin{array}{c}\text { REPORTING } \\
\text { LIMIT } \\
\end{array}$ & D. F. & METHOD & $\begin{array}{c}\text { TCLP/STLC } \\
\text { EXTRACTION } \\
\text { DATE } \\
\end{array}$ & DIGESTED & ANALYZED \\
\hline Arsenic & ND & $0.1 \mathrm{mg} / \mathrm{L}$ & 1 & EPA 6010 & 7/8/99 & 7/9/99 & $7 / 12 / 99$ \\
\hline Barium & ND & $1 . \mathrm{mg} / \mathrm{L}$ & 1 & EPA 6010 & 7/8/99 & 7/9/99 & 7/12/99 \\
\hline Cadmium & ND & $0.01 \mathrm{mg} / \mathrm{L}$ & 1 & EPA 6010 & 7/8/99 & 7/9/99 & $7 / 12 / 99$ \\
\hline Chromium & ND & $0.01 \mathrm{mg} / \mathrm{L}$ & 1 & EPA 6010 & $7 / 8 / 99$ & 7/9/99 & $7 / 12 / 99$ \\
\hline Lead & ND & $0.05 \mathrm{mg} / \mathrm{L}$ & 1 & EPA 6010 & 7/8/99 & 7/9/99 & $7 / 12 / 99$ \\
\hline Mercury & ND & $0.002 \mathrm{mg} / \mathrm{L}$ & 10 & EPA 7470A & $7 / 8 / 99$ & 7/11/99 & $7 / 11 / 99$ \\
\hline Selenium & ND & $0.1 \mathrm{mg} / \mathrm{L}$ & 1 & EPA 6010 & $7 / 8 / 99$ & 7/9/99 & $7 / 12 / 99$ \\
\hline Silver & $\mathrm{ND}$ & $0.02 \mathrm{mg} / \mathrm{L}$ & 1 & EPA 6010 & 7/8/99 & $7 / 9 / 99$ & $7 / 12 / 99$ \\
\hline
\end{tabular}

D.F. - Dilution Factor

ND - Not Detected

This report shall not be reproduced except in full, without the written approval of the laboratory. 


\begin{tabular}{|c|c|c|c|c|c|c|c|}
\hline $\begin{array}{l}\text { CLIENT: } \\
\text { PROJECT ID: } \\
\text { PROJECT \#: }\end{array}$ & $\begin{array}{l}\text { Bechtel Nevada } \\
\text { V653 } \\
17777\end{array}$ & & & $\begin{array}{l}\text { CLIENT II } \\
\text { DATE SAI } \\
\text { NEL SAMI }\end{array}$ & $\begin{array}{l}\text { TAML - } \\
\text { IPLED: 6/29/99 } \\
\text { LE ID: } \text { L990704 }\end{array}$ & $\begin{array}{l}\text { VER - } 15 \\
6-15\end{array}$ & . \\
\hline $\begin{array}{l}\text { TEST: } \\
\text { MATRIX: }\end{array}$ & $\begin{array}{l}\text { TCLP-8 Metals } \\
\text { Solid }\end{array}$ & & & & & & \\
\hline PARAMETER & $\begin{array}{c}\text { RESULT } \\
\mathrm{mg} / \mathrm{L} \\
\end{array}$ & $\begin{array}{c}\text { REPORTING } \\
\text { LIMIT } \\
\end{array}$ & D. F. & METHOD & $\begin{array}{c}\text { TCLPSTLC } \\
\text { EXTRACTION } \\
\text { DATE } \\
\end{array}$ & DIGESTED & ANALYZED \\
\hline Arsenic & ND & $0.1 \mathrm{mg} / \mathrm{L}$ & 1 & EPA 6010 & 7/8/99 & 7/9/99 & $7 / 12 / 99$ \\
\hline Barium & ND & 1. $\mathrm{mg} / \mathrm{L}$ & 1 & EPA 6010 & $7 / 8 / 99$ & 7/9/99 & $7 / 12 / 99$ \\
\hline Cadmium & ND & $0.01 \mathrm{mg} / \mathrm{L}$ & 1 & EPA 6010 & $7 / 8 / 99$ & $7 / 9 / 99$ & $7 / 12 / 99$ \\
\hline Chromium & $\mathrm{ND}$ & $0.01 \mathrm{mg} / \mathrm{L}$ & 1 & EPA 6010 & 7/8/99 & 7/9/99 & $7 / 12 / 99$ \\
\hline Lead & $\mathrm{ND}$ & $0.05 \mathrm{mg} / \mathrm{L}$ & 1 & EPA 6010 & $7 / 8 / 99$ & $7 / 9 / 99$ & $7 / 12 / 99$ \\
\hline Mercury & $\mathrm{ND}$ & $0.002 \mathrm{mg} / \mathrm{L}$ & 10 & EPA $7470 A$ & $7 / 8 / 99$ & $7 / 11 / 99$ & $7 / 11 / 99$ \\
\hline Selenium & ND & $0.1 \mathrm{mg} / \mathrm{L}$ & 1 & EPA 6010 & $7 / 8 / 99$ & 7/9/99 & $7 / 12 / 99$ \\
\hline Silver & ND & $0.02 \mathrm{mg} / \mathrm{L}$ & 1 & EPA 6010 & $7 / 8 / 99$ & $7 / 9 / 99$ & $7 / 12 / 99$ \\
\hline
\end{tabular}

D.F. - Dilution Factor

ND - Not Detected

This report shall not be reproduced except in full, without the written approval of the laboratory. 
CLIENT: Bechtel Nevada

PROJECT ID: V653

PROJECT \#: $\quad 17777$

TEST:

MATRIX: Solid
CLIENT ID:

DATE SAMPLED: $6 / 29 / 99$

NEL SAMPLE ID: L9907046-16

\begin{tabular}{|c|c|c|c|c|c|c|c|}
\hline PARAMETER & $\begin{array}{c}\text { RESULT } \\
\mathrm{mg} / \mathrm{L} \\
\end{array}$ & $\begin{array}{c}\text { REPORTING } \\
\text { LIMIT } \\
\end{array}$ & D. F. & METHOD & $\begin{array}{c}\text { TCLP/STLC } \\
\text { EXTRACTION } \\
\text { DATE } \\
\end{array}$ & DIGESTED & ANALYZED \\
\hline Arsenic & ND & $0.1 \mathrm{mg} / \mathrm{L}$ & 1 & EPA 6010 & $7 / 8 / 99$ & 7/9/99 & $7 / 12 / 99$ \\
\hline Barium & ND & 1. $\mathrm{mg} / \mathrm{L}$ & 1 & EPA 6010 & $7 / 8 / 99$ & 7/9/99 & $7 / 12 / 99$ \\
\hline Cadmium & ND & $0.01 \mathrm{mg} / \mathrm{L}$ & 1 & EPA 6010 & $7 / 8 / 99$ & 7/9/99 & $7 / 12 / 99$ \\
\hline Jhromium & $\mathrm{ND}$ & $0.01 \mathrm{mg} / \mathrm{L}$ & 1 & EPA 6010 & $7 / 8 / 99$ & 7/9/99 & $7 / 12 / 99$ \\
\hline Lead & ND & $0.05 \mathrm{mg} / \mathrm{L}$ & 1 & EPA 6010 & $7 / 8 / 99$ & $7 / 9 / 99$ & $7 / 12 / 99$ \\
\hline Mercury & $\mathrm{ND}$ & $0.002 \mathrm{mg} / \mathrm{L}$ & 10 & EPA 7470A & $7 / 8 / 99$ & $7 / 11 / 99$ & $7 / 11 / 99$ \\
\hline jelenium & ND & $0.1 \mathrm{mg} / \mathrm{L}$ & 1 & EPA 6010 & $7 / 8 / 99$ & 7/9/99 & $7 / 12 / 99$ \\
\hline Silver & $\mathrm{ND}$ & $0.02 \mathrm{mg} / \mathrm{L}$ & 1 & EPA 6010 & $7 / 8 / 99$ & 7/9/99 & 7/12/99 \\
\hline
\end{tabular}

I.F. - Dilution Factor

ND - Not Detected

his report shall not be reproduced except in full, without the written approval of the laboratory. 


\begin{tabular}{|c|c|c|}
\hline $\begin{array}{l}\text { CLIENT: } \\
\text { PROJECT ID: } \\
\text { PROJECT \#: }\end{array}$ & $\begin{array}{l}\text { Bechtel Nevada } \\
\text { V653 } \\
17777\end{array}$ & $\begin{array}{ll}\text { CLIENT ID: } & \text { TAML - VER - } 17 \\
\text { DATE SAMPLED: } & \text { 6/29/99 } \\
\text { NEL SAMPLE ID: } & \text { L9907046-17 }\end{array}$ \\
\hline $\begin{array}{l}\text { TEST: } \\
\text { MATRIX: }\end{array}$ & $\begin{array}{l}\text { TCLP-8 Metals } \\
\text { Solid }\end{array}$ & \\
\hline
\end{tabular}

\begin{tabular}{|c|c|c|c|c|c|c|c|}
\hline PARAMETER & $\begin{array}{c}\text { RESULT } \\
\text { mg/L } \\
\end{array}$ & $\begin{array}{l}\text { REPORTING } \\
\text { LIMIT } \\
\end{array}$ & D. F. & METHOD & $\begin{array}{c}\text { TCLP/STLC } \\
\text { EXTRACTION } \\
\text { DATE } \\
\end{array}$ & DIGESTED & ANALYZED \\
\hline Arsenic & ND & $0.1 \mathrm{mg} / \mathrm{L}$ & 1 & EPA 6010 & 7/8/99 & 7/9/99 & $7 / 12 / 99$ \\
\hline Barium & ND & 1. $\mathrm{mg} / \mathrm{L}$ & 1 & EPA 6010 & 7/8/99 & 7/9/99 & $7 / 12 / 99$ \\
\hline Cadmium & ND & $0.01 \mathrm{mg} / \mathrm{L}$ & 1 & EPA 6010 & 7/8/99 & 7/9/99 & $7 / 12 / 99$ \\
\hline Chromium & ND & $0.01 \mathrm{mg} / \mathrm{L}$ & 1 & EPA 6010 & $7 / 8 / 99$ & $7 / 9 / 99$ & $7 / 12 / 99$ \\
\hline Lead & $\mathrm{ND}$ & $0.05 \mathrm{mg} / \mathrm{L}$ & 1 & EPA 6010 & 7/8/99 & 7/9/99 & $7 / 12 / 99$ \\
\hline Mercury & ND & $0.002 \mathrm{mg} / \mathrm{L}$ & 10 & EPA 7470A & 7/8/99 & $7 / 11 / 99$ & $7 / 11 / 99$ \\
\hline Selenium & ND & $0.1 \mathrm{mg} / \mathrm{L}$ & 1 & EPA 6010 & $7 / 8 / 99$ & 7/9/99 & $7 / 12 / 99$ \\
\hline Silver & ND & $0.02 \mathrm{mg} / \mathrm{L}$ & 1 & EPA 6010 & $7 / 8 / 99$ & 7/9/99 & $7 / 12 / 99$ \\
\hline
\end{tabular}

D.F. - Dilution Factor

ND - Not Detected

This report shall not be reproduced except in full, without the written approval of the laboratory. 


\section{CLIENT: Bechtel Nevada}

PROJECT ID: V653

PROJECT \#: $\quad 17777$
CLIENT ID: $\quad$ TAMI - VER - 18

DATE SAMPLED: 6/28/99

NEL SAMPLE ID: L9907046-18

\section{TEST: \\ TCLP-8 Metals \\ MATRIX: Solid}

\begin{tabular}{|c|c|c|c|c|c|c|c|}
\hline PARAMETER & $\begin{array}{c}\text { RESULT } \\
\text { mg/L } \\
\end{array}$ & $\begin{array}{l}\text { REPORTING } \\
\text { LIMIT } \\
\end{array}$ & D. F. & METHOD & $\begin{array}{c}\text { TCLP/STLC } \\
\text { EXTRACTION } \\
\text { DATE } \\
\end{array}$ & DIGESTED & ANALYZED \\
\hline Arsenic & ND & $0.1 \mathrm{mg} / \mathrm{L}$ & 1 & EPA 6010 & $7 / 8 / 99$ & 7/9/99 & $7 / 12 / 99$ \\
\hline 3arium & $\mathrm{ND}$ & 1. mg/L & 1 & EPA 6010 & 7/8/99 & 7/9/99 & 7/12/99 \\
\hline Cadmium & ND & $0.01 \mathrm{mg} / \mathrm{L}$ & 1 & EPA 6010 & $7 / 8 / 99$ & 7/9/99 & $7 / 12 / 99$ \\
\hline Zhromium & $\mathrm{ND}$ & $0.01 \mathrm{mg} / \mathrm{L}$ & 1 & EPA 6010 & 7/8/99 & 7/9/99 & $7 / 12 / 99$ \\
\hline Lead & ND & $0.05 \mathrm{mg} / \mathrm{L}$ & 1 & EPA 6010 & 7/8/99 & $7 / 9 / 99$ & $7 / 12 / 99$ \\
\hline Mercury & ND & $0.002 \mathrm{mg} / \mathrm{L}$ & 10 & EPA 7470A & $7 / 8 / 99$ & 7/11/99 & $7 / 11 / 99$ \\
\hline jelenium & ND & $0.1 \mathrm{mg} / \mathrm{L}$ & 1 & EPA 6010 & $7 / 8 / 99$ & $7 / 9 / 99$ & $7 / 12 / 99$ \\
\hline iilver & $\mathrm{ND}$ & $0.02 \mathrm{mg} / \mathrm{L}$ & 1 & EPA 6010 & $7 / 8 / 99$ & 7/9/99 & $7 / 12 / 99$ \\
\hline
\end{tabular}

F. - Dilution Factor

ND - Not Detected

tis report shall not be reproduced except in full, without the written approval of the laboratory. 


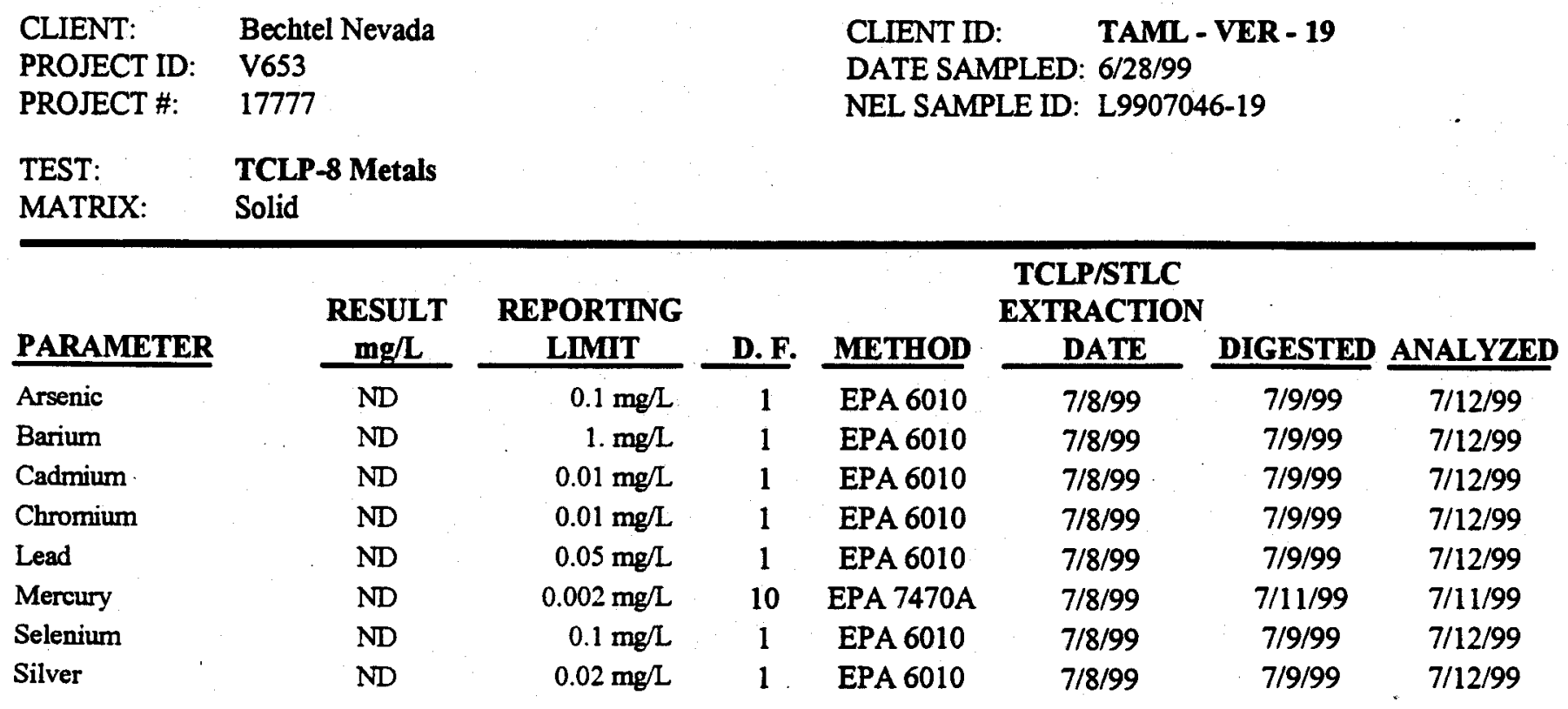

D.F. - Dilution Factor

ND - Not Detected

This report shall not be reproduced except in full, without the written approval of the laboratory. 


$\begin{array}{ll}\text { :LIENT: } & \text { Bechtel Nevada } \\ \text {-ROJECT ID: } & \text { V653 } \\ \text { PROJECT \#: } & 17777\end{array}$

EST: $\quad$ TCLP-8 Metals MATRIX: Solid
CLIENT ID: $\quad$ TAML - VER - 20

DATE SAMPLED: 6/29/99

NEL SAMPLE ID: L9907046-20

\begin{tabular}{|c|c|c|c|c|c|c|c|}
\hline PARAMETER & $\begin{array}{c}\text { RESULT } \\
\mathrm{mg} / \mathrm{L}\end{array}$ & $\begin{array}{l}\text { REPORTING } \\
\text { LIMIT } \\
\end{array}$ & D. F. & METHOD & $\begin{array}{c}\text { TCLP/STLC } \\
\text { EXTRACTION } \\
\text { DATE } \\
\end{array}$ & DIGESTED & ANALYZED \\
\hline rsenic & $\mathrm{ND}$ & $0.1 \mathrm{mg} / \mathrm{L}$ & 1 & EPA 6010 & $7 / 8 / 99$ & 7/9/99 & $7 / 12 / 99$ \\
\hline -arium & $\mathrm{ND}$ & 1. $\mathrm{mg} / \mathrm{L}$ & 1 & EPA 6010 & 7/8/99 & 7/9/99 & $7 / 12 / 99$ \\
\hline Cadmium & ND & $0.01 \mathrm{mg} / \mathrm{L}$ & 1 & EPA 6010 & $7 / 8 / 99$ & 7/9/99 & $7 / 12 / 99$ \\
\hline hromium & ND & $0.01 \mathrm{mg} / \mathrm{L}$ & 1 & EPA 6010 & $7 / 8 / 99$ & 7/9/99 & $7 / 12 / 99$ \\
\hline ead & $\mathrm{ND}$ & $0.05 \mathrm{mg} / \mathrm{L}$ & 1 & EPA 6010 & 7/8/99 & 7/9/99 & $7 / 12 / 99$ \\
\hline Mercury & ND & $0.002 \mathrm{mg} / \mathrm{L}$ & 10 & EPA 7470A & 7/8/99 & $7 / 11 / 99$ & $7 / 11 / 99$ \\
\hline elenium & ND & $0.1 \mathrm{mg} / \mathrm{L}$ & 1 & EPA 6010 & 7/8/99 & $7 / 9 / 99$ & $7 / 12 / 99$ \\
\hline ilver & $\mathrm{ND}$ & $0.02 \mathrm{mg} / \mathrm{L}$ & 1 & EPA 6010 & $7 / 8 / 99$ & 7/9/99 & $7 / 12 / 99$ \\
\hline
\end{tabular}

F. - Dilution Factor .

ND - Not Detected

is report shall not be reproduced except in full, without the written approval of the laboratory. 


\begin{tabular}{|c|c|}
\hline $\begin{array}{l}\text { CLIENT: } \\
\text { PROJECT ID: } \\
\text { PROJECT \#: }\end{array}$ & $\begin{array}{l}\text { Bechtel Nevada } \\
\text { V653 } \\
17777\end{array}$ \\
\hline $\begin{array}{l}\text { TEST: } \\
\text { MATRIX: }\end{array}$ & $\begin{array}{l}\text { TCLP-8 Metals } \\
\text { Solid }\end{array}$ \\
\hline
\end{tabular}

CLIENT ID: $\quad$ TAML - VER - 21

DATE SAMPLED: 6/29/99

NEL SAMPLE ID: L9907046-21

\section{MATRIX: Solid}

\begin{tabular}{|c|c|c|c|c|c|c|c|}
\hline PARAMETER & $\begin{array}{c}\text { RESULT } \\
\text { mg/L } \\
\end{array}$ & $\begin{array}{l}\text { REPORTING } \\
\text { LIMIT } \\
\end{array}$ & D. F. & METHOD & $\begin{array}{c}\text { TCLP/STLC } \\
\text { EXTRACTION } \\
\text { DATE } \\
\end{array}$ & DIGESTED & ANALYZED \\
\hline Arsenic & ND & $0.1 \mathrm{mg} / \mathrm{L}$ & 1 & EPA 6010 & 7/8/99 & 7/9/99 & 7/12/99 \\
\hline Barium & ND & 1. $\mathrm{mg} / \mathrm{L}$ & 1 & EPA 6010 & $7 / 8 / 99$ & 7/9/99 & 7/12/99 \\
\hline Cadmium & ND & $0.01 \mathrm{mg} / \mathrm{L}$ & 1 & EPA 6010 & $7 / 8 / 99$ & 7/9/99 & $7 / 12 / 99$ \\
\hline Chromium & ND & $0.01 \mathrm{mg} / \mathrm{L}$ & 1 & EPA 6010 & $7 / 8 / 99$ & 7/9/99 & 7/12/99 \\
\hline Lead & ND & $0.05 \mathrm{mg} / \mathrm{L}$ & 1 & EPA 6010 & $7 / 8 / 99$ & 7/9/99 & $7 / 12 / 99$ \\
\hline Mercury & ND & $0.002 \mathrm{mg} / \mathrm{L}$ & 10 & EPA 7470A & $7 / 8 / 99$ & $7 / 11 / 99$ & $7 / 11 / 99$ \\
\hline Selenium & $\mathrm{ND}$ & $0.1 \mathrm{mg} / \mathrm{L}$ & 1 & EPA 6010 & $7 / 8 / 99$ & 7/9/99 & $7 / 12 / 99$ \\
\hline Silver & $\mathrm{ND}$ & $0.02 \mathrm{mg} / \mathrm{L}$ & 1 & EPA 6010 & $7 / 8 / 99$ & 7/9/99 & $7 / 12 / 99$ \\
\hline
\end{tabular}

D.F. - Dilution Factor

ND - Not Detected

This report shall not be reproduced except in full, without the written approval of the laboratory. 


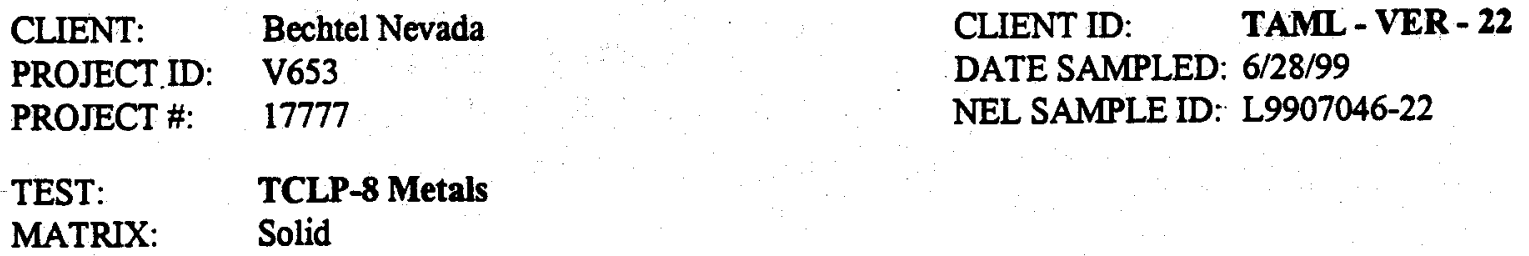

\begin{tabular}{|c|c|c|c|c|c|c|c|}
\hline PARAMETER & $\begin{array}{c}\text { RESULT } \\
\text { mg/L } \\
\end{array}$ & $\begin{array}{c}\text { REPORTING } \\
\text { LIMIT } \\
\end{array}$ & D. F. & METHOD & $\begin{array}{c}\text { TCLP/STLC } \\
\text { EXTRACTION } \\
\text { DATE } \\
\end{array}$ & DIGESTED & ANAL YZED \\
\hline Arsenic & $\mathrm{ND}$ & $0.1 \mathrm{mg} / \mathrm{L}$ & 1 & EPA 6010 & 7/8/99 & 7/9/99 & $7 / 12 / 99$ \\
\hline Barium & ND & 1. $\mathrm{mg} / \mathrm{L}$ & 1 & EPA 6010 & 7/8/99 & $7 / 9 / 99$ & $7 / 12 / 99$ \\
\hline Cadmium & $\mathrm{ND}$ & $0.01 \mathrm{mg} / \mathrm{L}$ & 1 & EPA 6010 & $7 / 8 / 99$ & 7/9/99 & $7 / 12 / 99$ \\
\hline Chromium & ND & $0.01 \mathrm{mg} / \mathrm{L}$ & 1 & EPA 6010 & $7 / 8 / 99$ & 7/9/99 & $7 / 12 / 99$ \\
\hline Lead & ND & $0.05 \mathrm{mg} / \mathrm{L}$ & 1 & EPA 6010 & $7 / 8 / 99$ & $7 / 9 / 99$ & $7 / 12 / 99$ \\
\hline Mercury & ND & $0.002 \mathrm{mg} / \mathrm{L}$ & 10 & EPA $7470 A$ & $7 / 8 / 99$ & $7 / 11 / 99$ & $7 / 11 / 99$ \\
\hline Selenium & $\mathrm{ND}$ & $0.1 \mathrm{mg} / \mathrm{L}$ & 1 & EPA 6010 & $7 / 8 / 99$ & $7 / 9 / 99$ & $7 / 12 / 99$ \\
\hline Silver & $\mathrm{ND}$ & $0.02 \mathrm{mg} / \mathrm{L}$ & 1 & EPA 6010 & $7 / 8 / 99$ & 7/9/99 & $7 / 12 / 99$ \\
\hline
\end{tabular}

J.F. - Dilution Factor

ND - Not Detected

his report shall not be reproduced except in full, without the written approval of the laboratory. 
CLIENT: Bechtel Nevada

PROJECT ID: V653

PROJECT \#: $\quad 17777$
CLIENT ID: $\quad$ TAML - VER - 23

DATE SAMPLED: 6/28/99

NEL SAMPLE ID: L9907046-23

\section{TEST: \\ MATRIX: \\ TCLP-8 Metals \\ Solid}

\section{TCLP/STLC EXTRACTION}

PARAMETER

RESULT REPORTING

Arsenic

Barium

$\mathbf{m g} / \mathbf{L}$

LIMIT

D. F. METHOD

DATE

DIGESTED ANALYZED

Cadmium

Chromium

Lead

Mercury

Selenium

Silver
ND

ND

ND

$\mathrm{ND}$

ND

ND

ND

ND

\section{$0.1 \mathrm{mg} / \mathrm{L}$}

1. $\mathrm{mg} / \mathrm{L}$

$0.01 \mathrm{mg} / \mathrm{L}$.

$0.01 \mathrm{mg} / \mathrm{L}$

$0.05 \mathrm{mg} / \mathrm{L}$

$0.002 \mathrm{mg} / \mathrm{L}$

$0.1 \mathrm{mg} / \mathrm{L}$

$0.02 \mathrm{mg} / \mathrm{L}$
EPA 6010

EPA 6010

EPA 6010

EPA 6010

EPA 7470A

EPA 6010

EPA 6010
EPA 6010
7/8/99

$7 / 8 / 99$

$7 / 8 / 99$

$7 / 8 / 99$

$7 / 8 / 99$

$7 / 8 / 99$

7/8/99

$7 / 8 / 99$
7/9/99

7/9/99

$7 / 9 / 99$

7/9/99

7/9/99

7/11/99

7/9/99

7/9/99
$7 / 12 / 99$

$7 / 12 / 99$

$7 / 12 / 99$

7/12/99

$7 / 12 / 99$

$7 / 11 / 99$

7/12/99

7/12/99

D.F. - Dilution Factor

ND - Not Detected

This report shall not be reproduced except in full, without the written approval of the laboratory. 


$\begin{array}{ll}\text { JLIENT: } & \text { Bechtel Nevada } \\ \text {-ROJECT ID: } & \text { V653 } \\ \text { PROJECT \#: } & 17777\end{array}$

CLIENT ID: $\quad$ TAML - VER - 24

DATE SAMPLED: 6/28/99

TEST: TCLP-8 Metals

MATRIX: Solid

\begin{tabular}{|c|c|c|c|c|c|c|c|}
\hline PARAMETER & $\begin{array}{c}\text { RESULT } \\
\mathrm{mg} / \mathrm{L} \\
\end{array}$ & $\begin{array}{c}\text { REPORTING } \\
\text { LIMIT } \\
\end{array}$ & D. F. & METHOD & $\begin{array}{c}\text { TCLPISTLC } \\
\text { EXTRACTION } \\
\text { DATE } \\
\end{array}$ & DIGESTED & ANALYZED \\
\hline Irsenic & $\mathrm{ND}$ & $0.1 \mathrm{mg} / \mathrm{L}$ & 1 & EPA 6010 & $7 / 8 / 99$ & 7/9/99 & $7 / 12 / 99$ \\
\hline Jarium & $\mathrm{ND}$ & 1. $\mathrm{mg} / \mathrm{L}$ & 1 & EPA 6010 & $7 / 8 / 99$ & $7 / 9 / 99$ & $7 / 12 / 99$ \\
\hline Cadmium & $\mathrm{ND}$ & $0.01 \mathrm{mg} / \mathrm{L}$ & 1 & EPA 6010 & $7 / 8 / 99$ & $7 / 9 / 99$ & $7 / 12 / 99$ \\
\hline 'hromium & ND & $0.01 \mathrm{mg} / \mathrm{L}$ & 1 & EPA 6010 & $7 / 8 / 99$ & 7/9/99 & $7 / 12 / 99$ \\
\hline ead & ND & $0.05 \mathrm{mg} / \mathrm{L}$ & 1 & EPA 6010 & $7 / 8 / 99$ & $7 / 9 / 99$ & $7 / 12 / 99$ \\
\hline Mercury & ND & $0.002 \mathrm{mg} / \mathrm{L}$ & 10 & EPA $7470 A$ & $7 / 8 / 99$ & $7 / 11 / 99$ & $7 / 11 / 99$ \\
\hline elenium & ND & $0.1 \mathrm{mg} / \mathrm{L}$ & 1 & EPA 6010 & $7 / 8 / 99$ & $7 / 9 / 99$ & $7 / 12 / 99$ \\
\hline ilver & $\mathrm{ND}$ & $0.02 \mathrm{mg} / \mathrm{L}$ & 1 & EPA 6010 & $7 / 8 / 99$ & $7 / 9 / 99$ & $7 / 12 / 99$ \\
\hline
\end{tabular}

I F. - Dilution Factor

ND - Not Detected

is is report shall not be reproduced except in full, without the written approval of the laboratory. 


$\begin{array}{ll}\text { CLIENT: } & \text { Bechtel Nevada } \\ \text { PROJECT ID: } & \text { V653 } \\ \text { PROJECT \#: } & 17777 \\ & \\ \text { TEST: } & \text { TCLP-8 Metals } \\ \text { MATRIX: } & \text { Solid }\end{array}$

MATRIX: Solid

\begin{tabular}{|c|c|c|c|c|c|c|c|}
\hline PARAMETER & $\begin{array}{c}\text { RESULT } \\
\mathrm{mg} / \mathrm{L} \\
\end{array}$ & $\begin{array}{c}\text { REPORTING } \\
\text { LIMIT } \\
\end{array}$ & D. F. & METHOD & $\begin{array}{c}\text { TCLP/STLC } \\
\text { EXTRACTION } \\
\text { DATE } \\
\end{array}$ & DIGESTED & ANALYZED \\
\hline Arsenic & $\mathrm{ND}$ & $0.1 \mathrm{mg} / \mathrm{L}$ & 1 & EPA 6010 & $7 / 8 / 99$ & 7/9/99 & $7 / 12 / 99$ \\
\hline Barium & ND & 1. $\mathrm{mg} / \mathrm{L}$ & 1 & EPA 6010 & $7 / 8 / 99$ & $7 / 9 / 99$ & $7 / 12 / 99$ \\
\hline Cadmium & ND & $0.01 \mathrm{mg} / \mathrm{L}$ & 1 & EPA 6010 & $7 / 8 / 99$ & 7/9/99 & $7 / 12 / 99$ \\
\hline Chromium & ND & $0.01 \mathrm{mg} / \mathrm{L}$ & 1 & EPA 6010 & $7 / 8 / 99$ & $7 / 9 / 99$ & $7 / 12 / 99$ \\
\hline Lead & ND & $0.05 \mathrm{mg} / \mathrm{L}$ & 1 & EPA 6010 & $7 / 8 / 99$ & $7 / 9 / 99$ & $7 / 12 / 99$ \\
\hline Mercury & ND & $0.002 \mathrm{mg} / \mathrm{L}$ & 10 & EPA 7470A & $7 / 8 / 99$ & 7/11/99 & $7 / 11 / 99$ \\
\hline Selenium & ND & $0.1 \mathrm{mg} / \mathrm{L}$ & 1 & EPA 6010 & $7 / 8 / 99$ & $7 / 9 / 99$ & $7 / 12 / 99$ \\
\hline Silver & $\mathrm{ND}$ & $0.02 \mathrm{mg} / \mathrm{L}$ & 1. & EPA 6010 & $7 / 8 / 99$ & 7/9/99 & $7 / 12 / 99$ \\
\hline
\end{tabular}

D.F. - Dilution Factor

ND - Not Detected

This report shall not be reproduced except in full, without the written approval of the laboratory. 
CLIENT: Bechtel Nevada

PROJECT ID: V653

PROJECT \#: 17777
CLIENT ID:

DATE SAMPLED: 6/28/99

NEL SAMPLE ID: L9907046-26

\section{TEST: $\quad$ TCLP-8 Metals \\ MATRIX: Solid}

\begin{tabular}{|c|c|c|c|c|c|c|c|}
\hline PARAMETER & $\begin{array}{c}\text { RESULT } \\
\mathrm{mg} / \mathrm{L} \\
\end{array}$ & $\begin{array}{c}\text { REPORTING } \\
\text { LIMIT } \\
\end{array}$ & D. F. & METHOD & $\begin{array}{c}\text { TCLP/STLC } \\
\text { EXTRACTION } \\
\text { DATE } \\
\end{array}$ & DIGESTED & ANALYZED \\
\hline Arsenic & ND & $0.1 \mathrm{mg} / \mathrm{L}$ & 1 & EPA 6010 & 7/8/99 & 7/9/99 & $7 / 12 / 99$ \\
\hline Barium & ND & 1. $\mathrm{mg} / \mathrm{L}$ & 1 & EPA 6010 & $7 / 8 / 99$ & 7/9/99 & $7 / 12 / 99$ \\
\hline Cadmium & ND & $0.01 \mathrm{mg} / \mathrm{L}$ & 1 & EPA 6010 & $7 / 8 / 99$ & $7 / 9 / 99$ & $7 / 12 / 99$ \\
\hline Shromium & ND & $0.01 \mathrm{mg} / \mathrm{L}$ & 1 & EPA 6010 & 7/8/99 & 7/9/99 & $7 / 12 / 99$ \\
\hline Lead & ND & $0.05 \mathrm{mg} / \mathrm{L}$ & 1 & EPA 6010 & $7 / 8 / 99$ & 7/9/99 & $7 / 12 / 99$ \\
\hline Mercury & ND & $0.002 \mathrm{mg} / \mathrm{L}$ & 10 & EPA 7470A & $7 / 8 / 99$ & $7 / 11 / 99$ & $7 / 11 / 99$ \\
\hline Selenium & ND & $0.1 \mathrm{mg} / \mathrm{L}$ & 1 & EPA 6010 & $7 / 8 / 99$ & $7 / 9 / 99$ & $7 / 12 / 99$ \\
\hline Silver & ND & $0.02 \mathrm{mg} / \mathrm{L}$ & 1 & EPA 6010 & 7/8/99 & 7/9/99 & $7 / 12 / 99$ \\
\hline
\end{tabular}

I.F. - Dilution Factor

ND - Not Detected

his report shall not be reproduced except in full, without the written approval of the laboratony. 


\begin{tabular}{|c|c|c|}
\hline CLIENT: & Bechtel Nevada & CLIENT ID: $\quad$ TAML - VER - 27 \\
\hline PROJECT ID: & V653 & DATE SAMPLED: $6 / 28 / 99$ \\
\hline PROJECT \#: & 17777 & NEL SAMPLE ID: L9907046-27 \\
\hline
\end{tabular}

TEST: $\quad$ TCLP-8 Metals

MATRIX: Solid

\begin{tabular}{|c|c|c|c|c|c|c|c|}
\hline PARAMETER & $\begin{array}{c}\text { RESULT } \\
\mathrm{mg} / \mathrm{L} \\
\end{array}$ & $\begin{array}{c}\text { REPORTING } \\
\text { LIMIT } \\
\end{array}$ & D. F. & METHOD & $\begin{array}{c}\text { TCLP/STLC } \\
\text { EXTRACTION } \\
\text { DATE } \\
\end{array}$ & DIGESTED & ANALYZED \\
\hline Arsenic & ND & $0.1 \mathrm{mg} / \mathrm{L}$ & 1 & EPA 6010 & 7/8/99 & 7/9/99 & $7 / 12 / 99$ \\
\hline Barium & ND & 1. $\mathrm{mg} / \mathrm{L}$ & 1 & EPA 6010 & 7/8/99 & 7/9/99 & $7 / 12 / 99$ \\
\hline Cadmium & ND & $0.01 \mathrm{mg} / \mathrm{L}$ & 1 & EPA 6010 & $7 / 8 / 99$ & 7/9/99 & $7 / 12 / 99$ \\
\hline Chromium & ND & $0.01 \mathrm{mg} / \mathrm{L}$ & 1 & EPA 6010 & 7/8/99 & 7/9/99 & $7 / 12 / 99$ \\
\hline Lead & $\mathrm{ND}$ & $0.05 \mathrm{mg} / \mathrm{L}$ & 1 & EPA 6010 & $7 / 8 / 99$ & $7 / 9 / 99$ & $7 / 12 / 99$ \\
\hline Mercury & ND & $0.002 \mathrm{mg} / \mathrm{L}$ & 10 & EPA 7470A & $7 / 8 / 99$ & $7 / 11 / 99$ & $7 / 11 / 99$ \\
\hline Selenium & ND & $0.1 \mathrm{mg} / \mathrm{L}$ & 1 & EPA 6010 & 7/8/99 & $7 / 9 / 99$ & $7 / 12 / 99$ \\
\hline Silver & ND & $0.02 \mathrm{mg} / \mathrm{L}$ & 1 & EPA 6010 & $7 / 8 / 99$ & $7 / 9 / 99$ & $7 / 12 / 99$ \\
\hline
\end{tabular}

D.F. - Dilution Factor

ND - Not Detected

This report shall not be reproduced except in full, without the written approval of the laboratory. 


\begin{tabular}{|c|c|c|c|c|c|c|c|}
\hline $\begin{array}{l}\text { CLIENT: } \\
\text { PROJECT ID: } \\
\text { PROJECT \#: }\end{array}$ & $\begin{array}{l}\text { Bechtel Nevada } \\
\text { V653 } \\
17777\end{array}$ & 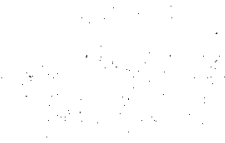 & & \multicolumn{3}{|c|}{$\begin{array}{ll}\text { CLIENT ID: } & \text { TAML - VER - } 28 \\
\text { DATE SAMPLED: } & \text { 6/28/99 } \\
\text { NEL SAMPLE ID: } & \text { L9907046-28 }\end{array}$} & \\
\hline $\begin{array}{l}\text { IEST: } \\
\text { MATRIX: }\end{array}$ & $\begin{array}{l}\text { TCLP-8 Metals } \\
\text { Solid }\end{array}$ & & & 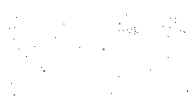 & & & \\
\hline PARAMETER & $\begin{array}{c}\text { RESULT } \\
\mathrm{mg} / \mathbf{L} \\
\end{array}$ & $\begin{array}{c}\text { REPORTING } \\
\text { LIMIT } \\
\end{array}$ & D. F. & METHOD & $\begin{array}{c}\text { TCLP/STLC } \\
\text { EXTRACTION } \\
\text { DATE } \\
\end{array}$ & DIGESTED & ANALYZED \\
\hline Arsenic & ND & $0.1 \mathrm{mg} / \mathrm{L}$ & 1 & EPA 6010 & 7/8/99 & 7/9/99 & $7 / 12 / 99$ \\
\hline 3arium & $\mathrm{ND}$ & 1. $\mathrm{mg} / \mathrm{L}$ & 1 & EPA 6010 & $7 / 8 / 99$ & 7/9/99 & $7 / 12 / 99$ \\
\hline Cadmium & ND & $0.01 \mathrm{mg} / \mathrm{L}$ & 1 & EPA 6010 & $7 / 8 / 99$ & 7/9/99 & $7 / 12 / 99$ \\
\hline 'hromium & $\mathrm{ND}$ & $0.01 \mathrm{mg} / \mathrm{L}$ & 1 & EPA 6010 & $7 / 8 / 99$. & 7/9/99 & $7 / 12 / 99$ \\
\hline ead & $\mathrm{ND}$ & $0.05 \mathrm{mg} / \mathrm{L}$ & 1 & EPA 6010 & $7 / 8 / 99$ & $7 / 9 / 99$ & $7 / 12 / 99$ \\
\hline Mercury & 0.0021 & $0.002 \mathrm{mg} / \mathrm{L}$ & 10 & EPA $7470 A$ & $7 / 8 / 99$ & $7 / 11 / 99$ & $7 / 11 / 99$ \\
\hline glenium & $\mathrm{ND}$ & $0.1 \mathrm{mg} / \mathrm{L}$ & 1 & EPA 6010 & $7 / 8 / 99$ & 7/9/99 & $7 / 12 / 99$ \\
\hline ilver & $\mathrm{ND}$ & $0.02 \mathrm{mg} / \mathrm{L}$ & 1 & EPA 6010 & $7 / 8 / 99$ & 7/9/99 & $7 / 12 / 99$ \\
\hline
\end{tabular}

I. 7. - Dilution Factor.

ND - Not Detected

7 is report shall not be reproduced except in full, without the written approval of the laboratory. 


$\begin{array}{llll}\text { CLIENT: } & \text { Bechtel Nevada } & \text { CLIENT ID: } & \text { Method Blank } \\ \text { PROJECT ID: } & \text { V653 } & \text { DATE SAMPLED: NA } \\ \text { PROJECT \#: } & 17777 & \text { NEL SAMPLE ID: } & \text { L07046-15-Hg-BLK }\end{array}$

TEST: $\quad$ TCLP by EPA 1311, July 1992 \& Mercury by EPA 7470A, July 1992 MATRIX: $\quad$ TCLP EXtract

\begin{tabular}{|c|c|c|c|c|c|c|c|}
\hline \multirow[b]{2}{*}{ PARAMETER } & \multirow{2}{*}{\multicolumn{2}{|c|}{$\begin{array}{l}\text { REPORTING } \\
\text { LIMIT }\end{array}$}} & \multicolumn{4}{|c|}{$\begin{array}{c}\text { TCLP/STLC } \\
\text { EXTRACTION }\end{array}$} & \\
\hline & & & D. F. & METHOD & DATE & DIGESTED & ANALYZED \\
\hline Mercury & ND & $0.002 \mathrm{mg} / \mathrm{L}$ & 10 & EPA 7470A & 7/8/99 & 7/11/99 & 7/11/99 \\
\hline
\end{tabular}

D.F. - Dilution Factor

ND - Not Detected

This report shall not be reproduced except in full, without the written approval of the laboratory. 


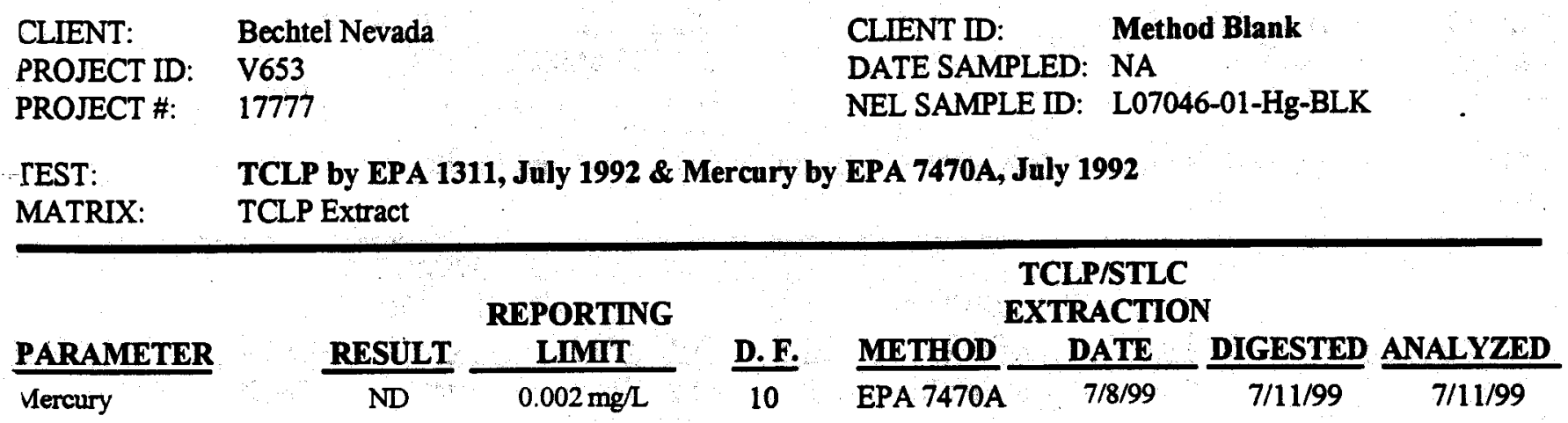

3.F. - Dilution Factor

ND - Not Detected

- his report shall not be reproduced except in full, without the written approval of the laboratory.

60 


$\begin{array}{llll}\text { CLIENT: } & \text { Bechtel Nevada } & \text { CLIENT ID: } & \text { Method Blank } \\ \text { PROJECT ID: } & \text { V653 } & \text { DATE SAMPLED: NA } \\ \text { PROJECT \#: } & 17777 & \text { NEL SAMPLE ID: } & \text { L07046-15I-BLK }\end{array}$

TEST: $\quad$ TCLP by EPA 1311, July 1992 \& 7 Metals by EPA 6010A, July 1992 MATRIX: $\quad$ TCLP EXtract

\begin{tabular}{|c|c|c|c|c|c|c|c|}
\hline \multirow[b]{2}{*}{ PARAMETER } & \multirow[b]{2}{*}{ RESULT } & \multirow{2}{*}{$\begin{array}{l}\text { REPORTING } \\
\text { LIMIT } \\
\end{array}$} & \multicolumn{5}{|c|}{$\begin{array}{l}\text { TCLP/STLC } \\
\text { EXTRACTION }\end{array}$} \\
\hline & & & D. F. & METHOD & DATE & DIGESTED & ANALYZED \\
\hline Arsenic & $\mathrm{ND}$ & $0.1 \mathrm{mg} / \mathrm{L}$ & 1 & EPA 6010 & $7 / 8 / 99$ & $7 / 9 / 99$ & $7 / 12 / 99$ \\
\hline Barium & ND & $1 \mathrm{mg} / \mathrm{L}$ & 1 & EPA 6010 & $7 / 8 / 99$ & 7/9/99 & $7 / 12 / 99$ \\
\hline Cadmium & $\mathrm{ND}$ & $0.01 \mathrm{mg} / \mathrm{L}$ & 1 & EPA 6010 & $7 / 8 / 99$ & 7/9/99 & $7 / 12 / 99$ \\
\hline Chromium & ND & $0.01 \mathrm{mg} / \mathrm{L}$ & 1 & EPA 6010 & $7 / 8 / 99$ & $7 / 9 / 99$ & $7 / 12 / 99$ \\
\hline Lead & ND & $0.05 \mathrm{mg} / \mathrm{L}$ & 1 & EPA 6010 & $7 / 8 / 99$ & 7/9/99 & $7 / 12 / 99$ \\
\hline Selenium & $\mathrm{ND}$ & $0.1 \mathrm{mg} / \mathrm{L}$ & 1 & EPA 6010 & $7 / 8 / 99$ & 7/9/99 & $7 / 12 / 99$ \\
\hline Silver & $\mathrm{ND}$ & $0.02 \mathrm{mg} / \mathrm{L}$ & 1 & EPA 6010 & $7 / 8 / 99$ & $7 / 9 / 99$ & $7 / 12 / 99$ \\
\hline
\end{tabular}

D.F. - Dilution Factor

ND - Not Detected

This report shall not be reproduced except in full, without the written approval of the laboratory. 


\begin{tabular}{|c|c|c|c|}
\hline $\begin{array}{l}\text { CLIENT: } \\
\text { PROJECT ID: } \\
\text { PROJECT \#: }\end{array}$ & $\begin{array}{l}\text { Bechtel Nevada } \\
\text { V653 } \\
17777\end{array}$ & $\begin{array}{l}\text { CLIENT ID: } \\
\text { DATE SAMPLED: } \\
\text { NEL SAMPLE ID: }\end{array}$ & $\begin{array}{l}\text { Method Blank } \\
\text { NA } \\
\text { L07046I-BLK }\end{array}$ \\
\hline
\end{tabular}

TEST: $\quad$ TCLP by EPA 1311, July 1992 \& 7 Metals by EPA 6010A, July 1992

MATRIX: TCLP Extract

\begin{tabular}{|c|c|c|c|c|c|c|c|}
\hline \multirow[b]{2}{*}{ PARAMETER } & \multirow[b]{2}{*}{ RESULT } & \multirow{2}{*}{$\begin{array}{l}\text { REPORTING } \\
\text { LMMT }\end{array}$} & \multicolumn{5}{|c|}{$\begin{array}{l}\text { TCLP/STLC } \\
\text { EXTRACTION }\end{array}$} \\
\hline & & & D. F. & METHOD & DATE & DIGESTED & ANAL YZED \\
\hline Arsenic & $\mathrm{ND}$ & $0.1 \mathrm{mg} / \mathrm{L}$ & 1 & EPA 6010 & $7 / 8 / 99$ & $.7 / 9 / 99$ & $7 / 12 / 99$ \\
\hline Barium & ND & $1 \mathrm{mg} / \mathrm{L}$ & 1 & EPA 6010 & $7 / 8 / 99$ & 7/9/99 & $7 / 12 / 99$ \\
\hline Cadmium & $\mathrm{ND}$ & $0.01 \mathrm{mg} / \mathrm{L}$ & 1 & EPA 6010 & $7 / 8 / 99$ & $7 / 9 / 99$ & $7 / 12 / 99$ \\
\hline Thromium & ND & $0.01 \mathrm{mg} / \mathrm{L}$ & 1 & EPA 6010 & $7 / 8 / 99$ & $7 / 9 / 99$ & $7 / 12 / 99$ \\
\hline Lead & ND & $0.05 \mathrm{mg} / \mathrm{L}$ & 1 & EPA 6010 & $7 / 8 / 99$ & $7 / 9 / 99$ & $7 / 12 / 99$ \\
\hline Selenium & $\mathrm{ND}$ & $0.1 \mathrm{mg} / \mathrm{L}$ & 1 & EPA 6010 & $7 / 8 / 99$ & 7/9/99 & $7 / 12 / 99$ \\
\hline Silver & ND & $0.02 \mathrm{mg} / \mathrm{L}$ & 1 & EPA 6010 & 7/8/99 & $7 / 9 / 99$ & $7 / 12 / 99$ \\
\hline
\end{tabular}

\section{I.F. - Dilution Factor \\ IV - Not Detected}

This report shall not be reproduced except in full, without the written approval of the laboratory. 


$\begin{array}{llll}\text { CLIENT: } & \text { Bechtel Nevada } & \text { CLIENT ID: } & \text { TAML - VER - 01 } \\ \text { PROJECT ID: } & \text { V653 } & \text { DATE SAMPLED: } & \text { 6/28/99 } \\ \text { PROJECT \#: } & 17777 & \text { NEL SAMPLE ID: } & \text { L9907046-01 } \\ & & & \\ \text { TEST: } & \text { TCLP by EPA 1311, July } 1992 & \text { \& Semivolatile Organics by EPA Method 8270C, Dec. } 1996 \\ \text { METHOD: } & \text { EPA } 8270 & \text { TCLP EXTRACT DATE: } & 7 / 8 / 99 \\ \text { MATRIX: } & \text { Solid } & \text { EXTRACTED } & 7 / 9 / 99 \\ \text { DILUTION: } & 1 & \text { ANALYZED: } & 7 / 17 / 99\end{array}$

\section{PARAMETER}

1,4-Dichlorobenzene (p-DCB)

2,4-Dinitrotoluene (DNT)

Hexachlorobenzene

Hexachlorobutadiene

Hexachloroethane

2-Methylphenol

3,4-Methylphenol (isomeric pair)

Nitrobenzene

Pentachlomphenol

Pyridine

2,4,5-Trichlorophenol

2,4,6-Trichlorophenol

\begin{tabular}{c}
$\begin{array}{c}\text { Result } \\
\mathbf{m g} / \mathbf{L}\end{array}$ \\
\hline $\mathrm{ND}$ \\
$\mathrm{ND}$ \\
$\mathrm{ND}$ \\
$\mathrm{ND}$ \\
$\mathrm{ND}$ \\
$\mathrm{ND}$ \\
$\mathrm{ND}$ \\
$\mathrm{ND}$ \\
$\mathrm{ND}$ \\
$\mathrm{ND}$ \\
$\mathrm{ND}$ \\
$\mathrm{ND}$
\end{tabular}

Reporting

Limit

$0.1 \mathrm{mg} / \mathrm{L}$

$0.1 \mathrm{mg} / \mathrm{L}$

$0.1 \mathrm{mg} / \mathrm{L}$

$0.1 \mathrm{mg} / \mathrm{L}$

$0.1 \mathrm{mg} / \mathrm{L}$

$0.1 \mathrm{mg} / \mathrm{L}$

$0.1 \mathrm{mg} / \mathrm{L}$

$0.1 \mathrm{mg} / \mathrm{L}$

$0.1 \mathrm{mg} / \mathrm{L}$

$0.1 \mathrm{mg} / \mathrm{L}$

$0.1 \mathrm{mg} / \mathrm{L}$

$0.1 \mathrm{mg} / \mathrm{L}$

\section{QUALITY CONTROL DATA:}

Surrogate

2,4,6-Tribromophenol

2-Fluorobiphenyl

2-Fluorophenol

Nitrobenzene-d5

p-Terphenyl-d14

Phenol-d5
$\%$ Recovery

61

70

39

76

120

24

\section{Acceptable Range}

$10-123$

$43-116$

$21-100$

$35-114$

$33-141$

$10-94$

ND - Not Detected

This report shall not be reproduced except in full, without the written approval of the laboratory. 


$\begin{array}{llll}\text { CLIENT: } & \text { Bechtel Nevada } & \text { CLIENT ID: } & \text { TAMI - VER - 02 } \\ \text { PROJECT ID: } & \text { V653 } & \text { DATE SAMPLED: 6/28/99 } \\ \text { PROJECT \#: } & 17777 & \text { NEL SAMPLE ID: } & \text { L9907046-02 }\end{array}$

TEST:

TCLP by EPA 1311, July 1992 \& Semivolatile Organics by EPA Method 8270C, Dec 1996

METHOD: . EPA 8270

TCLP EXTRACT DATE: 7/8/99

MATRIX: $\quad$ Solid

EXTRACTED

$7 / 9 / 99$

DILUTION: $\quad 1$

ANALYZED:

$7 / 17 / 99$

\begin{tabular}{lcc}
\hline PARAMETER & $\begin{array}{c}\text { Result } \\
\mathbf{m g} / \mathrm{L}\end{array}$ & $\begin{array}{c}\text { Reporting } \\
\text { Limit }\end{array}$ \\
\cline { 2 - 3 } 1,4-Dichlorobenzene (p-DCB) & $\mathrm{ND}$ & $0.1 \mathrm{mg} / \mathrm{L}$ \\
2,4-Dinitrotoluene (DNT) & $\mathrm{ND}$ & $0.1 \mathrm{mg} / \mathrm{L}$ \\
Jexachlorobenzene & $\mathrm{ND}$ & $0.1 \mathrm{mg} / \mathrm{L}$ \\
fexachlorobutadiene & $\mathrm{ND}$ & $0.1 \mathrm{mg} / \mathrm{L}$ \\
Hexachloroethane & $\mathrm{ND}$ & $0.1 \mathrm{mg} / \mathrm{L}$ \\
?-Methylphenol & $\mathrm{ND}$ & $0.1 \mathrm{mg} / \mathrm{L}$ \\
3,4-Methylphenol (isomeric pair) & $\mathrm{ND}$ & $0.1 \mathrm{mg} / \mathrm{L}$ \\
Nitrobenzene & $\mathrm{ND}$ & $0.1 \mathrm{mg} / \mathrm{L}$ \\
Pentachlorophenol & $\mathrm{ND}$ & $0.1 \mathrm{mg} / \mathrm{L}$ \\
'yridine & $\mathrm{ND}$ & $0.1 \mathrm{mg} / \mathrm{L}$ \\
Z,4,5-Trichlorophenol & $\mathrm{ND}$ & $0.1 \mathrm{mg} / \mathrm{L}$ \\
2,4,6-Trichlorophenol & $\mathrm{ND}$ & $0.1 \mathrm{mg} / \mathrm{L}$
\end{tabular}

\footnotetext{
TULITY CONTROL DATA:

irrogate

2,4,6-Tribromophenol

-Fluorobiphenyl

-Fluorophenol

Nitrobenzene-d5

- Terphenyl-d14

renol-d5
}

\% Recovery

Acceptable Range

62

79

42

82

119

26
$10-123$

$43-116$

$21-100$

$35-114$

$33-141$

$10-94$

ND - Not Detected

is report shall not be reproduced except in full, without the written approval of the laboratory: 


$\begin{array}{llll}\text { CLIENT: } & \text { Bechtel Nevada } & \text { CLIENT ID: } & \text { TAML - VER - 03 } \\ \text { PROJECT ID: } & \text { V653 } & \text { DATE SAMPLED: } 6 / 28 / 99 \\ \text { PROJECT \#: } & 17777 & \text { NEL SAMPLE ID: } & \text { L9907046-03 }\end{array}$

TEST: $\quad$ TCLP by EPA 1311, July 1992 \& Semivolatile Organics by EPA Method 8270C, Dec. 1996

METHOD: $\quad$ EPA $8270 \quad$ TCLP EXTRACT DATE: $7 / 8 / 99$

MATRIX: Solid $\quad$ EXTRACTED $\quad 7 / 9 / 99$

$\begin{array}{llll}\text { DILUTION: } 1 & \text { ANALYZED: } & \text { 7/17/99 }\end{array}$

\section{PARAMETER}

1,4-Dichlorobenzene (p-DCB)

2,4-Dinitrotoluene (DNT)

Hexachlorobenzene

Hexachlorobutadiene

Hexachloroethane

2-Methylphenol

3,4-Methylphenol (isomeric pair)

Nitrobenzene

Pentachlorophenol

Pyridine

2,4,5-Trichlorophenol

2,4,6-Trichlorophenol

\begin{tabular}{c}
$\begin{array}{c}\text { Result } \\
\text { mg/L }\end{array}$ \\
\hline $\mathrm{ND}$ \\
$\mathrm{ND}$ \\
$\mathrm{ND}$ \\
$\mathrm{ND}$ \\
$\mathrm{ND}$ \\
$\mathrm{ND}$ \\
$\mathrm{ND}$ \\
$\mathrm{ND}$ \\
$\mathrm{ND}$ \\
$\mathrm{ND}$ \\
$\mathrm{ND}$ \\
$\mathrm{ND}$
\end{tabular}

Reporting

Limit

$0.1 \mathrm{mg} / \mathrm{L}$

$0.1 \mathrm{mg} / \mathrm{L}$

$0.1 \mathrm{mg} / \mathrm{L}$

$0.1 \mathrm{mg} / \mathrm{L}$

$0.1 \mathrm{mg} / \mathrm{L}$

$0.1 \mathrm{mg} / \mathrm{L}$

$0.1 \mathrm{mg} / \mathrm{L}$

$0.1 \mathrm{mg} / \mathrm{L}$

$0.1 \mathrm{mg} / \mathrm{L}$

$0.1 \mathrm{mg} / \mathrm{L}$

$0.1 \mathrm{mg} / \mathrm{L}$

$0.1 \mathrm{mg} / \mathrm{L}$

QUALITY CONTROL DATA:

\section{Surrogate}

2,4,6-Tribromophenol

2-Fluorobiphenyl

2-Fluorophenol

Nitrobenzene-d5

p-Terphenyl-d14

Phenol-d5
\% Recovery

60

61

34

64

117

20

\section{Acceptable Range}

$10-123$

$43-116$

$21-100$

$35-114$

$33-141$

$10-94$

ND - Not Detected

This report shall not be reproduced except in full, without the written approval of the laboratory. 


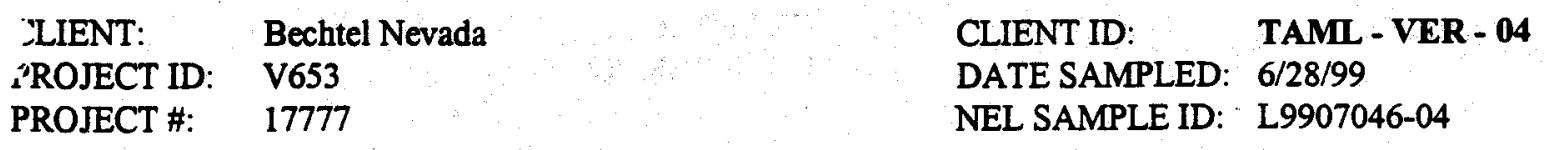

EST: $\quad$ TCLP by EPA 1311, July 1992 \& Semivolatile Organics by EPA Method 8270C, Dec. 1996

METHOD: $\quad$ EPA 8270

MATRIX: Solid

IILUTION: 1

TCLP EXTRACT DATE: $7 / 8 / 99$

EXTRACTED $\quad 7 / 9 / 99$

ANALYZED: $\quad 7 / 17 / 99$

\begin{tabular}{|c|c|c|}
\hline 'ARAMETER & $\begin{array}{c}\text { Result } \\
\mathrm{mg} / \mathrm{L}\end{array}$ & $\begin{array}{c}\text { Reporting } \\
\text { Limit }\end{array}$ \\
\hline , $\overline{4 \text { 4-Dichlorobenzene (p-DCB) }}$ & ND & $0.1 \mathrm{mg} / \mathrm{L}$ \\
\hline 2,4-Dinitrotoluene (DNT) & ND & $0.1 \mathrm{mg} / \mathrm{L}$ \\
\hline iexachlorobenzene & ND & $0.1 \mathrm{mg} / \mathrm{L}$ \\
\hline exachlorobutadiene & ND & $0.1 \mathrm{mg} / \mathrm{L}$ \\
\hline Hexachloroethane & ND & $0.1 \mathrm{mg} / \mathrm{L}$ \\
\hline -Methylphenol & ND & $0.1 \mathrm{mg} / \mathrm{L}$ \\
\hline 4-Methylphenol (isomeric pair) & ND & $0.1 \mathrm{mg} / \mathrm{L}$ \\
\hline Nitrobenzene & ND & $0.1 \mathrm{mg} / \mathrm{L}$ \\
\hline Dentachlorophenol & ND & $0.1 \mathrm{mg} / \mathrm{L}$ \\
\hline yridine & ND & $0.1 \mathrm{mg} / \mathrm{L}$ \\
\hline Z,4,5-Trichlorophenol & ND & $0.1 \mathrm{mg} / \mathrm{L}$ \\
\hline 2,4,6-Trichlorophenol & $\mathrm{ND}$ & $0.1 \mathrm{mg} / \mathrm{L}$ \\
\hline
\end{tabular}

"JALITY CONTROL DATA:

Irrogate

\% Recovery

Acceptable Range

2.4,6-Tribromophenol

66

84

47

91

117

28
$10-123$

$43-116$

$21-100$

$35-114$

$33-141$

$10-94$ 


$\begin{array}{llll}\text { CLIENT: } & \text { Bechtel Nevada } & \text { CLIENT ID: } & \text { TAML - VER - 05 } \\ \text { PROJECT ID: } & \text { V653 } & \text { DATE SAMPLED: } & 6 / 28 / 99 \\ \text { PROJECT \#: } & 17777 & \text { NEL SAMPLE ID: } & \text { L9907046-05 }\end{array}$

TEST: $\quad$ TCLP by EPA 1311, July 1992 \& Semivolatile Organics by EPA Method 8270C, Dec. 1996

METHOD: EPA $8270 \quad$ TCLP EXTRACT DATE: 7/8/99

MATRIX: Solid EXTRACTED $\quad$ T/9/99

$\begin{array}{llll}\text { DILUTION: } 1 & \text { ANALYZED: } & 7 / 17 / 99\end{array}$

\section{PARAMETER}

1,4-Dichlorobenzene (p-DCB)

2,4-Dinitrotoluene (DNT)

Hexachlorobenzene

Hexachlorobutadiene

Hexachloroethane

2-Methylphenol

3,4-Methylphenol (isomeric pair)

Nitrobenzene

Pentachlorophenol

Pyridine

2,4,5-Trichlorophenol

2,4,6-Trichlorophenol

\begin{tabular}{c}
$\begin{array}{c}\text { Result } \\
\mathbf{m g} / \mathbf{L}\end{array}$ \\
\hline $\mathrm{ND}$ \\
$\mathrm{ND}$ \\
$\mathrm{ND}$ \\
$\mathrm{ND}$ \\
$\mathrm{ND}$ \\
$\mathrm{ND}$ \\
$\mathrm{ND}$ \\
$\mathrm{ND}$ \\
$\mathrm{ND}$ \\
$\mathrm{ND}$ \\
$\mathrm{ND}$ \\
$\mathrm{ND}$
\end{tabular}

Reporting Limit

$0.1 \mathrm{mg} / \mathrm{L}$

$0.1 \mathrm{mg} / \mathrm{L}$

$0.1 \mathrm{mg} / \mathrm{L}$

$0.1 \mathrm{mg} / \mathrm{L}$

$0.1 \mathrm{mg} / \mathrm{L}$

$0.1 \mathrm{mg} / \mathrm{L}$

$0.1 \mathrm{mg} / \mathrm{L}$

$0.1 \mathrm{mg} / \mathrm{L}$

$0.1 \mathrm{mg} / \mathrm{L}$

$0.1 \mathrm{mg} / \mathrm{L}$

$0.1 \mathrm{mg} / \mathrm{L}$

$0.1 \mathrm{mg} / \mathrm{L}$

QUALITY CONTROL DATA:

\section{Surrogate}

2,4,6-Tribromophenol

2-Fluorobiphenyl

2-Fluorophenol

Nitrobenzene-d5

p-Terphenyl-d14

Phenol-d5
\% Recovery

61

72

41

78

120

25
Acceptable Range

$10-123$

$43-116$

$21-100$

$35-114$

$33-141$

$10-94$ 


$\begin{array}{llll}\text { CLIENT: } & \text { Bechtel Nevada } & \text { CLIENT ID: } & \text { TAML - VER - 06 } \\ \text { PROJECT ID: } & \text { V653 } & \text { DATE SAMPLED: } 6 / 28 / 99 \\ \text { PROJECT \#: } & 17777 & \text { NEL SAMPLE ID: } & \text { L9907046-06 }\end{array}$

TEST: $\quad$ TCLP by EPA 1311, July 1992 \& Semivolatile Organies by EPA Method 8270C, Dec. 1996

METHOD: $\quad$ EPA $8270 \quad$ TCLP EXTRACT DATE: $7 / 8 / 99$

MATRIX: Solid $\quad$ EXTRACTED $\quad 7 / 9 / 99$

$\begin{array}{llll}\text { DILUTION: } & 1 & \text { ANALYZED: } & 7 / 18 / 99\end{array}$

\begin{tabular}{|c|c|c|}
\hline PARAMETER & $\begin{array}{c}\text { Result } \\
\text { mg/L }\end{array}$ & $\begin{array}{c}\text { Reporting } \\
\text { Limit }\end{array}$ \\
\hline 1,4-Dichlorobenzene (p-DCB) & $\mathrm{ND}$ & $\overline{0.1 \mathrm{mg} / \mathrm{L}}$ \\
\hline 2,4-Dinitrotoluene (DNT) & ND & $0.1 \mathrm{mg} / \mathrm{L}$ \\
\hline Hexachlorobenzene & ND & $0.1 \mathrm{mg} / \mathrm{L}$ \\
\hline Aexachlorobutadiene & ND & $0.1 \mathrm{mg} / \mathrm{L}$ \\
\hline Hexachloroethane & ND & $0.1 \mathrm{mg} / \mathrm{L}$ \\
\hline 2-Methylphenol & ND & $0.1 \mathrm{mg} / \mathrm{L}$ \\
\hline 3,4-Methylphenol (isomeric pair) & $\mathrm{ND}$ & $0.1 \mathrm{mg} / \mathrm{L}$ \\
\hline Nitrobenzene & ND & $0.1 \mathrm{mg} / \mathrm{L}$ \\
\hline Pentachlorophenol & ND & $0.1 \mathrm{mg} / \mathrm{L}$ \\
\hline 2yridine & ND & $0.1 \mathrm{mg} / \mathrm{L}$ \\
\hline 2,4,5-Trichlorophenol & ND & $0.1 \mathrm{mg} / \mathrm{L}$ \\
\hline 2,4,6-Trichlorophenol & ND & $0.1 \mathrm{mg} / \mathrm{L}$ \\
\hline
\end{tabular}

?UALITY CONTROL DATA:

urrogate

2,4.6-Tribromophenol

-Fluorobiphenyl

-Fluorophenol

Nitrobenzene-d5

-Terphenyl-d14

henol-d5
\% Recovery

62

65

35

67

123

24
Acceptable Range

$10-123$

$43-116$

$21-100$

$35-114$

$33-141$

$10-94$

ND - Not Detected

is report shall not be reproduced except in full, without the written approval of the laboratory. 


$\begin{array}{llll}\text { CLIENT: } & \text { Bechtel Nevada } & \text { CLIENT ID: } & \text { TAML - VER - 07 } \\ \text { PROJECT ID: } & \text { V653 } & \text { DATE SAMPLED: } & 6 / 28 / 99 \\ \text { PROJECT \#: } & 17777 & \text { NEL SAMPLE ID: } & \text { L9907046-07 }\end{array}$

TEST: $\quad$ TCLP by EPA 1311, July 1992 \& Semivolatile Organics by EPA Method 8270C, Dec. 1996

METHOD: EPA $8270 \quad$ TCLP EXTRACT DATE: $7 / 8 / 99$

MATRIX: $\quad$ Solid $\quad$ EXTRACTED $\quad 7 / 9 / 99$

$\begin{array}{llll}\text { DILUTION: } & 1 & \text { ANALYZED: } & 7 / 18 / 99\end{array}$

\section{PARAMETER}

1,4-Dichlorobenzene (p-DCB)

2,4-Dinitrotoluene (DNT)

Hexachlorobenzene

Hexachlorobutadiene.

Hexachloroethane

2-Methylphenol

3,4-Methyiphenol (isomeric pair)

Nitrobenzene

Pentachlorophenol

Pyridine

2,4,5-Trichlorophenol

2,4,6-Trichlorophenol

\begin{tabular}{c}
$\begin{array}{c}\text { Result } \\
\mathbf{m g} / \mathbf{L}\end{array}$ \\
\hline $\mathrm{ND}$ \\
$\mathrm{ND}$ \\
$\mathrm{ND}$ \\
$\mathrm{ND}$ \\
$\mathrm{ND}$ \\
$\mathrm{ND}$ \\
$\mathrm{ND}$ \\
$\mathrm{ND}$ \\
$\mathrm{ND}$ \\
$\mathrm{ND}$ \\
$\mathrm{ND}$ \\
$\mathrm{ND}$
\end{tabular}

Reporting

Limit

$0.1 \mathrm{mg} / \mathrm{L}$

$0.1 \mathrm{mg} / \mathrm{L}$

$0.1 \mathrm{mg} / \mathrm{L}$

$0.1 \mathrm{mg} / \mathrm{L}$

$0.1 \mathrm{mg} / \mathrm{L}$

$0.1 \mathrm{mg} / \mathrm{L}$

$0.1 \mathrm{mg} / \mathrm{L}$

$0.1 \mathrm{mg} / \mathrm{L}$

$0.1 \mathrm{mg} / \mathrm{L}$

$0.1 \mathrm{mg} / \mathrm{L}$

$0.1 \mathrm{mg} / \mathrm{L}$

$0.1 \mathrm{mg} / \mathrm{L}$

QUALITY CONTROL DATA:

\section{Surrogate}

2,4,6-Tribromophenol

2-Fluorobiphenyl

2-Fluorophenol

Nitrobenzene-d5

p-Terphenyl-d14

Phenol-d5

\section{$\%$ Recovery}

61

70

37

73

121

26

\section{Acceptable Range}

$10-123$

$43-116$

$21-100$

$35-114$

$33-141$

10- 94 


$\begin{array}{llll}\text { CLIENT: } & \text { Bechtel Nevada } & \text { CLIENT ID: } & \text { TAML - VER - 08 } \\ \text { PROJECT ID: } & \text { V653 } & \text { DATE SAMPLED: } 6 / 28 / 99 \\ \text { PROJECT \#: } & 17777 & \text { NEL SAMPLE ID: } & \text { L9907046-08 }\end{array}$

TEST: $\quad$ TCLP by EPA 1311, July 1992 \& Semivolatile Organics by EPA Method 8270C, Dec. 1996

METHOD: EPA $8270 \quad$ TCLP EXTRACT DATE: 7/8/99

MATRIX: Solid $\quad$ EXTRACTED $\quad 7 / 9 / 99$

ANALYZED: $\quad 7 / 18 / 99$

\section{PARAMETER}

1,4-Dichlorobenzene (p-DCB)

2,4-Dinitrotoluene (DNT)

.Jexachlorobenzene

Texachlorobutadiene

Hexachloroethane

?-Methylphenol

,4-Methyiphenol (isomeric pair)

Nitrobenzene

Pentachlorophenol

'yridine

2,4,5-Trichlorophenol

2,4,6-Trichlorophenol

\begin{tabular}{c}
$\begin{array}{c}\text { Result } \\
\text { mg/L }\end{array}$ \\
\hline $\mathrm{ND}$ \\
$\mathrm{ND}$ \\
$\mathrm{ND}$ \\
$\mathrm{ND}$ \\
$\mathrm{ND}$ \\
$\mathrm{ND}$ \\
$\mathrm{ND}$ \\
$\mathrm{ND}$ \\
$\mathrm{ND}$ \\
$\mathrm{ND}$ \\
$\mathrm{ND}$ \\
$\mathrm{ND}$
\end{tabular}

Reporting

Limit

$0.1 \mathrm{mg} / \mathrm{L}$

$0.1 \mathrm{mg} / \mathrm{L}$

$0.1 \mathrm{mg} / \mathrm{L}$

$0.1 \mathrm{mg} / \mathrm{L}$

$0.1 \mathrm{mg} / \mathrm{L}$

$0.1 \mathrm{mg} / \mathrm{L}$

$0.1 \mathrm{mg} / \mathrm{L}$

$0.1 \mathrm{mg} / \mathrm{L}$

$0.1 \mathrm{mg} / \mathrm{L}$

$0.1 \mathrm{mg} / \mathrm{L}$

$0.1 \mathrm{mg} / \mathrm{L}$

$0.1 \mathrm{mg} / \mathrm{L}$

TUALITY CONTROL DATA:

urrogate

2,4,6-Tribromophenol

-Fluorobiphenyl

-Fluorophenol

Nitrobenzene-d5

Terphenyl-d14

benol-d5
$\%$ Recovery

57

71

37

72

124

25
Acceptable Range

$10-123$

$43-116$

$21-100$

$35-114$

$33-141$

$10-94$

ND - Not Detected

is report shall not be reproduced except in full, without the written approval of the laboratory. 


$\begin{array}{llll}\text { CLIENT: } & \text { Bechtel Nevada } & \text { CLIENT ID: } & \text { TAML - VER - 09 } \\ \text { PROJECT ID: } & \text { V653 } & \text { DATE SAMPLED: } 6 / 28 / 99 \\ \text { PROJECT \#: } & 17777 & \text { NEL SAMPLE ID: } & \text { L9907046-09 }\end{array}$

TEST: $\quad$ TCLP by EPA 1311, July 1992 \& Semivolatile Organics by EPA Method 8270C, Dec. 1996

METHOD: EPA 8270

MATRIX: Solid TCLP EXTRACT DATE: 7/8/99

DILUTION: : 1

EXTRACTED 7/9/99

ANALYZED: $\quad 7 / 18 / 99$

\section{PARAMETER}

1,4-Dichlorobenzene (p-DCB)

2,4-Dinitrotoluene (DNT)

Hexachlorobenzene

Hexachlorobutadiene

Hexachloroethane

2-Methylphenol

3,4-Methylphenol (isomeric pair)

Nitrobenzene

Pentachlorophenol

Pyridine

2,4,5-Trichlorophenol

2,4,6-Trichlorophenol

\begin{tabular}{c}
$\begin{array}{c}\text { Result } \\
\mathbf{m g} / \mathbf{L}\end{array}$ \\
\hline $\mathrm{ND}$ \\
$\mathrm{ND}$ \\
$\mathrm{ND}$ \\
$\mathrm{ND}$ \\
$\mathrm{ND}$ \\
$\mathrm{ND}$ \\
$\mathrm{ND}$ \\
$\mathrm{ND}$ \\
$\mathrm{ND}$ \\
$\mathrm{ND}$ \\
$\mathrm{ND}$ \\
$\mathrm{ND}$
\end{tabular}

Reporting

Limit

$0.1 \mathrm{mg} / \mathrm{L}$

$0.1 \mathrm{mg} / \mathrm{L}$

$0.1 \mathrm{mg} / \mathrm{L}$

$0.1 \mathrm{mg} / \mathrm{L}$

$0.1 \mathrm{mg} / \mathrm{L}$

$0.1 \mathrm{mg} / \mathrm{L}$

$0.1 \mathrm{mg} / \mathrm{L}$

$0.1 \mathrm{mg} / \mathrm{L}$

$0.1 \mathrm{mg} / \mathrm{L}$

$0.1 \mathrm{mg} / \mathrm{L}$

$0.1 \mathrm{mg} / \mathrm{L}$

$0.1 \mathrm{mg} / \mathrm{L}$

QUALITY CONTROL DATA:

\section{Surrogate}

2,4,6-Tribromophenol

2-Fluorobiphenyl

2-Fluorophenol

Nitrobenzene-d5

p-Terphenyl-d14

Phenol-d5
\% Recovery

63

77

41

81

107

28

\section{Acceptable Range}

$10-123$

$43-116$

$21-100$

$35-114$

$33-141$

$10-94$

ND - Not Detected

This report shall not be reproduced except in full, without the written approval of the laboraton. 


$\begin{array}{llll}\text { CLIENT: } & \text { Bechtel Nevada } & \text { CLIENT ID: } & \text { TAML - VER - 10 } \\ \text { PROJECT ID: } & \text { V653 } & \text { DATE SAMPLED: } 6 / 28 / 99 \\ \text { PROJECT \#: } & 17777 & \text { NEL SAMPLE ID: } & \text { L9907046-10 }\end{array}$

IEST: $\quad$ TCLP by EPA 1311, July 1992 \& Semivolatile Organics by EPA Method 8270C, Dec. 1996

METHOD: EPA $8270 \quad$ TCLP EXTRACT DATE: $7 / 8 / 99$

MATRIX: $\quad$ Solid $\quad$ EXTRACTED $\quad 7 / 9 / 99$

OILUTION: $1 \quad$ ANALYZED: $\quad 7 / 18 / 99$

\begin{tabular}{lcc}
\hline & $\begin{array}{c}\text { Result } \\
\text { mg/L }\end{array}$ & $\begin{array}{c}\text { Reporting } \\
\text { Limit }\end{array}$ \\
\cline { 2 - 3 } :ARAMETER & $\mathrm{ND}$ & $0.1 \mathrm{mg} / \mathrm{L}$ \\
2,4-Dichlorobenzene (p-DCB) & $\mathrm{ND}$ & $0.1 \mathrm{mg} / \mathrm{L}$ \\
lexachiorobenzene & $\mathrm{ND}$ & $0.1 \mathrm{mg} / \mathrm{L}$ \\
Jexachlorobutadiene & $\mathrm{ND}$ & $0.1 \mathrm{mg} / \mathrm{L}$ \\
Hexachloroethane & $\mathrm{ND}$ & $0.1 \mathrm{mg} / \mathrm{L}$ \\
T-Methylphenol & $\mathrm{ND}$ & $0.1 \mathrm{mg} / \mathrm{L}$ \\
,4-Methylphenol (isomeric pair) & $\mathrm{ND}$ & $0.1 \mathrm{mg} / \mathrm{L}$ \\
Nitrobenzene & $\mathrm{ND}$ & $0.1 \mathrm{mg} / \mathrm{L}$ \\
Pentachlorophenol & $\mathrm{ND}$ & $0.1 \mathrm{mg} / \mathrm{L}$ \\
'yridine & $\mathrm{ND}$ & $0.1 \mathrm{mg} / \mathrm{L}$ \\
2,4,5-Trichlorophenol & $\mathrm{ND}$ & $0.1 \mathrm{mg} / \mathrm{L}$ \\
2,4,6-Trichlorophenol & $\mathrm{ND}$ & $0.1 \mathrm{mg} / \mathrm{L}$
\end{tabular}

\begin{tabular}{lcc}
\hline \hline UUALITY CONTROL DATA: & \% Recovery & Acceptable Range \\
Irrogate & 69 & $10-123$ \\
\hline 2,4,6-Tribromophenol & 84 & $43-116$ \\
Fluorobiphenyl & 47 & $21-100$ \\
Fluorophenol & 84 & $35-114$ \\
Nitrobenzene-d5 & 122 & $33-141$ \\
- Terphenyl-d14 & 31 & $10-94$ \\
1enol-d5 & &
\end{tabular}

ND - Not Detected

is report shall not be reproduced except in full, without the written approval of the laboratory. 


$\begin{array}{llll}\text { CLIENT: } & \text { Bechtel Nevada } & \text { CLIENT ID: } & \text { TAML - VER - } 11 \\ \text { PROJECT ID: } & \text { V653 } & \text { DATE SAMPLED: } & 6 / 28 / 99 \\ \text { PROJECT \#: } & 17777 & \text { NEL SAMPLE ID: } & \text { L9907046-11 }\end{array}$

TEST: $\quad$ TCLP by EPA 1311, July 1992 \& Semivolatile Organics by EPA Method 8270C, Dec. 1996

METHOD: $\quad$ EPA $8270 \quad$ TCLP EXTRACT DATE: 7/8/99

MATRIX: Solid $\quad$ EXTRACTED $\quad 7 / 9 / 99$

DILUTION: 1 ANALYZED: 7/18/99

\section{PARAMETER}

1,4-Dichlorobenzene (p-DCB)

2,4-Dinitrotoluene (DNT)

Hexachlorobenzene

Hexachlorobutadiene

Hexachloroethane

2-Methylphenol

3,4-Methylphenol (isomeric pair)

Nitrobenzene

Pentachlorophenol

Pyridine

2,4,5-Trichlorophenol

2,4,6-Trichlorophenol

\begin{tabular}{c}
$\begin{array}{c}\text { Result } \\
\mathbf{m g} / \mathbf{L}\end{array}$ \\
\hline $\mathrm{ND}$ \\
$\mathrm{ND}$ \\
$\mathrm{ND}$ \\
$\mathrm{ND}$ \\
$\mathrm{ND}$ \\
$\mathrm{ND}$ \\
$\mathrm{ND}$ \\
$\mathrm{ND}$ \\
$\mathrm{ND}$ \\
$\mathrm{ND}$ \\
$\mathrm{ND}$ \\
$\mathrm{ND}$
\end{tabular}

Reporting

Limit

$0.1 \mathrm{mg} / \mathrm{L}$

$0.1 \mathrm{mg} / \mathrm{L}$

$0.1 \mathrm{mg} / \mathrm{L}$

$0.1 \mathrm{mg} / \mathrm{L}$

$0.1 \mathrm{mg} / \mathrm{L}$

$0.1 \mathrm{mg} / \mathrm{L}$

$0.1 \mathrm{mg} / \mathrm{L}$

$0.1 \mathrm{mg} / \mathrm{L}$

$0.1 \mathrm{mg} / \mathrm{L}$

$0.1 \mathrm{mg} / \mathrm{L}$

$0.1 \mathrm{mg} / \mathrm{L}$

$0.1 \mathrm{mg} / \mathrm{L}$

QUALITY CONTROL DATA:

\section{Surrogate}

2,4,6-Tribromophenol

2-Fluorobiphenyl

2-Fluorophenol

Nitrobenzene-d5

p-Terphenyl-d14

Phenol-d5

\section{\% Recovery}

70

74

43

80

118

29

\section{Acceptable Range}

$10-123$

$43-116$

$21-100$

$35-114$

$33-141$

$10-94$

ND - Not Detected

This report shall not be reproduced except in full, without the written approval of the laboratory. 


$\begin{array}{llll}\text { ILIENT: } & \text { Bechtel Nevada } & \text { CLIENT ID: } & \text { TAML - VER - } 12 \\ \text { PROJECT ID: } & \text { V653 } & \text { DATE SAMPLED: } & 6 / 29 / 99 \\ \text { PROJECT \#: } & 17777 & \text { NEL SAMPLE ID: } & \text { L9907046-12 }\end{array}$

[EST: $\quad$ TCLP by EPA 1311, July 1992 \& Semivolatile Organics by EPA Method 8270C, Dec, 1996

METHOD: $\quad$ EPA 8270

MATRIX: Solid

TCLP EXTRACT DATE: $7 / 8 / 99$

ILUTION: 1

EXTRACTED

$7 / 9 / 99$

ANALYZED:

$7 / 18 / 99$

\begin{tabular}{lcc}
\hline & $\begin{array}{c}\text { Result } \\
\text { mg/L }\end{array}$ & $\begin{array}{c}\text { Reporting } \\
\text { 'ARAmit }\end{array}$ \\
\hline .,4-Dichlorobenzene (p-DCB) & ND & $0.1 \mathrm{mg} / \mathrm{L}$ \\
2,4-Dinitrotoluene (DNT) & $\mathrm{ND}$ & $0.1 \mathrm{mg} / \mathrm{L}$ \\
lexachlorobenzene & $\mathrm{ND}$ & $0.1 \mathrm{mg} / \mathrm{L}$ \\
Iexachlorobutadiene & $\mathrm{ND}$ & $0.1 \mathrm{mg} / \mathrm{L}$ \\
Hexachloroethane & $\mathrm{ND}$ & $0.1 \mathrm{mg} / \mathrm{L}$ \\
'-Methylphenol & $\mathrm{ND}$ & $0.1 \mathrm{mg} / \mathrm{L}$ \\
„4-Methylphenol (isomeric pair) & $\mathrm{ND}$ & $0.1 \mathrm{mg} / \mathrm{L}$ \\
Nitrobenzene & $\mathrm{ND}$ & $0.1 \mathrm{mg} / \mathrm{L}$ \\
Pentachlorophenol & $\mathrm{ND}$ & $0.1 \mathrm{mg} / \mathrm{L}$ \\
yridine & $\mathrm{ND}$ & $0.1 \mathrm{mg} / \mathrm{L}$ \\
z,4,5-Trichlorophenol & $\mathrm{ND}$ & $0.1 \mathrm{mg} / \mathrm{L}$ \\
2,4,6-Trichlorophenol & $\mathrm{ND}$ & $0.1 \mathrm{mg} / \mathrm{L}$
\end{tabular}

\begin{tabular}{lcc}
\hline \hline UALITY CONTROL DATA: & Acceptable Range \\
Irrogate & \% Recovery & $10-123$ \\
\hline 2,4,6-Tribromophenol & 72 & $43-116$ \\
Fluorobiphenyl & 79 & $21-100$ \\
Fluorophenol & 44 & $35-114$ \\
Nitrobenzene-d5 & 85 & $33-141$ \\
- Terphenyl-d14 & 128 & $10-94$ \\
lenol-d5 & 29 & 9
\end{tabular}




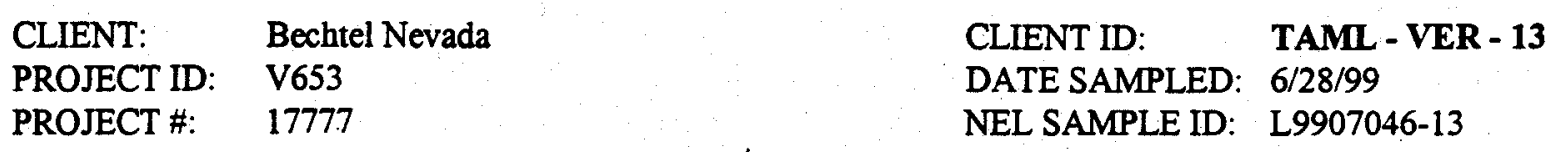

TEST: $\quad$ TCLP by EPA 1311, July 1992 \& Semivolatile Organics by EPA Method 8270C, Dec. 1996

METHOD: EPA 8270

MATRIX: Solid

DILUTION: 1

TCLP EXTRACT DATE: $7 / 8 / 99$

EXTRACTED $\quad 7 / 9 / 99$

ANALYZED: $\quad 7 / 18 / 99$

\section{PARAMETER}

1,4-Dichlorobenzene (p-DCB)

2,4-Dinitrotoluene (DNT)

Hexachlorobenzene

Hexachlorobutadiene

Hexachloroethane

2-Methylphenol

3,4-Methylphenol (isomeric pair)

Nitrobenzene

Pentachlomphenol

Pyridine

2,4,5-Trichlorophenol

2,4,6-Trichlorophenol

\begin{tabular}{c}
$\begin{array}{c}\text { Result } \\
\mathbf{m g} / \mathbf{L}\end{array}$ \\
\hline $\mathrm{ND}$ \\
$\mathrm{ND}$ \\
$\mathrm{ND}$ \\
$\mathrm{ND}$ \\
$\mathrm{ND}$ \\
$\mathrm{ND}$ \\
$\mathrm{ND}$ \\
$\mathrm{ND}$ \\
$\mathrm{ND}$ \\
$\mathrm{ND}$ \\
$\mathrm{ND}$ \\
$\mathrm{ND}$
\end{tabular}

Reporting

Limit

$0.1 \mathrm{mg} / \mathrm{L}$

$0.1 \mathrm{mg} / \mathrm{L}$

$0.1 \mathrm{mg} / \mathrm{L}$

$0.1 \mathrm{mg} / \mathrm{L}$

$0.1 \mathrm{mg} / \mathrm{L}$

$0.1 \mathrm{mg} / \mathrm{L}$

$0.1 \mathrm{mg} / \mathrm{L}$

$0.1 \mathrm{mg} / \mathrm{L}$

$0.1 \mathrm{mg} / \mathrm{L}$

$0.1 \mathrm{mg} / \mathrm{L}$

$0.1 \mathrm{mg} / \mathrm{L}$

$0.1 \mathrm{mg} / \mathrm{L}$

QUALITY CONTROL DATA:

\section{Surrogate}

2,4,6-Tribromophenol

2-Fluorobiphenyl

2-Fluorophenol

Nitrobenzene-d5

p-Terphenyl-d14

Phenol-d5

\section{\% Recovery}

67

78

42

83

118

28
Acceptable Range

$10-123$

$43-116$

$21-100$

$35-114$

$33-141$

$10-94$ 
NEL LABORATORIES

$\begin{array}{ll}\text { CLIENT: } & \text { Bechtel Nevada } \\ \text { PROJECT ID: } & \text { V653 } \\ \text { PROJECT \#: } & 17777\end{array}$

CLIENT ID: $\quad$ TAML - VER - 14

DATE SAMPLED: 6/28/99

NEL SAMPLE ID: L9907046-14
TEST:

METHOD:

MATRIX:

DILUTION:
TCLP by
EPA 8270

Solid

PARAMETER
1,4-Dichlorobenzene (p-DCB)
2,4-Dinitrotoluene (DNT)
Hexachlorobenzene
Hexachlorobutadiene
Hexachloroethane
2-Methylphenol
3,4-Methylphenol (isomeric pair)
Nitrobenzene
Pentachlorophenol
Pyridine
2,4,5-Trichlorophenol
2,4,6-Trichlorophenol

QUALITY CONTROL DATA:

Surrogate

2.4,6-Tribromophenol

2-Fluorobiphenyl

2-Fluorophenol

Nitrobenzene-d5

p-Terphenyl-d14

Phenol-d5

\section{$\%$ Recovery}

65

70

37

72

107

25
Reporting

Limit

$0.1 \mathrm{mg} / \mathrm{L}$

$0.1 \mathrm{mg} / \mathrm{L}$

$0.1 \mathrm{mg} / \mathrm{L}$

$0.1 \mathrm{mg} / \mathrm{L}$

$0.1 \mathrm{mg} / \mathrm{L}$

$0.1 \mathrm{mg} / \mathrm{L}$

$0.1 \mathrm{mg} / \mathrm{L}$

$0.1 \mathrm{mg} / \mathrm{L}$

$0.1 \mathrm{mg} / \mathrm{L}$

$0.1 \mathrm{mg} / \mathrm{L}$

$0.1 \mathrm{mg} / \mathrm{L}$

$0.1 \mathrm{mg} / \mathrm{L}$
Acceptable Range

$10-123$

$43-116$

$21-100$

$35-114$

$33-141$

10- 94

ND - Not Detected

This report shall not be reproduced except in full. without the written approval of the laboratory. 


$\begin{array}{llll}\text { CLIENT: } & \text { Bechtel Nevada } & \text { CLIENT ID: } & \text { TAML - VER - 15 } \\ \text { PROJECT ID: } & \text { V653 } & \text { DATE SAMPLED: } 6 / 29 / 99 \\ \text { PROJECT \#: } & 17777 & \text { NEL SAMPLE ID: } & \text { L9907046-15 }\end{array}$

TEST:

TCLP by EPA 1311, July 1992 \& Semivolatile Organics by EPA Method 8270C, Dec. 1996

METHOD: EPA 8270

MATRIX: Solid

DILUTION: 1

TCLP EXTRACT DATE: $7 / 8 / 99$

EXTRACTED $\quad 7 / 12 / 99$

ANALYZED: $\quad 7 / 19 / 99$

\section{PARAMETER}

1,4-Dichlorobenzene (p-DCB)

2,4-Dinitrotoluene (DNT)

Hexachlorobenzene

\begin{tabular}{c}
$\begin{array}{c}\text { Result } \\
\text { mg/L }\end{array}$ \\
\hline $\mathrm{ND}$ \\
$\mathrm{ND}$ \\
$\mathrm{ND}$ \\
$\mathrm{ND}$ \\
$\mathrm{ND}$ \\
$\mathrm{ND}$ \\
$\mathrm{ND}$ \\
$\mathrm{ND}$ \\
$\mathrm{ND}$ \\
$\mathrm{ND}$ \\
$\mathrm{ND}$ \\
$\mathrm{ND}$
\end{tabular}

Reporting

Hexachlorobutadiene

Hexachloroethane

2-Methylphenol

3,4-Methylphenol (isomeric pair)

Nitrobenzene

Pentachlorophenol

Pyridine

2,4,5-Trichlorophenol

ND

\section{Limit}

$0.1 \mathrm{mg} / \mathrm{L}$

$0.1 \mathrm{mg} / \mathrm{L}$

$0.1 \mathrm{mg} / \mathrm{L}$

$0.1 \mathrm{mg} / \mathrm{L}$

$0.1 \mathrm{mg} / \mathrm{L}$

$0.1 \mathrm{mg} / \mathrm{L}$

$0.1 \mathrm{mg} / \mathrm{L}$

$0.1 \mathrm{mg} / \mathrm{L}$

$0.1 \mathrm{mg} / \mathrm{L}$

$0.1 \mathrm{mg} / \mathrm{L}$

$0.1 \mathrm{mg} / \mathrm{L}$

$0.1 \mathrm{mg} / \mathrm{L}$

QUALITY CONTROL DATA:

Surrogate

2,4,6-Tribromophenol

2-Fluorobiphenyl

2-Fluorophenol

Nitrobenzene-d5

p-Terphenyl-d14

Phenol-d5
$\%$ Recovery

61

71

41

71

122

28
Acceptable Range

$10-123$

$43-116$

$21-100$

$35-114$

$33-141$

$10-94$ 


$\begin{array}{llll} & & & \\ \text { CLIENT: } & \text { Bechtel Nevada } & \text { CLIENT ID: } & \text { TAML - VER - } 16 \\ \text { PROJECT ID: } & \text { V653 } & \text { DATE SAMPLED: } 6 / 29 / 99 \\ \text { PROJECT \#: } & 17777 & \text { NEL SAMPLE ID: } & \text { L9907046-16 }\end{array}$

PROJECT \#: $\quad 17777$

TEST:

METHOD:

MATRIX:

DILUTION:
TCLP by EPA 1311, July 1992 \& Semivolatil EPA 8270

Solid

1

TCLP EXTRACT DATE: 7/8/99

EXTRACTED

ANALYZED:

$7 / 19 / 99$

\begin{tabular}{c}
$\begin{array}{c}\text { Reporting } \\
\text { Limit }\end{array}$ \\
\hline $0.1 \mathrm{mg} / \mathrm{L}$ \\
$0.1 \mathrm{mg} / \mathrm{L}$ \\
$0.1 \mathrm{mg} / \mathrm{L}$ \\
$0.1 \mathrm{mg} / \mathrm{L}$ \\
$0.1 \mathrm{mg} / \mathrm{L}$ \\
$0.1 \mathrm{mg} / \mathrm{L}$ \\
$0.1 \mathrm{mg} / \mathrm{L}$ \\
$0.1 \mathrm{mg} / \mathrm{L}$ \\
$0.1 \mathrm{mg} / \mathrm{L}$ \\
$0.1 \mathrm{mg} / \mathrm{L}$ \\
$0.1 \mathrm{mg} / \mathrm{L}$ \\
$0.1 \mathrm{mg} / \mathrm{L}$
\end{tabular}

Reporting

Result

$\frac{\mathrm{mg} / \mathbf{L}}{\mathrm{ND}}$

ND

ND

ND

ND

ND

ND

ND

ND

ND

ND

ND
$7 / 12 / 99$
Pentachlorophenol

Pyridine

2,4,5-Trichlorophenol

2,4,6-Trichlorophenol

\section{$\%$ Recovery}

60

71

36

71

122

25
Acceptable Range

$10-123$

43- 116

$21-100$

$35-114$

33- 141

$10-94$

p-Terphenyl-d14

Phenol-d5

ND - Not Detected

This report shall not be reproduced except in full. without the written approval of the laboratory 


$\begin{array}{llll}\text { CLIENT: } & \text { Bechtel Nevada } & \text { CLIENT ID: } & \text { TAML - VER - 17 } \\ \text { PROJECT ID: } & \text { V653 } & \text { DATE SAMPLED: } 6 / 29 / 99 \\ \text { PROJECT \#: } & 17777 & \text { NEL SAMPLE ID: } & \text { L9907046-17 }\end{array}$

TEST: $\quad$ TCLP by EPA 1311, July 1992 \& Semivolatile Organics by EPA Method 8270C, Dec. 1996

METHOD: $\quad$ EPA 8270

MATRIX: Solid

DILUTION: 1

TCLP EXTRACT DATE: $7 / 8 / 99$

EXTRACTED $\quad 7 / 12 / 99$

ANALYZED: $\quad 7 / 19 / 99$

\section{PARAMETER}

1,4-Dichlorobenzene (p-DCB)

2,4-Dinitrotoluene (DNT)

Hexachlorobenzene

Hexachlorobutadiene

Hexachloroethane

2-Methylphenol

3,4-Methylphenol (isomeric pair)

Nitrobenzene

Pentachlorophenol

Pyridine

2,4,5-Trichlorophenol

2,4,6-Trichlorophenol

\begin{tabular}{c}
$\begin{array}{c}\text { Result } \\
\mathbf{m g} / \mathbf{L}\end{array}$ \\
\hline $\mathrm{ND}$ \\
$\mathrm{ND}$ \\
$\mathrm{ND}$ \\
$\mathrm{ND}$ \\
$\mathrm{ND}$ \\
$\mathrm{ND}$ \\
$\mathrm{ND}$ \\
$\mathrm{ND}$ \\
$\mathrm{ND}$ \\
$\mathrm{ND}$ \\
$\mathrm{ND}$ \\
$\mathrm{ND}$
\end{tabular}

Reporting

Limit

$0.1 \mathrm{mg} / \mathrm{L}$

$0.1 \mathrm{mg} / \mathrm{L}$

$0.1 \mathrm{mg} / \mathrm{L}$

$0.1 \mathrm{mg} / \mathrm{L}$

$0.1 \mathrm{mg} / \mathrm{L}$

$0.1 \mathrm{mg} / \mathrm{L}$

$0.1 \mathrm{mg} / \mathrm{L}$

$0.1 \mathrm{mg} / \mathrm{L}$

$0.1 \mathrm{mg} / \mathrm{L}$

$0.1 \mathrm{mg} / \mathrm{L}$

$0.1 \mathrm{mg} / \mathrm{L}$

$0.1 \mathrm{mg} / \mathrm{L}$

\section{QUALITY CONTROL DATA:}

\section{Surrogate}

2,4,6-Tribromophenol

2-Fluorobiphenyl

2-Fluorophenol

Nitrobenzene-d5

p-Terphenyl-d14

Phenol-d5

\section{$\%$ Recovery}

55

63

35

67

117

24

\section{Acceptable Range}

$10-123$

$43-116$

$21-100$

$35-114$

$33-141$

$10-94$

ND - Not Detected

This report shall not be reproduced except in full. without the uritten approval of the laboraton: 


$\begin{array}{llll}\text { CLIENT: } & \text { Bechtel Nevada } & \text { CLIENT ID: } & \text { TAML - VER - } 18 \\ \text { PROJECT ID: } & \text { V653 } & \text { DATE SAMPLED: } 6 / 28 / 99 \\ \text { PROJECT \#: } & 17777 & \text { NEL SAMPLE ID: } & \text { L9907046-18 }\end{array}$

TEST:

TCLP by EPA 1311, July 1992 \& Semivolatile Organics by EPA Method 8270C, Dec. 1996

METHOD: $\quad$ EPA 8270

MATRIX: Solid

JILUTION: 1

TCLP EXTRACT DATE: $7 / 8 / 99$

EXTRACTED $\quad 7 / 12 / 99$

ANALYZED: $\quad 7 / 19 / 99$

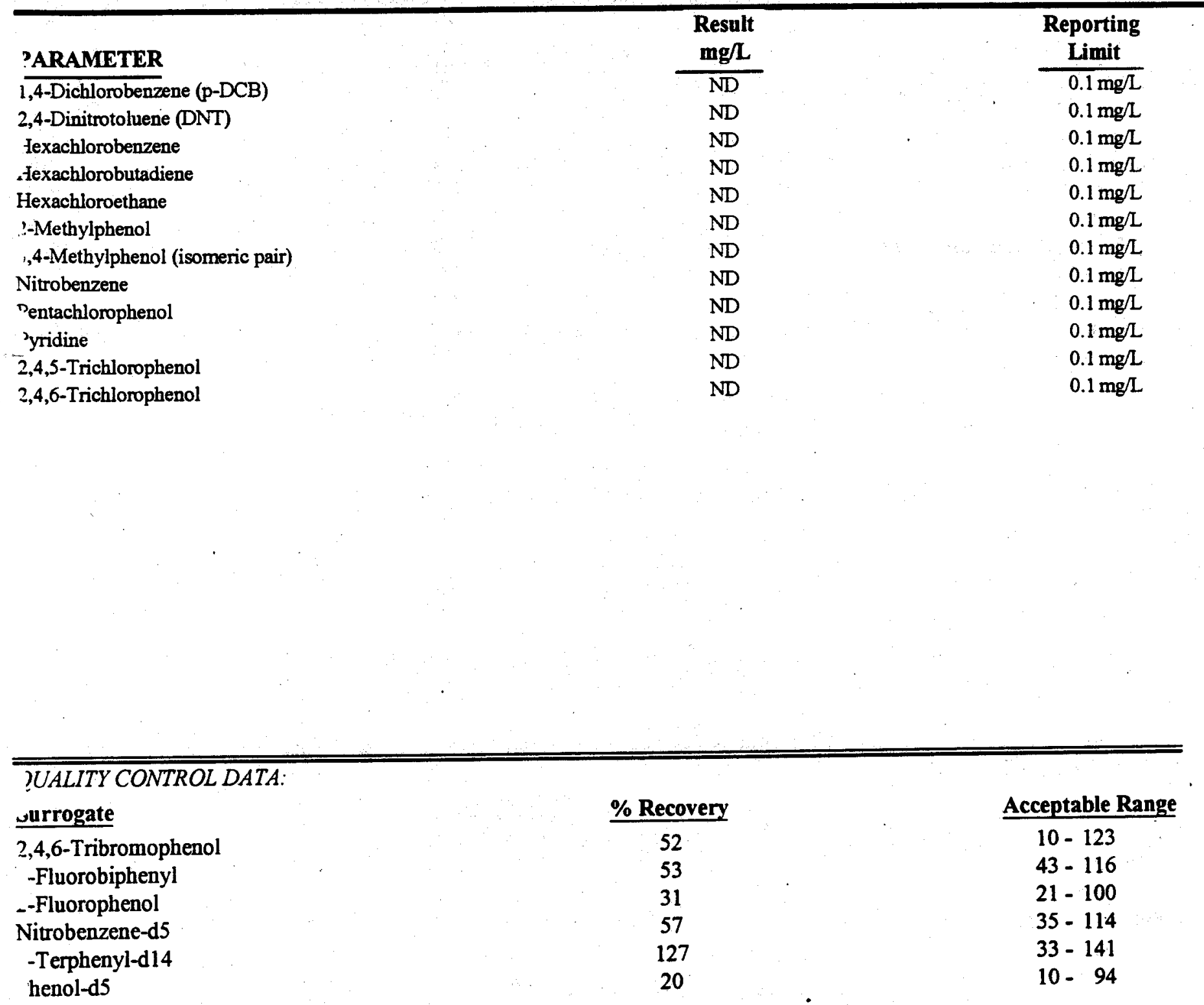




$\begin{array}{llll}\text { CLIENT: } & \text { Bechtel Nevada } & \text { CLIENT ID: } & \text { TAML - VER - 19 } \\ \text { PROJECT ID: } & \text { V653 } & \text { DATE SAMPLED: } 6 / 28 / 99 \\ \text { PROJECT \#: } & 17777 & \text { NEL SAMPLE ID: } & \text { L9907046-19 }\end{array}$

TEST: $\quad$ TCLP by EPA 1311, July 1992 \& Semivolatile Organics by EPA Method 8270C, Dec. 1996

METHOD: $\quad$ EPA 8270

TCLP EXTRACT DATE: 7/8/99

MATRIX: Solid

EXTRACTED $\quad 7 / 12 / 99$

$\begin{array}{llll}\text { DILUTION: } 1 & \text { ANALYZED: } & \cdot 7 / 19 / 99\end{array}$

\section{PARAMETER}

1,4-Dichlorobenzene (p-DCB)

2,4-Dinitrotoluene (DNT)

Hexachlorobenzene

Hexachlorobutadiene

Hexachloroethane

2-Methylphenol

3,4-Methylphenol (isomeric pair)

Nitrobenzene

Pentachlorophenol

Pyridine

2,4,5-Trichlorophenol

2,4,6-Trichlorophenol

\begin{tabular}{c}
$\begin{array}{c}\text { Result } \\
\mathbf{m g} / \mathbf{L}\end{array}$ \\
\hline $\mathrm{ND}$ \\
$\mathrm{ND}$ \\
$\mathrm{ND}$ \\
$\mathrm{ND}$ \\
$\mathrm{ND}$ \\
$\mathrm{ND}$ \\
$\mathrm{ND}$ \\
$\mathrm{ND}$ \\
$\mathrm{ND}$ \\
$\mathrm{ND}$ \\
$\mathrm{ND}$ \\
$\mathrm{ND}$
\end{tabular}

Reporting

Limit

$0.1 \mathrm{mg} / \mathrm{L}$

$0.1 \mathrm{mg} / \mathrm{L}$

$0.1 \mathrm{mg} / \mathrm{L}$

$0.1 \mathrm{mg} / \mathrm{L}$

$0.1 \mathrm{mg} / \mathrm{L}$

$0.1 \mathrm{mg} / \mathrm{L}$

$0.1 \mathrm{mg} / \mathrm{L}$

$0.1 \mathrm{mg} / \mathrm{L}$

$0.1 \mathrm{mg} / \mathrm{L}$

$0.1 \mathrm{mg} / \mathrm{L}$

$0.1 \mathrm{mg} / \mathrm{L}$

$0.1 \mathrm{mg} / \mathrm{L}$

QUALITY CONTROL DATA:

\section{Surrogate}

2,4,6-Tribromophenol

2-Fluorobiphenyl

2-Fluorophenol

Nitrobenzene-d5

p-Terphenyl-d14

Phenol-d5

\section{\% Recovery}

50

61

32

65

115

21
Acceptable Range

$10-123$

$43-116$

$21-100$

$35-114$

$33-141$

10- 94

ND - Not Detected

This report shall not be reproduced except in full, without the written approval of the laboratory. 


$\begin{array}{llll}\text { CLIENT: } & \text { Bechtel Nevada } & \text { CLIENT ID: } & \text { TAML - VER - 20 } \\ \text { PROJECT ID: } & \text { V653 } & \text { DATE SAMPLED: } 6 / 29 / 99 \\ \text { PROJECT \#: } & 17777 & \text { NEL SAMPLE ID: } & \text { L9907046-20 }\end{array}$

TEST: $\quad$ TCLP by EPA 1311, July 1992 \& Semivolatile Organics by EPA Method 8270C, Dec. 1996

METHOD: $\quad$ EPA 8270

MATRIX: Solid

TCLP EXTRACT DATE: 7/8/99

DILUTION: 1

EXTRACTED $\quad 7 / 12 / 99$

ANALYZED: $\quad 7 / 19 / 99$

\section{PARAMETER}

1,4-Dichlorobenzene (p-DCB)

2,4-Dinitrotoluene (DNT)

fexachlorobenzene

Jexachlorobutadiene

Hexachloroethane

?-Methylphenol

, 4-Methylphenol (isomeric pair)

Nitrobenzene

Pentachlorophenol

'yridine

2,4,5-Trichlorophenol

2,4,6-Trichlorophenol

\begin{tabular}{c}
$\begin{array}{c}\text { Result } \\
\mathbf{m g} / \mathbf{L}\end{array}$ \\
\hline $\mathrm{ND}$ \\
$\mathrm{ND}$ \\
$\mathrm{ND}$ \\
$\mathrm{ND}$ \\
$\mathrm{ND}$ \\
$\mathrm{ND}$ \\
$\mathrm{ND}$ \\
$\mathrm{ND}$ \\
$\mathrm{ND}$ \\
$\mathrm{ND}$ \\
$\mathrm{ND}$ \\
$\mathrm{ND}$
\end{tabular}

Reporting

Limit

$0.1 \mathrm{mg} / \mathrm{L}$

$0.1 \mathrm{mg} / \mathrm{L}$

$0.1 \mathrm{mg} / \mathrm{L}$

$0.1 \mathrm{mg} / \mathrm{L}$

$0.1 \mathrm{mg} / \mathrm{L}$

$0.1 \mathrm{mg} / \mathrm{L}$

$0.1 \mathrm{mg} / \mathrm{L}$

$0.1 \mathrm{mg} / \mathrm{L}$

$0.1 \mathrm{mg} / \mathrm{L}$

$0.1 \mathrm{mg} / \mathrm{L}$

$0.1 \mathrm{mg} / \mathrm{L}$

$0.1 \mathrm{mg} / \mathrm{L}$

\section{TUALITY CONTROL DATA:}

-urrogate

2.4,6-Tribromophenol

-Fluorobiphenyl

-Fluorophenol

Nitrobenzene-d5

-Terphenyl-d14

henol-d5
\% Recovery

54

66

35

67

112

24
Acceptable Range

$10-123$

$43-116$

$21-100$

$35-114$

$33-141$

$10-94$

ND - Not Detected

his report shall not be reproduced except in full, without the written approval of the laboratory. 


$\begin{array}{llll}\text { CLIENT: } & \text { Bechtel Nevada } & \text { CLIENT ID: } & \text { TAML - VER - 21 } \\ \text { PROJECT ID: } & \text { V653 } & \text { DATE SAMPLED: } & \text { 6/29/99 } \\ \text { PROJECT \#: } & 17777 & \text { NEL SAMPLE ID: } & \text { L9907046-21 }\end{array}$

TEST: $\quad$ TCLP by EPA 1311, July 1992 \& Semivolatile Organics by EPA Method 8270C, Dec 1996

METHOD: $\quad$ EPA 8270

MATRIX: Solid

DILUTION: 1

TCLP EXTRACT DATE: $7 / 8 / 99$

EXTRACTED $\quad 7 / 12 / 99$

ANALYZED: $\quad 7 / 19 / 99$

\section{PARAMETER}

1,4-Dichlorobenzene (p-DCB)

2,4-Dinitrotoluene (DNT)

Hexachlorobenzene

Hexachlorobutadiene

Hexachloroethane

2-Methylphenol

3,4-Methylphenol (isomeric pair)

Nitrobenzene

Pentachlorophenol

Pyridine

2,4,5-Trichlorophenol

2,4,6-Trichlorophenol

\begin{tabular}{c}
$\begin{array}{c}\text { Result } \\
\mathbf{m g} / \mathbf{L}\end{array}$ \\
\hline $\mathrm{ND}$ \\
$\mathrm{ND}$ \\
$\mathrm{ND}$ \\
$\mathrm{ND}$ \\
$\mathrm{ND}$ \\
$\mathrm{ND}$ \\
$\mathrm{ND}$ \\
$\mathrm{ND}$ \\
$\mathrm{ND}$ \\
$\mathrm{ND}$ \\
$\mathrm{ND}$ \\
$\mathrm{ND}$
\end{tabular}

Reporting

Limit

$0.1 \mathrm{mg} / \mathrm{L}$

$0.1 \mathrm{mg} / \mathrm{L}$

$0.1 \mathrm{mg} / \mathrm{L}$

$0.1 \mathrm{mg} / \mathrm{L}$

$0.1 \mathrm{mg} / \mathrm{L}$

$0.1 \mathrm{mg} / \mathrm{L}$

$0.1 \mathrm{mg} / \mathrm{L}$

$0.1 \mathrm{mg} / \mathrm{L}$

$0.1 \mathrm{mg} / \mathrm{L}$

$0.1 \mathrm{mg} / \mathrm{L}$

$0.1 \mathrm{mg} / \mathrm{L}$

$0.1 \mathrm{mg} / \mathrm{L}$

QUALITY CONTROL DATA:

\section{Surrogate}

2,4,6-Tribromophenol

2-Fluorobiphenyl

2-Fluorophenol

Nitrobenzene-d5

p-Terphenyl-d14

Phenol-d5

\section{$\%$ Recovery}

52

61

33

61

117

22
Acceptable Range

$10-123$

$43-116$

$21-100$

$35-114$

$33-141$

$10-94$

ND - Not Detected

This report shall not be reproduced except in full, without the written approval of the laboratory. 


$\begin{array}{llll}\text { ILIENT: } & \text { Bechtel Nevada } & \text { CLIENT ID: } & \text { TAML - VER - 22 } \\ \text { PROJECT ID: } & \text { V653 } & \text { DATE SAMPLED: } & 6 / 28 / 99 \\ \text { PROJECT \#: } & 17777 & \text { NEL SAMPLE ID: } \text { L9907046-22 }\end{array}$

IEST:

TCLP by EPA 1311, July 1992 \& Semivolatile Organics by EPA Method 8270C, Dec. 1996

METHOD: $\quad$ EPA 8270

MATRIX: Solid

TCLP EXTRACT DATE: $7 / 8 / 99$

IILUTION: 1

EXTRACTED

$7 / 12 / 99$

ANALYZED:

$7 / 20 / 99$

\begin{tabular}{lcc}
\hline & $\begin{array}{c}\text { Result } \\
\text { mg/L }\end{array}$ & $\begin{array}{c}\text { Reporting } \\
\text { Limit }\end{array}$ \\
\hline 1,4-Dichlorobenzene (p-DCB) & $\mathrm{ND}$ & $0.1 \mathrm{mg} / \mathrm{L}$ \\
2,4-Dinitrotoluene (DNT) & $\mathrm{ND}$ & $0.1 \mathrm{mg} / \mathrm{L}$ \\
lexachlorobenzene & $\mathrm{ND}$ & $0.1 \mathrm{mg} / \mathrm{L}$ \\
-lexachlorobutadiene & $\mathrm{ND}$ & $0.1 \mathrm{mg} / \mathrm{L}$ \\
Hexachloroethane & $\mathrm{ND}$ & $0.1 \mathrm{mg} / \mathrm{L}$ \\
-Methylphenol & $\mathrm{ND}$ & $0.1 \mathrm{mg} / \mathrm{L}$ \\
,4-Methylphenol (isomeric pair) & $\mathrm{ND}$ & $0.1 \mathrm{mg} / \mathrm{L}$ \\
Nitrobenzene & $\mathrm{ND}$ & $0.1 \mathrm{mg} / \mathrm{L}$ \\
Dentachlorophenol & $\mathrm{ND}$ & $0.1 \mathrm{mg} / \mathrm{L}$ \\
yridine & $\mathrm{ND}$ & $0.1 \mathrm{mg} / \mathrm{L}$ \\
2,4,5-Trichlorophenol & $\mathrm{ND}$ & $0.1 \mathrm{mg} / \mathrm{L}$ \\
2,4,6-Trichlorophenol & $\mathrm{ND}$ & $0.1 \mathrm{mg} / \mathrm{L}$
\end{tabular}

UALITY CONTROL DATA:

-irrogate

2,4,6-Tribromophenol

Fluorobiphenyl

- Fluorophenol

Nitrobenzene-d5

Terphenyl-d14

ienol-d5
\% Recovery

59

67

35

68

92

26
Acceptable Range

$10-123$

$43-116$

$21-100$

$35-114$

$33-141$

$10-94$

ND - Not Detected

is report shall not be reproduced except in full, without the written approval of the laboratory. 


$\begin{array}{llll}\text { CLIENT: } & \text { Bechtel Nevada } & \text { CLIENT ID: } & \text { TAML - VER - 23 } \\ \text { PROJECT ID: } & \text { V653 } & \text { DATE SAMPLED: } 6 / 28 / 99 \\ \text { PROJECT \#: } & 17777 & \text { NEL SAMPLE ID: } & \text { L9907046-23 }\end{array}$

TEST: $\quad$ TCLP by EPA 1311, July 1992 \& Semivolatile Organics by EPA Method 8270C, Dec. 1996

METHOD: $\quad$ EPA 8270

MATRIX: Solid

DILUTION: 1

TCLP EXTRACT DATE: 7/8/99

EXTRACTED 7/12/99

ANALYZED: $\quad 7 / 20 / 99$

\section{PARAMETER}

1,4-Dichlorobenzene (p-DCB)

2,4-Dinitrotoluene (DNT)

Hexachlorobenzene

Hexachlorobutadiene

Hexachloroethane

2-Methylphenol

3,4-Methylphenol (isomeric pair)

Nitrobenzene

Pentachlorophenol

Pyridine

2,4,5-Trichlorophenol

2,4,6-Trichlorophenol

\begin{tabular}{c}
$\begin{array}{c}\text { Result } \\
\text { mg/L }\end{array}$ \\
\hline ND \\
ND \\
ND \\
ND \\
ND \\
ND \\
ND \\
ND \\
ND \\
ND \\
ND \\
ND
\end{tabular}

Reporting

Limit

$0.1 \mathrm{mg} / \mathrm{L}$

$0.1 \mathrm{mg} / \mathrm{L}$

$0.1 \mathrm{mg} / \mathrm{L}$

$0.1 \mathrm{mg} / \mathrm{L}$

$0.1 \mathrm{mg} / \mathrm{L}$

$0.1 \mathrm{mg} / \mathrm{L}$

$0.1 \mathrm{mg} / \mathrm{L}$

$0.1 \mathrm{mg} / \mathrm{L}$

$0.1 \mathrm{mg} / \mathrm{L}$

$0.1 \mathrm{mg} / \mathrm{L}$

$0.1 \mathrm{mg} / \mathrm{L}$

$0.1 \mathrm{mg} / \mathrm{L}$

\section{QUALITY CONTROL DATA:}

\section{Surrogate}

2,4,6-Tribromophenol

2-Fluorobiphenyl

2-Fluorophenol

Nitrobenzene-d5

p-Terphenyl-d14

Phenol-d5

\section{\% Recovery}

64

72

35

70

102

24

\section{Acceptable Range}

$10-123$

$43-116$

$21-100$

$35-114$

$33-141$

$10-94$ 
CLIENT: Bechtel Nevada

PROJECT ID: V653

PROJECT \#: $\quad 17777$
CLIENT ID:

DATE SAMPLED: $6 / 28 / 99$

NEL SAMPLE ID: $\quad$ L9907046-24
TEST:

METHOD:

MATRIX:

DILUTION:
TCLP by EPA 1311, July 1992 \& Semivolatile Organies by EPA Method 8270C, Dec. 1996 EPA 8270

Solid

1
TCLP EXTRACT DATE: 7/8/99

EXTRACTED

$7 / 12 / 99$

ANALYZED:

\begin{tabular}{lcc}
\hline PARAMETER & $\begin{array}{c}\text { Result } \\
\mathbf{m g} / \mathrm{L}\end{array}$ & $\begin{array}{c}\text { Reporting } \\
\text { Limit }\end{array}$ \\
\cline { 2 - 3 } & $\mathrm{ND}$ & $0.1 \mathrm{mg} / \mathrm{L}$ \\
\hline 1,4-Dichlorobenzene (p-DCB) & $\mathrm{ND}$ & $0.1 \mathrm{mg} / \mathrm{L}$ \\
2,4-Dinitrotoluene (DNT) & $\mathrm{ND}$ & $0.1 \mathrm{mg} / \mathrm{L}$ \\
Jexachlorobenzene & $\mathrm{ND}$ & $0.1 \mathrm{mg} / \mathrm{L}$ \\
Jexachlorobutadiene & $\mathrm{ND}$ & $0.1 \mathrm{mg} / \mathrm{L}$ \\
Hexachloroethane & $\mathrm{ND}$ & $0.1 \mathrm{mg} / \mathrm{L}$ \\
3-Methylphenol & $\mathrm{ND}$ & $0.1 \mathrm{mg} / \mathrm{L}$ \\
3,4-Methylphenol (isomeric pair) & $\mathrm{ND}$ & $0.1 \mathrm{mg} / \mathrm{L}$ \\
Nitrobenzene & $\mathrm{ND}$ & $0.1 \mathrm{mg} / \mathrm{L}$ \\
Pentachlorophenol & $\mathrm{ND}$ & $0.1 \mathrm{mg} / \mathrm{L}$ \\
Jyridine & $\mathrm{ND}$ & $0.1 \mathrm{mg} / \mathrm{L}$ \\
2,4,5-Trichlorophenol & $\mathrm{ND}$ & $0.1 \mathrm{mg} / \mathrm{L}$ \\
2,4,6-Trichlorophenol & &
\end{tabular}

TUALITY CONTROL DATA:

urrogate

2,4,6-Tribromophenol

-Fluorobiphenyl

-Fluorophenol

Nitrobenzene-d5

-Terphenyl-d14

thenol-d5
\% Recovery

58

73

37

72

99

25

\section{Acceptable Range}

$10-123$

$43-116$

$21-100$

$35-114$

$33-141$

$10-94$

ND - Not Detected

is report shall not be reproduced except in full, without the written approval of the laboratory. 


$\begin{array}{llll}\text { CLIENT: } & \text { Bechtel Nevada } & \text { CLIENT ID: } & \text { TAMI - VER - 25 } \\ \text { PROJECT ID: } & \text { V653 } & \text { DATE SAMPLED: } 6 / 28 / 99 \\ \text { PROJECT \#: } & 17777 & \text { NEL SAMPLE ID: } & \text { L9907046-25 }\end{array}$

TEST: $\quad$ TCLP by EPA 1311, July 1992 \& Semivolatile Organics by EPA Method 8270C, Dec. 1996

METHOD: EPA 8270 TCLP EXTRACT DATE: $7 / 8 / 99$

MATRIX: Solid

DILUTION: 1

EXTRACTED

$7 / 12 / 99$

ANALYZED:

$7 / 20 / 99$

\section{PARAMETER}

1,4-Dichlorobenzene (p-DCB)

2,4-Dinitrotoluene (DNT)

Hexachlorobenzene

Hexachlorobutadiene

Hexachloroethane

2-Methylphenol

3,4-Methylphenol (isomeric pair)

Nitrobenzene

Pentachlorophenol

Pyridine

2,4,5-Trichlorophenol

2,4,6-Trichlorophenol

\begin{tabular}{c}
$\begin{array}{c}\text { Result } \\
\text { mg/L }\end{array}$ \\
\hline $\mathrm{ND}$ \\
$\mathrm{ND}$ \\
$\mathrm{ND}$ \\
$\mathrm{ND}$ \\
$\mathrm{ND}$ \\
$\mathrm{ND}$ \\
$\mathrm{ND}$ \\
$\mathrm{ND}$ \\
$\mathrm{ND}$ \\
$\mathrm{ND}$ \\
$\mathrm{ND}$ \\
$\mathrm{ND}$
\end{tabular}

Result

$\mathrm{mg} / \mathrm{L}$

ND

ND

ND

D

$\mathrm{ND}$

D

$\mathrm{ND}$
Reporting

Limit

$0.1 \mathrm{mg} / \mathrm{L}$

$0.1 \mathrm{mg} / \mathrm{L}$

$0.1 \mathrm{mg} / \mathrm{L}$

$0.1 \mathrm{mg} / \mathrm{L}$

$0.1 \mathrm{mg} / \mathrm{L}$

$0.1 \mathrm{mg} / \mathrm{L}$

$0.1 \mathrm{mg} / \mathrm{L}$

$0.1 \mathrm{mg} / \mathrm{L}$

$0.1 \mathrm{mg} / \mathrm{L}$

$0.1 \mathrm{mg} / \mathrm{L}$

$0.1 \mathrm{mg} / \mathrm{L}$

$0.1 \mathrm{mg} / \mathrm{L}$

QUALITY CONTROL DATA:

\section{Surrogate}

2,4,6-Tribromophenol

2-Fluorobiphenyl

2-Fluorophenol

Nitrobenzene-d5

p-Terphenyl-d14

Phenol-d5
\% Recovery

56

69

35

68

85

26
Acceptable Range

$10-123$

$43-116$

$21-100$

$35-114$

$33-141$

$10-94$

ND - Not Detected

This report shall not be reproduced except in full, without the written approval of the laboratory. 


$\begin{array}{llll}\text { CLIENT: } & \text { Bechtel Nevada } & \text { CLIENT ID: } & \text { TAML - VER - 26 } \\ \text { PROJECT ID: } & \text { V653 } & \text { DATE SAMPLED: 6/28/99 } \\ \text { PROJECT \#: } & 17777 & \text { NEL SAMPLE ID: } & \text { L9907046-26 }\end{array}$

IEST: TCLP by EPA 1311, July 1992 \& Semivolatile Organics by EPA Method 8270C, Dec. 1996

METHOD: EPA 8270 TCLP EXTRACT DATE: 7/8/99

MATRIX: Solid $\quad$ EXTRACTED $\quad 7 / 12 / 99$

$\begin{array}{llll}\text { OILUTION: } & 1 & \text { ANALYZED: } & 7 / 20 / 99\end{array}$

\begin{tabular}{lcc}
\hline PARAMETER & $\begin{array}{c}\text { Result } \\
\text { mg/L }\end{array}$ & $\begin{array}{c}\text { Reporting } \\
\text { Limit }\end{array}$ \\
\cline { 2 - 2 } ,,4-Dichlorobenzene (p-DCB) & $\mathrm{ND}$ & $0.1 \mathrm{mg} / \mathrm{L}$ \\
2,4-Dinitrotoluene (DNT) & $\mathrm{ND}$ & $0.1 \mathrm{mg} / \mathrm{L}$ \\
lexachlorobenzene & $\mathrm{ND}$ & $0.1 \mathrm{mg} / \mathrm{L}$ \\
lexachlorobutadiene & $\mathrm{ND}$ & $0.1 \mathrm{mg} / \mathrm{L}$ \\
Hexachloroethane & $\mathrm{ND}$ & $0.1 \mathrm{mg} / \mathrm{L}$ \\
--Methylphenol & $\mathrm{ND}$ & $0.1 \mathrm{mg} / \mathrm{L}$ \\
,4-Methylphenol (isomeric pair) & $\mathrm{ND}$ & $0.1 \mathrm{mg} / \mathrm{L}$ \\
Nitrobenzene & $\mathrm{ND}$ & $0.1 \mathrm{mg} / \mathrm{L}$ \\
Pentachlorophenol & $\mathrm{ND}$ & $0.1 \mathrm{mg} / \mathrm{L}$ \\
'yridine & $\mathrm{ND}$ & $0.1 \mathrm{mg} / \mathrm{L}$ \\
z,4,S-Trichlorophenol & $\mathrm{ND}$ & $0.1 \mathrm{mg} / \mathrm{L}$
\end{tabular}

"UALITY CONTROL DATA:

irrogate

2,4,6-Tribromophenol Fluorobiphenyl

Fluorophenol

Nitrobenzene-d5

Terphenyl-d14 ienol-d5

\section{\% Recovery}

61

68

34

67

102

24
Acceptable Range

$10-123$

$43-116$

$21-100$

$35-114$

$33-141$

$10-94$ 


$\begin{array}{llll}\text { CLIENT: } & \text { Bechtel Nevada } & \text { CLIENT ID: } & \text { TAML - VER - 27 } \\ \text { PROJECT ID: } & \text { V653 } & \text { DATE SAMPLED: } & 6 / 28 / 99 \\ \text { PROJECT \#: } & 17777 & \text { NEL SAMPLE ID: } & \text { L9907046-27 }\end{array}$

TEST: $\quad$ TCLP by EPA 1311, July 1992 \& Semivolatile Organics by EPA Method 8270C, Dec. 1996

METHOD: $\quad$ EPA 8270

MATRIX: $\quad$ Solid

DILUTION: . 1

TCLP EXTRACT DATE: 7/8/99

EXTRACTED $\quad 7 / 12 / 99$

ANALYZED: $\quad 7 / 20 / 99$

\section{PARAMETER}

1,4-Dichlorobenzene (p-DCB)

2,4-Dinitrotoluene (DNT)

Hexachlorobenzene

Hexachlorobutadiene

Hexachloroethane

2-Methylphenol

3,4-Methylphenol (isomeric pair)

Nitrobenzene

Pentachlorophenol

Pyridine

2,4,5-Trichlorophenol

2,4,6-Trichlorophenol

\begin{tabular}{c}
$\begin{array}{c}\text { Result } \\
\mathbf{m g} / \mathbf{L}\end{array}$ \\
\hline $\mathrm{ND}$ \\
$\mathrm{ND}$ \\
$\mathrm{ND}$ \\
$\mathrm{ND}$ \\
$\mathrm{ND}$ \\
$\mathrm{ND}$ \\
$\mathrm{ND}$ \\
$\mathrm{ND}$ \\
$\mathrm{ND}$ \\
$\mathrm{ND}$ \\
$\mathrm{ND}$ \\
$\mathrm{ND}$
\end{tabular}

Reporting

Limit

$0.1 \mathrm{mg} / \mathrm{L}$

$0.1 \mathrm{mg} / \mathrm{L}$

$0.1 \mathrm{mg} / \mathrm{L}$

$0.1 \mathrm{mg} / \mathrm{L}$

$0.1 \mathrm{mg} / \mathrm{L}$

$0.1 \mathrm{mg} / \mathrm{L}$

$0.1 \mathrm{mg} / \mathrm{L}$

$0.1 \mathrm{mg} / \mathrm{L}$

$0.1 \mathrm{mg} / \mathrm{L}$

$0.1 \mathrm{mg} / \mathrm{L}$

$0.1 \mathrm{mg} / \mathrm{L}$

$0.1 \mathrm{mg} / \mathrm{L}$

\section{QUALITY CONTROL DATA:}

\section{Surrogate}

2,4,6-Tribromophenol

2-Fluorobiphenyl

2-Fluorophenol

Nitrobenzene-d5

p-Terpheny1-d14

Phenol-d5
\% Recovery

64

71

34

71

99

24
Acceptable Range

$10-123$

$43-116$

$21-100$

$35-114$

$33-141$

$10-94$ 


$\begin{array}{llll}\text { CLIENT: } & \text { Bechtel Nevada } & \text { CLIENT ID: } & \text { TAML - VER - 28 } \\ \text { PROJECT ID: } & \text { V653 } & \text { DATE SAMPLED: } 6 / 28 / 99 \\ \text { PROJECT \#: } & 17777 & \text { NEL SAMPLE ID: } \text { L9907046-28 }\end{array}$

TEST: $\quad$ TCLP by EPA 1311, July 1992 \& Semivolatile Organics by EPA Method 8270C, Dec. 1996

METHOD: EPA 8270

MATRIX: , Solid

DILUTION: 1

TCLP EXTRACT DATE: $7 / 8 / 99$

EXTRACTED 7/12/99

ANALYZED: $\quad 7 / 20 / 99$

\section{PARAMETER}

1,4-Dichlorobenzene (p-DCB)

2,4-Dinitrotoluene (DNT)

Iexachlorobenzene

Iexachlorobutadiene

Hexachloroethane

?-Methylphenol

,4-Methylphenol (isomeric pair)

Nitrobenzene

Pentachlorophenol

'yridine

2,4,5-Trichlorophenol

2,4,6-Trichlorophenol

\begin{tabular}{c}
$\begin{array}{c}\text { Result } \\
\text { mg/L }\end{array}$ \\
\hline ND \\
ND \\
ND \\
ND \\
ND \\
ND \\
ND \\
ND \\
ND \\
ND \\
ND \\
ND
\end{tabular}

Reporting

Limit

$0.1 \mathrm{mg} / \mathrm{L}$

$0.1 \mathrm{mg} / \mathrm{L}$

$0.1 \mathrm{mg} / \mathrm{L}$

$0.1 \mathrm{mg} / \mathrm{L}$

$0.1 \mathrm{mg} / \mathrm{L}$

$0.1 \mathrm{mg} / \mathrm{L}$

$0.1 \mathrm{mg} / \mathrm{L}$

$0.1 \mathrm{mg} / \mathrm{L}$

$0.1 \mathrm{mg} / \mathrm{L}$

$0.1 \mathrm{mg} / \mathrm{L}$

$0.1 \mathrm{mg} / \mathrm{L}$

$0.1 \mathrm{mg} / \mathrm{L}$

UALITY CONTROL DATA:

urrogate

2,4,6-Tribromophenol

Fluorobiphenyl

Fluorophenol

Nitrobenzene-d5

Terphenyl-d14

ienol-d5

\section{$\%$ Recovery}

59

67

33

66

107

23

\section{Acceptable Range}

10 - 123

$43-116$

$21-100$

$35-114$

$33-141$

$10-94$

ND - Not Detected

is report shall not be reproduced except in full, without the written approval of the laboratory. 


$\begin{array}{lll}\text { CLIENT: } & \text { Bechtel Nevada } & \text { CLIENT ID: } \\ \text { PROJECT ID: } & \text { V653 } & \text { DATE SAMPLED: NA } \\ \text { PROJECT \#: } & 17777 & \text { NEL SAMPLE ID: 070999-E1-BLK }\end{array}$

TEST:

METHOD: $\quad$ EPA 8270

MATRIX: TCLP Extract
TCLP by EPA 1311, July 1992 \& Semivolatile Organics by EPA Method 8270C, Dec 1996

TCLP EXTRACT DATE: $7 / 8 / 99$

EXTRACTED $\quad 7 / 9 / 99$

ANALYZED: $\quad 7 / 21 / 99$

\section{PARAMETER}

1,4-Dichlorobenzene (p-DCB)

2,4-Dinitrotoluene (DNT)

Hexachlorobenzene

Hexachlorobutadiene

Hexachloroethane

2-Methylphenol

3,4-Methylphenol (isomeric pair)

Nitrobenzene

Pentachlorophenol

Pyridine

2,4,5-Trichlorophenol

2,4,6-Trichlorophenol

\section{Result}

$\frac{\mathrm{mg} / \mathbf{L}}{\mathrm{ND}}$

ND

ND

ND

ND

ND

ND

ND

ND

ND

ND

$\mathrm{ND}$
Reporting

Limit

$0.1 \mathrm{mg} / \mathrm{L}$

$0.1 \mathrm{mg} / \mathrm{L}$

$0.1 \mathrm{mg} / \mathrm{L}$

$0.1 \mathrm{mg} / \mathrm{L}$

$0.1 \mathrm{mg} / \mathrm{L}$

$0.1 \mathrm{mg} / \mathrm{L}$

$0.1 \mathrm{mg} / \mathrm{L}$

$0.1 \mathrm{mg} / \mathrm{L}$

$0.1 \mathrm{mg} / \mathrm{L}$

$0.1 \mathrm{mg} / \mathrm{L}$

$0.1 \mathrm{mg} / \mathrm{L}$

$0.1 \mathrm{mg} / \mathrm{L}$

QUALITY CONTROL DATA:

\section{Surrogate}

2,4,6-Tribromophenol

2-Fluorobiphenyl

2-Fluorophenol

Nitrobenzene-d5

p-Terphenyl-d 14

Phenol-d5
\% Recovery

62

68

43

77

126

27
Acceptable Range

$10-123$

$43-116$

$21-100$

$35-114$

$33-141$

$10-94$

ND - Not Detected

This report shall not be reproduced except in full, without the written approval of the laboratory. 


$\begin{array}{llll}\text { ILIENT: } & \text { Bechtel Nevada } & \text { CLIENT ID: } & \text { Method Blank } \\ \text { PROJECT ID: } & \text { V653 } & \text { DATE SAMPLED: NA } \\ \text { PROJECT \#: } & 17777 & \text { NEL SAMPLE ID: 071299-E1-BLK }\end{array}$

[EST: $\quad$ TCLP by EPA 1311, July 1992 \& Semivolatile Organics by EPA Method 8270C, Dec. 1996

METHOD: EPA 8270

MATRIX: TCLP Extract

TCLP EXTRACT DATE: 7/8/99

EXTRACTED $\quad 7 / 12 / 99$

ANALYZED: $\quad 7 / 19 / 99$

\begin{tabular}{|c|c|c|}
\hline 'ARAMETER & $\begin{array}{c}\text { Result } \\
\mathrm{mg} / \mathrm{L}\end{array}$ & $\begin{array}{c}\text { Reporting } \\
\text { Limit }\end{array}$ \\
\hline s,4-Dichlorobenzene (p-DCB) & $\mathrm{ND}$ & $0.1 \mathrm{mg} / \mathrm{L}$ \\
\hline 2,4-Dinitrotoluene (DNT) & ND & $0.1 \mathrm{mg} / \mathrm{L}$ \\
\hline lexachlorobenzene & ND & $0.1 \mathrm{mg} / \mathrm{L}$ \\
\hline lexachlorobutadiene & ND & $0.1 \mathrm{mg} / \mathrm{L}$ \\
\hline Hexachloroethane & ND & $0.1 \mathrm{mg} / \mathrm{L}$ \\
\hline -Methylphenol & ND & $0.1 \mathrm{mg} / \mathrm{L}$ \\
\hline ,4-Methylphenol (isomeric pair) & ND & $0.1 \mathrm{mg} / \mathrm{L}$ \\
\hline Nitrobenzene & ND & $0.1 \mathrm{mg} / \mathrm{L}$ \\
\hline Pentachlorophenol & ND & $0.1 \mathrm{mg} / \mathrm{L}$ \\
\hline yridine & ND & $0.1 \mathrm{mg} / \mathrm{L}$ \\
\hline$z, 4,5$-Trichlorophenol & ND & $0.1 \mathrm{mg} / \mathrm{L}$ \\
\hline 2,4,6-Trichlorophenol & ND & $0.1 \mathrm{mg} / \mathrm{L}$ \\
\hline
\end{tabular}

UALITY CONTROL DATA:

Surrogate

4,6-Tribromophenol

Fluorobiphenyl

2-Fluorophenol

Nitrobenzene-d5

Terphenyl-d14

rnenol-d5
\% Recovery

59

61

38

63

109

26
Acceptable Range

$10-123$

$43-116$

$21-100$

$35-114$

$33-141$

$10-94$

ND - Not Detected

is report shall not be reproduced except in full, without the written approval of the laboratory. 


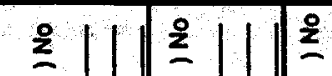

영

을

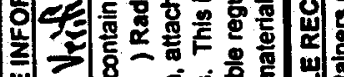

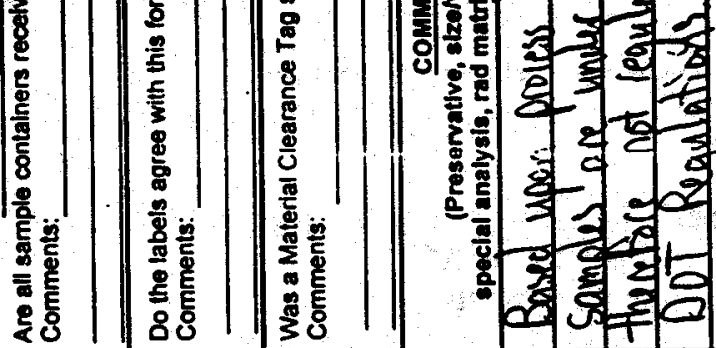

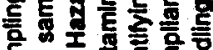
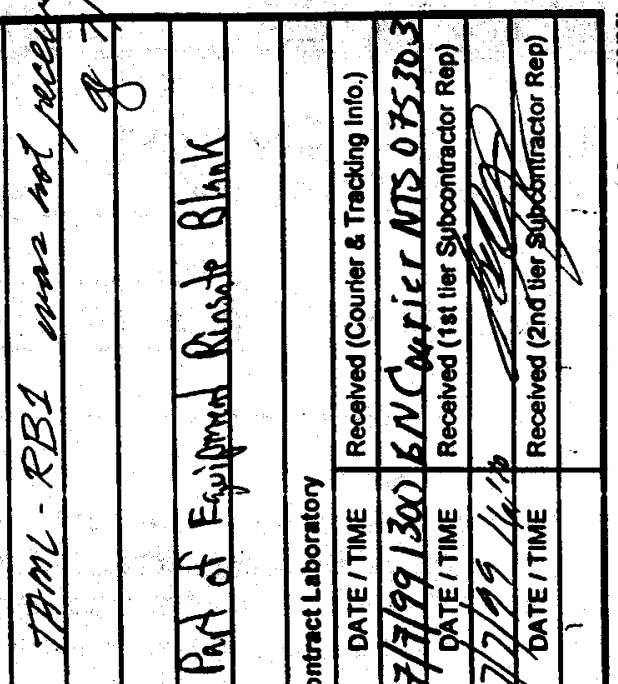

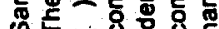

8
8
$\frac{1}{\alpha}$

๙ิㅇํㅇ

要

$\$ \$$

○

ง $\frac{m}{5}$

$+\quad w \geq$

岁

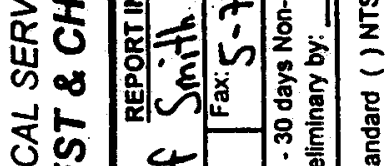

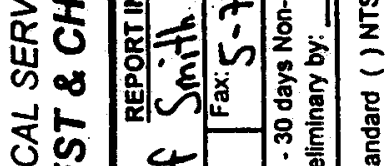

ऽ。

$-\geq \frac{1}{8}$

) $\sum \frac{\pi}{\alpha}$

$\frac{n}{0}$

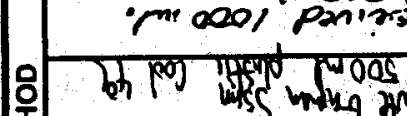

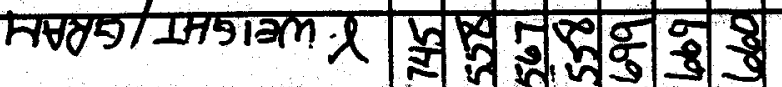
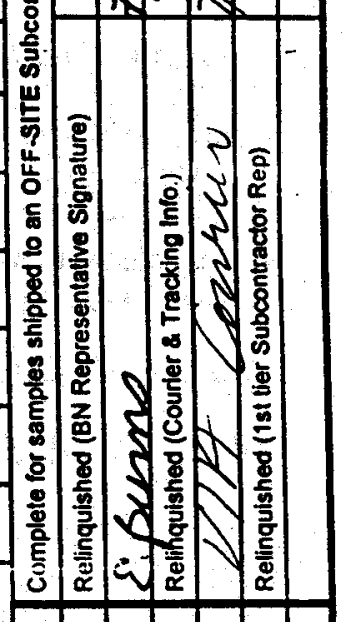

$\frac{1}{5}$

Q10? supu dnI

E.

E

$-6$

吾廉

2

\$.

毛

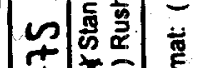

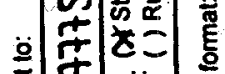

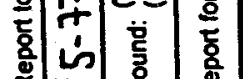

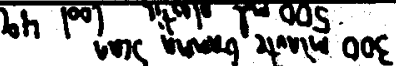

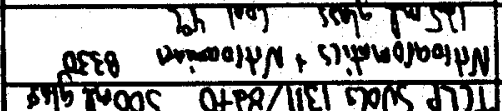

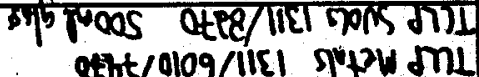

弯 है
施

mo

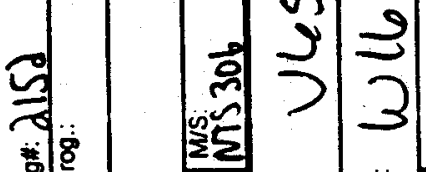

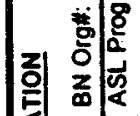

iे

>

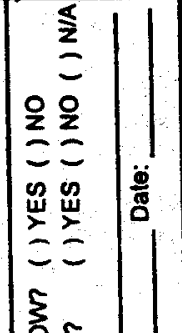

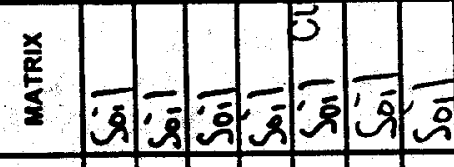

$\frac{\bar{m}}{3}$

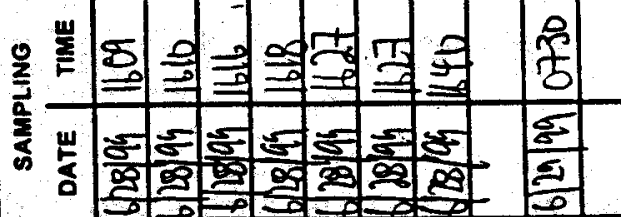

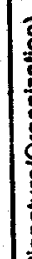

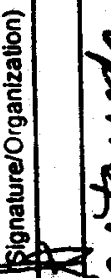

$\rightarrow 2$

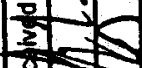

- 1

옹

wै

ज्ञात

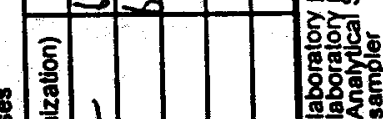

8

密

장하

ㅎํำ

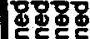

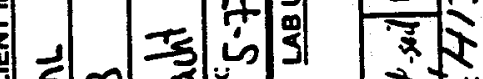

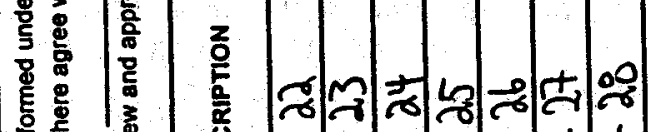

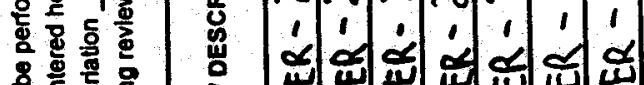

a)

密

害

응

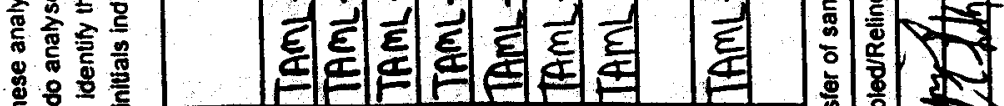

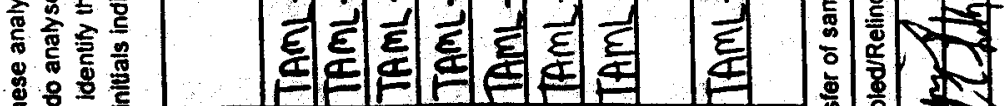

9.

을 $\supset \supset \Longrightarrow 引$

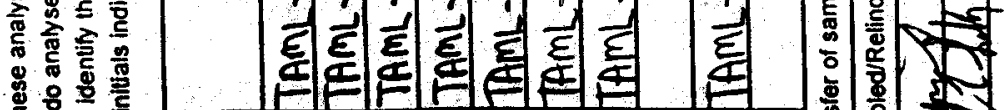

.

产总范

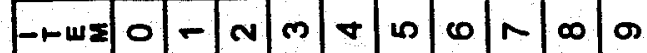




\section{Project (AU 401 TAM)}

Charge No: (7PQSHB

BN Org*: 2152 ASLProg:

\begin{tabular}{|l|l}
\hline Send \\
\hline Pho \\
\hline Tu \\
\hline Fin
\end{tabular}

\section{REPORT INFORMATION}

Sinith

Project Manager: Steve Naht

\begin{tabular}{l|l|l|} 
Phone: $5-7234$ & Fax: $5-7761$ \\
\hline
\end{tabular} Nis: 306 $\left.\right|^{\text {Fax: } 5-776} \quad{ }^{\text {M/S: NTS } 3 \alpha}$ Turnaround: Standard - 30 days Non-rad, 60 Days Rad, Other. () Rush Preliminary by:__ Final by: Final report format: () Standard () NTS-WAC \& Other form I, UP

\section{Sampling site: (lear Verificition Sneples)}

The samples submitted contain (check): (I) Unknown

() Hazardous () Radloactlve () Unknown contamination. If known, attach a brlef narrattve summary identffying contaminants. This information will ensure compliance with applicable regulations and allow for the safe handling of the sample materials.
Rad SGD: $\quad C 519^{\frac{\text { LAB USE ONLY }}{\text { Non-Rad SDG: }}}$ V6S3 Rad Packet $41332{ }^{\text {Non-Rad Packet }}$ W 1689

Client Services Representative:

Will these analyses be performed under a signed SOWh () YES ( ) NO

If so, do analyses entered here agree with the SOW?

if not, identify the variation

CSR initlals indicating review and approval:

E ID IOESCRIPTION

M

09 TAML-VER - Q9

101 TAML-VER- 10

II 2 TAML-VER - II

23 TAML - VER - 12

134 TAML - VER - 13

145 TAML - VER - 14

156 TAML - VER - 15

167 TAML - VER - 16

\begin{tabular}{|l|}
\hline 8 \\
\hline 9 \\
\hline
\end{tabular}

Transfer of samples submitted for analyses

Sampled/Relinquishod (Signatiuge/Organizallon)

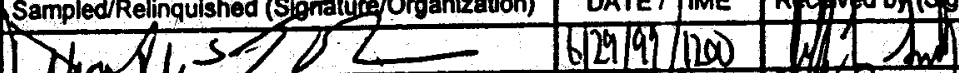
max (628) 194 15OB SOi. 120.199 1510 boil $17897991535 \quad 50.1$ 6ixing 10839 Soil 16199 991142 Soil 1281991164 Soi 6291990846 Soi 629990839 Sal

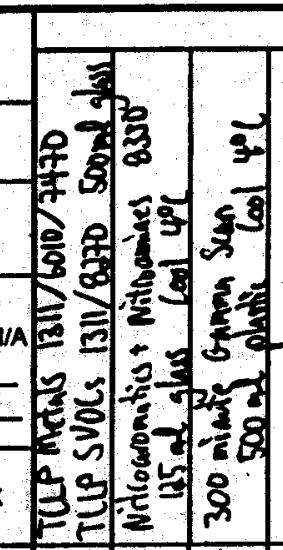

ANALYSES \& METHOD SAMPLE RECEIPT INFORMATION Are all sample containers recelved intact $\$ 2$ Yes $($ ) No Comments:

Do the labels agree with this form? Deyes () No Comments:

Was a Material Clearance Tag submitted? Wyes ( ) No Comments:

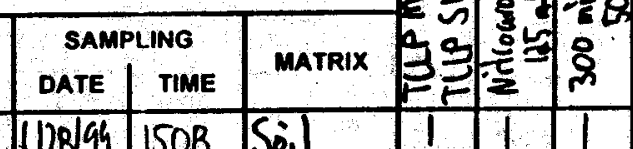
COMMENTS

(Preservative, size/volume, mshisD

\begin{tabular}{l|l|l|l|l|} 
& & & 1 & 1 \\
& & & &
\end{tabular}

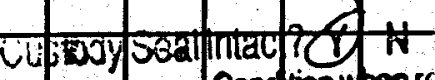
Based upin ploies knoulatue all 753 Samples ine undes $70 \mathrm{~B}$ lamen and 714 theritale not regulated under 573 DOT Regulations

639 Ter 6,9 ara 8

Complete for samples shipped to an OFF. SITE Subcontract Laboratory Relinquished (BN Representative Signature) \begin{tabular}{|l|l|}
\hline DATE / TIME & Received (Courier \& Tracking Info.) \\
\hline
\end{tabular} E. Buno

Rellnquished (Courter \& Tracking Into.)

$7 / 7 / 991300$ BNCowrier NTSO 75303

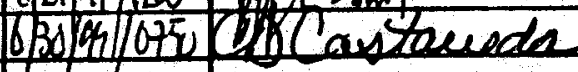

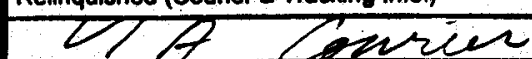
Relinquished (ist tier Subcontractor Rep)

DATE / TIME Received (1st tier Subcontractor Rep) 73isalece 


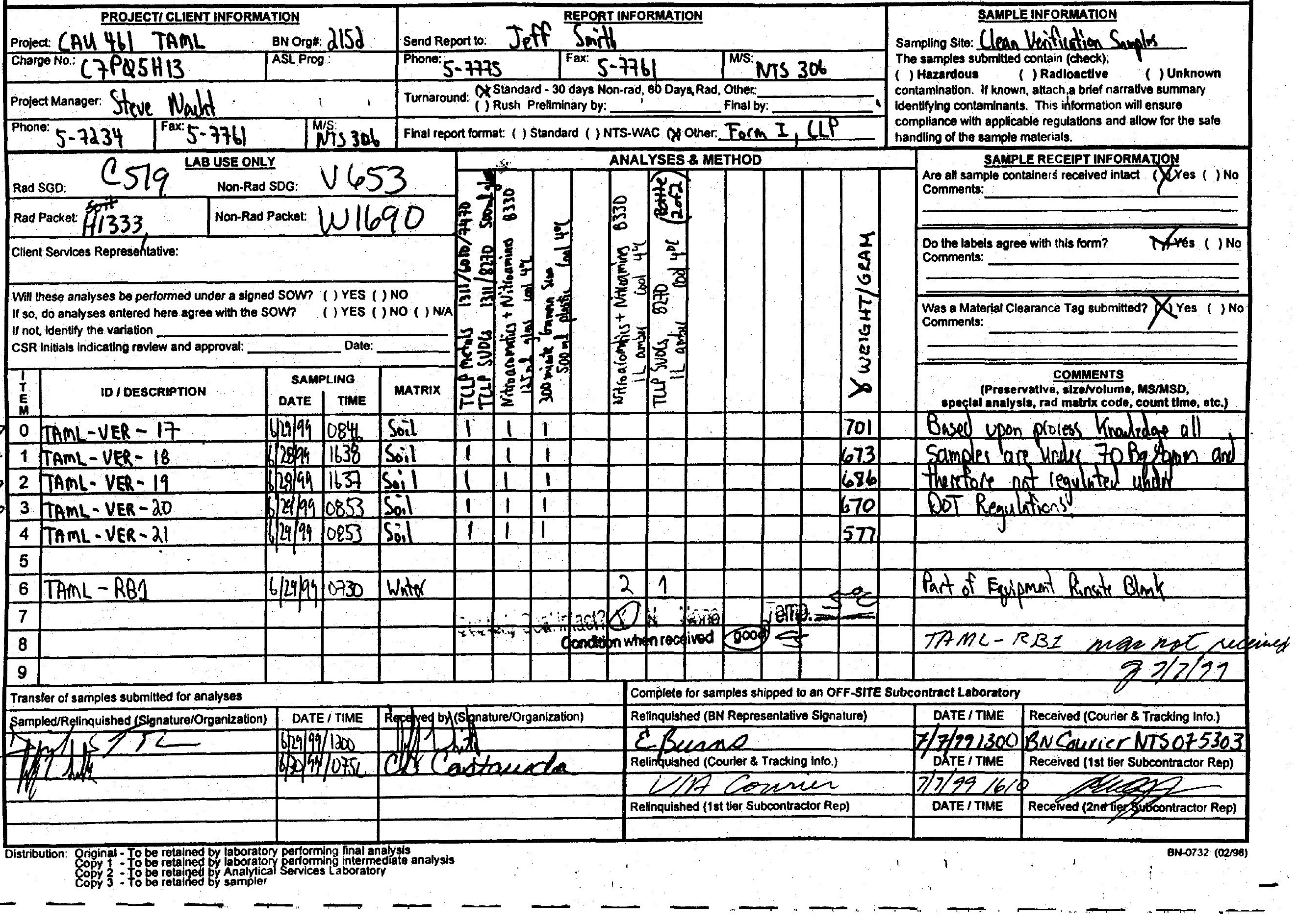





\section{GENERAL INFORMATION}

Sample Delivery Group: V653

Client: Bechtel Nevada

Laboratory:

NEL

Reviewed by:

Marsha C. Webb

Methods: Extraction 1311

Requested Analysis: TCLP SVO

Matrix: Soil

II. COMPLETENESS

A. Were all deliverables submitted?

B. Are forms properly completed and legible?

C. Were all requested analyses performed by the laboratory?

D. Were the requested analytical methods used for analysis?

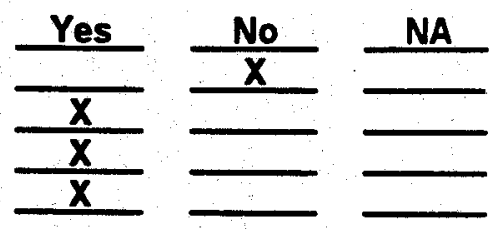

III. DATA ASSESSMENT SUMMARY

( ) A. Field and QAVQC sample data have been generated in accordance with method requirements and within quality control limits.

( ) B. QAVC problems were encountered during analysis of the samples. However, the data are considered acceptable.

(x) C. Significant QAQC problems were encountered during analysis of the samples. Data for the following samples may be rejected.

TCLP extraction fluid \#1 had a pH of 4.63. The limit is 4.88 to 4.98 - Potentiolly hich biosed. $T$

IV. HOLDING TIME COMPLIANCE
A. Sampled
B. ASL Received:
C. Contract Lab Received:
D. Latest TCLP Extraction:
E. Latest Preparative Extraction:
F. Latest Date Analyzed:
Were holding time criteria met?

$\frac{\text { Date }}{\frac{6 / 28 / 99}{6 / 30 / 99}}$
$\frac{7 / 199}{\frac{7 / 8 / 99}{7 / 12 / 99}}$
(X) Yes $\frac{7 / 20 / 99}{2 \text { () No }}$

Date

$6 / 30 / 99$

() No

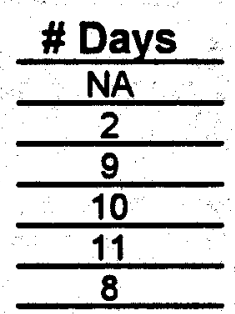

\begin{tabular}{c}
$\frac{\text { Acceptable }}{0-2}$ \\
\hline 1 \\
\hline$\frac{14}{14}$ \\
\hline 40 \\
\hline
\end{tabular}

\section{INSTRUMENT \& SAMPLE QAQC CRITERIA}

\section{A. INSTRUMENT QUALITY CONTROL}

1. Were all samples analyzed within a valid 12 -hour tune period?

2. Tune - Decafluorotriphenylphosphine

3. Calibrations Initial:

Verification:
Ion Abundances

SPCC RRF values

CCC \% RSD, CofV $>0.99$

SPCC RRF values

CCC \% D, Conc. \% Drift

is Area and Retention Times

B. SAMPLE ASSOCIATED QUALITY CONTROL

1. Blanks: Were target compounds detected in field or laboratory blanks?

If so, were they detected:

a. at $>5$ times the CRDL / PQL / Reporting Limit?

b. in the samples at $>5$ times the blank value (non-common lab contaminants) or $>10$ times the blank value (common lab contaminants)?

2. Lab Control Sample: Were recoveries within acceptable limits?

3. Samples

a. Were target compounds detected in the samples?

b. If so, were the raw EICPs provided which confirm the qualification?

c. Were concentrations calculated accurately?

4. Were all Surrogate recoveries acceptable?

5. Were all Internal Standard Areas and Retention Times within control limits?

Criteria met

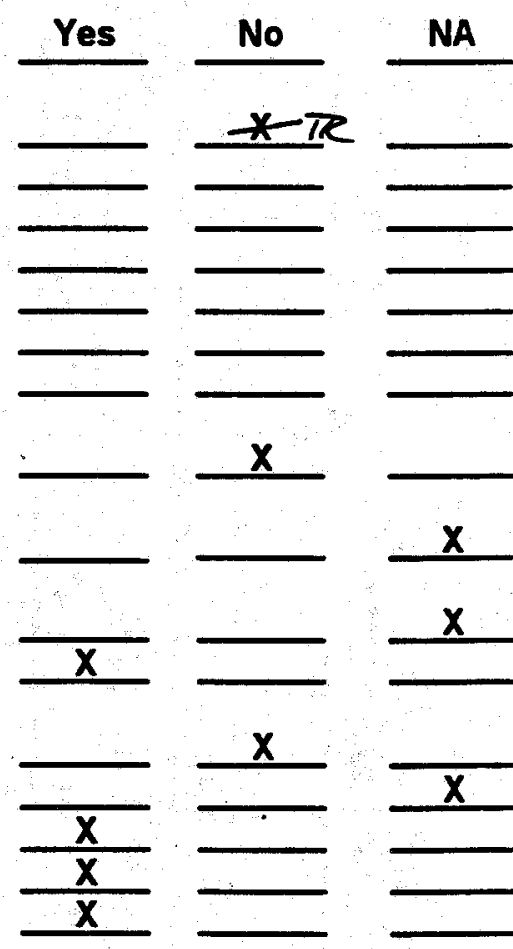


COMMENTS: This data is not defensible since the TCLP extraction was not done correctly. The pH of Extraction fluid 1 was measured at 4.63. The limit is 4.88 to 4.98; The measurement of the pH after the additon of $3.5 \mathrm{ml}$ of $1 \mathrm{~N} \mathrm{HCL}$ is questionable (very low). There is no range for the room temperature; there is no time noted for the Extraction Off. There is no initial and date for the cross out of the temperature.

Mash Cabo

Auqust 31.1999 (9:15am)

Reviewer Signature:

Date: 


\section{RADIOMETRIC DATA ANALYSIS PACKAGE}

BECHTEL NEVADA Analytical Services

Method Type: RAGMA

Sample Numbers

\begin{tabular}{lc} 
DOE No. & Lab ID No. \\
TAML-RB1 & $H 1335-0-75000$ \\
TAML-VER-11 & $H 1332-2-75042$ \\
TAML-VER-12 & $H 1332-3-75043$ \\
TAML-VER-13 & $H 1332-4-75044$ \\
TAML-VER-14 & $H 1332-5-75045$ \\
TAML-VER-15 & $H 1332-6-75046$ \\
TAML-VER-16 & $H 1332-7-75047$ \\
TAML-VER-17 & $H 1333-0-75048$ \\
TAML-VER-18 & $H 1333-1-75049$ \\
TAML-VER-19 & $H 1333-2-75050$ \\
TAML-VER-20 & $H 1333-3-75051$ \\
TAML-VER-23 & $H 1334-1-75054$ \\
TAML-VER-24 & $H 1334-2-75055$ \\
TAML-VER-25 & $H 1334-3-75056$ \\
TAML-VER-21 & $H 1333-4-75052$ \\
\hline
\end{tabular}

Case No.: CAU 461 TAML Verif.

SDG No.: C519

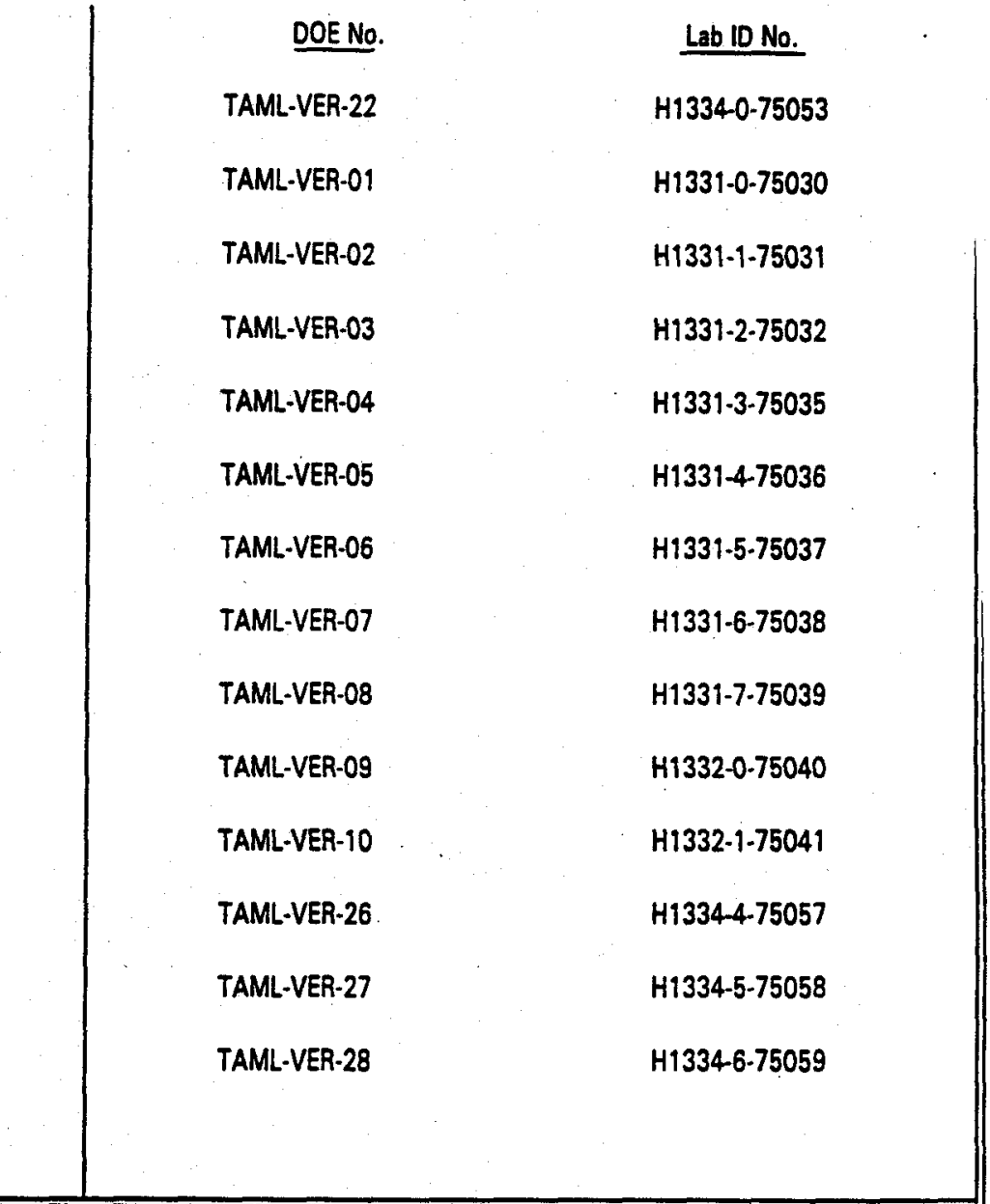

Comments: Data generated from the $\mathbf{3 0 0}$ minute gamma spectroscopy scans of samples submitted to the laboratory on June 30, 1999.

Release of the data contained in this data package has been authorized by the Laboratory Manager or the Manager's designee, as verified by the following signature:

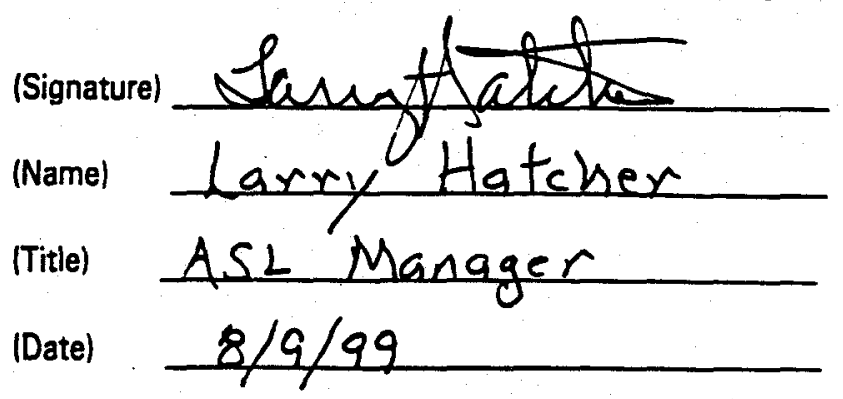




\section{GENERAL INFORMATION}

Sample Delivery Group: V653

Client: Bechtel Nevada

Laboratory:

\begin{tabular}{l} 
NEL \\
\hline RCRA Metals (TCLP) \\
\hline Soil
\end{tabular}

Reviewed by: Marsha C. Webb

Date: August 31, 1999

Requested Analysis:

Methods:

Extraction 1311

Analysis 6010

Matrix:

Soil

II. COMPLETENESS

A. Were all deliverables submitted?

B. Are forms properly completed and legible?

C. Were all requested analyses performed by the laboratory?

D. Were the requested analytical methods used for analysis?

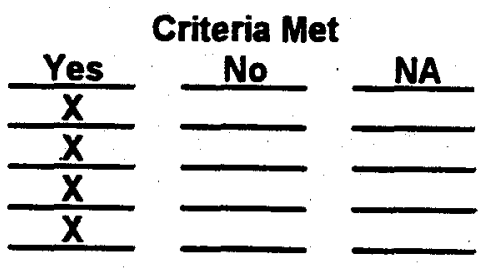

III. DATA ASSESSMENT SUMMARY

( ) A. Field and QAQC sample data have been generated in accordance with method requirements and within quality control limits.

X B. QAVC problems were encountered during analysis of the samples. However, the data are considered acceptable.

PXR C. Significant QAQC problems were encountered during analysis of the samples. Data for the following samples may be rejected.

TCLP extraction fluid \#1 had a pH of 4.63. The limit is 4.88 to 4.98 . Poteutidly high bizsed. IR

IV. HOLDING TIME COMPLIANCE

A. Sampled

B. ASL Received:

C. Contract Lab Received:

D. Latest Extraction: TCLP

E. Latest Date Analyzed:

Were holding time criteria met?
Date
$6 / 28 / 99$
$6 / 30 / 99$
$7 \Pi 199$
$7 / 8 / 99$
(X) Yes
$7 / 12 / 99$
( ) No

\section{INSTRUMENT \& SAMPLE QAQC COMPLIANCE}

\section{A. INSTRUMENT QUALITY CONTROL}

1. Calibrations

$\frac{\text { Blank: }}{\text { Initial: }}$

Within $3 X$ of the IDL

Minimum of Cal Blank and 1 Standard

ICV, CCVs, check standard within $\pm 10 \%$

RSD $<5 \%$ for 22 replicate integrations

2. Interference Check Sample

within $\pm 20 \%$ true value

B. SAMPLE ASSOCIATED QUALITY CONTROL

1. Blanks: Were target compounds detected in field or laboratory blanks?

If so, were they detected:

a. at levels 2 CRDL?

b. in the samples at $<5$ times the blank value?

2. Lab Control Sample: Were recoveries within acceptable limits?

3. Samples

a. Were target compounds detected in the samples?

b. Were concentrations calculated correctly?

4. As appropriate, were the following within quality control limits:

a. MSMSD recoveries and RPDs

b. Lab and/or field duplicate RPDs

c. Serial dilution within $\pm 10 \%$ for undiluted concentrations $\geq 10 X$ the IDL

Criteria met

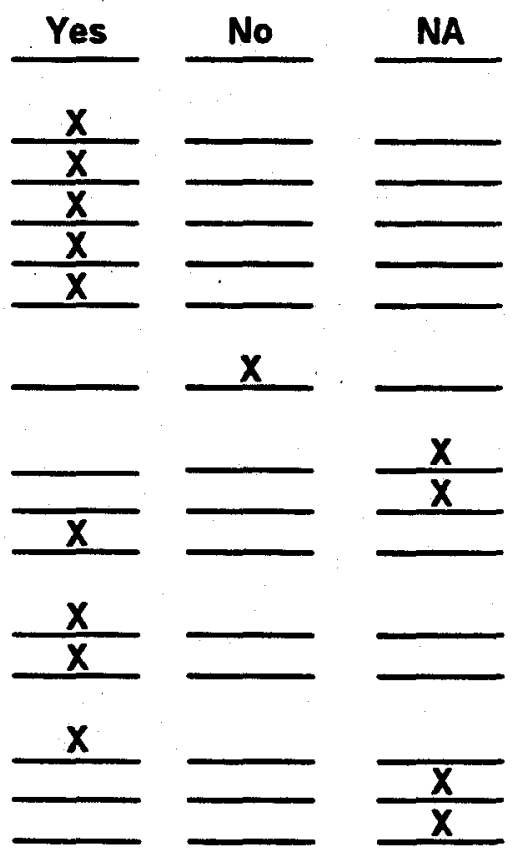


Bechtel Nevada

VALYTICAL SERVICES LABORAT TY DATA PACKAGE REVIEW CHECKLIST

COMMENTS: No difficulties were encountered during validation of this data FYI The laboratory should be reporting the surrogate recovery on each Reporting form.

Marsala Cclebto

Reviewer Signature:
August 31. 1999 (9:06am) Date: 


\section{GENERAL INFORMATION}

Sample Delivery Group: V653

Client: Bechtel Nevada

Laboratory:

NEL

Reviewed by: Marsha C. Webb

Date: $8 / 26 / 99$

Requested Analysis:

$\mathrm{Hg}$

Methods:

Extraction 1311

Analysis 7470

Matrix:

Soil

II. COMPLETENESS

A. Were all deliverables submitted?

B. Are forms properly completed and legible?

C. Were all requested analyses performed by the laboratory?

D. Were the requested analytical methods used for analysis?

Criteria Met

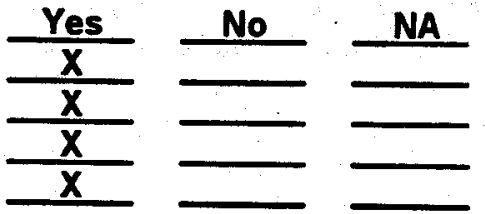

III. DATA ASSESSMENT SUMMARY

( ) A. Field and QAVC sample data have been generated in accordance with method requirements and within quality control limits.

B. QAQC problems were encountered during analysis of the samples. However, the data are considered acceptable.

(a) 7 . Significant QAQC problems were encountered during analysis of the samples. Data for the following samples may be rejected.

TCLP extraction fluid \#1 had a pH of 4.63. The limit is 4.88 to 4.98 - Pofestid lly high $b 1+5 e d$. 조

IV. HOLDING TIME COMPLIANCE

A. Sampled

B. ASL Received:

C. Contract Lab Received:

D. Latest Extraction:TCLP

E. Latest Date Analyzed:

Were holding time criteria met?

$\frac{\frac{\text { Date }}{6 / 28 / 99}}{\frac{6 / 30 / 99}{7 / 7 / 99}} \frac{\frac{\text { \# Days }}{\text { NA }}}{\frac{7}{\frac{7}{7 / 11 / 99}}} \frac{\frac{9}{10}}{\frac{3}{3}}$

(X) Yes

( ) No

V. INSTRUMENT \& SAMPLE QAQC COMPLIANCE

A. INSTRUMENT QUALITY CONTROL

1. Calibrations Blank: Within $3 X$ of the IDL

Initial: $\quad$ Cal Blank and 5 Standards

Verification: ICV within $\pm 10 \%$

B. SAMPLE ASSOCIATED QUALITY CONTROL

CCV every 10 samples within $\pm 20 \%$

1. Prep blank: Were target compounds detected in field or laboratory blanks?

2. Lab Control Sample: Were recoveries within acceptable limits? $(80-120 \%)$

3. Samples

a. Were target compounds detected in the samples?

b. Were concentrations calculated correctly?

4. As appropriate, were the following within quality control limits:

a. MSMSD recoveries $(75-125 \%)$

b. Lab and/or field duplicate RPDs ( $<20 \%)$

c. Serial dilution within $\pm 10 \%$ for undiluted concentrations $\geq 10 X$ the IDL
Criteria met
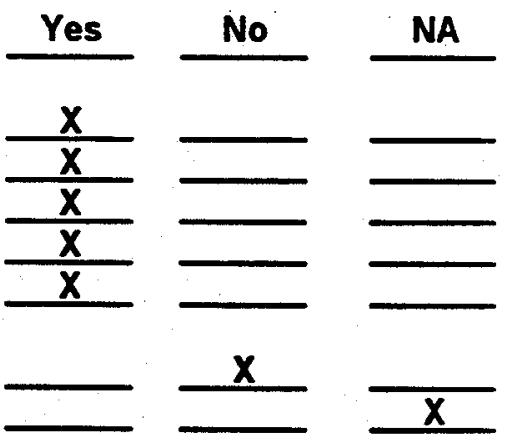

$\frac{\mathbf{x}}{\mathbf{x}}-$

$\frac{x}{x}=-$ 
COMMENTS: $\quad$ Cr was detected in L9907046-01 at 0.071; the duplicate was 0.017; RPD was not met. Both results are $>$ than RL. -03 was done at 16:20; CB at 16:24; and -04 at 16:27; A CCV was not performed;

11 samples were analyzed before the next CB and CCV were performed. Procedure was not followed.

This data is not defensible since the TCLP extraction was not done correctly. The pH of Extraction fluid \#1 was measured at 4.63. The limit is 4.88 to 4.98. The measurement of the pH after the additon of $3.5 \mathrm{ml}$ of $1 \mathrm{~N} \mathrm{HCl}$ is questionable (too low). There is no range for the temperature; There is no time noted for the Extraction Off; There are no initials and date for the crossout of the temperature.

Marsh Cabet

Reviewer Signature:
Auqust $31.1999(9 ; 43 \mathrm{am})$

Date: 


\section{GENERAL INFORMATION}

Sample Delivery Group: V653

Laboratory:

NEL

Client:

Bechtel Nevada

Requested Analysis:

Nitroaromatics

Reviewed by:

Marsha C. Webb

Date: $8 / 31 / 99$

Matrix:

Soils

II. COMPLETENESS
A. Were all deliverables submitted?
B. Are forms properly completed and legible?
C. Were all requested analyses performed by the laboratory?
D. Were the requested analytical methods used for analysis?

Extraction NA Analysis 8330

III. DATA ASSESSMENT SUMMARY

(X) A. Field and QAQC sample data have been generated in accordance with method requirements and within quality control limits.

0 B. QAVC problems were encountered during analysis of the samples. However, the data are considered acceptable.

0 . C. Significant QAVC problems were encountered during analysis of the samples. Data for the following samples may be rejected.

IV. HOLDING TIME COMPLIANCE
A. Sampled
B. ASL Received:
C. Contract Lab Received:
D. Latest Extraction:
E. Latest Date Analyzed:

Were holding time criteria met?

V. INSTRUMENT \& SAMPLE QAQC COMPLIANCE

A. INSTRUMENT QUALITY CONTROL
1. Calibrations
Blank:
Verification:
Within $3 X$ of the IDL (< reporting limit)
3 Standards minimum
$R^{2}>0.995$ or \%RSD met
ICV within $\pm 20 \%$
CCV every 10 samples within $\pm 20 \%$

B. SAMPLE ASSOCIATED QUALITY CONTROL

1. Prep blank: Were target compounds detected in field or laboratory blanks?

2. Lab Control Sample: Were recoveries within acceptable limits? $( \pm 20 \%)$

3. Samples

a. Were target compounds detected in the samples?

b. Were concentrations calculated correctly?

4. As appropriate, were the following within quality control limits:

a. MSMSD recoveries (70-130\%) MSMSD RPD (20\%)

b. Lab and/or field duplicate RPDs (<20\%)

c. Surrogate recoveries within quality control limits
Acceptable

\begin{tabular}{c}
$\frac{0-2}{1}$ \\
\hline$\frac{1}{14}$ \\
\hline 40 \\
\hline
\end{tabular}

Criteria met
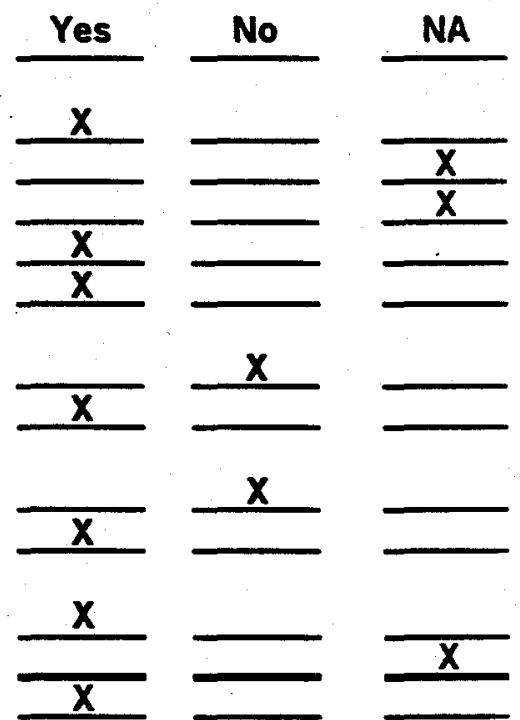


\section{INSTRUMENT \& SAMPLE QAQC CRITERIA}

6. As appropriate, were the following within quality control limits:

Criteria met

a. MSMSD recoveries and RPDs

b. Lab and/or field duplicate RPDs

$1^{*}=$ indeterminant

COMMENTS: Samples were run on 7/16-7/20; no tune for each day of analysis.

On 7/19, the $50 \mathrm{ng}$ std was run at 7:02 am; the last sample was run at 7:50 pm-outside the 12 hour tune (sample -21)

$50 \mathrm{ng}$ stds were done on each day, but the continuing calibration summary was not included in the package.

This data is not defensible since the TCLP extraction was not done correctly. The pH of Extraction fluid \#1 was measured at 4.63. The limit is 4.88 to 4.98 . The measurement of the pH after the additon of $3.5 \mathrm{ml}$ of $1 \mathrm{~N} \mathrm{HCl}$ is questionable (too low). There is no range for the temperature; There is no time noted for the Extraction Off; There are no initials and date for the crossout of the temperature. 
RADIOMETRIC ANALYSIS RESULTS

BECHTEL Analytical Services

Method Type: RAGMA
Case No.: CAU 461 TAML Verif.

SDG No.: C519

\begin{tabular}{|c|c|c|c|c|c|c|c|c|c|c|}
\hline ...... Sample No. ....... & $\begin{array}{l}\text { Sample } \\
\text { Type }\end{array}$ & $\begin{array}{l}\text { Radio- } \\
\text { nuclido }\end{array}$ & Result & $\begin{array}{l}\text { Uncer- } \\
\text { tainty }\end{array}$ & $\begin{array}{l}\text { Result } \\
\text { Units }\end{array}$ & $\begin{array}{c}\text { Analysis } \\
\text { Date }\end{array}$ & $\begin{array}{l}\text { Sample } \\
\text { Matrix }\end{array}$ & $\begin{array}{l}\text { Sample } \\
\text { Size }\end{array}$ & Method No. & Instrument ID \\
\hline TAML-RB1 & REG & No Nucl Det] & $0.00 E+00$ & $0.0 E+00$ & pCi/kg & 07.01 .99 & WATER & $500.00 \mathrm{ml}$ & E10602.PC & $08-01$ \\
\hline TAML-VER-11 & REG & K 40 & $2.79 E+04$ & $2.6 E+03$ & pCi/kg & 07-01-99 & SOlL & $714.00 \mathrm{gm}$ & E10602.PC & 06-01 \\
\hline TAML-VER-11 & REG & AA226 & $1.84 E+03$ & $2.0 E+02$ & $\mathrm{pC} / \mathrm{kg}$ & 07-01.99 & SOIL & $714.00 \mathrm{gm}$ & E10602.PC & $06-01$ \\
\hline TAML-VEA-11 & REG & TH228 & $2.11 E+03$ & $2.1 E+02$ & pCi/kg & $07-01-99$ & SOIL & $714.00 \mathrm{gm}$ & E10602.PC & 06.01 \\
\hline TAML-VER-11 & REG & TH232 & $1.88 E+03$ & $2.4 E+02$ & pCi/kg & 07-01-99 & SOIL & $714.00 \mathrm{gm}$ & E10602.PC & $06-01$ \\
\hline TAML-VER-11 & REG & U 235 & $2.96 E+02$ & $1.5 E+02$ & pCikg & $07-01-99$ & SOIL & $714.00 \mathrm{gm}$ & E10602.PC & $06-01$ \\
\hline TAML-VER-11 & REG & U 238 & $7.81 E+03$ & $3.3 E+03$ & pCi/kg & $07.01-99$ & SOlL & $714.00 \mathrm{gm}$ & E10602.PC & $06-01$ \\
\hline TAML-VER-12 & REG & K 40 & $2.70 E+04$ & $2.5 E+03$ & pCi/kg & $07-02-99$ & SOlL. & $573.00 \mathrm{gm}$ & E10602.PC & $06-01$ \\
\hline TAML-VER-12 & REG & RA226 & $1.06 E+03$ & $1.3 E+02$ & pCi/kg & 07.02 .99 & soll & $573.00 \mathrm{gm}$ & E10602.PC & $06-01$ \\
\hline TAML-VER-12 & REG & TH22B & $2.00 E+03$ & $2.0 E+02$ & pCi/kg & $07.02-99$ & SOlL & $573.00 \mathrm{gm}$ & E10602.PC & $06-01$ \\
\hline TAML-VER-12 & REG & TH232 & $1.74 E+03$ & $2.3 E+02$ & pCilkg & $07-02-99$ & SOIL & $573.00 \mathrm{gm}$ & E10602.PC & 06.01 \\
\hline TAML-VER-12 & REG & U 238 & $0.00 E+00$ & $0.0 E+00$ & $\mathrm{pC} / \mathrm{kg}$ & 07.02 .99 & SOIL & $573.00 \mathrm{gm}$ & E10602.PC & $06-01$ \\
\hline TAML-VER-13 & REG & CS137 & $5.25 E+01$ & $2.2 E+01$ & pCi/kg & $07-02-99$ & SOIL & $732.00 \mathrm{gm}$ & E10602.PC & $.06-01$ \\
\hline TAML-VER-13 & REG & K 40 & $2.96 E+04$ & $2.7 E+03$ & pCi/kg & 07.02.99 & SOIL & $732.00 \mathrm{gm}$ & E10602.PC & 06.01 \\
\hline TAML-VER-13 & AEG & RA226 & $1.20 E+03$ & $1.4 E+02$ & pCi/kg & $07.02-99$ & SOIL & $732.00 \mathrm{gm}$ & E10602.PC & $06-01$ \\
\hline
\end{tabular}

Comments: 
RADIOMETRIC ANALYSIS RESULTS

BECHTEL Analytical Services

Method Type: RAGMA
Case No.: CAU 461 TAML Verif.

SDG No.: C519

\begin{tabular}{|c|c|c|c|c|c|c|c|c|c|c|}
\hline 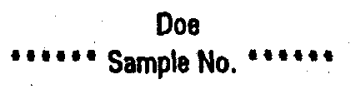 & $\begin{array}{l}\text { Sample } \\
\text { Type }\end{array}$ & $\begin{array}{l}\text { Radio- } \\
\text { nuclide }\end{array}$ & Result & $\begin{array}{l}\text { Uncer- } \\
\text { tainty }\end{array}$ & $\begin{array}{l}\text { Result } \\
\text { Units }\end{array}$ & $\begin{array}{c}\text { Analysis } \\
\text { Date }\end{array}$ & $\begin{array}{l}\text { Sample } \\
\text { Matrix }\end{array}$ & $\begin{array}{l}\text { Sample } \\
\text { Size }\end{array}$ & Method No. & Instrument ID \\
\hline TAML-VER-13 & REG & TH228 & $2.14 E+03$ & $2.2 E+02$ & $\mathrm{pCi} / \mathrm{kg}$ & 07-02-99 & SOIL. & $732.00 \mathrm{gm}$ & E10602.PC & $06-01$ \\
\hline TAML-VER-13 & REG & TH232 & $1.93 E+03$ & $2.5 E+02$ & pCi/kg & 07-02-99 & SOIL & $732.00 \mathrm{gm}$ & E10602.PC & $06-01$ \\
\hline TAML-VER-13 & REG & U 238 & $0.00 E+00$ & $0.0 E+00$ & pCi/kg & $07.02-99$ & SOIL. & $732.00 \mathrm{gm}$ & E10602.PC & $06-01$ \\
\hline TAML-VER-14 & REG & CS137 & $5.51 E+01$ & $2.2 E+01$ & pCi/kg & $07.02-99$ & SOIL & $639.00 \mathrm{gm}$ & E10602.PC & $06-01$ \\
\hline TAML-VER-14 & REG & $K 40$ & $2.73 E+04$ & $2.5 E+03$ & pCi/kg & 07.02-99 & SOIL & $639.00 \mathrm{gm}$ & E10602.PC & $06-01$ \\
\hline TAML-VER-14 & REG & RA226 & $1.08 E+03$ & $1.3 E+02$ & pCi/kg & $07.02-99$ & SOIL & $639.00 \mathrm{gm}$ & E10602.PC & $06-01$ \\
\hline TAML-VER-14 & REG & TH228 & $1.94 E+03$ & $2.0 E+02$ & pCing & $07.02-99$ & SOIL. & $639.00 \mathrm{gm}$ & E10602.PC & $06-01$ \\
\hline TAML-VER-14 & REG & TH232 & $1.78 E+03$ & $2.3 E+02$ & pCi/kg & 07.02 .99 & SOIL & $639.00 \mathrm{gm}$ & E10602.PC & $06-01$ \\
\hline TAML-VER-14 & REG & U 238 & $1.12 E+04$ & $3.7 E+03$ & $\mathrm{pC} / \mathrm{kg}$ & 07.02 .99 & SOIL & $639.00 \mathrm{gm}$ & E10602.PC & 06.01 \\
\hline TAML-VER-15 & REG & CS137 & $7.74 E+01$ & $2.5 E+01$ & $\mathrm{pCi} / \mathrm{kg}$ & $07.02-99$ & SOIL & $603.00 \mathrm{gm}$ & E10602.PC & $06-01$ \\
\hline TAML-VER-15 & REG & K 40 & $2.62 E+04$ & $2.5 E+03$ & pCi/kg & 07.02 .99 & SOIL & $603.00 \mathrm{gm}$ & E10602.PC & 06.01 \\
\hline TAML-VER-15 & REG & RA226 & $9.73 E+02$ & $1.3 E+02$ & $\mathrm{pC} / \mathrm{kg}$ & 07-02-99 & SOIL & $603.00 \mathrm{gm}$ & E10602.PC & 06.01 \\
\hline TAML-VER-15 & REG & TH228 & $1.92 E+03$ & $2.0 E+02$ & pCi/kg & $07-02-99$ & SOIL & $603.00 \mathrm{gm}$ & E10602.PC & $06-01$ \\
\hline TAML-VER-15 & REG & TH232 & $1.67 E+03$ & $2.2 E+02$ & $\mathrm{pC} / \mathrm{kg}$ & $07.02-99$ & SOIL & $603.00 \mathrm{gm}$ & E10602.PC & $06-01$ \\
\hline TAML-VER-16 & REG & U 238 & $6.54 E+03$ & $3.0 E+03$ & pCi/kg & 07.02-99 & SOlL & $603.00 \mathrm{gm}$ & E10602.PC & 06.01 \\
\hline
\end{tabular}

Comments: 
RADIOMETRIC ANALYSIS RESULTS

BECHTEL Analytical Services

Method Type: RAGMA
Case No.: CAU 461 TAML Verif.

SDG No.: C519

\begin{tabular}{|c|c|c|c|c|c|c|c|c|c|c|}
\hline ....... Sample No. ....... & $\begin{array}{l}\text { Sample } \\
\text { Type }\end{array}$ & $\begin{array}{l}\text { Radio- } \\
\text { nuclide }\end{array}$ & Result & $\begin{array}{l}\text { Uncer- } \\
\text { tainty }\end{array}$ & $\begin{array}{l}\text { Result } \\
\text { Units }\end{array}$ & $\begin{array}{c}\text { Analysis } \\
\text { Date }\end{array}$ & $\begin{array}{l}\text { Sample } \\
\text { Matrix }\end{array}$ & $\begin{array}{l}\text { Sample } \\
\text { Size }\end{array}$ & Method No. & Instrument ID \\
\hline TAML-VER-16 & REG & CS137 & $5.17 \mathrm{E}+01$ & $2.2 E+01$ & pCi/kg & $07-02.99$ & SOIL & $747.00 \mathrm{gm}$ & E10602.PC & 06-01 \\
\hline TAML-VER-16 & REG & K 40 & $2.86 E+04$ & $2.6 E+03$ & $\mathrm{pC} / \mathrm{kg}$ & $07.02-99$ & SOIL & $747.00 \mathrm{gm}$ & E10602.PC & $06-01$ \\
\hline TAML-VER-16 & REG & RA226 & $1.14 E+03$ & $1.4 E+02$ & pCi/kg & 07-02-99 & SOIL & $747.00 \mathrm{gm}$ & E10602.PC & $06-01$ \\
\hline TAML-VER-16 & REG & TH228 & $2.01 E+03$ & $2.0 E+02$ & pCi/kg & 07-02-99 & soll & $747.00 \mathrm{gm}$ & E10602.PC & $06-01$ \\
\hline TAML-VER-16 & REG & TH232 & 1.83E +03 & $2.4 E+02$ & pCikg & $07.02-99$ & SOlL & $747.00 \mathrm{gm}$ & E10602.PC & 06.01 \\
\hline TAML-VER-16 & REG & U 235 & $5.40 E+01$ & $1.4 E+02$ & pCi/kg & $07.02-99$ & SOIL & $747.00 \mathrm{gm}$ & E10602.PC & $06-01$ \\
\hline TAML-VER-16 & REG & U 238 & $7.27 E+03$ & $3.2 E+03$ & pCi/kg & $07.02-99$ & SOIL & $747.00 \mathrm{gm}$ & E10602.PC & $06-01$ \\
\hline TAML-VER-17 & REG & CS137 & $7.92 E+01$ & $2.5 E+01$ & pCi/kg & $07-03-99$ & SOIL & $701.00 \mathrm{gm}$ & E10602.PC & 06-01 \\
\hline TAML-VER-17 & REG & $K 40$ & $2.78 E+04$ & $2.6 E+03$ & pCi/kg & $07.03-99$ & SOIL & $701.00 \mathrm{gm}$ & E10602.PC & 06.01 \\
\hline TAML-VER-17 & REG & RA226 & $1.10 E+03$ & $1.4 E+02$ & pCi/kg & 07-03-99 & SOIL: & $701.00 \mathrm{gm}$ & E10602.PC & 06-01 \\
\hline TAML-VER-17 & REG & TH228 & $2.04 E+03$ & $2.1 E+02$ & pCi/kg & 07-03.99 & SOIL & $701.00 \mathrm{gm}$ & E10602.PC & $06-01$ \\
\hline TAML-VER-17 & REG & TH232 & $1.91 E+03$ & $2.4 E+02$ & pCi/kg & 07.03.99 & SOIL & $701.00 \mathrm{gm}$ & E10602.PC & $06-01$ \\
\hline TAML-VER-17 & REG & U 238 & $1.17 E+04$ & $3.4 E+03$ & pCi/kg & 07-03-99. & SOIL & $701.00 \mathrm{gm}$ & E10602.PC & $06-01$ \\
\hline TAML-VER-18 & REG & CS137 & $9.06 E+01$ & $2.6 E+01$ & pCi/kg & 07-03-99 & SOIL & $673.00 \mathrm{gm}$ & E10602.PC & 06.01 \\
\hline TAML-VER-18 & REG & K 40 & $3.07 E+04$ & $2.8 E+03$ & pCi/kg & 07-03-99 & SOIL & $673.00 \mathrm{gm}$ & E10602.PC & $06-01$ \\
\hline
\end{tabular}

Comments: 
RADIOMETRIC ANALYSIS RESULTS

BECHTEL Analytical Services

Method Type: RAGMA
Case No.: CAU 461 TAML Verif.

SDG No.: C519

\begin{tabular}{|c|c|c|c|c|c|c|c|c|c|c|}
\hline 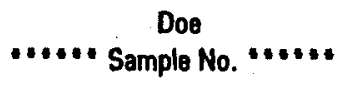 & $\begin{array}{l}\text { Sample } \\
\text { Type }\end{array}$ & $\begin{array}{l}\text { Radio- } \\
\text { nuclide }\end{array}$ & Result & $\begin{array}{l}\text { Uncer- } \\
\text { tainty }\end{array}$ & $\begin{array}{l}\text { Result } \\
\text { Units }\end{array}$ & $\begin{array}{c}\text { Analysis } \\
\text { Date }\end{array}$ & $\begin{array}{l}\text { Sample } \\
\text { Matrix }\end{array}$ & $\begin{array}{l}\text { Sample } \\
\text { Size }\end{array}$ & Method No. & Instrument 10 \\
\hline TAML-VER-18 & REG & RA226 & $1.26 E+03$ & $1.5 E+02$ & pCi/kg & 07-03-99 & SOIL & $673.00 \mathrm{gm}$ & E10602.PC & 06-01 \\
\hline TAML-VER-18 & REG & TH228 & $2.09 E+03$ & $2.1 E+02$ & pCi/kg & 07-03-99 & soll & $673.00 \mathrm{gm}$ & E10602.PC & 06-01 \\
\hline TAML-VER-18 & REG & TH232 & $1.97 \mathrm{E}+03$ & $2.5 E+02$ & pCi/kg & 07-03-99 & soll & $673.00 \mathrm{gm}$ & E10602.PC & $06-01$ \\
\hline TAML-VER-18 & REG & U 238 & $0.00 E+00$ & $0.0 E+00$ & pCi/kg & 07-03-99 & SOIL & $673.00 \mathrm{gm}$ & E10602.PC & $06-01$ \\
\hline TAML-VER-19 & REG & K 40 & $2.84 E+04$ & $2.6 E+03$ & pCi/kg & 07-03-99 & SOIL & $686.00 \mathrm{gm}$ & E10602.PC & $06-01$ \\
\hline TAML-VEA-19 & REG & AA226 & $1.12 E+03$ & $1.4 E+02$ & pCi/kg & 07.03-99 & soll & $686.00 \mathrm{gm}$ & E10602.PC & $06-01$ \\
\hline TAML-VER-19 & REG & TH228 & $2.06 E+03$ & $2.1 E+02$ & pCi/kg & 07.03-99 & SOIL & $686.00 \mathrm{gm}$ & E10602.PC & $06-01$ \\
\hline TAML-VER-19 & REG & TH 232 & $1.93 E+03$ & $2.5 E+02$ & pCi/kg & $07.03-99$ & SOIL & $686.00 \mathrm{gm}$ & E10602.PC & $06-01$ \\
\hline TAML-VER-19 & REG & U 238 & $3.95 E+03$ & $2.9 E+03$ & pCi/kg & 07.03.99 & soll & $686.00 \mathrm{gm}$ & E10602.PC & $06-01$ \\
\hline TAML-VER-20 & REG & $K 40$ & $2.50 E+04$ & $2.3 E+03$ & pCi/kg & 07.03-99 & SOll & $670.00 \mathrm{gm}$ & E10602.PC & $06-01$ \\
\hline TAML-VER-20 & REG & RA226 & $1.04 E+03$ & $1.3 E+02$ & pCirkg & 07.03-99 & SOIL & $670.00 \mathrm{gm}$ & E10602.PC & $06-01$ \\
\hline TAML-VER-20 & REG & TH228 & $1.84 E+03$ & $1.9 E+02$ & pCi/kg & 07.03-99 & soll & $670.00 \mathrm{gm}$ & E10602.PC & $06-01$ \\
\hline TAML-VER-20 & REG & TH232 & $1.72 E+03$ & $2.3 E+02$ & $\mathrm{pCi} / \mathrm{kg}$ & $07.03-99$ & SOIL & $670.00 \mathrm{gm}$ & E10602.PC & $06-01$ \\
\hline TAML-VER-20 & REG & U 238 & $0.00 E+00$ & $0.0 E+00$ & pCi/kg & 07.03-99 & SOIL & $670.00 \mathrm{gm}$ & E10602.PC & 06.01 \\
\hline TAML-VEA-23 & REG & CS137 & $3.66 E+02$ & $5.3 E+01$ & pCl/kg & $07-06-99$ & SOIL & $558.00 \mathrm{gm}$ & E10602.PC & $06-01$ \\
\hline
\end{tabular}

Comments: 
RADIOMETRIC ANALYSIS RESULTS

BECHTEL Analytical Services

Method Type: RAGMA
Case No.: CAU 461 TAML Verif.

SDG No.: C519

\begin{tabular}{|c|c|c|c|c|c|c|c|c|c|c|}
\hline ...... Sample No. ...... & $\begin{array}{l}\text { Sample } \\
\text { Type }\end{array}$ & $\begin{array}{l}\text { Radio- } \\
\text { nuclide }\end{array}$ & Result & $\begin{array}{l}\text { Uncer- } \\
\text { tainty }\end{array}$ & $\begin{array}{l}\text { Result } \\
\text { Units }\end{array}$ & $\begin{array}{c}\text { Analysis } \\
\text { Date }\end{array}$ & $\begin{array}{l}\text { Sample } \\
\text { Matrix }\end{array}$ & $\begin{array}{l}\text { Sample } \\
\text { Size }\end{array}$ & Method No. & Instrument ID \\
\hline TAML-VER-23 & AEG & K 40 & $3.22 E+04$ & $2.9 \mathrm{E}+03$ & pCi/kg & 07-06-99 & SOIL & $558.00 \mathrm{gm}$ & E10602.PC & $06-01$ \\
\hline TAML-VER-23 & REG & RA226 & $1.37 E+03$ & $1.6 E+02$ & $\mathrm{pC} / \mathrm{kg}$ & 07-06-99 & SOIL & $558.00 \mathrm{gm}$ & E10602.PC & 06.01 \\
\hline TAML-VER-23 & REG & TH228 & $2.18 E+03$ & $2.2 E+02$ & pCi/kg & $07.06-99$ & SOIL & $558.00 \mathrm{gm}$ & E10602.PC & $06-01$ \\
\hline TAML-VER-23 & REG & TH232 & $2.04 E+03$ & $2.6 E+02$ & pCi/kg & 07.06 .99 & SOIL & $558.00 \mathrm{gm}$ & E10602.PC & $06-01$ \\
\hline TAML-VER-23 & REG & U 238 & $4.54 E+03$ & $3.1 E+03$ & pCi/kg & 07-06-99 & SOIL. & $558.00 \mathrm{gm}$ & E10602.PC & $06-01$ \\
\hline TAML-VER-24 & REG & BE7 & 6.34E-01 & $3.0 \mathrm{E}-01$ & pCi/kg & 07-06-99 & soll & $567.00 \mathrm{gm}$ & E10602.PC & $06-01$ \\
\hline TAML-VER-24 & REG & CS137 & $3.63 E+01$ & $2.3 E+01$ & pCi/kg & $07.06-99$ & SOIL & $567.00 \mathrm{gm}$ & E10602.PC & 06.01 \\
\hline TAML-VER-24 & REG & K 40 & $2.99 E+04$ & $2.8 E+03$ & pCi/kg & 07-06-99 & SOIL & $567.00 \mathrm{gm}$ & E10602.PC & $06-01$ \\
\hline TAML-VER-24 & REG & RA226 & $1.20 E+03$ & $1.5 E+02$ & pCi/kg & $07-06-99$ & SOIL & $567.00 \mathrm{gm}$ & E10602.PC & 06-01 \\
\hline TAML-VER-24 & REG & TH228 & $2.11 E+03$ & $2.1 E+02$ & pCi/kg & $07-06-99$ & SOIL & $567.00 \mathrm{gm}$ & E10602.PC & 06.01 \\
\hline TAML-VER-24 & REG & TH232 & $1.84 E+03$ & $2.4 E+02$ & pCi/kg & 07-06-99 & SOIL & $567.00 \mathrm{gm}$ & E10602.PC & $06-01$ \\
\hline TAML-VER-24 & REG & U 238 & $7.97 E+03$ & $3.3 E+03$ & pCilkg & $.07-06-99$ & SOIL & $567.00 \mathrm{gm}$ & E10602.PC & $06-01$ \\
\hline TAML-VER-25 & REG & CS137 & $9.97 E+01$ & $2.7 E+01$ & pCi/kg & 07-06-99 & soll. & $558.00 \mathrm{gm}$ & E10602.PC & $06-01$ \\
\hline TAML-VER-25 & REG & K 40 & $3.14 E+04$ & $2.9 E+03$ & pCi/kg & 07-06.99 & SOIL & $558.00 \mathrm{gm}$ & $E 10602 . P C$ & $06-01$ \\
\hline TAML-VER-25 & REG & RA226 & $1.29 E+03$ & $1.5 E+02$ & pCi/kg & 07-06-99 & soll. & $558.00 \mathrm{gm}$ & E10602.PC & $06-01$ \\
\hline
\end{tabular}

Comments: 
RADIOMETRIC ANALYSIS RESULTS

\section{BECHTEL Analytical Services}

Method Type: RAGMA
Case No.: CAU 461 TAML Verif.

SDG No.: C519

\begin{tabular}{|c|c|c|c|c|c|c|c|c|c|c|}
\hline ...... Sample No. ...... & $\begin{array}{l}\text { Sample } \\
\text { Type }\end{array}$ & $\begin{array}{l}\text { Radio- } \\
\text { nuclide }\end{array}$ & Result & $\begin{array}{l}\text { Uncer- } \\
\text { tainty }\end{array}$ & $\begin{array}{l}\text { Result } \\
\text { Units }\end{array}$ & $\begin{array}{c}\text { Analysis } \\
\text { Date }\end{array}$ & $\begin{array}{l}\text { Sample } \\
\text { Matrix }\end{array}$ & $\begin{array}{l}\text { Sample } \\
\text { Size }\end{array}$ & Method No. & Instrument ID \\
\hline TAML-VER-25 & REG & TH228 & $2.12 E+03$ & $2.2 E+02$ & pCi/kg & 07-06-99 & SOlL & $558.00 \mathrm{gm}$ & E10602.PC & $06-01$ \\
\hline TAML-VER-25 & REG & TH232 & $2.08 E+03$ & $2.6 E+02$ & pCi/kg & 07-06-99 & SOIL & $558.00 \mathrm{gm}$ & E10602.PC & 06-01 \\
\hline TAML-VER-25 & REG & U 238 & $8.64 E+03$ & $3.5 E+03$ & pCi/kg & 07-06-99 & SOIL & $558.00 \mathrm{gm}$ & E10602.PC & 06-01 \\
\hline TAML-VER-21 & AEG & K 40 & $9.15 E+03$ & $8.8 E+02$ & pCi/kg & 07.01-99 & SOIL & $577.00 \mathrm{gm}$ & E10602.PC & $07-01$ \\
\hline TAML-VER-21 & REG & RA226 & $1.07 E+03$ & $1.4 E+02$ & pCi/kg & $07.01-99$ & soll & $577.00 \mathrm{gm}$ & E10602.PC & 07.01 \\
\hline TAML-VER-21 & REG & TH228 & $2.04 E+03$ & $2.1 E+02$ & $\mathrm{pCi} / \mathrm{kg}$ & $07.01-99$ & SOIL & $577.00 \mathrm{gm}$ & E10602.PC & 07.01 \\
\hline TAML-VER-21 & AEG & TH232 & $1.79 E+03$ & $2.4 E+02$ & pCilkg & $07.01-99$ & soll & $577.00 \mathrm{gm}$ & E10602.PC & 07.01 \\
\hline TAML-VER-21 & REG & U 238 & $4.73 E+03$ & $3.4 E+03$ & $\mathrm{pC}$ Cikg & $07.01-99$ & SOll. & $577.00 \mathrm{gm}$ & E10602.PC & 07.01 \\
\hline TAML-VER-22 & REG & K 40 & 3.17E+04 & $3.0 E+03$ & pCi/kg & $07.06-99$ & SOIL & $745.00 \mathrm{gm}$ & E10602.PC & 07.01 \\
\hline TAML-VER-22 & REG & RA226 & $2.44 E+03$ & $2.6 E+02$ & pCi/kg & 07.06-99 & SOIL & $745.00 \mathrm{gm}$ & E10602.PC & $07-01$ \\
\hline TAML-VER-22 & REG & TH228 & $2.44 E+03$ & $2.5 E+02$ & pCi/kg & 07.06-99 & SOIL & $745.00 \mathrm{gm}$ & E10602.PC & 07.01 \\
\hline TAML-VER-22 & REG & TH232 & $2.07 E+03$ & $2.7 E+02$ & pCi/kg & 07-06-99 & SOIL & $745.00 \mathrm{gm}$ & E10602.PC & 07.01 \\
\hline TAML-VER-22 & REG & U 238 & $5.55 E+03$ & $3.7 E+03$ & $\mathrm{pCi} / \mathrm{kg}$ & $07-06-99$ & SOIL & $745.00 \mathrm{gm}$ & E10602.PC & $07-01$ \\
\hline TAML-VER-01 & REG & $K 40$ & $3.21 E+04$ & $3.1 E+03$ & $\mathrm{pCi} / \mathrm{kg}$ & $07.01-99$ & SOIL & $774.00 \mathrm{gm}$ & E10602.PC & 05-01 \\
\hline TAML-VER-01 & REG & RA226 & $2.22 E+03$ & $2.4 E+02$ & pCi/kg & 07.01 .99 & SOIL & $774.00 \mathrm{gm}$ & E10602.PC & $05-01$ \\
\hline
\end{tabular}

Comments: 
RADIOMETRIC ANALYSIS RESULTS

BECHTEL Analytical Services

Method Type: RAGMA
Case No.: CAU 461 TAML Verif.

SDG No.: C519

\begin{tabular}{|c|c|c|c|c|c|c|c|c|c|c|}
\hline ....... Sample No. ....... & $\begin{array}{l}\text { Sample } \\
\text { Type }\end{array}$ & $\begin{array}{l}\text { Radio- } \\
\text { nuclide }\end{array}$ & Result & $\begin{array}{l}\text { Uncer- } \\
\text { tainty }\end{array}$ & $\begin{array}{l}\text { Result } \\
\text { Units }\end{array}$ & $\begin{array}{c}\text { Analysis } \\
\text { Date }\end{array}$ & $\begin{array}{l}\text { Sample } \\
\text { Matrix }\end{array}$ & $\begin{array}{l}\text { Sample } \\
\text { Size }\end{array}$ & Method No. & Instrument ID \\
\hline TAML-VER-01 & REG & TH228 & $2.76 E+03$ & $2.7 E+02$ & pCi/kg & 07.01-99 & soll & $774.00 \mathrm{gm}$ & E10602.PC & $05-01$ \\
\hline TAML-VER-01 & REG & TH232 & $2.24 E+03$ & $3.1 E+02$ & pCi/kg & 07.01-99 & SOIL & $774.00 \mathrm{gm}$ & E10602.PC & $05-01$ \\
\hline TAML-VER-01 & REG & U 238 & $0.00 E+00$ & $0.0 E+00$ & pCi/kg & $07-01-99$ & soll & $774.00 \mathrm{gm}$ & E10602.PC & $05-01$ \\
\hline TAML-VER-02 & REG & K 40 & $3.00 E+04$ & $2.9 E+03$ & pCi/kg & 07.02 .99 & SOIL & $678.00 \mathrm{gm}$ & E10602.PC & $05-01$ \\
\hline TAML-VER-02 & REG & RA226 & $1.80 E+03$ & $2.1 E+02$ & $\mathrm{pCl} / \mathrm{kg}$ & $07-02-99$ & SOIL & $678.00 \mathrm{gm}$ & E10602.PC & 05-01 \\
\hline TAML-VER-02 & REG & TH228 & $2.36 E+03$ & $2.4 E+02$ & pCi/kg & 07.02-99 & SOIL & $678.00 \mathrm{gm}$ & E10602.PC & $05-01$ \\
\hline TAML-VER-02 & REG & TH232 & $1.90 E+03$ & $2.7 E+02$ & pCi/kg & $07-02-99$ & SOIL & $678.00 \mathrm{gm}$ & E10602.PC & $05-01$ \\
\hline TAML-VER-02 & REG & U 238 & $0.00 E+00$ & $0.0 E+00$ & pCi/kg & $07-02-99$ & SOIL & $678.00 \mathrm{gm}$ & E10602.PC & $05-01$ \\
\hline TAML-VER-03 & REG & K 40 & 3.15E +04 & $3.1 E+03$ & pCi/kg & 07-02-99 & SOIL & $770.00 \mathrm{gm}$ & E10602.PC & 05-01 \\
\hline TAML-VER-03 & REG & RA226 & $1.61 E+03$ & $1.9 E+02$ & pCi/kg & $07-02-99$ & SOIL & $770.00 \mathrm{gm}$ & E10602.PC & $05-01$ \\
\hline TAML-VER-03 & REG & TH228 & $2.44 E+03$ & $2.5 E+02$ & pCi/kg & 07-02-99 & SOIL & $770.00 \mathrm{gm}$ & E10602.PC & 05-01 \\
\hline TAML-VER-03 & REG & TH232 & $2.05 E+03$ & $2.9 E+02$ & pCi/kg & $07-02-99$ & Soll & $770.00 \mathrm{gm}$ & E10602.PC & $05-01$ \\
\hline TAML-VER-03 & REG & U 238 & $0.00 E+00$ & $0.0 E+00$ & pCi/kg & 07.02 .99 & SOIL & $770.00 \mathrm{gm}$ & E10602.PC & 05-01 \\
\hline TAML-VER-04 & REG & K 40 & $3.07 E+04$ & $3.0 E+03$ & pCi/kg & 07.02.99 & SOIL & $751.00 \mathrm{gm}$ & E10602.PC & 05.01 \\
\hline TAML-VER-04 & REG & RA226 & $1.87 E+03$ & $2.1 E+02$ & pCi/kg & 07-02-99 & SOIL & $751.00 \mathrm{gm}$ & E10602.PC & 05-01 \\
\hline
\end{tabular}

Comments: 
RADIOMETRIC ANALYSIS RESULTS

BECHTEL Analytical Services

Method Type: RAGMA
Case No.: CAU 461 TAML Verif.

SDG No.: C519

\begin{tabular}{|c|c|c|c|c|c|c|c|c|c|c|}
\hline ....... Samplo No. ....... & $\begin{array}{l}\text { Sample } \\
\text { Type }\end{array}$ & $\begin{array}{l}\text { Radio- } \\
\text { nuclide }\end{array}$ & Result & $\begin{array}{l}\text { Uncer- } \\
\text { tointy }\end{array}$ & $\begin{array}{l}\text { Result } \\
\text { Units }\end{array}$ & $\begin{array}{l}\text { Analysis } \\
\text { Date }\end{array}$ & $\begin{array}{l}\text { Sample } \\
\text { Matrix }\end{array}$ & $\begin{array}{l}\text { Sample } \\
\text { Size }\end{array}$ & Mothod No. & Instrument ID \\
\hline TAML-VER-04 & REG & TH228 & $2.43 E+03$ & $2.5 E+02$ & pci/kg & 07-02-99 & SOIL & $751.00 \mathrm{gm}$ & E10602.PC & 05.01 \\
\hline TAML-VER-04 & REG & TH232 & $1.96 E+03$ & $2.8 E+02$ & pCi/kg & 07.02 .99 & SOIL' & $751.00 \mathrm{gm}$ & E10602.PC & 05.01 \\
\hline TAML-VER-04 & REG & U 238 & $0.00 E+00$ & $0.0 E+00$ & pCikkg & 07.02-99 & SOIL & $751.00 \mathrm{gm}$ & E10602.PC & $05-01$ \\
\hline TAML-VER-05 & REG & K 40 & $2.94 E+04$ & $2.9 E+03$ & pCi/kg & $07-02-99$ & SOIL & $665.00 \mathrm{gm}$ & E10602.PC & $05-01$ \\
\hline TAML-VER-05 & REG & RA226 & $1.55 E+03$ & $1.9 E+02$ & pCi/kg & 07.02-99 & SOIL & $665.00 \mathrm{gm}$ & E10602.PC & 05.01 \\
\hline TAML-VER-05 & REG & TH228 & $2.32 E+03$ & $2.4 E+02$ & pCilkg & $07.02-99$ & SOIL & $665.00 \mathrm{gm}$ & E10602.PC & 05.01 \\
\hline TAML-VER-05 & REG & TH232 & $1.93 E+03$ & $2.7 E+02$ & pCi/kg & 07-02-99 & SOIL & $665.00 \mathrm{gm}$ & E10602.PC & 05.01 \\
\hline TAML-VER-05 & REG & U 238 & $0.00 E+00$ & $0.0 E+00$ & pCi/kg & 07.02.99 & SOIL & $665.00 \mathrm{gm}$ & E10602.PC & 05.01 \\
\hline TAML-VER-06 & REG & K 40 & $2.65 E+04$ & $2.6 E+03$ & pCi/kg & 07.02-99 & SOIL & $624.00 \mathrm{gm}$ & E10602.PC & 05-01 \\
\hline TAML-VER-06 & REG & RA226 & $1.15 E+03$ & $1.5 E+02$ & pCinkg & 07.02-99 & SOIL & $624.00 \mathrm{gm}$ & E10602.PC & 05.01 \\
\hline TAML-VER-06 & REG & TH228 & $2.18 E+03$ & $2.3 E+02$ & pCi/kg & 07.02.99 & SOIL & $624.00 \mathrm{gm}$ & E10602.PC & 05.01 \\
\hline TAML-VER-06 & REG & TH232 & $1.77 E+03$ & $2.6 E+02$ & pCi/kg & $07.02-99$ & SOIL & $624.00 \mathrm{gm}$ & E10602.PC & 05.01 \\
\hline TAML-VER-06 & REG & U 238 & $0.00 E+00$ & $0.0 E+00$ & pCi/kg & $07.02-99$ & SOIL & $624.00 \mathrm{gm}$ & E10602.PC & 05.01 \\
\hline TAML-VER-07 & REG & K 40 & $3.31 E+04$ & $3.2 E+03$ & pCi/kg & 07.03-99 & SOIL & $759.00 \mathrm{gm}$ & E10602.PC & 05.01 \\
\hline TAML-VER-07 & REG & RA226 & $1.97 E+03$ & $2.2 E+02$ & pCi/kg & 07.03-99 & SOIL & $759.00 \mathrm{gm}$ & E10602.PC & 05.01 \\
\hline
\end{tabular}

Comments: 
RADIOMETRIC ANALYSIS RESULTS

BECHTEL Analytical Services

Case No.: CAU 461 TAML Verif.

Method Type: RAGMA

SDG No.: C519

\begin{tabular}{|c|c|c|c|c|c|c|c|c|c|c|}
\hline ...... Sample No. ....... & $\begin{array}{l}\text { Sample } \\
\text { Type }\end{array}$ & $\begin{array}{l}\text { Radio- } \\
\text { nuclide }\end{array}$ & Result & $\begin{array}{l}\text { Uncer- } \\
\text { teinty }\end{array}$ & $\begin{array}{l}\text { Result } \\
\text { Units }\end{array}$ & $\begin{array}{l}\text { Analysis } \\
\text { Date }\end{array}$ & $\begin{array}{l}\text { Sample } \\
\text { Matrix }\end{array}$ & $\begin{array}{l}\text { Sample } \\
\text { Size }\end{array}$ & Method No. & Instrument ID \\
\hline TAML-VER-07 & REG & TH228 & $2.65 E+03$ & $2.7 E+02$ & pCi/kg & 07-03-99 & soll & $759.00 \mathrm{gm}$ & E10602.PC & 05-01 \\
\hline TAML-VER-07 & REG & TH232 & $2.20 E+03$ & $3.0 E+02$ & pCi/kg & 07-03-99 & SOIL & $759.00 \mathrm{gm}$ & E10602.PC & 05-01 \\
\hline TAML-VER-07 & REG & U 238 & $0.00 E+00$ & $0.0 E+00$ & pCi/kg & 07-03-99 & SOIL & $759.00 \mathrm{gm}$ & E10602.PC & $05-01$ \\
\hline TAML-VER-08 & REG & K 40 & $2.99 \mathrm{E}+04$ & $2.9 E+03$ & pCi/kg & 07-03-99 & SOIL & $621.00 \mathrm{gm}$ & E10602.PC & $05-01$ \\
\hline TAML-VER-08 & REG & RA226 & $3.56 E+03$ & $3.6 E+02$ & pCi/kg & 07-03-99 & SOIL & $621.00 \mathrm{gm}$ & E10602.PC & $05-01$ \\
\hline TAML-VER-08 & REG & TH228 & $2.25 E+03$ & $2.4 E+02$ & pCikg & 07-03-99 & SOIL & $621.00 \mathrm{gm}$ & E10602.PC & $05-01$ \\
\hline TAML-VER-08 & REG & TH232 & $1.90 E+03$ & $2.8 E+02$ & pCi/kg & 07-03-99 & SOIL & $621.00 \mathrm{gm}$ & E10602.PC & $05-01$ \\
\hline TAML-VER-08 & REG & U 235 & $6.43 E+02$ & $2.5 E+02$ & pCi/kg & 07-03-99 & SOIL & $621.00 \mathrm{gm}$ & E10602.PC & 05.01 \\
\hline TAML-VER-08 & REG & U 238 & $1.91 E+04$ & $6.0 E+03$ & pCi/kg & 07-03-99 & SOIL & $621.00 \mathrm{gm}$ & E10602.PC & $05-01$ \\
\hline TAML-VER-09 & REG & K 40 & $3.32 E+04$ & $3.2 E+03$ & pCi/kg & 07.03-99 & SOIL & $773.00 \mathrm{gm}$ & E10602.PC & 05-01 \\
\hline TAML-VER-09 & REG & RA226 & $2.32 E+03$ & $2.5 E+02$ & pCi/kg & 07-03-99 & SOIL & $773.00 \mathrm{gm}$ & E10602.PC & 05-01 \\
\hline TAML-VER-09 & REG & TH228 & $2.70 E+03$ & $2.7 E+02$ & pCilkg & 07-03-99 & SOIL & $773.00 \mathrm{gm}$ & E10602.PC & $05-01$ \\
\hline TAML-VER-09 & REG & TH232 & $2.12 E+03$ & $3.0 E+02$ & pCi/kg & 07-03-99 & SOIL & $773.00 \mathrm{gm}$ & E10602.PC & $05-01$ \\
\hline TAML-VER-09 & REG & U 238 & $1.34 E+04$ & $4.7 E+03$ & pCi/kg & 07.03.99 & SOIL & $773.00 \mathrm{gm}$ & E10602.PC & $05-01$ \\
\hline TAML-VER-10 & REG & K 40 & $3.27 E+04$ & $3.2 E+03$ & pCilkg & 07-03-99 & SOIL & $753.00 \mathrm{gm}$ & E10602.PC & $05-01$ \\
\hline
\end{tabular}

Comments: 
RADIOMETRIC ANALYSIS RESULTS

BECHTEL Analytical Services

Method Type: RAGMA
Case No.: CAU 461 TAML Verif.

SDG No.: C519

\begin{tabular}{|c|c|c|c|c|c|c|c|c|c|c|}
\hline $\begin{array}{c}\text { Doe } \\
\ldots . . . . \text { Sample No. ...... }\end{array}$ & $\begin{array}{l}\text { Sample } \\
\text { Type }\end{array}$ & $\begin{array}{l}\text { Radio- } \\
\text { nuclide }\end{array}$ & Result & $\begin{array}{l}\text { Uncer- } \\
\text { tainty }\end{array}$ & $\begin{array}{l}\text { Result } \\
\text { Units }\end{array}$ & $\begin{array}{c}\text { Analysis } \\
\text { Date }\end{array}$ & $\begin{array}{l}\text { Sample } \\
\text { Matrix }\end{array}$ & $\begin{array}{l}\text { Sample } \\
\text { Size }\end{array}$ & Mothod No. & Instrument ID \\
\hline TAML-VER-10 & REG & RA226 & $2.42 E+03$ & $2.6 E+02$ & pCi/kg & 07-03-99 & SOIL & $753.00 \mathrm{gm}$ & E10602.PC & 05-01 \\
\hline TAML-VER-10 & REG & TH228 & $2.83 E+03$ & $2.8 E+02$ & pCi/kg & 07-03-99 & SOIL & $753.00 \mathrm{gm}$ & E10602.PC & $05-01$ \\
\hline TAML-VER-10 & REG & TH232 & $2.22 E+03$ & $3.1 E+02$ & pCi/kg & 07-03-99 & soll. & $753.00 \mathrm{gm}$ & E10602.PC & 05-01. \\
\hline TAML-VER-10 & REG & U 238 & $0.00 E+00$ & $0.0 E+\infty 0$ & pCi/kg & 07-03-99 & SOIL & $753.00 \mathrm{gm}$ & E10602.PC & $05-01$ \\
\hline TAML-VER-26 & REG & K 40 & $2.45 E+04$ & $2.5 E+03$ & pCi/kg & 07-06-99 & SOIL & $699.00 \mathrm{gm}$ & E10602.PC & $05-01$ \\
\hline TAML-VER-26 & REG & RA226 & $1.09 E+03$ & $1.4 E+02$ & pCi/kg & 07-06-99 & SOIL & $699.00 \mathrm{gm}$ & E10602.PC & 05-01 \\
\hline TAML-VER-26 & REG & TH228 & $2.02 E+03$ & $2.1 E+02$ & pCi/kg & 07-06-99 & SOIL & $699.00 \mathrm{gm}$ & E10602.PC & $05-01$ \\
\hline TAML-VER-26 & REG & TH232 & $1.59 E+03$ & $2.4 E+02$ & pCi/kg & 07-06-99 & SOIL & $699.00 \mathrm{gm}$ & E10602.PC & 05.01 \\
\hline TAML-VER-26 & REG & U 235 & $1.16 E+02$ & $1.4 E+02$ & pCi/kg & 07-06-99 & Soll & $699.00 \mathrm{gm}$ & E10602.PC & 05.01 \\
\hline TAML-VER-26 & REG & U 238 & $7.82 E+03$ & $3.9 E+03$ & pCi/kg & $07-06-99$ & SOIL & $699.00 \mathrm{gm}$ & E10602.PC & 05-01 \\
\hline TAML-VER-27 & REG & K 40 & $2.37 E+04$ & $2.4 E+03$ & pCi/kg & $07-06-99$ & SOIL & $669.00 \mathrm{gm}$ & E10602.PC & 05.01 \\
\hline TAML-VER-27 & REG & RA226 & $1.32 E+03$ & $1.6 E+02$ & pCilkg & $07.06-99$ & SOIL & $669.00 \mathrm{gm}$ & E10602.PC & $05-01$ \\
\hline TAML-VER-27 & REG & TH228 & $2.10 E+03$ & $2.2 E+02$ & $\mathrm{pCi} / \mathrm{kg}$ & 07.06-99 & SOIL & $669.00 \mathrm{gm}$ & E10602.PC & $05-01$ \\
\hline TAML-VER-27 & REG & TH232 & $1.73 E+03$ & $2.5 E+02$ & pCi/kg & $07.06-99$ & Soll & $669.00 \mathrm{gm}$ & E10602.PC & $05-01$ \\
\hline TAML-VER-27 & REG & U 235 & $5.35 E+02$ & $1.8 E+02$ & pCi/kg & 07.06-99 & SOIL & $669.00 \mathrm{gm}$ & E10602.PC & 05-01 \\
\hline
\end{tabular}

Comments: 
RADIOMETRIC ANALYSIS RESULTS

BECHTEL Analytical Services

Method Type: RAGMA
Case No.: CAU 461 TAML Verif.

SDG No.: C519

\begin{tabular}{|c|c|c|c|c|c|c|c|c|c|c|}
\hline 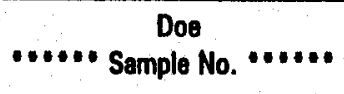 & $\begin{array}{l}\text { Sample } \\
\text { Type }\end{array}$ & $\begin{array}{l}\text { Radio- } \\
\text { nuclide }\end{array}$ & Result & $\begin{array}{l}\text { Uncer- } \\
\text { tainty }\end{array}$ & $\begin{array}{l}\text { Result } \\
\text { Units }\end{array}$ & $\begin{array}{c}\text { Analysis } \\
\text { Date }\end{array}$ & $\begin{array}{l}\text { Sample } \\
\text { Matrix }\end{array}$ & $\begin{array}{l}\text { Sample } \\
\text { Sizo }\end{array}$ & Method No. & Instrument ID \\
\hline TAML-VER-27 & REG & U 238 & $2.38 E+04$ & $5.7 E+03$ & pCi/kg & 07-06-99 & SOlL. & $669.00 \mathrm{gm}$ & E10602.PC & 05.01 \\
\hline TAML-VER-28 & AEG & K 40 & $2.95 E+04$ & $2.9 E+03$ & $\mathrm{pC} / \mathrm{kg}$ & 07-06-99 & SOIL & $660.00 \mathrm{gm}$ & E10602.PC & 05.01 \\
\hline TAML-VER-28 & REG & AA226 & 1.18E+03 & $1.5 E+02$ & $\mathrm{pCl} / \mathrm{kg}$ & $07-06-99$ & SOIL & $660.00 \mathrm{gm}$ & E10602.PC & 05.01 \\
\hline TAML-VER-28 & REG & TH228 & $2.51 E+03$ & $2.6 E+02$ & $\mathrm{pC} / \mathrm{kg}$ & $07-06-99$ & SOIL & $660.00 \mathrm{gm}$ & E10602.PC & $05-01$ \\
\hline TAML-VER-28 & AEG & $\mathrm{TH} 232$ & $1.86 E+03$ & $2.7 E+02$ & $\mathrm{pC} / \mathrm{kg}$ & $07-06-99$ & SOIL & $660.00 \mathrm{gm}$ & E10602.PC & 05-01 \\
\hline TAML-VER-28 & REG & U 238 & $0.00 E+00$ & $0.0 E+00$ & PCi/kg & 07-06-99 & SOIL & $660.00 \mathrm{gm}$ & E10602.PC & $05-01$ \\
\hline
\end{tabular}

Comments: 

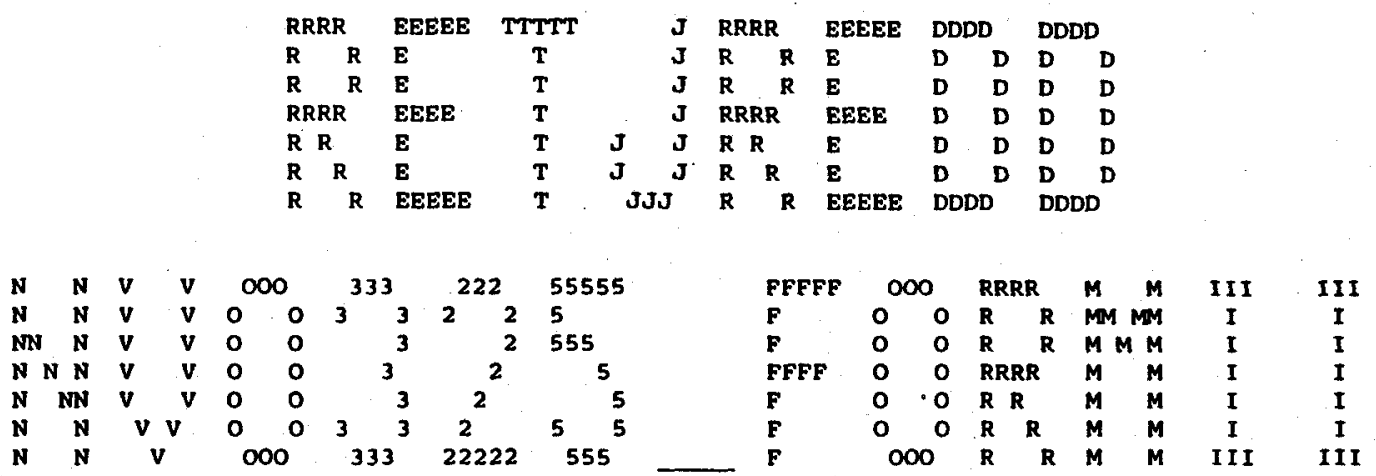

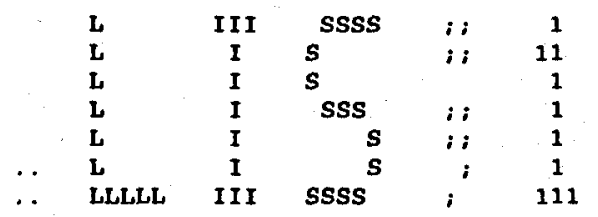

File PLUCKY1 \$SDIA1: [ESDLAB.RETJREDD] NVO325 FORMII.LIS; 1 (14812,71,0), last revised on 9-AUG-1999 09:08, is a 19 block sequent1al file owned by UIC [ESDLAB, RETJREDD]. The records are variable length with FORTRAN (FTN) carriage control. The longest record is 114 bytes.

Job NVO325_FORMII (435) queued to A23_650_22_HPLN3 on 9-AUG-1999 09:08 by user RETJREDD, UIC [ESDLAB, RETJREDD], Under aCCOUnt at priority 100. started on printer NLP6: on 9-ÄUG-1999 09:15 from queue A23_650_22_HPLJ3.

KKKKKKKKKK 111111111111111111111111111111111111111112111111111111111111111111111111111111111111111111111111111111111111 KKKKKKKKKK 111111111111111111111111 Digital Equipment Corporation - VAX/VMS Version V5.5-2 1111111111111111111111111 
RADIOMETRIC QA/OC RESULTS

BECHTEL Analytical Services

Method Type: RAGMA
Case No.: CAU 461 TAML Verif.

SDG No.: C519

\begin{tabular}{|c|c|c|c|c|c|c|c|c|c|c|}
\hline $\begin{array}{l}\text { Control Sample } \\
\text { Identification }\end{array}$ & $\begin{array}{l}\text { Sample } \\
\text { Type }\end{array}$ & $\begin{array}{l}\text { Radio- } \\
\text { nuclido }\end{array}$ & $\begin{array}{c}\text { Peak Energy } \\
\mathrm{KoV}\end{array}$ & $\begin{array}{l}\text { Measured } \\
\text { Value }\end{array}$ & $\begin{array}{l}\text { Known } \\
\text { Value }\end{array}$ & \% Diff. & Units & Mothod No. & Instrument ID & $\begin{array}{l}\text { Date of } \\
\text { Check }\end{array}$ \\
\hline QA BKG WATER UOB & CNTPL & No Nuclide & Not Appl. & $0.00 E+00$ & $0.00 E+00$ & & & E10602.PC & 08-01 & 070699 \\
\hline OA BKG WATER UOB & CNTRL & AM241 & Not Appl. & $0.00 E+00$ & $0.00 E+00$ & & pCi/kg & E10602.PC & $08-01$ & 070699 \\
\hline OA BKG WATER UOB. & CNTRL & 10060 & Not Appl. & $0.00 E+00$ & $0.00 E+00$ & & pCi/kg & E10602.PC & 08-01 & 070699 \\
\hline OA BKG WATER UOB & CNTRL. & CS137 & Not Appl. & $0.00 E+\infty 0$ & $0.00 E+00$ & & pCikg & E10602.PC & 08-01 & 070699 \\
\hline QA SP:OAW-8A & CNTRL & AM241 & 69.2 & $3.07 E+03$ & $3.29 E+03$ & -6.7 & pCikg & E10602.PC & 08-01 & 070799 \\
\hline QA SP:QAW-8A & CNTRL & 10060 & 1173.4 & $5.19 E+03$ & $5.36 E+03$ & -3.2 & pCi/kg & E10602.PC & $08-01$ & 070799 \\
\hline QA SP:OAW-BA & CNTPL & CS137 & 661.8 & $3.84 E+03$ & $3.96 \mathrm{E}+03$ & -3.1 & pCikg & E10602.PC & $08-01$ & 070799 \\
\hline QA BKG EMPTY BTL U06 & CNTRL & No Nuclide & Not Appl. & $0.00 E+\infty 0$ & $0.00 E+00$ & & pCi/kg & E10602.PC & $06-01$ & 070699 \\
\hline OA BKG EMPTY BTL U06 & CNTRL & AM241 & Not Appl. & $0.00 E+00$ & $0.00 E+00$ & & pCinkg. & E10602.PC & 06-01 & $070699^{\circ}$ \\
\hline QA BKG EMPTY BTL U06 & CNTRL & Co60 & Not Appl. & $0.00 E+\infty 0$ & $0.00 E+00$ & & pCi/kg & E10602.PC & $06-01$ & 070699 \\
\hline QA BKG EMPTY BTL U06 & CNTRL & CS137 & Not Appl. & $0.00 E+00$ & $0.00 E+\infty 0$ & & pCi/kg & E10602.PC & $06-01$ & 070699 \\
\hline QA SP:NAS-A0269 & CNTRL & AM241 & 59.7 & $1.73 E+05$ & $1.80 E+05$ & -3.8 & pCikg & E10602.PC & $06-01$ & 070699 \\
\hline QA SP:NAS-A0269 & CNTRL & |c060 & 1173.4 & $3.41 E+05$ & $3.32 E+05$ & 2.7. & $\mathrm{pCi} / \mathrm{kg}$ & E10602.PC & $06-01$ & 070699 \\
\hline QA SP:NAS-A0269 & CNTRL & CS137 & 661.7 & $2.29 E+05$ & $2.25 E+05$ & 1.8 & pCikg & E10602.PC & $06-01$ & 070699 \\
\hline QA BKG EMPTY BTL UOT & CNTRL & No Nuclide & Not Appl. & $0.00 E+00$ & $0.00 E+\infty$ & & pCikg & E10602.PC & $07-01$ & 070699 \\
\hline
\end{tabular}

Comments: 
RADIOMETRIC QA/OC RESULTS

BECHTEL Analytical Services

Method Type: RAGMA
Case No.: CAU 461 TAML Verif.

SDG No.: C519

\begin{tabular}{|c|c|c|c|c|c|c|c|c|c|c|}
\hline $\begin{array}{l}\text { Control Sample } \\
\text { Identification }\end{array}$ & $\begin{array}{l}\text { Sample } \\
\text { Type }\end{array}$ & $\begin{array}{l}\text { Radio- } \\
\text { nuclido }\end{array}$ & $\begin{array}{c}\text { Peak Energy } \\
\mathrm{KeV}\end{array}$ & $\begin{array}{l}\text { Measured } \\
\text { Value }\end{array}$ & $\begin{array}{l}\text { Known } \\
\text { Value }\end{array}$ & \% Diff. $\mid$ & Units & Method No. & Instrument ID & $\begin{array}{l}\text { Date of } \\
\text { Check }\end{array}$ \\
\hline OA BKG EMPTY BTL U07 & CNTRL & AM241 & Not Appl. & $0.00 E+00$ & $0.00 E+\infty 0$ & & pCi/kg & E10602.PC & 07.01 & 070699 \\
\hline QA BKG EMPTY BTL U07 & CNTRL & 10060 & Not Appl. & $0.00 E+00$ & $0.00 E+00$ & & pCikg & E10602.PC & 07.01 & 070699 \\
\hline QA BKG EMPTY BTL U07 & CNTRR & Cs137 & Not Appl. & $0.00 E+\infty 0$ & $0.00 E+00$ & & pCikg & E10602.PC & $.07-01$ & 070699 \\
\hline QA SP:NAS-A0270 & CNTRL & AM241 & 59.3 & $1.65 E+05$ & $1.79 E+05$ & -7.6 & pCing & E10602.PC & $07-01$ & 070799 \\
\hline QA SP:NAS-A0270 & CNTRL & 10060 & 1173.4 & $3.16 E+05$ & $3.30 E+05$ & -4.0 & pCikg & E10602.PC & $07-01$ & 070799 \\
\hline QA SP:NAS-A0270 & CNTRL & CS137 & 661.6 & $2.15 E+05$ & $2.24 E+05$ & -4.2 & pCikg & E10602.PC & 07.01 & 070799 \\
\hline QA BKG EMPTY BTL UO5 & CNTRL & No Nuclide & Not Appl. & $0.00 E+00$ & $0.00 E+00$ & & PCikg & E10602.PC & $05-01$ & 070699 \\
\hline OA BKG EMPTY BTL UO5 & CNTRL & AM241 & Not Appl. & $0.00 E+\infty$ & $0.00 E+00$ & & pCing & E10602.PC & $05-01$ & 070699 \\
\hline QA BKG EMPTY BTL U05 & CNTRL & $c 060$ & Not Appl. & $0.00 E+\infty$ & $0.00 E+00$ & & pCikg & E10602.PC & $05-01$ & 070699 \\
\hline OA BKG EMPTY BTL U05 & CNTRL & CS137 & Not Appl. & $0.00 E+00$ & $0.00 E+00$ & & $\mathrm{pCl} / \mathrm{kg}$ & E10602.PC & $05-01$ & 070699 \\
\hline OA SP:NAS-A0271 & CNTRL & AM241 & 59.3 & 2.01E + 05 & $1.80 E+05$ & 11.4 & pCikg & E10602.PC & 05-01 & 070699 \\
\hline QA SP:NAS-A0271 & CNTRL & $C 060$ & 1173.0 & $3.35 E+05$ & $3.31 E+05$ & 1.3 & pCI/kg & E10602.PC & $05-01$ & 070699 \\
\hline QA SP:NAS-A0271 & CNTRL & Cs137 & 661.5 & $2.29 E+05$ & $2.26 E+05$ & 1.3 & pCI/kg & E10602.PC & $05-01$ & 070699 \\
\hline
\end{tabular}

Comments: 
APPENDIX E

\section{RADIOLOGICAL SURVEY RESULTS}


THIS PAGE LEFT INTENTIONALLY BLANK 


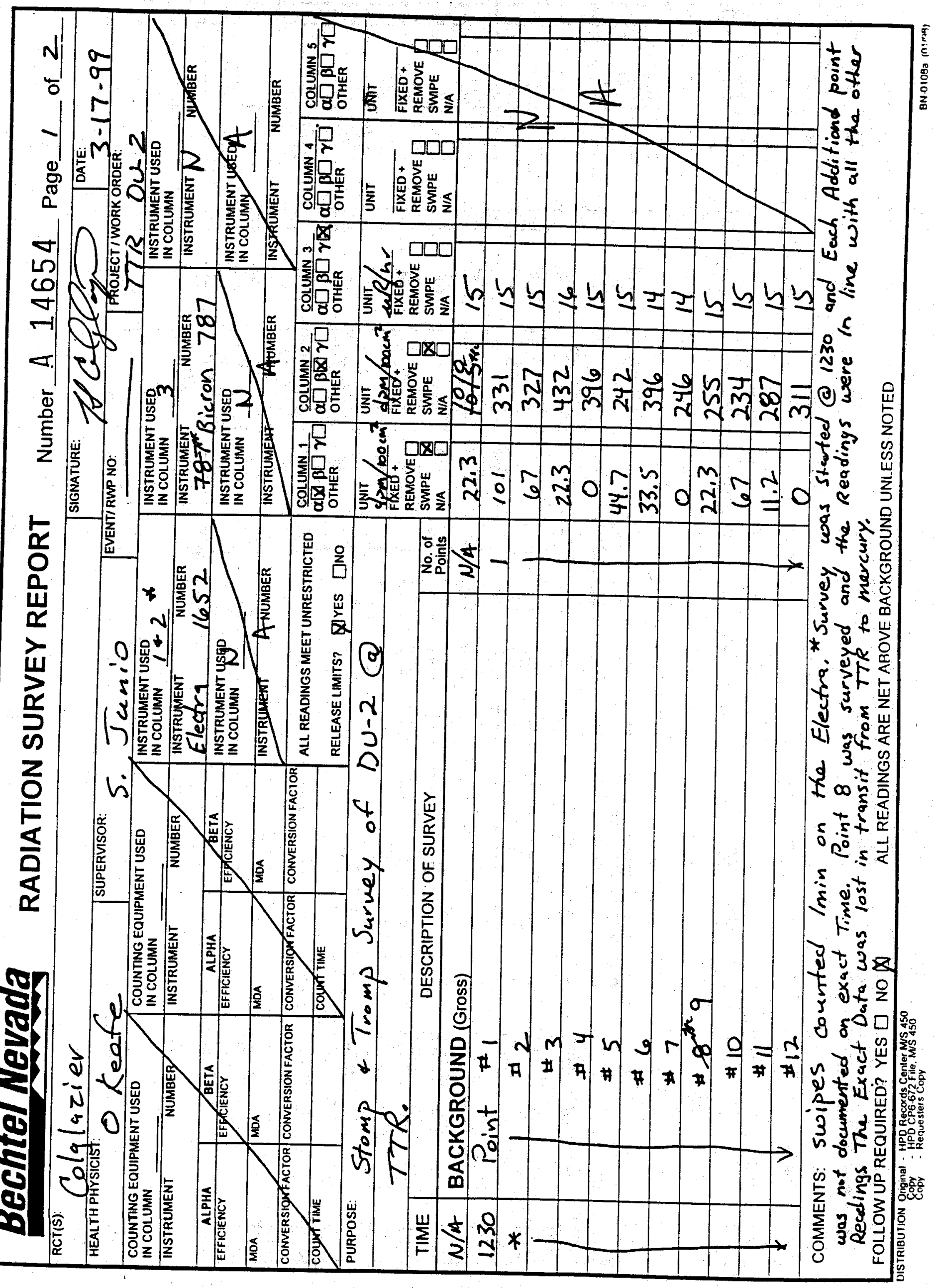




\section{Bechtel Nevada RADIATION SURVEY REPORT}

(SUPPLEMENT)

Number $A 14654$ Page 2 of 2

RCis): Colglazier

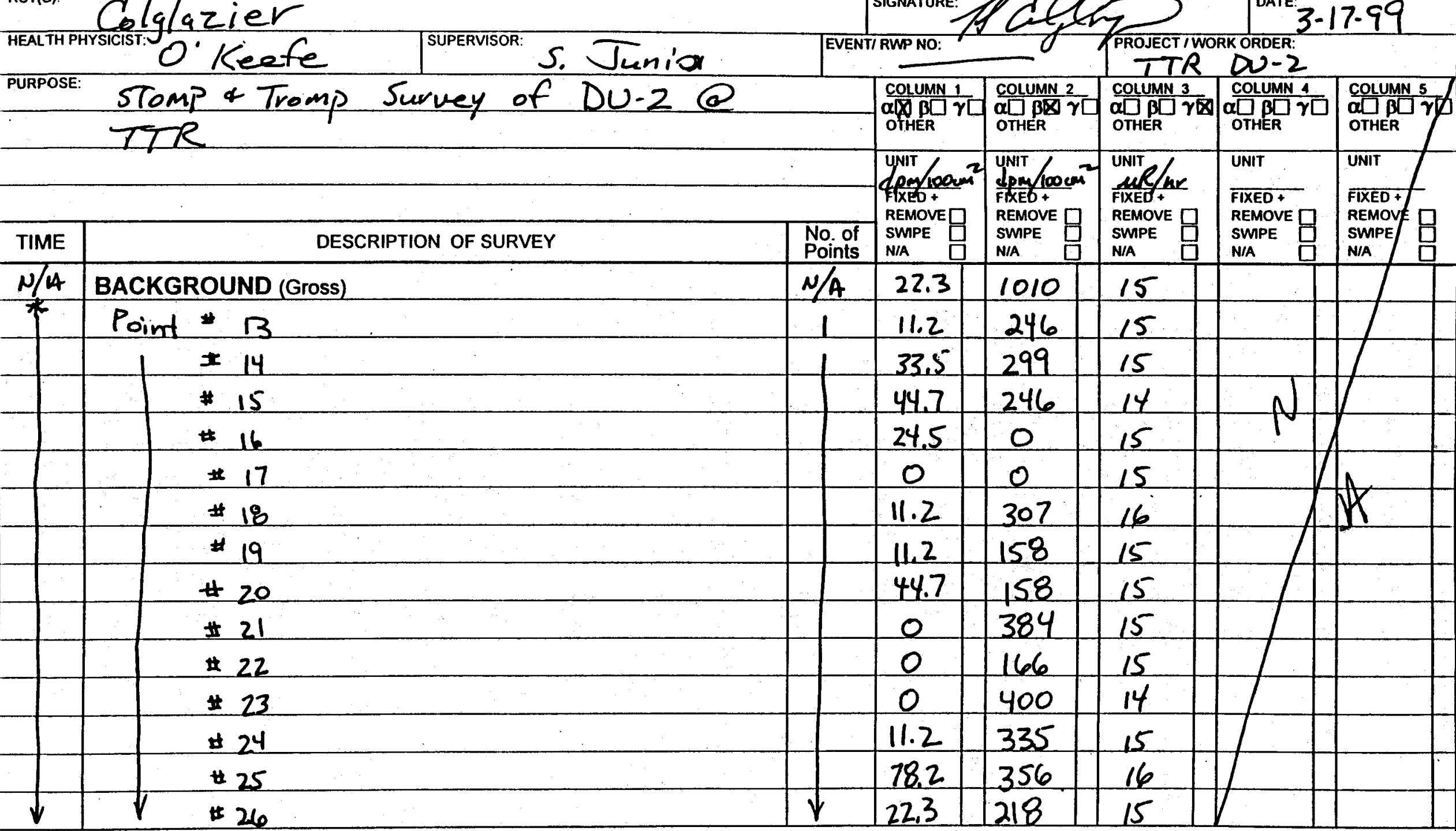

COMMENTS:

FOLLOW UP REQUIRED? YES ${ }^{x}$ NOW

ALL READINGS ARE NET ABOVE BACKGROUND UNLESS NOTED

OISIRIBUTION Original : HPDRecords Cenler MVS 450 


\section{Bechtel Nevada}

RCT(S):

Colglazier/Morris

RADIATION SURVEY REPORT

HEALTH PHYSICIST:

Lyons

COUNTING EQUIPMENT USED
IN COLUMN

INSTRUMENT NUMBER

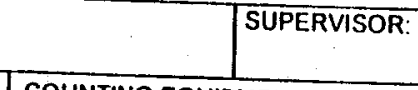

Junio

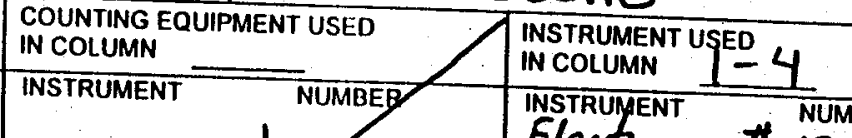

\begin{tabular}{|l|l|l|}
\hline EFFICIENCY & BLPAA \\
\hline CONOA & \\
\hline CONNT TIME & \\
\hline
\end{tabular}

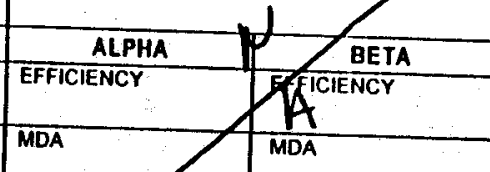

PURPOSE:

Stomp + Tromp for deposting CAO 461

INSTRUMENT USED

IN COLUMN

CONVERSIO FACTOR CONVERSION FACTOR

INSTRUMATI F FUMBER

ALL READINGS MEET UNRESTRICTED

ALL READINGSMEETUNRESTRICTED

CTED

Number $\triangle 02762$

Page 1 of SIGNATURE:

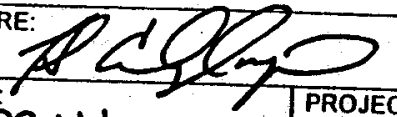

DATE:

6.24 .99

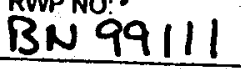

INSTRUMENT USED

IN COLUMN

INSTRUMENT $N$ NUMBER

CAU HLRER

ANSTRUMENT USED

IN COLUMN

INSTRUMENT USEO A
IN COLUMN

INST

INSPRUMENT NUMBER

\begin{tabular}{l|l|l|l|}
\hline COLUMN 1 & COLUMN 2 & COLUMN 3 & COLUMN 4 \\
\hline
\end{tabular}

OTHER $\gamma \square \frac{1 \bar{\alpha} \beta \bar{\square} \square}{\alpha \bar{a}}$

UNIT

dis / $2 \sin ^{2}$

OTHER

TIME

DESCRIPTION OF SURVEY

FXED

PPM 100 an

SMPE $\square$ REMOVE $\square$

SWPE $\square$ SWPE

N/A $\quad$ BACKGROUND (Gross)

1 Sample Point

it

$\pm 2$

+3

\#4

\# 5

$\# 6$

\# 7

\#8

\# 9

\# 10

\# 11

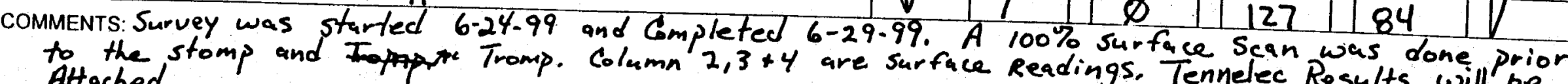

Attached.

FOLLOWUP REQUIRED? YES $\square$ NO $\varnothing$

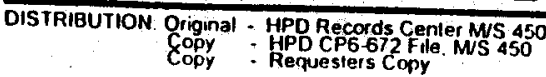

6-24-99 and Cmpleted 6-29.99. A $100 \%$ surf
ALOML READINGS ARE NET ABOVE BACKGROUND UNLESS NOTED

$\begin{array}{lll}79 & 7 & 114\end{array}$

\begin{tabular}{ll|l|l}
29 & 14 & 15 & 30
\end{tabular}

\begin{tabular}{l|l|l|l}
38 & 7 & 127 & 134
\end{tabular}

36

0

72

7 || 129

7

$\varnothing 0$

\begin{tabular}{l|l|l|l|}
$\varnothing$ & 138 \\
$\varnothing$ & 127
\end{tabular}

\begin{tabular}{l|l|l}
29 & 193 & 222
\end{tabular}

50

7

$\varnothing$

202

46

209

124

$2 7 \longdiv { 8 4 }$

121

34

$N$ 


\section{Bechtel Nevada}

\section{RCT(S):}

RADIATION SURVEY REPORT Number A 02763

\begin{tabular}{|c|c|c|c|c|}
\hline \multicolumn{3}{|l|}{ HEALTH PHYSICIST: } & \multicolumn{2}{|l|}{ SUPERVISOR: } \\
\hline \multicolumn{2}{|c|}{$\begin{array}{l}\text { COUNTING EQUIPMENT USED } \\
\text { IN COLUMN }\end{array}$} & \multicolumn{2}{|c|}{$\begin{array}{l}\text { COUNTING EQUIPMENT USED } \\
\text { IN COLUMN }\end{array}$} & $\begin{array}{l}\text { INSTRUMENT USED } \\
\text { IN COLUMN }\end{array}$ \\
\hline INSTRUMENT & NUMBER & INSTRUMENT & NUMBER & STTRUMENT \\
\hline ALPHA & BEFICIENCY & ALPHA & Siterder & $\begin{array}{l}\text { INSTRUMENT USED } \\
\text { IN COLUMN }\end{array}$ \\
\hline MDA & $\overline{M D A}$ & MDA & MDA & INSTRUMENT \\
\hline CONVERSION FACTOR & CONVERSION FACTOR & CONVERSIONFACTOA & CONVERSION FACTOR & ALL READINGS MEET UNRESTRICTED \\
\hline COUNT TIME & & COUNT TIME & & RELEASE LIMITS? XYYES $\square$ NO \\
\hline
\end{tabular}

SIGNATURE:

\begin{tabular}{|c|c|c|}
\hline TiME & DESCRPTTION OF SURVEV & 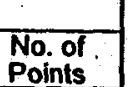 \\
\hline & BACKGROUND (Goss) & $\frac{N / 4}{N / 4}$ \\
\hline & Sample Point \#12 & \\
\hline & 14 & \\
\hline & 15 & \\
\hline & 11 & \\
\hline & 18 & \\
\hline & 19 & \\
\hline & $\frac{20}{21}$ & \\
\hline
\end{tabular}

\begin{tabular}{l|l}
\hline RWP NO: & PROJECT I WORK ORDER: \\
\hline
\end{tabular}

2 of 6

DATE:

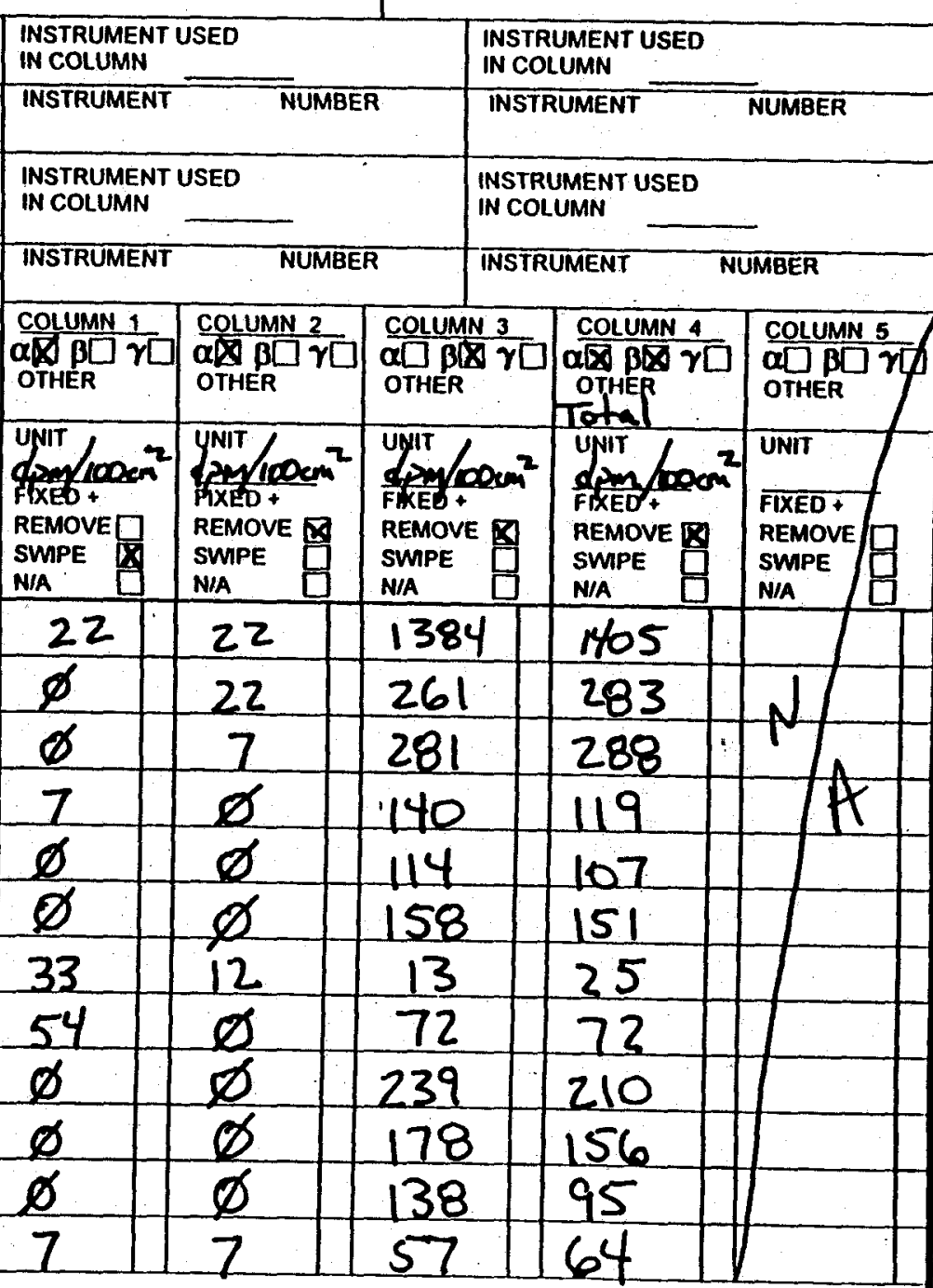

COMMENTS:

FOLLOW UP REQUIRED? YES $\square$ NO

ALL READINGS ARE NET ABOVE BACKGROUND UNLESS NOTED

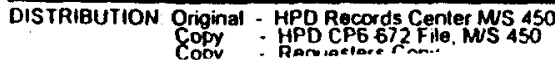




\section{Bechtel Nevada}

RCT(S)

RADIATION SURVEY REPORT Number A 02764 Page_ 3 of 6

HEALTHPHYSICIST:

SUPERVISOR:

SIGNATURE:

COUNTING EQUIPMENT USED

IN COLUMN

INSTRUMENT NUMBER

\begin{tabular}{|c|c|c|c|}
\hline \multicolumn{3}{|l|}{ HEALTH PHYSICIST: } & SUPERVISOR: \\
\hline \multicolumn{2}{|c|}{$\begin{array}{l}\text { COUNIING EQUIPMENT USED } \\
\text { IN COLUMN }\end{array}$} & \multicolumn{2}{|c|}{$\begin{array}{l}\text { COUNTING EQUIPMENT USED } \\
\text { IN COLUMN }\end{array}$} \\
\hline INSTRUMENT & NUMBER & INSTRUMENT & \\
\hline ALPHA & BETA & & \\
\hline EFFICIENCY & EFFICIENCY & & EFFICIENCY \\
\hline MOA & MDA & MDA & MDA \\
\hline CONVERSION FACTOR & CONVERSION FACTOR & CONVERSION FACTOR & CONVERSION \\
\hline COUNT TIME & & COUNT TIME & \\
\hline
\end{tabular}

PURPOSE:

INSTRUMENT USED
IN COLUMY

EVENT/RWP NO:

PROJECT/WORK ORDER

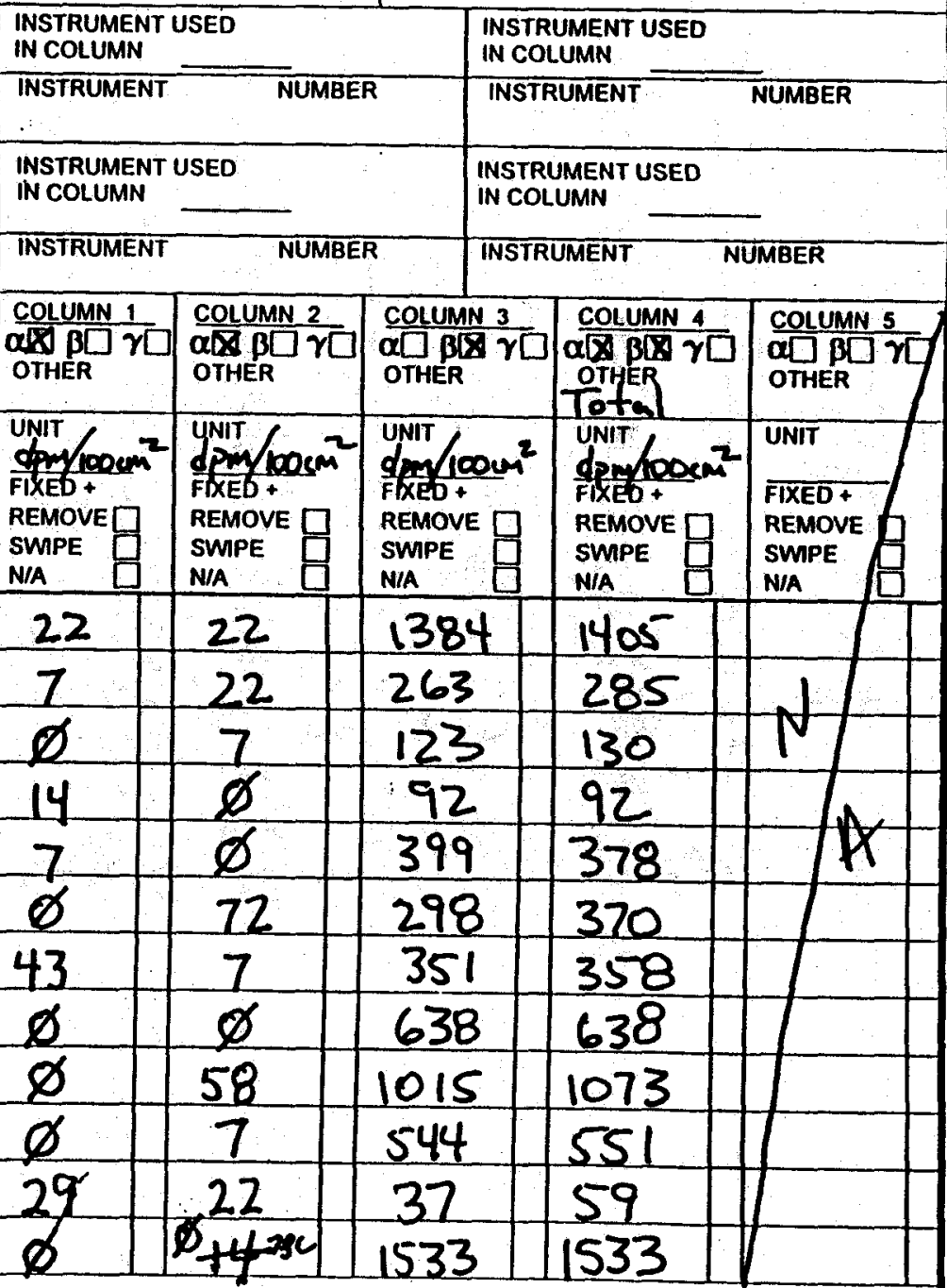

COMMENTS:

FOLLOW UP REQUIRED? YES $\square$ NO

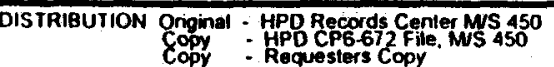

23

25

26

27

28

29

30

31

32

33

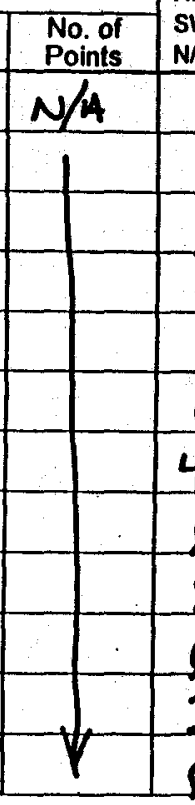

1533

1533 


\section{Bechtel Nevada}

RCT(S):

\section{RADIATION SURVEY REPORT}

Number A 02765

Page 4 of 6

HEALTH PHYSICIST

COUNTING EQUIPMENT USED

IN COLUMN

SUPERVISOR:

SIGNATURE:

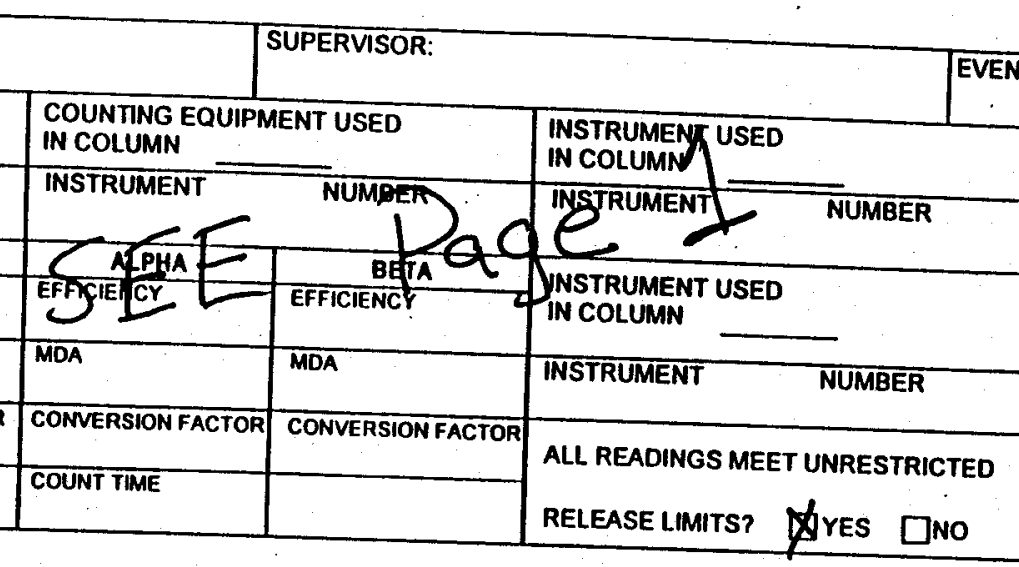

EVENT/RWP NO

DATE:

INSTRUMENT NUMBER

\begin{tabular}{l|l|}
\hline \multicolumn{1}{|c|}{ ALPHA } & \multicolumn{1}{|c|}{ BETA } \\
\hline EFFICIENCY & EFFICIENCY \\
\hline MDA & MOA \\
\hline CONUERSION FACTOR & CONVERSION FACTOR \\
\hline COUNT TIME & \\
\hline PURPOSE & \\
\hline
\end{tabular}

PURPOSE: RELEASE LIMITS? AYES DNO

\begin{tabular}{|c|r|}
\hline TIME & DESCRIPT \\
\hline N/A & BACKGROUND (Gross) \\
\hline & Sample Point H \\
\hline & 34 \\
& 35 \\
& 36 \\
& 37 \\
& 38 \\
& 39 \\
& 40 \\
& 41 \\
\hline & 42 \\
& 43 \\
\hline
\end{tabular}

COMMENTS:

34

36

37

38

39

40

41

42

43

\begin{tabular}{|c|c|c|c|c|}
\hline $\begin{array}{l}\text { INSTRUMENT } \\
\text { IN COLUMN } \\
\text { NSTOUMENT }\end{array}$ & USED & & $\begin{array}{l}\text { INSTRUMMENT USED } \\
\text { IN COLUMN }\end{array}$ & \\
\hline & NUMBE & & INSTRUMENT & NUMBER \\
\hline $\begin{array}{l}\text { INSTRUMENT } \\
\text { IN COLUMN }\end{array}$ & USED & & INSTRUMENT USED & \\
\hline INSTRUMENT & NUMEE & & INSTRUMENT & JUMBER \\
\hline 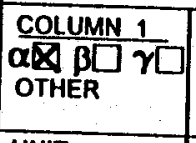 & 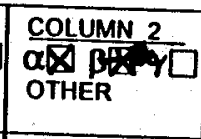 & 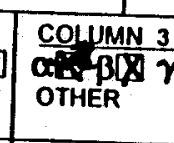 & $\frac{3}{\gamma \square} \mid \frac{\text { COLUMN } 4}{\alpha \bar{Q} \beta \bar{X} \gamma[}$ & $\begin{array}{l}\text { COLUMN } 5 \\
\alpha[D \beta D \gamma \\
\text { OTHER }\end{array}$ \\
\hline 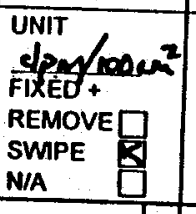 & 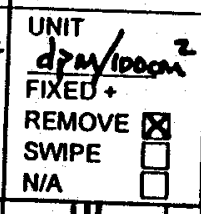 & 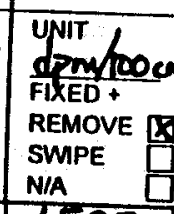 & 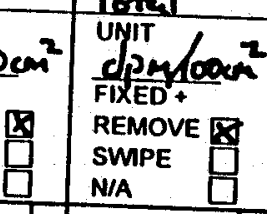 & 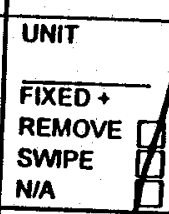 \\
\hline 14 & +5000 & t5im & 1514 & \\
\hline$\varnothing$ & 14 & 456 & 471 & \\
\hline$\varnothing$ & $\varnothing$ & 15 & $\varnothing$ & \\
\hline$\varnothing$ & $\phi$ & $\varnothing$ & $\varnothing$ & 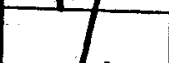 \\
\hline$\varnothing$ & 7 & $\varnothing$ & $\varnothing$ & X \\
\hline$\varnothing$ & $\varnothing$ & 217 & 210 & \\
\hline$\varnothing$ & $\varnothing$ & 2 & $\varnothing$ & \\
\hline$\varnothing$ & $\varnothing$ & $\varnothing$ & $\phi$ & \\
\hline 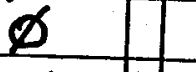 & $\varnothing$ & 415 & 400 & \\
\hline 4 & $\varnothing$ & 790 & 754 & \\
\hline$\gamma$ & 14 & $\varnothing$ & $\varnothing$ & \\
\hline 14 & 7 & $\varnothing$ & $\varnothing$ & \\
\hline
\end{tabular}

FOLLOW UP REQUIRED? YES $\square$ NO

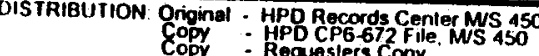


Bechtel Nevada RCT(S):

RADIATION SURVEY REPORT . Number_A 02766

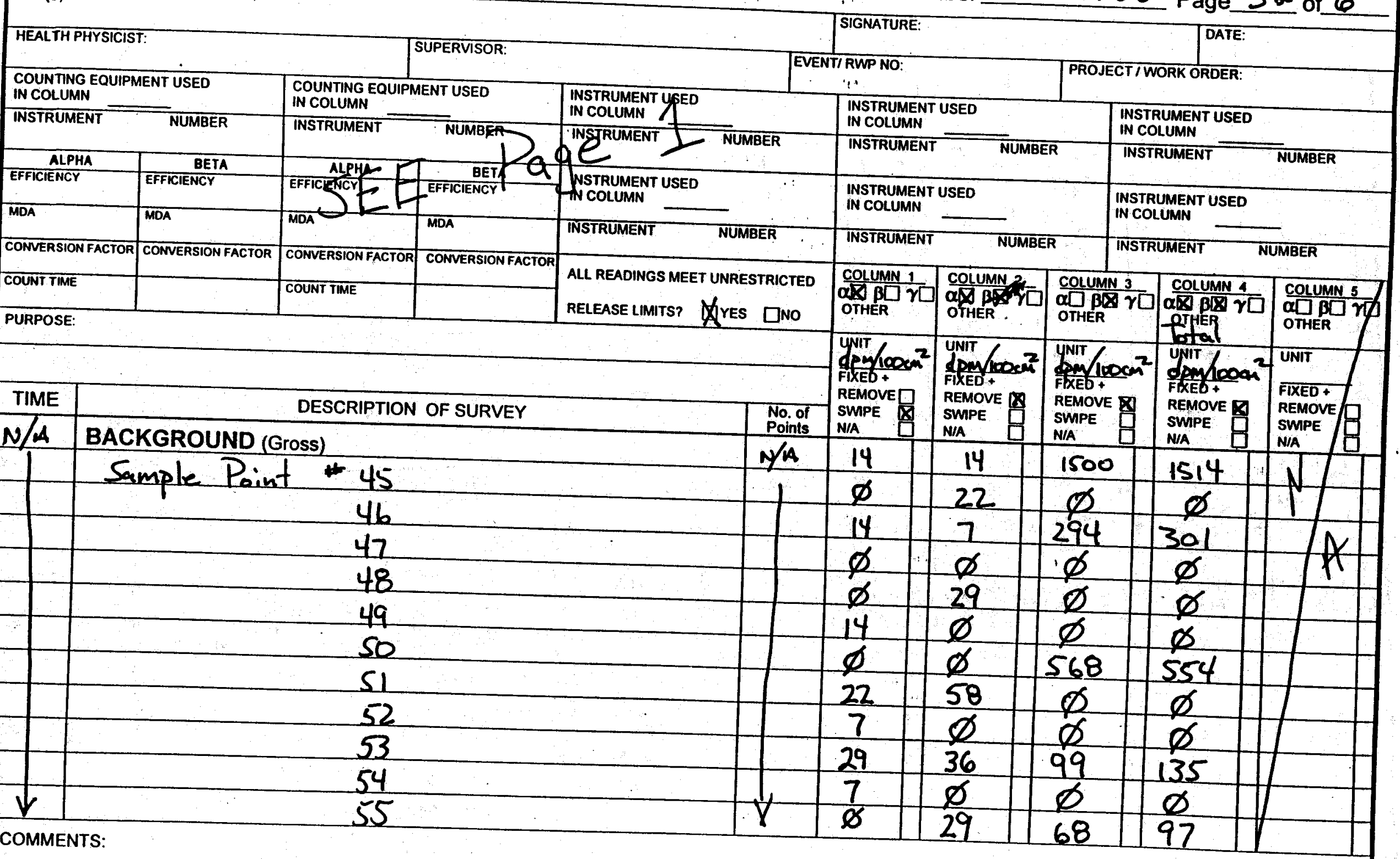

FOLLOWUP REQUIRED? YES $\square$ NO

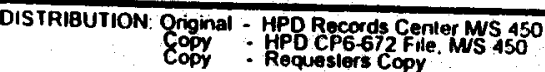




\section{Bechtel Nevada}

\section{RCT(S):}

RADIATION SURVEY REPORT Number A 02767

6

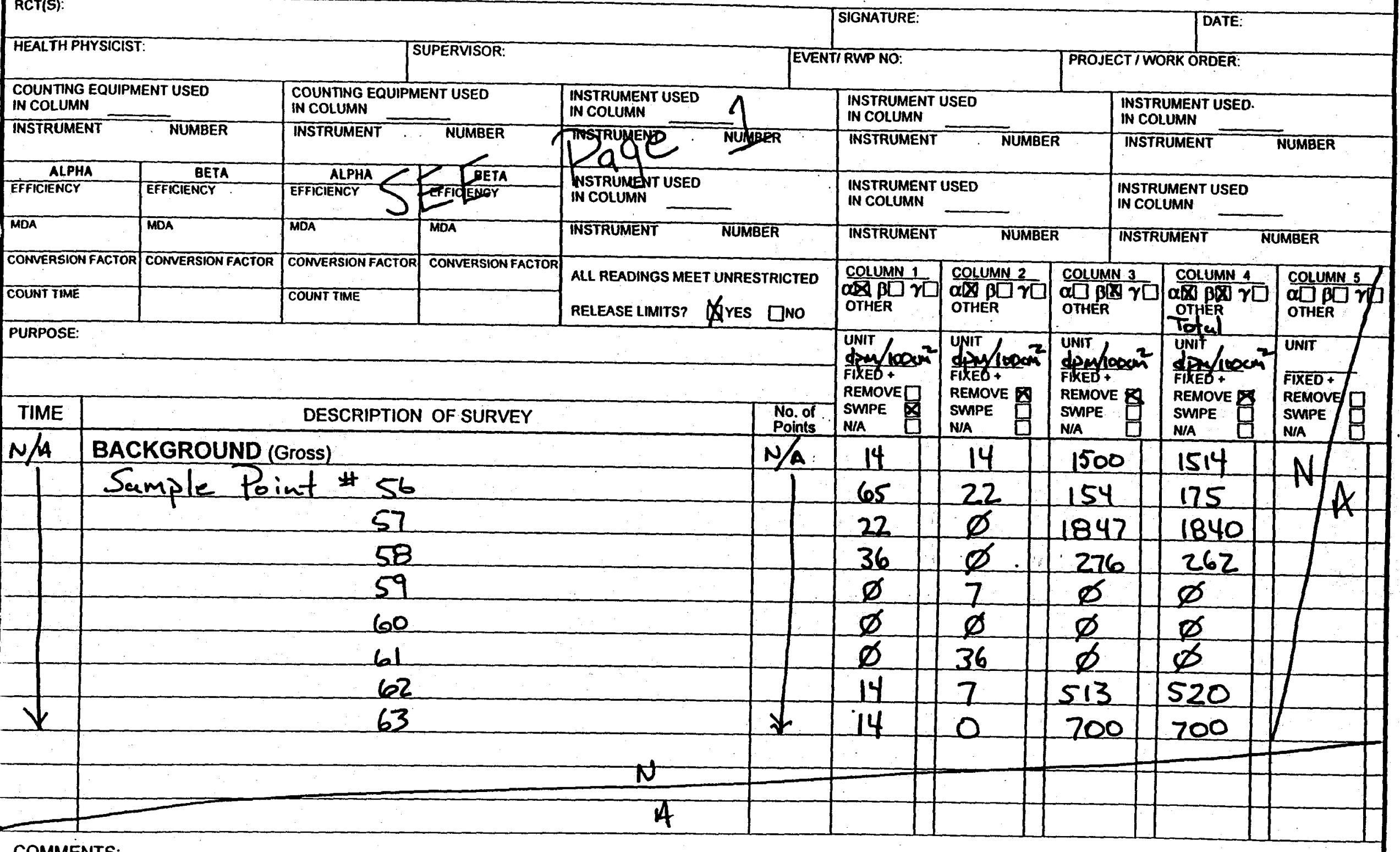

COMMENTS:

FOLLOW UP REQUIRED? YES $\square$ NO

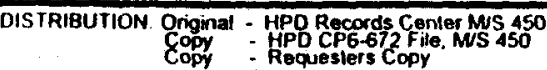




\section{APPENDIX F}

\section{TECHNICAL CHANGES}


THIS PAGE LEFT INTENTIONALLY BLANK 


\section{RECORD OF TECHNICAL FIELD CHANGE \\ TO APPROVED WORK PLANS}

\begin{tabular}{|l|l|l|}
\hline Technical Change No: TCN-001-99 & Project/Job No: CAU 461 & Date: 3/4/99 \\
\hline Project/Job Name: SAFER Closure for CAS TA-52-002-TAML & \\
\hline Work Plan Title: SAFER for CAU 461 \& CAU 495, TTR, Nevada & Document Date: August 1998 \\
\hline The following Work Plan technical changes are requested by: & Title: Task Manager & Signature: \\
\hline Printed Name: Jeffrey L. Smith & The \\
\hline Technical Change (list specific page/section/paragraph as appropriate): &
\end{tabular}

$\operatorname{Pg}$ 23/3.4.1.1

This Technical Amendment addresses a modification to the TA-52-002-TAML (TAML) site verification sampling process portion of the Nevada Division of Environmental Protection (NDEP) approved Streamlined Approach for Environmental Restoration (SAFER) Work Plan. The approved verification sampling activity consists of the collection of eight biased surface soil samples from previously identified DU impacted areas. The remainder of the site was to be divided into $3 \mathrm{~m}(10 \mathrm{ft})$ by $3 \mathrm{~m}$ (10 ft) grids. Five percent (16 samples) of the grids will be randomly selected using a random number generator (each cell would be assigned a number and computer program will select 14 of the cells). This verification sampling strategy was based upon limited pre-characterization data that indicated that the DU impacted soil was primarily located in the near surface. The SAFER Work Plan verification sampling process did not address a large subsurface excavation.

At the DU artillery round sites (Pg 26/3.4.1.2), a methodology bad been developed for large subsurface excavations. Five verification samples are collected, one sample from each side of the excavation. Since the TAML site excavation would be larger than the DU artillery round excavations, additional samples would be required for the TAML site to confirm that all of the impacted soil had been excavated. Therefore, the following verification sampling strategy will be employed at the TAML site:

- Ten verification samples will be collected from the $9 \mathrm{~m}(30 \mathrm{ft})$ by $12 \mathrm{~m}(40 \mathrm{ft})$ "hot spot". Two samples will be collected from each surface. Each surface of the excavation will be divided into six squares. Two of these squares will be randomly selected using a random number generator.

- The remaining 14 verification samples will be randomly selected (using a random number generator) from the cells created by dividing the site into $3 \mathrm{~m}(10 \mathrm{ft})$ by $3 \mathrm{~m}$ (10 ft) grids.

These modifications along with the radiological instrument survey over the entire site, will provide sufficient information to verify that all DU impacted soil above the SAFER Work Plan release criteria of $500 \mathrm{pCi} / \mathrm{g}$ has been removed from the site. 
Justification (list specific reasons for change and the effects on project schedule/costs/personnel):

Corrective Action Unit (CAU) 461 consists of three Corrective Action Sites (CAS). Field work following the Streamlined Approach for Environmental Restoration (SAFER) Work Plan was completed in December 1998 for the TTR-001 (Depleted Uranium (DU) Round Number 1) and TA-52-003-0960 (DU Round Number 2) CASs. Work on the final CAU 461 CASs, TA52-002-TAML (TAML), was initiated on January 11, 1999. The closure activities, outlined within the SAFER Work Plan, for the TAMI site was based upon information that indicated that the vertical extent of DU impacted soil was limited to a depth of no greater than two feet below ground surface at a few small (less than one meter (m) [3 feet (ft)] in diameter) "hot spots".

The SAFER Work Plan estimated that the total of 153 cubic meters $\left(m^{3}\right)$ (200 cubic yards [yd $\left.d^{3}\right]$ ) of DU impacted soil would be removed from the TAML site. Thirteen $15.3-\mathrm{m}^{3}\left(20-\mathrm{yd}^{3}\right)$ trailers were contracted to be on-site to transport the DU impacted soil from the Tonopah Test Range (TTR) to the Nevada test Site (NTS) for disposal. During the TAML closure activities a $9 \mathrm{~m}$ (30 $\mathrm{ft}$ ) by $12 \mathrm{~m}$ ( $40 \mathrm{ft}$ ) "hot spot" was identified. Investigation of the "hot spot" delineated a vertical depth of $3 \mathrm{~m}$ ( $10 \mathrm{ft})$. The volume of DU impacted soil increased from approximately $153 \mathrm{~m}^{3}\left(200 \mathrm{yd}^{3}\right)$ to $573 \mathrm{~m}^{3}\left(750 \mathrm{yd}^{3}\right)$. Work was stopped at the site and an extension was requested by the Department of Energy, Nevada Operations Office (DOE/NV) to the Nevada Division of Environmental Protection (NDEP) for the Federal Facility Agreement Consent Order (FFACO) Closure Report milestone date.

Will other project documents be affected? $\square$ Yes $\otimes$ No Specify:

APPROV 1 . S.

\begin{tabular}{|c|c|c|c|}
\hline BN Program Manager: & Printed Name: Dave Cowser & Signature: & Date: $3 / 8 / 99$ \\
\hline BN Project Manager: & Printed Name: Steve Nacht & Signature & Date $3 / 8 / 99$ \\
\hline BN Task Manager: & Printed Name: Jeff Smith & Signature: & Date: 314199 \\
\hline \multicolumn{4}{|l|}{ CONCURRENGES? } \\
\hline DOE/NV Project Manager: & Printed Name: Janet Appenzeller-Wing & & Date: 10100 \\
\hline \multicolumn{4}{|c|}{ EFFECTIVE DATE OF FIELD CHANGE: } \\
\hline Verbal Approval Provided to: & Printed Name: N/A & Title: & Date: \\
\hline
\end{tabular}




\section{RECORD OF TECHNICAL CHANGE NOTICE \\ TO APPROVED WORK PLANS}

\begin{tabular}{|l|l|l|}
\hline Technical Change No: TCN-002-99 & Project/Job No: CAU 461 & Date: 3/29/99 \\
\hline Project/Job Name: SAFER Closưre for CAS TA-52-002-TAML & Document Date: August 1998 \\
\hline Work Plan Title: SAFER for CAU 461 \& CAU 495, TTR, Nevada & Signature: \\
\hline The following Work Plan technical changes are requested by: & Title: Task Manager \\
\hline Printed Name: Jeffrey L. Smith & Technical Change (list specific page/section/paragraph as appropriate):
\end{tabular}

Since the SAFER Work Plan did not anticipate large scale subsurface excavation activity at the TAML site, there is no site specific excavation process for the TAML site outlined within the Work Plan. However, the process for subsurface excavation at the other CASs in CAU 495 and 461 is specified in Section 3.3.2 of the SAFER Work Plan and Attachments A and G of the SAFER Site Specific Health and Safety Plan. The methodologies proposed within the plans will be implemented at the TAML site. The following modifications to the SAFER Work Plan are proposed:

- Section 3.3.1, page 21, 3rd paragraph, 3rd sentence - change sentence to indicate subsurface activities will be confined to upper $3 \mathrm{~m}(10 \mathrm{ft})$.

- Section 3.3.1, page 21, 4th paragraph, 3rd sentence - Change sentence to indicate soil will not be transferred into the trailers using a conveyor system.

- Section 3.3.1, page 21, 4th paragraph, 4th sentence - Need to clarify that $153 \mathrm{~m}^{3}\left(200 \mathrm{yd}^{3}\right)$ of soil will be generated during the surface scraping activity and an additional $420 \mathrm{~m}^{3}\left(550 \mathrm{yd}^{3}\right)$ of DU impacted soil will be generated during subsurface excavation.

- Section 3.3.1, page 21, 4th paragraph, 7th sentence - Modify sentence to indicate that "hot spots" excavation can be conducted using a shovel as well as a backhoe. Further modify sentence to indicate that "hot spot" soil will be placed into burrito lined trailers instead of a B-25 box.

- Section 3.3.2, page 22, 3rd paragraph, 2nd sentence - Modify sentence to indicate that non-impacted soil will not be placed on plastic or covered with plastic. Instead, the soil will be stockpiled on the uncovered ground and sprayed with water. The water spray will form an outer crust which will inhibit wind dispersion of the soil from the stockpile.

Section 3.3.2, page 22, 6th paragraph - The entire paragraph after the first sentence will be deleted and replaced with the following: Field investigation during the TAML closure activities have determined that the

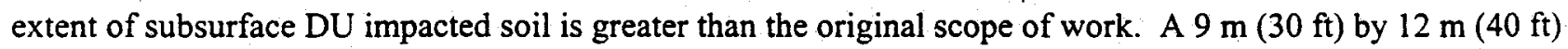
by $3 \mathrm{~m}$ ( $10 \mathrm{ft})$ deep "hot spot" was identified. Approximately $420 \mathrm{~m}^{3}\left(550 \mathrm{yd}^{3}\right)$ of DU impacted soil will be generated during subsurface excavation of this "hot spot". The DU impacted soil will be excavated following the procedure outlined in Section 3.1 of this SAFER Work Plan. The DU impacted soil will be stockpiled directly onto plastic and sprayed with water. The water spray will form a outer crust which will inhibit wind dispersion of the DU impacted soil from the stockpile. The burrito lined trailers will be filled directly by a front end loader which will be used to transport the soil from the stockpile. 
Justification (list specific reasons for change and the effects on project schedule/costs/personnel):

Corrective Action Unit (CAU) 461 consists of three Corrective Action Sites (CAS). Field work following the Streamlined Approach for Environmental Restoration (SAFER) Work Plan was completed in December 1998 for the TTR-001 (Depleted Uranium (DU) Round Number 1) and TA-52-003-0960 (DU Round Number 2) CASs. Work on the remaining CAU 461 CAS, TA-52-002-TAML (TAML), was initiated on January 11, 1999. The closure activities for the TAML site, as outlined within the SAFER Work Plan, was based upon information which indicated that the vertical extent of DU impacted soil was limited to a depth of no greater than two feet below ground surface at a few small (less than one meter (m) [3 feet (ft)] in diameter) "hot spots".

The SAFER Work Plan estimated that the total of 153 cubic meters $\left(\mathrm{m}^{3}\right)\left(200\right.$ cubic yards $\left.\left[\mathrm{yd}^{3}\right]\right)$ of $\mathrm{DU}$ impacted soil would be removed from the TAML site. Thirteen $15.3-\mathrm{m}^{3}\left(20-\mathrm{yd}^{3)}\right.$ trailers were contracted to be on-site to transport the DU impacted soil from the Tonopah Test Range (TTR) to the Nevada Test Site (NTS) for disposal. During the TAML closure activities a $9 \mathrm{~m}$ (30 $\mathrm{ft}$ ) by $12 \mathrm{~m}$ ( $40 \mathrm{ft})$ "hot spot" was identified. Investigation of the "hot spot" delineated a vertical depth of $3 \mathrm{~m}(10 \mathrm{ft})$. The volume of DU impacted soil increased from approximately $153 \mathrm{~m}^{3}\left(200 \mathrm{yd}^{3}\right)$ to $573 \mathrm{~m}^{3}\left(750 \mathrm{yd}^{3}\right)$. Work was stopped at the site and an extension was requested by the Department of Energy, Nevada Operations Office (DOE/NV) to the Nevada Division of Environmental Protection (NDEP) for the Federal Facility Agreement Consent Order (FFACO) Closure Report milestone date.

Will other project documents be affected? $\square$ Yes $₫$ No Specify:

APPROVALS:

\begin{tabular}{|l|l|l|l|}
\hline BN Program Manager: & Printed Name: Dave Cowser & Signature: & Signature:sate: $4-1-94$ \\
\hline BN Project Manager: & Printed Name: Steve Nacht & Signature: & Date:3/3//99 \\
\hline BN Task Manager: & Printed Name: Jeff Smith & Date:3/31/99 \\
\hline CONCURRENCES: & &
\end{tabular}

CONCURRENCES:

DOE/NV Project Manager: Printed Name: Janet Appenzeller-Wing

Signature: Hew Cold ter $\mid \begin{aligned} & \text { Date: } \\ & 4 / 1 / 99\end{aligned}$ EFFECTIVE DATE OF FIELD CHANGE: 


\section{RECORD OF TECENICAI EDHD CEANGI} TO APPROVID WORK FLANS

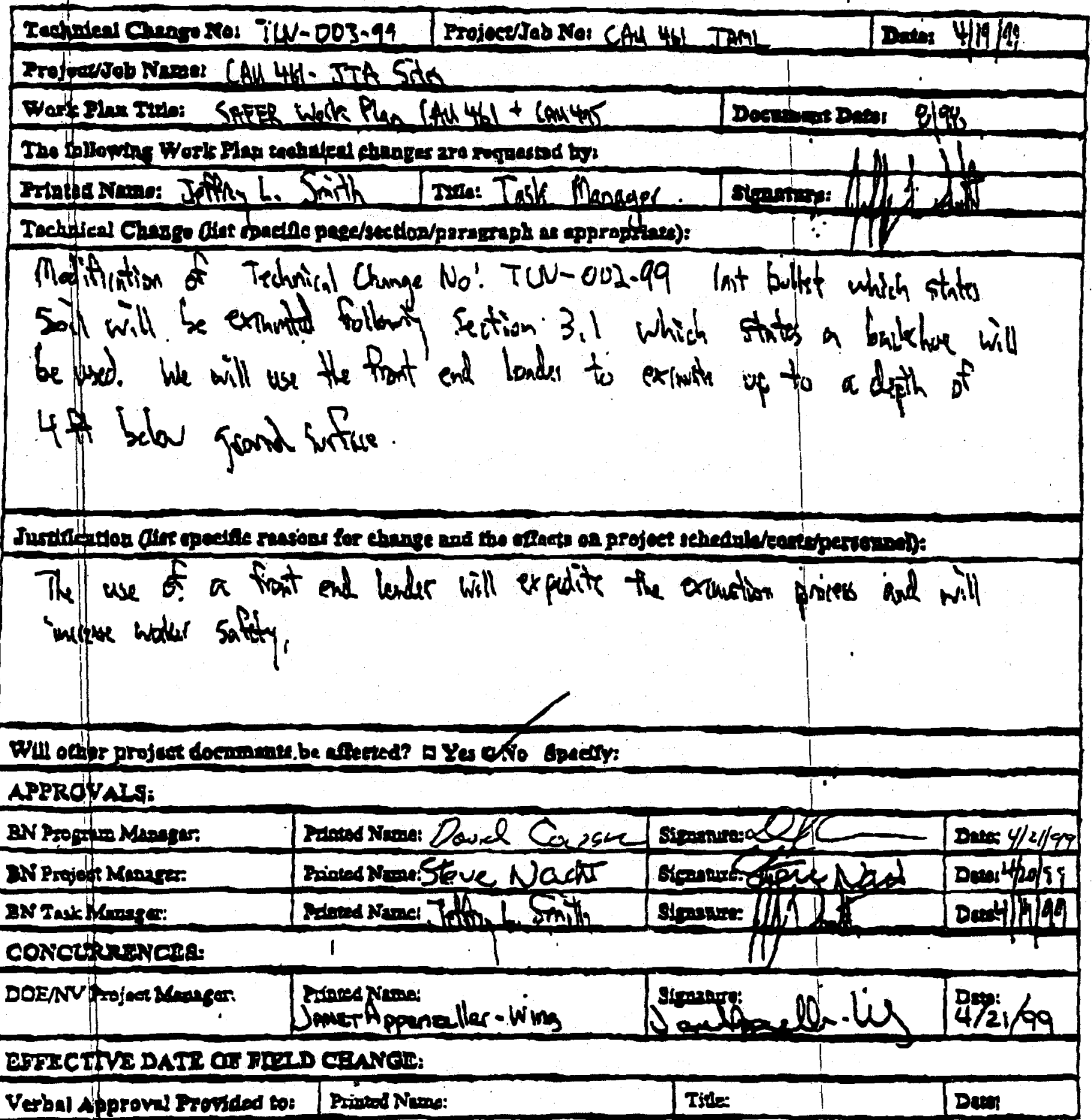


THIS PAGE INTENTIONALLY LEFT BLANK 


\section{APPENDIX G}

\section{SHIPPING MANIFESTS}


THIS PAGE INTENTIONALLY LEFT BLANK 
SRAIGKT BIII OF IADING - SHORT FORM - ORIGINAI - NOt NegOtiable

H:- :OR: BECHTEL NEVADA FOR US DOE

CAU 461 TAIVL SITEI TTR

TONO=AA NV EะO43.
Purchasel

Customer Order No
Shipper No. NTS073636

Date: $04-19.99$

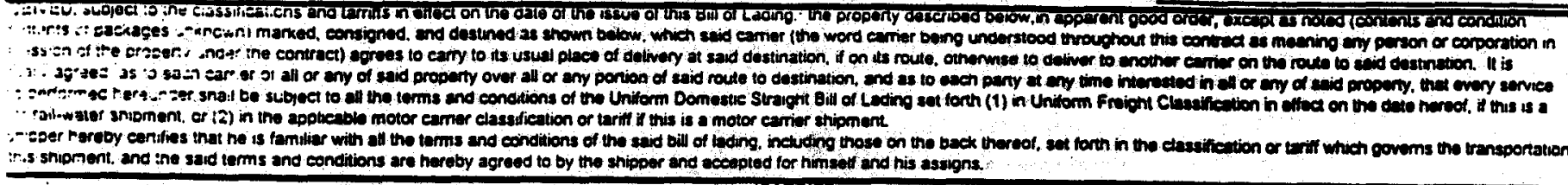

Insignee

3ECHTEL NEVADA FOR USDOE

7-3 RWMS. ZONE 2

NEVADA TEST SITE

MERCURY NV 89023.

VIKE SMITH / (702) 295-7365

CARlilER ETON I ENVIRO TRANSPORT OF NV

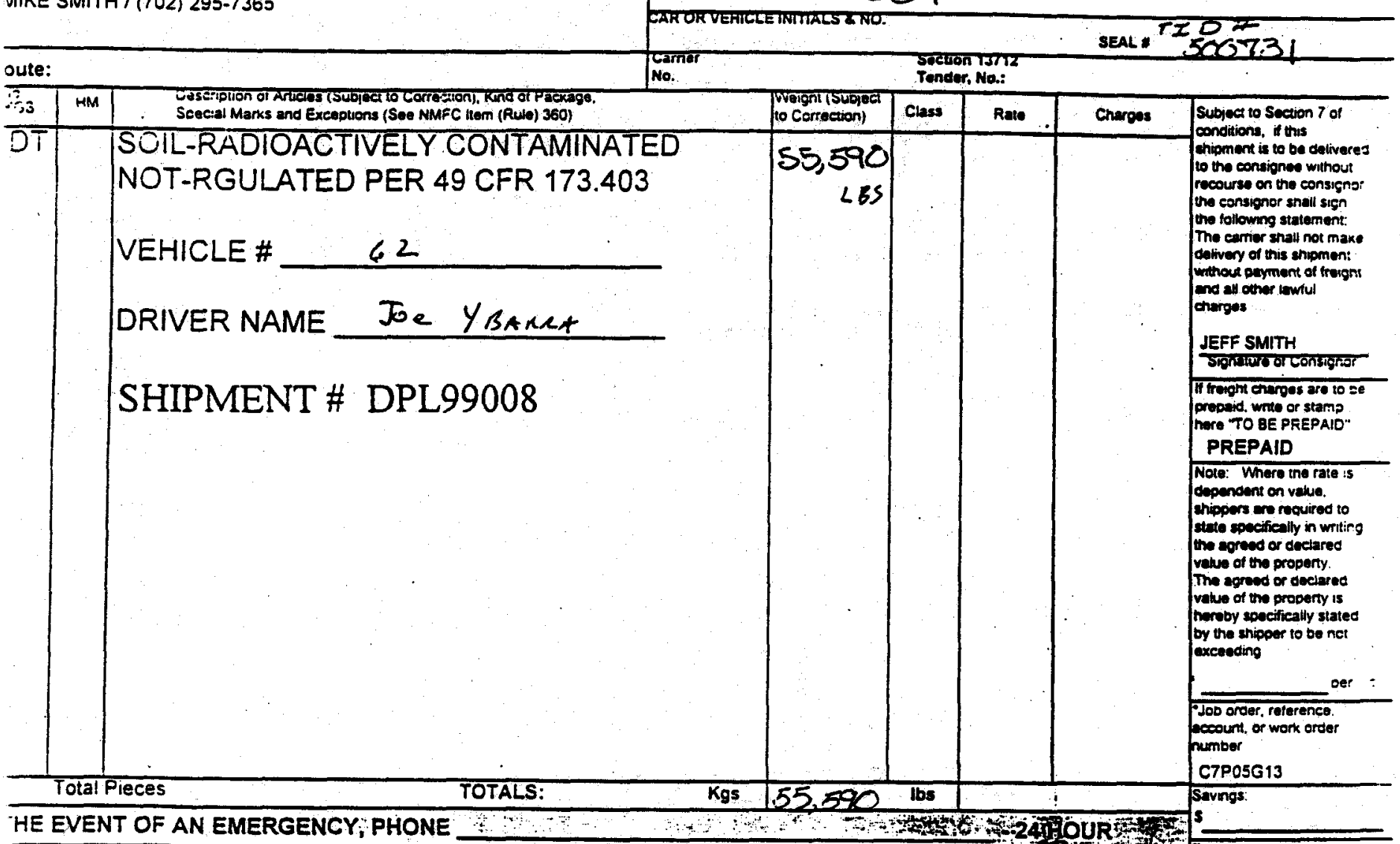

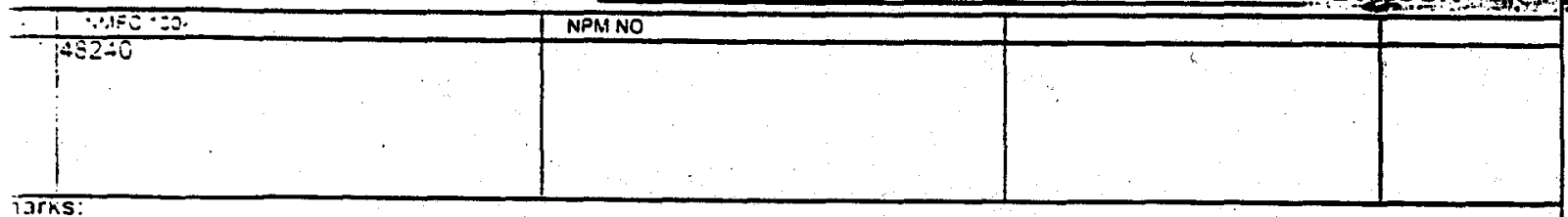

INICAL CONTACT

is to cenify that the above named matenals fre propenty classtied.

zribeo. Dackaged. marked. ane tabeled, and are in proper condition tor soonation according to the applicable regulations of the Department of :sDoration. (Applicadle tor Hazardous Matenats Onty.)

BECHTEL NEVADA. P O. BOX 98521, LAS VEGAS. NV 89193

- under contract DC ACOS 96NVI1718

$\therefore$ IAN R MARCHANO

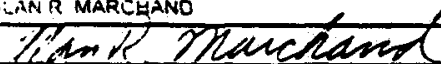

with U.S. Dept. of Energy

Date: 04108/1999

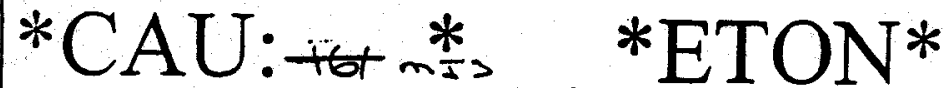




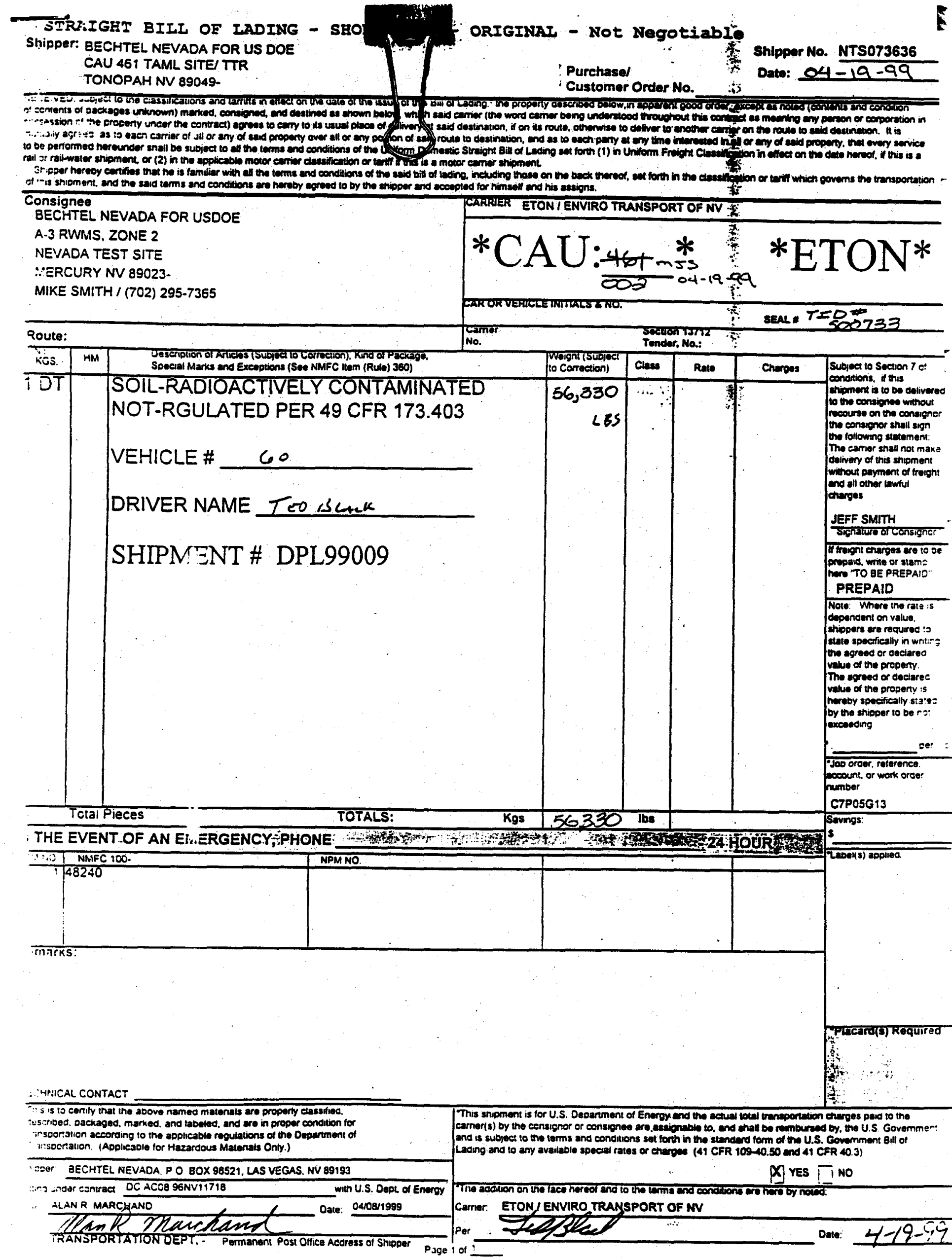


- $C E$ - $=2 \geq Y 1$

VEVIADA FOF. US DOE

AIIL SITE/TI

inV 89049

(1)

Purchasel

' Customer Order No.

Shipper No. NTS073636

Date: $04-19-94$

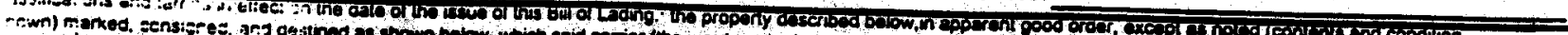

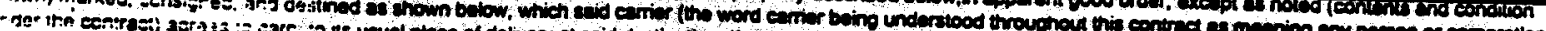

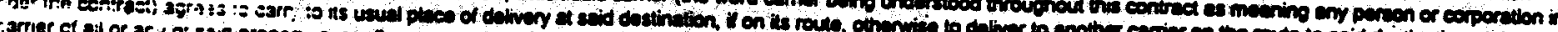

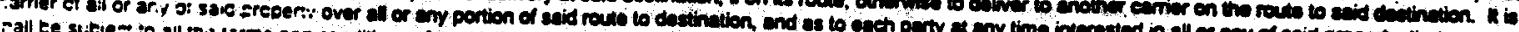

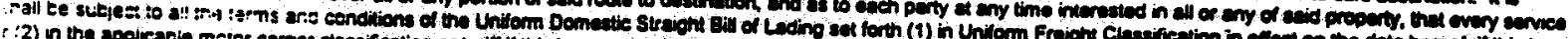

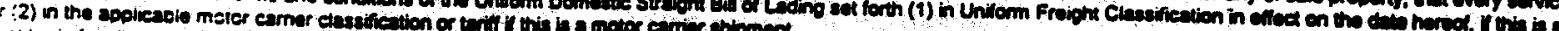

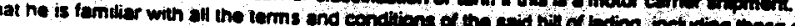

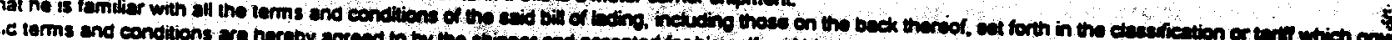

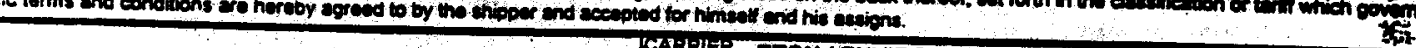

A FOR USDOE

2

TE

:23.

.. 295-7365

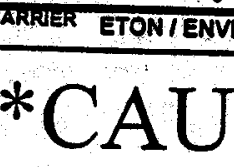

is treneporition

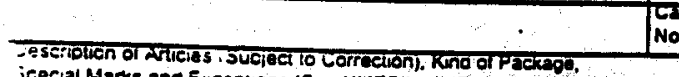

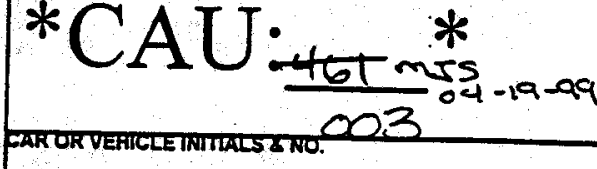
*ETON* jeectal Marks and Exeeduers (See NMFC Hem (Rule) 360)

--RADIOACTIVELY CONTAMINATED -RGULATED PER 49 CFR 173.403

ICLE\# 59

IER NAME Tom CAxTex PMENT \# DPL99010 Solvon TSTI
No.
Tonder, No.: SEAL 720 TH

\begin{tabular}{l|l|l|l|l|l} 
Woght(Subjec & Class & Rote & Charges & Subject to Suction 7 of
\end{tabular}
$207 / 5$

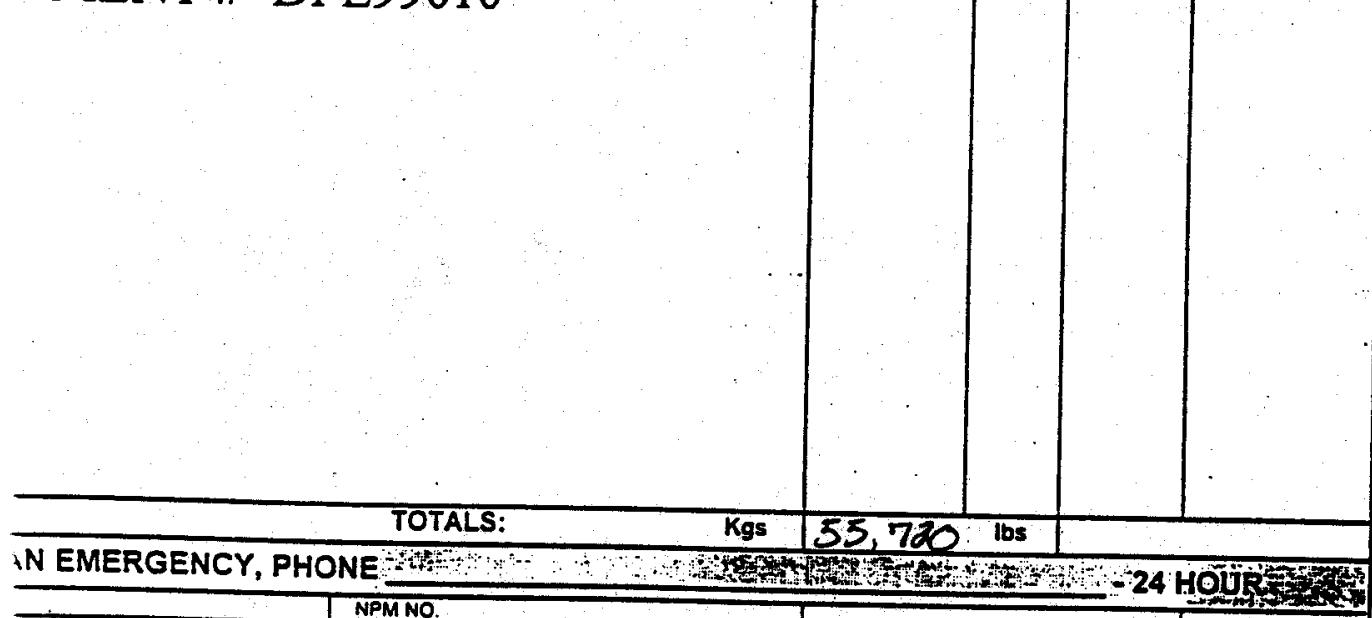

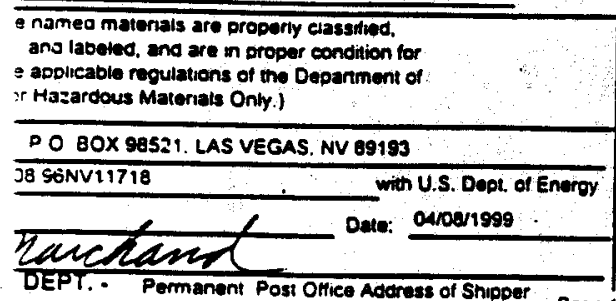

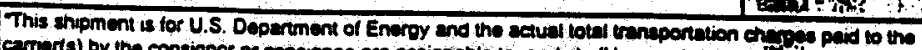
and is subje the consignor or consignes are essignable to, and hall be rumberied by U.S. Covemment and ss subject to the terms and conditions sot forn in the standard form of the U.S. Cosimnems Bill of Leding and to eny avalable spocial rates or charges (41 CFR $109-10.50$ and 41 CFR 4a.3) eonctitions, it this chenent is to be delivered rever consonse withour recourse on the consignor. thiyollowing shall sign This exvier smais not make delivery of this snipment Wrovit peyment of freught nd all other inwhl tritse its JEFF'SMITH

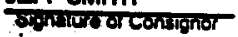
H.turent chares are to be oripaid. write or etamp nim TO BE PREPAD" BREPAID

Map: Where the rate s" dpoendert on value. - tippers are required to ots specilically in writing tripereed or declared vife of the property. The pread or dectared vitio of the property is hepby soceifeally staled by the shipper to be not tonding.

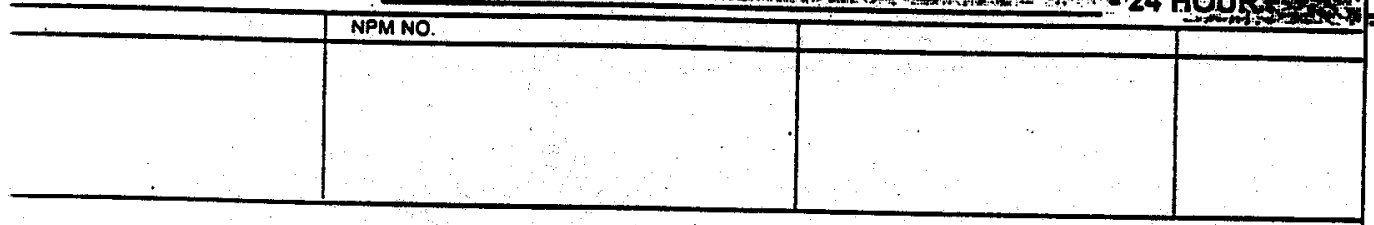

.




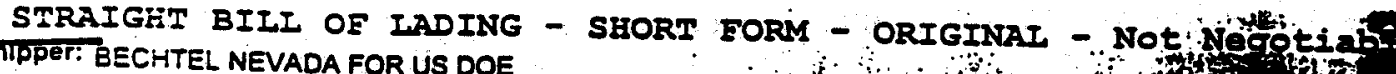
SIIPPET: BECHTEL NEVADA FOR US DOE CAU 461 TAML SITE/TTR TONCPAH NV 89049-

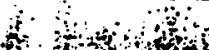
aration

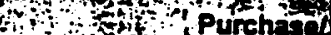

\section{- $\quad \therefore$}

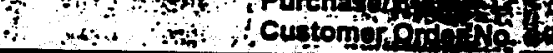

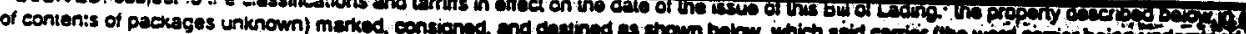

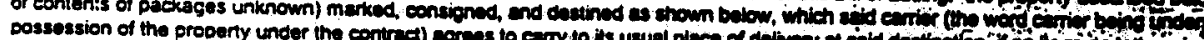

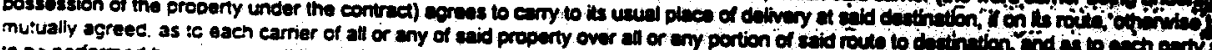

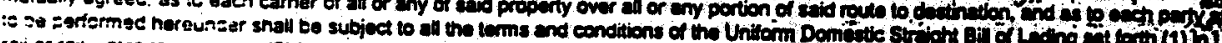

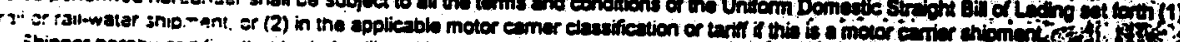

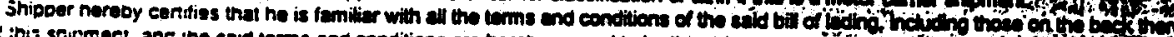

Consignes sniem a

BECHTEL NEVADA FOR USDOE

A-3 RWMS. ZONE 2

NEVADA TEST SITE

MERCURY NV 89023.

MIKE SMITH / (702) 295-7365

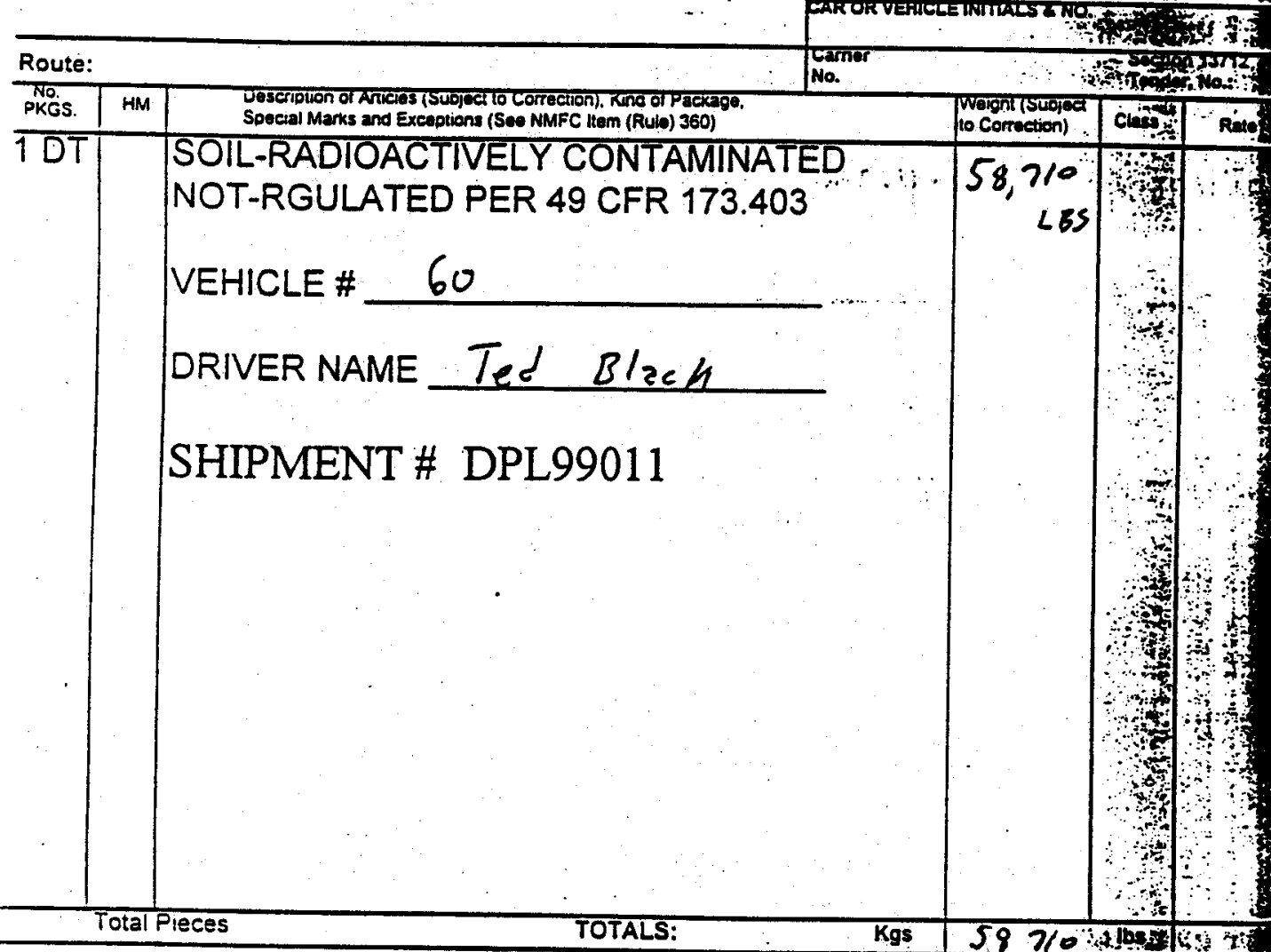

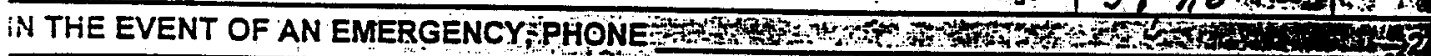

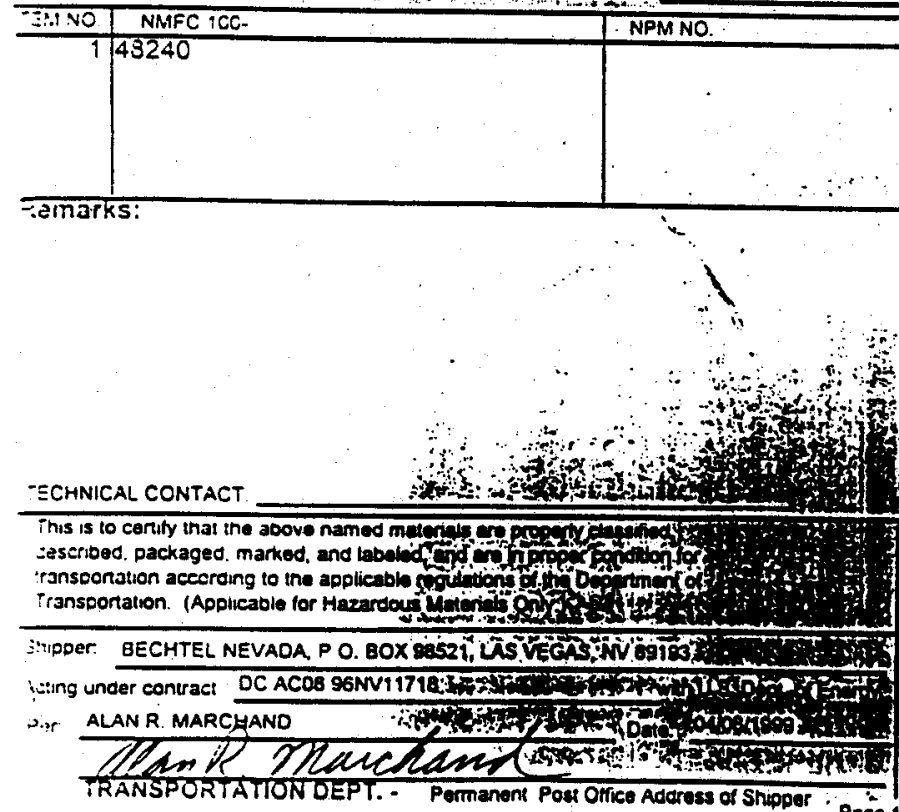

$$
\begin{gathered}
n \\
\vdots \\
\because
\end{gathered}
$$

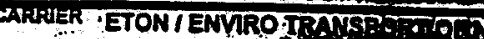

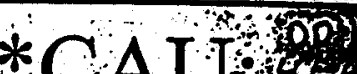

its

Ig

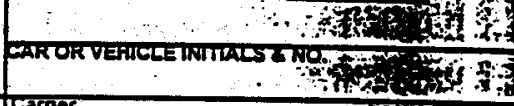
Purchace :

\section{(1)}

ppor NTS07363i tontogy $-20-99$

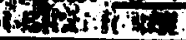

$7 x+2 y$ ow

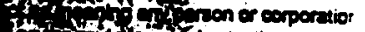

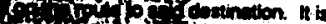

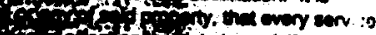

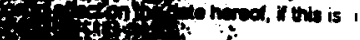

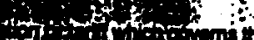

F. If if .

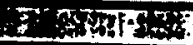

7x

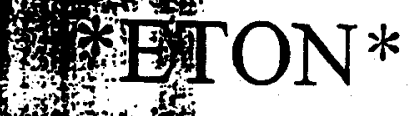

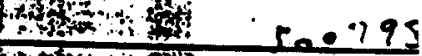

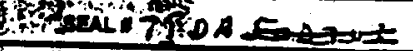

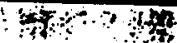

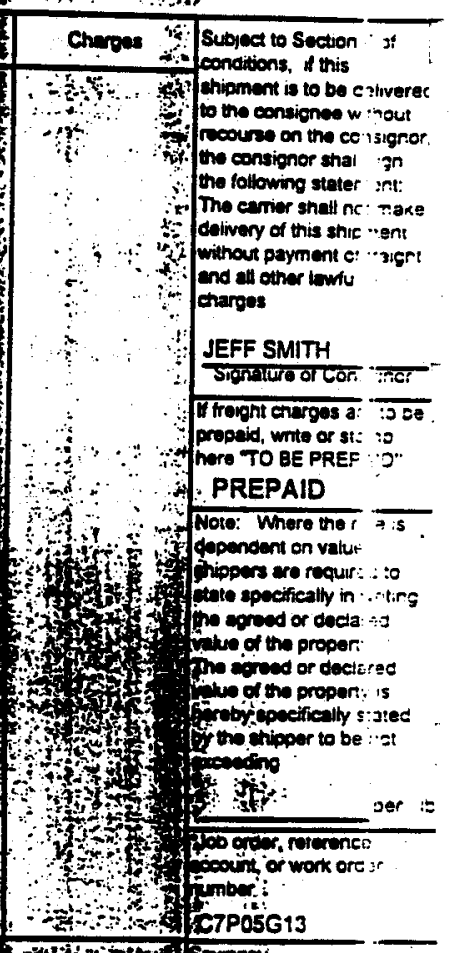

xitutisats

imnoex:

OIdF:
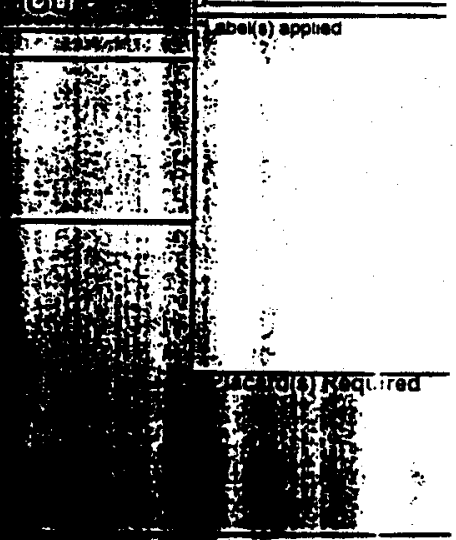

100

Thits Gover: ant

menters biul of

40.54

19.?

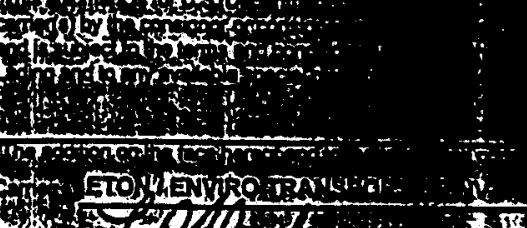

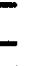


SFRAIGHI BILI OE IADING - SHORT EORM - ORIGINAI - NOt NeğOtigS 1

Shipper: BECHTEL NEVADAFOR SS DOE

CAU 461 TAML SITE TTR

TONOPAH NV 89049-

ans

Purchesat in

Customerotortin

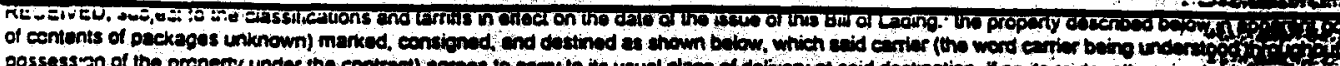

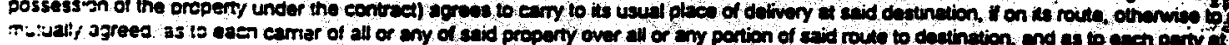

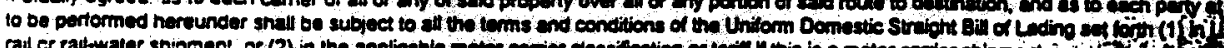

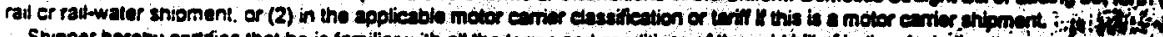

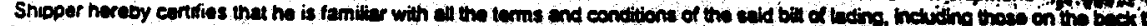

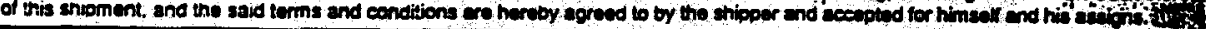

Consignee

BECHTEL NEVADA FOR USDOE

A.3 RWMS. ZONE 2

NEVADA TEST SITE

MERCURY NV a9023-

MIKE SMITH I (702) 295-7365

Route:

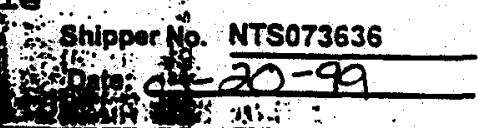

I.t.

tenow in

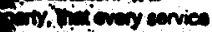

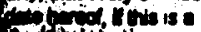

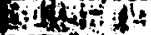

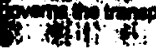

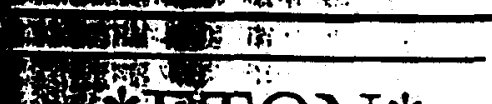
4. Sal TIDF

\begin{tabular}{|c|c|c|}
\hline \multirow{4}{*}{$\frac{\text { PKGS }}{1 D T}$} & HM & 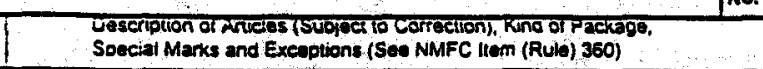 \\
\hline & & $\begin{array}{l}\text { SOIL-RADIOACTIVELY CONTAMINATED } \\
\text { NOT-RGULATED PER } 49 \text { CFR } 173.403\end{array}$ \\
\hline & & $V E H I C L E \# \quad 6^{2}$ \\
\hline & & DRIVER NAME $J_{0} e$ Ybares \\
\hline
\end{tabular}

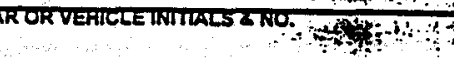

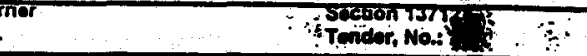

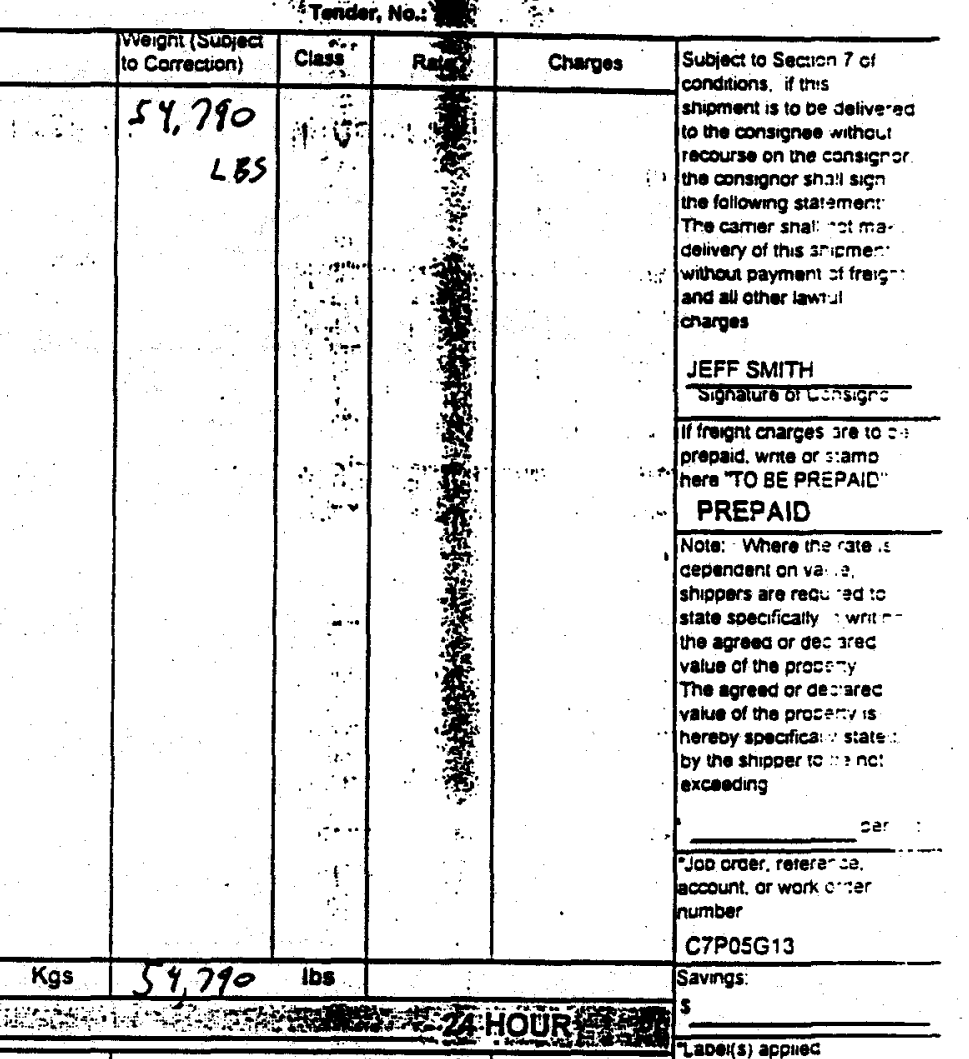

\begin{tabular}{l|l|l|l|l}
$\triangle N O$ & NMFE : & NPM NO & \\
\hline
\end{tabular}

Toral P!eces

TOTALS:

$+1$

1

emarks:

ECHNICAL CONTAE:

ihis is to centify that ine above named matenals are property ctessitiad. Festribed. packagec. marked. and labeled. and are in proper condition for ionsocitation aceorcing io the acplicable regulations of the Depanment of Transoenation (Apciıesole for Hazaroous Matenals Only.)

RiEDer BECHTEL NEVADA P O BOX 98521. LAS VEGAS NV B9193 zing unger contros: CEACOd $93 \mathrm{NV} 11718$

er MLAN R. MARCYAND

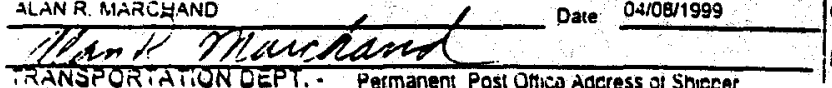

winn U.S. Dept of Energy

Date $04108 / 1999$
This shipment is for U.S. Department of Eneroy and the ectual totel treneporetion crarget pact to the

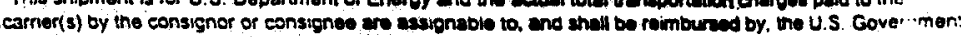
jnd is sueject to the terms and conditions set forth in the stendard form of tho U.S. Govermment Bill $\mathrm{e}$ ! Lading and to any availaole speciat rates or cherpes (41 CFR .109-10.50 end 41 CFR 40 3)

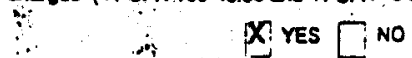

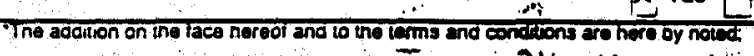

CTON I ENVIRO TRANSPORT OF NV $7 \cdots \cdots ?$

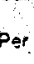

P.

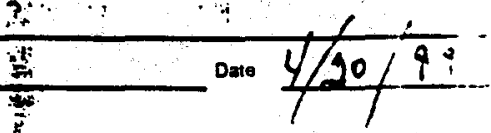


STRAIGIIF BIII OE IADING - SHORT FORM - ORIGINAI - NOt NJEG̈Otiabi

Shipper: BECHTEL NEVADA FOR US DOE

-... CaU 4öi TAML SITE/TTR

TONOPAH NV 89049.
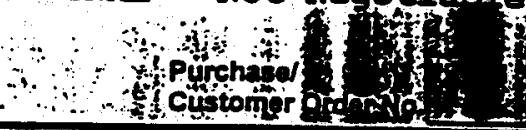

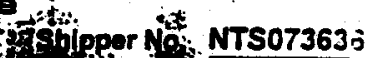

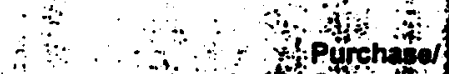
a $2 \frac{20}{20-99}$

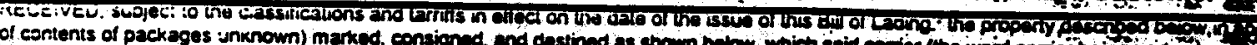

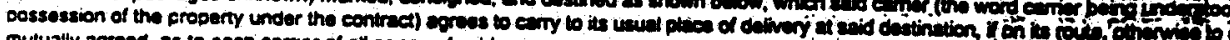

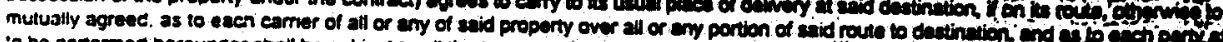

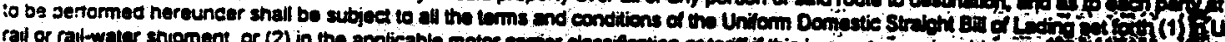

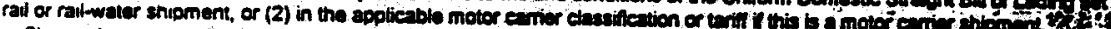

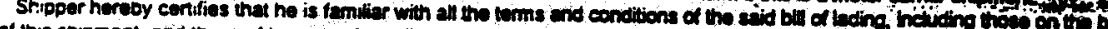

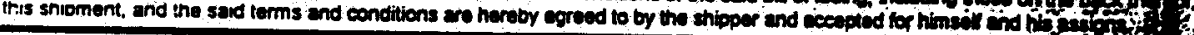

Consignee

BECHTEL NEVADA FOR USDOE

A.3 RWMS. ZONE 2

NEVADA TEST SITE

MERCURY NV 39023

MIKE SMITH / (702) 295-7365

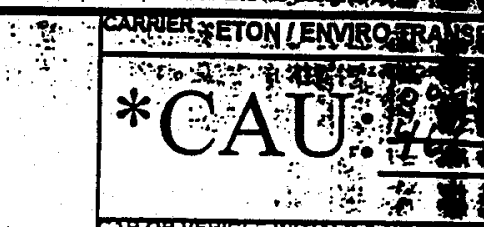

Ty

torion

torition

1)

(5) in: $x_{i}$

Fin

Fif: In:

雍

B. a.sicen

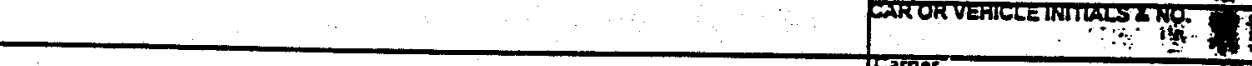

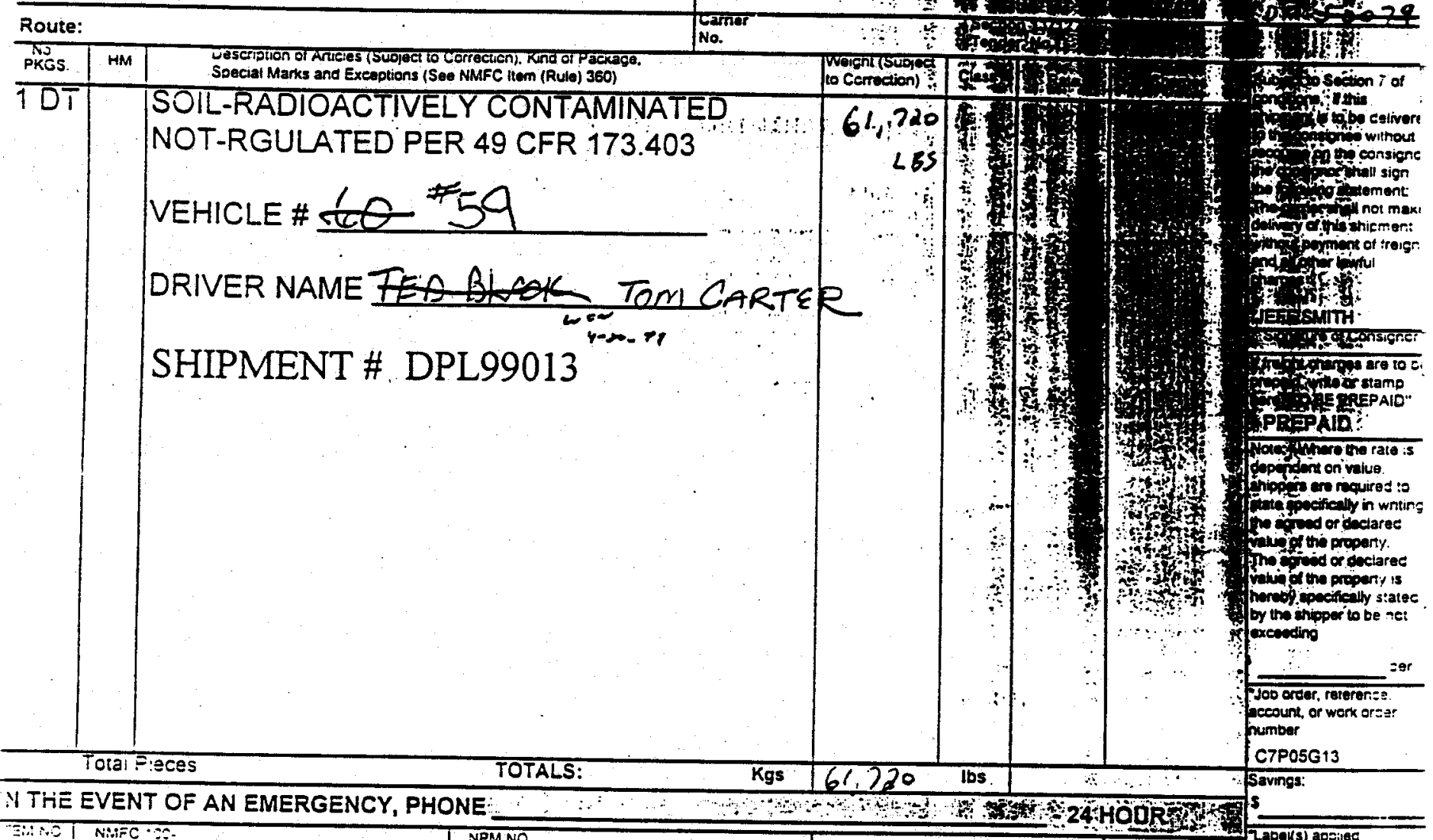

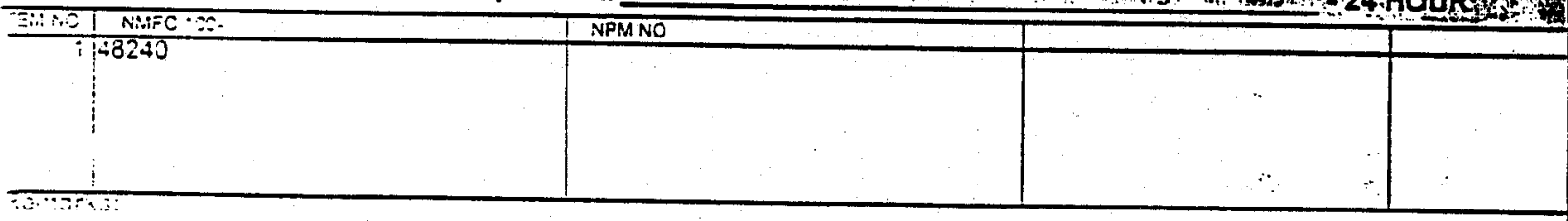
Labots) apsilec

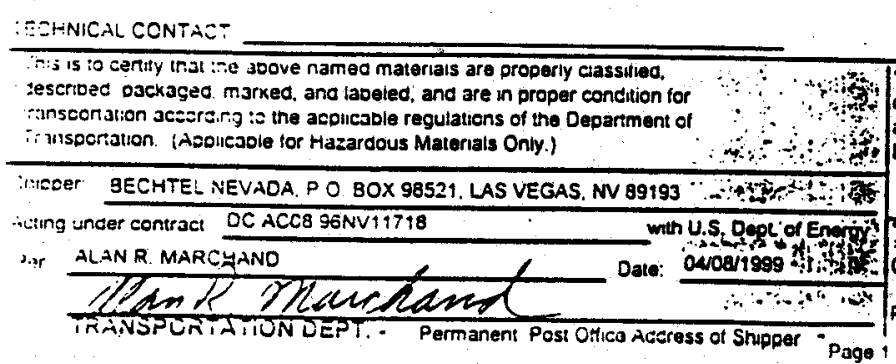

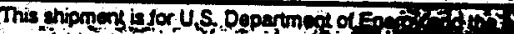
carnoris by the consionor ar consionee and is cubject to ino terms and condition

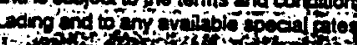

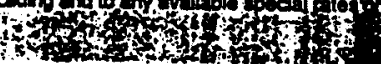

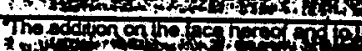

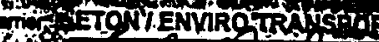
$\frac{1+2=2}{4+\infty}$ Los 1.2 
STRAIGHT BIIL OF LADING - SHORT FORM - ORIGINAL - NOt Négotiabile
Shipper: BECHTEL NEVADA FOR US DOE CAU 461 TAML SITE/TTR TONOPAH NV 89049

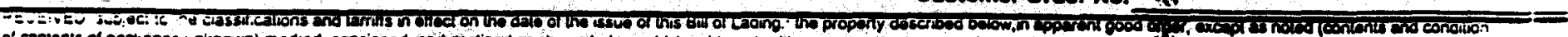

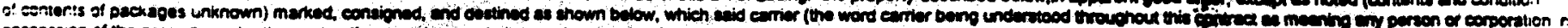

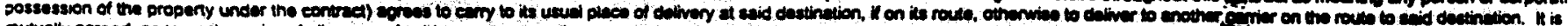

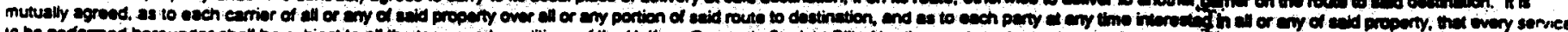

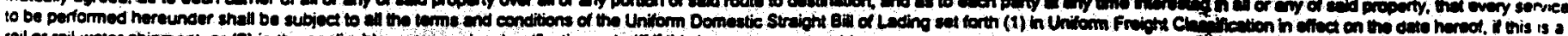

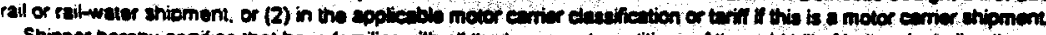
x:

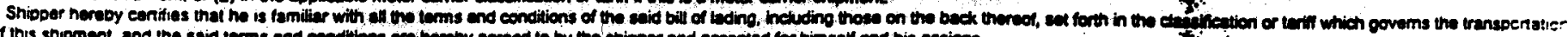

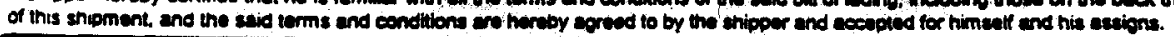

Consignee

BECHTEL NEVADA FOR USDOE

A-3 RWMS, ZONE 2

NEVADA TEST SITE

MERCURY NV 89023-

MIKE SMITH / (702) 295-7365 CARRUER ETON I ENVIRO TRANSPORT OF WY ti.
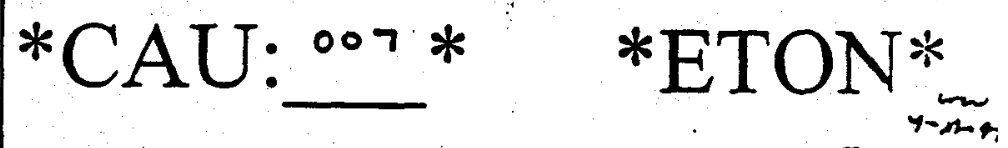

SEN. TLD $T \operatorname{con} 3 e^{2} x$

Route:

Camor
No.
Tonder, No:

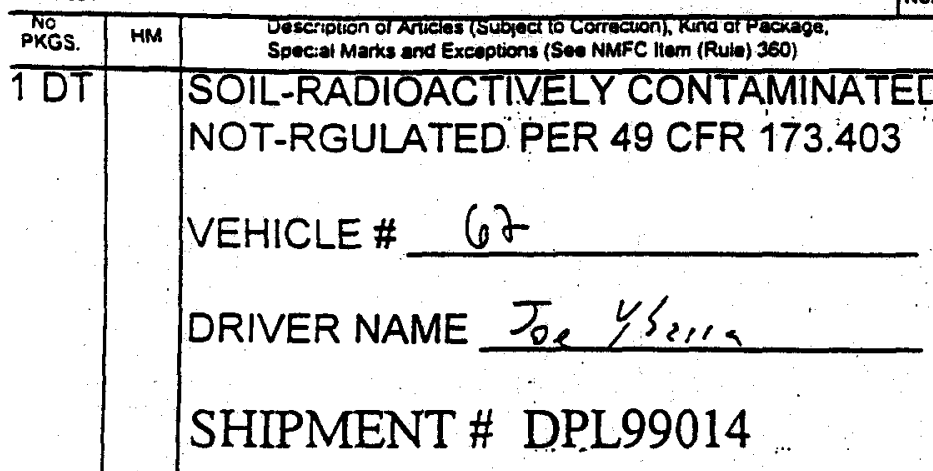

Total Pieces

TOTALS:

Kgs 55,50 to Correction)

\begin{tabular}{|c|c|c|c|}
\hline Clase & Rave & Cherges \\
\hline & $\ddots$ & $\ddots$ &
\end{tabular}

Subject to Sectien 7 :: condtions. It this

shipment is to be delivs-ted to the consignee withe: recourse on the cens: $₹-$ "? the consignor shrit sian the following sta: i -2 .

The camer shain The camer shal :.. delivery of this sr : $=-7$. and all other lawt.: charges

JEFF SMITH STh

fif frocom charges are :: : : propact. write of siar: here TO BE PREOAに PREPAID Note: Where the :a: depencent on vaice. shippers are rectit: shippers are reclit: es :z state spectically : in wri. value of the property

The egreed or deciarec value of the propeny :s hereby specificall; s:a':-

by the shieper tc $\Rightarrow ?-$ ? exceeding

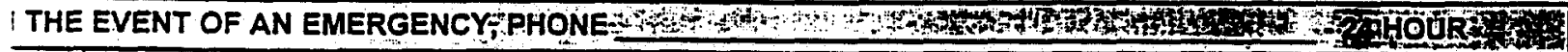

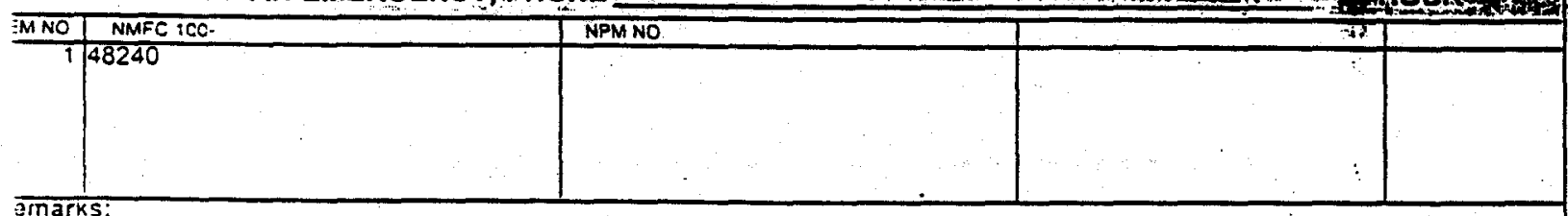

OHNICAL CONTACT

his is to certily that the above named materials afe property clessitied. escribec. packaged, marked. and labeled, and are $n$ proper condition for ansoortition according to the applicable reguiations of the Department of ranscortation. (Appicable for Hazaroous Matorials Onty.)

DET BECHTEL NEVADA. P.O. gOX 99521. LAS VEGAS. NV 89193 ing under contract DC ACDB $96 \mathrm{NV} 11718$ with U.S. Dept of Enargy ir ALANR MARCHAND Dase 0408/1999

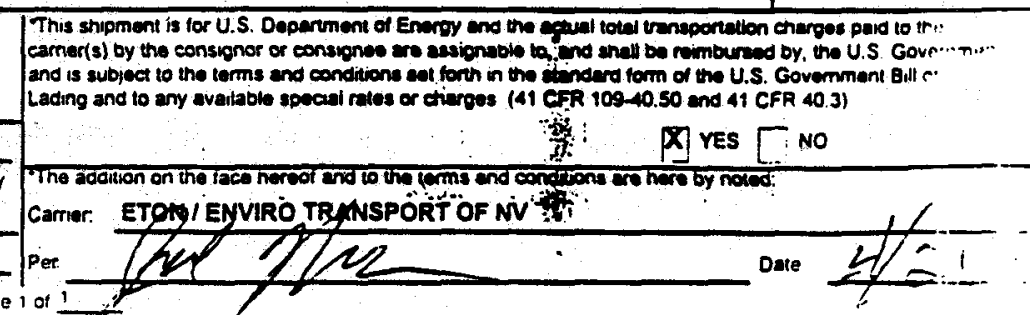


STRAIGHT BIII OF IADING - SHORT FORM - ORIGINAL - NOE NegOtiable

Shipper: BECHTEL NEVADA FOR US DOE CAU 461 TAML SITE/ TTR TONOPAH NV 89049

:Purchasel

'Customer Order No.

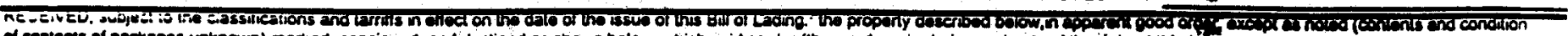

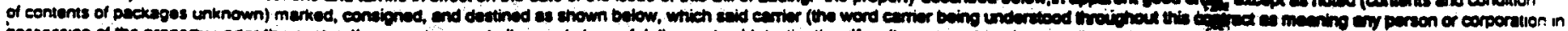

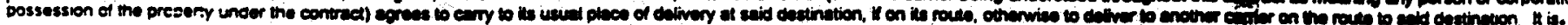

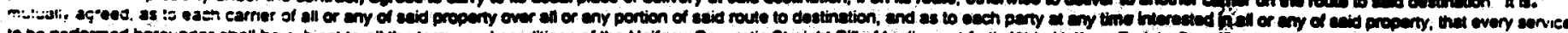

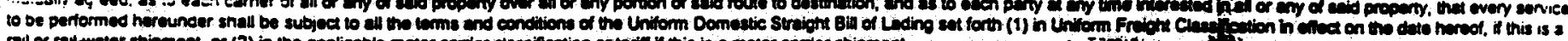

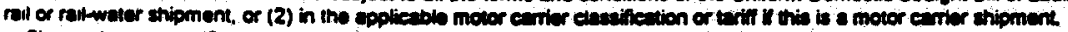

Tht

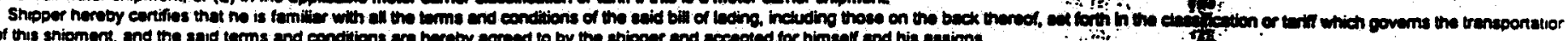

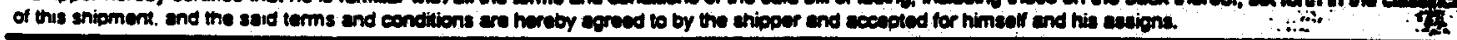

Consignee
BECHTEL NEVADA FOR USDOE

A.3 RWMS. ZONE 2

NEVADA TEST SITE

MERCURY NV 39023 .

$\rightarrow$ MIIKE SMITH I (702) 295-7365 * A U. oog * : * *

Route:

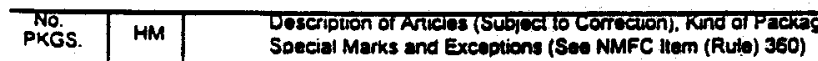
No.

1DT SOIL-RADIOACTIVELY CONTAMINATED NOT-RGULATED PER 49 CFR 173.403

VEHICLE \# 59

DRIVER NAME Tom Cinte SHIPMENT \# DPL99015 Tender, No.:

IN THE EVENT OF AN EMERGENCY,PHONE

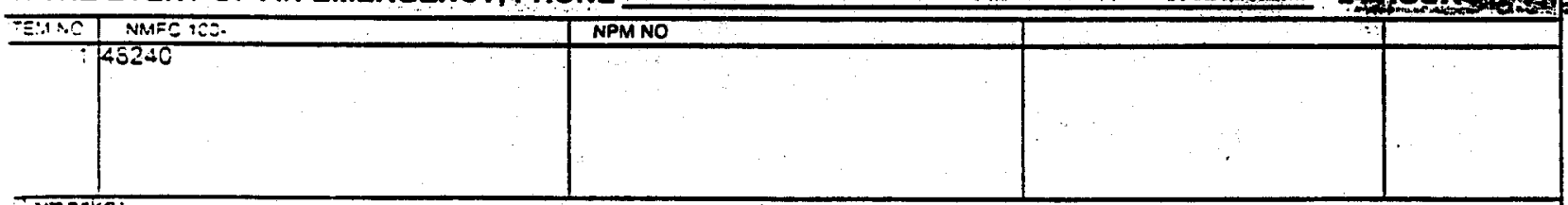

jinccer BECHTEL NEVADA. P.O. BOX 98521 . LAS VEGAS. NV 89193 
Customer Order No.

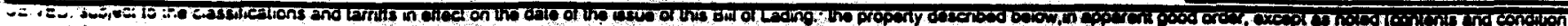

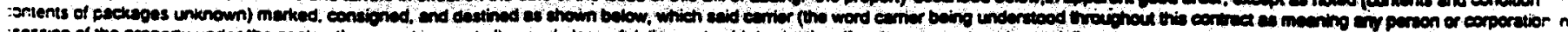

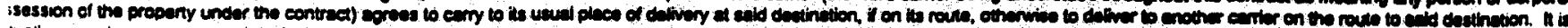

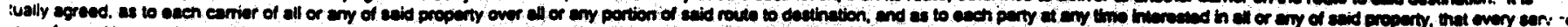

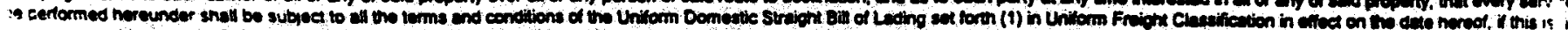

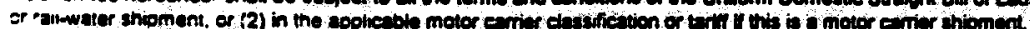

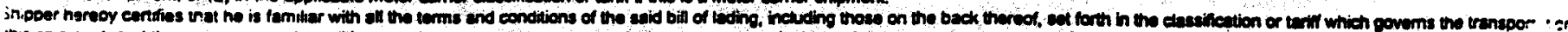

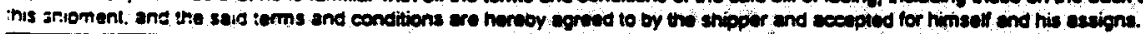

Insignee

SECHTEL NEVADA FOR USDOE

4.J RIVMS, ZONE 2

VEVACA TEST SITE

HERCURY NV 8SC23.

SIKE SMITH / (702) 295.7365

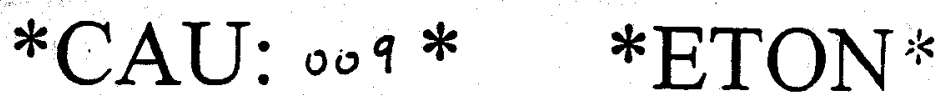

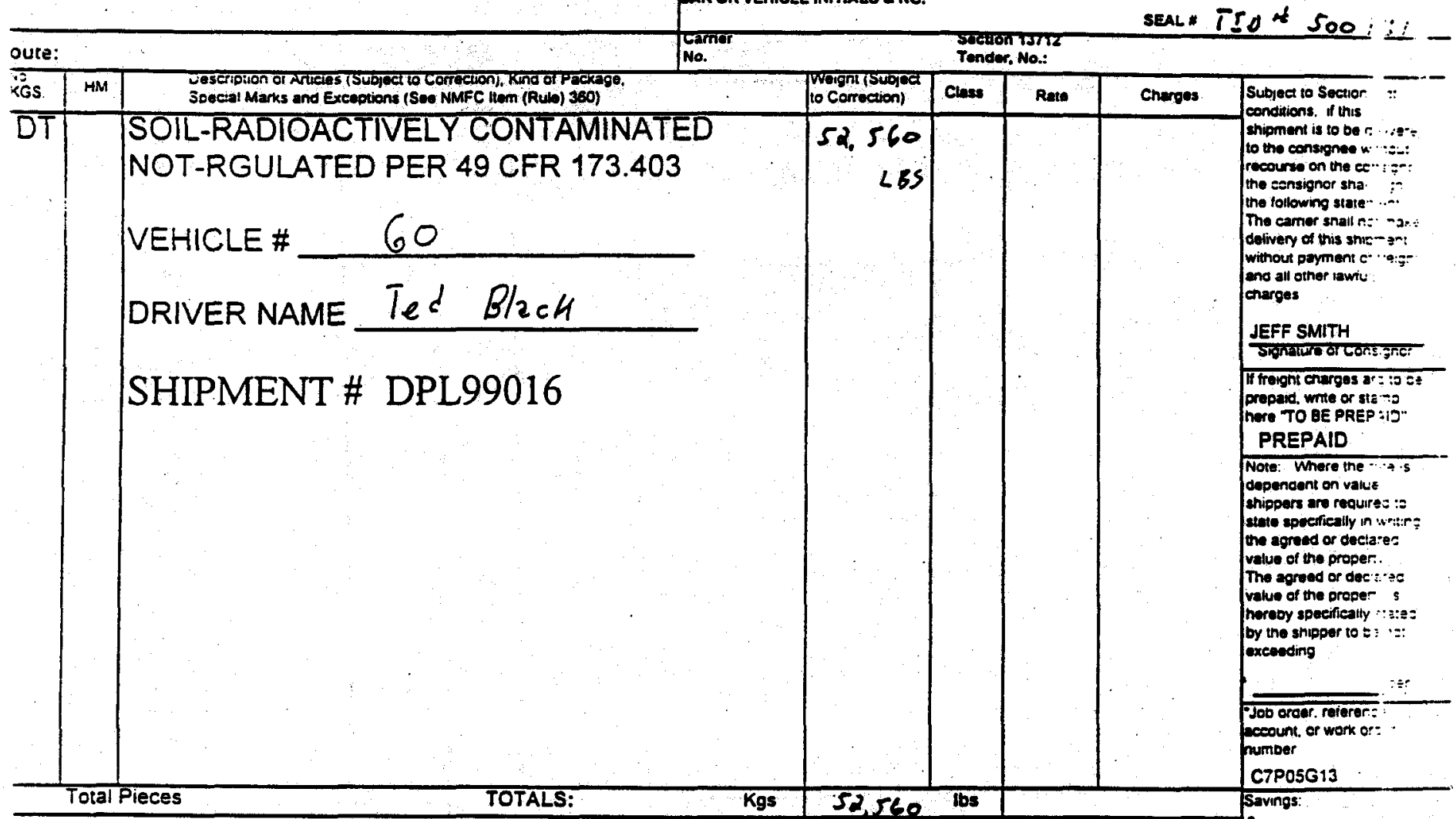

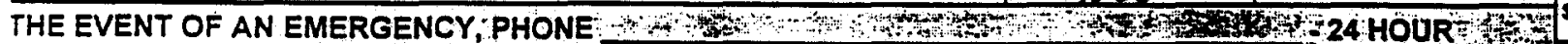

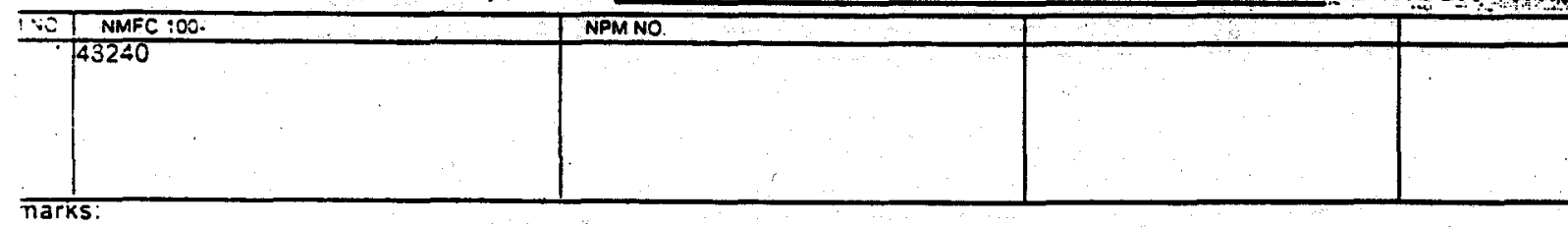

:HNICAL CONTACT

is is to cenily that the above named materials are property ctassived. 5:- :es. sackaged. marked. and labeled, and are in proper condition for 75:-onziton according so the applicable requations of the Depantment of insponation (Applicable for Hazardous Matenals Only.)

cer BECHITEL NEVADA, P.O. BOX 98521. LAS VEGAS. NV 89193 19. uncer contract DC ACOB 96 NV 11718 ALAN R. MARCHAND

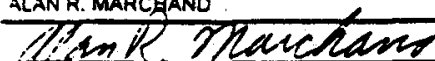
1 with U.S. Dept. of Energy

TRANSPORTATION DEPT. Permanent Post Oftice Aodress of Shipger
Date: 0410a/1999

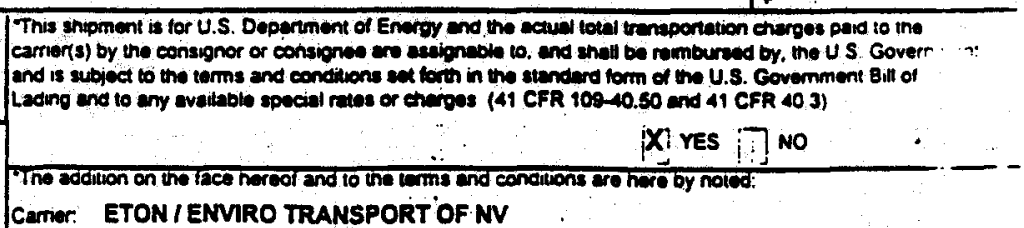
Carrier: ETON/ENMRO TRANSPORT OF.NV 
STRAIGHT BILI OF IADING - SHORT FORM - ORIGINAL - Not Negogitiable

H.TPper: BECHTEL NEVADA FOR US DOE

CAU 461 TAML SITEITTR

TONOPAH NV 89049 .

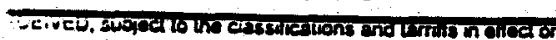

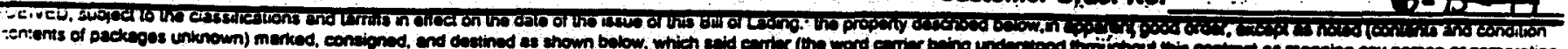

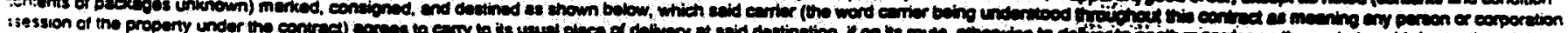

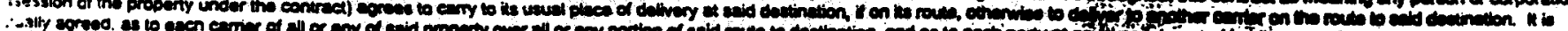

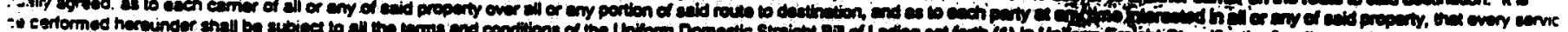

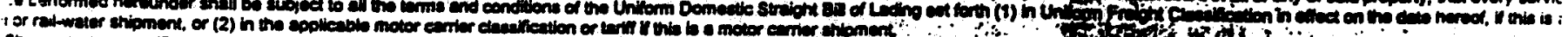

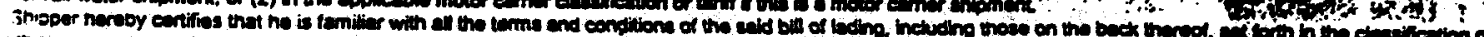

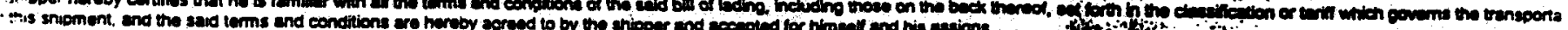
:onsignee

3ECHTEL NEVADA FOR USDOE

A.3 RWMS, ZONE 2

NEVADA TEST SITE

MERCURY NV 89023.

MIKE SMITH / (702) 295-7365

Zoute:

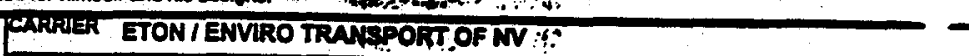

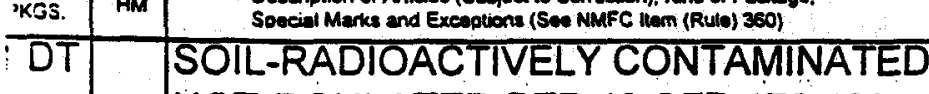

NOT-RGULATED PER 49 CFR 173.403

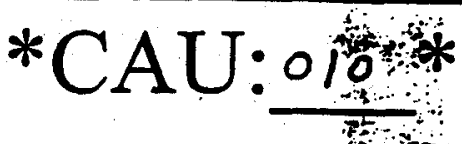

*ETON:

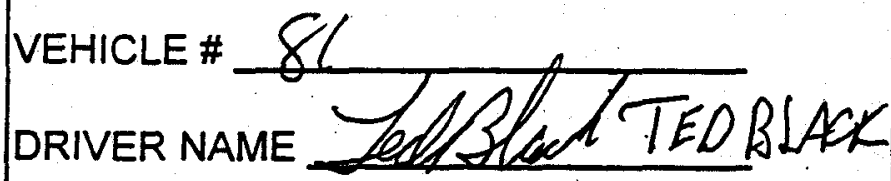

SHIPMENT \# DPL99017

Total Pieces

TOTALS:

Kgs 1460

lbes:

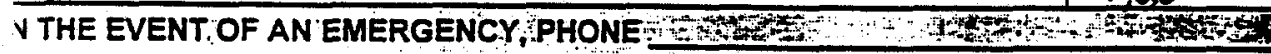

BNO I NMFC ICO.

$: 48240$

emarks:

NPMNO.

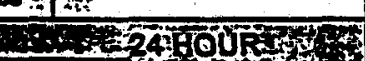

$\Rightarrow$

Subject to Section 7 conditions, if this

thipment is to be de res

to the consignes wit: :

neourre on the con: -or

the convignor shall

the following staterr

The carriar shalt ne:

withous paymens of.

end all other lawul

JEFF SMITH

STonduret or cons: -

Wh froghn enarges are

prepaid. write or star

hero TO BE PREP;

PREPAID

Note: Where trie rá

dependent on vatue.

chipoers are requree

thate soveifieally in u

the agreed or toctar.

the equer dectar.

vive of the proporty

the eqreed or ceclar

value of the propenty.

homby specifieally s:

by the shipper to be :

excesting

Job order, reterence

ccoount, or work ore

number

C7P05G13

sevings:

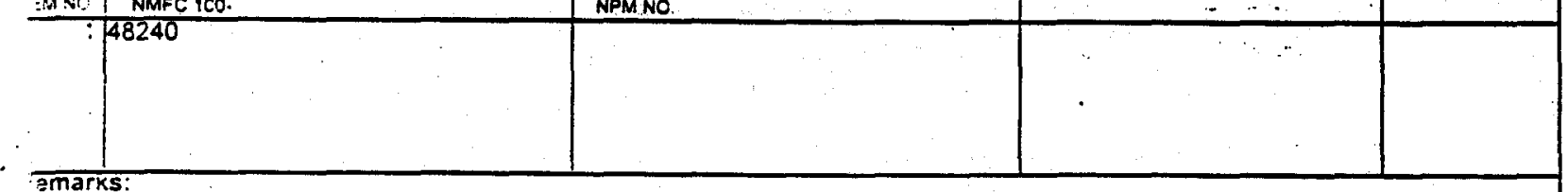

CHNICAL CONTACT

Tis is to centify that the acove named matenals ere property ciassited.

:ase:0ed. paekaged. marked. and labeled, and tre in proper condition lor

insogriation aceording 10 ine applieable regulations of the Department of

:ansoortation. (Applicable for Hazardous Matenats Only.)

oder BECHTEL NEVADA, P. O. 80X \$8521, LAS VEGAS, NV 89193

$\because:$ ing under contract DC ACOB 96NV11718

with U.S. Dept of Energy

* ALAN R MARCHAND

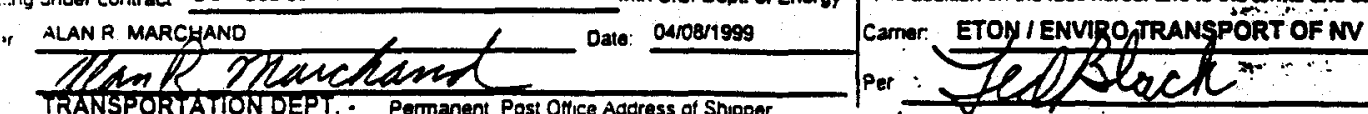

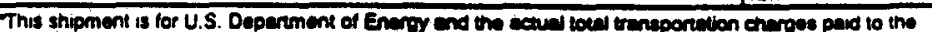

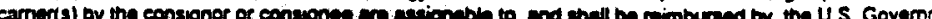
and is subject io ine terms and conditions get form hn the stunderd form of the US. Conernment Bill of Lading and to eny avaitable speciel retes or cheres (4t CFR $109-10.50$ and 41 CFR 40.3)

TRANSPORTATION DEPT. - Permanent Post Oftice Address of Shipper

$$
\text { - }
$$

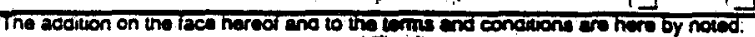

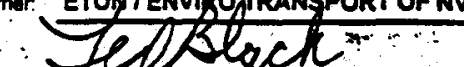


STRAIGET BIIL OF IADING - SHORT FORM - ORIGINAT - NOt Negotiable
ipper: BECHTEL NEVADA FOR US DOE

CAU 461 TAML SITEI TTR

TONOPAH NV 89049.

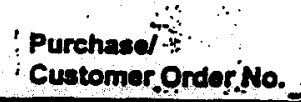

Shippor No. NTS0736:

Dato:

4 -inte

Customar OrdoriNo.

$\overline{-}$

AETVE. SUb

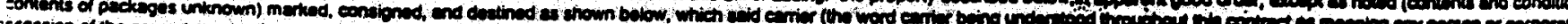

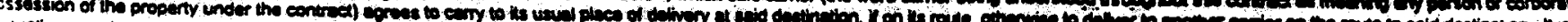

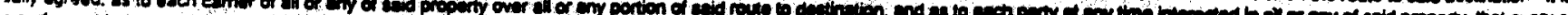

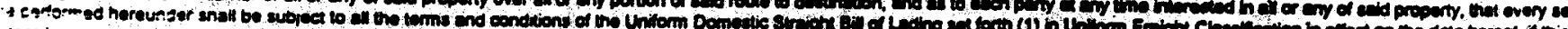

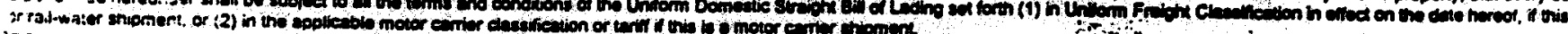

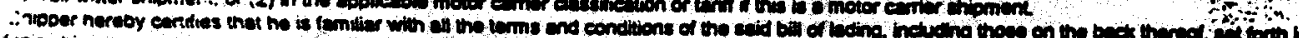

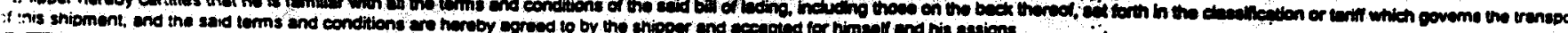
ionsignee

BECHTEL NEVADA FOR USDOE

A-3 RWMS, ZONE 2

NEVADA TEST SITE

MERCURY NV 89023 -

MIKE SMITH / (702) 295-7365

WARLUER ETONIENVIRO TRAMSPOQTT OF NV

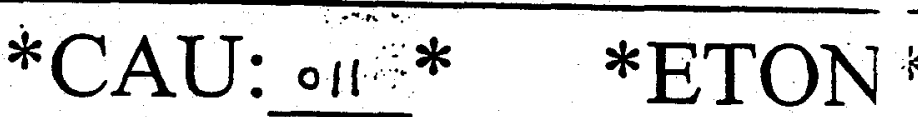

is

Route:

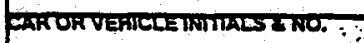

Grimor
No.

SEAL: TIO 4 Sing.

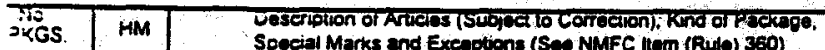

-

$50+9$

DT SOIL-RADIOACTIVELY CONTAMINATED NOT-RGULATED.PER 49 CFR 173.403

VEHICLE \#

102

DRIVER NAME

Tack Perna

SHIPMENT \# DPL99018

Total Pieces

TOTALS:

to Correction)

\begin{tabular}{|l|l|l|}
\hline Cleses & Rate & Cherpes \\
\hline
\end{tabular}

Subject to Sectior eonitions if this condivions. If this

to the consignee $r$

to the consigned $r$

recourse on the e.

the lollowing sat

The canter snalt

dolivery of this ar

winhour payment

and all other lawt

charves

JEFF SMITH

Signaluto dCE

if trught charges

prepact. write or here TO BE PRE

PREPAID

Note: Where ine

dependent on vali:

shipoers are requ:

state specifieally ir

the egreed or dec.

value of the proce.

The agreed or des

value of the prope.

hereoy apocifiesily

by the shipper to $\mathrm{s}$

exceoding

(2)

Jos orcer, rererer

sceount, of work $\mathrm{C}$.

number

C7P05G:3

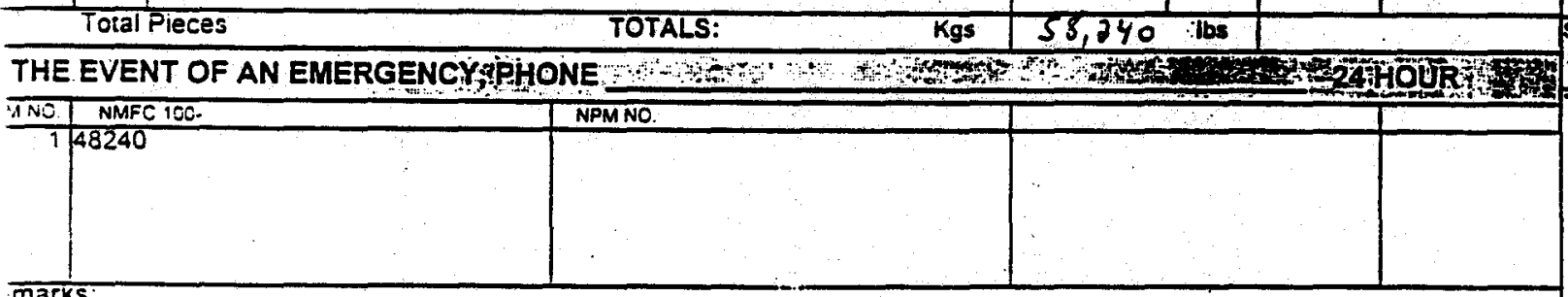

ZHNICAL CONTACT

is is to cently that tha aoove namec matenals ere property classitued escribed. packaged, marked. and labeled, and are in proper condition tor Insoonation according to the applicable regulations of the Deparment of anscontation. (Applezale for Mazardous Matorials Only.)

goer BECHTEL NEVAOA, P.O. BOX 98521 , LAS VEGAS, NV 89193 ing under contract DC ACOB $96 N V T 1718$ with U.S. Dept. of Energy ALAN R MARCHAND Date: 04/0erigs9 Savongs: s s.

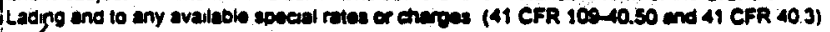




\section{-}

STRAIGET BILL OE IADING - SEORT FORM - ORIGINAI - NOt NegOtiable

Shipper: BECHTEL NEVADA FOR US DOE CAU 461 TAML STTE TTR

TONOPAH NV 89049.

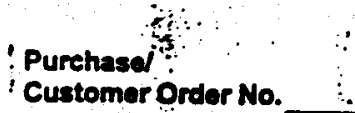

Shippor No. NTS07363

Customar Ordor No.

Date: $4+\frac{4-1}{6-1}$ $6-1,-.48$.

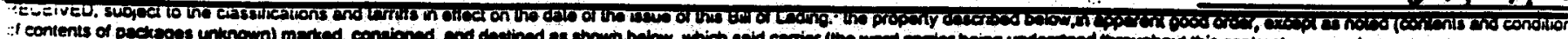

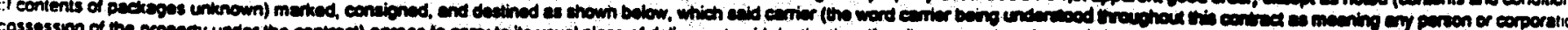

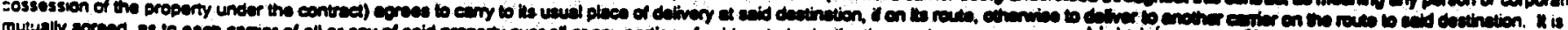

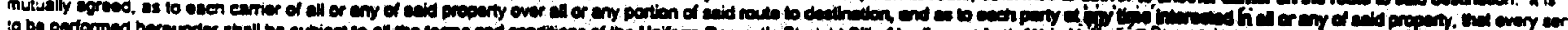

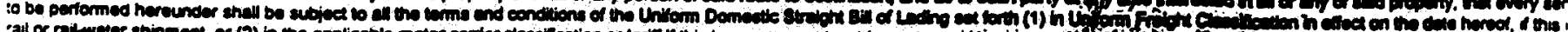

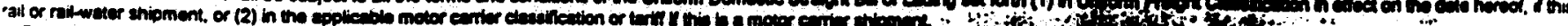

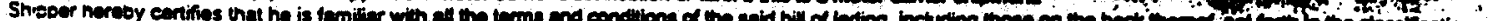

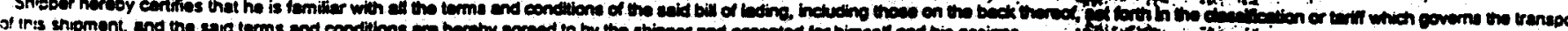

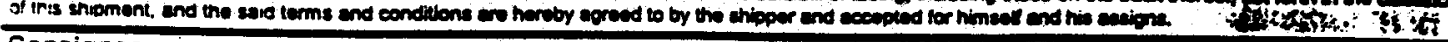

CONsignee BECHTEL NEVADA FOR USDOE

A.3 RWMS, ZONE 2

NEVADA TEST SITE

MERCURY NV 89023-

MIKE SMITH / (702) 295-7365

\begin{tabular}{l|l}
\hline Route: & No. \\
\hline
\end{tabular}

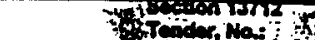

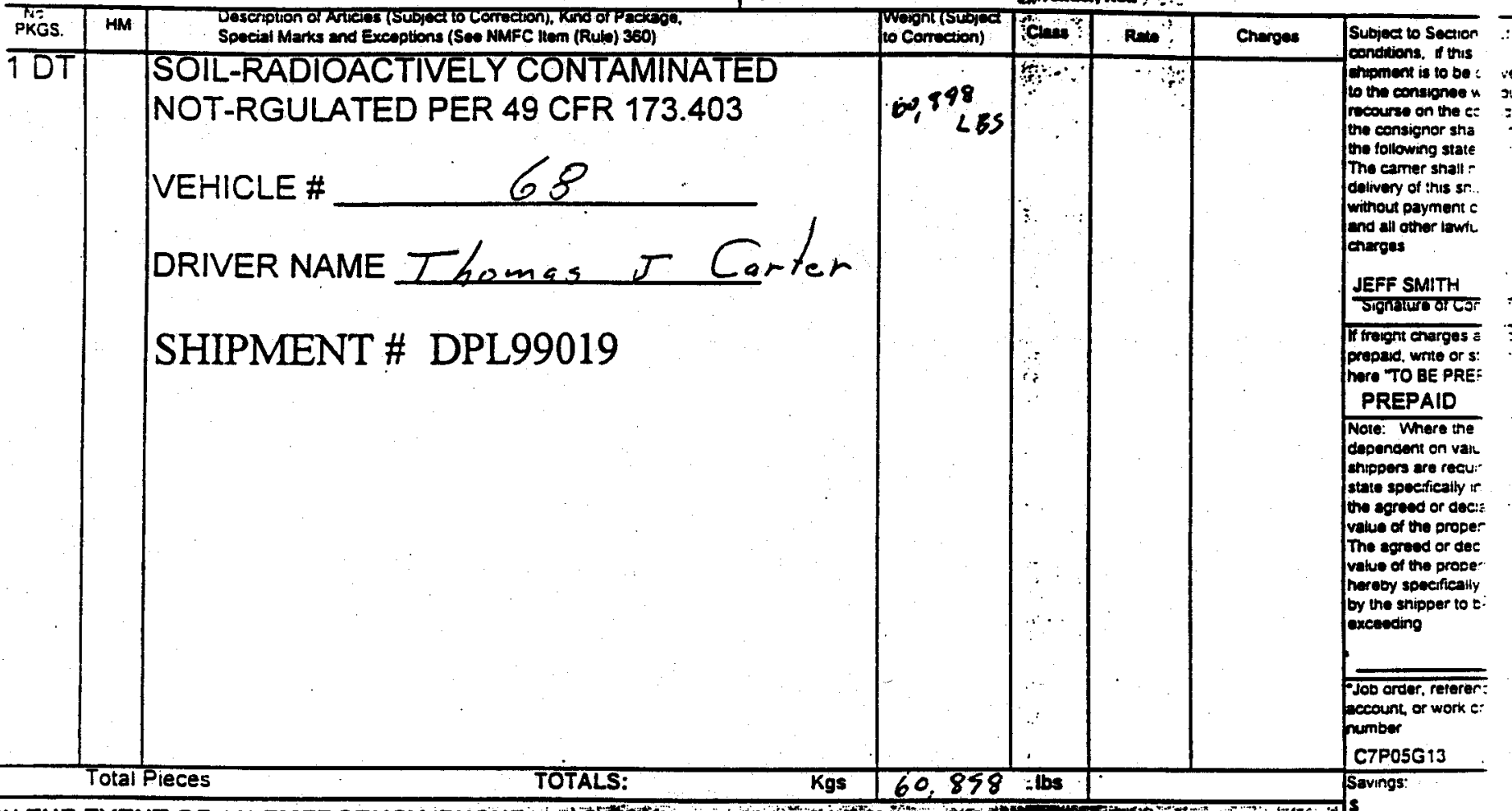

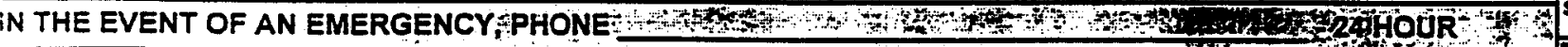

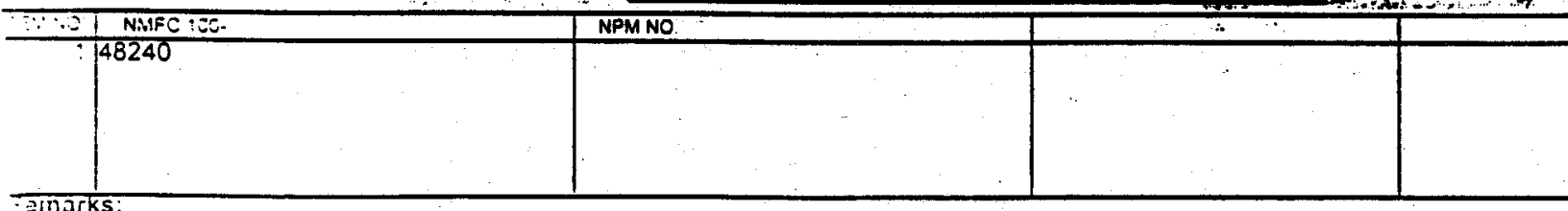

EEMNICAL CONTACT

T-is is to contry that ine above named materais are property elasstied.

: sce:10ed. packaged, marked. and labeled, end are in proper condition for

inseortation according to the acplicabte regulations of the Depanment of

insponation. (Applicadie for Mazardous Matenalis Only.)

, $3: 38$ BECHTEL NEVADA. P O. BOX 98521 , LAS VEGAS. NV 89193

$\because-3$ orser contract DC ACOB 96NV11718

with U.S. Dept of Energy

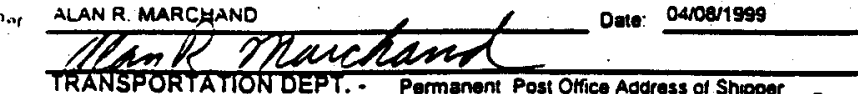

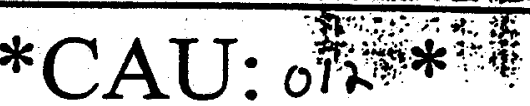

*4. 
FRAIGET BIIL OF IADING - SHORT FORM - ORIGINAI - NOt NOGOLIablo

per: BECHTEL NEVADA FOR USDOE

CAU 461 TAML SITE/ TTR

TONOPAH NV 89049.

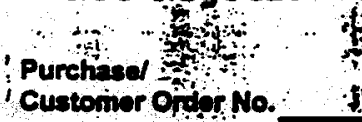

shippor No. NTS073636

Data: $\frac{6-17-11}{}$

t)

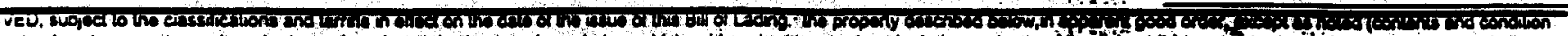

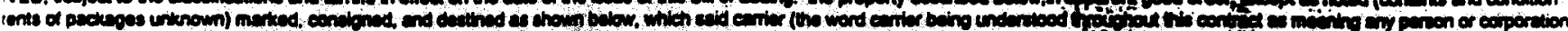

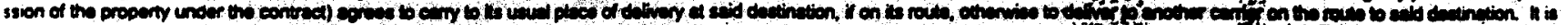

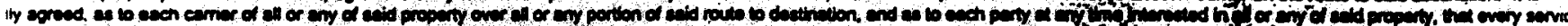

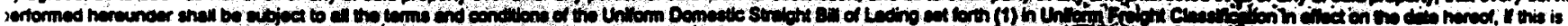

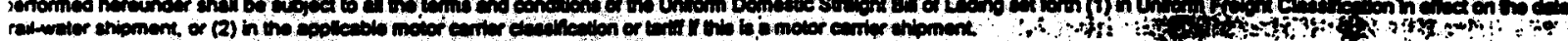

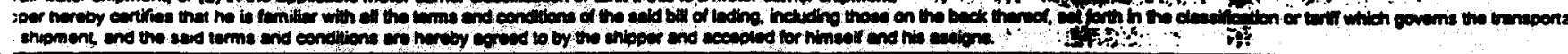

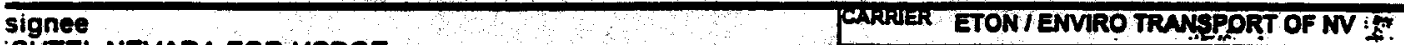

CHTEL NEVADA FOR USDOE

3 RWMS, ZONE 2

VVADA TEST SITE

IRCURY NV 89023.

. KE SMITH / (702) 295-7365

\begin{tabular}{ll|l}
\hline Ite: & No \\
\hline
\end{tabular}

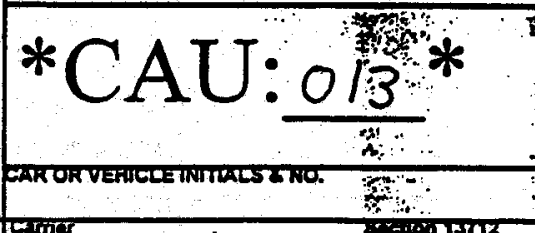

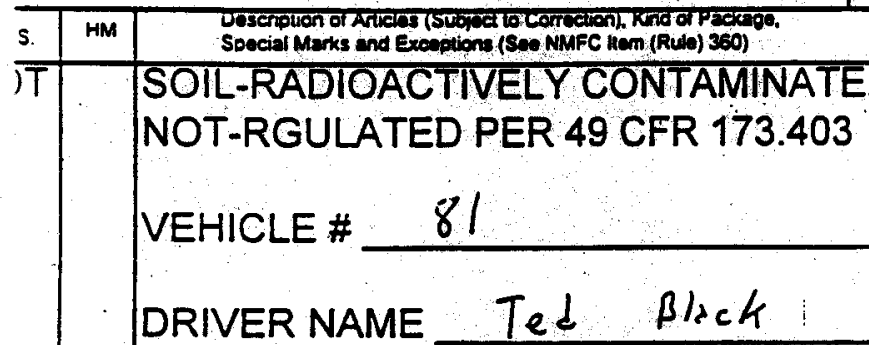

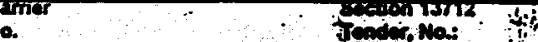

$* \mathrm{ETON}$ :

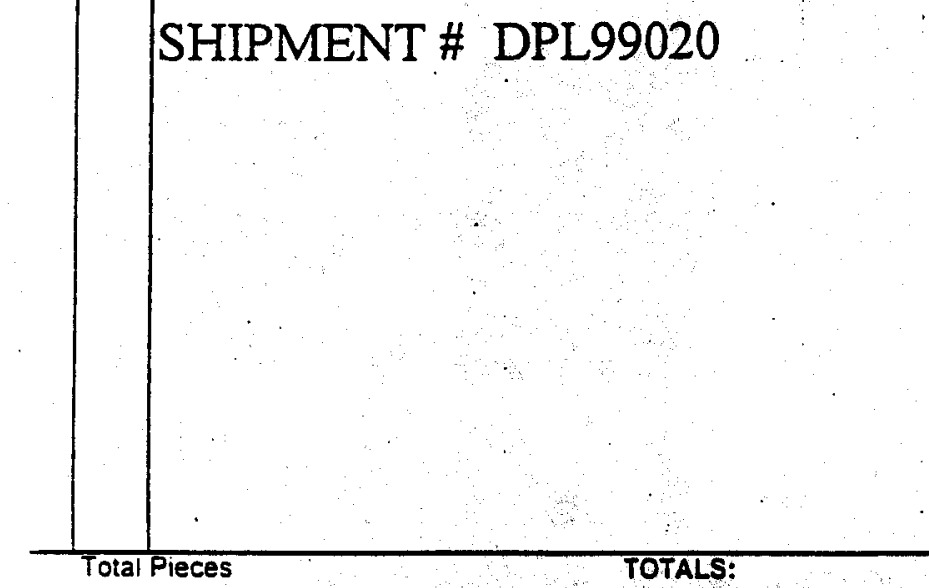

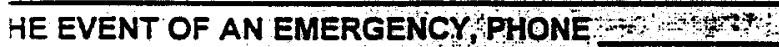

Kgs $5 \% 910.105$ $\rightarrow$

sen: 500966

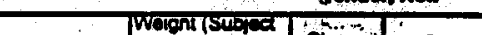

is

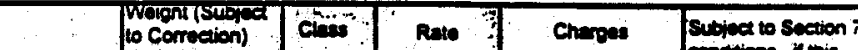

Subject to Suction

shipment is oo bo de leies

thipmant is to be de ceice

recourse on the cen: :- :-

the convioner shall :

the following statem.

The carrier shall net

delivery of this anipr

witnout osyment of:

winout desment of or

chinges

JEFF SMITH

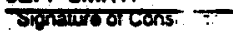

Thenght charges are

prepaid, white or sta:

hero TO BE PREP:

PREPAID

Note: Whers the ra

dependent on value

athippers are require.

thate specificatly in is

ine agreed or cecla:

vilue of the proper

The apreed or decla

value of the proper:

heroby epecifically $s$ :

by the shiper to be

exeending

\begin{tabular}{l|l|l|l|l|}
\hline O. NMFC $100-$ & NPMNO & & \\
\hline 1.48240 & & & & \\
& & & & \\
\hline
\end{tabular}

arks:

Job order, roference

cocount, $\propto x$ work orct

number

C7P05G13

Suings:

$s$

Label(s) applied

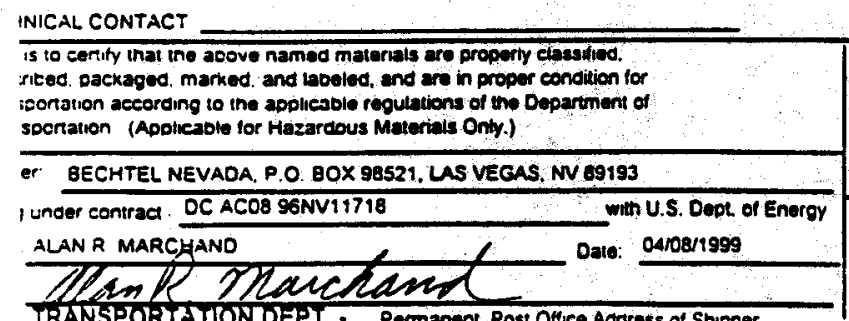

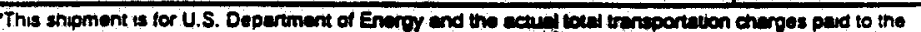

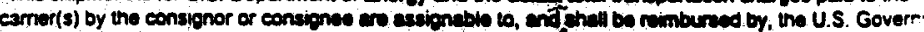
and is subject to the terms and conditons eat forth in the suend ond lorm of the U.S. Covernment Bill of Lading and to any available apecial rates or cherese (41 CFR 10040.50 end 41 CFR 40.3)

f: XI Yes No

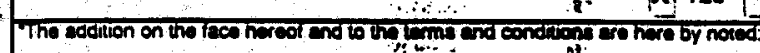

Camer ETON I ENVIRP TRAYSPORT OF NV ":

TRANSPORTATION DEPT. - Permanent POst Offuce Address of Shipoer 


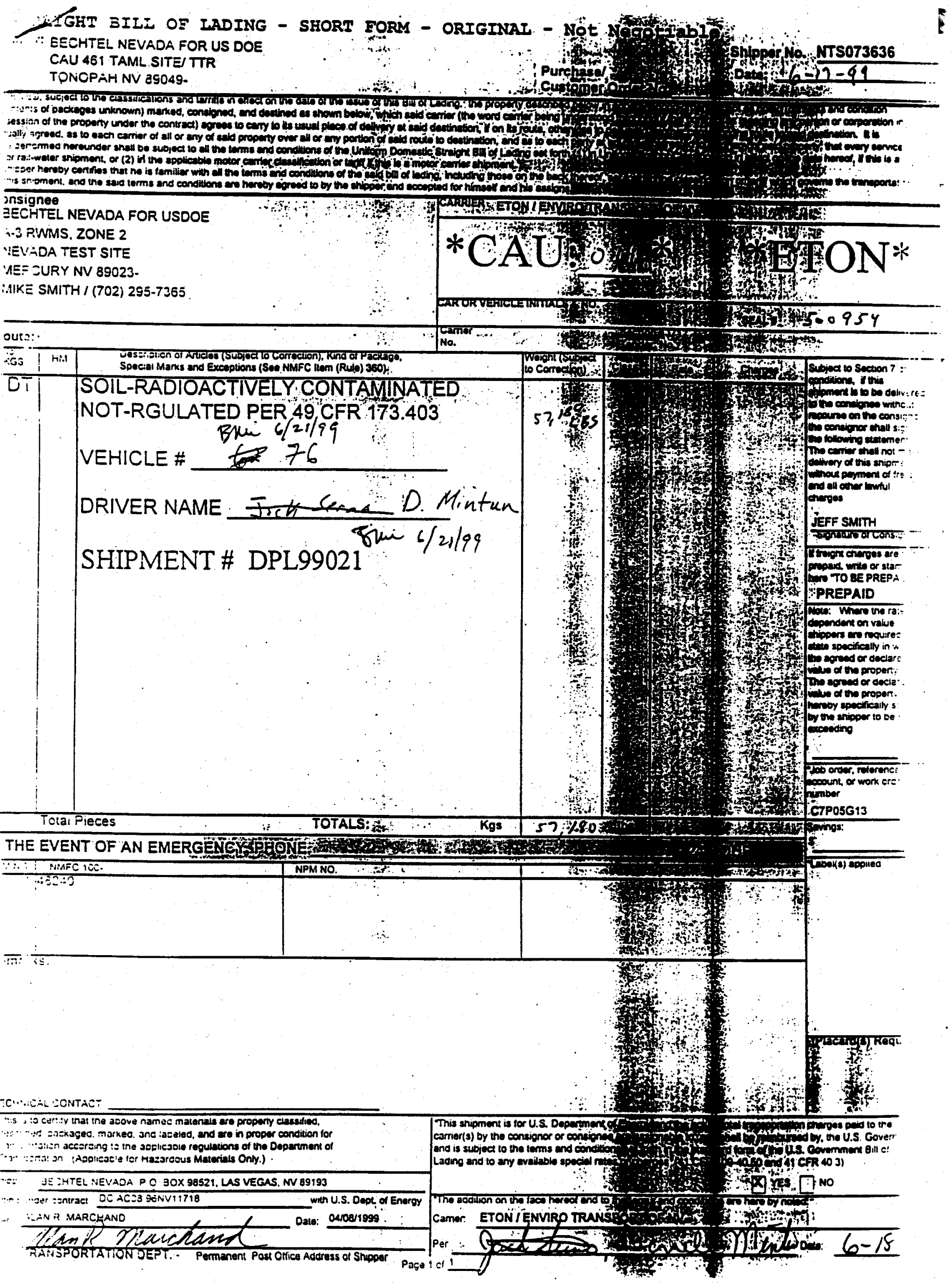


STREIGHT BIIL OF IADING - SHORT FORM - ORIGINAI - NOE NegOtiable
HQPER: BECHTEL NEVADA FOR USDOE

CAU 461 TAML SITE TTR

TONOPAH NV 89049 .

Purchased $2+1$

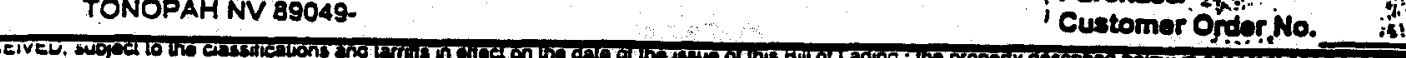

Shipper No. NTS073636

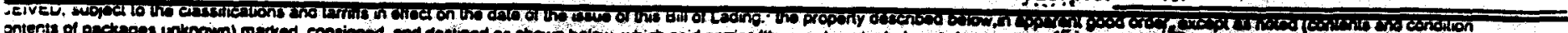

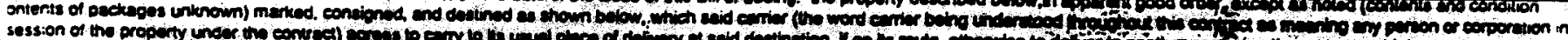

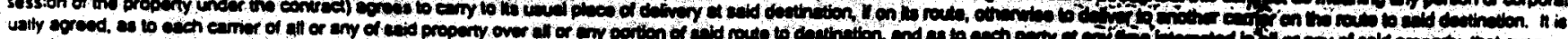

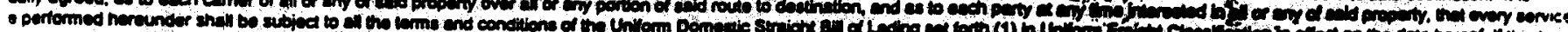

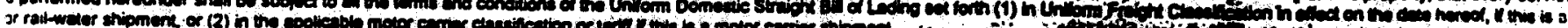

nicser nerooy contiog or

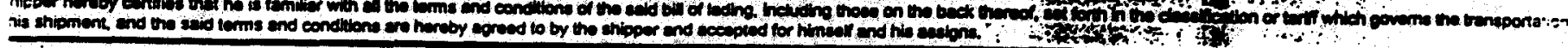
nsignee

3ECHTEL NEVADA FOR. USDOE

1.3 RWMS. ZONE 2

IEVADA TEST SITE

IERCURY NV 89023.

IIKE SMITH / (702) 295-7365

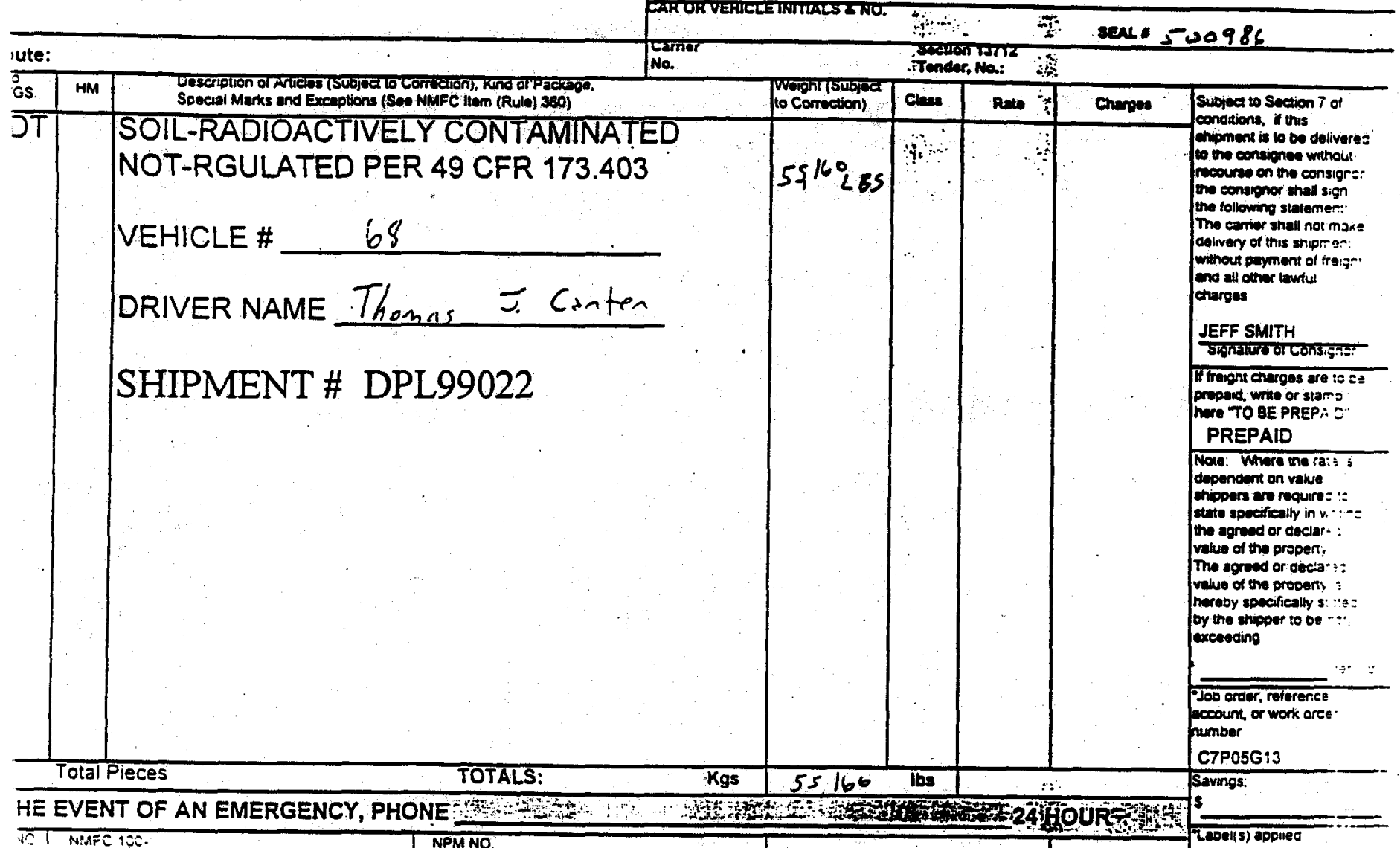

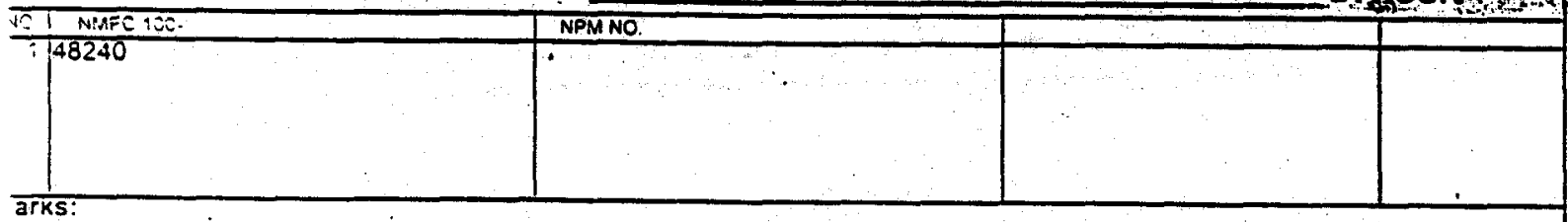

NICAL CONTACT

is to centify that the acove named materials are property classtied

ribed. packaged, marked. and labeled. and aro in proper condition for Donation according :o the apptreabie regulations of the Department of soonation (Applicable for Mazarcous Matenals Only.)

\# BECHTEL NEVAOA, PO BOX 98521 , LAS VEGAS. NV 89193 uncer sontract $D C A C C 896 \mathrm{NV} 11718$

ALAN R MARCHAND

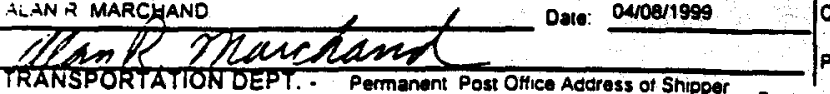

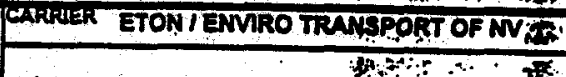

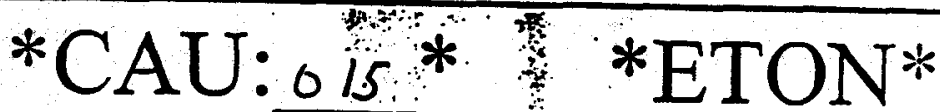




\section{DTPAIGHT BILL OF IADING - SHORT FORM - ORIGINAI - NOt Negotiable
ShPDER: BECHTEL NEVADA FOR US DOE}

CAU 461 TAML SITE/TTR TONOPAH NV B9049.

: Purchased

Customer OrdërNo. Ant
Shippor No. NTS073636

Dato: $6-17.81$

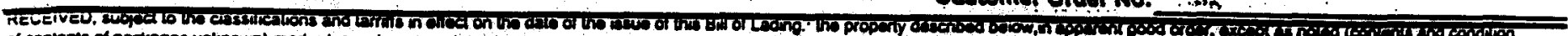

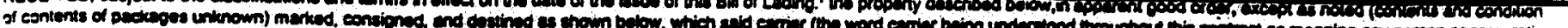

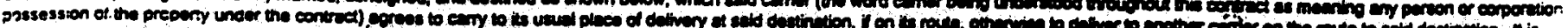

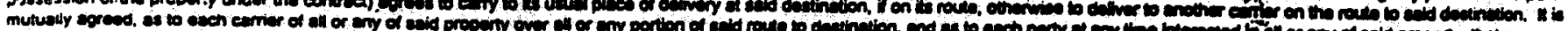

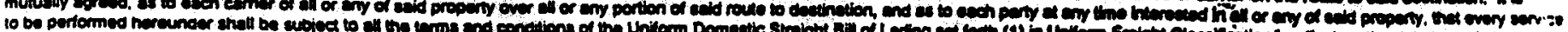

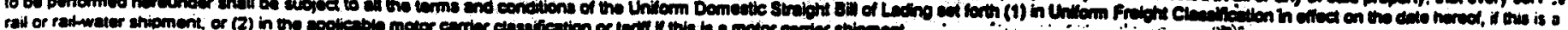

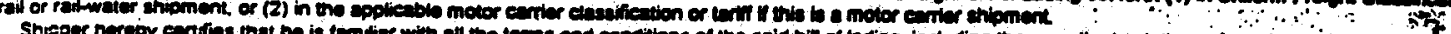

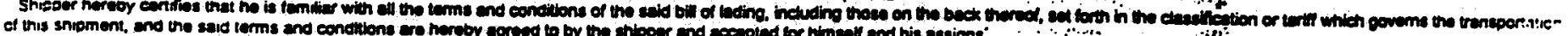
Consignee

BECHTEL NEVADA FOR USDOE

A.3 RWMS, ZONE 2

NEVADA TEST SITE

MERCURY NV 89023.

MIKE SMITH / (702) 295-7365

*CARRIER ETONI ENMIRO TRAMSPORT OF NV

Route:

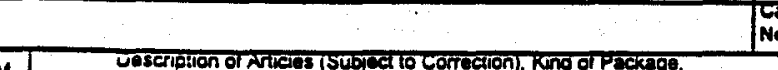
PAROFVERICLE MNITALSTNO.

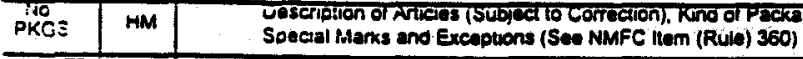

1DT SOIL-RADIOACTIVELY CONTAMINATED NOT-RGULATED PER 49 CFR 173.403

VEHICLE\# \# 81

DRIVER NAME Ted Black

SHIPMENT \# .. DPL99023

Total Pieces

TOTALS:

Kgs $5 \% 760$. las

Tendor, No.:

SEAL: 500955

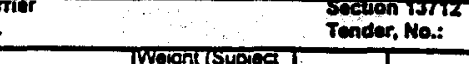

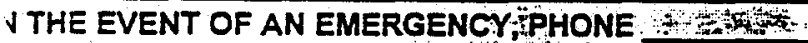

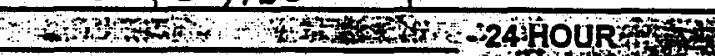
EMNO TMFC 100 . i 48240

emarks:

ECHNICAL CONTACT

his -s to cenify that the adove nameo matenats are propenty classitied. :asefited packaged. marked. and labeled. and are in proper condition for insecnation acceraing to the appticable regulations of the Depanment of iranseortation. (Apolicable for Hazaroous Matentis Only.)

rioder BECHTEL NEVADA. P O. BOX 98521 . LAS VEGAS, NV 99193 zung onder contract DC ACOO 96NV11718

$\because \frac{\text { LANR MARCAANO }}{\text { TRPALA Date: OANOB/1999 }}$
-This shiement is for U.S. Depertment of Energy and the ecanl totel trensportion cherges perd to the

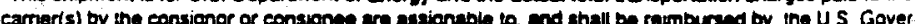
and is subject to the terms and conditions set forth in the standerd torm of the U.S. Covernment Bill $\mathrm{Cf}^{f}$ Lading and to any aveitabie special rates or cheress (41 CFR 109-40.50 and 41 CFR 40 3)

$$
\text { X) Yes } 7 \text { No }
$$

The addition on the face hereor and to th terms ano condions are here oy nold:

Camer ETON/ENVIRO TRANSPORT OF NV 


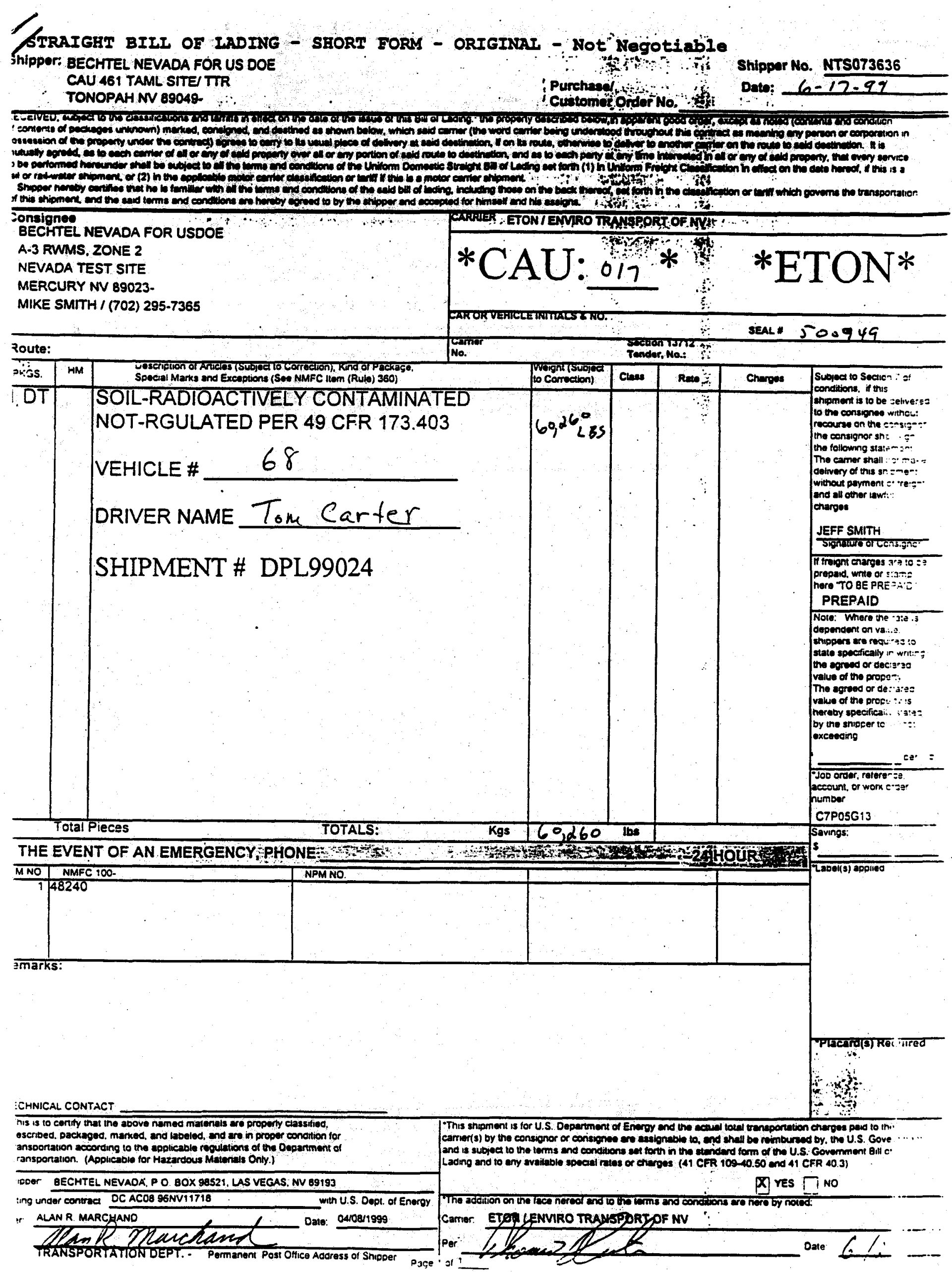


STRAIGET BILI OF IADING - SHORT EORM - ORIGINAL - NothiJegotiable

Shipper: BECHTEL NEVADA FOR US DOE

CAU 461 TAML SITE TTR

TONOPAH NV 89049

Nitis:

shist

Purched o

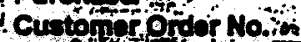

Shippor No. NTSO7:

Dato: $0 6 \longdiv { 2 1 / 6 }$

36

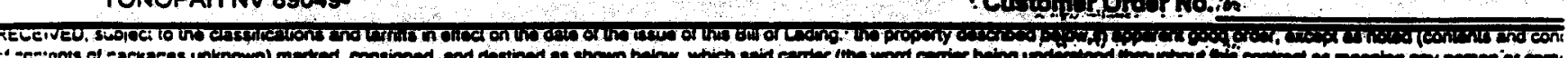

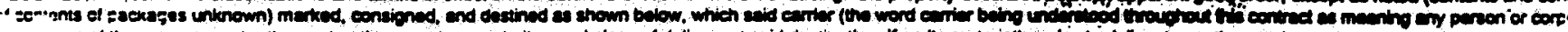

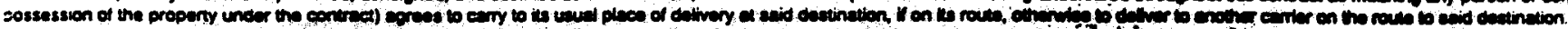

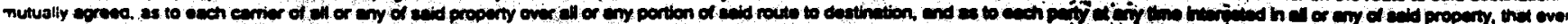

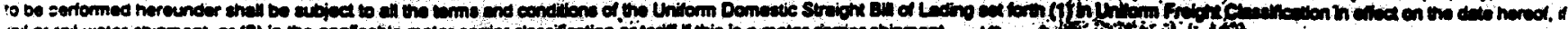

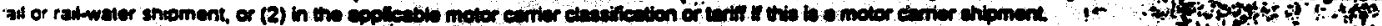

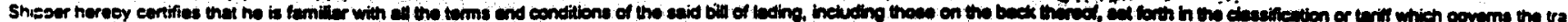

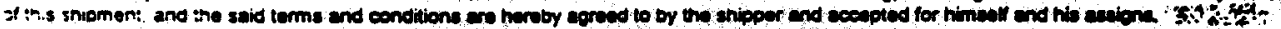

Consignee

BECHTEL NEVADA FOR USDOE

A-3 RWMS, ZONE 2

NEVADA TEST SITE

MERCURY NV 89023-

MIKE SMITH / (702) 295-7365

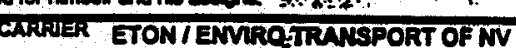

\begin{tabular}{ll|l}
\hline Route: & \\
\hline
\end{tabular}
$* \cap A T 0_{0}^{*} q^{*}$

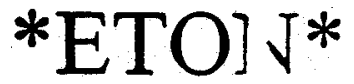

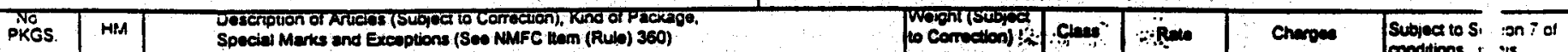

TDT SOIL-RADIOACTIVELY CONTAMINATED NOT-RGULATED PER 49 CFR 173.403 •

VEHICLE \#

68

DRIVER NAME Tom Carter

SHIPMENT \# DPL99026

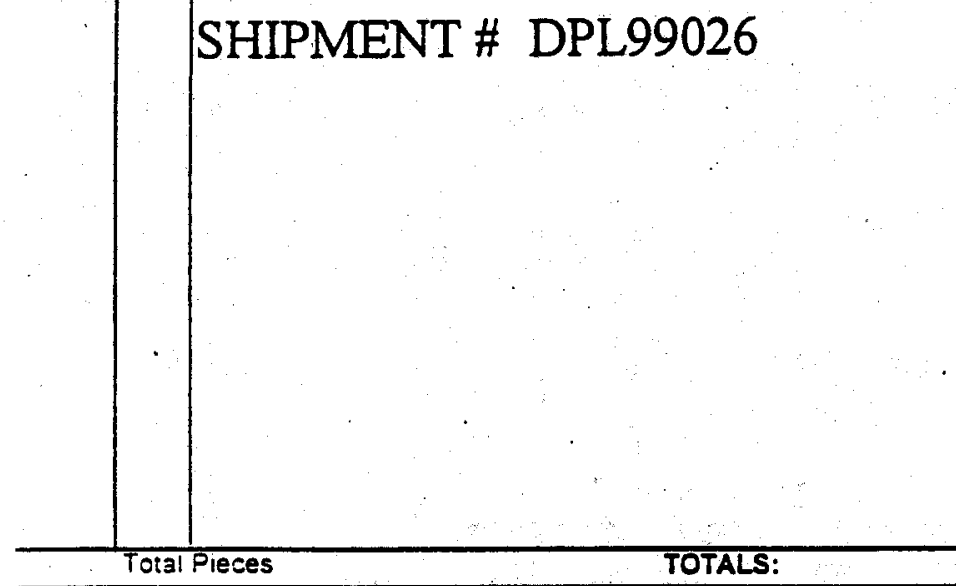

7079

\begin{tabular}{|c|c|c|}
\hline Clanes" & مسMR & Cheromen \\
\hline 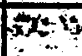 & $9 x$ & \\
\hline
\end{tabular}

chipment is! is coliveres

to the consic a withel:

recoures on. consigne

recurse on "consign

the following is -3 . st:

tho camer in:

dalivery of :- $::-:-$

whithout pay'

and all othe

cherpes

JEFF SMI

SThature

If troichnt ense

prepaid. wri

here TO BE

PREPA

Note: Whe: $7: 2$;

dopendent $c$

shipoers are

stete spocifi

the agreed:

value of the

The equed

velue of the

hereby spec

by the ships

exceeding

Joo srcer. account. or

mimoer

C7POSG.

Savings:

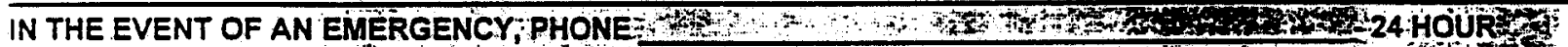

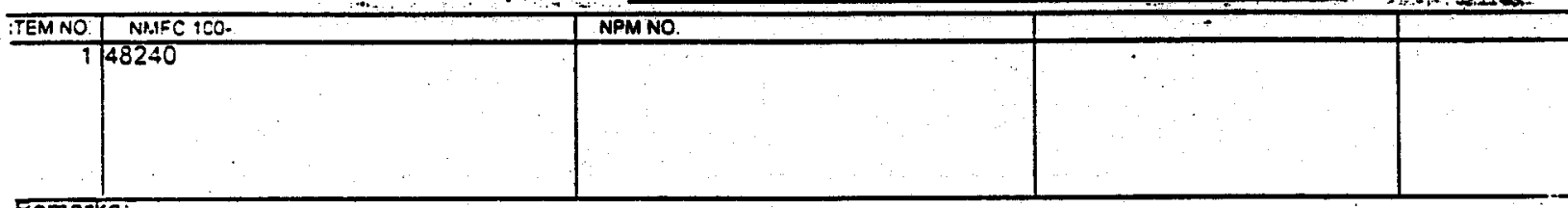

Femarks:

TECHNICAL CONTAET

This is to cerity that the above named matenals ere property classtied. described. packaged. marked. and labeled, and are in proper coodition tor Iransoortatien accoraing to the applicable regulations of the Departinent of Transponation (Applicable for Hezardous Materiats Only.)

Shipoer BECHTEL NEVADA. P.O. BOX 98521. LAS VEGAS, NV 89193 acting under contrac: DC ACOB 96 NV11718 wath U.S. Dept. of Energy Per ALAN R MARCHAND Oate: O40or1999

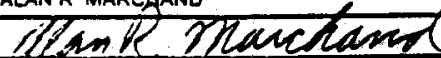

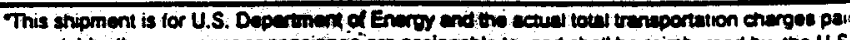

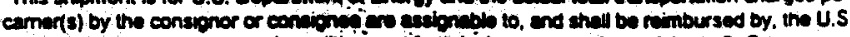

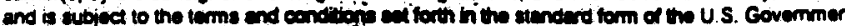

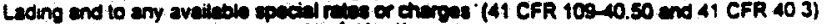

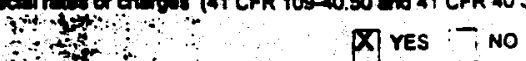

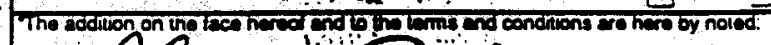

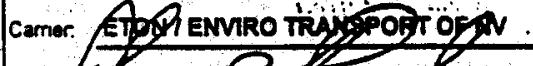


OARAIGHT BIIL OF IADING - SHORT FORM - ORIGINAL - NOt iNegiotiable

Shipper: BECHTEL NEVADA FOR US DOE

CAU 461 TAML STTE/ TTR

TONOPAH NV 89049

Purchasd of

Customorofordio. :-

Shipper No. NTS0736:

Data: $6 / 22 \sqrt{99}$ -

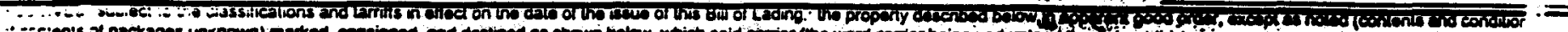

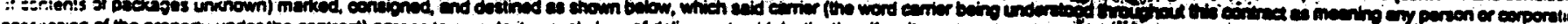

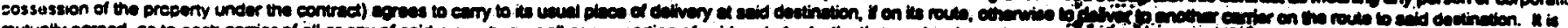

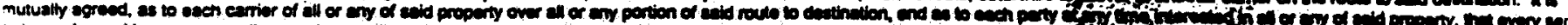

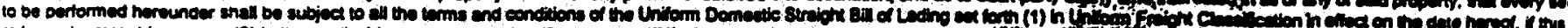

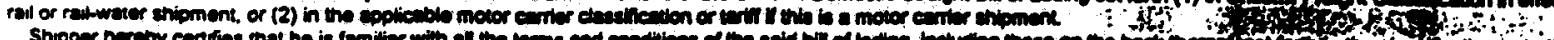

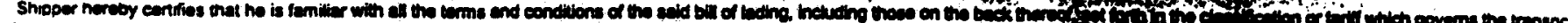

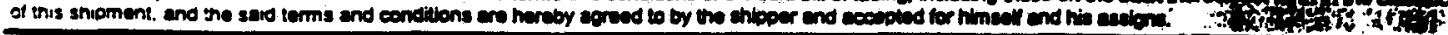

Consignee

BECHTEL NEVADA FOR USDOE

A.-3 RWMS. ZONE 2

NEVADA TEST SITE

MERCURY NV 89023-

MIKE SMITH / (702) 295-7365

\begin{tabular}{l|l|l|l|}
\hline Route: & \\
\hline No. & HM & \\
\hline
\end{tabular}

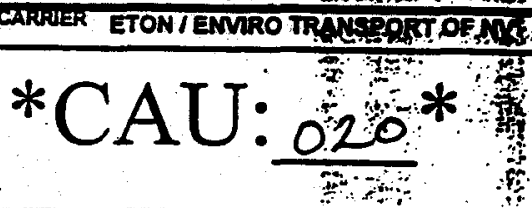

S.:- : :

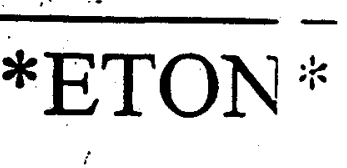

$1 D T$ SOIL-RADIOACTIVELY CONTAMINATED NOT-RGULATED PER 49 CFR 173.403

VEHICLE \# 76

DRIVER NAME Darrel Mintan

SHIPMENT \# DPL99027

Total Pieces

TOTALS:

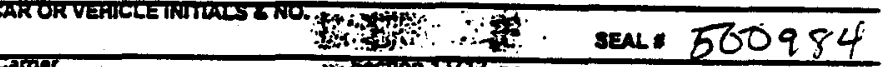

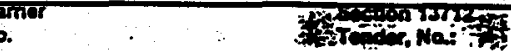

$-$

Subject to Sectior io Correction 67580

\begin{tabular}{|c|c|c|}
\hline Clas & Fato: & Chareas \\
\hline & $\therefore$ & \\
\hline
\end{tabular}

thiprent is to be

the coneriones $v e r$

recoures on the c -....

recourse on the $c$

the corriginne st

the following stat

T-.e camer shall .
deivery of this $3 n$

without payment

and all other taw:

charges

JEFF SMITH

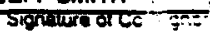

ff frevgnt enarges: : $:=$

prepaid, wrte or 5 here TO BE PRE

PREPAID

Note: Where the

dependent on val

snippers are requ: : :

state spectically if $\because:$

the soreed of ooc. ?"

value of the prope

Tha eroed or dee. is

value of the prope ":

hereby specificail, ::a

by the shipper to:

exceeding

jop orater retere

number

C7P05G:3

IN THE EVENT OF AN EMERGENCX PHONE- SPREX

Kgs $67580-700 \%$

$\therefore$ Savings:

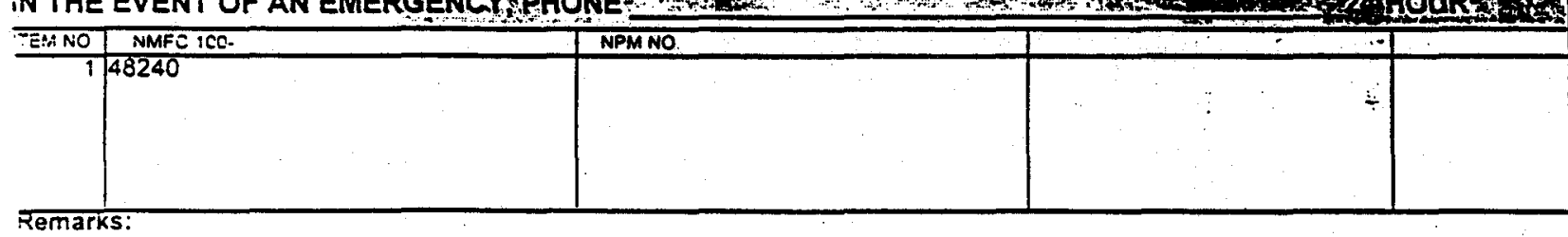

Remarks:

PractrolstRe तE:

TECHNICAL CONTACT

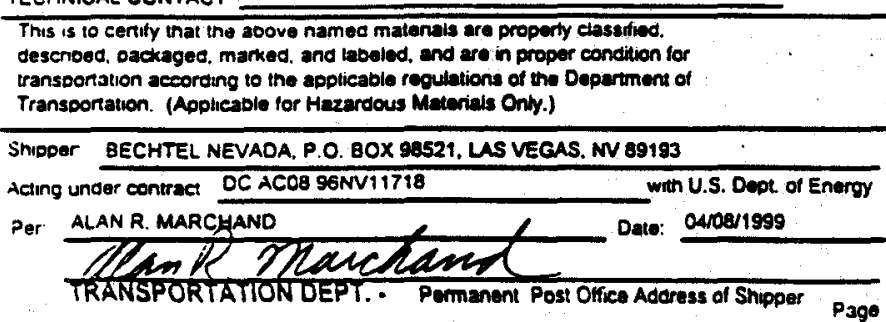

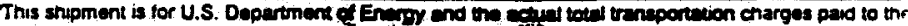
camer(s) by the contignor or conagnes ere essiorabie to nd andl be rembursed by, the U.S. Gove and is subjoct to the terms and condition ent to h the

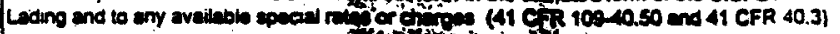

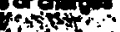
isivitis: [X] Yes [i NO

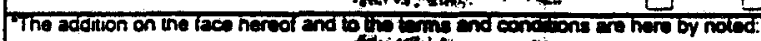
Camer. ETON/ENVRO TRANSPORT OF iN "?

Label(s) acsilec

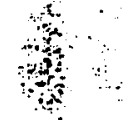
Per Pary mintens Date $6 / 2:$ 
TIRAIGKT BIIL OF IADING - SHORT FORM - ORIGINAL - NOt F̂töOtiable

hipper: BECHTEL NEVADA FOR US DOE

CAU 461 TAML SITE/TTR

TONOPAH NV 89049.

Hot $\rightarrow$

Purchasel

Cuatomar Oresino.

Shipper No. NTSO7363i

Date: $06 / 21 / 99$

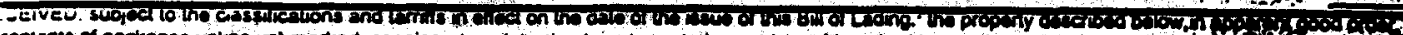

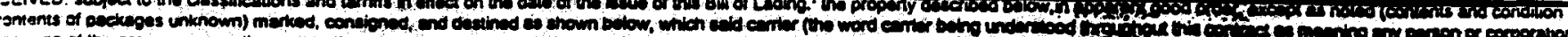

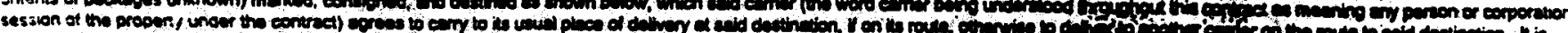

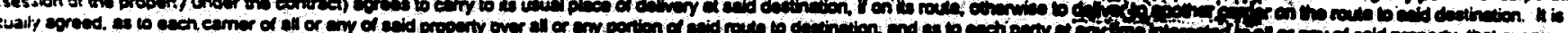

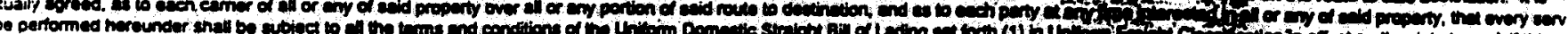

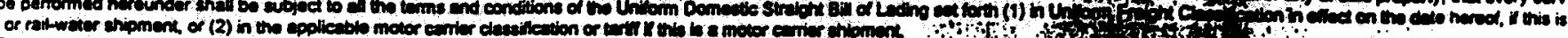

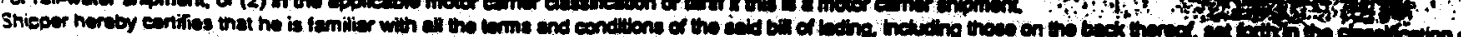

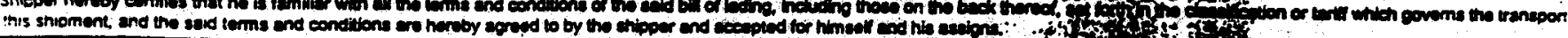
onsignee

BECHTEL NEVADA FOR USDOE

A.3 RWMS. ZONE 2

NEVADA TEST SITE

MERCURY NV 89023-

MIKE SMITH / (702) 295-7365

oute:

$$
\text { som }
$$

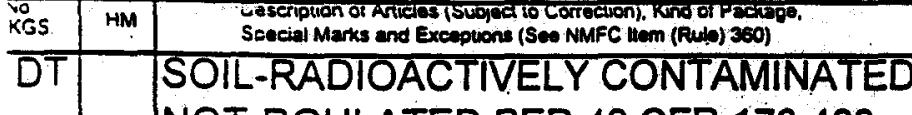

NOT-RGULATED PER 49 CFR 173.403

VEHICLE\# 68

DRIVER NAME Tom Carter

SHIPMENT \# DPL99028

Total Pieces

TOTALS:

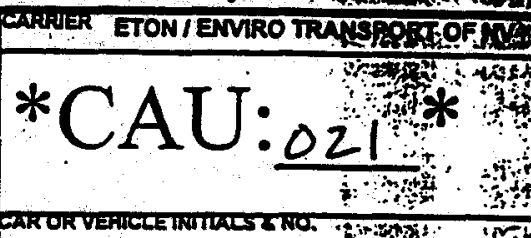

*ETON

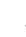

Trats seal 500962

tis. thend

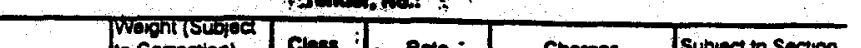

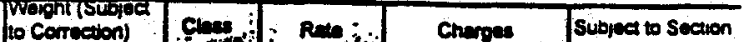

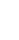

58200

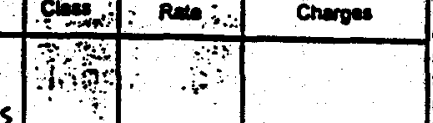

conditions toction

chupment is to be di. , erec

ito the consignes wi - sul

meoirve on the corr zret

the comengor shal:

the following stater.

The earrer snall ne 2.e

delivery of this snif

without payment of :-1

enc all orver iawiul

charges

JEFF SMITH

Sighamoricosn:

if freight charges or $=$

predaid. white or sta

hers "TO BE PREF

PREPAID

No:e Whera ina

dependent on valu

chicoers ere requir

state specifically in

the agreed or deeta

valus of the proper.

thilus of the proper.

whilue of the orooer

mereay specifieally

by tre shipper to be

excesaing

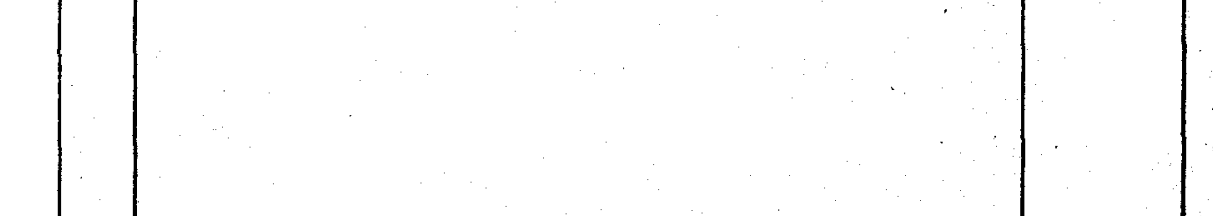

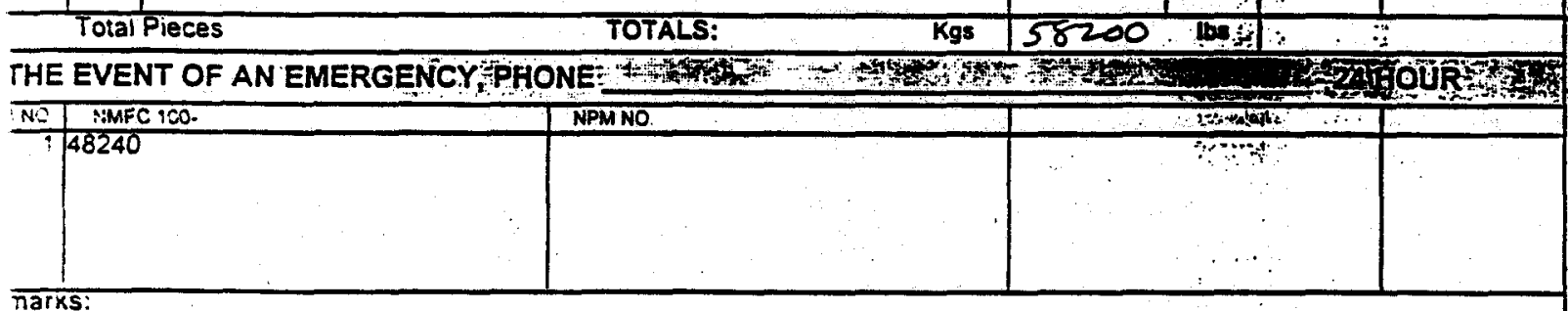

Joc Jister, retaren:

cocount. or work ore

numeer

C7P05G13

Sovings:

narks

HNICAL CONTACT

$\therefore a$

5

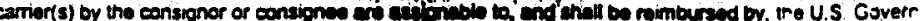

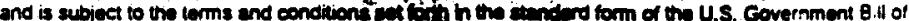

Lading and to any availabie speciel ratee of anfe (41 CFR' $109-10.50$ and 41 CFR 403 )
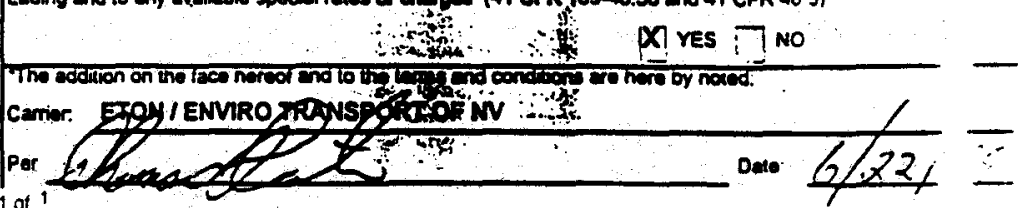
STRAIGHT BILI OF IADING - SBORT FORM - ORIGINAL - NOt Negotiable
ShIPPER: BECHTEL NEVADA FOR US DOE CAU 461 TAML SITE TTR TONOPAH NV 89049.

'Purchasel

'Customar Order No.'
Shlpper No. NTS07363t

Date: $0 6 \longdiv { 7 2 / 9 9 }$

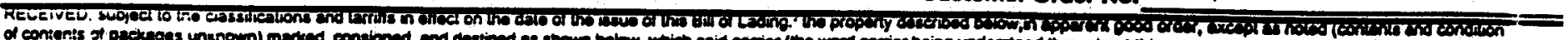

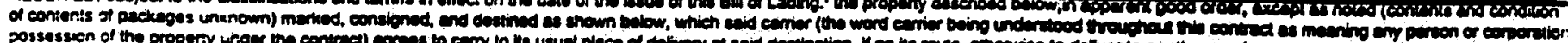

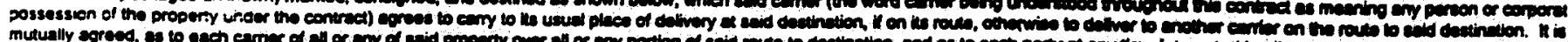

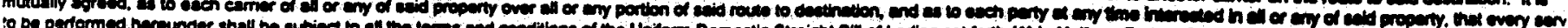

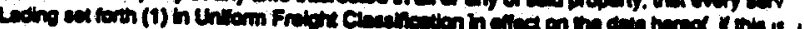

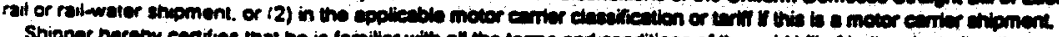

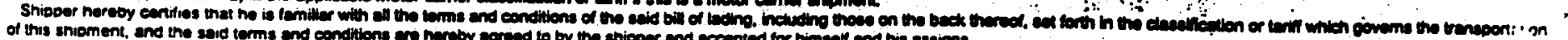
Consignee

BECHTEL NEVADA FOR USDOE

A.3 RWMS, ZONE 2

NEVADA TEST SITE

MERCURY NV 39023 -

MIKE SMITH / (702) 295-7365 CARRUIER ETONIENVIRO TRANGPORT OF NV

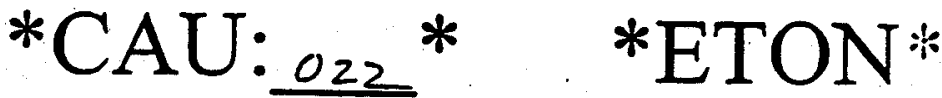

Route:

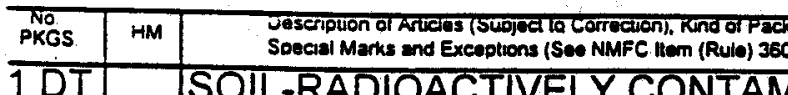

1DT SOIL-RADIOACTIVELY CONTAMINATED

$\left\{\begin{array}{l}\text { NOT-RGULATED PER } 49 \text { CFR } 173.403 \\ \text { VEHICLE\# } \frac{68}{68} \\ \text { DRIVER NAME Thomas Canter }\end{array}\right.$

$\left\{\begin{array}{l}\text { NOT-RGULATED PER } 49 \text { CFR } 173.403 \\ \text { VEHICLE\# } \\ \text { DRIVER NAME Thomas Carter }\end{array}\right.$

$\left\{\begin{array}{l}\text { NOT-RGULATED PER } 49 \text { CFR } 173.403 \\ \text { VEHICLE\# } \\ \text { DRIVER NAME Thomas Canter }\end{array}\right.$ CARUR VEHICLETMITALSTKO. 580978

Noind
No. Tendor, Ma.:

SHIPMENT \# DPL99029

Total Pieces

TOTALS:

Kgs 60080

los .

\begin{tabular}{|c|c|c|c|}
\hline $\begin{array}{c}\text { Waighitswojoct } \\
\text { 10 Cortoction) }\end{array}$ & Class & Rato & Charges \\
\hline 60080 & & & \\
255 & & & \\
\hline
\end{tabular}

Subject to Section conditions. If this

shipment is to be C seres

to the consignee w'..'.

recourse on the cor ir:

the consignor shail

the following stater

The camer shall $n$ :

detivery of this snic

without poyment of

charges

JEFF SMITH

Signawe or Cons Fht

If freight charges ar : : : $\neq$ prepaid. wrte or ste hers TO BE PREF

PREPAID

Note: Where the rz 2:3

dependent on value

shippers are require: :

stato specrieally in :...:-?

the agreed or decta-

value of the preper:

The agreed or decte :

value of the proper.

hereby spectieally $5 \cdot: ?$

by tha shipper to be

exceeding

ป THE EVENT OF AN EMERGENCY,PHONE

\begin{tabular}{|l|l|l|l|l|}
\hline$\equiv M A N O$ & NMFC :CC. & & & \\
\hline 148240 & & & & \\
& & & & \\
\hline xemarks: & & & \\
\hline
\end{tabular}

"ECHNICAL CONTACT

This is to sertity that the above named matenals are property classified. iescribed. Dackaged. manked, and babeled, and are in proper condition for 'ranscortation aceording to the applicabie regulations of the Department of

iransoonation. (Applicaole tor Hazardous Matenals Only.)

mipper BECHTEL NEVAOA. P.O. BOX 98521. LAS VEGAS, NV 89193 wing under contract DC ACC896NV11718

Iof ALAN R MARCHAND

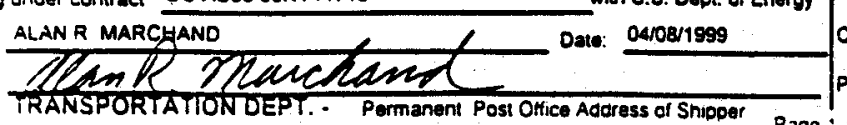

Jos orcer, referenc account. or work or numeer

C7P05G13 Savings:

Shipment is for U.S. Department of Energy and ins sctual total transportation charges pand to the and is subjt consignor or consignea are easignable to, and shall be rembursed by. Lading and to any avauble special rates of churges (At CFR, 109-40.50 and 41 CFR 40.3)

$$
X \text { Y Y }\lceil\text {. No }
$$

The acbition on the fece nereor and to tho toms and conditions are hero by noted?

Carnar. RTg I ENVIRO TRAMPOQg OF NV

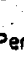




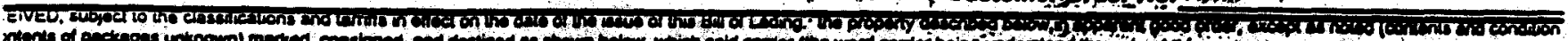

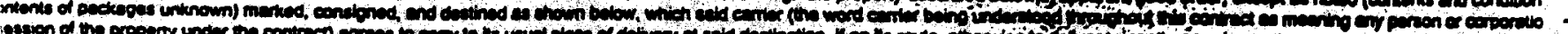

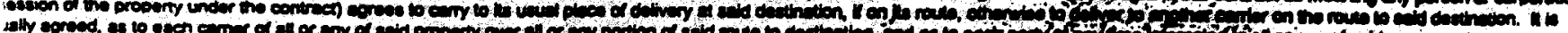

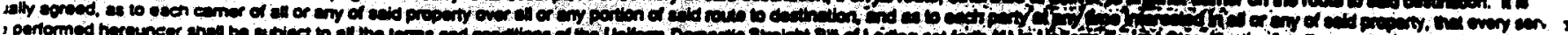

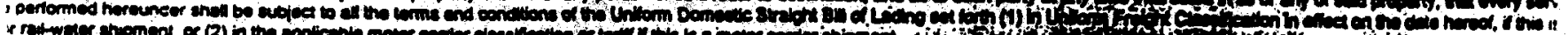

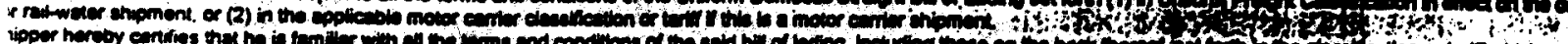

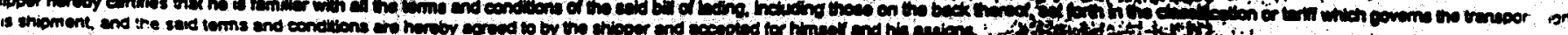
(a) nsignee ECHTEL NEVADA FOR USDOE

3 RWMS, ZONE 2

EVADA TEST SITE

IERCURY NV 39023

IIKE SMITH / (702) 295-7365

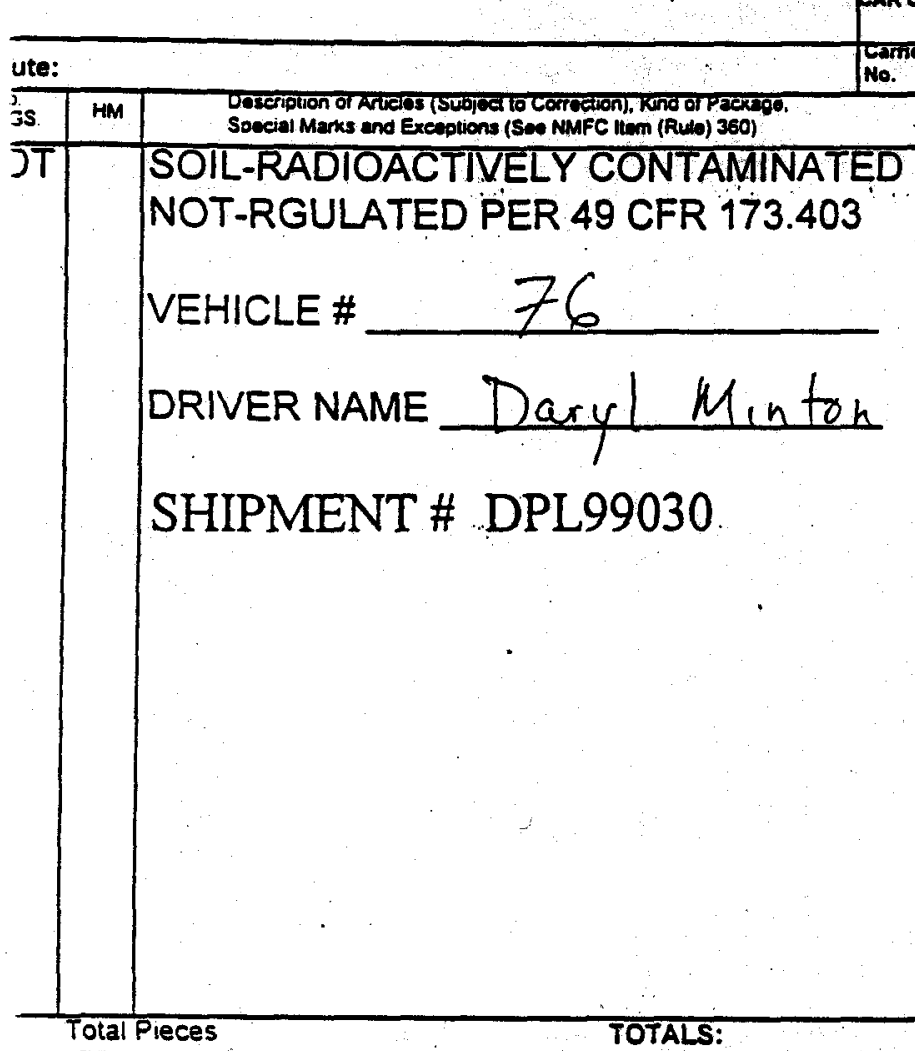

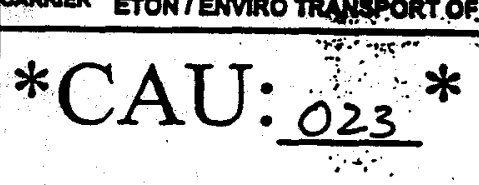

*ETON*

sal $: 500923$

No.
Teader, Mo.:

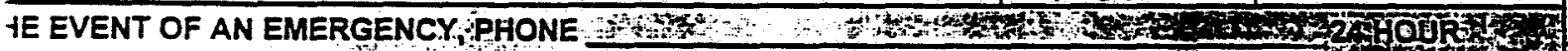

\begin{tabular}{|l|l|l|l|l|l|}
\hline NMFC ISC. & NPMNO & & \\
\hline 1 & 48240 & & & & \\
& & & & \\
\hline
\end{tabular}

VICAL CONTACT

$s$ to centify that the aoove named matorals aro property etatsified. bed, packaged, manked and labeled, and are in proper condition for iortation according 10 the applicabie regulations of the Department of Dortation. (Appltcsole for Hazardous Matenials Onty.)

$r$ BECHTEL NEVADA. P.O. BOX 98521 , LAS VEGAS. NV 89193 under contract DC ACO896NV11718_wth U.S. Dept. of Energy

charges

Subject to Section conditions if this shipment is to be $c$ verec to the consignes w reut rucourse on the co "signor.

the comionor shal: gn

the following state: in

The comer snati $r$-3ro

delivery of this sti: in:

without payment: : :

and all other lawh.

charges

JEFF SMITH STonkwortes :F

fifregin charges a preosid wnte or $\mathrm{si}$ prepace whe or s: PREPAID Note: Where ins : deoendient on valu shiopers are recuir :o state spoctically if $\quad-$; the agreed er dec:e value of the prover The agreed or oec: if velue of the proper: hereoy specitically : her the specitically. exceeding

Joo oroer rererer cosums C7P05G 13 Savings: 3 . $\frac{}{\text { Labells) aps1140 }}=$

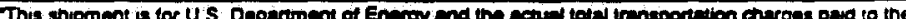

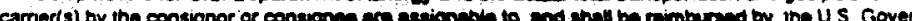
and is subject to the terms and conditions cet torth in the dinderd form of the U.S. Government Bill of Lading and to any avaitabie specivi rates or cherges (41 GFR 109-40.50 and A1 CFR 40 3)

XI VES 7 No

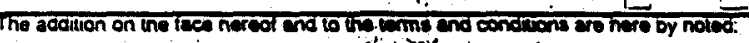
Camiar. ETON/ENVIRO TRANSPORT ÖF NV Pare 202 Date 


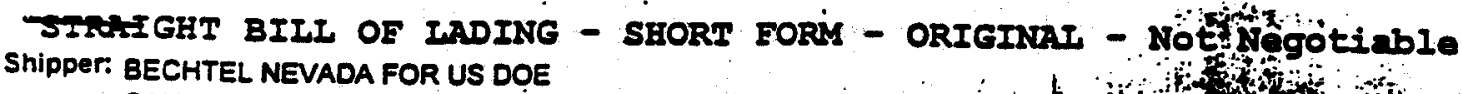
CAU 461 TAML SITE/TTR TONOPAH NV 89049 .

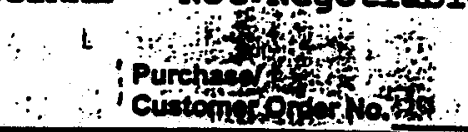

Shippor No. NTSO73i S

Deto: $0<\longdiv { 2 2 \sqrt { 2 } }$ j

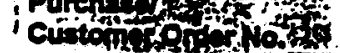

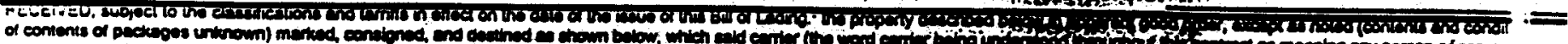

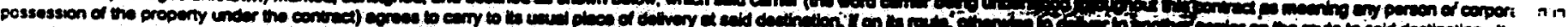

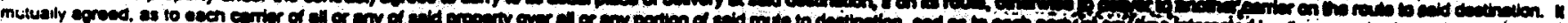

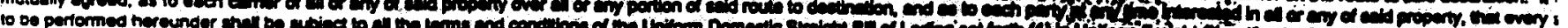

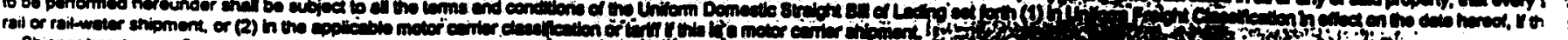

Shoor nor a

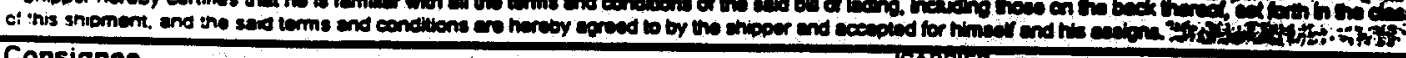

BECHTEL NEVADA FOR USDOE

A-3 RWMS, ZONE 2

NEVADA TEST SITE

MERCURY NV 89023.

MIKE SMITH / (702) 295-7365

Route:

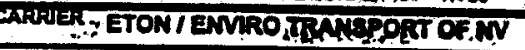

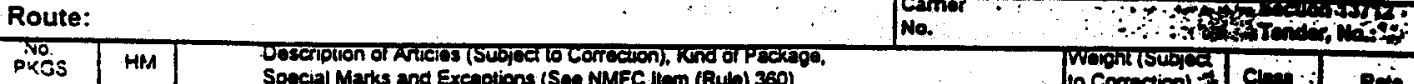

Special Marks and Exeaptions (Ses NMFC Item (Ruy) 360)

SOIL-RADIOACTIVELY CONTAMINATED

NOT-RGULATED PER 49 CFR 173.403

VEHICLE \#

81

Driver name Ted Black

SHIPMENT \# DPL99031

Tolal Pieces

TOTALS:

Kgs $\quad 57170 \quad$ Las

ISAL: 500992

\begin{tabular}{|c|c|c|c|c|}
\hline & 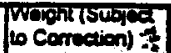 & $c$ & Ren & Charges \\
\hline$\therefore \cdots_{-}$ & & $=$ & th: Jat & \\
\hline
\end{tabular}

Subiect to Sec:

shipment is to

mipment is tot isive
to me conargne. ithes

recourse on the :ins: gr

the consignor $s$

the following st: - ve-

delivery of this

without Dayme

and all other ia

enarges

JEFF SMITT-

Stgrasure or:

If trought crarce

orepaid, wrte $\mathrm{C}$.

Te TO BE $P=$

PREPAID

Note: Whare:

dependent on v:

shippers are re: $2 z$ :

stato specticall. A.

the agreac or $d:$ : $" s:$

value of the pro:

The eqreed or 5 ire:

value of the pre: $\because \mathrm{s}$

hereoy specifie: $\quad:-\overline{3} \cdot$

by the shipper :

oxeseding

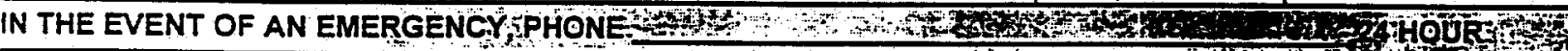

\begin{tabular}{l|l|l|l|l|}
\hline ITEM NO. & NMFC IOC. & NPMNO. & & \\
\hline 1 & 48240 & & & \\
& & & & \\
& & & & \\
\hline
\end{tabular}

Remarks:

TECHNICAL CONTACT

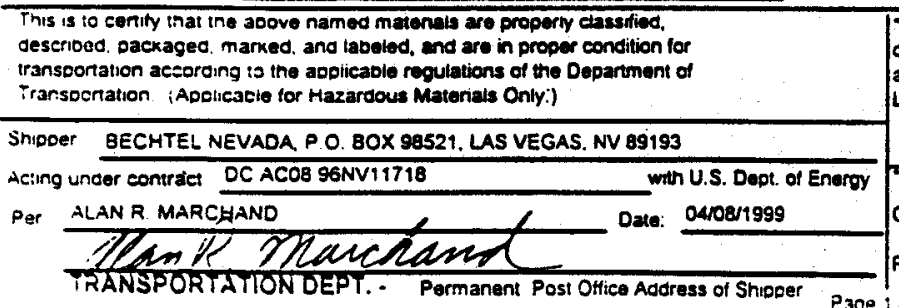

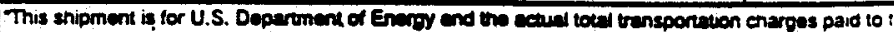

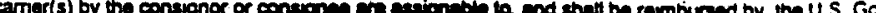
and is subject to the toims and conditions ent forth in the etandard form of the U.S. Govemment Bil Lading and to any evalubis speceal rates or Cherpes (41 CFR $109-40.50$ and 41 CFR 40.3 )

$$
\therefore \quad[X] \text { VES - NO }
$$

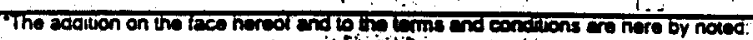

Camier: ETON $/$ ENYRO TRANSPORT of NV :-

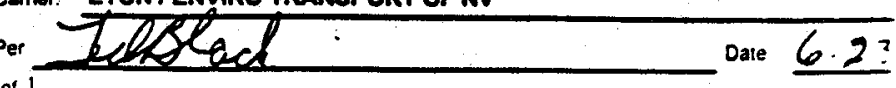


STRAIGHT BIIL OF IADING - SHORT FORM - ORIGINAI - NOt Negotiable
Shipper: BECHTEL NEVADA FOR US DOE CAU 461 TAML SITE TTR TONOPAH NV 89049 .

Purchaid of

Shippor No. NTS073636

Date: $0 6 \longdiv { 2 2 / 5 9 }$

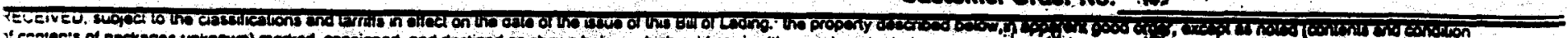

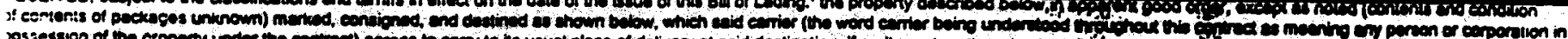

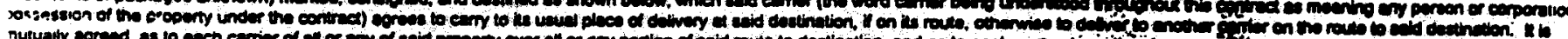

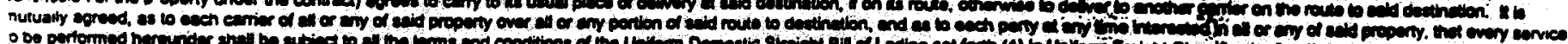

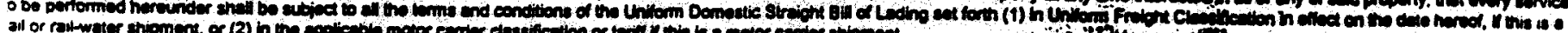

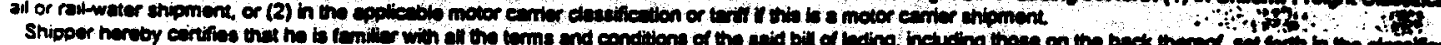

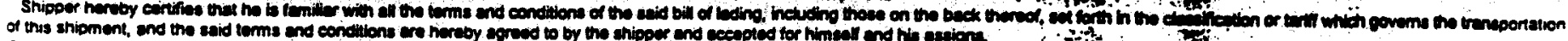
Sonsignee

BECHTEL NEVADA FOR USDOE

A-3 RWMS, ZONE 2

NEVADA TEST SITE

MERCURY NV'89023.

MIKE SMITH / (702) 295.7365

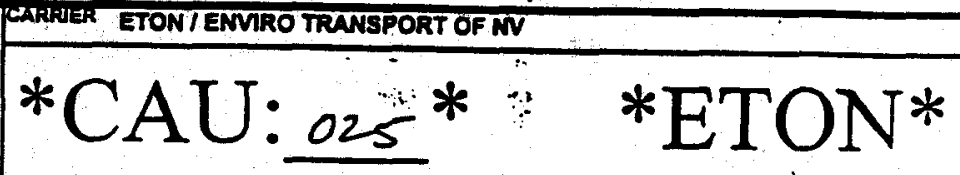

ROUte:

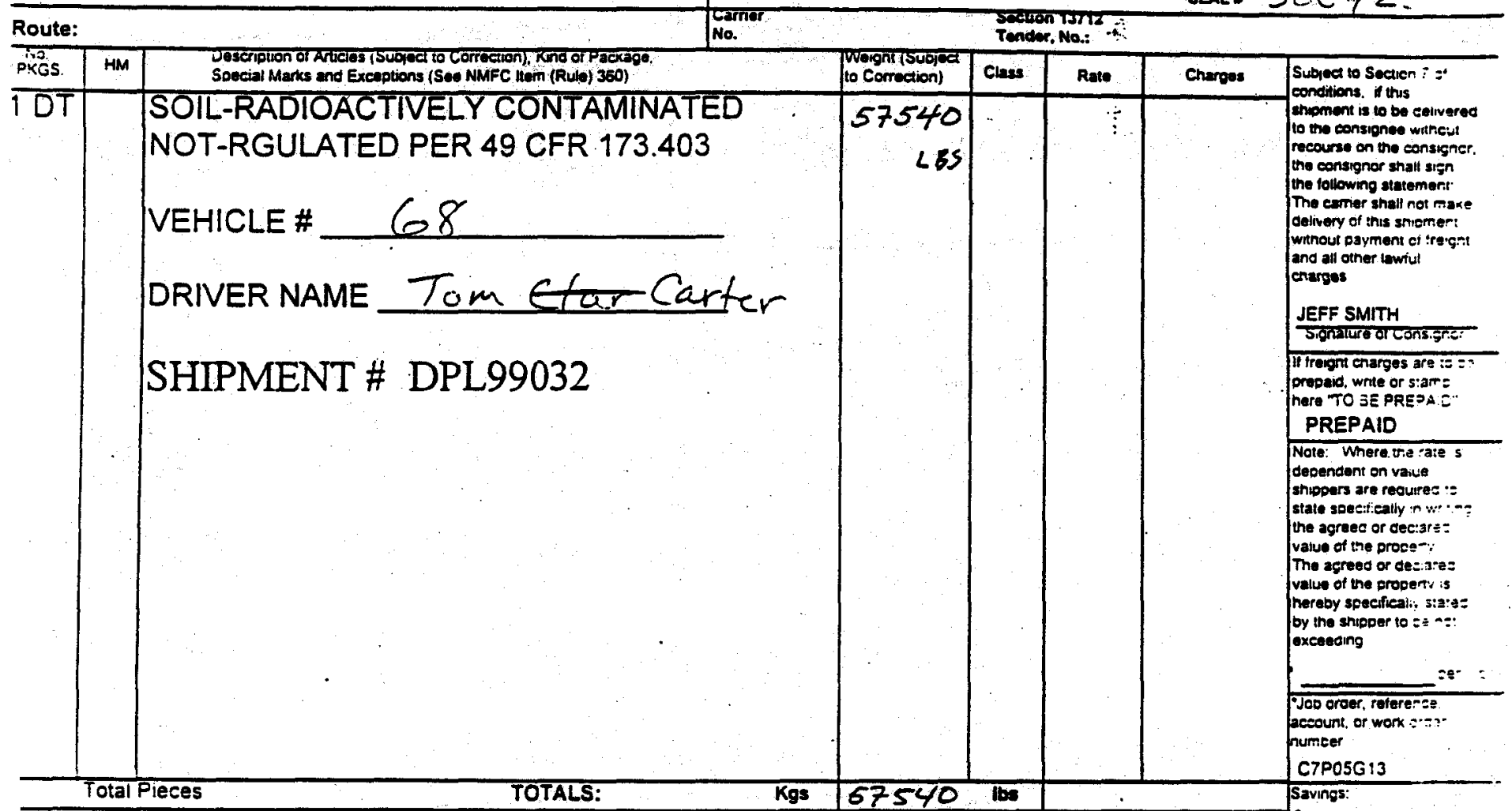

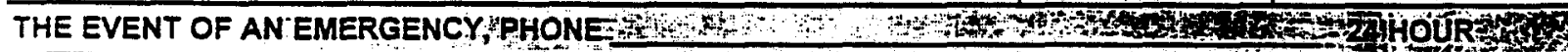

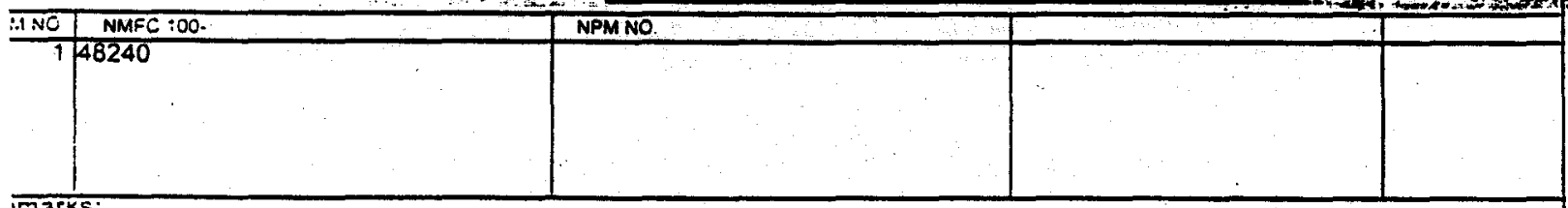

marks:

CHNICAL CONTACT

II is to certity that the above named materale are property classified iseriboc. packaged, marked, and laboliod, and are in proper condition for insporation according to the applicable regulations of the Department of anseornation. (ADpliciole for Hezarcous Matorals Onty.)

CDer BECHTEL NEVAOA, P.O. BOX 99521, LAS VEGAS. NV 89193 ng uncer contract OCACOB 96NVI1718

aLAN R MARCHAND

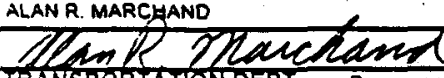

TRANSPORTATION UEPT." Permanent POSt OHice Adoress of Shipper with U.S. Dept of Energy

Dato: $0408 / 1999$
ARORVERICLEINITALS NO.

s.

Label(s) acolieo

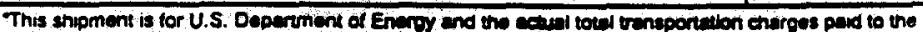

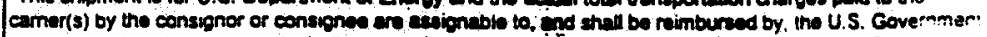
and is subjost to the torms and conditions aet forth in the thiderd form of the U.S. Govemment Bill o: Lading and to any avalable special ratee or cherpes (4t CFR 10040.50 and 41 CFR 40 3)

$$
\text { (X) YES } T \text { No }
$$

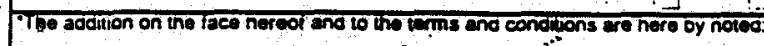

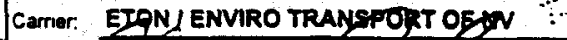

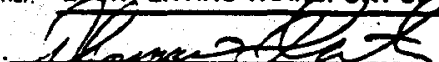


Shipper: BECHTEL NEVADA FOR US DOE CAU 461 TAML SITE/ TTR

TONOPAH NV 89049.
Purchasel inition

Customer Ordior No.
Shipper No. NTS07363

Date: $0</ 25 / 9$.

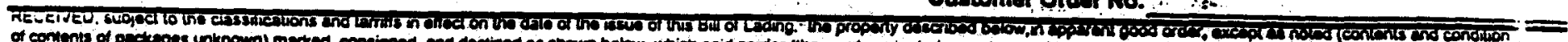

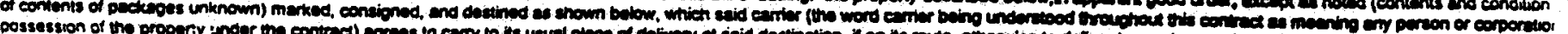

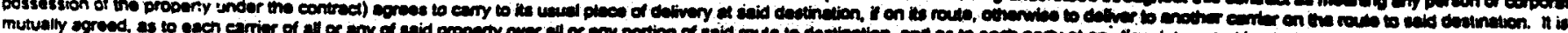

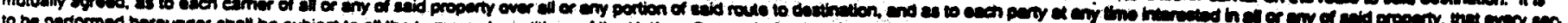

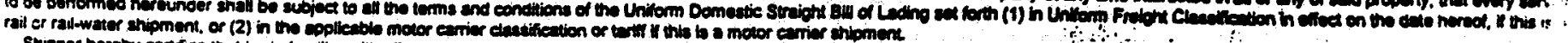

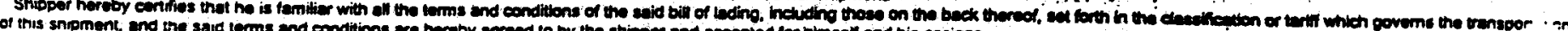

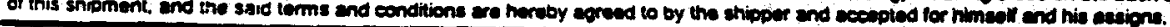

Consignee

BECHTEL NEVADA FOR USDOE

A-3 RWMS, ZONE 2

NEVADA TEST SITE

MERCURY NV 89023 .

MIKE SMITH / (702) 295-7365

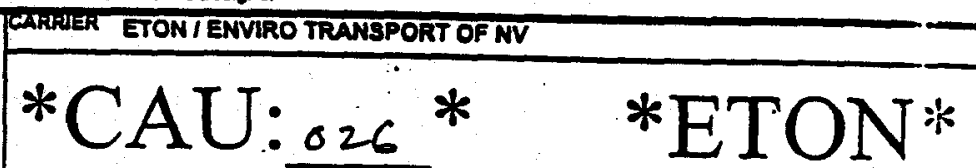

Route:

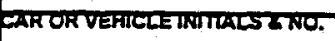

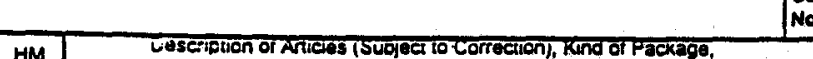

No. SCEvontor

9EAL* 500991

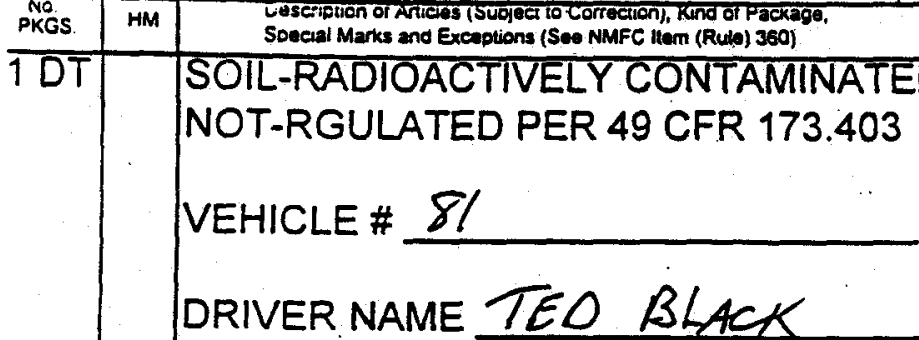

SHIPMENT \# DPL99033

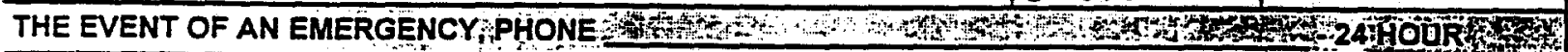

MNO $/$ NMFC 100

\begin{tabular}{l|l} 
MNO NMFC 100. \\
\hline$: 48240$ \\
marks
\end{tabular}

EHNICAL CONTACT

us is lo centry that the above named materats are properyy casstived iscribec. oackaged. marxed. anc labeled, and are in proper condition for irsecriation accoraing :0 the applicable regubations of the Department of ansportation. (Apphcadie tor Hazardous Matenals Only.)

soer BECHTEL NEVADA, P O. BOX 98521. LAS VEGÁS, NV 89193 runder contract DC ACO896NVi1710 with U.S. Dept. of Energy Oate: 0408/1990 Peante Prucectery

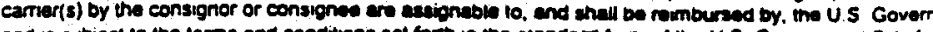
and is subject to the terms and conditions sel forth in the sunderd form of the U.S. Government Bill of Lading and to any avalable special rates or cherges (41 CFR 109-40.50 and 11 CFR 40 3) 
SIRAIGHT BIIL OF IADING - SHORT FORM - ORIGINAI - NOt Negotiable

PPER: BECHTEL NEVADA FOR US DOE

CAU 46: TAML SITE/TTR

TONOPAH NV 89049-

\author{
$\because$.
}

Purchased sin...

Customor ordorivo.
Shlpper No. NTS07363i

Date: $0 / \sqrt{23 \sqrt{95}}$

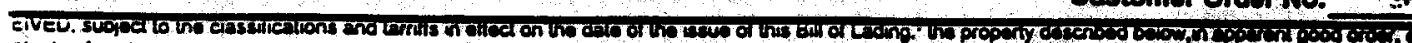

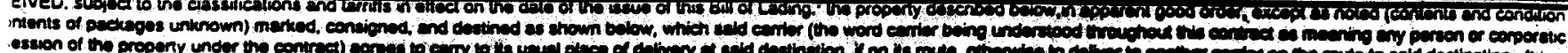

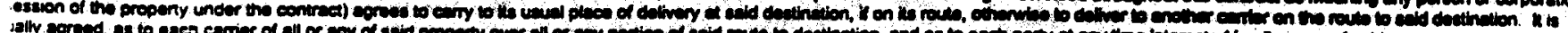

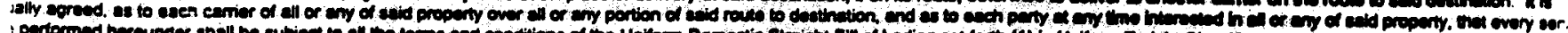

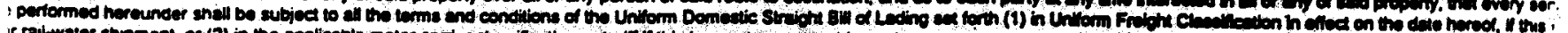

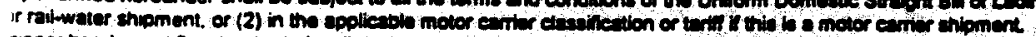

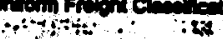

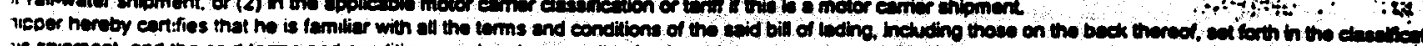

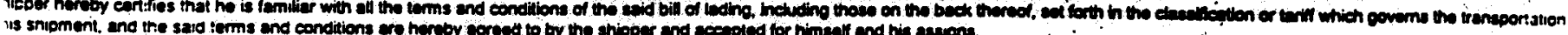
nsignee

IECHTEL NEVADA FOR USDOE

-3 RWMS, ZONE 2

IEVADA TEST SITE

IERCURY NV 89023.

IIKE SMITH / (702) 295-7365

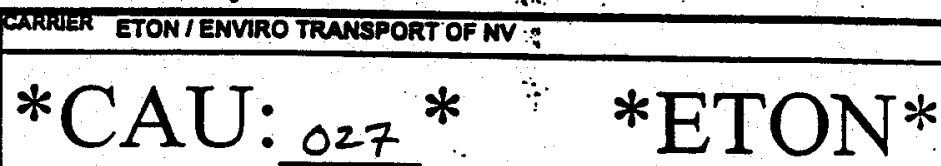

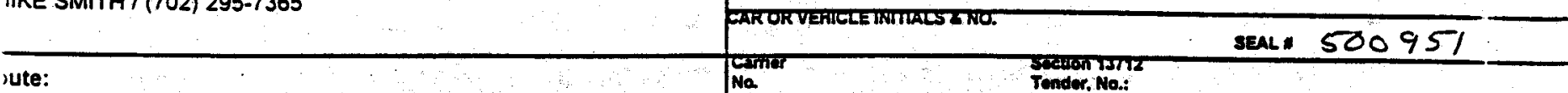

J TM

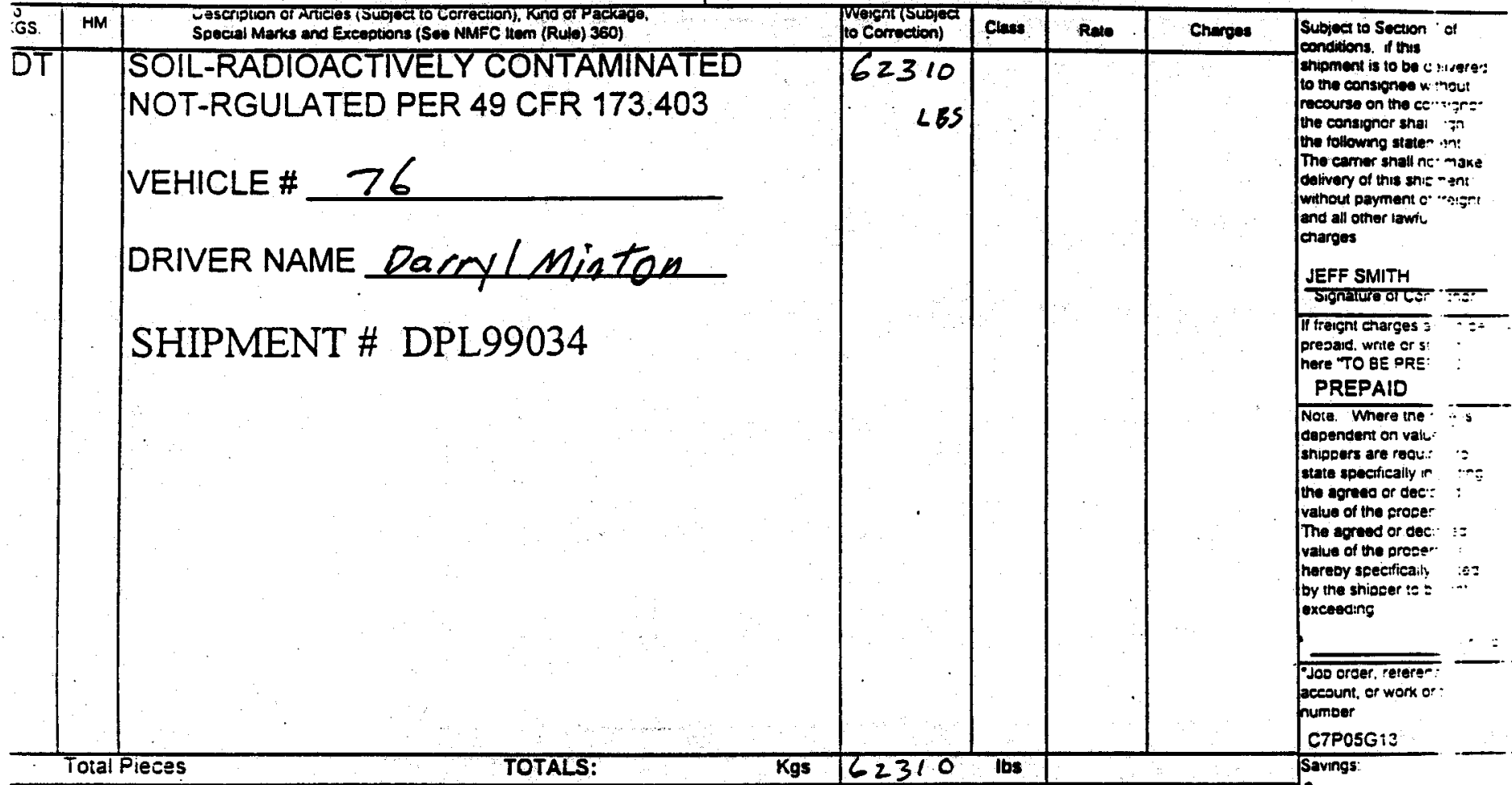

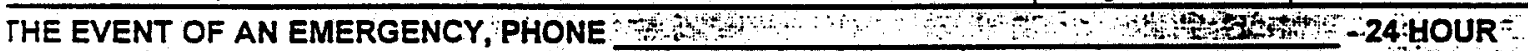

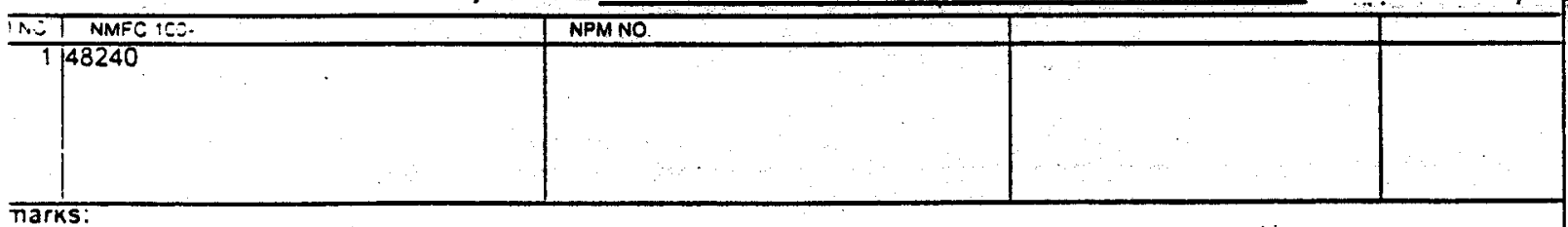

:HNICAL CONTAC:

is is to certify that the above named matenals are property classified.

scribed. Dackaged. marked. and labeled, and are in proper condition for

nspontition accoraing to the applicabie regulations of the Depantment of insecriation (Applicaole to: Hazardous Matenals Only.)

icer BECHTEL NEVADA. P O. 6OX 98521. LAS VEGAS, NV 89193

nq under contract DC ACOB $96 N V 11718$

with U.S. Dept of Energy

ILAN R MARCHAND

Date: 04/08/1999

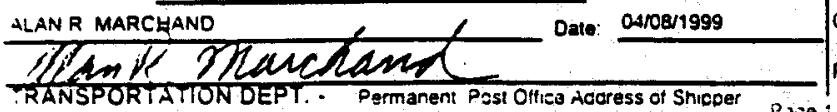

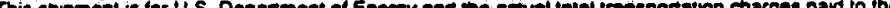
camer(s) by the consionor or consignes ere ancionable to, and thall be rembursed by, the US Gover and is subject to the termis and conditions sel forth in the stenderd form of the U.S. Government Bill et Lading and to any avalable special rates or charges (41 CFR 109-40.50 and 41 CFR 40 3)

$$
X \mid \text { YES : No }
$$

The additon on the iaco herbol and to th toms and cond
Camer. ETON / ENVIRO TRANSPORT OF NV

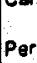

per

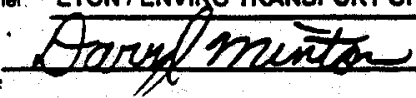

ons 
Shipper: BECHTEL NEVADA FOR US DOE CAU 461 TAML SITE/ TTR TONOPAH NV 89049. iz.

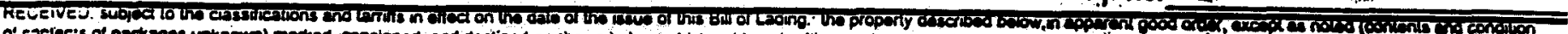

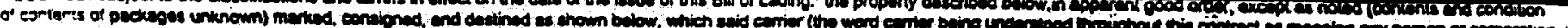

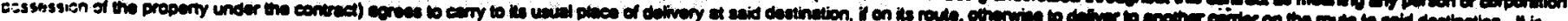

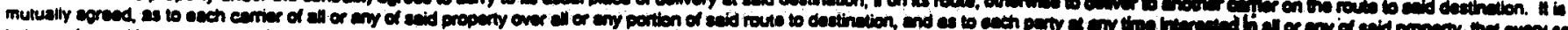

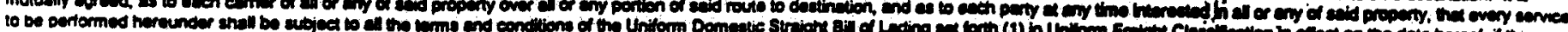

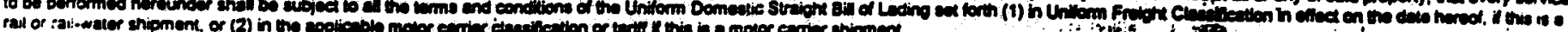

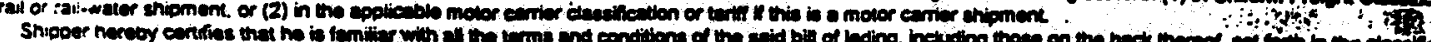

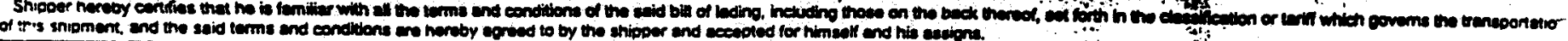
Consignee

BECHTEL NEVADA FOR USDOE

A-3 RWMS. ZONE 2

NEVADA TEST SITE

MERCURY NV B9023-

PAIKE SMITH / (702) 295-7365

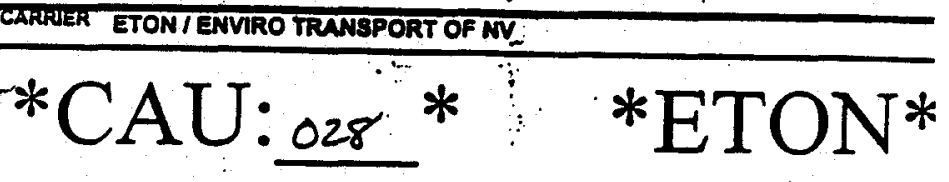

\begin{tabular}{l|l|l|}
\hline Route: & \\
\hline
\end{tabular}

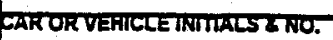

SEAL $500915^{\circ}$

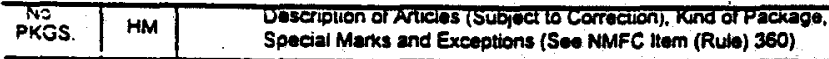

1 DT SOIL-RADIOACTIVELY CONTAMINATED

NOT-RGULATED PER 49 CFR 173.403

VEHICLE\# 76

DRIVER NAME Dary I Minton

SHIPMENT \# DPL99035

Total Pieces

TOTALS:

Kgs

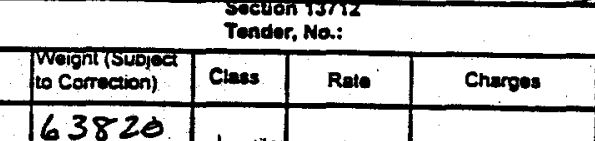

Subject to Secticr 7 of conditions. It this

thipment is to be acive

to the consignee witheu

recoures on the consigr

the consigner shall sign

the followng stat

The camer shat -1 in:

The carner shal ": $\mathrm{AT}$

without payment -! fre:

anc all other kewt

enargos

JEFF SMITH

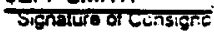

If troight charges ate 10 । prepand. write or starrio

nero TO BE PREPAID-

PREPAID

Note: Where the -are :s

dependent on valce.

shippers we reeurec :

state soectically in wrtit

the agreed of dectaree

value of the properiy

The agresd or declares

value of the prope $\rightarrow \mathrm{V}$ is

hareby specfica:. : : 3 !?

by the shipeer to $=2,-\infty$

excesoring

$\frac{1}{2}=e r$

aceount or wonk $=\cdot$ :

number

CiPosG13

Savings:

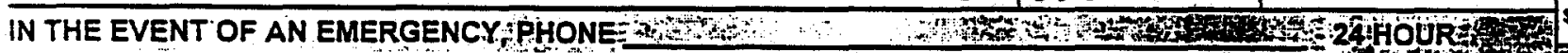

\begin{tabular}{|l|l|l|l|l|l|}
\hline ITEMINO & NMFC 100 & NPM NO & & \\
\hline 148240 & & & & \\
\hline
\end{tabular}

$\longrightarrow$

Label(s) acoitec

- TPI+NICAL CONTACT

Ir's is : 0 certity that the above named matenals are proparty classitied. gescripec. packaged. mankec. and laboled, and are in proper condition for transsortation accoroing to the applicable requlations of the Deparnent of Transponation. (Applicapio for Hazardous Materials Only.)

Shicoer BECHTEL NEVADA. P.O. BOX 98521 . LAS VEGAS. NV 89193 Actirg under contract DC ACD8 96 NVIIT18

Der ALAN R. MARCHAND IOZ with U.S. Dept. of Energy

Camer. ETON / ENVIRO TRANSPORT OF NV

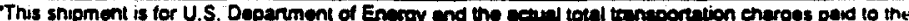

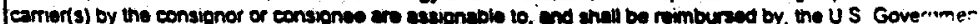
and is subject to the terms and conditions est forth in the stenderd form of the U.S. Govemment Bill of Lading and to any available special rates or charges (41 CFR 109-40.50 end 41 CFR 40.3)

\section{R] YES T NO}

Por

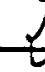

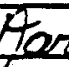

lame

materas Date 


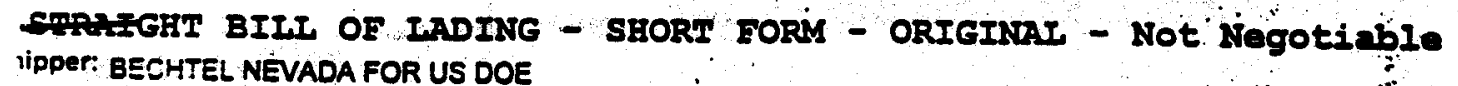

CAU 461 TAML SITE TIR

TONOPAH NV 89049-

i Purchased is

i. Shippor No. NTS073636

Cintomarogion No. is ?

Data: $0 6 \longdiv { 2 4 / 8 }$

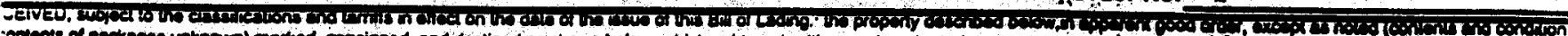

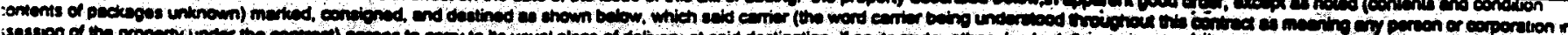

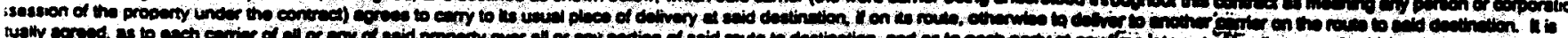

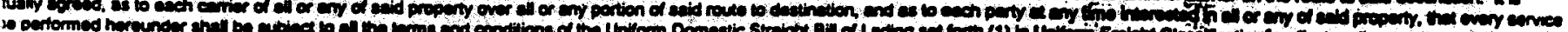

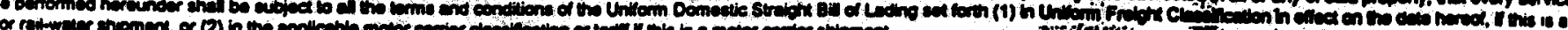

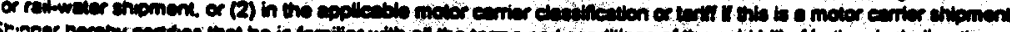

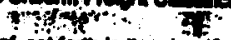

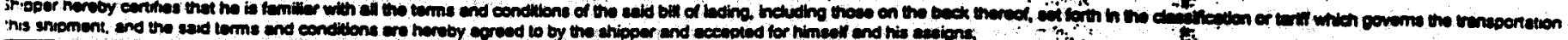
Insignee

BECHTEL NEVADA FOR USDOE

A.3 RWMS, ZONE 2

NEVADA TEST SITE

MERCURY NV 89023-

MIKE SMITH / (702) 295-7365

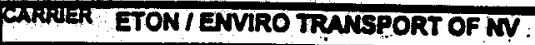

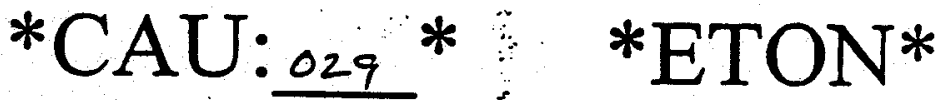

\begin{tabular}{l|l}
\hline oute: & L \\
\hline No.
\end{tabular}

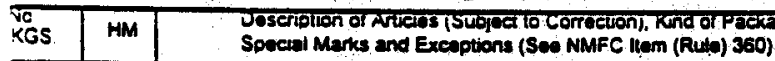

DT SOIL-RADIOACTIVELY CONTAMINATED

NOT-RGULATED PER 49 CFR 173.403

VEHICLE \# 81

DRIVER NAME, Ted Black

SHIPMENT \# DPL 99036

Total Pieces

TOTALS:

Kgs 161660 . lbs

GARORVERCLETMITLESTWO. SEAL 500997

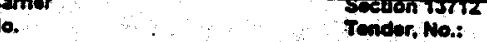

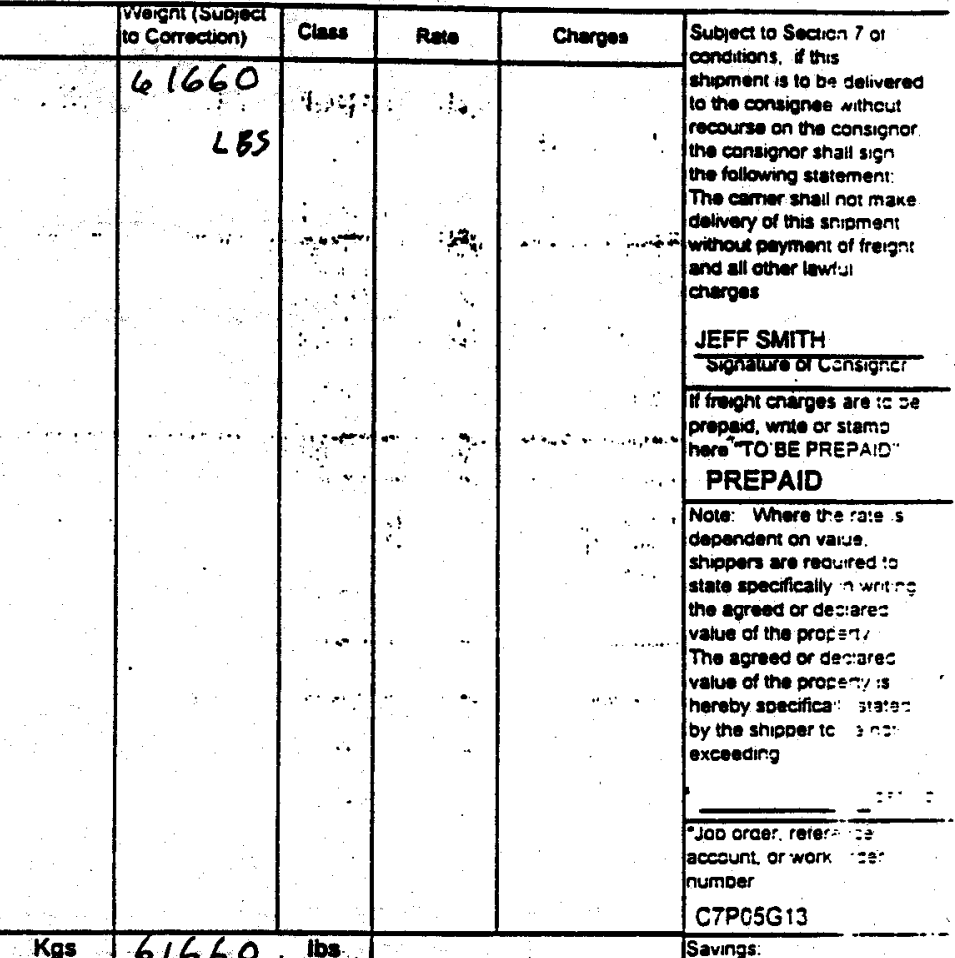

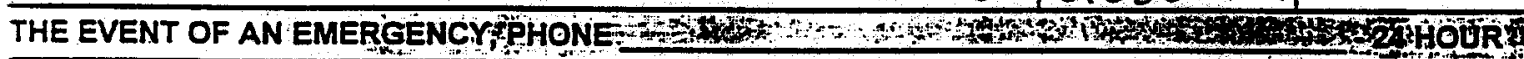

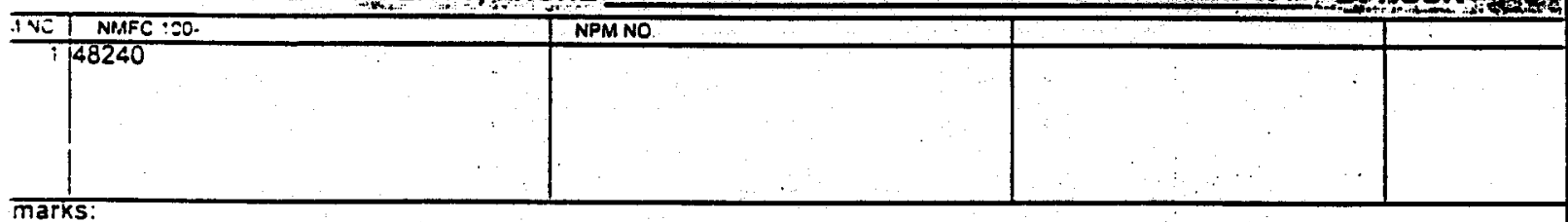

marks:

SHNICAL CONTACT

715 is to certify that the above nameo materats are property classified. iscribed. Dackaged, manked, and labeled, and are in proper condition for insoortation according to the applicable requtations of the Depariment of unsportation. (Applicable for Hazardous Matenais Only.)

DDOr BECHTEL NEVADA.P.O. BOX 98521 . LAS VEGAS. NV 89193 ing uncer contratt OC ACOB 96NVI1718

, ALAN R. MARCEAAND

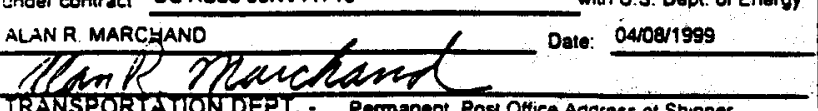

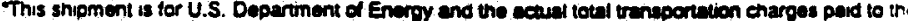

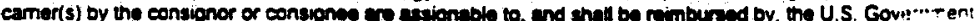
and is subjec to the terms and condtions set forth in the stendard form of the U.S. Govemment Bill $e^{\prime}$ Lading and to any aveulablo specal rates or cherges (41 CFR 109-6.50 and 41 CFR 40.3)

$$
\text { X) YES To }
$$

The acdition on we fece hered and to the tarma and condruons are here by notw: Camer ETON / ENVIRO TRANSPORT Ö NV 
STRAIGHT BIIL OE IADING - SHORT FORM - ORIGINAL - NOE Negotiable
Shipper: BECHTEL NEVADA FOR US DOE CAU 461 TAML SITEITTR

TONOPAH NV 89049 -

Purchased : .

i Cuatomer Ordor No.

Shipper No. NTS0736j3

Date: of $\longdiv { 2 5 1 8 i }$

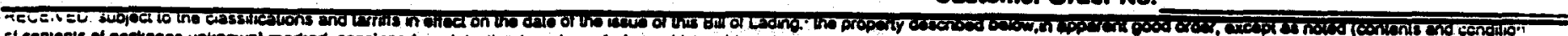

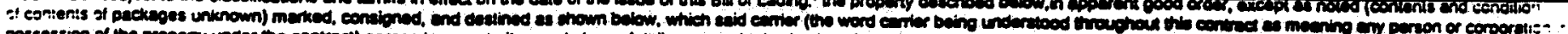

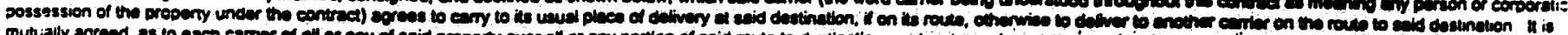

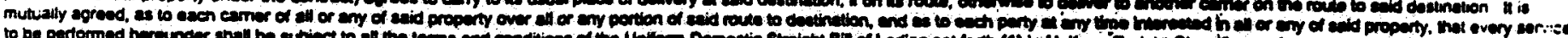

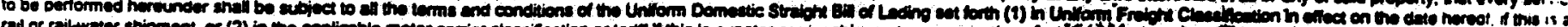

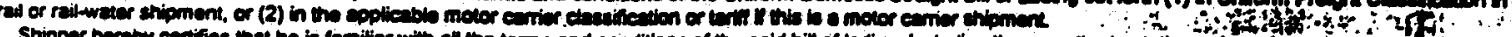

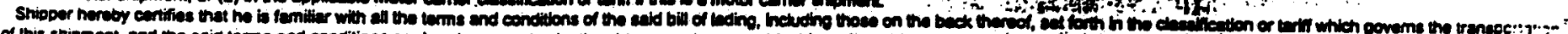

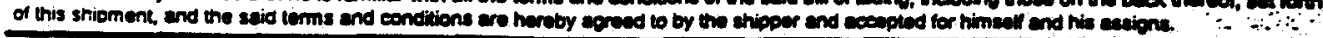

Consignee

BECHTEL NEVADA FOR USDOE

A-3 RWMS. ZONE 2

NEVADA TEST SITE

MERCURY NV 89023-

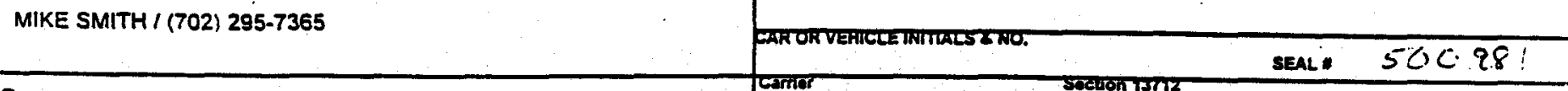

Route:

Soction tsth:
No.
Nonder, No.:

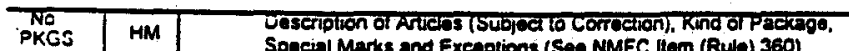

* $\mathrm{C} \cup: 030$ * : * *

1 DT SOIL-RADIOACTIVELY CONTAMINATED NOT-RGULATED PER 49 CFR 173.403

VEHICLE \#

DRIVER NAME Darry minton

SHIPMENT \# DPL99037.

Total Pieces

TOTALS:

\begin{tabular}{l|l} 
Kgs & $56 / 40$ \\
\hline
\end{tabular}

\begin{tabular}{|c|c|c|c|c|}
\hline $\begin{array}{l}\text { Worghin(subjoa } \\
\text { to Cornation) }\end{array}$ & Clases & Rme & Chergos: & \multirow{3}{*}{ 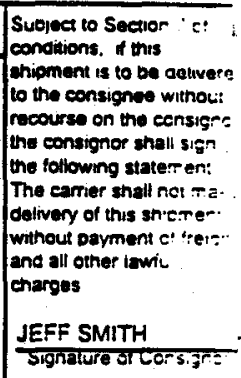 } \\
\hline \multirow[t]{6}{*}{$\begin{array}{r}56140 \\
465\end{array}$} & & \multirow[t]{6}{*}{. } & \multirow{6}{*}{. } & \\
\hline & & & & \\
\hline & & & & 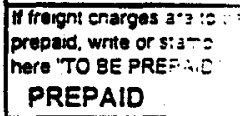 \\
\hline & & & & 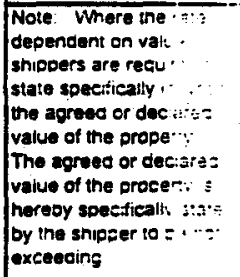 \\
\hline & & & & $\begin{array}{l}\text { Jeb orcer, rererer } 5 \\
\text { aceount, or wonk } \mathrm{c:}: 3^{-} \\
\text {humber }\end{array}$ \\
\hline & & & & C7P05G:3 \\
\hline
\end{tabular}

'N THE EVENT OF ANEMERGENCY SPONEN

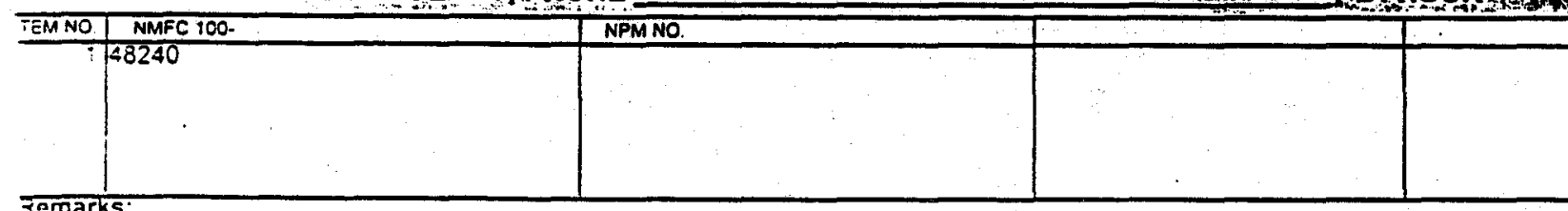

रemarks:

TECHNICAL CONTACT

This is to certify that the above named matenats are property ctassifiec. described. packaged, marked. and labeled, and are in proper condition for Iransoortation secording to the applicable regulations of the Department of Transportation. (Applicable for Hazardous Matenats Only.)

inidoet gECHTEL NEVADA, P O. BOX 98521. LAS VEGAS, NV 89193 a.ting incer contraet OC ACOB $96 \mathrm{NV} 11718$

LeP ALAN R MARCAAND

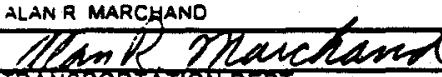

TRANSPORTATION DEPT. - Pemanent Post OHice Addross of Shipeer wth U.S. Dept. of Energy Cate: Q4/08/1999
This shipment is for U.S. Department of Energy ard the activet total transportation cherges paid to the carner(s) by the consignor or consignes ere aseipnable to, end shall be rembursed by. the U S Goverrm: . and is subject to the lerms and condtions set forth in the standard form of the U.S. Government Bitl of Lacing and to any available spectal ples or cherges (4t CFR $109-40.50$ and 41 CFR 403 )

$$
\text { X! YES : NO }
$$

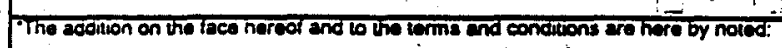

Carrier. ETON / ENVIRO TRANSPORT OF NV
Date $6 / 25 i$ 
STRAIGHT BIIL OF IADING - SHORT FORM - ORIGINAI - NOt-NegOtiable

hipper: BECHTEL NEVADA FOR US DOE

CAU 461 TAML SITE TTR

TONOPAH NV 89049 .

$\therefore \quad: \quad-$

Purehasel

Customer Ordor No.
Shippor No. NTS073636

Dato: $0672519 ;$

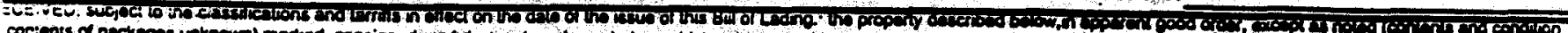

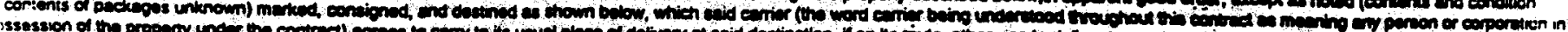

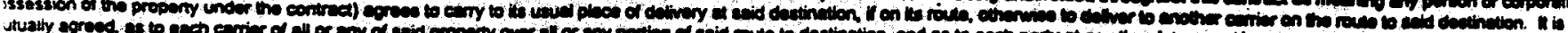

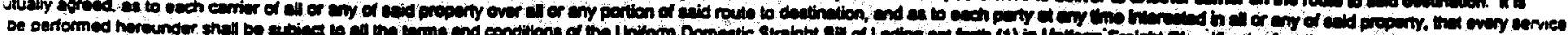

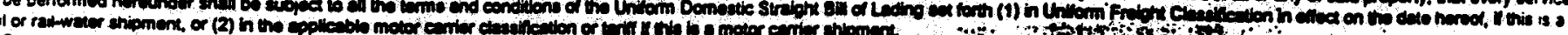

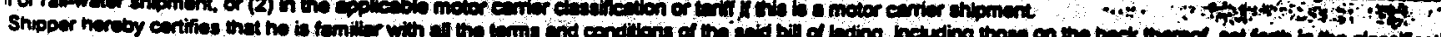

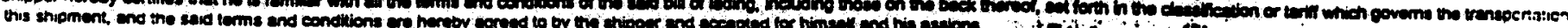
Onsignee

BECHTEL NEVADA FOR USDOE

A.3 RWMS, ZONE 2

NEVADA TEST SITE

H:ERCURY NV 29023-

MIKE SMITH / (702) 295-7365

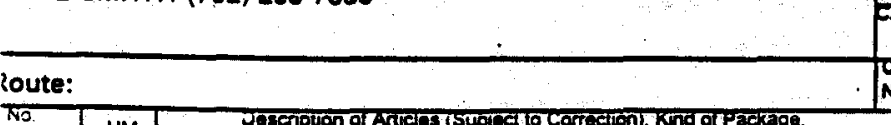

$$
\begin{aligned}
& \text { PAARFIER ETON/ENVIRO TRMNSPORT OF MV. }
\end{aligned}
$$

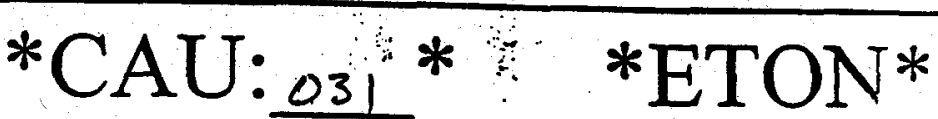

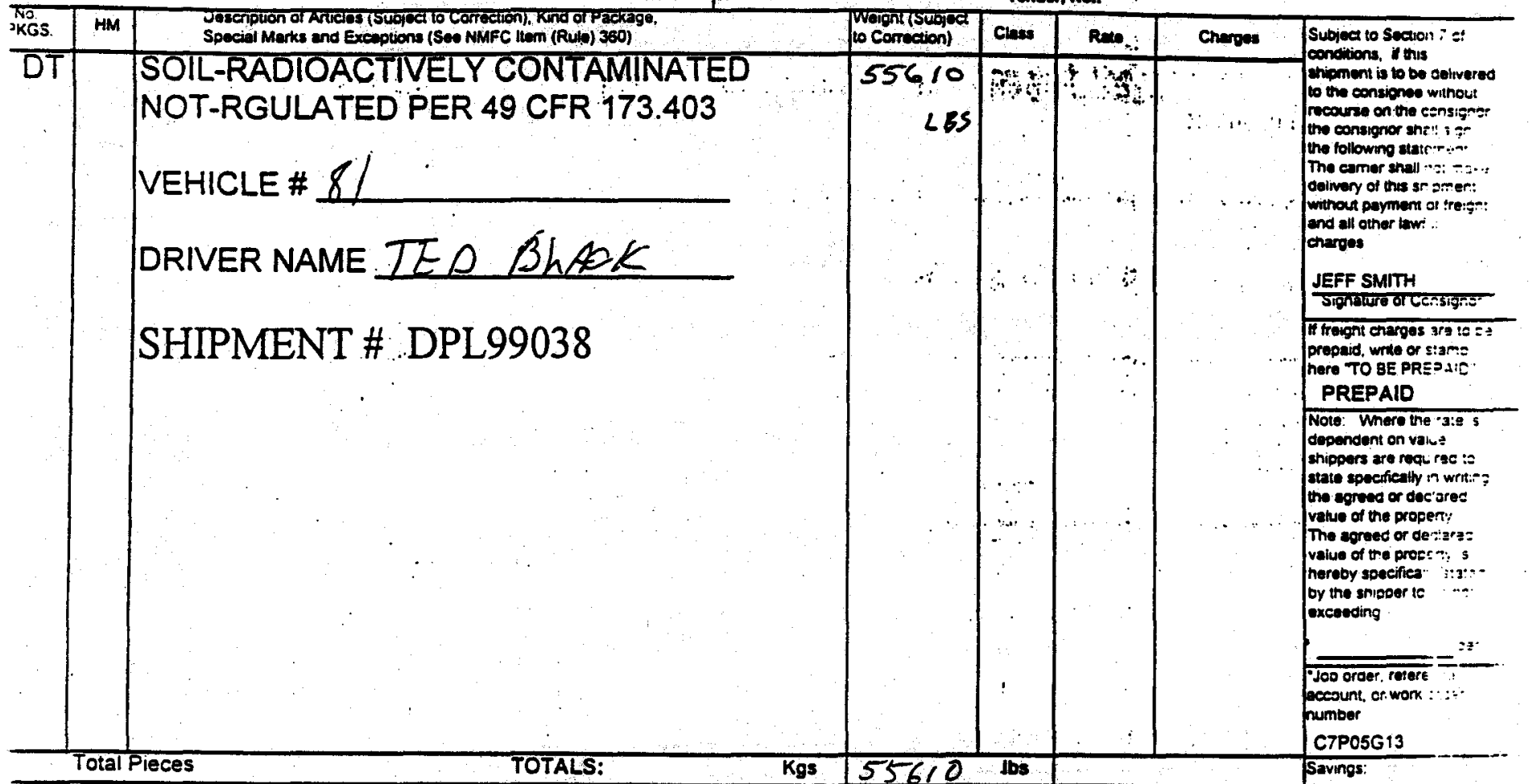

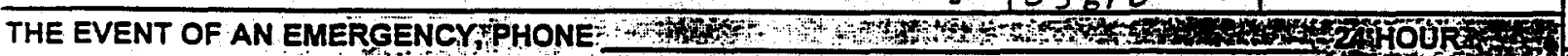

\begin{tabular}{|l|l|l|l|l|l|}
\hline UNO & NMFC $: C C-$ & NPM NO & & \\
\hline i 13240 & & & & \\
\hline
\end{tabular}

\section{$s$}

(aDei(s) acoinod

\section{SHNICAL CONTACT}

is is to centity that the above named matonals are property classifiad. escribed. packaged. marked. and laboled, and are in proper condition to arssen ation according to the applicatis reoulations of the Department of - Insportation. (Applicadie for Hazardous Matarials Only.)

Doer: 8ECHTEL NEVADA: P.O. 8OX 98521. LAS VEGAS, NV 89193 :rrg under contract DC ACOQ S6NV11718

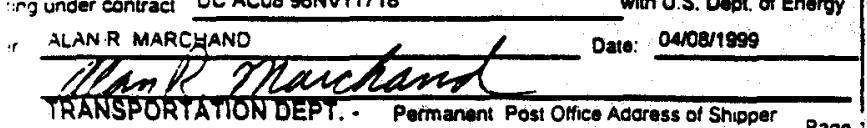

with U.S. Dept. of Energy

TRANSPORIATIONDEPT. - Permanent Post Olfice Adaress of Shipper
This shipment is for U.S. Department of Enevey end the ectud lotul treneportetion enarges pard to th."

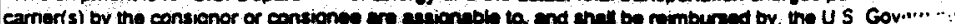

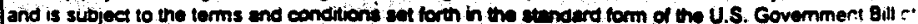
Lading and to any aveitabia speciat rates or cherpes (41 CFR 10940.50 and 41 CFR 40.3)

$$
\text { X) Yes } \Gamma_{i} \text { no }
$$

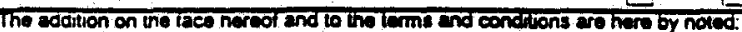
Camer. ETON I ENVIRO TRANSPORT OF NY 
STRAIGHT BIII OF IIADING - SHORT FORM - ORIGINAI - NOE Niegotiable
Shipper: BECHTEL NEVADA FOR US DOE CAU $\triangle 61$ TAML SITE TTR TONOPAH NV 89049

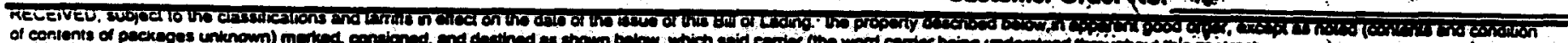

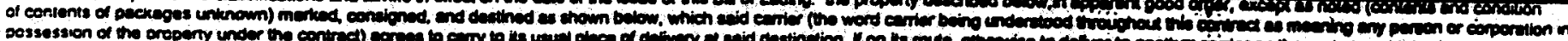

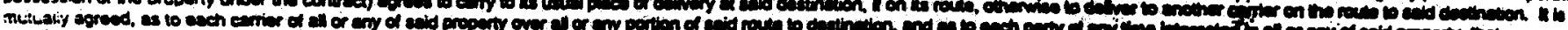

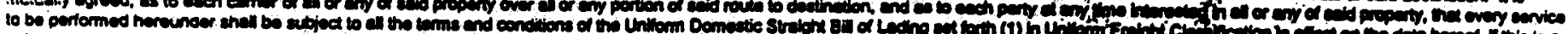

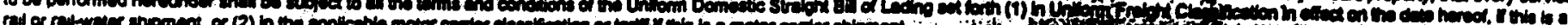

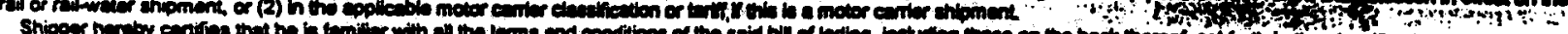

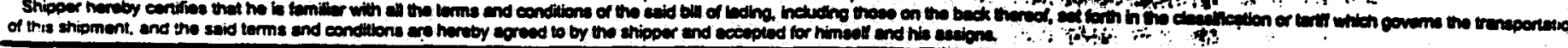

Consignee
BECHTEL NEVADA FOR USDOE

A-3 RWMS. ZONE 2

NEVADA TEST SITE

MERCURY NV 89023-

MIKE SMITH / (702) 295-7365

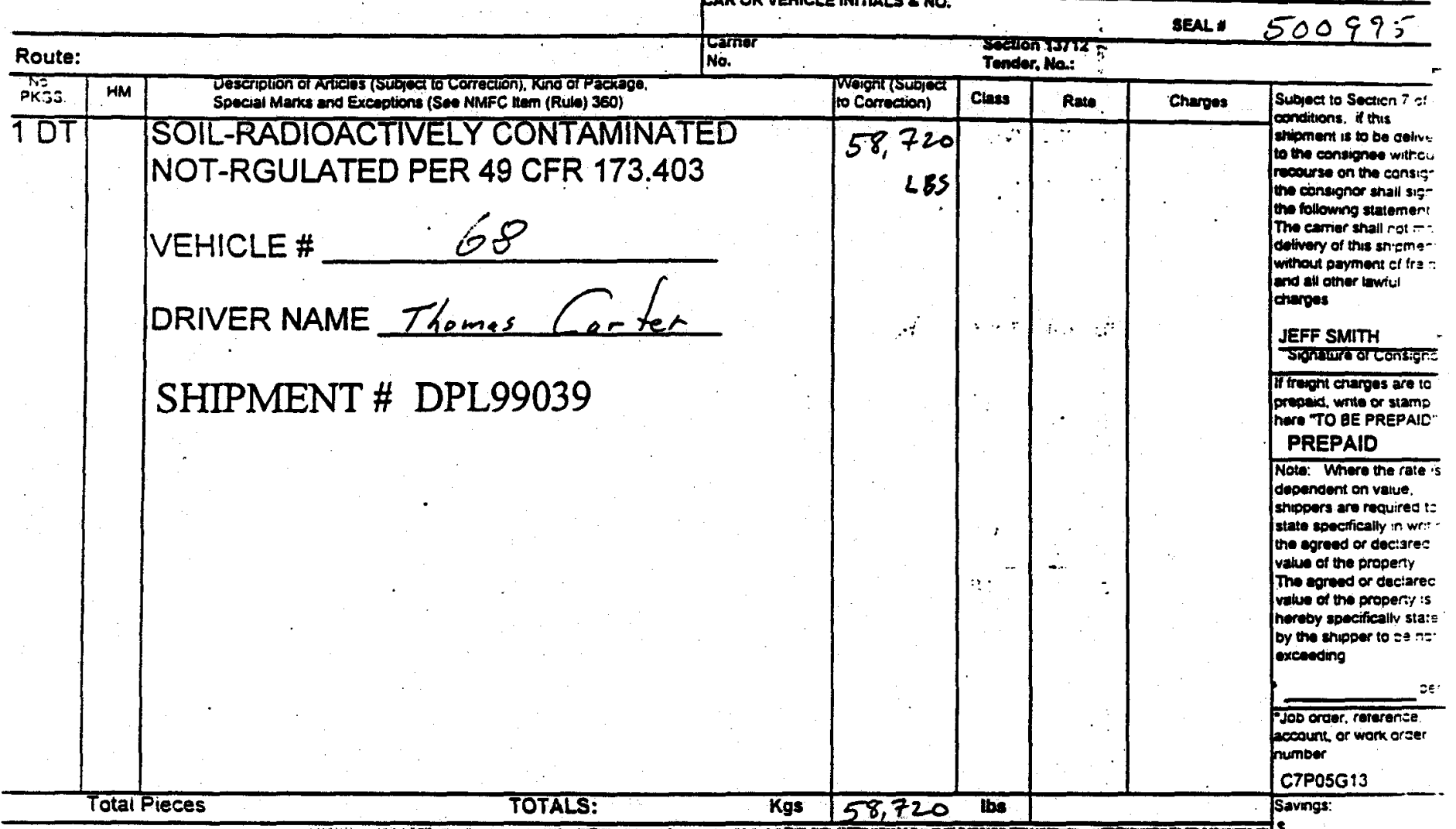

IN THE EVENT OF AN EMERCENGY

\begin{tabular}{|l|l|l|l|l|l|}
\hline TEEI NO. & NMFC 100. & NPM NO & & \\
\hline 1 & 48240 & & & & \\
& & & & & \\
\hline
\end{tabular}

TECHNICAL CONTACT

This is to centify that the above named matenals are property clasulied. Jescribed, paekaged. marked. and labuled, and are in proper condition for iransoonation accoroing to the appticable repulations of the Department of Transcortation. (Acplicabie tor Hazardous Matenals Only.)

in:DeE BECHTEL NEVADA, P.O. BOX 98521. LAS VEGAS, NV 89193 deting inder contract DC AC0896NV1171B Per HLAN R. MARCMAND $\ln$ ACO8 96NV1171B
with U.S. Dept. of Energy TRANSPORTATIONDEPT." Pemenont Post Otice Acaress of Shipper "Pane of

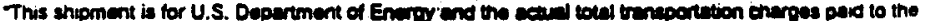

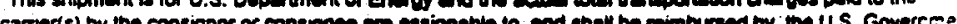
and is suoject to the terms and condtions ent forth in the rundard form of the U.S. Covernment Bill of Lading and to any available special mtes or cherpes (41 CFR'108-40.50 end 41 CFR 40.3)

$$
\text { X] YES TO }
$$

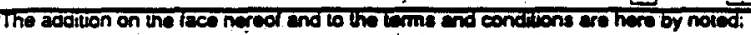

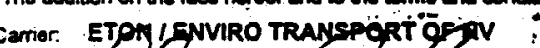


MOAFCHTEL NEVADA FOR US DOE CAU 461 TAML SITEI TTR

TONOPAH NV 89049 .

- SHORT EORM - ORIGINAY - Not Negotiable

\section{TONOPAH NV 89049 \\ Cuntomor Ordor No.}

Purchased

Shipper No. NTS07363:

Date: $6-25-99$

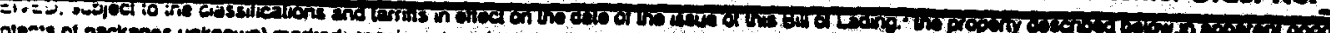

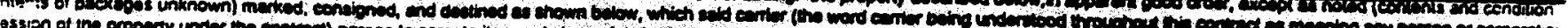

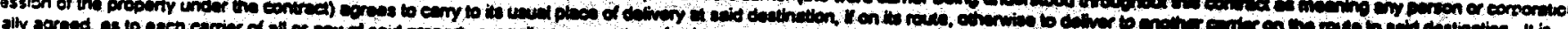

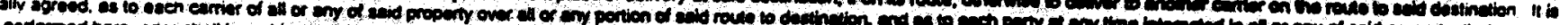

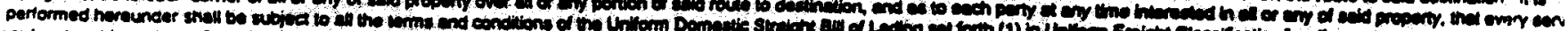

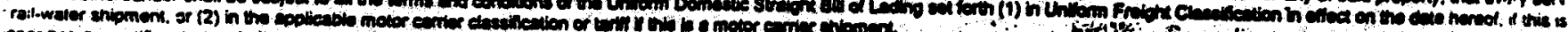

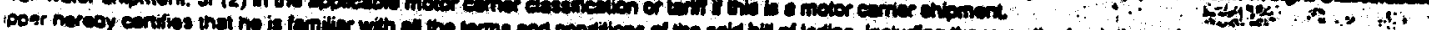

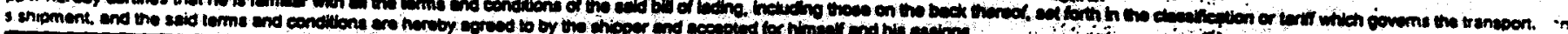
signee

¿HTEL NEVADA FOR USDOE

3 RWMS, ZONE 2

:VADA TEST SITE

IRCURY NV 89023.

KE SMITH / (702) 295-7365

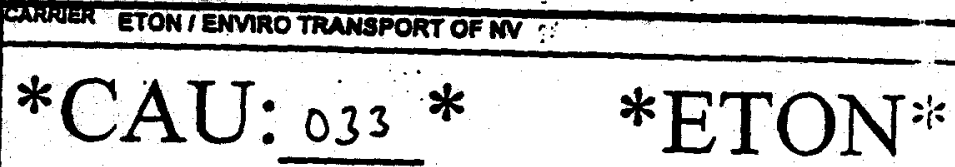

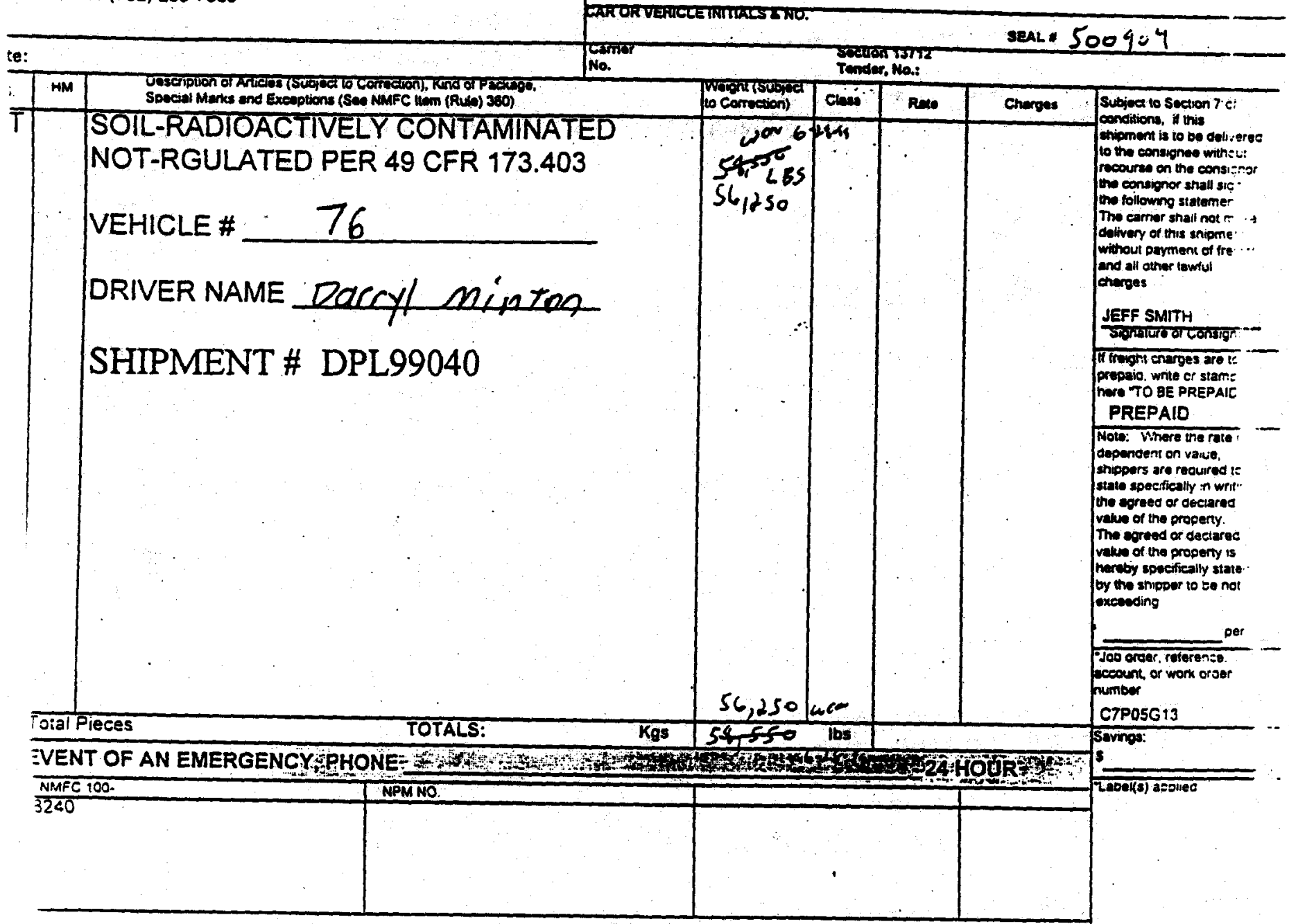

:ONTACT.

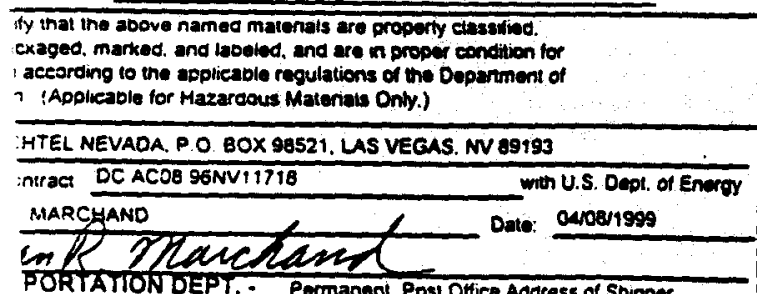

PORTATIONDLPT.. Permanent Post Office Acoress of Shipoer Per

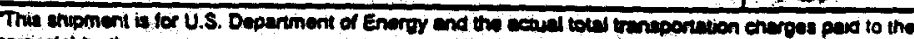

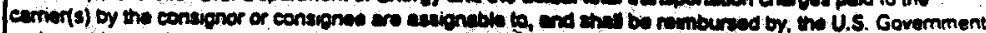

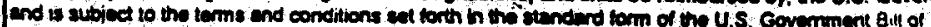
Leding and to eny aveilablis special rates or charges (41 CFR $109-40.50$ end 41 CFR 40.3)

$$
\text { XIVES - NO }
$$

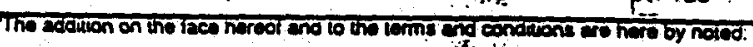

Canier. ETON/ENVRO TRANSPORT OF NV 
STRAIGHT BILL OF IADING - SHORT FORM - ORIGINAI - NOE NegOtiabIe

Shipper: BECHTEL NEVADA FOR US DOE CAU $\triangle 61$ TAML SITE/TTR TONOPAH NV 89049 -

Purchased

Customer Ordar No.
Shipper No. NTS073636

Dat: $46-28-49$

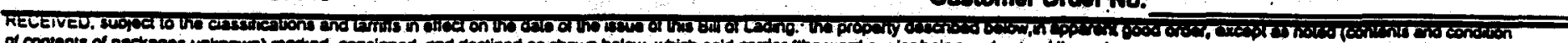

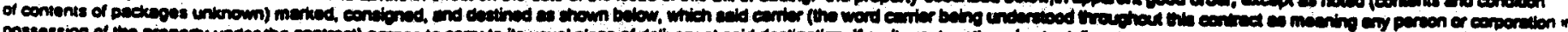

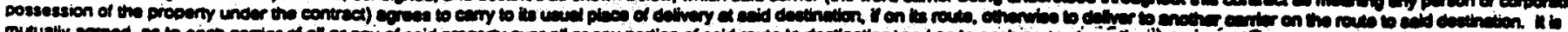

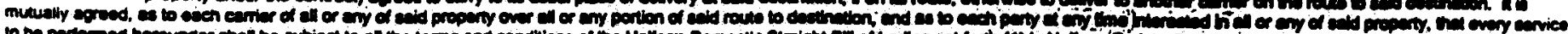

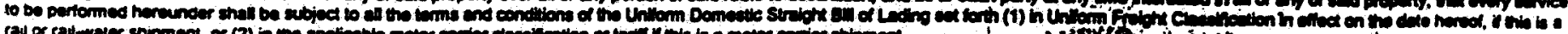

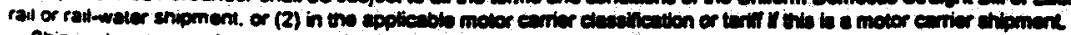

Shipoer hare eanties

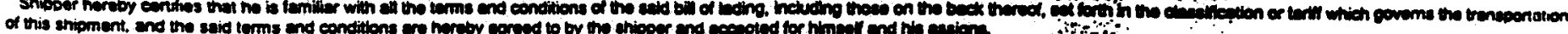

Consignee

BECHTEL NEVADA FOR USDOE

A.3 RWMS, ZONE 2

NEVADA TEST SITE

MERCURY NV 89023.

MIKE SMITH / (702) 295-7365

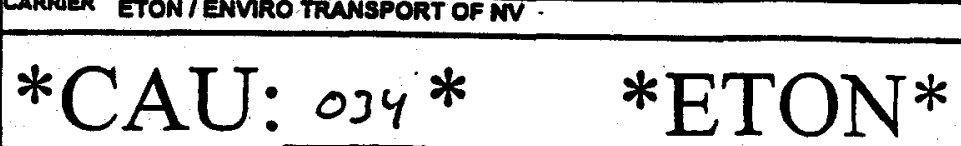

Route:

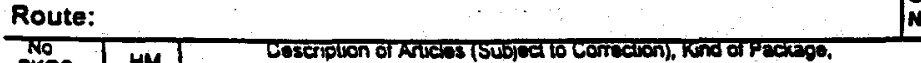

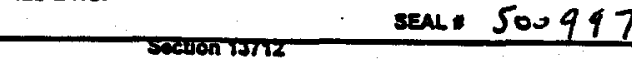

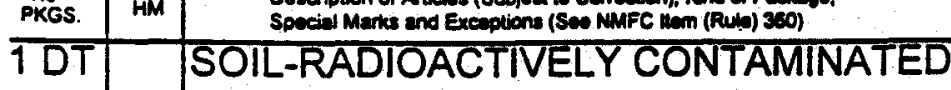

NOT-RGULATED PER 49 CFR 173.403

VEHICLE \# $8 l$

DRIVER NAME TEO BLACK

SHIPMENT \# DPL99041

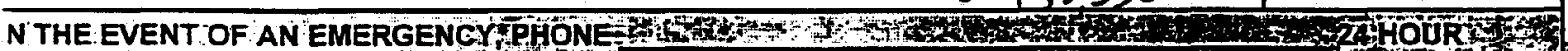

\begin{tabular}{|l|l|l|l|l|}
\hline EM NO & NMFC 100. & NPM NO & \\
\hline 1 & 48240 & & & \\
& & & & \\
\hline
\end{tabular}

TECHNICAL CONTACT

This is to cenify that the above named matenate are property ciassified. zese: ibed. packaged. marked. and labeled, end are in proper condition for transportation according to the applicabie reoulations of thi Department of Transportation. (Applicable for Hazardous Matenats Onty.)

Shipder: BECHTEL NEVADA. P.O. BOX 98521, LAS VEGAS, NV 89193 icting under contrae DC ACO8 96NV11718 with U.S. Dept. of Energy Per ALAN R. MARCHAND

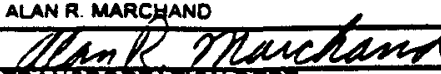
TRANSPOORTATION DEPT. - Permanent Post OHtiee Adaress of Shupper Oate: ovos/1989 in

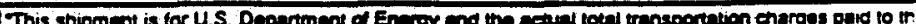

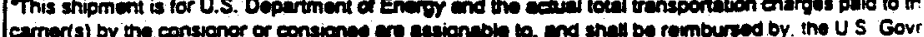
ander(s) by the consignor or consignes ene elaioneble b, and shall be rembured by. the US Govr Lading and to any available spectal revos or cherees (41 CFR 109-40.50 and 41 CFR 40.3)

$$
\text { X] YES Ti No }
$$

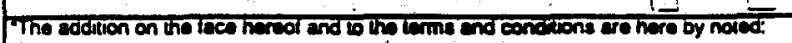

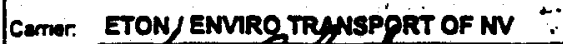

Subiect to Section :

conditions. this

shipment is to be ootivered

the consignee withou:

the consignor athall sign

in following tatement:

The carrar thall net make

without poyment et trogert

and all ather bertul

JEFF SMITH

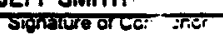

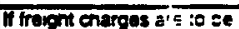

prepaid, wate or si:ro

hor TO 0 PREF

dependent en vall

shippers are requ' ': :

the ageed or decares

value of the prope-

The eareed or des: :-a

value of the orope - . I

hareby opacifiealy itise

exceecting

Job oroer, referer:

wecount, or work c:'?:?

Savings:

(1) 
-..... SLL OE IADING - SEORT FORM - ORIGINTI - NOE

ihipper: BECHTEL NEVADA FOR US DOE CAU 461 TAML STIETTR

TONOPAH NV 89049

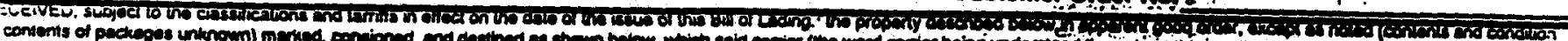

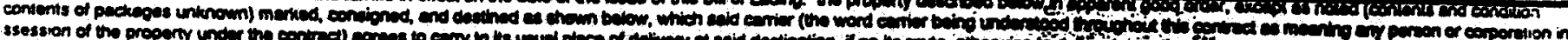

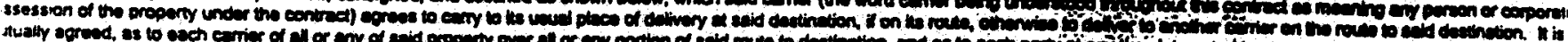

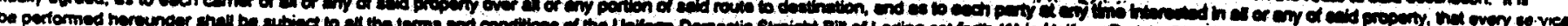

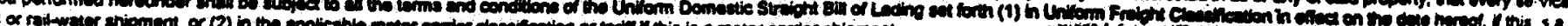

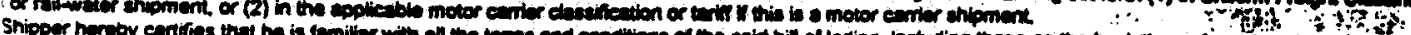

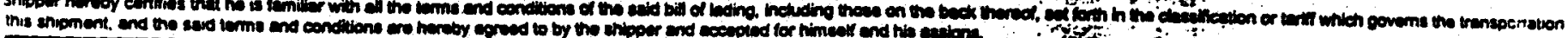
Insignee

3ECHTEL NEVADA FOR USDOE

1-3 RWMS. ZONE 2

VEVADA TEST SITE

MERCURY NV 89023.

AIKE SMITH / (702) 295-7365

WAR2NER ETONI ENVRO TRASPORT OF NV

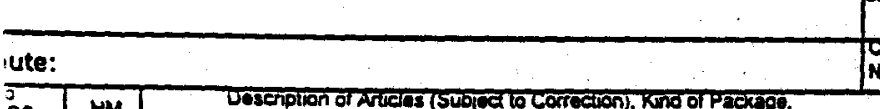

$$
\text { *CAUU: } \frac{035}{*}
$$

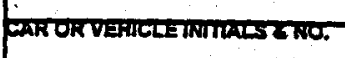
*ETON*
$i_{n} \rightarrow i-i 1$
i. "N

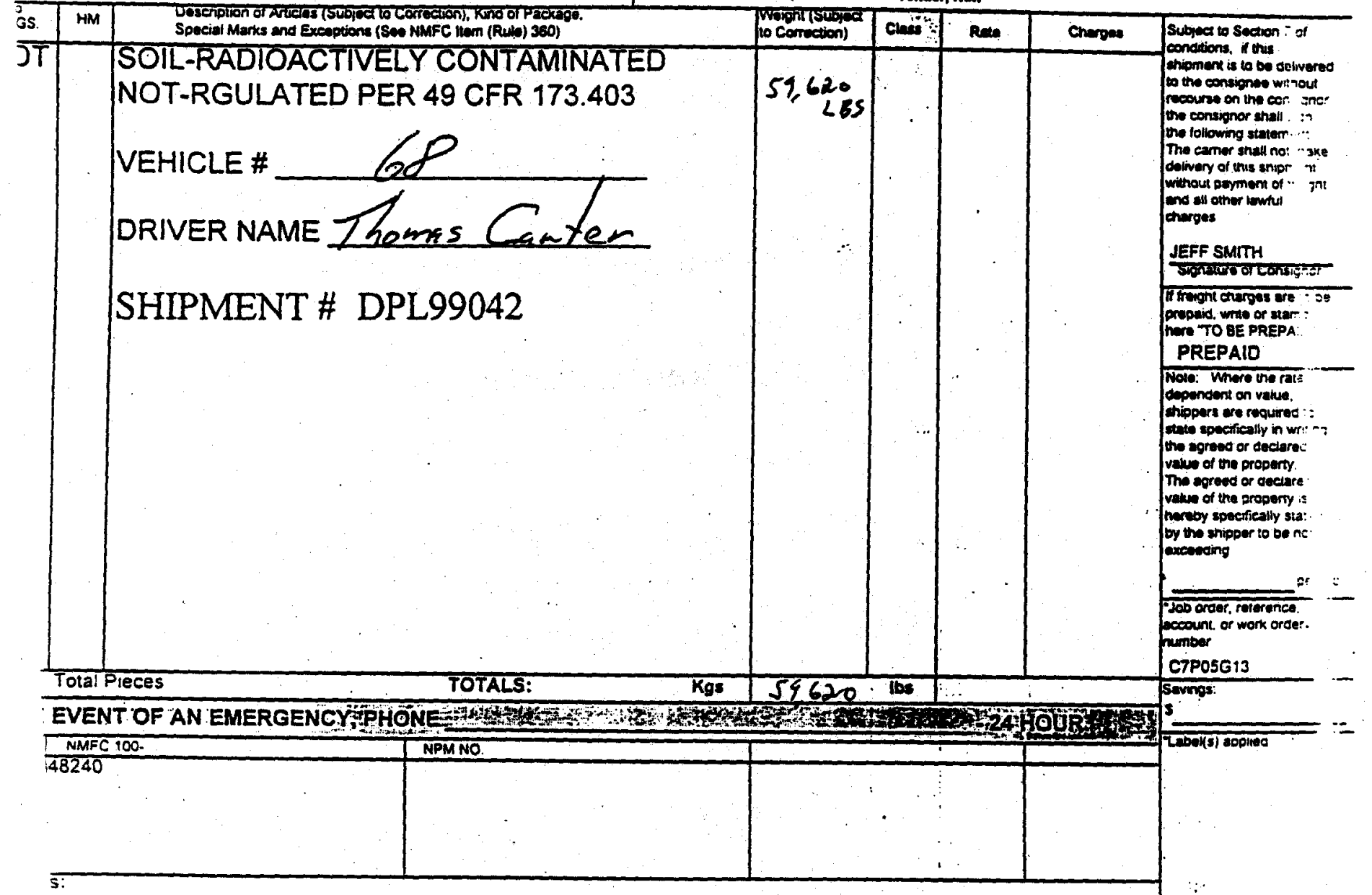

CONTACT. saekaged. manked, and labeled, and are in preper condition for on aceording to the applicable regulations of the Deparment of ich. (Apolicable for Hazardous Matonals Only.)

:CHTEL NEVADA, P O . 8OX 98521. LAS VEGAS. NV 89193 eontract OCACC8 9oNV11718

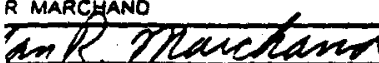
with U.S. Dept. of Energy

SPORTATIONDEPT. - Permanent Post Ofice Address of Snipper

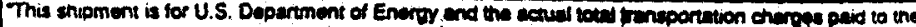

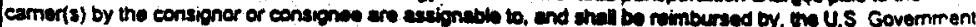
ano is subject to the terms and conditions set forth in the standerd form of the U.S. Government Bill of Lading and to any avalable special rates or cheres (41 CFR 109-40.50 and 41 CFR 40.3)

Q] Yes 7 No

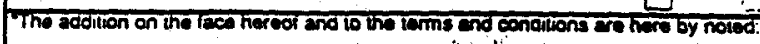

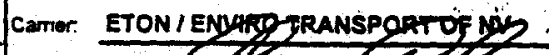
Per 
THIS PAGE INTENTIONALLY LEFT BLANK 


\section{APPENDIX H}

\section{NDEP COMMENT RESPONSE}


THIS PAGE INTENTIONALLY LEFT BLANK 


\section{DOCUMENT REVIEW SHEET}

\begin{tabular}{|c|c|c|c|c|}
\hline \multicolumn{5}{|c|}{$\begin{array}{l}\text { Document Title/Number: Draft SAFER Closure Report, Corrective Acti } \\
\text { Document Date: August } 1999 \\
\text { Revision Number: 0 } \\
\text { Originator: Jeff Smith, BN (295-7775) } \\
\text { Date Comments Due: September 13, } 1999 \\
\text { Reviewer/Organization: Michael McKinnion, NDEP }\end{array}$} \\
\hline $\begin{array}{l}\text { Comment } \\
\text { Number/ } \\
\text { Location }\end{array}$ & Type $^{a}$ & Comment & Comment Response & Accept \\
\hline $\begin{array}{l}\text { 1) Pg. 12, } \\
\text { Table 1, } \\
\text { 7th row, } \\
\text { 2nd column }\end{array}$ & м & $\begin{array}{l}\text { "...DU not an airborne hazard..." DU can be an airborne hazard } \\
\text { depending on several factors. e.g., amount of oxidation present, } \\
\text { wind velocity, distance to receptor, etc. Since these factors were } \\
\text { not addressed in the Record of Technical Change, this statement } \\
\text { may not be correct. NDEP requires additional justification on this } \\
\text { point prior to approving the final document. }\end{array}$ & $\begin{array}{l}\text { Extensive air monitoring was conducted during the field } \\
\text { activities. A continuous air monitor (CAM) and breathing } \\
\text { zone air (BZA) samplers were running during field operations. } \\
\text { The filters from these monitoring devices were counted on a } \\
\text { daily basis. No elevated radiological readings were detected } \\
\text { on any filter. In addition, nasal swipes were collected from } \\
\text { the field personnel and these also had no elevated readings. } \\
\text { The soil pile was checked on a daily basis during field } \\
\text { activities and on a weekly basis when the field work was shut } \\
\text { down. The crusted soil was never observed to have soil } \\
\text { particles entrained by the wind. } \\
\text { The Table } 1 \text { of the report has been modified to read as } \\
\text { follows: DU was determined not to be an airborne hazard } \\
\text { based on the comtimuous air monitoring filter data collected } \\
\text { during the exravation activities. In addition, no elevated DU } \\
\text { levels were detected in any on-site persommel's BZA filter or } \\
\text { nasal swipe. }\end{array}$ & Yes \\
\hline
\end{tabular}
a. Comment Types: M=Mandatory S=Suggested
Page 1 of 1 
THIS PAGE INTENTIONALLY LEFT BLANK 


\section{DISTRIBUTION LIST}


THIS PAGE INTENTIONALLY LEFT BLANK 


\section{DISTRIBUTION LIST}

Paul Liebendorfer, P.E., Chief

2 (Controlled)

Department of Conservation and Natural Resources

Division of Environmental Protection

333 W. Nye Lane, Room 138

Carson City, NV 89706-0851

Office Administrator

1 (Controlled)

Bureau of Federal Facilities

Division of Environmental Protection

555E. Washington, Suite 4300

Las Vegas, NV 89101

\section{U.S. Department of Energy, Nevada Operations Office}

Environmental Restoration Division

P. O. Box 98518

Las Vegas, NV 89193-8518

J. L. Appenzeller-Wing

1 (Uncontrolled)

K. J. Cabble

1 (Uncontrolled)

S. Lawrence

1 (Controlled)

Public Reading Facility

1 (Controlled)

P.O. Box 98521, M/S NLV040

Las Vegas, NV 89193-8521

Technical Information Resource Center

1 (Uncontrolled)

P.O. Box 98518, M/S 505

Las Vegas, NV 89193-8518

\section{U.S. Department of Energy,}

175 Oak Ridge Turnpike

P. O. Box 62

Oak Ridge, TN 37831-0062 


\section{Bechtel Nevada}

P. O. Box 98521

Las Vegas, NV 89193-8521

Correspondence Control

1 (Uncontrolled)

E.M. Correspondence

1 (Uncontrolled)

D. K. Cowser

1 (Uncontrolled)

W. F. Johnson

1 (Uncontrolled)

S. J. Nacht

1 (Uncontrolled)

W. C. Nicosia

1 (Uncontrolled)

J. L. Smith

1 (Uncontrolled)

A. M. Heidema

1 (Uncontrolled)

R. S. Remington

1 (Uncontrolled)

\section{IT Corporation}

P.O. Box 93838

Las Vegas, NV 89183-8521

P. Gretsky 